Supporting Online Material for

\title{
Selective Halogenation of Pyridines Using Designed Phosphine Reagents
}

Jeffrey N. Levy, Juan V. Alegre-Requena, Renrong Liu, Robert S. Paton, and Andrew McNally*

Department of Chemistry, Colorado State University,

Fort Collins, Colorado 80523, United States.

*andy.mcnally@colostate.edu

Table of Contents

1. General Information $\quad$ S2

2. Optimization Studies $\quad$ S4

$\begin{array}{ll}\text { 3. Problematic Substrates } & \text { S7 }\end{array}$

4. Preparation of Heterocyclic Phosphines $\quad$ S8

5. Preparation of Heterocyclic Phosphonium Salt Precursors $\quad$ S9

$\begin{array}{lr}\text { 6. Preparation of Heterocyclic Phosphonium Salts } & \text { S13 }\end{array}$

$\begin{array}{lr}\text { 7. Preparation of Chlorinated Heterocycles } & \text { S39 }\end{array}$

8. Preparation of Brominated Heterocycles $\quad$ S57

9. Preparation of Iodinated Heterocycles

10. Computation Studies $\quad$ S66

$\begin{array}{ll}\text { 11. References } & \text { S87 }\end{array}$

12. NMR Data $\quad$ S89 


\section{General Information}

Proton nuclear magnetic resonance $\left({ }^{1} \mathrm{H}\right.$ NMR) spectra were recorded at ambient temperature on either a Bruker Ultrashield-400 (400 MHz) spectrometer, a Varian $400 \mathrm{MR}(400 \mathrm{MHz})$ spectrometer or an Agilent Inova $400(400 \mathrm{MHz})$ spectrometer. Chemical shifts $(\delta)$ are reported in ppm and quoted to the nearest $0.01 \mathrm{ppm}$ relative to the residual protons in $\mathrm{CDCl}_{3}(7.26 \mathrm{ppm})$, $\mathrm{C}_{6} \mathrm{D}_{6}(7.16 \mathrm{ppm}),\left(\mathrm{CD}_{3}\right)_{2} \mathrm{SO}(2.50 \mathrm{ppm}), \mathrm{CD}_{3} \mathrm{OD}(3.31 \mathrm{ppm})$ or $\mathrm{CD}_{3} \mathrm{CN}$ (1.94 ppm) and coupling constants $(J)$ are quoted in Hertz $(\mathrm{Hz})$. Data are reported as follows: Chemical shift (number of protons, multiplicity, coupling constants). Coupling constants were quoted to the nearest $0.1 \mathrm{~Hz}$ and multiplicity reported according to the following convention: $\mathrm{s}=$ singlet, $\mathrm{d}=$ doublet, $\mathrm{t}=$ triplet, $\mathrm{q}=$ quartet, $\mathrm{qn}=$ quintet, sext $=$ sextet, $\mathrm{sp}=$ septet, $\mathrm{m}=$ multiplet, $\mathrm{br}=$ broad. Where coincident coupling constants have been observed, the apparent (app) multiplicity of the proton resonance has been reported. Carbon nuclear magnetic resonance $\left({ }^{13} \mathrm{C} N M R\right)$ spectra were recorded at ambient temperature on either a Bruker Ultrashield-400 (400 MHz) spectrometer, a Varian $400 \mathrm{MR}$ spectrometer (100 MHz) or an Agilent Inova $400(100 \mathrm{MHz})$ spectrometer. Chemical shift $(\delta)$ was measured in ppm and quoted to the nearest $0.1 \mathrm{ppm}$ relative to the residual solvent peaks in $\mathrm{CDCl}_{3}(77.16 \mathrm{ppm}), \mathrm{C}_{6} \mathrm{D}_{6}(128.06 \mathrm{ppm}),\left(\mathrm{CD}_{3}\right)_{2} \mathrm{SO}$ (39.51 ppm), $\mathrm{CD}_{3} \mathrm{OD}(49.00 \mathrm{ppm})$ or $\mathrm{CD}_{3} \mathrm{CN}(1.32 \mathrm{ppm})$.

Low-resolution mass spectra (LRMS) were measured on an Agilent 6310 Quadrupole Mass Spectrometer. Infrared (IR) spectra were recorded on a Bruker Tensor 27 FT-IR spectrometer as either solids or neat films, either through direct application or deposited in $\mathrm{CHCl}_{3}$, with absorptions reported in wavenumbers $\left(\mathrm{cm}^{-1}\right)$.

Analytical thin layer chromatography (TLC) was performed using pre-coated Merck glass backed silica gel plates (Silicagel 60 F254). Flash column chromatography was undertaken on Fluka or Material Harvest silica gel (230-400 mesh) under a positive pressure of air. Visualization was achieved using ultraviolet light $(254 \mathrm{~nm})$ and chemical staining with ceric ammonium molybdate or basic potassium permanganate solutions as appropriate.

Tetrahydrofuran (THF), toluene, hexane, diethyl ether and dichloromethane were dried and distilled using standard methods. ${ }^{1}$ Ethyl acetate (EtOAc), 1,2-Dichloroethane (DCE), chloroform, and acetone were purchased anhydrous from Sigma Aldrich chemical company. All reagents were purchased at the highest commercial quality and used without further purification. Reactions were carried out under an atmosphere of nitrogen unless otherwise stated. All reactions were monitored by $\mathrm{TLC},{ }^{1} \mathrm{H}$ NMR spectra taken from reaction samples, gas chromatography (GC) and gas chromatography-mass spectrometry (GCMS) using an Agilent 5977A fitted with an Agilent J\&W HP-5ms Ultra Inert Column $(30 \mathrm{~m}, 0.25 \mathrm{~mm}, 0.25 \mu \mathrm{m}$ film) for MS analysis and an Agilent J\&W VF-5ms column ( $10 \mathrm{~m}, 0.15 \mathrm{~mm}, 0.15 \mu \mathrm{m}$ film) for FID analysis or liquid chromatography mass spectrometry (LCMS) using an Agilent 6310 Quadrupole Mass Spectrometer. Melting points $(\mathrm{mp})$ were recorded using a Büchi B-450 melting point apparatus and are reported uncorrected.

Lithium halide salts were dried under vacuum at $120^{\circ} \mathrm{C}$ before being stored in a glovebox. $\mathrm{HCl}$ (4.0 $\mathrm{M}$ in Dioxane) and trifluoromethanesulfonic acid (98\%) were purchased from Sigma Aldrich chemical company and used without further purification but were routinely stored in a $20 \quad{ }^{\circ} \mathrm{C}$ fridge. Anhydrous 1,4-Dioxane was purchased from EMD Millipore. 
Chlorodiphenylphosphine was purchased from Strem Chemicals and stored in a glovebox. Dichlorophenylphosphine was purchased from BeanTown Chemical and stored in a glovebox. $\mathrm{NEt}_{3}$ and DBU were distilled before use. 


\title{
2. Optimization Studies
}

Table S1: Optimization of General Reaction Conditions ${ }^{\mathrm{a}}$<smiles>FC(F)(F)c1ccc(P(c2ccc(C(F)(F)F)nc2)c2ccnc(-c3ccccc3)c2)nc1</smiles>

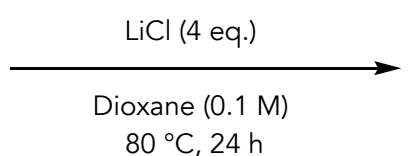<smiles>Pc1cc(Cl)ccn1</smiles>

$\begin{array}{ccc}\begin{array}{c}\text { Entry } \\ 1\end{array} & \text { Change from Standard Conditions } & \text { Yield } \\ 2 & \text { None } & 70 \\ 3 & 100^{\circ} \mathrm{C} & 68 \\ 4 & 60^{\circ} \mathrm{C} & 36 \\ 5 & \text { EtOAc } & 45 \\ 6 & \text { EtOAc, } 60^{\circ} \mathrm{C} & 12 \\ 7 & \text { Toluene } & 58 \\ 8 & \text { DME } & 38 \\ 9 & \text { DCE } & \text { n.d. } \\ 10 & \text { Dibutyl Ether } & \text { n.d. } \\ 11 & \text { EtOH } & \text { trace } \\ 12 & \text { DMF, } 60{ }^{\circ} \mathrm{C} & \text { n.d. } \\ 13 & 1: 1 \text { Dioxane:EtOAc } & 52 \\ 14 & 0.05 \mathrm{M} & 62 \\ 15 & 0.2 \mathrm{M} & 49 \\ 1 & 0.5 \mathrm{M} & 25\end{array}$

${ }^{a}$ Yields by GCMS using 1,3,5-Trimethoxybenzene as an internal standard. Reactions run on 0.05 mmol scale.

Table S2: Optimization of Chloride Source ${ }^{\mathrm{a}}$<smiles>FC(F)(F)c1ccc(P(c2ccc(C(F)(F)F)nc2)c2ccnc(-c3ccccc3)c2)nc1</smiles>

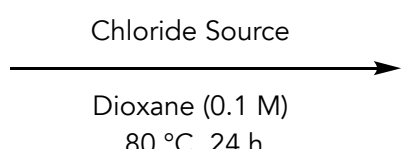<smiles>Pc1cc(Cl)ccn1</smiles>

Entry
1
2
3
4
5
6
7
8
9
10
11
12

\author{
Chloride Source \\ $\mathrm{LiCl}(4$ eq.) \\ $\mathrm{LiCl}$ (4 eq.) + 12-crown-4 (2 eq.) \\ $\mathrm{LiCl}$ (4 eq.) $+\mathrm{TBAC}$ ( 2 eq.) \\ $\mathrm{LiCl}(4$ eq. $)+4 \AA \mathrm{MS}$ \\ $\mathrm{LiCl}+\mathrm{H}_{2} \mathrm{O}$ (5 eq.) \\ $\mathrm{KCl}(4$ eq.) \\ $\mathrm{KCl}$ (4 eq.) +18 -crown-6 (2 eq.) \\ $\mathrm{NaCl}(4$ eq.) \\ $\mathrm{NaCl}$ (4 eq.) at $100{ }^{\circ} \mathrm{C}$ \\ $\mathrm{NaCl}$ (4 eq.) +15 -crown-5 (2 eq.) \\ $\mathrm{HCl}$ in Dioxane (1 eq.) \\ TBAC (4 eq.)
}



13
$\mathrm{CsCl}$
$\mathrm{AgCl}$
30
n.d.

${ }^{\text {ay }}$ ields determined by GCMS using 1,3,5-Trimethoxybenzene as an internal standard. Reactions run on $0.05 \mathrm{mmol}$ scale.

Table S3: Optimization of Bromination Conditions ${ }^{\mathrm{a}}$<smiles>FC(F)(F)c1ccc(P(c2ccc(C(F)(F)F)nc2)c2ccnc(-c3ccccc3)c2)nc1</smiles>
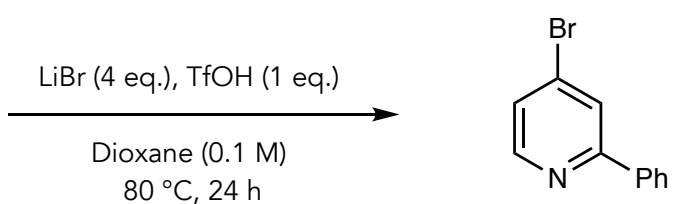

Entry
1
2
3
4
5

6
7
8
9
10

\begin{tabular}{|c|c|}
\hline Bromide Source & Yield \\
\hline $\operatorname{LiBr}(4$ eq.) & 21 \\
\hline $\mathrm{LiBr}(4$ eq. $)$ at $120^{\circ} \mathrm{C}$ & 45 \\
\hline $\operatorname{LiBr}(4$ eq. $)+12$-crown-4 (2 eq.) & 9 \\
\hline $\mathrm{LiBr}(4$ eq. $)+\mathrm{TfOH}(1$ eq. $)$ & 79 \\
\hline $\begin{array}{c}\mathrm{LiBr}(4 \text { eq. })+\mathrm{TfOH}(1 \text { eq. })+ \\
12 \text {-crown-4 ( } 2 \text { eq. })\end{array}$ & 54 \\
\hline $\mathrm{LiBr}(4$ eq. $)+\mathrm{AcOH}(1$ eq. $)$ & 13 \\
\hline $\operatorname{LiBr}(4$ eq. $)+\mathrm{TFA}(1$ eq. $)$ & 9 \\
\hline $\mathrm{KBr}(4$ eq. $)$ & 4 \\
\hline $\operatorname{KBr}(4$ eq. $)+18$-crown-6 (2 eq. $)$ & 23 \\
\hline TBAB & 8 \\
\hline
\end{tabular}

${ }^{\text {a }}$ Yields determined by ${ }^{1} \mathrm{H}$ NMR using 1,3,5-Trimethoxybenzene as an internal standard. Reactions run on $0.05 \mathrm{mmol}$ scale.

Table S4: Control Reactions on 2-phenylpyridine phosphonium salt ${ }^{\mathrm{a}, \mathrm{b}}$<smiles>[PbH2]c1ccnc(-c2ccccc2)c1</smiles>

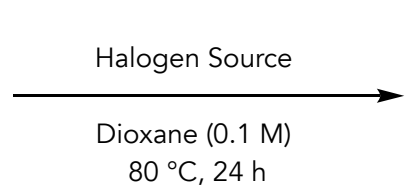<smiles>[AlH2]c1ccnc(P)c1</smiles>

$\begin{array}{cc}\text { Entry } & \text { Halogen Source } \\ 1 & \mathrm{LiCl} \text { (4 eq.) } \\ 2 & \mathrm{NaCl} \text { (4 eq.) } \\ 3 & \mathrm{KCl} \text { (4 eq.) } \\ 4 & \mathrm{TBAC} \text { (4 eq.) } \\ 5 & \mathrm{HCl} \text { (1 eq.) } \\ 7 & \mathrm{LiBr}(4 \text { eq.) } \\ 8^{\mathrm{b}} & \mathrm{LiI} \text { (4 eq.) }\end{array}$

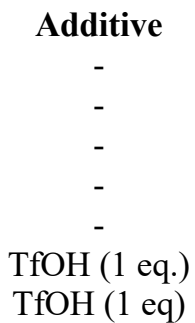

Yield
n.d.
n.d.
n.d.
1
$<1$
n.d.
n.d.

${ }^{\mathrm{a}} \mathrm{All}$ reactions run on $0.05 \mathrm{mmol}$ scale. Yields determined by ${ }^{1} \mathrm{H}$ NMR using 1,3,5trimethoxybenzene as an internal standard. ${ }^{\mathrm{b}}$ Iodination Ran at $120{ }^{\circ} \mathrm{C}$.

Table S5: Control Reactions on 3-phenylpyridine phosphonium salt ${ }^{\mathrm{a}, \mathrm{b}}$ 

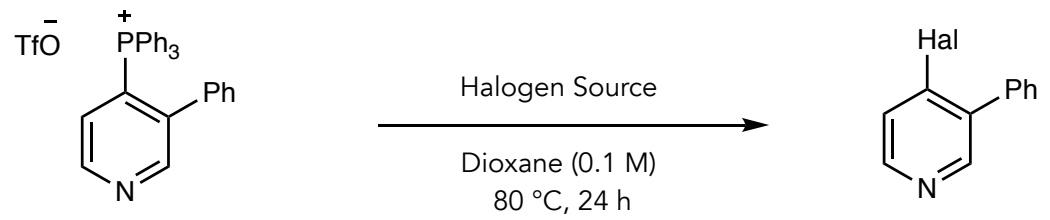

$\begin{array}{cc}\text { Entry } & \text { Halogen Source } \\ 1 & \mathrm{LiCl}(4 \text { eq.) } \\ 2 & \mathrm{NaCl}(4 \text { eq.) } \\ 3 & \mathrm{KCl}(4 \text { eq.) } \\ 4 & \mathrm{TBAC}(4 \text { eq.) } \\ 5 & \mathrm{HCl}(1 \text { eq. }) \\ 7 & \mathrm{LiBr}(4 \text { eq.) } \\ 8^{\mathrm{b}} & \mathrm{LiI}(4 \text { eq.) }\end{array}$

$\begin{array}{cc}\text { Additive } & \text { Yield } \\ - & 5 \\ - & 1 \\ - & \text { n.d. } \\ - & 7 \\ - & 39 \\ \text { TfOH (1 eq. }) & 2 \\ \text { TfOH (1 eq) } & \text { n.d. }\end{array}$

${ }^{\mathrm{a}} \mathrm{All}$ reactions run on $0.05 \mathrm{mmol}$ scale. Yields determined by ${ }^{1} \mathrm{H}$ NMR using 1,3,5trimethoxybenzene as an internal standard. ${ }^{b}$ Iodination Ran at $120^{\circ} \mathrm{C}$.

Table S6: One-pot reactions on 2-phenylpyridine ${ }^{\mathrm{a}}$
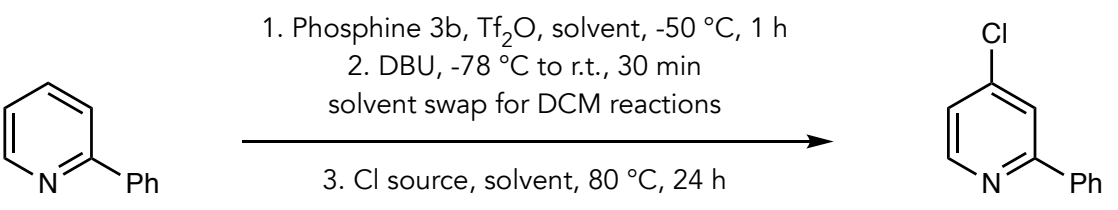

$\begin{array}{ccccc}\text { Entry } & \text { Halogen Source } & \begin{array}{c}\text { Solvent for Steps 1 } \\ \text { and 2 }\end{array} & \begin{array}{c}\text { Solvent for } \\ \text { Step 3 }\end{array} & \text { Yield } \\ 1^{\mathrm{b}} & \mathrm{LiCl}(4 \text { eq. }) & \mathrm{DCM} & \text { Dioxane } & 47 \\ 2^{\mathrm{b}} & \mathrm{LiCl}(4 \text { eq. } & \text { EtOAc } & \text { EtOAc } & 48 \\ 3^{\mathrm{b}} & \mathrm{HCl}(2 \text { eq.) } & \text { DCM } & \text { Dioxane } & 37 \\ 4^{\mathrm{HCl}} & \mathrm{HCq} \text { ) } & \text { EtOAc } & \text { EtOAc } & 21\end{array}$

${ }^{a}$ All reactions run on $0.1 \mathrm{mmol}$ scale. Yields determined by ${ }^{1} \mathrm{H}$ NMR using $1,3,5-$

trimethoxybenzene as an internal standard. ${ }^{b}$ Between steps 2 and 3, the reaction mixture was concentrated and Dioxane was added. 


\section{Problematic Substrates}

Major Problems: Pyridines with fluorines, multiple electron-withdrawing groups, electrondonating groups (alkoxy), and substrates with basic amines anywhere on the molecule.

Phosphonium Salt Formation is Succesful. Problems with Chlorination:<smiles>COc1cccnc1</smiles>

Dealkylation<smiles>c1cnc2ncsc2c1</smiles>

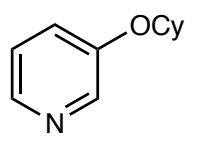

Salt Unreactive<smiles>Fc1cccnc1</smiles>

Degradation<smiles>COC(=O)c1cccnc1</smiles>

Decarboxylation<smiles>Cc1cccnc1C#N</smiles>

Salt Unreactive<smiles>Cc1cscc1-c1cccnc1F</smiles><smiles>FC(F)(F)c1ccncc1</smiles><smiles>N#Cc1c(Cl)cncc1Cl</smiles>

$$
\text { Degradation Dealkylation Salt Unreactive Salt Unreactive }
$$

Low Phosphonium Salt Formation:<smiles>COc1ccccn1</smiles><smiles>Fc1cnc(Br)c(F)c1</smiles><smiles>Fc1ccccn1</smiles><smiles>CN1CCC[C@H]1c1cccnc1</smiles><smiles>O=C1COc2cccnc2N1</smiles><smiles>Brc1cnc2ncccn12</smiles><smiles>COc1cc2ncnc(Cl)c2cc1OC</smiles><smiles>Clc1nccc2ccc(Br)cc12</smiles><smiles>CCOC(=O)c1ncc(Cl)cc1C</smiles>

$(\sim 20 \%)$<smiles>CCOC(=O)c1cccnc1Sc1ccc(Cl)cc1</smiles><smiles>ClC1=CCCC=N1</smiles><smiles>COc1ccccc1N1CCN(Cc2ccccn2)CC1</smiles>

$(\sim 15 \%)$ 


\section{Preparation of Heterocyclic Phosphines}

\section{5-(diphenylphosphaneyl)-2-(trifluoromethyl)pyridine (I)}<smiles>FC(F)(F)c1ccc(-c2ccccc2)cn1</smiles>

An oven-dried $250 \mathrm{~mL}$ round bottom flask was charged with 2-trifluoromethyl-5-bromo pyridine (4.97 g, $22.00 \mathrm{mmol})$ and diethyl ether $(80 \mathrm{~mL})$. The colorless solution was cooled to $-78{ }^{\circ} \mathrm{C}$, and $\mathrm{n}$-BuLi (1.6 M in Hexanes, $22.00 \mathrm{mmol} 13.75 \mathrm{~mL}$ ) was added dropwise. After 30 minutes, diphenylphosphine chloride $(3.56 \mathrm{~mL}, 20.00 \mathrm{mmol})$ was added dropwise, and the flask was allowed to warm to room temperature. After 4 hours of stirring, the reaction was quenched with water $(80 \mathrm{~mL})$. The organic layer was separated, and the aqueous layer was extracted with $\mathrm{CH}_{2} \mathrm{Cl}_{2}(3 \times 50 \mathrm{~mL})$. The combined organic extracts were dried $\left(\mathrm{MgSO}_{4}\right)$, filtered and concentrated in vacuo. The crude material was purified by flash chromatography (silica gel: 2.5\% EtOAc in Hexanes) to provide the title compound as a white solid (5.63 g, $17.01 \mathrm{mmol}$, $85 \%$ yield). $\operatorname{mp~} 67-70^{\circ} \mathrm{C}$. IR $v_{\max } / \mathrm{cm}^{-1}$ (film): 3058, 3013, 1479, 1433, 1331, 1142, 1073, 746, 695; ${ }^{1} \mathrm{H}$ NMR $\left(400 \mathrm{MHz}, \mathrm{CDCl}_{3}\right) \delta: 8.56(1 \mathrm{H}, \mathrm{s}), 7.72-7.66(1 \mathrm{H}, \mathrm{m}), 7.61(1 \mathrm{H}, \mathrm{d}, J=8.0 \mathrm{~Hz})$, 7.42-7.30 (10H, m); $\left.{ }^{13} \mathrm{C} \mathrm{NMR} \mathrm{(100} \mathrm{MHz,} \mathrm{CDCl}_{3}\right) \delta: 153.9(\mathrm{~d}, J=22.2 \mathrm{~Hz}), 147.8(\mathrm{q}, J=34.8$ $\mathrm{Hz}), 142.1(\mathrm{~d}, J=16.4 \mathrm{~Hz}), 138.7(\mathrm{~d}, J=20.1 \mathrm{~Hz}), 134.7(\mathrm{~d}, J=9.8 \mathrm{~Hz}), 134.1$ (d, $J=20.5 \mathrm{~Hz})$, 129.8, 129.2 (d, $J=7.2 \mathrm{~Hz}), 121.7$ (q, $J=274.0 \mathrm{~Hz}), 120.2-120.0(\mathrm{~m}) ;{ }^{19} \mathrm{~F}$ NMR (365 MHz, $\left.\mathrm{CDCl}_{3}\right) \delta:-68.07 ;{ }^{31} \mathrm{P}$ NMR $\left(162 \mathrm{MHz}, \mathrm{CDCl}_{3}\right) \delta:-11.63 ; \mathrm{m} / \mathrm{z}$ LRMS (ESI + APCI) found [M + $\mathrm{H}]^{+} 332.1, \mathrm{C}_{18} \mathrm{H}_{14} \mathrm{~F}_{3} \mathrm{NP}^{+}$requires 332.1.

\section{5,5'-(phenylphosphanediyl)bis(2-(trifluoromethyl)pyridine) (II)<smiles>FC(F)(F)c1ccc(P(c2ccccc2)c2ccc(C(F)(F)F)nc2)cn1</smiles>

An oven-dried $250 \mathrm{~mL}$ round bottom flask was charged with 2-trifluoromethyl-5-bromo pyridine (16.65 g, $73.68 \mathrm{mmol})$ and diethyl ether $(60 \mathrm{~mL})$. The colorless solution was cooled to $-78{ }^{\circ} \mathrm{C}$, n-BuLi (2.5 $\mathrm{M}$ in hexanes, $73.68 \mathrm{mmol}, 29.5 \mathrm{~mL}$ ) was added dropwise. After 30 minutes, dichlorophenyphosphine $(4.76 \mathrm{~mL}, 35.10 \mathrm{mmol})$ was added dropwise, and the flask was allowed to warm to room temperature. After 8 hours of stirring, the reaction was quenched with water $(80$ $\mathrm{mL})$, organic layer separated, and aqueous layer extracted with $\mathrm{CH}_{2} \mathrm{Cl}_{2}(3 \times 50 \mathrm{~mL})$. The combined organic extracts were dried $\left(\mathrm{MgSO}_{4}\right)$, filtered and concentrated in vacuo. The crude material was purified by flash chromatography (silica gel: 5\% EtOAc in Hexanes) to provide the title compound as a white solid (12.06 g, $30.10 \mathrm{mmol}, 86 \%$ yield). mp $65-66{ }^{\circ} \mathrm{C}$. IR $v_{\max } / \mathrm{cm}^{-1}$ (film): 3076, 1368, 1331, 1234, 1128, 1070, 834, 713; $\left.{ }^{1} \mathrm{H} \mathrm{NMR} \mathrm{(400} \mathrm{MHz,} \mathrm{CDCl}_{3}\right) \delta: 8.61(2 \mathrm{H}$, app t, $J=2.4 \mathrm{~Hz}), 7.75(2 \mathrm{H}$, ddd, $J=7.9,6.1,1.9 \mathrm{~Hz}), 7.68(2 \mathrm{H}, \mathrm{d}, J=8.0 \mathrm{~Hz}), 7.52-7.43(3 \mathrm{H}$, $\mathrm{m}), 7.40-7.33(2 \mathrm{H}, \mathrm{m}) ;{ }^{13} \mathrm{C} \mathrm{NMR}\left(100 \mathrm{MHz}, \mathrm{CDCl}_{3}\right) \delta: 154.0(\mathrm{~d}, J=24.0 \mathrm{~Hz}), 148.8(\mathrm{q}, J=35.3$ $\mathrm{Hz}), 142.3(\mathrm{~d}, J=16.6 \mathrm{~Hz}), 136.1(\mathrm{~d}, J=19.0 \mathrm{~Hz}), 134.2(\mathrm{~d}, J=21.3 \mathrm{~Hz}), 132.1(\mathrm{~d}, J=9.1 \mathrm{~Hz})$, 130.8, 129.7 (d, $J=8.0 \mathrm{~Hz}), 121.4$ (q, $J=121.4 \mathrm{~Hz}), 120.6-120.5(\mathrm{~m}) ;{ }^{19} \mathrm{~F}$ NMR $(365 \mathrm{MHz}$, 
$\left.\mathrm{CDCl}_{3}\right) \delta:-68.20 ;{ }^{31} \mathrm{P}$ NMR $\left(162 \mathrm{MHz}, \mathrm{CDCl}_{3}\right) \delta:-18.24 ; \mathrm{m} / \mathrm{z}$ LRMS (ESI + APCI) found [M + $\mathrm{H}]^{+}$401.2, $\mathrm{C}_{18} \mathrm{H}_{12} \mathrm{~F}_{6} \mathrm{~N}_{2} \mathrm{P}^{+}$requires 401.1.

\section{Preparation of Heterocyclic Phosphonium Salt Precursors}

\section{3-(1-Methyl-1H-pyrazol-5-yl)pyridine}<smiles>Cn1nccc1-c1cccnc1</smiles>

An oven-dried $250 \mathrm{~mL}$ round bottom flask was charged with 3-bromopyridine $(771 \mu \mathrm{L}, 8.00$ mmol), 1-methyl-5-(4,4,5,5-tetramethyl-1,3,2-dioxaborolan-2-yl)-1H-pyrazole (2.33 g, 11.20 $\mathrm{mmol}), \mathrm{Pd}\left(\mathrm{PPh}_{3}\right)_{4}(462 \mathrm{mg}, 0.40 \mathrm{mmol}), \mathrm{K}_{3} \mathrm{PO}_{4}(3.06 \mathrm{~g}, 14.40 \mathrm{mmol}), \mathrm{DMF}(80 \mathrm{~mL})$, and $\mathrm{H}_{2} \mathrm{O}$ $(780 \mu \mathrm{L})$. The reaction was heated at $80{ }^{\circ} \mathrm{C}$ for 24 hours. After cooling to room temperature, the reaction was diluted with EtOAc and $\mathrm{H}_{2} \mathrm{O}$ and the organic layer was separated. The aqueous layer was extracted from with EtOAc $(2 \times 50 \mathrm{~mL})$. The combined organic layers were then washed with $\mathrm{H}_{2} \mathrm{O}(5 \times 50 \mathrm{~mL})$, dried over $\mathrm{MgSO}_{4}$, concentrated in vacuo, and purified by flash chromatography (silica gel gradient elution: 50\% EtOAc in Hexanes to 100\% EtOAc) to provide the title compound as a clear oil (484 mg, $3.04 \mathrm{mmol}, 38 \%$ yield). IR $v_{\max } / \mathrm{cm}^{-1}$ (film): 3032 , 2946, 2226, 1717, 1470, 1409, 927, 711; ${ }^{1} \mathrm{H}$ NMR (400 MHz, $\left.\mathrm{CDCl}_{3}\right) \delta: 8.71(1 \mathrm{H}, \mathrm{dd}, J=2.2$, $0.7 \mathrm{~Hz}), 8.66(1 \mathrm{H}, \mathrm{dd}, J=4.8,1.6 \mathrm{~Hz}), 7.74(1 \mathrm{H}, \mathrm{ddd}, J=7.8,5.7,1.8 \mathrm{~Hz}), 7.55(1 \mathrm{H}, \mathrm{d}, J=1.9$ $\mathrm{Hz}), 7.40(1 \mathrm{H}, \mathrm{ddd}, J=7.8,3.0,0.8 \mathrm{~Hz}), 6.38(1 \mathrm{H}, \mathrm{d}, J=1.9 \mathrm{~Hz}), 3.91(3 \mathrm{H}, \mathrm{s}) ;{ }^{13} \mathrm{C}$ NMR $(100$ $\mathrm{MHz}_{\mathrm{CDCl}}$ ) $\delta: 149.6,149.5,140.2,138.9,136.0,126.9,123.5,106.9,37.7 ; \mathrm{m} / \mathrm{z}$ LRMS (ESI + APCI) found $[\mathrm{M}+\mathrm{H}]^{+} 160.1, \mathrm{C}_{9} \mathrm{H}_{10} \mathrm{~N}_{3}{ }^{+}$requires 160.1 .

\section{5-(3-Chloro-5-fluorophenyl)-2-methylpyridine}

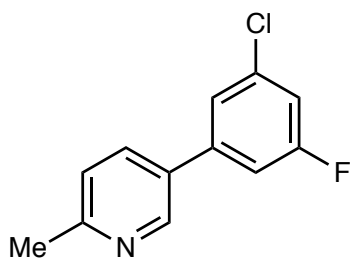

An oven-dried $25 \mathrm{~mL}$ sealed tube was charged with 5-bromo-2-methylpyridine (860 mg, 5.00 $\mathrm{mmol}$ ), (3-chloro-5-fluorophenyl)boronic acid (870 $\mathrm{mg}, 7.50 \mathrm{mmol}), \mathrm{Pd}\left(\mathrm{PPh}_{3}\right)_{4}(58 \mathrm{mg}, 0.05$ $\mathrm{mmol}), \mathrm{K}_{2} \mathrm{CO}_{3}(1.38 \mathrm{~g}, 10.00 \mathrm{mmol})$, EtOH $(10 \mathrm{~mL})$. The reaction was heated at $100{ }^{\circ} \mathrm{C}$ for 17 hours. After cooling to room temperature, the reaction was diluted with EtOAc and $\mathrm{H}_{2} \mathrm{O}$ and the organic layer was separated. The aqueous layer was extracted from with EtOAc $(2 \times 20 \mathrm{~mL})$. The combined organic layers were then washed with $\mathrm{H}_{2} \mathrm{O}$, dried over $\mathrm{MgSO}_{4}$, concentrated in vacuo, and purified by flash chromatography (silica gel gradient elution: $25 \%$ EtOAc in Hexanes) to provide the title compound as a white solid (946 mg, $4.28 \mathrm{mmol}, 86 \%$ yield). $\mathrm{mp} 80-81{ }^{\circ} \mathrm{C}$; IR $v_{\max } / \mathrm{cm}^{-1}$ (film): 3023, 1608, 1497, 1430, 1366, 1183, 918, 842, 779; ${ }^{1} \mathrm{H}$ NMR (400 MHz, $\left.\mathrm{CDCl}_{3}\right) \delta: 8.68(1 \mathrm{H}, \mathrm{dd}, J=2.5,0.8 \mathrm{~Hz}), 7.73(1 \mathrm{H}, \mathrm{dd}, J=8.0,2.5 \mathrm{~Hz}), 7.34(1 \mathrm{H}, \mathrm{td}, J=1.7,0.6$ $\mathrm{Hz}), 7.26-7.21(2 \mathrm{H}, \mathrm{m}), 7.17(1 \mathrm{H}, \mathrm{ddd}, J=9.3,2.4,1.5 \mathrm{~Hz}), 7.13-7.02(1 \mathrm{H}, \mathrm{m}), 2.62(3 \mathrm{H}, \mathrm{s}) ;{ }^{13} \mathrm{C}$ $\operatorname{NMR}\left(100 \mathrm{MHz}, \mathrm{CDCl}_{3}\right) \delta: 163.1(\mathrm{~d}, J=249.8 \mathrm{~Hz}), 158.8,147.4(\mathrm{~d}, J=2.1 \mathrm{~Hz}), 141.3(\mathrm{~d}, J=$ 
$8.6 \mathrm{~Hz}), 135.8(\mathrm{~d}, J=11.3 \mathrm{~Hz}), 134.7(\mathrm{~d}, J=3.9 \mathrm{~Hz}), 131.5,123.4(\mathrm{~d}, J=2.0 \mathrm{~Hz}), 123.1(\mathrm{~d}, J=$ $3.3 \mathrm{~Hz}), 115.5(\mathrm{~d}, J=25.0 \mathrm{~Hz}), 112.5(\mathrm{~d}, J=22.0 \mathrm{~Hz}), 24.3 ;{ }^{19} \mathrm{~F}$ NMR $\left(365 \mathrm{MHz}, \mathrm{CDCl}_{3}\right) \delta$ : $-110.19 ; \mathrm{m} / \mathrm{z}$ LRMS (ESI + APCI) found $[\mathrm{M}+\mathrm{H}]^{+} 222.1, \mathrm{C}_{12} \mathrm{H}_{10} \mathrm{ClFN}^{+}$requires 222.0.

\section{2-(3-(Pentafluoro- $\lambda^{6}$-sulfaneyl)phenyl)pyridine}<smiles>FS(F)(F)(F)(F)c1cccc(-c2ccccn2)c1</smiles>

An oven-dried $50 \mathrm{~mL}$ round bottom flask was charged with (3-bromophenyl)pentafluoro- $\lambda^{6}$ sulfane $(546 \mu \mathrm{L}, 3.53 \mathrm{mmol}), 2$-(tributylstannyl)pyridine (1.37 mL, $\left.4.24 \mathrm{mmol}), \mathrm{Pd}_{(\mathrm{PPh}}\right)_{4}(205$ $\mathrm{mg}, 0.18 \mathrm{mmol})$, and toluene $(15 \mathrm{~mL})$. The reaction was refluxed for 40 hours. After cooling to room temperature, the mixture was concentrated in vacuo. Flash chromatography (silica gel gradient elution: $33 \% \mathrm{CH}_{2} \mathrm{Cl}_{2}$ in Hexanes to $50 \% \mathrm{CH}_{2} \mathrm{Cl}_{2}$ in Hexanes) followed by a second and third flash chromatography column (silica gel gradient elution: $100 \%$ Hexanes to $50 \% \mathrm{CH}_{2} \mathrm{Cl}_{2}$ in Hexanes) afforded the title compound (761 mg, $2.70 \mathrm{mmol}, 77 \%$ yield) as an amorphous solid. IR $v_{\max } / \mathrm{cm}^{-1}$ (film): 3086, 3011, 1588, 1462, 1113, 832, 648, 595; ${ }^{1} \mathrm{H} \mathrm{NMR}\left(400 \mathrm{MHz}, \mathrm{CDCl}_{3}\right) \delta$ : $8.72(1 \mathrm{H}, \mathrm{d}, J=4.8 \mathrm{~Hz}), 8.50(1 \mathrm{H}$, app t,$J=1.9 \mathrm{~Hz}), 8.11(1 \mathrm{H}, \mathrm{d}, J=7.8 \mathrm{~Hz}), 7.82-7.70(3 \mathrm{H}$, $\mathrm{m}), 7.54(1 \mathrm{H}$, app t, $J=8.0 \mathrm{~Hz}), 7.28(1 \mathrm{H}$, ddd, $J=7.3,5.2,1.2 \mathrm{~Hz}) ;{ }^{13} \mathrm{C} \mathrm{NMR}(100 \mathrm{MHz}$, $\mathrm{CDCl}_{3}$ ) $\delta: 155.2,154.6(\mathrm{qn}, J=16.8 \mathrm{~Hz}), 150.0,140.5,137.1,129.8,129.1,126.2$ (qn, $J=4.5$ $\mathrm{Hz}), 124.6$ (qn, $J=4.7 \mathrm{~Hz}), 123.1,120.6 ;{ }^{19} \mathrm{~F} \mathrm{NMR}\left(365 \mathrm{MHz}, \mathrm{CDCl}_{3}\right) \delta:(84.27(1 \mathrm{~F}, \mathrm{qn}, J=$ $151.2 \mathrm{~Hz}), 62.72(4 \mathrm{~F}, \mathrm{~d}, J=150.1 \mathrm{~Hz}) ; \mathrm{m} / \mathrm{z}$ LRMS $(\mathrm{ESI}+\mathrm{APCI})$ found $[\mathrm{M}+\mathrm{H}]^{+} 282.1$, $\mathrm{C}_{11} \mathrm{H}_{9} \mathrm{~F}_{5} \mathrm{NS}^{+}$requires 282.0.

\section{((3S,4R)-4-(4-Fluorophenyl)-1-methylpiperidin-3-yl)methyl 2-methylnicotinate}<smiles>Cc1ncccc1C(=O)OC[C@@H]1CN(C)CC[C@@H]1c1ccc(F)cc1</smiles>

An oven-dried $100 \mathrm{~mL}$ round bottom flask was charged with 2-methylnicotinic acid (686 mg, $5.00 \mathrm{mmol}),((3 S, 4 R)-4-(4-f l u o r o p h e n y l)-1-m e t h y l p i p e r i d i n-3-y l) m e t h a n o l ~(1.23 \mathrm{~g}, 5.50 \mathrm{mmol})$, N,N'-Dicyclohexylcarbodiimide $(1.13 \mathrm{~g}, 5.50 \mathrm{mmol})$, DMAP (61 mg, $0.50 \mathrm{mmol})$, and $\mathrm{CH}_{2} \mathrm{Cl}_{2}$ $(25 \mathrm{~mL})$. After 20 hours of stirring at room temperature, the reaction was concentrated in vacuo, and purified by flash chromatography (silica gel: $10 \% \mathrm{MeOH}$ in $\mathrm{CH}_{2} \mathrm{Cl}_{2}$ ) to provide the title compound as a clear oil (1.25 g, $3.65 \mathrm{mmol}, 73 \%$ yield). IR $v_{\max } / \mathrm{cm}^{-1}$ (film): 2936, 2785, 1721, 1510, 1244, 1077, 754; ${ }^{1} \mathrm{H}$ NMR $\left(400 \mathrm{MHz}_{\mathrm{CDCl}}\right) \delta: 8.61(1 \mathrm{H}, \mathrm{dd}, J=4.8,1.8 \mathrm{~Hz}), 8.03(1 \mathrm{H}$, $\mathrm{dd}, J=7.9,1.8 \mathrm{~Hz}), 7.22-7.14(3 \mathrm{H}, \mathrm{m}), 7.02-6.95(2 \mathrm{H}, \mathrm{m}), 4.09-4.05(1 \mathrm{H}, \mathrm{m}), 3.94-3.88(1 \mathrm{H}$, m), $3.17(1 \mathrm{H}, \mathrm{d}, J=11.7 \mathrm{~Hz}), 3.00(1 \mathrm{H}, \mathrm{d}, J=10.3 \mathrm{~Hz}), 2.80(3 \mathrm{H}, \mathrm{s}), 2.42-2.30(5 \mathrm{H}, \mathrm{m}), 2.13-$ $1.80(4 \mathrm{H}, \mathrm{m}) ;{ }^{13} \mathrm{C} \mathrm{NMR}\left(100 \mathrm{MHz}, \mathrm{CDCl}_{3}\right) \delta: 163.3,161.8(\mathrm{~d}, \mathrm{~J}=244.6 \mathrm{~Hz}), 160.0,152.0$, 139.3, 138.4, 128.9 (d, $J=7.8 \mathrm{~Hz}), 125.3,121.0,115.8$ (d, $J=21.3 \mathrm{~Hz}), 66.3,59.6,56.3,46.6$, 
44.4, 41.5, 34.7, 25.1; ${ }^{19} \mathrm{~F}$ NMR (365 MHz, $\left.\mathrm{CDCl}_{3}\right) \delta:-116.2 ; \mathrm{m} / \mathrm{z}$ LRMS (ESI + APCI) found $[\mathrm{M}+\mathrm{H}]^{+}$343.2, $\mathrm{C}_{20} \mathrm{H}_{24} \mathrm{FN}_{2} \mathrm{O}_{2}{ }^{+}$requires 343.2.

((3S,4R)-1-(Ethoxycarbonyl)-4-(4-fluorophenyl)piperidin-3-yl)methyl 2-methylnicotinate<smiles>CCOC(=O)N1CC[C@@H](c2ccc(F)cc2)[C@H](COC(=O)c2cccnc2[N+](=O)[O-])C1</smiles>

An oven-dried $50 \mathrm{~mL}$ round bottom flask was charged with ((3S,4R)-4-(4-fluorophenyl)-1methylpiperidin-3-yl)methyl 2-methylnicotinate (1.10 g, $3.20 \mathrm{mmol}), \mathrm{Na}_{2} \mathrm{CO}_{3}(34 \mathrm{mg}, 0.032$ $\mathrm{mmol})$, ethyl chloroformate $(609 \mu \mathrm{L}, 6.40 \mathrm{mmol})$, and toluene $(16 \mathrm{~mL})$. The reaction was heated at $80{ }^{\circ} \mathrm{C}$ for 1 hour. After cooling down to room temperature, the reaction was diluted with EtOAc, poured into $\mathrm{H}_{2} \mathrm{O}$, and extracted with EtOAc $(3 \times 20 \mathrm{~mL})$. The combined organic layers were dried over $\mathrm{MgSO}_{4}$, concentrated in vacuo, and purified by flash chromatography (silica gel gradient elution: $50 \%$ EtOAc in Hexanes to $100 \%$ EtOAc) to provide the title compound as a colorless oil (1.01 g, $2.52 \mathrm{mmol}, 79 \%$ yield). IR $v_{\max } / \mathrm{cm}^{-1}$ (film): 2939, 2859, 2250, 1692, 1437 , 1222, 910, 728; ${ }^{1} \mathrm{H}$ NMR (400 MHz, $\left.\mathrm{CDCl}_{3}\right) \delta: 8.59(1 \mathrm{H}, \mathrm{dd}, J=4.8,1.6 \mathrm{~Hz}), 8.06(1 \mathrm{H}, \mathrm{dd}, J=$ 7.9, $1.7 \mathrm{~Hz}), 7.20-7.10(3 \mathrm{H}, \mathrm{m}), 7.01-6.95(2 \mathrm{H}, \mathrm{m}), 4.50(1 \mathrm{H}$, br s), 4.38-4.05 $(4 \mathrm{H}, \mathrm{m}), 3.89(1 \mathrm{H}$, $\mathrm{dd}, J=11.4,7.7 \mathrm{~Hz}), 2.93-2.65(5 \mathrm{H}, \mathrm{m}), 2.54(1 \mathrm{H}, \mathrm{td}, J=11.7,3.8), 2.21-2.09(1 \mathrm{H}, \mathrm{m}), 1.85-$ $1.76(1 \mathrm{H}, \mathrm{m}), 1.69(1 \mathrm{H}, \mathrm{qd}, J=12.9,4.2), 1.26(3 \mathrm{H}, \mathrm{t}, J=7.1 \mathrm{~Hz}) ;{ }^{13} \mathrm{C} \mathrm{NMR}\left(100 \mathrm{MHz}, \mathrm{CDCl}_{3}\right)$ $\delta: 166.2,161.8(\mathrm{~d}, J=245.3 \mathrm{~Hz}), 159.6,155.5,152.0,138.6(\mathrm{~d}, J=3.2 \mathrm{~Hz}), 138.4,128.7(\mathrm{~d}, J=$ $7.9 \mathrm{~Hz}), 125.1,121.0,115.8(\mathrm{~d}, J=21.3), 65.7,61.6,47.3,45.0,44.4,41.1,34.3,25.1,14.8 ;{ }^{19} \mathrm{~F}$ NMR $\left(365 \mathrm{MHz}, \mathrm{CDCl}_{3}\right) \delta:-115.66-(-115.74) ; \mathrm{m} / \mathrm{z}$ LRMS (ESI + APCI) found [M + H] ${ }^{+}$ 401.3, $\mathrm{C}_{22} \mathrm{H}_{26} \mathrm{FN}_{2} \mathrm{O}_{4}{ }^{+}$requires 401.2.

\section{(R)-1-(3,5-Bis(trifluoromethyl)phenyl)ethyl 5-methylpicolinate}

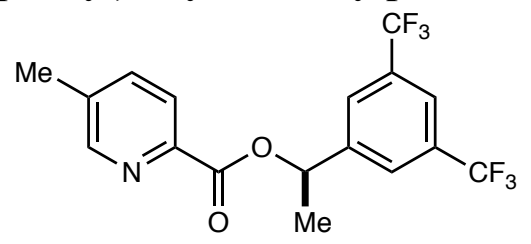

An oven-dried $100 \mathrm{~mL}$ round bottom flask was charged with 5-methylpicolinic acid (686 mg, $5.00 \mathrm{mmol}),(R)-1-\left(3,5-b i s\left(\right.\right.$ trifluoromethyl)phenyl)ethan-1-ol $(1.42 \mathrm{~g}, 5.50 \mathrm{mmol}), \mathrm{N}, \mathrm{N}^{\prime}-$ Dicyclohexylcarbodiimide (1.13 g, $5.50 \mathrm{mmol})$, DMAP (61 mg, $0.50 \mathrm{mmol})$, and $\mathrm{CH}_{2} \mathrm{Cl}_{2}(25$ $\mathrm{mL}$ ). After 18 hours of stirring at room temperature, the reaction was concentrated in vacuo, and purified by flash chromatography (silica gel: 15\% EtOAc in Hexanes) to provide the title compound as a colorless oil (596 mg, $1.58 \mathrm{mmol}, 32 \%$ yield). IR $v_{\max } / \mathrm{cm}^{-1}$ (film): 2988, 2234, 1723, 1383, 1277, 1131, 607, 728; ${ }^{1} \mathrm{H}$ NMR $\left(400 \mathrm{MHz}, \mathrm{CDCl}_{3}\right) \delta: 8.63-8.60(1 \mathrm{H}, \mathrm{m}), 8.02(1 \mathrm{H}$, $\mathrm{d}, J=8.0 \mathrm{~Hz}), 7.92(2 \mathrm{H}, \mathrm{s}), 7.82(1 \mathrm{H}, \mathrm{s}), 7.66-7.63(1 \mathrm{H}, \mathrm{m}), 6.26(1 \mathrm{H}, \mathrm{q}, J=6.7 \mathrm{~Hz}), 2.43(3 \mathrm{H}$, s), $1.77(3 \mathrm{H}, \mathrm{d}, J=6.7 \mathrm{~Hz}) ;{ }^{13} \mathrm{C} \mathrm{NMR}\left(100 \mathrm{MHz}, \mathrm{CDCl}_{3}\right) \delta: 164.6,150.9,145.2,144.2,138.0$, 
$137.5,132.1$ (q, $J=33.3 \mathrm{~Hz}), 126.8-126.6(\mathrm{~m}), 125.2,123.3$ (q, $J=272.8 \mathrm{~Hz}), 122.3-122.1(\mathrm{~m})$, 72.3, 22.3, 18.9; ${ }^{19} \mathrm{~F}$ NMR (365 MHz, $\left.\mathrm{CDCl}_{3}\right) \delta:-62.87(\mathrm{~m}) ; \mathrm{m} / \mathrm{z}$ LRMS (ESI + APCI) found [M $+\mathrm{H}]^{+}$378.1, $\mathrm{C}_{17} \mathrm{H}_{14} \mathrm{~F}_{6} \mathrm{NO}_{2}{ }^{+}$requires 378.1.

Ethyl (6R,10S)-6,7,9,10-tetrahydro-8H-6,10-methanoazepino[4,5-g]quinoxaline-8carboxylate

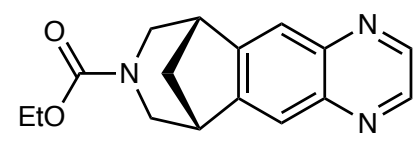

A $25 \mathrm{~mL}$ round bottom flask was charged with varenicline tartrate (488 $\mathrm{mg}, 1.35 \mathrm{mmol}$ ), DMAP (17 mg, $0.14 \mathrm{mmol})$, and $\mathrm{CH}_{2} \mathrm{Cl}_{2}(10 \mathrm{~mL})$. Ethyl Chloroformate $(257 \mu \mathrm{L}, 2.70 \mathrm{mmol})$ and $N, N$ Diisopropylethylamine $(941 \mu \mathrm{L}, 5.40 \mathrm{mmol})$ were added dropwise. After stirring at room temperature for 16 hours, the reaction was concentrated in vacuo and purified by flash chromatography ( $80 \%$ EtOAc in Hexanes) to afford the title product as an amorphous solid (314 $\mathrm{mg}, 1.11 \mathrm{mmol}, 82 \%$ yield). IR $v_{\max } / \mathrm{cm}^{-1}$ (film): 2952, 2866, 1687, 1417, 1212, 1113, 914, 731; ${ }^{1} \mathrm{H}$ NMR $\left(400 \mathrm{MHz}, \mathrm{CDCl}_{3}\right) \delta: 8.76(2 \mathrm{H}, \mathrm{d}, J=3.9 \mathrm{~Hz}), 7.87(2 \mathrm{H}, \mathrm{d}, J=6.9 \mathrm{~Hz}), 4.16-3.78(4 \mathrm{H}$, m), 3.50-3.27 (4H, m), 2.45-2.37 (1H, m), $2.00(1 \mathrm{H}, \mathrm{d}, J=11.1 \mathrm{~Hz}), 1.03(3 \mathrm{H}, \mathrm{t}, J=7.1 \mathrm{~Hz}) ;{ }^{13} \mathrm{C}$ NMR $\left(100 \mathrm{MHz}, \mathrm{CDCl}_{3}\right) \delta: 156.7,148.7(\mathrm{~d}, J=2.7 \mathrm{~Hz}), 144.0(\mathrm{~d}, J=6.8 \mathrm{~Hz}), 143.5(\mathrm{~d}, J=8.3$ $\mathrm{Hz}), 122.4(\mathrm{~d}, J=46.4 \mathrm{~Hz}), 61.3,49.8(\mathrm{~d}, J=27.4 \mathrm{~Hz}), 41.2,40.0(\mathrm{~d}, J=1.4 \mathrm{~Hz}), 14.6 ; \mathrm{m} / \mathrm{z}$ LRMS (ESI + APCI) found [M + H] $]^{+} 284.1, \mathrm{C}_{16} \mathrm{H}_{18} \mathrm{~N}_{3} \mathrm{O}_{2}{ }^{+}$requires 284.1 . 


\section{Preparation of Heterocyclic Phosphonium Salts}

\section{General Procedure A:}

An oven dried round bottom flask equipped with a stir bar was charged with the heterocycle (1.0 equiv), phosphine (1.1 equiv), and placed under a nitrogen atmosphere. $\mathrm{CH}_{2} \mathrm{Cl}_{2}(0.1 \mathrm{M})$ was added, the reaction vessel cooled to $-50{ }^{\circ} \mathrm{C}$ and $\mathrm{Tf}_{2} \mathrm{O}$ (1.0 equiv) was added dropwise. After stirring for 1 hour, the reaction was cooled to $-78{ }^{\circ} \mathrm{C}$ and DBU (1.0 equiv) was added dropwise via syringe. The cooling bath was removed and the reaction was allowed to warm to room temperature while stirring (approximately 20-30 minutes). The reaction mixture was diluted with $\mathrm{CH}_{2} \mathrm{Cl}_{2}$ and washed with $\mathrm{H}_{2} \mathrm{O}(3 \mathrm{x})$. The organic layer was dried $\left(\mathrm{MgSO}_{4}\right)$, filtered and concentrated in vacuo to approximately $2-5 \mathrm{~mL}$ (depending on the scale of the reaction). The concentrated reaction mixture was added dropwise to an excess of a 50:50 $\mathrm{Et}_{2} \mathrm{O}:$ Hexanes solution that was then placed in a $-20^{\circ} \mathrm{C}$ refrigerator until the solid has settled to the bottom of the flask. The suspension was filtered on a frit, the solid washed with chilled $\mathrm{Et}_{2} \mathrm{O}\left(0^{\circ} \mathrm{C}\right)$ and dried in vacuo to provide the pure product.

\section{Notes.}

1) To maximize the yield, vigorous stirring is required.

2) For long term storage ( $>2$ weeks) it is best to keep the heteroaryl phosphonium salt product in $\mathrm{a}-20{ }^{\circ} \mathrm{C}$ fridge.

\section{Triphenyl(3-phenylpyridin-4-yl)phosphonium trifluoromethanesulfonate (1)}<smiles>O=S(=O)([O-])C(F)(F)F</smiles>

Prepared according to our previous report. ${ }^{11}{ }^{1} \mathrm{H}$ NMR $\left(400 \mathrm{MHz}, \mathrm{CDCl}_{3}\right): 8.95(1 \mathrm{H}$, app t, $J=4.7$ $\mathrm{Hz}), 8.74(1 \mathrm{H}, \mathrm{d}, J=6.8 \mathrm{~Hz}), 7.85-7.73(3 \mathrm{H}, \mathrm{m}), 7.73-7.40(13 \mathrm{H}, \mathrm{m}), 7.11(1 \mathrm{H}, \mathrm{t}, J=7.6 \mathrm{~Hz})$, $6.91(2 \mathrm{H}$, app t, $J=7.6 \mathrm{~Hz}), 6.71(2 \mathrm{H}, \mathrm{d}, J=7.5 \mathrm{~Hz}) ;{ }^{13} \mathrm{C} \mathrm{NMR}\left(100 \mathrm{MHz}, \mathrm{CDCl}_{3}\right): 153.6(\mathrm{~d}, J=$ $8.0 \mathrm{~Hz}), 150.0(\mathrm{~d}, J=10.4 \mathrm{~Hz}), 141.7(\mathrm{~d}, J=7.3 \mathrm{~Hz}), 135.4(\mathrm{~d}, J=3.0 \mathrm{~Hz}), 134.4(\mathrm{~d}, J=4.5$ $\mathrm{Hz}), 134.2(\mathrm{~d}, J=10.3 \mathrm{~Hz}), 130.6(\mathrm{~d}, J=13.0 \mathrm{~Hz}), 129.2,128.9,128.3,128.2,126.4$ (d, $J=83.4$ $\mathrm{Hz}), 120.8$ (q, $J=321.2 \mathrm{~Hz}), 116.9(\mathrm{~d}, J=89.2 \mathrm{~Hz}) ;{ }^{19} \mathrm{~F}$ NMR $\left(365 \mathrm{MHz}, \mathrm{CDCl}_{3}\right):-77.68 ;{ }^{31} \mathrm{P}$ NMR (162 MHz, $\left.\mathrm{CDCl}_{3}\right): 21.73$. The spectroscopic data is in agreement with our reported synthesis.

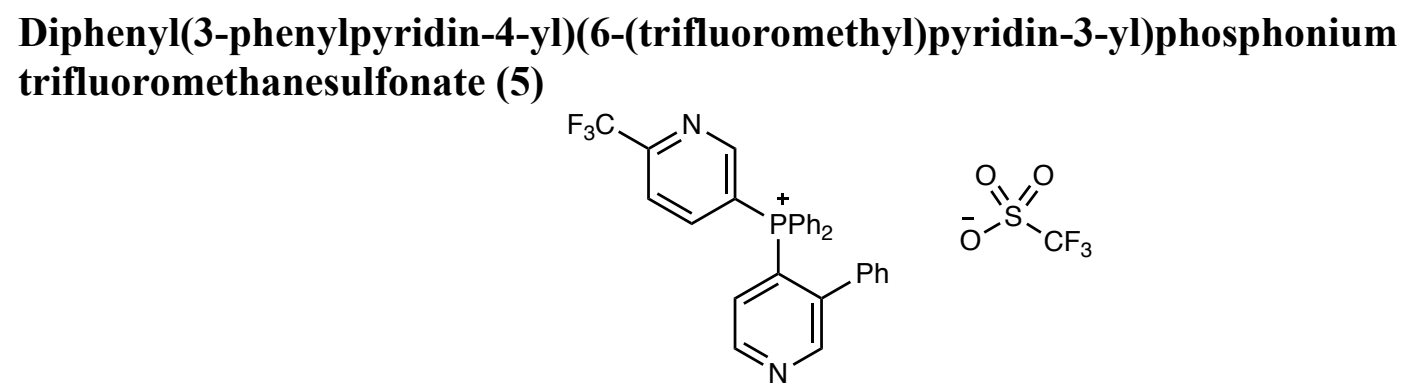


Prepared according to general procedure A, using 3-phenylpyridine ( $282 \mu \mathrm{L}, 2.00 \mathrm{mmol}), \mathrm{Tf}_{2} \mathrm{O}$ (336 $\mu \mathrm{L}, 2.00 \mathrm{mmol}), 5$-(diphenylphosphaneyl)-2-(trifluoromethyl)pyridine (728 $\mathrm{mg}, 2.20$ mmol), DBU $(298 \mu \mathrm{L}, 2.00 \mathrm{mmol})$, and $\mathrm{CH}_{2} \mathrm{Cl}_{2}(20 \mathrm{~mL})$. After purification by the standard procedure (except that two crash-outs were required), the title compound was isolated as a white solid (964 mg, $1.52 \mathrm{mmol}, 76 \%$ yield). $\mathrm{mp} 54-57^{\circ} \mathrm{C}$. IR $v_{\max } / \mathrm{cm}^{-1}$ (film): $3064,1440,1336$, 1258, 1140, 1029, 722, 635; ${ }^{1} \mathrm{H}$ NMR $\left(400 \mathrm{MHz}, \mathrm{CDCl}_{3}\right) \delta: 8.99(1 \mathrm{H}$, app t, $J=4.0 \mathrm{~Hz}), 8.89$ $8.70(2 \mathrm{H}, \mathrm{m}), 8.38(1 \mathrm{H}, \mathrm{dd}, J=5.9 \mathrm{~Hz}, 2.1 \mathrm{~Hz}), 7.97-7.87(3 \mathrm{H}, \mathrm{m}), 7.82-7.70(8 \mathrm{H}, \mathrm{m}), 7.49(1 \mathrm{H}$, $\mathrm{d}, J=15.4,5.2 \mathrm{~Hz}), 7.17(1 \mathrm{H}$, app t, $J=7.5 \mathrm{~Hz}), 6.96(2 \mathrm{H}$, app t, $J=8.0 \mathrm{~Hz}), 6.80(2 \mathrm{H}, \mathrm{dd}, J=$ 8.3, $1.3 \mathrm{~Hz}) ;{ }^{13} \mathrm{C}$ NMR $\left(100 \mathrm{MHz}, \mathrm{CDCl}_{3}\right) \delta: 153.7(\mathrm{~d}, J=7.9 \mathrm{~Hz}), 152.7(\mathrm{~d}, J=12.6 \mathrm{~Hz}), 151.9$ $(\mathrm{qd}, J=35.8,2.4 \mathrm{~Hz}), 150.2(\mathrm{~d}, J=10.6 \mathrm{~Hz}), 145.1(\mathrm{~d}, J=9.3 \mathrm{~Hz}), 141.7(\mathrm{~d}, J=7.0 \mathrm{~Hz}), 136.4$ $(\mathrm{d}, J=3.1 \mathrm{~Hz}), 134.6(\mathrm{~d}, J=10.7 \mathrm{~Hz}), 134.3(\mathrm{~d}, J=4.6 \mathrm{~Hz}), 131.2(\mathrm{~d}, J=13.4 \mathrm{~Hz}), 129.6$, 129.4, 128.6, $128.3(\mathrm{~d}, J=3.1 \mathrm{~Hz}), 124.8(\mathrm{~d}, J=84.7 \mathrm{~Hz}), 122.0-121.8(\mathrm{~m}), 120.7(\mathrm{q}, J=321.0$ $\mathrm{Hz}), 120.4(\mathrm{qd}, J=275.3,1.7 \mathrm{~Hz}), 119.2(\mathrm{~d}, J=86.4 \mathrm{~Hz}), 115.3(\mathrm{~d}, J=89.3 \mathrm{~Hz}) ;{ }^{19} \mathrm{~F}$ NMR $(365$ $\left.\mathrm{MHz}, \mathrm{CDCl}_{3}\right) \delta:-68.89,-78.29 ;{ }^{31} \mathrm{P} \mathrm{NMR}\left(162 \mathrm{MHz}, \mathrm{CDCl}_{3}\right) \delta: 19.31 ; \mathrm{m} / \mathrm{z} \mathrm{LRMS}(\mathrm{ESI}+$ APCI) found $[\mathrm{M}-\mathrm{OTf}]^{+} 485.2, \mathrm{C}_{27} \mathrm{H}_{21} \mathrm{~F}_{3} \mathrm{~N}_{4} \mathrm{P}^{+}$requires 485.1 .

\section{Triphenyl(2-phenylpyridin-4-yl)phosphonium trifluoromethanesulfonate (2)}

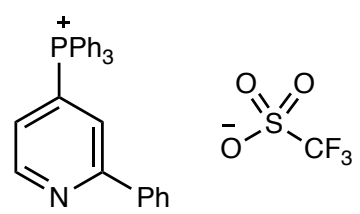

Prepared according to our previous report. ${ }^{1}{ }^{1} \mathrm{H}$ NMR (400 MHz, CDCl3): 9.01 (1H, app t, $J=5.1$ $\mathrm{Hz}), 7.93-7.54(18 \mathrm{H}, \mathrm{m}), 7.50(1 \mathrm{H}, \mathrm{ddd}, J=17.8,5.1,1.1 \mathrm{~Hz}), 7.42-7.36(3 \mathrm{H}, \mathrm{m}) ;{ }^{13} \mathrm{C}$ NMR (100 MHz, CDCl3): 159.1 (d, $J=9.9 \mathrm{~Hz}), 151.6(\mathrm{~d}, J=10.7 \mathrm{~Hz}), 136.7$ (d, $J=1.5 \mathrm{~Hz}), 136.1$ (d, $J=3.2 \mathrm{~Hz}), 134.3$ (d, $J=9.8 \mathrm{~Hz}), 130.9$ (d, $J=13.0 \mathrm{~Hz}), 130.4,129.2$ (d, $J=84.1 \mathrm{~Hz}), 129.0$, 127.0, $125.3(\mathrm{~d}, J=7.8 \mathrm{~Hz}), 123.1$, (d, $J=8.4 \mathrm{~Hz}), 120.7(\mathrm{q}, J=321.1 \mathrm{~Hz}), 115.5$ (d, $J=89.1$ $\mathrm{Hz}) ;{ }^{19} \mathrm{~F}$ NMR (365 MHz, CDCl3): -78.1; ${ }^{31} \mathrm{P}$ NMR (162 MHz, CDCl3): 22.7. The spectroscopic data is in agreement with our reported synthesis.

\section{Diphenyl(2-phenylpyridin-4-yl)(6-(trifluoromethyl)pyridin-3-yl)phosphonium trifluoromethanesulfonate (6)}

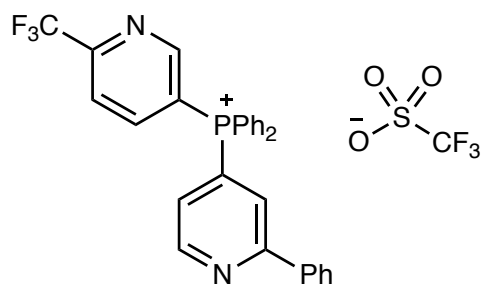

Prepared according to general procedure A, using 2-phenylpyridine (571 $\mu \mathrm{L}, 4.00 \mathrm{mmol}), \mathrm{Tf}_{2} \mathrm{O}$ (676 $\mu \mathrm{L}, 4.00 \mathrm{mmol}), 5$-(diphenylphosphaneyl)-2-(trifluoromethyl)pyridine $(1.460 \mathrm{~g}, 4.40$ mmol), DBU $(598 \mu \mathrm{L}, 4.00 \mathrm{mmol})$, and $\mathrm{CH}_{2} \mathrm{Cl}_{2}(40 \mathrm{~mL})$. After purification by the standard procedure (except that two crash-outs were required), the title compound was isolated as a white solid (1.906 g, $3.00 \mathrm{mmol}, 75 \%$ yield). $\mathrm{mp} 68-72{ }^{\circ} \mathrm{C}$. IR $v_{\max } / \mathrm{cm}^{-1}$ (film): $3063,1573,1440$, 
1258, 1141, 1029, 724, 635; ${ }^{1} \mathrm{H}$ NMR (400 MHz, $\left.\mathrm{CDCl}_{3}\right) \delta: 9.07(1 \mathrm{H}$, app t, $J=5.3 \mathrm{~Hz}), 8.83$ $(1 \mathrm{H}, \mathrm{dd}, J=6.1,1.5 \mathrm{~Hz}), 8.68(1 \mathrm{H}, \mathrm{ddd}, J=12.7,8.5,2.2 \mathrm{~Hz}), 8.18(1 \mathrm{H}, \mathrm{dd}, J=8.3,1.2 \mathrm{~Hz})$, 7.98-7.68 $(13 \mathrm{H}, \mathrm{m}), 7.56(1 \mathrm{H}, \mathrm{ddd}, J=13.3,5.1,1.1 \mathrm{~Hz}), 7.47-7.42(3 \mathrm{H}, \mathrm{m}) ;{ }^{13} \mathrm{C}$ NMR $(100$ $\left.\mathrm{MHz}, \mathrm{CDCl}_{3}\right) \delta: 159.4(\mathrm{~d}, J=10.8 \mathrm{~Hz}), 153.3(\mathrm{~d}, J=13.0 \mathrm{~Hz}), 153.1(\mathrm{qd}, J=36.1,2.7 \mathrm{~Hz})$, $151.9(\mathrm{~d}, J=11.0 \mathrm{~Hz}), 145.7(\mathrm{~d}, J=9.4 \mathrm{~Hz}), 136.7(\mathrm{~d}, J=2.0 \mathrm{~Hz}), 134.6(\mathrm{~d}, J=11.0 \mathrm{~Hz}), 131.3$ $(\mathrm{d}, J=13.4 \mathrm{~Hz}), 130.5,129.0,128.0,127.1,125.4$ (d, $J=8.5 \mathrm{~Hz}), 123.2(\mathrm{~d}, J=9.2 \mathrm{~Hz}), 122.6-$ $122.4(\mathrm{~m}), 120.5$ (q, $J=321.2 \mathrm{~Hz}), 120.4$ (qd, $J=275.2,1.7 \mathrm{~Hz}), 118.0(\mathrm{~d}, J=87.4 \mathrm{~Hz}), 113.8$ $(\mathrm{d}, J=89.9 \mathrm{~Hz}) ;{ }^{19} \mathrm{~F}$ NMR $\left(365 \mathrm{MHz}, \mathrm{CDCl}_{3}\right) \delta:-68.53,-78.34 ;{ }^{31} \mathrm{P} \mathrm{NMR}\left(162 \mathrm{MHz}, \mathrm{CDCl}_{3}\right)$ $\delta: 20.38 ; \mathrm{m} / \mathrm{z}$ LRMS (ESI $+\mathrm{APCI})$ found $[\mathrm{M}-\mathrm{OTf}]^{+} 485.2, \mathrm{C}_{27} \mathrm{H}_{21} \mathrm{~F}_{3} \mathrm{~N}_{4} \mathrm{P}^{+}$requires 485.1 .

\section{Phenyl(2-phenylpyridin-4-yl)bis(6-(trifluoromethyl)pyridin-3-yl)phosphonium} trifluoromethanesulfonate (7)
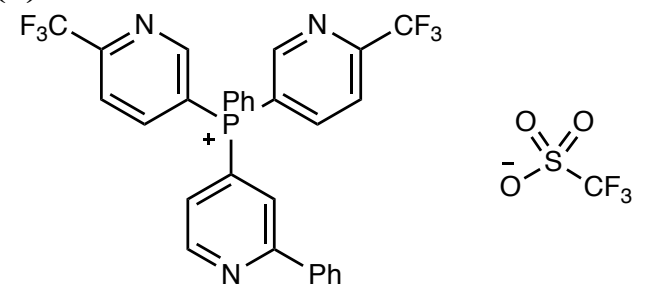

Prepared according to general procedure A, using 2-phenylpyridine ( $282 \mu \mathrm{L}, 2.00 \mathrm{mmol}), \mathrm{Tf}_{2} \mathrm{O}$ (336 $\mu \mathrm{L}, 2.00 \mathrm{mmol}), 5,5$-(phenylphosphanediyl)bis(2-(trifluoromethyl)pyridine (881 $\mathrm{mg}, 2.20$ $\mathrm{mmol}), \mathrm{DBU}(299 \mu \mathrm{L}, 2.00 \mathrm{mmol})$, and $\mathrm{CH}_{2} \mathrm{Cl}_{2}(20 \mathrm{~mL})$. After purification by the standard procedure (except that two crash-outs were required), the title compound was isolated as a white solid (1.146 g, $1.64 \mathrm{mmol}, 81 \%$ yield). $\mathrm{mp} 85-88^{\circ} \mathrm{C}$. IR $v_{\max } / \mathrm{cm}^{-1}$ (film): $3063,1573,1333$, 1139, 1075, 1029, 725, 635; ${ }^{1} \mathrm{H}$ NMR (400 MHz, $\left.\mathrm{CDCl}_{3}\right) \delta: 9.05(1 \mathrm{H}$, app t, $J=5.3 \mathrm{~Hz}), 8.90$ $(2 \mathrm{H}, \mathrm{dd}, J=6.1,1.7 \mathrm{~Hz}), 8.60(2 \mathrm{H}, \mathrm{ddd}, J=13.2,8.4,2.1 \mathrm{~Hz}), 8.09(2 \mathrm{H}, \mathrm{dd}, J=8.3,1.9 \mathrm{~Hz})$, 7.99-7.90 (4H, m), 7.85-7.71 (4H, m), $7.55(1 \mathrm{H}, \mathrm{ddd}, J=13.7,5.0,1.3 \mathrm{~Hz}), 7.45-7.38(3 \mathrm{H}, \mathrm{m})$; ${ }^{13} \mathrm{C}$ NMR $\left(100 \mathrm{MHz}, \mathrm{CDCl}_{3}\right) \delta: 159.8(\mathrm{~d}, J=11.0 \mathrm{~Hz}), 153.7(\mathrm{~d}, J=13.4 \mathrm{~Hz}), 153.6(\mathrm{qd}, J=$ $35.8,2.4 \mathrm{~Hz}), 152.2(\mathrm{~d}, J=11.5 \mathrm{~Hz}), 146.0(\mathrm{~d}, J=9.9 \mathrm{~Hz}), 137.3(\mathrm{~d}, J=2.9 \mathrm{~Hz}), 136.6(\mathrm{~d}, J=$ $1.7 \mathrm{~Hz}), 134.9(\mathrm{~d}, J=11.2 \mathrm{~Hz}), 131.6(\mathrm{~d}, J=13.6 \mathrm{~Hz}), 130.8,129.2,127.5,126.1(\mathrm{~d}, J=85.4$ $\mathrm{Hz}), 125.4(\mathrm{~d}, J=8.9 \mathrm{~Hz}), 123.5(\mathrm{~d}, J=9.4 \mathrm{~Hz}), 122.6-122.4(\mathrm{~m}), 120.5(\mathrm{qd}, J=275.7,2.1 \mathrm{~Hz})$, $120.3(\mathrm{q}, J=320.7 \mathrm{~Hz}), 116.6(\mathrm{~d}, J=88.4 \mathrm{~Hz}), 112.5(\mathrm{~d}, J=90.2 \mathrm{~Hz}) ;{ }^{19} \mathrm{~F}$ NMR $(365 \mathrm{MHz}$, $\left.\mathrm{CDCl}_{3}\right) \delta:-68.74,-78.74 ;{ }^{31} \mathrm{P} \mathrm{NMR}\left(162 \mathrm{MHz}, \mathrm{CDCl}_{3}\right) \delta: 18.52 ; \mathrm{m} / \mathrm{z}$ LRMS (ESI + APCI) found $[\mathrm{M}-\mathrm{OTf}]^{+}$554.2, $\mathrm{C}_{27} \mathrm{H}_{21} \mathrm{~F}_{3} \mathrm{~N}_{4} \mathrm{P}^{+}$requires 554.1.

\section{(3-Bromo-5-methylpyridin-4-yl)diphenyl(6-(trifluoromethyl)pyridin-3-yl)phosphonium} trifluoromethanesulfonate (8)

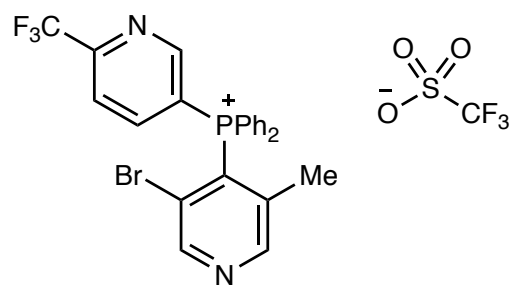

Prepared according to general procedure A, using 3-bromo-5-methylpyridine (116 $\mu \mathrm{L}, 1.00$ mmol), $\mathrm{Tf}_{2} \mathrm{O}(168 \mu \mathrm{L}, 1.00 \mathrm{mmol}), 5$-(diphenylphosphaneyl)-2-(trifluoromethyl)pyridine (364 $\mathrm{mg}, 1.10 \mathrm{mmol}), \mathrm{DBU}(149 \mu \mathrm{L}, 1.00 \mathrm{mmol})$, and $\mathrm{CH}_{2} \mathrm{Cl}_{2}(10 \mathrm{~mL})$. After purification by the 
standard procedure (except that two crash-outs were required), the title compound was isolated at 95\% purity as a white solid (172 $\mathrm{mg}, 0.26 \mathrm{mmol}, 26 \%$ yield). IR $v_{\max } / \mathrm{cm}^{-1}$ (film): 3065,1336 , $1258,1138,1075,1029,720,635 ;{ }^{1} \mathrm{H}$ NMR $\left(400 \mathrm{MHz}, \mathrm{CDCl}_{3}\right) \delta: 8.97-8.87(2 \mathrm{H}, \mathrm{m}), 8.84(1 \mathrm{H}$, $\mathrm{d}, J=5.7 \mathrm{~Hz}), 8.67(1 \mathrm{H}, \mathrm{d}, J=6.0 \mathrm{~Hz}), 8.19-8.15(1 \mathrm{H}, \mathrm{m}), 7.96-7.77(10 \mathrm{H}, \mathrm{m}), 1.92(3 \mathrm{H}, \mathrm{s}) ;{ }^{13} \mathrm{C}$ NMR (100 MHz, $\left.\mathrm{CDCl}_{3}\right) \delta: 154.2(\mathrm{~d}, J=8.6 \mathrm{~Hz}), 153.2(\mathrm{~d}, J=6.1 \mathrm{~Hz}), 152.9(\mathrm{~d}, J=12.7 \mathrm{~Hz})$, 152.8 (qd, $J=36.0,2.7 \mathrm{~Hz}), 145.7(\mathrm{~d}, J=9.3 \mathrm{~Hz}), 142.7(\mathrm{~d}, J=7.4 \mathrm{~Hz}), 136.6(\mathrm{~d}, J=3.3 \mathrm{~Hz})$, $134.8(\mathrm{~d}, J=11.0 \mathrm{~Hz}), 131.5(\mathrm{~d}, J=13.7 \mathrm{~Hz}), 125.1(\mathrm{~d}, J=8.5 \mathrm{~Hz}), 124.6(\mathrm{~d}, J=94.1 \mathrm{~Hz})$, 122.7-122.5 (m), $121.1(\mathrm{~d}, J=87.7 \mathrm{~Hz}), 120.8(\mathrm{q}, J=321.0 \mathrm{~Hz}), 120.6(\mathrm{qd}, J=275.6,2.2 \mathrm{~Hz})$, $116.6(\mathrm{~d}, J=89.1 \mathrm{~Hz}), 20.9(\mathrm{~d}, J=4.2 \mathrm{~Hz}) ;{ }^{19} \mathrm{~F} \mathrm{NMR}\left(365 \mathrm{MHz}, \mathrm{CDCl}_{3}\right) \delta:-68.55,-78.39 ;{ }^{31} \mathrm{P}$ NMR $\left(162 \mathrm{MHz}, \mathrm{CDCl}_{3}\right) \delta: 19.35 ; \mathrm{m} / \mathrm{z}$ LRMS (ESI + APCI) found [M - OTf $]^{+}$501.1, $\mathrm{C}_{27} \mathrm{H}_{21} \mathrm{~F}_{3} \mathrm{~N}_{4} \mathrm{P}^{+}$requires 501.0.

(3-(((Tert-butoxycarbonyl)(methyl)amino)methyl)pyridin-4-yl)diphenyl(6(trifluoromethyl)pyridin-3-yl)phosphonium trifluoromethanesulfonate<smiles>CN(Cc1cnccc1[P+](=O)c1ccc(C(F)(F)F)nc1)C(=O)OS(=O)(=O)C(F)(F)F</smiles>

Prepared according to general procedure A, using tert-butyl methyl(pyridin-3ylmethyl)carbamate $(222 \mathrm{mg}, 1.00 \mathrm{mmol}), \mathrm{Tf}_{2} \mathrm{O}(168 \mu \mathrm{L}, 1.00 \mathrm{mmol}), 5$-(diphenylphosphaneyl)2-(trifluoromethyl)pyridine (364 mg, $1.10 \mathrm{mmol}), \mathrm{DBU}(149 \mu \mathrm{L}, 1.00 \mathrm{mmol})$, and $\mathrm{CH}_{2} \mathrm{Cl}_{2}(10$ $\mathrm{mL})$. After purification by the standard procedure, the title compound was isolated as a brown solid (560 mg, $0.80 \mathrm{mmol}, 80 \%$ yield). $\mathrm{mp} 83-87^{\circ} \mathrm{C}$. IR $v_{\max } / \mathrm{cm}^{-1}$ (film): $2978,2932,1690$, 1260, 1140, 1029, 723, 636; ${ }^{1} \mathrm{H}$ NMR $\left(400 \mathrm{MHz} \mathrm{CDCl}_{3}\right) \delta: 8.88-8.70(3 \mathrm{H}, \mathrm{m}), 8.62(1 \mathrm{H}, \mathrm{ddd}, J$ $=12.8,8.4,2.1 \mathrm{~Hz}), 8.12(1 \mathrm{H}, \mathrm{d}, J=7.6 \mathrm{~Hz}), 7.95-7.88(2 \mathrm{H}, \mathrm{m}), 7.85-7.76(8 \mathrm{H}, \mathrm{m}), 7.23(1 \mathrm{H}, \mathrm{d}$, $J=15.3 \mathrm{~Hz}), 3.90(2 \mathrm{H}, \mathrm{s}), 2.61(3 \mathrm{H}, \mathrm{s}), 1.34(9 \mathrm{H}, \mathrm{s}) ;{ }^{13} \mathrm{C} \mathrm{NMR}\left(100 \mathrm{MHz}, \mathrm{CDCl}_{3}\right) \delta: 155.6$, $153.4(\mathrm{~d}, J=12.7 \mathrm{~Hz}), 153.3(\mathrm{qd}, J=36.1,2.3 \mathrm{~Hz}), 150.8,150.4,146.0(\mathrm{~d}, J=9.6 \mathrm{~Hz}), 137.3-$ $136.5(2 \mathrm{C}, \mathrm{m}), 134.5$ (d, $J=11.0 \mathrm{~Hz}), 131.7(\mathrm{~d}, J=13.4 \mathrm{~Hz}), 128.8,124.1(\mathrm{~d}, J=82.5 \mathrm{~Hz})$, 122.9-122.5 (m), 120.6 (q, $J=321.0 \mathrm{~Hz}), 120.5(\mathrm{qd}, J=275.6,1.7 \mathrm{~Hz}), 118.3(\mathrm{~d}, J=86.8 \mathrm{~Hz})$, $114.1(\mathrm{~d}, J=88.3 \mathrm{~Hz}), 81.1,50.4,35.6,28.2 ;{ }^{19} \mathrm{~F}$ NMR $\left(365 \mathrm{MHz}, \mathrm{CDCl}_{3}\right) \delta$ : $-68.63,-78.40$; ${ }^{31} \mathrm{P}$ NMR $\left(162 \mathrm{MHz}, \mathrm{CDCl}_{3}\right) \delta: 18.92 ; \mathrm{m} / \mathrm{z}$ LRMS (ESI + APCI) found [M - OTf $]^{+} 552.2$, $\mathrm{C}_{30} \mathrm{H}_{30} \mathrm{~F}_{3} \mathrm{~N}_{3} \mathrm{O}_{2} \mathrm{P}^{+}$requires 552.2.

(3-(1-Methyl-1H-pyrazol-5-yl)pyridin-4-yl)diphenyl(6-(trifluoromethyl)pyridin-3yl)phosphonium trifluoromethanesulfonate<smiles>Cn1nccc1-c1cnccc1[PH2+](=O)[O-]</smiles> 
Prepared according to general procedure A, using 3-(1-methyl-1H-pyrazol-5-yl)pyridine (159 $\mathrm{mg}, \quad 1.00 \mathrm{mmol}), \quad \mathrm{Tf}_{2} \mathrm{O} \quad(168 \mu \mathrm{L}, \quad 1.00 \quad \mathrm{mmol}), \quad 5-($ diphenylphosphaneyl)-2(trifluoromethyl)pyridine (364 mg, $1.10 \mathrm{mmol})$, DBU $(149 \mu \mathrm{L}, 1.00 \mathrm{mmol})$, and $\mathrm{CH}_{2} \mathrm{Cl}_{2}(10$ $\mathrm{mL}$ ). After purification by the standard procedure (except that two crash-outs were required), the title compound was isolated as a white solid (362 mg, $0.57 \mathrm{mmol}, 57 \%$ yield). $\mathrm{mp} 154-149{ }^{\circ} \mathrm{C}$. IR $v_{\max } / \mathrm{cm}^{-1}$ (film): 3066, 1440, 1259, 1142, 1029, 722, 636; ${ }^{1} \mathrm{H}$ NMR $\left(400 \mathrm{MHz}, \mathrm{CDCl}_{3}\right) \delta$ : $9.06(1 \mathrm{H}$, app t, $J=4.6 \mathrm{~Hz}), 8.89-8.76(2 \mathrm{H}, \mathrm{m}), 8.64(1 \mathrm{H}, \mathrm{d}, J=5.8 \mathrm{~Hz}), 8.08(1 \mathrm{H}, \mathrm{d}, J=8.2$ $\mathrm{Hz}), 7.96-7.66(10 \mathrm{H}, \mathrm{m}), 7.57(1 \mathrm{H}, \mathrm{dd}, J=15.4,4.8 \mathrm{~Hz}), 7.11(1 \mathrm{H}, \mathrm{s}), 5.68(1 \mathrm{H}, \mathrm{s}), 3.28(3 \mathrm{H}, \mathrm{s})$; ${ }^{13} \mathrm{C}$ NMR $\left(100 \mathrm{MHz}, \mathrm{CDCl}_{3}\right) \delta: 153.5(\mathrm{~d}, J=7.4 \mathrm{~Hz}), 153.0(\mathrm{~d}, J=12.8 \mathrm{~Hz}), 152.6(\mathrm{qd}, J=$ 36.1, $2.2 \mathrm{~Hz}), 152.1(\mathrm{~d}, J=10.5 \mathrm{~Hz}), 145.1(\mathrm{~d}, J=9.2 \mathrm{~Hz}), 138.6,136.5(\mathrm{~d}, J=2.7 \mathrm{~Hz}), 134.5$ $(\mathrm{d}, J=10.7 \mathrm{~Hz}), 134.3(\mathrm{~d}, J=4.9 \mathrm{~Hz}), 131.1(\mathrm{~d}, J=13.4 \mathrm{~Hz}), 130.1(\mathrm{~d}, J=6.2 \mathrm{~Hz}), 129.4(\mathrm{~d}, J=$ $9.3 \mathrm{~Hz}), 127.3$ (d, $J=85.8 \mathrm{~Hz}), 121.9-121.6(\mathrm{~m}), 120.6$ (q, $J=320.7 \mathrm{~Hz}), 120.5$ (q, $J=275.0$ $\mathrm{Hz}), 118.6$ (d, $J=87.0 \mathrm{~Hz}), 114.5$ (d, $J=89.3 \mathrm{~Hz}), 110.9,37.0 ;{ }^{19} \mathrm{~F}$ NMR $\left(365 \mathrm{MHz}, \mathrm{CDCl}_{3}\right) \delta$ : -68.69, -78.36; ${ }^{31} \mathrm{P}$ NMR (162 $\left.\mathrm{MHz}, \mathrm{CDCl}_{3}\right) \delta: 19.01 ; \mathrm{m} / \mathrm{z}$ LRMS (ESI + APCI) found [M $\mathrm{OTf}^{+} 489.2, \mathrm{C}_{27} \mathrm{H}_{21} \mathrm{~F}_{3} \mathrm{~N}_{4} \mathrm{P}^{+}$requires 489.1 .

\section{Diphenyl(3-(phenylethynyl)pyridin-4-yl)(6-(trifluoromethyl)pyridin-3-yl)phosphonium trifluoromethanesulfonate}

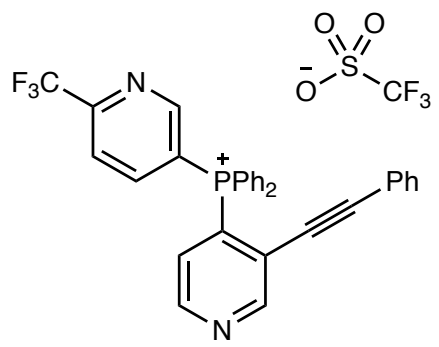

Prepared according to general procedure A, using 3-(phenylethynyl)pyridine (179 mg, 1.00 mmol), $\mathrm{Tf}_{2} \mathrm{O}(168 \mu \mathrm{L}, 1.00 \mathrm{mmol}), 5$-(diphenylphosphaneyl)-2-(trifluoromethyl)pyridine (364 $\mathrm{mg}, 1.10 \mathrm{mmol})$, DBU $(149 \mu \mathrm{L}, 1.00 \mathrm{mmol})$, and $\mathrm{CH}_{2} \mathrm{Cl}_{2}(10 \mathrm{~mL})$. After purification by the standard procedure, the title compound was isolated as a brown solid (444 mg, $0.67 \mathrm{mmol}, 67 \%$ yield). $\mathrm{mp} 69-72^{\circ} \mathrm{C}$. IR $v_{\max } / \mathrm{cm}^{-1}$ (film): 3062, 2361, 2213, 1439, 1335, 1259, 1142, 722, 563; ${ }^{1} \mathrm{H}$ NMR (400 MHz, $\left.\mathrm{CDCl}_{3}\right) \delta: 9.10(1 \mathrm{H}, \mathrm{d}, J=6.2 \mathrm{~Hz}), 8.93(1 \mathrm{H}$, app t, $J=4.8 \mathrm{~Hz}), 8.85(1 \mathrm{H}$, $\mathrm{dd}, J=6.1,2.1 \mathrm{~Hz}), 8.79(1 \mathrm{H}$, ddd, $J=12.9,4.5,2.0 \mathrm{~Hz}), 8.11(1 \mathrm{H}, \mathrm{dd}, J=7.7,1.5 \mathrm{~Hz}), 7.95-$ $7.87(2 \mathrm{H}, \mathrm{m}), 7.84-7.76(8 \mathrm{H}, \mathrm{m}), 7.42-7.30(2 \mathrm{H}, \mathrm{m}), 7.22-7.17(2 \mathrm{H}, \mathrm{m}), 6.69-6.65(2 \mathrm{H}, \mathrm{m}) ;{ }^{13} \mathrm{C}$ NMR $\left(100 \mathrm{MHz}, \mathrm{CDCl}_{3}\right) \delta: 154.6(\mathrm{~d}, J=6.7 \mathrm{~Hz}), 153.2(\mathrm{~d}, J=12.9 \mathrm{~Hz}), 153.0(\mathrm{qd}, J=35.9,2.5$ $\mathrm{Hz}), 150.6$ (d, $J=10.5 \mathrm{~Hz}), 145.7$ (d, $J=9.5 \mathrm{~Hz}), 136.5(\mathrm{~d}, J=3.1 \mathrm{~Hz}), 134.7$ (d, $J=11.0 \mathrm{~Hz})$, $131.2(\mathrm{~d}, J=13.5 \mathrm{~Hz}), 131.0,130.6,129.1(\mathrm{~d}, J=9.1 \mathrm{~Hz}), 128.7,127.1(\mathrm{~d}, J=87.6 \mathrm{~Hz}), 123.39$ $(\mathrm{d}, J=4.6 \mathrm{~Hz}), 122.5-122.3(\mathrm{~m}), 120.7$ (q, $J=321.0 \mathrm{~Hz}), 120.5(\mathrm{qd}, J=275.4,2.0 \mathrm{~Hz}), 119.4$, $118.2(\mathrm{~d}, J=88.3 \mathrm{~Hz}), 114.0(\mathrm{~d}, J=90.8 \mathrm{~Hz}), 105.2,84.1(\mathrm{~d}, J=6.5 \mathrm{~Hz}) ;{ }^{19} \mathrm{~F} \mathrm{NMR}(365 \mathrm{MHz}$, $\left.\mathrm{CDCl}_{3}\right) \delta:-68.68,-78.31 ;{ }^{31} \mathrm{P} \mathrm{NMR}\left(162 \mathrm{MHz}, \mathrm{CDCl}_{3}\right) \delta: 20.23 ; \mathrm{m} / \mathrm{z}$ LRMS (ESI + APCI) found $[\mathrm{M} \text { - OTf }]^{+}$509.2, $\mathrm{C}_{31} \mathrm{H}_{21} \mathrm{~F}_{3} \mathrm{~N}_{2} \mathrm{P}^{+}$requires 509.1 .

\section{Diphenyl(3-(pyridin-2-yloxy)pyridin-4-yl)(6-(trifluoromethyl)pyridin-3-yl)phosphonium trifluoromethanesulfonate}




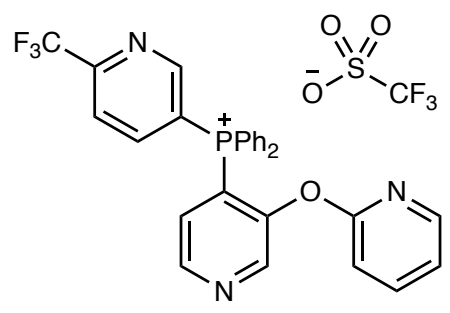

Prepared according to general procedure A, using 2-(pyridin-3-yloxy)pyridine (258 $\mathrm{mg}, 1.50$ $\mathrm{mmol}), \mathrm{Tf}_{2} \mathrm{O}(252 \mu \mathrm{L}, 1.50 \mathrm{mmol}), 5$-(diphenylphosphaneyl)-2-(trifluoromethyl)pyridine (547 $\mathrm{mg}, 1.65 \mathrm{mmol})$, DBU $(224 \mu \mathrm{L}, 1.50 \mathrm{mmol})$, and $\mathrm{CH}_{2} \mathrm{Cl}_{2}(15 \mathrm{~mL})$. After purification by the standard procedure, the title compound was isolated as a white solid ( $870 \mathrm{mg}, 1.34 \mathrm{mmol}, 89 \%$ yield). $\mathrm{mp} 50-55^{\circ} \mathrm{C}$. IR $v_{\max } / \mathrm{cm}^{-1}$ (film): 3064, 2248, 1430, 1529, 1140, 1029, 723, 635; ${ }^{1} \mathrm{H}$ NMR $\left(400 \mathrm{MHz}, \mathrm{CDCl}_{3}\right) \delta: 8.79-8.74(2 \mathrm{H}, \mathrm{m}), 8.68(1 \mathrm{H}$, app t, $J=4.3 \mathrm{~Hz}), 8.51(1 \mathrm{H}, \mathrm{ddd}, J=$ $13.1,8.2,2.2 \mathrm{~Hz}), 7.97(1 \mathrm{H}, \mathrm{ddd}, J=8.3,2.1,0.7 \mathrm{~Hz}), 7.88(1 \mathrm{H}$, ddd, $J=5.0,2.0,0.7 \mathrm{~Hz}), 7.83-$ $7.76(2 \mathrm{H}, \mathrm{m}), 7.72-7.64(8 \mathrm{H}, \mathrm{m}), 7.49(1 \mathrm{H}, \mathrm{ddd}, J=8.2,7.3,2.0 \mathrm{~Hz}), 7.32(1 \mathrm{H}, \mathrm{dd}, J=14.5,5.1$ $\mathrm{Hz}), 6.98(1 \mathrm{H}, \mathrm{ddd}, J=7.3,5.0,0.8 \mathrm{~Hz}), 6.33(1 \mathrm{H}, \mathrm{d}, J=8.3 \mathrm{~Hz}) ;{ }^{13} \mathrm{C} \mathrm{NMR}\left(100 \mathrm{MHz}, \mathrm{CDCl}_{3}\right)$ $\delta$ : $159.6,153.0(\mathrm{~d}, J=13.2 \mathrm{~Hz}), 152.8(\mathrm{qd}, J=34.7,2.7 \mathrm{~Hz}), 151.2,146.9,146.9(\mathrm{~d}, J=10.8$ $\mathrm{Hz}), 146.5(\mathrm{~d}, J=4.7 \mathrm{~Hz}), 145.2(\mathrm{~d}, J=9.7 \mathrm{~Hz}), 140.6,136.3(\mathrm{~d}, J=3.1 \mathrm{~Hz}), 134.3(\mathrm{~d}, J=11.4$ $\mathrm{Hz}), 131.1(\mathrm{~d}, J=13.6 \mathrm{~Hz}), 128.0(\mathrm{~d}, J=7.3 \mathrm{~Hz}), 122.3-122.1(\mathrm{~m}), 121.2,120.7(\mathrm{q}, J=321.0$ $\mathrm{Hz}), 120.4(\mathrm{qd}, J=275.7 \mathrm{~Hz}, 2.2 \mathrm{~Hz}), 118.3(\mathrm{~d}, J=87.2 \mathrm{~Hz}), 118.2(\mathrm{~d}, J=89.3 \mathrm{~Hz}), 114.0(\mathrm{~d}, J$ $=91.3 \mathrm{~Hz}), 111.2 ;{ }^{19} \mathrm{~F}$ NMR $\left(365 \mathrm{MHz}, \mathrm{CDCl}_{3}\right) \delta:-68.57,-78.30 ;{ }^{31} \mathrm{P} \mathrm{NMR}\left(162 \mathrm{MHz}, \mathrm{CDCl}_{3}\right)$ $\delta: 18.70 ; \mathrm{m} / \mathrm{z}$ LRMS (ESI + APCI) found [M - OTf] $]^{+} 502.2, \mathrm{C}_{28} \mathrm{H}_{20} \mathrm{~F}_{3} \mathrm{~N}_{3} \mathrm{OP}^{+}$requires 502.1.

\section{Diphenyl(5-(trifluoromethyl)-[2,3'-bipyridin]-4'-yl)(6-(trifluoromethyl)pyridin-3- yl)phosphonium trifluoromethanesulfonate}<smiles>O=S(=O)(O)C(F)(F)C(F)(F)F</smiles>

Prepared according to general procedure A using 5-(trifluoromethyl)-2,3'-bipyridine (336 mg, $1.50 \mathrm{mmol}), \mathrm{Tf}_{2} \mathrm{O}(252 \mu \mathrm{L}, 1.50 \mathrm{mmol}), 5$-(diphenylphosphanyl)-2-(trifluoromethyl)pyridine (546 mg, $1.65 \mathrm{mmol})$, DBU $(222 \mu \mathrm{L}, 1.50 \mathrm{mmol})$ and $\mathrm{CH}_{2} \mathrm{Cl}_{2}(15 \mathrm{~mL})$. After purification by the standard procedure, the title compound was isolated as a yellow solid $(720 \mathrm{mg}, 1.03 \mathrm{mmol}, 69 \%$ yield). $\mathrm{mp} 72-76^{\circ} \mathrm{C}$; IR $v_{\max } / \mathrm{cm}^{-1}$ (film): 1606, 1581, 1440, 1329, 1259, 1134, 1076, 1029, 719, $635 ;{ }^{1} \mathrm{H}$ NMR $\left(400 \mathrm{MHz}, \mathrm{CDCl}_{3}\right) \delta: 9.55(1 \mathrm{H}, \mathrm{d}, J=7.1 \mathrm{~Hz}), 9.02(1 \mathrm{H}, \mathrm{t}, J=4.9 \mathrm{~Hz}), 8.82(1 \mathrm{H}$, dd, $J=5.9,2.2 \mathrm{~Hz}), 8.60(1 \mathrm{H}$, ddd, $J=12.8,8.2,2.3 \mathrm{~Hz}), 8.28(1 \mathrm{H}, \mathrm{d}, J=8.4 \mathrm{~Hz}), 8.07-7.94$ $(2 \mathrm{H}, \mathrm{m}), 7.79(3 \mathrm{H}, \mathrm{qq}, J=4.3,2.0 \mathrm{~Hz}), 7.73-7.61(8 \mathrm{H}, \mathrm{m}), 7.39(1 \mathrm{H}, \mathrm{dd}, J=16.7,5.1 \mathrm{~Hz}) ;{ }^{13} \mathrm{C}$ NMR $\left(100 \mathrm{MHz}, \mathrm{CDCl}_{3}\right) \delta: 152.9(\mathrm{~d}, J=11.8 \mathrm{~Hz}), 152.7,151.8(\mathrm{q}, J=35.8 \mathrm{~Hz}), 151.2(\mathrm{~d}, J=$ $6.5 \mathrm{~Hz}), 144.6(\mathrm{~d}, J=8.8 \mathrm{~Hz}), 143.4,135.8(\mathrm{~d}, J=3.7 \mathrm{~Hz}), 135.1(\mathrm{~d}, J=3.1 \mathrm{~Hz}), 135.0,133.0$ (dd, $J=10.4,2.7 \mathrm{~Hz}), 131.45(\mathrm{~d}, J=10.6 \mathrm{~Hz}), 130.57(\mathrm{~d}, J=13.6 \mathrm{~Hz}), 126.97(\mathrm{q}, J=33.8 \mathrm{~Hz})$, $124.5,123.3(\mathrm{~d}, J=57.7 \mathrm{~Hz}), 122.2(\mathrm{~d}, J=273.0 \mathrm{~Hz}), 122.1,121.7(\mathrm{~d}, J=10.1 \mathrm{~Hz}), 120.9$, $120.5(\mathrm{q}, J=321.1 \mathrm{~Hz}), 120.4(\mathrm{qd}, J=275.1,1.9 \mathrm{~Hz}), 119.1(\mathrm{~d}, J=95.5 \mathrm{~Hz}) ;{ }^{19} \mathrm{~F}$ NMR $(365$ 
$\left.\mathrm{MHz}, \mathrm{CDCl}_{3}\right) \delta:-63.06,-68.64,-78.39 ;{ }^{31} \mathrm{P} \mathrm{NMR}\left(162 \mathrm{MHz}, \mathrm{CDCl}_{3}\right) \delta: 24.41 ; \mathrm{m} / z$ LRMS (ESI $+\mathrm{APCI})$ found $[\mathrm{M}-\mathrm{OTf}]^{+} 554.2, \mathrm{C}_{29} \mathrm{H}_{19} \mathrm{~F}_{6} \mathrm{~N}_{3} \mathrm{P}^{+}$requires 554.1 .

\section{(6,7-Dihydro-5H-cyclopenta[b]pyridin-4-yl)diphenyl(6-(trifluoromethyl)pyridin-3- yl)phosphonium trifluoromethanesulfonate}

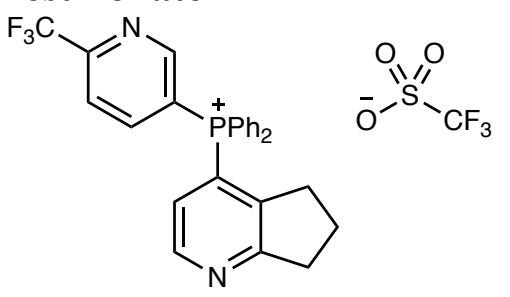

Prepared according to general procedure A using 6,7-dihydro-5H-cyclopenta[b]pyridine (119 mg, $1.00 \mathrm{mmol}), \mathrm{Tf}_{2} \mathrm{O}$ (169 $\left.\mu \mathrm{L}, 1.00 \mathrm{mmol}\right)$, 5-(diphenylphosphanyl)-2-(trifluoromethyl)pyridine (364 mg, $1.10 \mathrm{mmol})$, DBU $(147 \mu \mathrm{L}, 1.00 \mathrm{mmol})$ and $\mathrm{CH}_{2} \mathrm{Cl}_{2}(10 \mathrm{~mL})$. After purification by the standard procedure, the title compound was isolated as a white solid (300 mg, $0.50 \mathrm{mmol}, 50 \%$ yield). mp 65-68 ${ }^{\circ} \mathrm{C}$; IR $v_{\max } / \mathrm{cm}^{-1}$ (film): 1587, 1440, 1335, 1260, 1139, 1076, 1029, 724, 636; ${ }^{1} \mathrm{H}$ NMR $\left(400 \mathrm{MHz}, \mathrm{CDCl}_{3}\right) \delta: 8.87-8.64(3 \mathrm{H}, \mathrm{m}), 8.25(1 \mathrm{H}, \mathrm{ddd}, J=8.0,2.2,1.1 \mathrm{~Hz}), 8.02-7.90$ $(2 \mathrm{H}, \mathrm{m}), 7.84(4 \mathrm{H}, \mathrm{td}, J=7.8,3.9 \mathrm{~Hz}), 7.80-7.67(4 \mathrm{H}, \mathrm{m}), 7.02(1 \mathrm{H}, \mathrm{dd}, J=14.5,5.2 \mathrm{~Hz}), 3.17$ $(2 \mathrm{H}, \mathrm{t}, J=7.6 \mathrm{~Hz}), 2.37-2.23(2 \mathrm{H}, \mathrm{m}), 2.09(2 \mathrm{H}, \mathrm{q}, J=7.5 \mathrm{~Hz},) ;{ }^{13} \mathrm{C} \mathrm{NMR}\left(100 \mathrm{MHz}, \mathrm{CDCl}_{3}\right) \delta$ : $169.6(\mathrm{~d}, J=8.8 \mathrm{~Hz}), 153.2(\mathrm{qd}, J=36.1,2.5 \mathrm{~Hz}), 153.2(\mathrm{~d}, J=13.0 \mathrm{~Hz}), 150.0(\mathrm{~d}, J=9.9 \mathrm{~Hz})$, $145.8(\mathrm{~d}, J=9.4 \mathrm{~Hz}), 140.9(\mathrm{~d}, J=7.5 \mathrm{~Hz}), 136.7(\mathrm{~d}, J=3.2 \mathrm{~Hz}), 134.3(\mathrm{~d}, J=10.9 \mathrm{~Hz}), 131.5$ $(\mathrm{d}, J=13.4 \mathrm{~Hz}), 124.6(\mathrm{~d}, J=9.8 \mathrm{~Hz}), 122.8(\mathrm{dd}, J=10.1,2.6 \mathrm{~Hz}), 121.9(\mathrm{~d}, J=83.9 \mathrm{~Hz}), 120.6$ $(\mathrm{q}, J=321.0 \mathrm{~Hz}), 120.5(\mathrm{qd}, J=275.4,1.9 \mathrm{~Hz}), 118.1(\mathrm{~d}, J=86.5 \mathrm{~Hz}), 113.9(\mathrm{~d}, J=89.1 \mathrm{~Hz})$, $33.9(\mathrm{~d}, J=1.6 \mathrm{~Hz}), 32.4(\mathrm{~d}, J=2.5 \mathrm{~Hz}), 22.9 ;{ }^{19} \mathrm{~F} \mathrm{NMR}\left(365 \mathrm{MHz}, \mathrm{CDCl}_{3}\right) \delta$ : $-68.60,-78.37$; ${ }^{31} \mathrm{P}$ NMR (162 MHz, $\left.\mathrm{CDCl}_{3}\right) \delta: 17.86 ; \mathrm{m} / z$ LRMS (ESI + APCI) found [M - OTf] ${ }^{+} 449.2$, $\mathrm{C}_{26} \mathrm{H}_{21} \mathrm{~F}_{3} \mathrm{~N}_{2} \mathrm{P}^{+}$requires 449.1 .

\section{(3-Bromo-2-methylpyridin-4-yl)diphenyl(6-(trifluoromethyl)pyridin-3-yl)phosphonium} trifluoromethanesulfonate

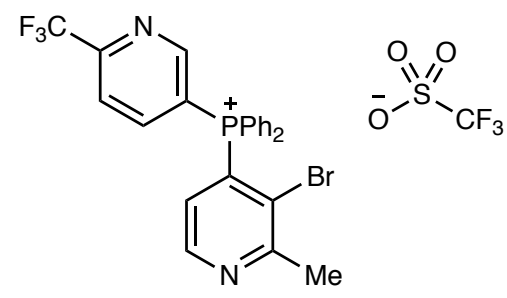

Prepared according to general procedure A using 3-bromo-2-methylpyridine (172 $\mathrm{mg}, 1.0 \mathrm{mmol}$ ), $\mathrm{Tf}_{2} \mathrm{O}(169 \mu \mathrm{L}, 1.00 \mathrm{mmol}), 5$-(diphenylphosphanyl)-2-(trifluoromethyl)pyridine (364 mg, 1.10 mmol), DBU $(147 \mu \mathrm{L}, 1.00 \mathrm{mmol})$ and $\mathrm{CH}_{2} \mathrm{Cl}_{2}(10 \mathrm{~mL})$. After purification by the standard procedure, the title compound was isolated as a yellow solid (360 mg, $0.56 \mathrm{mmol}, 56 \%$ yield). mp 56-60 ${ }^{\circ} \mathrm{C}$; IR $v_{\max } / \mathrm{cm}^{-1}$ (film): 3064, 1440, 1335, 1185, 1142, 1076, 1028, 722, 635; ${ }^{1} \mathrm{H}$ NMR $\left(400 \mathrm{MHz}, \mathrm{CDCl}_{3}\right) \delta:$ 8.93-8.78 $(3 \mathrm{H}, \mathrm{m}), 8.27-8.17(1 \mathrm{H}, \mathrm{m}), 7.99-7.90(2 \mathrm{H}, \mathrm{m}), 7.86-7.72(8 \mathrm{H}$, m), $7.12(1 \mathrm{H}, \mathrm{dd}, J=15.0,4.9 \mathrm{~Hz}), 2.83(3 \mathrm{H}, \mathrm{s}) ;{ }^{13} \mathrm{C} \mathrm{NMR}\left(100 \mathrm{MHz}, \mathrm{CDCl}_{3}\right) \delta: 161.9(\mathrm{~d}, J=$ $6.1 \mathrm{~Hz}), 153.2(\mathrm{~d}, J=12.9 \mathrm{~Hz}), 152.9(\mathrm{qd}, J=36.1,2.5 \mathrm{~Hz}), 149.9(\mathrm{~d}, J=11.6 \mathrm{~Hz}), 145.7(\mathrm{~d}, J=$ $9.4 \mathrm{~Hz}), 136.4$ (d, $J=3.2 \mathrm{~Hz}), 134.5$ (d, $J=10.9 \mathrm{~Hz}), 131.3(\mathrm{~d}, J=13.7 \mathrm{~Hz}), 129.3$ (d, $J=9.9$ $\mathrm{Hz}), 128.0(\mathrm{~d}, J=91.4 \mathrm{~Hz}), 124.8$ (d, $J=3.4 \mathrm{~Hz}), 122.5$ (dd, $J=10.4,2.9 \mathrm{~Hz}), 120.5$ (q, $J=$ 
$321.1 \mathrm{~Hz}), 120.4(\mathrm{qd}, J=275.2,1.6 \mathrm{~Hz}), 118.2(\mathrm{~d}, J=88.6 \mathrm{~Hz}), 114.0(\mathrm{~d}, J=91.0 \mathrm{~Hz}), 25.8(\mathrm{~d}$, $J=2.2 \mathrm{~Hz}) ;{ }^{19} \mathrm{~F}$ NMR $\left(365 \mathrm{MHz}, \mathrm{CDCl}_{3}\right) \delta:-68.60,-78.36 ;{ }^{31} \mathrm{P} \mathrm{NMR}\left(162 \mathrm{MHz}, \mathrm{CDCl}_{3}\right) \delta$ : 23.10; $\mathrm{m} / z$ LRMS (ESI + APCI) found [M - OTf] $]^{+}$501.1, $\mathrm{C}_{24} \mathrm{H}_{28} \mathrm{BrF}_{3} \mathrm{~N}_{2} \mathrm{P}^{+}$requires 501.0.

(2-Methyl-3-(thiophen-3-yl)pyridin-4-yl)diphenyl(6-(trifluoromethyl)pyridin-3yl)phosphonium trifluoromethanesulfonate

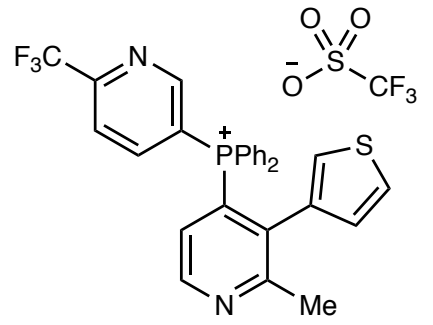

Prepared according to general procedure A, using 2-methyl-3-(thiophen-3-yl)pyridine (263 mg, $1.50 \mathrm{mmol}), \mathrm{Tf}_{2} \mathrm{O}(252 \mu \mathrm{L}, 1.50 \mathrm{mmol}), 5$-(diphenylphosphaneyl)-2-(trifluoromethyl)pyridine (547 mg, $1.65 \mathrm{mmol})$, DBU $(224 \mu \mathrm{L}, 1.50 \mathrm{mmol})$, and $\mathrm{CH}_{2} \mathrm{Cl}_{2}(15 \mathrm{~mL})$. After purification by the standard procedure (except that two crash-outs were done), the title compound was isolated as a white solid (489 mg, $0.75 \mathrm{mmol}, 50 \%$ yield). $\mathrm{mp} 66-71^{\circ} \mathrm{C}$. IR $v_{\max } / \mathrm{cm}^{-1}$ (film): 3066, 3013, 2248, $1439,1260,1144,1029,721,663 ;{ }^{1} \mathrm{H}$ NMR $\left(400 \mathrm{MHz}, \mathrm{CDCl}_{3}\right) \delta: 8.78(1 \mathrm{H}$, app t, $J=5.1 \mathrm{~Hz})$, $8.57(1 \mathrm{H}, \mathrm{ddd}, J=12.5,8.5,2.0 \mathrm{~Hz}), 8.49(1 \mathrm{H}, \mathrm{d}, J=5.7 \mathrm{~Hz}), 8.01-7.96(1 \mathrm{H}, \mathrm{m}), 7.91-7.82(2 \mathrm{H}$, m) 7.81-7.70 (4H, m), 7.70-7.61 (4H, m), $7.20(1 \mathrm{H}, \mathrm{dd}, J=15.1,5.2 \mathrm{~Hz}), 6.84(1 \mathrm{H}, \mathrm{ddd}, J=4.9$, 2.9, 0.4 Hz), 6.69-6.66 $(1 \mathrm{H}, \mathrm{m}), 6.27(1 \mathrm{H}, \mathrm{d}, J=5.0 \mathrm{~Hz}), 2.27(3 \mathrm{H}, \mathrm{s}) ;{ }^{13} \mathrm{C}$ NMR $(100 \mathrm{MHz}$, $\left.\mathrm{CDCl}_{3}\right) \delta: 162.4(\mathrm{~d}, J=8.0 \mathrm{~Hz}), 152.3(\mathrm{~d}, J=12.5 \mathrm{~Hz}), 151.9(\mathrm{qd}, J=35.7,2.4 \mathrm{~Hz}), 149.8(\mathrm{~d}, J$ $=11.9 \mathrm{~Hz}), 144.8(\mathrm{~d}, J=9.2 \mathrm{~Hz}), 136.2(\mathrm{~d}, J=5.1 \mathrm{~Hz}), 135.9(\mathrm{~d}, J=7.1 \mathrm{~Hz}), 134.9(\mathrm{~d}, J=5.5$ $\mathrm{Hz}), 134.4(\mathrm{~d}, J=10.5 \mathrm{~Hz}), 131.2(\mathrm{~d}, J=13.2 \mathrm{~Hz}), 128.4,127.0(\mathrm{~d}, J=66.1 \mathrm{~Hz}), 126.1(\mathrm{~d}, J=$ $86.2 \mathrm{~Hz}), 126.0(\mathrm{~d}, J=10.7 \mathrm{~Hz}), 122.1-121.9(\mathrm{~m}), 120.7$ (q, $J=321.0 \mathrm{~Hz}), 120.5(\mathrm{qd}, J=275.2$, $2.2 \mathrm{~Hz}), 119.6(\mathrm{~d}, J=87.0 \mathrm{~Hz}), 116.2(\mathrm{~d}, J=83.1 \mathrm{~Hz}), 115.3(\mathrm{~d}, J=83.9 \mathrm{~Hz}), 23.7(\mathrm{~d}, J=2.4$ $\mathrm{Hz}) ;{ }^{19} \mathrm{~F}$ NMR (365 MHz, $\left.\mathrm{CDCl}_{3}\right) \delta:-68.73,-78.30 ;{ }^{31} \mathrm{P} \mathrm{NMR}\left(162 \mathrm{MHz}, \mathrm{CDCl}_{3}\right) \delta: 19.25 ; \mathrm{m} / \mathrm{z}$ LRMS (ESI + APCI) found [M - OTf] $]^{+} 505.2, \mathrm{C}_{28} \mathrm{H}_{21} \mathrm{~F}_{3} \mathrm{~N}_{2} \mathrm{PS}^{+}$requires 515.1.

\section{(2-Cyano-3-methylpyridin-4-yl)diphenyl(6-(trifluoromethyl)pyridin-3-yl)phosphonium} trifluoromethanesulfonate

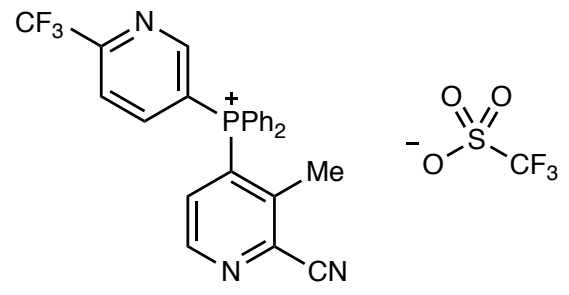

17:1 Mixture of Product:Impurity*

Prepared according to general procedure A, using 3-methylpicolinonitrile (118 $\mathrm{mg}, 1.00 \mathrm{mmol})$, $\mathrm{Tf}_{2} \mathrm{O}(168 \mu \mathrm{L}, 1.00 \mathrm{mmol})$, 5-(diphenylphosphaneyl)-2-(trifluoromethyl)pyridine (364 mg, 1.10 mmol), DBU $(149 \mu \mathrm{L}, 1.00 \mathrm{mmol})$, and $\mathrm{CH}_{2} \mathrm{Cl}_{2}(10 \mathrm{~mL})$. After purification by the standard procedure, the title compound was isolated as a yellow solid $(490 \mathrm{mg}, 0.82 \mathrm{mmol}, 82 \%$ yield, 17:1 mixture of product:impurity*); ${ }^{1} \mathrm{H}$ NMR $\left(400 \mathrm{MHz}, \mathrm{CDCl}_{3}\right) \delta: 8.86-8.80(2 \mathrm{H}, \mathrm{m}), 8.69(1 \mathrm{H}$, ddd, $J=13.0,8.4,2.2 \mathrm{~Hz}), 8.17(1 \mathrm{H}, \mathrm{dd}, J=8.3,1.8 \mathrm{~Hz}), 8.00-7.93(2 \mathrm{H}, \mathrm{m}), 7.87-7.75(8 \mathrm{H}, \mathrm{m})$, 
$7.51(1 \mathrm{H}, \mathrm{dd}, J=15.7,5.0 \mathrm{~Hz}), 2.23(3 \mathrm{H}, \mathrm{s}) ;{ }^{13} \mathrm{C} \mathrm{NMR}\left(100 \mathrm{MHz}, \mathrm{CD}_{3} \mathrm{CN}\right) \delta: 155.2(\mathrm{~d}, J=13.2$ hz), 153.5 (d, $J=35.6,2.4 \mathrm{~Hz}), 150.9$ (d, $J=11.7 \mathrm{~Hz}), 146.6(\mathrm{~d}, J=9.6 \mathrm{~Hz}), 142.7(\mathrm{~d}, J=8.2$ $\mathrm{Hz}), 138.8$ (d, $J=11.1 \mathrm{~Hz}), 137.5(\mathrm{~d}, J=3.2 \mathrm{~Hz}), 135.8$ (d, $J=11.2 \mathrm{~Hz}), 134.3$ (d, $J=10.4 \mathrm{~Hz})$, $132.1(\mathrm{~d}, J=13.9 \mathrm{~Hz}), 128.5(\mathrm{~d}, J=86.3 \mathrm{~Hz}), 123.2-123.3(\mathrm{~m}), 122.0(\mathrm{q}, J=321.0 \mathrm{~Hz}), 121.9$ $(\mathrm{qd}, J=276.0,3.2 \mathrm{~Hz}), 119.1(\mathrm{~d}, J=87.89 \mathrm{~Hz}), 116.4(\mathrm{~d}, J=7.6 \mathrm{~Hz}), 115.9(\mathrm{~d}, J=86.0 \mathrm{~Hz})$, $21.1(\mathrm{~d}, J=5.3 \mathrm{~Hz}) ;{ }^{19} \mathrm{~F}$ NMR $\left(365 \mathrm{MHz}, \mathrm{CD}_{3} \mathrm{CN}\right) \delta:-69.19,-79.20 ;{ }^{31} \mathrm{P} \mathrm{NMR}(162 \mathrm{MHz}$, $\left.\mathrm{CD}_{3} \mathrm{CN}\right) \delta: 20.21 ; \mathrm{m} / \mathrm{z}$ LRMS (ESI $\left.+\mathrm{APCI}\right)$ found $[\mathrm{M}-\mathrm{OTf}]^{+} 448.2, \mathrm{C}_{25} \mathrm{H}_{18} \mathrm{~F}_{3} \mathrm{~N}_{3} \mathrm{P}^{+}$requires 448.1 .

*based on LCMS analysis and ${ }^{31} \mathrm{P}$ NMR, we suspect that the impurity is the 2-position isomer.

\section{(2-Chloro-5-phenylpyridin-4-yl)diphenyl(6-(trifluoromethyl)pyridin-3-yl)phosphonium} trifluoromethanesulfonate

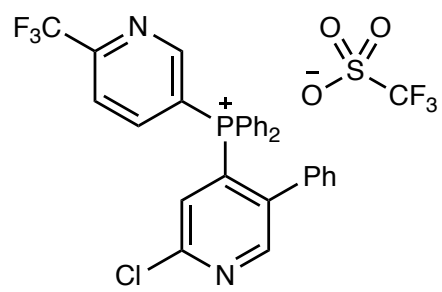

Prepared according to general procedure A, using 2-chloro-5-phenylpyridine (114 mg, 0.60 $\mathrm{mmol}), \mathrm{Tf}_{2} \mathrm{O}(101 \mu \mathrm{L}, 0.60 \mathrm{mmol}), 5$-(diphenylphosphaneyl)-2-(trifluoromethyl)pyridine (219 $\mathrm{mg}, 0.66 \mathrm{mmol})$, DBU $(90 \mu \mathrm{L}, 0.60 \mathrm{mmol})$, and $\mathrm{CH}_{2} \mathrm{Cl}_{2}(6 \mathrm{~mL})$. After purification by the standard procedure, the title compound was isolated as an amorphous solid (292 $\mathrm{mg}, 0.44 \mathrm{mmol}$, $73 \%$ yield). IR $v_{\max } / \mathrm{cm}^{-1}$ (film): 3604, 3013, 1560, 1441, 1259, 1029, 722, 666; ${ }^{1} \mathrm{H}$ NMR (400 $\left.\mathrm{MHz}, \mathrm{CDCl}_{3}\right) \delta: 8.80(1 \mathrm{H}, \mathrm{ddd}, J=12.8,8.2,2.1 \mathrm{~Hz}), 8.54(1 \mathrm{H}, \mathrm{d}, J=7.1 \mathrm{~Hz}), 8.35(1 \mathrm{H}, \mathrm{dd}, J=$ 5.9, $1.9 \mathrm{~Hz}), 7.97-7.91(3 \mathrm{H}, \mathrm{m}), 7.86-7.74(8 \mathrm{H}, \mathrm{m}), 7.38(1 \mathrm{H}, \mathrm{d}, J=15.2 \mathrm{~Hz}), 7.16(1 \mathrm{H}$, app td, $J$ $=7.5,1.0 \mathrm{~Hz}), 6.96(2 \mathrm{H}$, app t, $J=7.6 \mathrm{~Hz}), 6.81(2 \mathrm{H}, \mathrm{dd}, J=8.0,0.9 \mathrm{~Hz}) ;{ }^{13} \mathrm{C} \mathrm{NMR}(100 \mathrm{MHz}$, $\left.\mathrm{CDCl}_{3}\right) \delta: 153.9(\mathrm{~d}, J=9.1 \mathrm{~Hz}), 152.6(\mathrm{~d}, J=12.8 \mathrm{~Hz}), 152.4(\mathrm{~d}, J=15.7 \mathrm{~Hz}), 152.1(\mathrm{qd}, J=$ $36.4,2.7 \mathrm{~Hz}), 145.5(\mathrm{~d}, J=9.5 \mathrm{~Hz}), 140.5(\mathrm{~d}, J=7.2 \mathrm{~Hz}), 136.6(\mathrm{~d}, J=3.1 \mathrm{~Hz}), 134.7(\mathrm{~d}, J=$ $10.6 \mathrm{~Hz}), 133.2(\mathrm{~d}, J=3.9 \mathrm{~Hz}), 131.9(\mathrm{~d}, J=10.2 \mathrm{~Hz}), 131.3(\mathrm{~d}, J=13.4 \mathrm{~Hz}), 129.8,129.1(\mathrm{~d}, J$ $=80.9 \mathrm{~Hz}), 128.7(\mathrm{~d}, J=11.7 \mathrm{~Hz}), 128.2(\mathrm{~d}, J=84.9 \mathrm{~Hz}), 122.1-121.8(\mathrm{~m}), 120.7(\mathrm{q}, J=320.9$ $\mathrm{Hz}), 120.4$ (qd, $J=275.5,2.3 \mathrm{~Hz}), 118.4(\mathrm{~d}, J=87.4 \mathrm{~Hz}), 114.9(\mathrm{~d}, J=89.3 \mathrm{~Hz}) ;{ }^{19} \mathrm{~F}$ NMR $(365$ $\left.\mathrm{MHz}, \mathrm{CDCl}_{3}\right) \delta$ : $-68.97,-78.33 ;{ }^{31} \mathrm{P} \mathrm{NMR}\left(162 \mathrm{MHz} \mathrm{CDCl}_{3}\right) \delta: 19.20 ; \mathrm{m} / \mathrm{z} \mathrm{LRMS}(\mathrm{ESI}+$ APCI) found [M - OTf $]^{+} 519.2, \mathrm{C}_{29} \mathrm{H}_{20} \mathrm{ClF}_{3} \mathrm{~N}_{2} \mathrm{P}^{+}$requires 519.1 .

(5-(3-Chloro-5-fluorophenyl)-2-methylpyridin-4-yl)diphenyl(6-(trifluoromethyl)pyridin-3yl)phosphonium trifluoromethanesulfonate
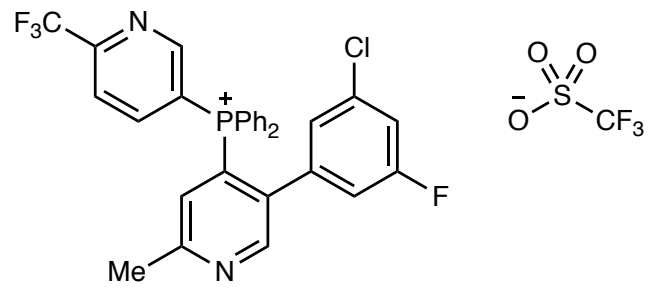

Prepared according to general procedure A using (3-chloro-5-fluorophenyl)-2-methylpyridine $(221 \mathrm{mg}, \quad 1.00 \mathrm{mmol}), \quad \mathrm{Tf}_{2} \mathrm{O} \quad(169 \mu \mathrm{L}, 1.00 \mu \mathrm{mmol}), \quad 5-($ diphenylphosphanyl)-2- 
(trifluoromethyl)pyridine (364 mg, $1.10 \mathrm{mmol})$, DBU (147 $\mu \mathrm{L}, 1.00 \mathrm{mmol})$ and $\mathrm{CH}_{2} \mathrm{Cl}_{2}(10 \mathrm{~mL})$. After purification by the standard procedure, the title compound was isolated as a white solid (434 mg, $0.62 \mathrm{mmol}, 62 \%$ yield). $\mathrm{mp} 100-102{ }^{\circ} \mathrm{C}$; IR $v_{\max } / \mathrm{cm}^{-1}$ (film): 1581, 1439, 1335, 1259, $1143,1075,1029,722,636 ;{ }^{1} \mathrm{H}$ NMR $\left(400 \mathrm{MHz}, \mathrm{CDCl}_{3}\right) \delta: 9.00-8.83(1 \mathrm{H}, \mathrm{m}), 8.63(1 \mathrm{H}, \mathrm{d}, J=$ $7.5 \mathrm{~Hz}), 8.52(1 \mathrm{H}, \mathrm{dd}, J=6.0,1.9 \mathrm{~Hz}), 8.10(1 \mathrm{H}, \mathrm{dd}, J=8.2,2.1 \mathrm{~Hz}), 7.98-7.86(2 \mathrm{H}, \mathrm{m}), 7.86-$ $7.69(8 \mathrm{H}, \mathrm{m}), 7.32(1 \mathrm{H}, \mathrm{d}, J=16.2 \mathrm{~Hz}), 6.85(1 \mathrm{H}, \mathrm{dt}, J=8.2,2.0 \mathrm{~Hz}), 6.54(1 \mathrm{H}, \mathrm{t}, J=1.7 \mathrm{~Hz})$, $6.44(1 \mathrm{H}, \mathrm{dt}, J=8.3,1.9 \mathrm{~Hz}), 2.72(3 \mathrm{H}, \mathrm{s}) ;{ }^{13} \mathrm{C} \mathrm{NMR}\left(100 \mathrm{MHz}, \mathrm{CDCl}_{3}\right) \delta: 161.7(\mathrm{~d}, J=254.1$ $\mathrm{Hz}), 161.3(\mathrm{~d}, J=10.5 \mathrm{~Hz}), 153.2-152.2(\mathrm{~m}), 152.6(\mathrm{qd}, J=36.0,2.4 \mathrm{~Hz}), 145.7(\mathrm{~d}, J=9.3 \mathrm{~Hz})$, $137.6(\mathrm{dd}, J=8.9,4.4 \mathrm{~Hz}), 136.5(\mathrm{~d}, J=3.2 \mathrm{~Hz}), 135.9-135.2(\mathrm{~m}), 134.6(\mathrm{~d}, J=10.7 \mathrm{~Hz}), 131.9$ $(\mathrm{d}, J=10.3 \mathrm{~Hz}), 131.2(\mathrm{~d}, J=13.3 \mathrm{~Hz}), 129.0(\mathrm{~d}, J=12.5 \mathrm{~Hz}), 128.1(\mathrm{~d}, J=9.7 \mathrm{~Hz}), 126.2(\mathrm{~d}, J$ $=3.3 \mathrm{~Hz}), 125.0(\mathrm{~d}, J=84.2 \mathrm{~Hz}), 122.1(\mathrm{~d}, J=10.1 \mathrm{~Hz}), 120.7(\mathrm{q}, J=320.8 \mathrm{~Hz}, 1 \mathrm{H}), 120.5(\mathrm{qd}$, $J=275.2,1.9 \mathrm{~Hz}), 119.4(\mathrm{~d}, J=65.1 \mathrm{~Hz}), 117.1(\mathrm{~d}, J=24.3 \mathrm{~Hz}), 115.6(\mathrm{~d}, J=22.6 \mathrm{~Hz}), 115.0$ (d, $J=89.0 \mathrm{~Hz}), 24.6(\mathrm{~d}, J=1.2 \mathrm{~Hz}) ;{ }^{19} \mathrm{~F}$ NMR $\left(365 \mathrm{MHz}, \mathrm{CDCl}_{3}\right) \delta:-68.80,-78.37,-108.46$; ${ }^{31} \mathrm{P}$ NMR $\left(162 \mathrm{MHz}, \mathrm{CDCl}_{3}\right) \delta: 18.93 ; \mathrm{m} / \mathrm{z}$ LRMS (ESI + APCI) found $[\mathrm{M}-\mathrm{OTf}]^{+} 551.2$, $\mathrm{C}_{30} \mathrm{H}_{21} \mathrm{ClF}_{4} \mathrm{~N}_{2} \mathrm{P}^{+}$requires 551.1.

\section{(2-Butyl-5-(trifluoromethyl)pyridin-4-yl)diphenyl(6-(trifluoromethyl)pyridin-3- yl)phosphonium trifluoromethanesulfonate (44)}

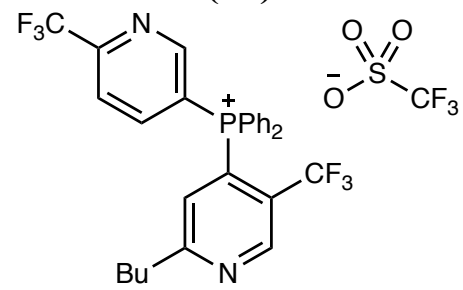

Prepared according to general procedure A, using 2-butyl-5-(trifluoromethyl)pyridine (305 $\mathrm{mg}$, $1.50 \mathrm{mmol}), \mathrm{Tf}_{2} \mathrm{O}(252 \mu \mathrm{L}, 1.50 \mathrm{mmol}), 5$-(diphenylphosphaneyl)-2-(trifluoromethyl)pyridine (547 mg, $1.65 \mathrm{mmol})$, DBU $(224 \mu \mathrm{L}, 1.50 \mathrm{mmol})$, and $\mathrm{CH}_{2} \mathrm{Cl}_{2}(15 \mathrm{~mL})$. After purification by the standard procedure (except that two crash-outs were required), the title compound was isolated as a white solid (723 mg, $1.06 \mathrm{mmol}, 71 \%$ yield). $\mathrm{mp} 58-60{ }^{\circ} \mathrm{C}$. IR $v_{\max } / \mathrm{cm}^{-1}$ (film): 2962,2874 , $1578,1259,1137,1029,722,636$; ${ }^{1} \mathrm{H}$ NMR $\left(400 \mathrm{MHz}, \mathrm{CDCl}_{3}\right) \delta: 9.19(1 \mathrm{H}, \mathrm{d}, J=7.4 \mathrm{~Hz}), 8.83-$ $8.76(2 \mathrm{H}, \mathrm{m}), 8.21-8.16(1 \mathrm{H}, \mathrm{m}), 7.97-7.91(2 \mathrm{H}, \mathrm{m}), 7.85-7.69(8 \mathrm{H}, \mathrm{m}), 7.22(1 \mathrm{H}, \mathrm{d}, J=17.6$ $\mathrm{Hz}), 2.95(2 \mathrm{H}, \mathrm{t}, J=7.8 \mathrm{~Hz}), 1.72-1.63(2 \mathrm{H}, \mathrm{m}), 1.37-1.27(2 \mathrm{H}, \mathrm{m}), 0.87(3 \mathrm{H}, \mathrm{t}, J=7.3 \mathrm{~Hz}) ;{ }^{13} \mathrm{C}$ NMR $\left(100 \mathrm{MHz}, \mathrm{CDCl}_{3}\right) \delta: 170.6(\mathrm{~d}, J=9.9 \mathrm{~Hz}), 153.2(\mathrm{qd}, J=35.5,2.5 \mathrm{~Hz}), 153.2(\mathrm{~d}, J=13.1$ Hz), 150.4-150.1 (m), 146.3 (d, $J=9.5 \mathrm{~Hz}), 136.7$ (d, $J=3.0 \mathrm{~Hz}), 134.7$ (d, $J=11.0 \mathrm{~Hz}), 131.2$ $(\mathrm{d}, J=13.7 \mathrm{~Hz}), 130.3(\mathrm{~d}, J=8.7 \mathrm{~Hz}), 124.1(\mathrm{~d}, J=80.8 \mathrm{~Hz}), 124.1(\mathrm{qd}, J=33.2,3.9 \mathrm{~Hz}), 122.6$ $(\mathrm{qd}, J=274.8,2.5 \mathrm{~Hz}), 122.6-122.3(\mathrm{~m}), 120.7(\mathrm{q}, J=321.0 \mathrm{~Hz}), 120.5(\mathrm{qd}, J=273.2,2.1 \mathrm{~Hz})$, $118.9(\mathrm{~d}, J=88.1 \mathrm{~Hz}), 114.9(\mathrm{~d}, J=90.5 \mathrm{~Hz}), 38.1,30.4,22.3,13.7 ;{ }^{19} \mathrm{~F}$ NMR $(365 \mathrm{MHz}$, $\left.\mathrm{CDCl}_{3}\right) \delta:-53,49,-68.68,-78.48 ;{ }^{31} \mathrm{P} \mathrm{NMR}\left(162 \mathrm{MHz}, \mathrm{CDCl}_{3}\right) \delta: 25.06 ; \mathrm{m} / \mathrm{z} \mathrm{LRMS}(\mathrm{ESI}+$ APCI) found $[\mathrm{M}-\mathrm{OTf}]^{+}$533.2, $\mathrm{C}_{28} \mathrm{H}_{24} \mathrm{~F}_{6} \mathrm{~N}_{2} \mathrm{P}^{+}$requires 533.2.

\section{(7-Bromoquinolin-4-yl)diphenyl(6-(trifluoromethyl)pyridin-3-yl)phosphonium trifluoromethanesulfonate}




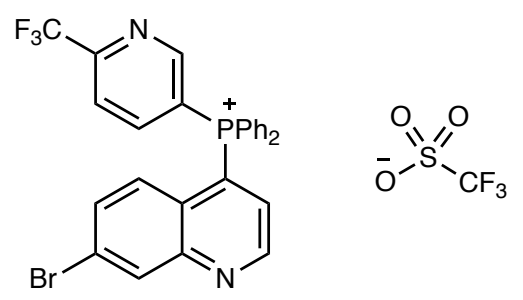

Prepared according to general procedure A (except that the reaction mixture was stirred for 60 min at $-30{ }^{\circ} \mathrm{C}$ instead of $\left.-50{ }^{\circ} \mathrm{C}\right)$ using 7-bromoquinoline $(208 \mathrm{mg}, 1.0 \mathrm{mmol}), \mathrm{Tf}_{2} \mathrm{O}(169 \mu \mathrm{L}$, $1.0 \mathrm{mmol}$ ), 5-(diphenylphosphanyl)-2-(trifluoromethyl)pyridine (364 mg, $1.1 \mathrm{mmol}), \mathrm{DBU}$ (147 $\mu \mathrm{L}, 1.0 \mathrm{mmol})$ and $\mathrm{CH}_{2} \mathrm{Cl}_{2}(10 \mathrm{~mL})$. After the purification procedure, the title compound was isolated as a white solid (560 mg, $0.82 \mathrm{mmol}, 82 \%$ yield). $\mathrm{mp} 134-137{ }^{\circ} \mathrm{C}$; IR $v_{\max } / \mathrm{cm}^{-1}$ (film): 1488, 1439, 1135, 1260, 1141, 1076, 1029, 723, 636; ${ }^{1} \mathrm{H}$ NMR (400 MHz, $\left.\mathrm{CDCl}_{3}\right) \delta: 9.22(1 \mathrm{H}, \mathrm{t}$, $J=4.4 \mathrm{~Hz}), 8.82-8.70(2 \mathrm{H}, \mathrm{m}), 8.56(1 \mathrm{H}, \mathrm{t}, J=2.0 \mathrm{~Hz}), 8.23-8.15(1 \mathrm{H}, \mathrm{m}), 7.98-7.89(2 \mathrm{H}, \mathrm{m})$, 7.86-7.70 (8H, m), 7.61-7.50 $(2 \mathrm{H}, \mathrm{m}), 7.20(1 \mathrm{H}, \mathrm{d}, J=9.0 \mathrm{~Hz}) ;{ }^{13} \mathrm{C} \mathrm{NMR}\left(100 \mathrm{MHz}, \mathrm{CDCl}_{3}\right) \delta$ : $153.4(\mathrm{qd}, J=36.2,2.4 \mathrm{~Hz}), 153.4(\mathrm{~d}, J=13.1 \mathrm{~Hz}), 151.7(\mathrm{~d}, J=12.3 \mathrm{~Hz}), 149.3(\mathrm{~d}, J=7.2 \mathrm{~Hz})$, $146.0(\mathrm{~d}, J=9.6 \mathrm{~Hz}), 136.8(\mathrm{~d}, J=3.2 \mathrm{~Hz}), 134.6(\mathrm{~d}, J=11.0 \mathrm{~Hz}), 134.3(\mathrm{~d}, J=2.3 \mathrm{~Hz}), 133.2$, $131.8(\mathrm{~d}, J=9.1 \mathrm{~Hz}), 131.6(\mathrm{~d}, J=13.5 \mathrm{~Hz}), 126.4(\mathrm{~d}, J=6.5 \mathrm{~Hz}), 125.7,124.3(\mathrm{~d}, J=6.7 \mathrm{~Hz})$, $122.8(\mathrm{dd}, J=10.3,2.7 \mathrm{~Hz}), 122.3(\mathrm{~d}, J=29.9 \mathrm{~Hz}), 120.6(\mathrm{q}, J=321.0 \mathrm{~Hz}), 120.5$ (qd, $J=275.3$, $1.8 \mathrm{~Hz}), 118.5(\mathrm{~d}, J=87.2 \mathrm{~Hz}), 114.6(\mathrm{~d}, J=89.1 \mathrm{~Hz}) ;{ }^{19} \mathrm{~F} \mathrm{NMR}\left(365 \mathrm{MHz}, \mathrm{CDCl}_{3}\right) \delta$ : $-68.63,-$ 78.39; ${ }^{31} \mathrm{P}$ NMR (162 MHz, $\left.\mathrm{CDCl}_{3}\right) \delta: 19.40 ; \mathrm{m} / z$ LRMS (ESI + APCI) found [M - OTf] ${ }^{+} 537.1$, $\mathrm{C}_{27} \mathrm{H}_{28} \mathrm{BrF}_{3} \mathrm{~N}_{2} \mathrm{P}^{+}$requires 537.0.

\section{Diphenyl(6-(thiophen-3-yl)quinolin-4-yl)(6-(trifluoromethyl)pyridin-3-yl)phosphonium trifluoromethanesulfonate}<smiles>O=S(=O)([O-])C(F)(F)F</smiles>

Prepared according to general procedure A using 6-(thiophen-3-yl)quinoline (211 mg, 1.00 mmol), $\mathrm{Tf}_{2} \mathrm{O}(169 \mu \mathrm{L}, 1.00 \mathrm{mmol}), 5$-(diphenylphosphanyl)-2-(trifluoromethyl)pyridine (382 $\mathrm{mg}, 1.10 \mathrm{mmol})$, DBU $(147 \mu \mathrm{L}, 1.00 \mathrm{mmol})$ and $\mathrm{CH}_{2} \mathrm{Cl}_{2}(10 \mathrm{~mL})$. After purification by the standard procedure, the title compound was isolated as a yellow solid (418 $\mathrm{mg}, 0.61 \mathrm{mmol}, 61 \%$ yield). $\mathrm{mp} 95-99^{\circ} \mathrm{C}$; IR $v_{\max } / \mathrm{cm}^{-1}$ (film): 1616, 1439, 1334, 1260, 1140, 1075, 1029, 723, 635; ${ }^{1} \mathrm{H}$ NMR $\left(400 \mathrm{MHz} \mathrm{CDCl}_{3}\right) \delta: 9.16(1 \mathrm{H}, \mathrm{t}, J=4.5 \mathrm{~Hz}), 8.86-8.72(2 \mathrm{H}, \mathrm{m}), 8.38(1 \mathrm{H}, \mathrm{dd}, J=8.8$, $2.2 \mathrm{~Hz}), 8.21(1 \mathrm{H}, \mathrm{dd}, J=8.7,2.3 \mathrm{~Hz}), 8.10(1 \mathrm{H}, \mathrm{dd}, J=8.8,1.9 \mathrm{~Hz}), 7.95(2 \mathrm{H}, \mathrm{ddt}, J=11.2$, 6.1, $2.8 \mathrm{~Hz}), 7.88-7.76(8 \mathrm{H}, \mathrm{m}), 7.53(1 \mathrm{H}, \mathrm{dd}, J=17.6,4.4 \mathrm{~Hz}), 7.39(1 \mathrm{H}, \mathrm{d}, J=1.9 \mathrm{~Hz}), 7.30$ $(1 \mathrm{H}, \mathrm{dd}, J=5.1,2.9 \mathrm{~Hz}), 7.12(1 \mathrm{H}, \mathrm{dd}, J=2.9,1.4 \mathrm{~Hz}), 6.68(1 \mathrm{H}, \mathrm{dd}, J=5.1,1.4 \mathrm{~Hz}){ }^{13} \mathrm{C} \mathrm{NMR}$ $\left(100 \mathrm{MHz}, \mathrm{CDCl}_{3}\right) \delta: 153.5(\mathrm{~d}, J=13.0 \mathrm{~Hz}), 153.4(\mathrm{~d}, J=35.5 \mathrm{~Hz}), 149.9(\mathrm{~d}, J=12.4 \mathrm{~Hz})$, $148.0(\mathrm{~d}, J=7.1 \mathrm{~Hz}), 146.1(\mathrm{~d}, J=9.5 \mathrm{~Hz}), 139.4,136.8,136.7(\mathrm{~d}, J=3.1 \mathrm{~Hz}), 134.4(\mathrm{~d}, J=$ $11.0 \mathrm{~Hz}), 132.6(\mathrm{~d}, J=2.3 \mathrm{~Hz}), 131.7,131.6(\mathrm{~d}, J=13.3 \mathrm{~Hz}), 130.5,127.8,126.3(\mathrm{~d}, J=6.5$ $\mathrm{Hz}), 125.3,123.2,122.8(\mathrm{~d}, J=10.4 \mathrm{~Hz}), 121.4,121.3(\mathrm{~d}, J=4.0 \mathrm{~Hz}), 120.7(\mathrm{q}, J=321.1 \mathrm{~Hz})$, 120.5 (q, $J=275.6 \mathrm{~Hz}), 118.7(\mathrm{~d}, J=68.3 \mathrm{~Hz}), 114.9(\mathrm{~d}, J=88.9 \mathrm{~Hz}) ;{ }^{19} \mathrm{~F}$ NMR $(365 \mathrm{MHz}$, 
$\left.\mathrm{CDCl}_{3}\right) \delta:-68.62,-78.35 ;{ }^{31} \mathrm{P} \mathrm{NMR}\left(162 \mathrm{MHz}, \mathrm{CDCl}_{3}\right) \delta: 19.32 ; \mathrm{m} / z$ LRMS (ESI + APCI) found $[\mathrm{M}-\mathrm{OTf}]^{+} 541.2, \mathrm{C}_{31} \mathrm{H}_{21} \mathrm{~F}_{3} \mathrm{~N}_{2} \mathrm{PS}^{+}$requires 541.1 .

\section{(4-Methylquinolin-2-yl)diphenyl(6-(trifluoromethyl)pyridin-3-yl)phosphonium trifluoromethanesulfonate}<smiles>Cc1cc([PH+](c2ccccc2)c2ccc(C(F)(F)F)nc2)nc2ccccc12</smiles>

Prepared according to general procedure A, using 4-methylquinoline (132 $\mu \mathrm{L}, 1.00 \mathrm{mmol}), \mathrm{Tf}_{2} \mathrm{O}$ (168 $\mu \mathrm{L}, 1.00 \mathrm{mmol})$, 5-(diphenylphosphaneyl)-2-(trifluoromethyl)pyridine (364 mg, 1.10 $\mathrm{mmol})$, DBU $(149 \mu \mathrm{L}, 1.00 \mathrm{mmol})$, and $\mathrm{CH}_{2} \mathrm{Cl}_{2}(10 \mathrm{~mL})$. After purification by the standard procedure, the title compound was isolated as a yellow solid (510 $\mathrm{mg}, 0.82 \mathrm{mmol}, 82 \%$ yield). mp 64-67 ${ }^{\circ} \mathrm{C}$. IR $v_{\max } / \mathrm{cm}^{-1}$ (film): $3065,1576,1334,1259,1140,1029,724,635 ;{ }^{1} \mathrm{H}$ NMR (400 $\left.\mathrm{MHz} \mathrm{CDCl}_{3}\right) \delta: 9.08(1 \mathrm{H}, \mathrm{dd}, J=5.5,1.7 \mathrm{~Hz}), 8.63(1 \mathrm{H}, \mathrm{ddd}, J=12.4,8.5,2.1 \mathrm{~Hz}), 8.19-8.15$ $(2 \mathrm{H}, \mathrm{m}), 8.13(1 \mathrm{H}, \mathrm{dd}, J=8.3,1.9 \mathrm{~Hz}), 7.95-7.87(3 \mathrm{H}, \mathrm{m}), 7.85-7.71(9 \mathrm{H}, \mathrm{m}), 7.65(1 \mathrm{H}, \mathrm{d}, J=$ $4.9 \mathrm{~Hz}), 2.83(3 \mathrm{H}, \mathrm{s}) ;{ }^{13} \mathrm{C} \mathrm{NMR}\left(100 \mathrm{MHz}, \mathrm{CDCl}_{3}\right) \delta: 154.2(\mathrm{~d}, J=11.7 \mathrm{~Hz}), 153.0$ (qd, $J=36.2$, 2.4), 149.5 (d, $J=11.1 \mathrm{~Hz}), 148.7(\mathrm{~d}, J=23.0), 145.9(\mathrm{~d}, J=8.8 \mathrm{~Hz}), 143.5,142.3,136.5(\mathrm{~d}, J=$ $3.0 \mathrm{~Hz}), 134.9(\mathrm{~d}, J=10.6 \mathrm{~Hz}), 132.1,131.2(\mathrm{~d}, J=13.1 \mathrm{~Hz}), 130.8(\mathrm{~d}, J=12.6 \mathrm{~Hz}), 129.2(\mathrm{~d}, J$ $=3.1 \mathrm{~Hz}), 125.2(\mathrm{~d}, J=109.5 \mathrm{~Hz}), 125.1(\mathrm{~d}, J=82.0 \mathrm{~Hz}), 122.2-122.0(\mathrm{~m}), 120.8(\mathrm{q}, J=321.0)$, $120.7(\mathrm{qd}, J=274.7,1.7 \mathrm{~Hz}), 119.8(\mathrm{~d}, J=87.3 \mathrm{~Hz}), 115.5(\mathrm{~d}, J=88.3 \mathrm{~Hz}), 19.3 ;{ }^{19} \mathrm{~F}$ NMR $(365$ $\left.\mathrm{MHz}, \mathrm{CDCl}_{3}\right) \delta:-68.71,-78.51 ;{ }^{31} \mathrm{P} \mathrm{NMR}\left(162 \mathrm{MHz}, \mathrm{CDCl}_{3}\right) \delta: 12.41 ; \mathrm{m} / \mathrm{z}$ LRMS (ESI + APCI) found $[\mathrm{M}-\mathrm{OTf}]^{+} 473.3, \mathrm{C}_{28} \mathrm{H}_{21} \mathrm{~F}_{3} \mathrm{~N}_{3} \mathrm{P}^{+}$requires 473.1 .

(5-Nitroisoquinolin-1-yl)diphenyl(6-(trifluoromethyl)pyridin-3-yl)phosphonium trifluoromethanesulfonate

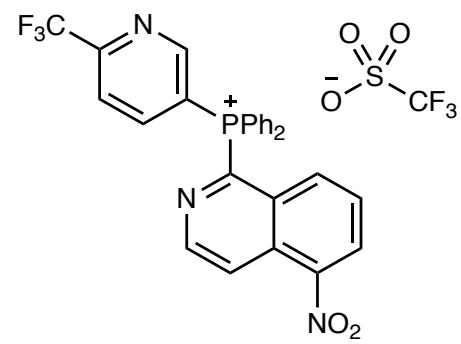

Prepared according to general procedure A, using 5-nitroisoquinoline (174 $\mathrm{mg}, 1.00 \mathrm{mmol})$, $\mathrm{Tf}_{2} \mathrm{O}(168 \mu \mathrm{L}, 1.00 \mathrm{mmol}), 5$-(diphenylphosphaneyl)-2-(trifluoromethyl)pyridine (364 mg, 1.10 mmol), DBU $(149 \mu \mathrm{L}, 1.00 \mathrm{mmol})$, and $\mathrm{CH}_{2} \mathrm{Cl}_{2}(10 \mathrm{~mL})$. After purification by the standard procedure, the title compound was isolated as an amorphous solid (471 mg, $0.72 \mathrm{mmol}, 72 \%$ yield). IR $v_{\max } / \mathrm{cm}^{-1}$ (film): $3067,3013,1530,1335,1262,1029,747,636 ;{ }^{1} \mathrm{H}$ NMR (400 MHz, $\left.\mathrm{CDCl}_{3}\right) \delta: 9.02(1 \mathrm{H}, \mathrm{d}, J=5.9 \mathrm{~Hz}), 8.89(1 \mathrm{H}, \mathrm{dd}, J=5.6,2.4 \mathrm{~Hz}), 8.83(1 \mathrm{H}, \mathrm{d}, J=4.7 \mathrm{~Hz}), 8.57$ $(1 \mathrm{H}, \mathrm{d}, J=7.7 \mathrm{~Hz}), 8.44(1 \mathrm{H}$, app t,$J=9.7 \mathrm{~Hz}), 8.05(1 \mathrm{H}, \mathrm{d}, J=8.1 \mathrm{~Hz}), 7.89-7.88(3 \mathrm{H}, \mathrm{m})$, 7.82-7.74 (9H, m); ${ }^{13} \mathrm{C} \mathrm{NMR}\left(100 \mathrm{MHz}, \mathrm{CDCl}_{3}\right) \delta: 154.5(\mathrm{~d}, J=12.1 \mathrm{~Hz}), 152.8(\mathrm{qd}, J=35.6$, $2.4 \mathrm{~Hz}), 146.3(\mathrm{~d}, J=42.7 \mathrm{~Hz}), 146.2(\mathrm{~d}, J=3.3 \mathrm{~Hz}), 146.1(\mathrm{~d}, J=29.4 \mathrm{~Hz}), 143.6$ (d, $J=121.2$ 
$\mathrm{Hz}), 136.8(\mathrm{~d}, J=3.1 \mathrm{~Hz}), 134.6(\mathrm{~d}, J=10.8 \mathrm{~Hz}), 131.7(\mathrm{~d}, J=13.3 \mathrm{~Hz}), 131.3(\mathrm{~d}, J=25.9 \mathrm{~Hz})$, 130.8 (d, $J=2.2 \mathrm{~Hz}), 130.0-129.9(2 \mathrm{C}, \mathrm{m}), 129.1$ (d, $J=12.6 \mathrm{~Hz}), 122.3$ (d, $J=3.8 \mathrm{~Hz}), 122.0-$ $121.7(\mathrm{~m}), 120.7$ (qd, $J=272.9,2.3 \mathrm{~Hz}), 120.7$ (q, $J=321.7 \mathrm{~Hz}), 120.4$ (d, $J=92.7 \mathrm{~Hz}), 115.2$ $(\mathrm{d}, J=85.7 \mathrm{~Hz}) ;{ }^{19} \mathrm{~F}$ NMR $\left(365 \mathrm{MHz}, \mathrm{CDCl}_{3}\right) \delta:-68.58,-78.37 ;{ }^{31} \mathrm{P} \mathrm{NMR}\left(162 \mathrm{MHz}, \mathrm{CDCl}_{3}\right) \delta$ : 19.30; m/z LRMS (ESI + APCI) found $[\mathrm{M}-\mathrm{OTf}]^{+}$504.2, $\mathrm{C}_{27} \mathrm{H}_{18} \mathrm{~F}_{3} \mathrm{~N}_{3} \mathrm{O}_{2} \mathrm{P}^{+}$requires 504.1.

\section{(1,5-Naphthyridin-4-yl)diphenyl(6-(trifluoromethyl)pyridin-3-yl)phosphonium} trifluoromethanesulfonate

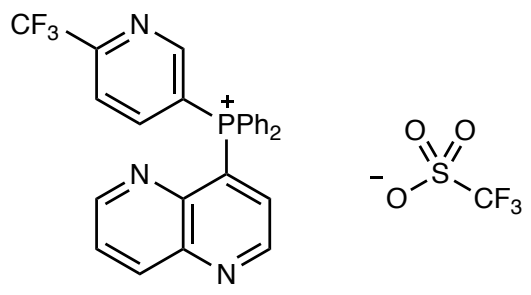

7:1 Mixture of Product:Impurity*

Prepared according to general procedure A using 1,5-naphthyridine (130 mg, $1.0 \mathrm{mmol}), \mathrm{Tf}_{2} \mathrm{O}$ (169 $\mu \mathrm{L}, 1.0 \mathrm{mmol})$, 5-(diphenylphosphanyl)-2-(trifluoromethyl)pyridine (382 $\mathrm{mg}, 1.1 \mathrm{mmol}$ ), DBU $(147 \mu \mathrm{L}, 1.0 \mathrm{mmol})$ and $\mathrm{CH}_{2} \mathrm{Cl}_{2}(10 \mathrm{~mL})$. After the purification procedure, the title compound was isolated as a yellow solid (440 $\mathrm{mg}, 0.73 \mathrm{mmol}, 73 \%$ yield, 7:1 mixture of product:impurity). $\mathrm{mp} 95-99{ }^{\circ} \mathrm{C}$; IR $v_{\max } / \mathrm{cm}^{-1}$ (film): 1622, 1519, 1324, 1265, 1240, 1175, 942, 729, 636; ${ }^{1} \mathrm{H}$ NMR (400 MHz, $\left.\mathrm{CDCl}_{3}\right) \delta: 9.36(1 \mathrm{H}, \mathrm{t}, J=4.6 \mathrm{~Hz}), 8.88(1 \mathrm{H}, \mathrm{dd}, J=6.4,2.1 \mathrm{~Hz})$, 8.74- $8.62(3 \mathrm{H}, \mathrm{m}), 7.89$ (3H, dq, $J=14.3,3.3,2.2 \mathrm{~Hz}), 7.84-7.64(10 \mathrm{H}, \mathrm{m}) ;{ }^{13} \mathrm{C} \mathrm{NMR}(100$ $\left.\mathrm{MHz} \mathrm{CDCl}_{3}\right) \delta: 153.2(\mathrm{~d}, J=13.2 \mathrm{~Hz}), 152.0,151.7(\mathrm{~d}, J=12.2 \mathrm{~Hz}), 145.1(\mathrm{~d}, J=9.6 \mathrm{~Hz})$, $144.5(\mathrm{~d}, J=5.8 \mathrm{~Hz}), 141.0(\mathrm{~d}, J=3.6 \mathrm{~Hz}), 138.6(\mathrm{~d}, J=2.4 \mathrm{~Hz}), 135.8$ (d, $J=3.3 \mathrm{~Hz}), 134.2(\mathrm{~d}$, $J=11.0 \mathrm{~Hz}), 133.2(\mathrm{~d}, J=7.7 \mathrm{~Hz}), 131.1(\mathrm{~d}, J=13.3 \mathrm{~Hz}), 130.7(\mathrm{~d}, J=13.6 \mathrm{~Hz}), 126.7,126.1$ $(\mathrm{d}, J=86.6 \mathrm{~Hz}), 123.2-121.2(\mathrm{~m}), 120.6(\mathrm{qd}, J=275.5,2.0 \mathrm{~Hz}), 120.5(\mathrm{q}, J=321.1 \mathrm{~Hz}), 120.0$ $(\mathrm{d}, J=90.1 \mathrm{~Hz}), 115.7(\mathrm{~d}, J=91.9 \mathrm{~Hz}) ;{ }^{19} \mathrm{~F} \mathrm{NMR}\left(365 \mathrm{MHz}, \mathrm{CDCl}_{3}\right) \delta:-68.59,-78.40 ;{ }^{31} \mathrm{P}$ NMR (162 MHz, $\left.\mathrm{CDCl}_{3}\right) \delta: 20.96 ; \mathrm{m} / z \mathrm{LRMS}(\mathrm{ESI}+\mathrm{APCI})$ found [M - OTf] $]^{+} 460.2$, $\mathrm{C}_{26} \mathrm{H}_{18} \mathrm{~F}_{3} \mathrm{~N}_{3} \mathrm{P}^{+}$requires 460.1 .

*based on LCMS analysis and ${ }^{31} \mathrm{P}$ NMR, we suspect that the impurity is the 2-position isomer.

\section{Diphenyl(quinoxalin-2-yl)(6-(trifluoromethyl)pyridin-3-yl)phosphonium trifluoromethanesulfonate}<smiles>O=S(=O)([O-])C(F)(F)F</smiles>

Prepared according to general procedure A, using quinoxaline (130 mg, $1.00 \mathrm{mmol}), \mathrm{Tf}_{2} \mathrm{O}(168$ $\mu \mathrm{L}, 1.00 \mathrm{mmol}$ ), 5-(diphenylphosphaneyl)-2-(trifluoromethyl)pyridine (364 mg, $1.10 \mathrm{mmol}$ ), DBU $(149 \mu \mathrm{L}, 1.00 \mathrm{mmol})$, and $\mathrm{CH}_{2} \mathrm{Cl}_{2}(10 \mathrm{~mL})$. After purification by the standard procedure, the title compound was isolated as a white solid (562 mg, $0.92 \mathrm{mmol}, 92 \%$ yield). mp 172-175 
${ }^{\circ} \mathrm{C}$. IR $v_{\max } / \mathrm{cm}^{-1}$ (film): 3065, 2922, 1440, 1260, 1141, 1029, 724, 634; ${ }^{1} \mathrm{H}$ NMR (400 MHz, $\left.\mathrm{CDCl}_{3}\right) \delta: 9.11-9.04(2 \mathrm{H}, \mathrm{m}), 8.74(1 \mathrm{H}, \mathrm{ddd}, J=12.3,8.6,1.4 \mathrm{~Hz}), 8.28-8.22(2 \mathrm{H}, \mathrm{m}), 8.17-8.11$ $(1 \mathrm{H}, \mathrm{dd}, J=8.2,1.1 \mathrm{~Hz}), 8.11-7.90(4 \mathrm{H}, \mathrm{m}), 7.87-7.75(8 \mathrm{H}, \mathrm{m}) ;{ }^{13} \mathrm{C} \mathrm{NMR}\left(100 \mathrm{MHz}, \mathrm{CDCl}_{3}\right) \delta$ : $154.0(\mathrm{~d}, J=12.1 \mathrm{~Hz}, 153.3(\mathrm{qd}, J=35.7,2.4 \mathrm{~Hz}), 146.4(\mathrm{~d}, J=7.3 \mathrm{~Hz}), 146.3(\mathrm{~d}, J=10.0 \mathrm{~Hz})$, $143.9(\mathrm{~d}, J=2.9 \mathrm{~Hz}), 142.9(\mathrm{~d}, J=17.5 \mathrm{~Hz}) 139.5(\mathrm{~d}, J=114.1 \mathrm{~Hz}), 136.9(\mathrm{~d}, J=3.1 \mathrm{~Hz})$, $135.4,135.1(\mathrm{~d}, J=10.9 \mathrm{~Hz}), 133.3,131.4(\mathrm{~d}, J=13.4 \mathrm{~Hz}), 130.3(\mathrm{~d}, J=1.0 \mathrm{~Hz}), 130.2(\mathrm{~d}, J=$ $2.6 \mathrm{~Hz}), 122.5-122.3(\mathrm{~m}), 120.7(\mathrm{qd}, J=275.2,1.7 \mathrm{~Hz}), 120.7(\mathrm{q}, J=321.0 \mathrm{~Hz}), 118.8(\mathrm{~d}, J=$ $88.0 \mathrm{~Hz}), 114.6(\mathrm{~d}, J=88.6 \mathrm{~Hz}) ;{ }^{19} \mathrm{~F} \mathrm{NMR}\left(365 \mathrm{MHz}, \mathrm{CDCl}_{3}\right) \delta:-68.62,-78.46 ;{ }^{31} \mathrm{P} \mathrm{NMR}(162$ $\left.\mathrm{MHz}, \mathrm{CDCl}_{3}\right) \delta: 11.78 ; \mathrm{m} / \mathrm{z}$ LRMS (ESI + APCI) found $[\mathrm{M}-\mathrm{OTf}]^{+} 460.2, \mathrm{C}_{26} \mathrm{H}_{18} \mathrm{~F}_{3} \mathrm{~N}_{3} \mathrm{P}^{+}$ requires 460.1 .

\section{Diphenyl(pyrazolo[1,5-a]pyrimidin-7-yl)(6-(trifluoromethyl)pyridin-3-yl)phosphonium} trifluoromethanesulfonate

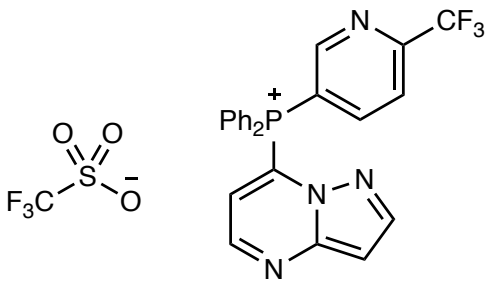

$>20: 1$ (Major:Unidentified Phosphonium) Mixture of Isomers

Prepared according to general procedure A using pyrazolo[1,5-a]pyrimidine (179 $\mathrm{mg}, 1.50$ $\mathrm{mmol}), \mathrm{Tf}_{2} \mathrm{O}(252 \mu \mathrm{L}, 1.50 \mathrm{mmol}), 5$-(diphenylphosphaneyl)-2-(trifluoromethyl)pyridine (546 $\mathrm{mg}, 1.65 \mathrm{mmol})$, DBU $(224 \mu \mathrm{L}, 1.50 \mathrm{mmol})$, and $\mathrm{CH}_{2} \mathrm{Cl}_{2}(15 \mathrm{~mL})$. After purification by the standard procedure, the title compound was isolated as a yellow solid (739 $\mathrm{mg}, 1.23 \mathrm{mmol}, 82 \%$ yield). Both Isomers, IR $v_{\max } / \mathrm{cm}^{-1}$ (film): 3066, 2925, 1726, 1603, 1260, 1121, 840, 766; Major Isomer: ${ }^{1} \mathrm{H} \mathrm{NMR}\left(400 \mathrm{MHz}, \mathrm{CDCl}_{3}\right) \delta: 8.95(1 \mathrm{H}, \mathrm{d}, J=6.4,2.1 \mathrm{~Hz}), 8.78-8.70(2 \mathrm{H}, \mathrm{m}), 8.07$ $(1 \mathrm{H}, \mathrm{ddd}, J=8.3,6.1,0.7 \mathrm{~Hz}), 7.98-7.91(3 \mathrm{H}, \mathrm{m}), 7.82-7.74(8 \mathrm{H}, \mathrm{m}), 7.29(1 \mathrm{H}, \mathrm{dd}, J=12.1,4.2$ $\mathrm{Hz}), 6.93(1 \mathrm{H}$, app t, $J=2.4 \mathrm{~Hz})$; Major Isomer: ${ }^{13} \mathrm{C} \mathrm{NMR}\left(100 \mathrm{MHz}, \mathrm{CDCl}_{3}\right) \delta: 153.8(\mathrm{~d}, J=$ $14.1 \mathrm{~Hz}), 153.5(\mathrm{qd}, J=36.4,2.8 \mathrm{~Hz}), 149.2(\mathrm{~d}, J=8.6 \mathrm{~Hz}), 148.9(\mathrm{~d}, J=1.4 \mathrm{~Hz}), 146.0(\mathrm{~d}, J=$ $9.8 \mathrm{~Hz}), 145.4,137.0$ (d, $J=3.2 \mathrm{~Hz}), 134.8(\mathrm{~d}, J=11.3 \mathrm{~Hz}), 131.4(\mathrm{~d}, J=14.2 \mathrm{~Hz}), 126.7(\mathrm{~d}, J=$ $99.5 \mathrm{~Hz}), 122.4-122.2(\mathrm{~m}), 121.3(\mathrm{~d}, J=8.8 \mathrm{~Hz}), 120.7(\mathrm{q}, J=320.7 \mathrm{~Hz}), 120.6(\mathrm{qd}, J=275.7$, $2.3 \mathrm{~Hz}), 116.8(\mathrm{~d}, J=90.9 \mathrm{~Hz}), 112.8(\mathrm{~d}, J=92.7 \mathrm{~Hz}), 100.2 ;{ }^{19} \mathrm{~F}$ NMR $\left(365 \mathrm{MHz}, \mathrm{CDCl}_{3}\right) \delta$ : 68.65, -78.43; ${ }^{31} \mathrm{P}$ NMR (162 MHz, $\left.\mathrm{CDCl}_{3}\right) \delta: 18.10$ (Major Isomer), 14.6 (Minor Isomer); $\mathrm{m} / \mathrm{z}$ LRMS (ESI + APCI) found [M - OTf] ${ }^{+} 449.3, \mathrm{C}_{24} \mathrm{H}_{17} \mathrm{~F}_{3} \mathrm{~N}_{4} \mathrm{P}^{+}$requires 449.1 .

Imidazo[1,5-a]pyrazin-8-yldiphenyl(6-(trifluoromethyl)pyridin-3-yl)phosphonium trifluoromethanesulfonate

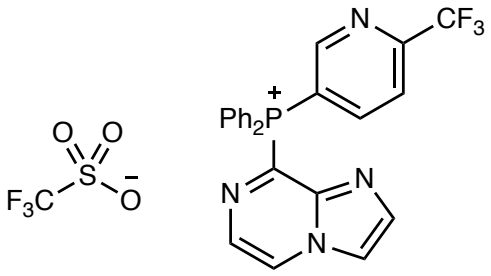


Prepared according to general procedure A, using imidazo[1,2-a]pyrazine (179 $\mathrm{mg}, 1.50 \mathrm{mmol}$ ), $\mathrm{Tf}_{2} \mathrm{O}(252 \mu \mathrm{L}, 1.50 \mathrm{mmol}), 5$-(diphenylphosphaneyl)-2-(trifluoromethyl)pyridine (547 mg, 1.65 mmol), DBU $(224 \mu \mathrm{L}, 1.50 \mathrm{mmol})$, and $\mathrm{CH}_{2} \mathrm{Cl}_{2}(15 \mathrm{~mL})$. After purification by the standard procedure (except that two crash-outs were done), the title compound was isolated as an amorphous solid (321 mg, $0.54 \mathrm{mmol}, 36 \%$ yield). IR $v_{\max } / \mathrm{cm}^{-1}$ (film): 3101, 3064, 2249, 1588, 1439, 1259, 1142, 1029, 724; ${ }^{1} \mathrm{H}$ NMR $\left(400 \mathrm{MHz}, \mathrm{CDCl}_{3}\right) \delta: 9.04(1 \mathrm{H}$, app t, $J=4.3 \mathrm{~Hz}), 8.99$ $(1 \mathrm{H}, \mathrm{dd}, J=5.9,2.1 \mathrm{~Hz}), 8.60(1 \mathrm{H}, \mathrm{ddd}, J=12.5,8.1,2.2 \mathrm{~Hz}), 8.35(1 \mathrm{H}, \mathrm{dd}, J=1.7,1.2 \mathrm{~Hz})$, $8.24(1 \mathrm{H}, \mathrm{dd}, J=4.4,1.0 \mathrm{~Hz}), 8.06(1 \mathrm{H}, \mathrm{ddd}, J=8.3,2.1,0.8 \mathrm{~Hz}), 7.93-7.87(2 \mathrm{H}, \mathrm{m}), 7.87-7.70$ $(9 \mathrm{H}, \mathrm{m}) ;{ }^{13} \mathrm{C} \mathrm{NMR}\left(100 \mathrm{MHz}, \mathrm{CDCl}_{3}\right) \delta: 154.6(\mathrm{~d}, J=12.5 \mathrm{~Hz}), 153.1(\mathrm{qd}, J=36.2,2.4 \mathrm{~Hz})$, $146.1(\mathrm{~d}, J=9.3 \mathrm{~Hz}), 140.5(\mathrm{~d}, J=33.9 \mathrm{~Hz}), 137.9,136.5(\mathrm{~d}, J=3.1 \mathrm{~Hz}), 135.0(\mathrm{~d}, J=11.1 \mathrm{~Hz})$, $133.8,130.9$ (d, $J=13.7 \mathrm{~Hz}), 130.6(\mathrm{~d}, J=22.1 \mathrm{~Hz}), 125.7(\mathrm{~d}, J=3.3 \mathrm{~Hz}), 121.7-121.5(\mathrm{~m})$, $120.8(\mathrm{q}, J=320.7 \mathrm{~Hz}), 120.7(\mathrm{qd}, J=275.2,1.6 \mathrm{~Hz}), 118.6(\mathrm{~d}, J=89.3 \mathrm{~Hz}), 116.9,114.8(\mathrm{~d}, J$ $=90.6 \mathrm{~Hz}) ;{ }^{19} \mathrm{~F}$ NMR $\left(365 \mathrm{MHz}, \mathrm{CDCl}_{3}\right) \delta:-68.62,-78.39 ;{ }^{31} \mathrm{P} \mathrm{NMR}\left(162 \mathrm{MHz}, \mathrm{CDCl}_{3}\right) \delta$ : $16.40 ; \mathrm{m} / \mathrm{z}$ LRMS (ESI + APCI) found $[\mathrm{M}-\mathrm{OTf}]^{+} 449.2, \mathrm{C}_{24} \mathrm{H}_{17} \mathrm{~F}_{3} \mathrm{~N}_{4} \mathrm{P}^{+}$requires 449.1 .

\section{(2-(3-(Pentafluoro- $\lambda^{6}$-sulfaneyl)phenyl)pyridin-4-yl)(phenyl)bis(6-(trifluoromethyl)pyridin-} 3-yl)phosphonium trifluoromethanesulfonate (41)

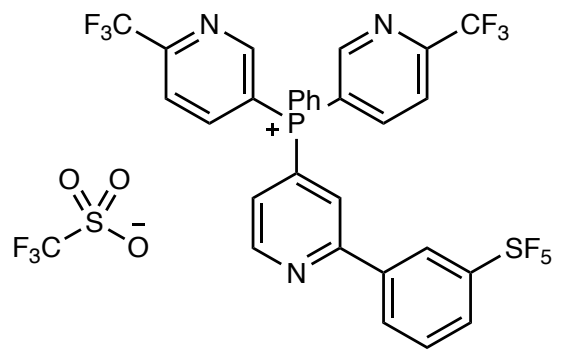

Prepared according to general procedure A (except that $\mathrm{Et}_{3} \mathrm{~N}$ was used as the base), using, 2-(3(pentafluoro- $\lambda 6$-sulfaneyl)phenyl)pyridine (422 $\mathrm{mg}, 1.50 \mathrm{mmol}), \mathrm{Tf}_{2} \mathrm{O}(252 \mu \mathrm{L}, 1.50 \mathrm{mmol})$, 5,5'-(phenylphosphanediyl)bis(2-(trifluoromethyl)pyridine (660 mg, $1.65 \mathrm{mmol}), \mathrm{Et}_{3} \mathrm{~N}(209 \mu \mathrm{L}$, $1.50 \mathrm{mmol})$, and $\mathrm{CH}_{2} \mathrm{Cl}_{2}(15 \mathrm{~mL})$. After purification by the standard procedure (except that a second crash-out using $100 \%$ diethyl ether was required), the title compound was isolated as a white solid (1.01 g, $1.22 \mathrm{mmol}, 80 \%$ yield). $\mathrm{mp} 82-86^{\circ} \mathrm{C}$. IR $v_{\max } / \mathrm{cm}^{-1}$ (film): $3066,1586,1441$, 1334, 1075, 1029, 839, 635; ${ }^{1} \mathrm{H}$ NMR (400 MHz, $\left.\mathrm{CDCl}_{3}\right) \delta: 9.09$ (1H, app t, $\left.J=5.4 \mathrm{~Hz}\right), 8.92$ $(2 \mathrm{H}, \mathrm{dd}, J=6.1,1.7 \mathrm{~Hz}), 8.60(2 \mathrm{H}, \mathrm{ddd}, J=13.1,4.8,2.0 \mathrm{~Hz}), 8.47(1 \mathrm{H}$, app t, $J=1.6 \mathrm{~Hz}), 8.13-$ $8.06(4 \mathrm{H}, \mathrm{m}), 8.01-7.94(1 \mathrm{H}, \mathrm{m}), 7.87-7.72(5 \mathrm{H}, \mathrm{m}), 7.60(1 \mathrm{H}, \mathrm{ddd}, J=13.7,8.7,1.4 \mathrm{~Hz}), 7.52$ $(1 \mathrm{H}$, app t, $J=8.0 \mathrm{~Hz}) ;{ }^{13} \mathrm{C} \mathrm{NMR}\left(100 \mathrm{MHz}, \mathrm{CDCl}_{3}\right) \delta: 158.1(\mathrm{~d}, J=12.0 \mathrm{~Hz}), 155.1-154.4$ $(\mathrm{m}), 154.1(\mathrm{qd}, J=36.3,2.4 \mathrm{~Hz}), 153.8(\mathrm{~d}, J=13.5 \mathrm{~Hz}), 152.3(\mathrm{~d}, J=11.3 \mathrm{~Hz}), 146.1(\mathrm{~d}, J=$ $10.0 \mathrm{~Hz}), 137.7,137.6(\mathrm{~d}, J=3.0 \mathrm{~Hz}), 135.0(\mathrm{~d}, J=11.1 \mathrm{~Hz}), 131.8(\mathrm{~d}, J=13.7 \mathrm{~Hz}), 130.7$, $129.9,128.0-127.8(\mathrm{~m}), 126.8(\mathrm{~d}, J=86.1 \mathrm{~Hz}), 126.3(\mathrm{~d}, J=8.9 \mathrm{~Hz}), 125.4-125.2(\mathrm{~m}) 124.1$ (d, $J=9.5 \mathrm{~Hz}), 122.8-122.5(\mathrm{~m}), 120.5(\mathrm{qd}, J=275.8,2.2 \mathrm{~Hz}), 120.4(\mathrm{q}, J=320.3 \mathrm{~Hz}), 116.5(\mathrm{~d}, J$ $=88.5 \mathrm{~Hz}), 112.6(\mathrm{~d}, J=86.2 \mathrm{~Hz}) ;{ }^{19} \mathrm{~F} \mathrm{NMR}\left(365 \mathrm{MHz}, \mathrm{CDCl}_{3}\right) \delta: 83.61(1 \mathrm{~F}, \mathrm{qn}, J=150.6 \mathrm{~Hz})$, 62.55 (4F, d, $J=150.0 \mathrm{~Hz}),-68.78,-78.80 ;{ }^{31} \mathrm{P}$ NMR $\left(162 \mathrm{MHz}, \mathrm{CDCl}_{3}\right) \delta: 18.64 ; \mathrm{m} / \mathrm{z} \mathrm{LRMS}$ $(\mathrm{ESI}+\mathrm{APCI})$ found $[\mathrm{M}-\mathrm{OTf}]^{+} 680.2, \mathrm{C}_{29} \mathrm{H}_{18} \mathrm{~F}_{11} \mathrm{~N}_{3} \mathrm{PS}^{+}$requires 680.1.

(2-(1,3-Dioxolan-2-yl)pyridin-4-yl)(phenyl)bis(6-(trifluoromethyl)pyridin-3yl)phosphonium trifluoromethanesulfonate 


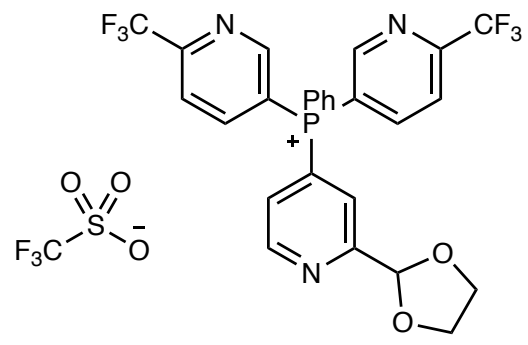

Prepared according to general procedure A, using 2-(1,3-dioxolan-2-yl)pyridine (151 mg, 1.00 $\mathrm{mmol}), \mathrm{Tf}_{2} \mathrm{O}(168 \mu \mathrm{L}, 1.00 \mathrm{mmol}), 5,5$ '-(phenylphosphanediyl)bis(2-(trifluoromethyl)pyridine (440 mg, $1.10 \mathrm{mmol})$, DBU $(149 \mu \mathrm{L}, 1.00 \mathrm{mmol})$, and $\mathrm{CH}_{2} \mathrm{Cl}_{2}(10 \mathrm{~mL})$. After purification by the standard procedure (except that two crash-outs were done), the title compound was isolated as a brown solid (330 mg, $0.47 \mathrm{mmol}, 47 \%$ yield). $\mathrm{mp} 67-72{ }^{\circ} \mathrm{C}$. IR $v_{\max } / \mathrm{cm}^{-1}$ (film): 3066, 2900, $1334,1258,1138,1074,725,636 ;{ }^{1} \mathrm{H}$ NMR $\left(400 \mathrm{MHz}, \mathrm{CDCl}_{3}\right) \delta: 8.99(1 \mathrm{H}$, app t, $J=4.9 \mathrm{~Hz})$, $8.87(2 \mathrm{H}, \mathrm{d}, J=5.0 \mathrm{~Hz}), 8.57(2 \mathrm{H}, \mathrm{ddd}, J=13.1,8.4,1.7 \mathrm{~Hz}), 8.10(2 \mathrm{H}, \mathrm{dd}, J=8.3,1.3 \mathrm{~Hz})$, $7.98(1 \mathrm{H}$, app t, $J=7.7 \mathrm{~Hz}), 7.86-7.78(2 \mathrm{H}, \mathrm{m}), 7.77-7.69(4 \mathrm{H}, \mathrm{m}), 5.86(1 \mathrm{H}, \mathrm{s}), 4.07-3.95(4 \mathrm{H}$, m); ${ }^{13} \mathrm{C}$ NMR (100 MHz, $\left.\mathrm{CDCl}_{3}\right) \delta: 160.4(\mathrm{~d}, J=10.1 \mathrm{~Hz}), 153.9(\mathrm{qd}, J=36.2,2.6 \mathrm{~Hz}), 153.7$ $(\mathrm{d}, J=13.5 \mathrm{~Hz}), 152.1(\mathrm{~d}, J=10.7 \mathrm{~Hz}), 146.1(\mathrm{~d}, J=9.8 \mathrm{~Hz}), 137.5(\mathrm{~d}, J=3.1 \mathrm{~Hz}), 135.0(\mathrm{~d}, J=$ $11.3 \mathrm{~Hz}), 131.7(\mathrm{~d}, J=13.6 \mathrm{~Hz}), 128.1(\mathrm{~d}, J=9.0 \mathrm{~Hz}), 126.3(\mathrm{~d}, J=85.5 \mathrm{~Hz}), 123.9(\mathrm{~d}, J=9.6$ $\mathrm{Hz}), 122.57-122.55(\mathrm{~m}), 122.55(\mathrm{qd}, J=275.3,2.2 \mathrm{~Hz}), 120.4(\mathrm{q}, J=320.8 \mathrm{~Hz}), 116.5(\mathrm{~d}, J=$ $88.5 \mathrm{~Hz}), 112.4(\mathrm{~d}, J=90.2 \mathrm{~Hz}), 101.2(\mathrm{~d}, J=1.9 \mathrm{~Hz}), 65.9 ;{ }^{19} \mathrm{~F} \mathrm{NMR}\left(365 \mathrm{MHz}, \mathrm{CDCl}_{3}\right) \delta:-$ $68.78,-78.78 ;{ }^{31} \mathrm{P}$ NMR $\left(162 \mathrm{MHz}, \mathrm{CDCl}_{3}\right) \delta: 18.42 ; \mathrm{m} / \mathrm{z}$ LRMS (ESI + APCI) found [M OTf] $]^{+} 550.2, \mathrm{C}_{26} \mathrm{H}_{19} \mathrm{~F}_{6} \mathrm{~N}_{3} \mathrm{O}_{2} \mathrm{P}^{+}$requires 550.1 .

\section{(2-(((Tert-butyldimethylsilyl)oxy)(phenyl)methyl)pyridin-4-yl)(phenyl)bis(6- (trifluoromethyl)pyridin-3-yl)phosphonium trifluoromethanesulfonate}

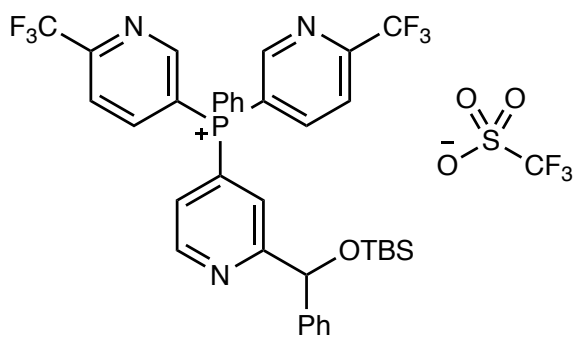

Prepared according to general procedure A using 2-(((tert-butyldimethylsilyl)oxy) (phenyl)methyl)pyridine $(300 \quad \mathrm{mg}, \quad 1.00 \quad \mathrm{mmol}), \quad \mathrm{Tf}_{2} \mathrm{O} \quad\left(\begin{array}{lllll}169 & \mu \mathrm{L}, & 1.00 & \mathrm{mmol}\end{array}\right), \quad 5,5^{\prime}-$ (phenylphosphanediyl) bis(2-(trifluoromethyl)pyridine) $(440 \mathrm{mg}, 1.10 \mathrm{mmol}), \mathrm{DBU}(147 \mu \mathrm{L}$, $1.00 \mathrm{mmol})$ and $\mathrm{CH}_{2} \mathrm{Cl}_{2}(10 \mathrm{~mL})$. After purification by the standard procedure, the title compound was isolated as a yellow solid $\left(510 \mathrm{mg}, 0.60 \mathrm{mmol}, 60 \%\right.$ yield). $\mathrm{mp} 70-74{ }^{\circ} \mathrm{C}$; IR $v_{\max } / \mathrm{cm}^{-1}$ (film): 2954, 2932, 2859, 1373, 1333, 1257, 1142, 1075, 1029, 838, 725, 636; ${ }^{1} \mathrm{H}$ NMR $\left(400 \mathrm{MHz}, \mathrm{CDCl}_{3}\right) \delta:{ }^{1} \mathrm{H}$ NMR $(400 \mathrm{MHz},) \delta 9.07(1 \mathrm{H}, \mathrm{t}, J=5.2 \mathrm{~Hz}), 8.94(2 \mathrm{H}$, ddd, $J=8.3$, $6.1,2.0 \mathrm{~Hz}), 8.87-8.74(2 \mathrm{H}, \mathrm{m}), 8.28(2 \mathrm{H}, \mathrm{dt}, J=8.4,2.7 \mathrm{~Hz}), 8.18-8.06(1 \mathrm{H}, \mathrm{m}), 8.00-7.77(5 \mathrm{H}$, m), $7.71(1 \mathrm{H}, \mathrm{ddd}, J=13.5,5.1,1.9 \mathrm{~Hz}), 7.52-7.39(5 \mathrm{H}, \mathrm{m}), 6.08(1 \mathrm{H}, \mathrm{s}), 0.84(9 \mathrm{H}, \mathrm{s}), 0.07(3 \mathrm{H}$, s), $0.00(3 \mathrm{H}, \mathrm{s}) ;{ }^{13} \mathrm{C}$ NMR $\left(100 \mathrm{MHz}, \mathrm{CDCl}_{3}\right) \delta: 167.4(\mathrm{~d}, J=9.7 \mathrm{~Hz}), 153.8(J=35.8,1.8 \mathrm{~Hz})$, $153.6(\mathrm{~d}, J=13.4 \mathrm{~Hz}), 151.7(\mathrm{~d}, J=10.9 \mathrm{~Hz}), 146.0(\mathrm{dd}, J=9.8,1.9 \mathrm{~Hz}), 142.1,137.3(\mathrm{~d}, J=$ $3.2 \mathrm{~Hz}), 134.9(\mathrm{~d}, J=11.3 \mathrm{~Hz}), 131.6(\mathrm{~d}, J=13.8 \mathrm{~Hz}), 128.7,128.1,126.5,126.0(\mathrm{~d}, J=84.9$ 
$\mathrm{Hz}), 125.9(\mathrm{~d}, J=9.0 \mathrm{~Hz}), 122.5(\mathrm{~d}, J=10.1 \mathrm{~Hz}), 120.5(\mathrm{qd}, J=275.4,1.8 \mathrm{~Hz}), 120.4(\mathrm{q}, J=$ $320.7 \mathrm{~Hz}), 116.5(\mathrm{dd}, J=88.3,5.3 \mathrm{~Hz}), 112.4(\mathrm{~d}, J=90.2 \mathrm{~Hz}), 77.2(\mathrm{~d}, J=1.7 \mathrm{~Hz}), 25.6,17.9,-$ $5.1(\mathrm{~d}, J=39.0 \mathrm{~Hz}) ;{ }^{19} \mathrm{~F}$ NMR $\left(365 \mathrm{MHz}, \mathrm{CDCl}_{3}\right) \delta:-68.70,-78.64 ;{ }^{31} \mathrm{P}$ NMR $(162 \mathrm{MHz}$, $\left.\mathrm{CDCl}_{3}\right) \delta: 18.49 ; \mathrm{m} / z$ LRMS (ESI $\left.+\mathrm{APCI}\right)$ found $[\mathrm{M}-\mathrm{OTf}]^{+} 698.3, \mathrm{C}_{36} \mathrm{H}_{35} \mathrm{~F}_{6} \mathrm{~N}_{3} \mathrm{OPSi}^{+}$requires 698.2.

(2-((4-Chlorophenyl)((1-(ethoxycarbonyl)piperidin-4-yl)oxy)methyl)pyridin-4yl)(phenyl)bis(6-(trifluoromethyl)pyridin-3-yl)phosphonium trifluoromethanesulfonate<smiles>CCON1CCC(OC(c2ccc(Cl)cc2)c2cc([P+](c3ccc(C(F)(F)F)nc3)c3ccc(C(F)(F)F)nc3)ccn2)CC1</smiles>

Prepared according to general procedure A using ethyl 4-((4-chlorophenyl) (pyridin-2yl)methoxy)piperidine-1-carboxylate $(450 \mathrm{mg}, 1.20 \mathrm{mmol}), \mathrm{Tf}_{2} \mathrm{O}(202 \mu \mathrm{L}, 1.20 \mathrm{mmol}), 5,5^{\prime}-$ (phenylphosphanediyl)bis(2-(trifluoromethyl)pyridine) $(528 \mathrm{mg}, 1.44 \mathrm{mmol}), \mathrm{DBU}(176 \mu \mathrm{L}$, $1.20 \mathrm{mmol})$ and $\mathrm{CH}_{2} \mathrm{Cl}_{2}(12 \mathrm{~mL})$. After purification by the standard procedure, the title compound was isolated as a yellow solid (700 $\mathrm{mg}, 0.94 \mathrm{mmol}, 63 \%$ yield). $\mathrm{mp} 103-105{ }^{\circ} \mathrm{C}$; IR $v_{\max } / \mathrm{cm}^{-1}$ (film): $3063,2930,1682,1439,1334,1259,1142,1075,1029,725,636 ;{ }^{1} \mathrm{H}$ NMR (400 $\left.\mathrm{MHz}, \mathrm{CDCl}_{3}\right) \delta: 9.01-8.80(3 \mathrm{H}, \mathrm{m}), 8.73-8.59(2 \mathrm{H}, \mathrm{m}), 8.14(2 \mathrm{H}, \mathrm{dt}, J=8.6,2.2 \mathrm{~Hz}), 8.07-7.93$ $(1 \mathrm{H}, \mathrm{m}), 7.92-7.64(5 \mathrm{H}, \mathrm{m}), 7.57(1 \mathrm{H}$, ddd, $J=13.5,5.1,1.7 \mathrm{~Hz}), 7.36-7.28(4 \mathrm{H}, \mathrm{m}), 5.73(1 \mathrm{H}$, s), $4.10(2 \mathrm{H}, \mathrm{q}, J=7.1 \mathrm{~Hz}), 3.72-3.44(3 \mathrm{H}, \mathrm{m}), 3.14(2 \mathrm{H}, \mathrm{ddt}, J=12.3,7.6,3.7 \mathrm{~Hz}), 1.78-1.56$ $(3 \mathrm{H}, \mathrm{m}), 1.45(2 \mathrm{H}, \mathrm{dtd}, J=12.4,7.9,3.8 \mathrm{~Hz}), 1.24(3 \mathrm{H}, \mathrm{t}, J=7.1 \mathrm{~Hz}) ;{ }^{13} \mathrm{C}$ NMR $(100 \mathrm{MHz}$, $\left.\mathrm{CDCl}_{3}\right) \delta: 165.1(\mathrm{~d}, J=10.1 \mathrm{~Hz}), 155.5,153.9(\mathrm{qd}, J=36.3,2.5 \mathrm{~Hz}), 153.7(\mathrm{~d}, J=13.4 \mathrm{~Hz})$, $151.7(\mathrm{~d}, J=10.8 \mathrm{~Hz}), 146.1(\mathrm{~d}, J=9.7 \mathrm{~Hz}), 138.5,137.4(\mathrm{~d}, J=3.2 \mathrm{~Hz}), 135.0(\mathrm{~d}, J=11.3 \mathrm{~Hz})$, 134.2, $131.6(\mathrm{~d}, J=13.6 \mathrm{~Hz}), 128.9(\mathrm{~d}, J=26.9 \mathrm{~Hz}), 126.3(\mathrm{~d}, J=38.4 \mathrm{~Hz}), 125.9$ (d, $J=38.0$ $\mathrm{Hz}), 123.7(\mathrm{~d}, J=10.0 \mathrm{~Hz}), 122.5(\mathrm{~d}, J=10.5 \mathrm{~Hz}), 120.5(\mathrm{qd}, J=275.6,2.1 \mathrm{~Hz}), 120.4(\mathrm{q}, J=$ $320.8 \mathrm{~Hz}), 116.5$ (dd, $J=88.3,3.8 \mathrm{~Hz}), 112.6(\mathrm{~d}, J=90.3 \mathrm{~Hz}), 80.0,73.1,61.4,40.8$ (d, $J=6.0$ $\mathrm{Hz}), 30.9(\mathrm{~d}, J=94.6 \mathrm{~Hz}), 14.7 ;{ }^{19} \mathrm{~F}$ NMR $\left(365 \mathrm{MHz}, \mathrm{CDCl}_{3}\right) \delta:-68.74,-78.68 ;{ }^{31} \mathrm{P}$ NMR $(162$ $\left.\mathrm{MHz}, \mathrm{CDCl}_{3}\right) \delta: 18.51 ; \mathrm{m} / z$ LRMS (ESI + APCI) found $[\mathrm{M}-\mathrm{OTf}]^{+} 773.2, \mathrm{C}_{38} \mathrm{H}_{33} \mathrm{ClF}_{6} \mathrm{~N}_{4} \mathrm{O}_{3} \mathrm{P}^{+}$ requires 773.2 .

(3-((((3S,4R)-1-(Ethoxycarbonyl)-4-(4-fluorophenyl)piperidin-3-yl)methoxy)carbonyl)-2methylpyridin-4-yl)diphenyl(6-(trifluoromethyl)pyridin-3-yl)phosphonium trifluoromethanesulfonate 


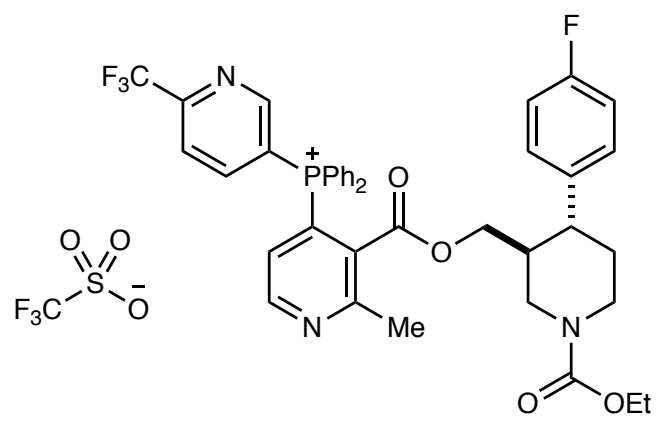

Prepared according to general procedure A, using ((3S,4R)-1-(ethoxycarbonyl)-4-(4fluorophenyl)piperidin-3-yl)methyl 2-methylnicotinate $(401 \mathrm{mg}, 1.00 \mathrm{mmol}), \mathrm{Tf}_{2} \mathrm{O}(169 \mu \mathrm{L}$, $1.00 \mathrm{mmol}), 5$-(diphenylphosphaneyl)-2-(trifluoromethyl)pyridine (364 mg, $1.10 \mathrm{mmol})$, DBU (149 $\mu \mathrm{L}, 1.00 \mathrm{mmol})$, and $\mathrm{CH}_{2} \mathrm{Cl}_{2}(10 \mathrm{~mL})$. After purification by the standard procedure, the title compound was isolated as a white solid ( $1^{\text {st }}$ run: $525 \mathrm{mg}, 0.60 \mathrm{mmol}, 60 \%$ yield, $2^{\text {nd }}$ run: $772 \mathrm{mg}$, $0.88 \mathrm{mmol}, 88 \%$ yield, Average $=74 \%$ ). $\mathrm{mp} 106-110^{\circ} \mathrm{C}$. IR $v_{\max } / \mathrm{cm}^{-1}$ (film): $3068,2985,1688$, 1439, 1261, 1139, 1030, 636; ${ }^{1} \mathrm{H} \mathrm{NMR}\left(400 \mathrm{MHz}, \mathrm{CDCl}_{3}\right) \delta: 8.99$ (1H, app t, $\left.J=4.9 \mathrm{~Hz}\right), 8.69$ $(1 \mathrm{H}, \mathrm{dd}, J=5.7,1.7 \mathrm{~Hz}), 8.59(1 \mathrm{H}$, app t, $J=10.6 \mathrm{~Hz}), 8.09(1 \mathrm{H}, \mathrm{dd}, J=8.1,1.7 \mathrm{~Hz}), 7.83-7.55$ $(10 \mathrm{H}, \mathrm{m}), 7.30-7.22(1 \mathrm{H}, \mathrm{m}), 7.05-6.95(4 \mathrm{H}, \mathrm{m}), 4.33-4.16(4 \mathrm{H}, \mathrm{m}), 3.53-3.08(2 \mathrm{H}, \mathrm{m}), 2.97(3 \mathrm{H}$, s), $2.79\left(1 \mathrm{H}\right.$, br s), 2.55-2.28 $(2 \mathrm{H}, \mathrm{m}), 1.92-1.50(3 \mathrm{H}, \mathrm{m}), 1.30(3 \mathrm{H}, \mathrm{t}, J=7.1 \mathrm{~Hz}) ;{ }^{13} \mathrm{C}$ NMR $(100$ $\left.\mathrm{MHz}, \mathrm{CDCl}_{3}\right) \delta: 166.6,166.6,161.7(\mathrm{~d}, J=245.5 \mathrm{~Hz}), 161.7-161.4(\mathrm{~m}), 155.4-155.1(\mathrm{~m}), 153.5$ $(\mathrm{dd}, J=40.4,12.2 \mathrm{~Hz}), 152.5(\mathrm{qd}, J=35.3,2.2 \mathrm{~Hz}), 145.9(\mathrm{~d}, J=9.1 \mathrm{~Hz}), 138.0(\mathrm{~d}, J=2.7 \mathrm{~Hz})$, $135.7(\mathrm{~d}, J=10.6 \mathrm{~Hz}), 133.9(\mathrm{dd}, J=26.3,10.5 \mathrm{~Hz}), 131.0-130.5(2 \mathrm{C}, \mathrm{m}), 128.7$ (d, $J=7.8 \mathrm{~Hz})$, $127.5(\mathrm{~d}, J=4.4 \mathrm{~Hz}), 122.2-121.9(\mathrm{~m}), 120.7$ (q, $J=321.0 \mathrm{~Hz}), 120.7(\mathrm{~d}, J=91.8 \mathrm{~Hz}), 120.6$ $(\mathrm{qd}, J=275.9,1.6 \mathrm{~Hz}), 117.0(\mathrm{~d}, J=92.0), 116.7(\mathrm{~d}, J=92.9 \mathrm{~Hz}), 115.8(\mathrm{~d}, J=21.2 \mathrm{~Hz}), 68.0$, 61.5, 47.0, 44.1, 44.0, 40.4, 34.0, 26.2, 14.6; ${ }^{19} \mathrm{~F}$ NMR (365 MHz, $\left.\mathrm{CDCl}_{3}\right) \delta$ : $-68.55,-78.33,-$ $115.40 ;{ }^{31} \mathrm{P}$ NMR $\left(162 \mathrm{MHz}, \mathrm{CDCl}_{3}\right) \delta: 26.58 ; \mathrm{m} / \mathrm{z}$ LRMS (ESI + APCI) found [M - OTf] ${ }^{+}$ $730.3, \mathrm{C}_{40} \mathrm{H}_{37} \mathrm{~F}_{4} \mathrm{~N}_{3} \mathrm{O}_{4} \mathrm{P}^{+}$requires 730.2 .

(R)-(2-((1-(3,5-Bis(trifluoromethyl)phenyl)ethoxy)carbonyl)-5-methylpyridin-4yl)diphenyl(6-(trifluoromethyl)pyridin-3-yl)phosphonium trifluoromethanesulfonate<smiles>[Y4]C(OC(=O)c1cc([PH+]([O-])c2ccc(C(F)(F)F)nc2)c(C)cn1)c1cc(C(F)(F)F)cc(C(F)(F)F)c1</smiles>

Prepared according to general procedure A, using (R)-1-(3,5-bis(trifluoromethyl)phenyl)ethyl 5methylpicolinate $(596 \mathrm{mg}, 1.58 \mathrm{mmol}), \mathrm{Tf}_{2} \mathrm{O}(266 \mu \mathrm{L}, 1.58 \mathrm{mmol}), 5$-(diphenylphosphaneyl)-2(trifluoromethyl)pyridine (576 mg, $1.74 \mathrm{mmol})$, DBU $(233 \mu \mathrm{L}, 1.58 \mathrm{mmol})$, and $\mathrm{CH}_{2} \mathrm{Cl}_{2}(16$ $\mathrm{mL}$ ). After purification by the standard procedure (except that two crash-outs were required), the title compound was isolated as a white solid ( $1.04 \mathrm{~g}, 1.21 \mathrm{mmol}, 77 \%$ yield). $\mathrm{mp} 106-108{ }^{\circ} \mathrm{C}$. IR $v_{\max } / \mathrm{cm}^{-1}$ (film): $3064,1743,1441,1277,1130,1076,724,636 ;{ }^{1} \mathrm{H} \mathrm{NMR}\left(400 \mathrm{MHz}, \mathrm{CDCl}_{3}\right) \delta$ : $8.93(1 \mathrm{H}, \mathrm{d}, J=6.6 \mathrm{~Hz}), 8.83(1 \mathrm{H}, \mathrm{d}, J=5.1 \mathrm{~Hz}), 8.69(1 \mathrm{H}$, app t, $J=9.2 \mathrm{~Hz}), 8.17(1 \mathrm{H}, \mathrm{d}, J=$ $7.9 \mathrm{~Hz}), 7.97-7.70(14 \mathrm{H}, \mathrm{m}), 6.10(1 \mathrm{H}, \mathrm{q}, J=6.5 \mathrm{~Hz}), 2.15(3 \mathrm{H}, \mathrm{s}), 1.64(3 \mathrm{H}, \mathrm{d}, J=6.5 \mathrm{~Hz}) ;{ }^{13} \mathrm{C}$ 
$\operatorname{NMR}\left(100 \mathrm{MHz}, \mathrm{CDCl}_{3}\right) \delta: 162.5,154.6(\mathrm{~d}, J=8.5 \mathrm{~Hz}), 153.3(\mathrm{q}, J=35.4 \mathrm{~Hz}), 153.2(\mathrm{~d}, J=$ $12.9 \mathrm{~Hz}), 147.4(\mathrm{~d}, J=11.1 \mathrm{~Hz}), 145.9(\mathrm{~d}, J=9.5 \mathrm{~Hz}), 143.3,141.4(\mathrm{~d}, J=7.1 \mathrm{~Hz}), 136.8(\mathrm{~d}, J=$ $1.8 \mathrm{~Hz}), 134.4(\mathrm{~d}, J=11.0 \mathrm{~Hz}), 131.8(\mathrm{q}, J=33.3 \mathrm{~Hz}), 131.6(\mathrm{~d}, J=13.7 \mathrm{~Hz}), 129.6(\mathrm{~d}, J=11.3$ $\mathrm{Hz}), 126.6,126.2(\mathrm{~d}, J=84.6 \mathrm{~Hz}), 123.1(\mathrm{q}, J=272.7 \mathrm{~Hz}), 122.9-122.6(\mathrm{~m}), 122.3-122.2(\mathrm{~m})$, $120.5(\mathrm{q}, J=321.3 \mathrm{~Hz}), 120.4(\mathrm{q}, J=275.1 \mathrm{~Hz}), 117.9(\mathrm{~d}, J=86.7 \mathrm{~Hz}), 113.8(\mathrm{~d}, J=89.1 \mathrm{~Hz})$, 73.2, 21.5, $20.6(\mathrm{~d}, J=4.7 \mathrm{~Hz}) ;{ }^{19} \mathrm{~F}$ NMR $\left(365 \mathrm{MHz}, \mathrm{CDCl}_{3}\right) \delta:-62.68,-68.69,-78.50 ;{ }^{31} \mathrm{P}$ NMR (162 MHz, $\left.\mathrm{CDCl}_{3}\right) \delta: 19.53 ; \mathrm{m} / \mathrm{z}$ LRMS (ESI + APCI) found [M - OTf] 707.2 , $\mathrm{C}_{35} \mathrm{H}_{25} \mathrm{~F}_{9} \mathrm{~N}_{2} \mathrm{O}_{2} \mathrm{P}^{+}$requires 707.2 .

(3-(3-Fluoro-5-(5-(trifluoromethyl)pyridin-2-yl)phenyl)pyridin-4-yl)diphenyl(6(trifluoromethyl)pyridin-3-yl)phosphonium trifluoromethanesulfonate

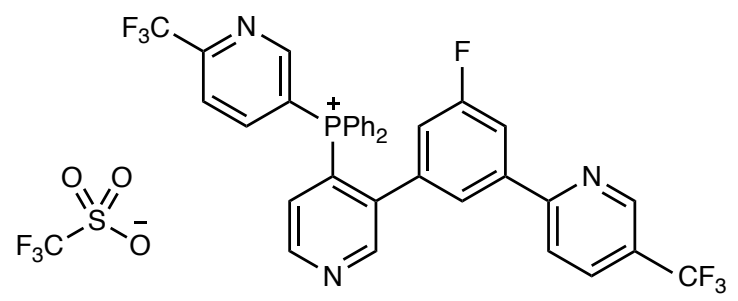

Prepared according to general procedure A, using 3-(3-fluoro-5-(5-(trifluoromethyl)pyridin-2yl)phenyl)pyridine $(318 \mathrm{mg}, 1.50 \mathrm{mmol}), \mathrm{Tf}_{2} \mathrm{O}(252 \mu \mathrm{L}, 1.50 \mathrm{mmol}), 5$-(diphenylphosphaneyl)2-(trifluoromethyl)pyridine (547 mg, $1.65 \mathrm{mmol}), \mathrm{DBU}(224 \mu \mathrm{L}, 1.50 \mathrm{mmol})$, and $\mathrm{CH}_{2} \mathrm{Cl}_{2}(15$ $\mathrm{mL}$ ). After purification by the standard procedure (except that two crash-outs were required), the title compound was isolated as a white solid (1.04 g, $1.30 \mathrm{mmol}, 87 \%$ yield $) . \mathrm{mp} 106-110^{\circ} \mathrm{C}$. IR $v_{\max } / \mathrm{cm}^{-1}$ (film): 3066, 1604, 1330, 1260, 1134, 1029, 722, 636; ${ }^{1} \mathrm{H}$ NMR $\left(400 \mathrm{MHz}, \mathrm{CDCl}_{3}\right) \delta$ : $8.93(1 \mathrm{H}$, app t, $J=4.8 \mathrm{~Hz}), 8.74(1 \mathrm{H}, \mathrm{d}, J=7.1 \mathrm{~Hz}), 8.69-8.57(3 \mathrm{H}, \mathrm{m}), 7.97-7.67(12 \mathrm{H}, \mathrm{m})$, $7.62(1 \mathrm{H}, \mathrm{d}, J=8.4 \mathrm{~Hz}), 7.57-7.44(3 \mathrm{H}, \mathrm{m}), 6.64(1 \mathrm{H}, \mathrm{d}, J=8.0 \mathrm{~Hz}) ;{ }^{13} \mathrm{C} \mathrm{NMR}(100 \mathrm{MHz}$, $\left.\mathrm{CDCl}_{3}\right) \delta: 162.4(\mathrm{~d}, J=250.7 \mathrm{~Hz}), 156.7,153.3(\mathrm{~d}, J=7.8 \mathrm{~Hz}), 153.0(\mathrm{~d}, \mathrm{~J}=12.5 \mathrm{~Hz}), 152.1$ (qd, $J=36.1,2.4 \mathrm{~Hz}), 150.7(\mathrm{~d}, J=10.6 \mathrm{~Hz}), 146.3(\mathrm{q}, J=3.9 \mathrm{~Hz}), 145.3(\mathrm{~d}, J=9.3 \mathrm{~Hz}), 140.1$ $(\mathrm{d}, J=8.1 \mathrm{~Hz}), 139.6(\mathrm{dd}, J=6.8,1.8 \mathrm{~Hz}), 137.0(\mathrm{dd}, J=8.1,4.5 \mathrm{~Hz}), 136.2(\mathrm{~d}, J=2.9 \mathrm{~Hz})$, 134.5-134.8 (2C, m), 131.1 (d, $J=13.3 \mathrm{~Hz}), 128.5$ (d, $J=9.8 \mathrm{~Hz}), 125.8$ (q, $J=33.2 \mathrm{~Hz}), 125.5$, 124.8-124.6 (m), 123.4 (q, $J=272.4 \mathrm{~Hz}), 121.9-121.6(\mathrm{~m}), 120.7$ (q, $J=320.9 \mathrm{~Hz}), 120.3,120.3$ $(\mathrm{qd}, J=275.3,2.2 \mathrm{~Hz}), 119.2(\mathrm{~d}, J=86.6 \mathrm{~Hz}), 117.6(\mathrm{~d}, J=23.1 \mathrm{~Hz}), 115.1(\mathrm{~d}, J=89.3 \mathrm{~Hz})$, $115.0(\mathrm{~d}, J=22.9 \mathrm{~Hz}) ;{ }^{19} \mathrm{~F}$ NMR $\left(365 \mathrm{MHz}, \mathrm{CDCl}_{3}\right) \delta:-62.49,-69.08,-78.38,-110.01(1 \mathrm{~F}, \mathrm{t}, J$ $=8.9 \mathrm{~Hz}) ;{ }^{31} \mathrm{P}$ NMR $\left(162 \mathrm{MHz}, \mathrm{CDCl}_{3}\right) \delta: 19.24 ; \mathrm{m} / \mathrm{z}$ LRMS (ESI + APCI) found [M - OTf] ${ }^{+}$ $648.2, \mathrm{C}_{35} \mathrm{H}_{22} \mathrm{~F}_{7} \mathrm{~N}_{3} \mathrm{P}^{+}$requires 648.1 .

(2-(Bis(4-acetoxyphenyl)methyl)pyridin-4-yl)(phenyl)bis(6-(trifluoromethyl)pyridin-3yl)phosphonium trifluoromethanesulfonate 


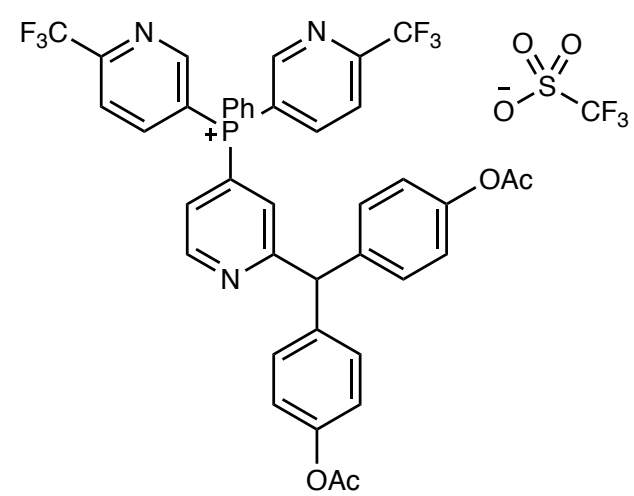

Prepared according to general procedure A using (pyridin-2-ylmethylene)bis(4,1-phenylene) diacetate $(362 \mathrm{mg}, 1.00 \mathrm{mmol}), \mathrm{Tf}_{2} \mathrm{O}(169 \mu \mathrm{L}, 1.00 \mathrm{mmol}), 5,5^{\prime}$-(phenylphosphanediyl)bis (2(trifluoromethyl)pyridine) $(440 \mathrm{mg}, 1.10 \mathrm{mmol})$, DBU $(147 \mu \mathrm{L}, 1.00 \mathrm{mmol})$ and $\mathrm{CH}_{2} \mathrm{Cl}_{2}(10$ $\mathrm{mL})$. After purification by the standard procedure, the title compound was isolated as a white solid (610 mg, $0.64 \mathrm{mmol}, 64 \%$ yield). $\mathrm{mp} 125-130{ }^{\circ} \mathrm{C}$; IR $v_{\max } / \mathrm{cm}^{-1}$ (film): $1751,1505,1372$, 1335, 1260, 1194, 1076, 725, 637; ${ }^{1} \mathrm{H}$ NMR $\left(400 \mathrm{MHz}, \mathrm{CDCl}_{3}\right) \delta: 8.93(1 \mathrm{H}, \mathrm{s}), 8.75(2 \mathrm{H}, \mathrm{d}, J=$ $6.1 \mathrm{~Hz}), 8.57(2 \mathrm{H}, \mathrm{s}), 8.09(2 \mathrm{H}, \mathrm{s}), 7.94(1 \mathrm{H}, \mathrm{d}, J=7.4 \mathrm{~Hz}), 7.77(2 \mathrm{H}, \mathrm{s}), 7.61(2 \mathrm{H}, \mathrm{dd}, J=13.4$, $7.2 \mathrm{~Hz}), 7.52(1 \mathrm{H}, \mathrm{s}), 7.24(1 \mathrm{H}, \mathrm{s}), 7.17(4 \mathrm{H}, \mathrm{d}, J=8.0 \mathrm{~Hz}), 6.96(4 \mathrm{H}, \mathrm{d}, J=8.0 \mathrm{~Hz}), 5.78(1 \mathrm{H}, \mathrm{s})$, $2.25(6 \mathrm{H}, \mathrm{s}) ;{ }^{13} \mathrm{C} \mathrm{NMR}\left(100 \mathrm{MHz}, \mathrm{CDCl}_{3}\right) \delta: 169.5,165.9(\mathrm{~d}, J=10.3 \mathrm{~Hz}), 153.7(\mathrm{~d}, J=13.6$ $\mathrm{Hz}), 153.6(\mathrm{qd}, J=36.0,2.3 \mathrm{~Hz}), 151.9(\mathrm{~d}, J=11.0 \mathrm{~Hz}), 149.7,145.8(\mathrm{~d}, J=9.7 \mathrm{~Hz}), 138.7$, $137.3,134.8(\mathrm{~d}, J=11.3 \mathrm{~Hz}), 131.5(\mathrm{~d}, J=13.7 \mathrm{~Hz}), 130.3,126.9(\mathrm{~d}, J=9.4 \mathrm{~Hz}), 125.3(\mathrm{~d}, J=$ $15.5 \mathrm{~Hz}), 122.4$ (d, $J=10.5 \mathrm{~Hz}), 121.9,120.6(\mathrm{qd}, J=275.1,1.9 \mathrm{~Hz}), 120.5(\mathrm{~d}, J=320.8 \mathrm{~Hz})$, 119.9-118.1 (m), $116.5(\mathrm{~d}, J=88.4 \mathrm{~Hz}), 112.3(\mathrm{~d}, J=90.1 \mathrm{~Hz}), 57.5,21.0 ;{ }^{19} \mathrm{~F}$ NMR $(365 \mathrm{MHz}$, $\left.\mathrm{CDCl}_{3}\right) \delta:-68.70,-78.58 ;{ }^{31} \mathrm{P}$ NMR $\left(162 \mathrm{MHz}, \mathrm{CDCl}_{3}\right) \delta: 18.19 ; \mathrm{m} / z$ LRMS (ESI + APCI) found $[\mathrm{M}-\mathrm{OTf}]^{+} 760.2, \mathrm{C}_{40} \mathrm{H}_{29} \mathrm{~F}_{6} \mathrm{~N}_{3} \mathrm{O}_{4} \mathrm{P}^{+}$requires 760.2 .

(2-(2-Chloro-5-(2-chloro-N-(ethoxycarbonyl)-4-(methylsulfonyl)benzamido)phenyl)pyridin4-yl)(phenyl)bis(6-(trifluoromethyl)pyridin-3-yl)phosphonium trifluoromethanesulfonate<smiles>CCOC(=O)N(C(=O)c1ccc(S(=O)(=O)O)cc1Cl)c1ccc(Cl)c(-c2cc(P(c3ccc(C(F)(F)F)cc3)c3ccc(C(F)(F)F)nc3)ccn2)c1</smiles>

Prepared according to general procedure A using ethyl (4-chloro-3-(pyridin-2-yl)phenyl) (2chloro-4-(methylsulfonyl)benzoyl)carbamate (492 mg, $1.00 \mathrm{mmol}), \mathrm{Tf}_{2} \mathrm{O}(169 \mu \mathrm{L}, 1.00 \mathrm{mmol})$, 5,5'-(phenylphosphanediyl)bis(2-(trifluoromethyl)pyridine) (440 mg, $1.10 \mathrm{mmol}), \mathrm{DBU}(147 \mu \mathrm{L}$, $1.00 \mathrm{mmol})$ and $\mathrm{CH}_{2} \mathrm{Cl}_{2}(10 \mathrm{~mL})$. After purification by the standard procedure, the title compound was isolated as a white solid (500 mg, $0.49 \mathrm{mmol}, 49 \%$ yield). $\mathrm{mp} 155-160{ }^{\circ} \mathrm{C}$; IR $v_{\max } / \mathrm{cm}^{-1}$ (film): 1750, 1689, 1373, 1335, 1259, 1148, 1075, 1030, 725, 637; ${ }^{1} \mathrm{H}$ NMR (400 MHz, $\left.\mathrm{CDCl}_{3}\right) \delta: 9.15(1 \mathrm{H}, \mathrm{t}, J=5.4 \mathrm{~Hz}), 8.92(2 \mathrm{H}, \mathrm{dd}, J=6.3,2.2 \mathrm{~Hz}), 8.68(2 \mathrm{H}, \mathrm{ddd}, J=13.4,8.3$, $2.3 \mathrm{~Hz}), 8.13(2 \mathrm{H}, \mathrm{dd}, J=8.4,2.2 \mathrm{~Hz}), 8.05-7.47(12 \mathrm{H}, \mathrm{m}), 7.35(1 \mathrm{H}, \mathrm{dd}, J=8.5,2.6 \mathrm{~Hz}), 4.09$ $(2 \mathrm{H}, \mathrm{q}, J=7.1 \mathrm{~Hz}), 3.06(3 \mathrm{H}, \mathrm{s}), 1.04(3 \mathrm{H}, \mathrm{t}, J=7.1 \mathrm{~Hz}) ;{ }^{13} \mathrm{C} \mathrm{NMR}\left(100 \mathrm{MHz}, \mathrm{CDCl}_{3}\right) \delta: 167.9$, 
$157.4(\mathrm{~d}, J=11.5 \mathrm{~Hz}), 154.0$ (dd, $J=36.4,2.3 \mathrm{~Hz}), 153.9(\mathrm{~d}, J=13.9 \mathrm{~Hz}), 152.6(\mathrm{~d}, J=11.3$ $\mathrm{Hz}), 152.5,146.0(\mathrm{~d}, J=9.9 \mathrm{~Hz}), 142.3(\mathrm{~d}, J=47.1 \mathrm{~Hz}), 137.0(\mathrm{~d}, J=114.0 \mathrm{~Hz}), 135.0(\mathrm{~d}, J=$ $11.3 \mathrm{~Hz}), 132.3(\mathrm{~d}, J=5.4 \mathrm{~Hz}), 131.7(\mathrm{~d}, J=13.8 \mathrm{~Hz}), 131.5,131.3,131.0(\mathrm{~d}, J=14.5 \mathrm{~Hz})$, $129.6(\mathrm{~d}, J=13.0 \mathrm{~Hz}), 129.4,129.0,128.1,128.0(\mathrm{~d}, J=9.8 \mathrm{~Hz}), 126.6(\mathrm{~d}, J=8.5 \mathrm{~Hz}), 126.1(\mathrm{~d}$, $J=9.7 \mathrm{~Hz}), 125.2,122.6(\mathrm{~d}, J=10.3 \mathrm{~Hz}), 122.1,121.9(\mathrm{~d}, J=2.0 \mathrm{~Hz}), 120.5(\mathrm{qd}, J=275.5,2.0$ $\mathrm{Hz}), 120.5(\mathrm{q}, J=320.7 \mathrm{~Hz}), 119.0(\mathrm{~d}, J=29.4 \mathrm{~Hz}), 116.5(\mathrm{~d}, J=88.5 \mathrm{~Hz}), 112.4(\mathrm{~d}, J=90.3$ $\mathrm{Hz}), 64.4,44.4,13.8 ;{ }^{19} \mathrm{~F}$ NMR $\left(365 \mathrm{MHz}, \mathrm{CDCl}_{3}\right) \delta$ : $-68.67,-78.62 ;{ }^{31} \mathrm{P}$ NMR $(162 \mathrm{MHz}$, $\left.\mathrm{CDCl}_{3}\right) \delta: 18.58 ; \mathrm{m} / z$ LRMS (ESI + APCI) found [M - OTf] ${ }^{+} 891.1, \mathrm{C}_{40} \mathrm{H}_{28} \mathrm{Cl}_{2} \mathrm{~F}_{6} \mathrm{~N}_{4} \mathrm{O}_{5} \mathrm{PS}^{+}$ requires 891.1 .

(5-Chloro-6'-methyl-3-(4-(methylsulfonyl)phenyl)-[2,3'-bipyridin]-4'-yl)diphenyl(6(trifluoromethyl)pyridin-3-yl)phosphonium trifluoromethanesulfonate

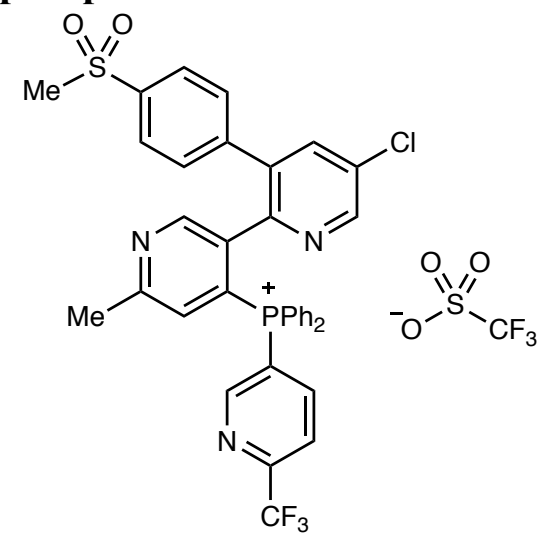

Prepared according to general procedure A using 5-chloro-6'-methyl-3-(4-(methylsulfonyl) phenyl)-2,3'-bipyridine (538 $\mathrm{mg}, \quad 1.50 \quad \mathrm{mmol}), \quad \mathrm{Tf}_{2} \mathrm{O} \quad(252 \quad \mu \mathrm{L}, \quad 1.50 \quad \mathrm{mmol}), \quad 5-$ (diphenylphosphanyl)-2-(trifluoromethyl)pyridine (546 mg, $1.65 \mathrm{mmol}$ ), DBU (147 $\mu \mathrm{L}, 1.50$ mmol) and $\mathrm{CH}_{2} \mathrm{Cl}_{2}(15 \mathrm{~mL})$. After purification by the standard procedure, the title compound was isolated as a white solid (620 mg, $0.75 \mathrm{mmol}, 50 \%$ yield). $\mathrm{mp} 160-164{ }^{\circ} \mathrm{C}$; IR $v_{\max } / \mathrm{cm}^{-1}$ (film): 1576, 1437, 1336, 1260, 1068, 1030, 772, $720 ;{ }^{1} \mathrm{H} \mathrm{NMR}\left(400 \mathrm{MHz}, \mathrm{CDCl}_{3}\right) \delta: 8.82(2 \mathrm{H}$, d, $J=5.6 \mathrm{~Hz}), 8.33(1 \mathrm{H}, \mathrm{d}, J=7.5 \mathrm{~Hz}), 8.09(3 \mathrm{H}, \mathrm{d}, J=8.0 \mathrm{~Hz}), 7.83(6 \mathrm{H}$, ddd, $J=27.8,10.5$, $6.8 \mathrm{~Hz}), 7.74-7.61(5 \mathrm{H}, \mathrm{m}), 7.60-7.44(3 \mathrm{H}, \mathrm{m}), 7.21(1 \mathrm{H}, \mathrm{d}, J=17.1 \mathrm{~Hz}), 3.11(3 \mathrm{H}, \mathrm{s}), 2.55(3 \mathrm{H}$, s); ${ }^{13} \mathrm{C}$ NMR $\left(100 \mathrm{MHz}, \mathrm{CDCl}_{3}\right) \delta: 161.5(\mathrm{~d}, J=11.5 \mathrm{~Hz}), 153.7(\mathrm{~d}, J=12.1 \mathrm{~Hz}), 152.5(\mathrm{~d}, J=$ $7.4 \mathrm{~Hz}), 152.2$ (q, $J=35.8 \mathrm{~Hz}), 147.2(\mathrm{~d}, J=2.3 \mathrm{~Hz}), 146.1,145.6$ (d, $J=8.8 \mathrm{~Hz}), 141.4$ (d, $J=$ $38.5 \mathrm{~Hz}), 139.5,136.1,135.5(\mathrm{~d}, J=3.1 \mathrm{~Hz}), 134.1(\mathrm{~d}, J=10.3 \mathrm{~Hz}), 133.1(\mathrm{~d}, J=3.5 \mathrm{~Hz})$, $132.6,131.2$ (d, $J=10.3 \mathrm{~Hz}), 130.6(\mathrm{~d}, J=13.5 \mathrm{~Hz}), 130.1,128.8,126.4(\mathrm{~d}, J=88.2 \mathrm{~Hz}), 122.7$, $121.8,120.8(\mathrm{q}, J=321.1 \mathrm{~Hz}), 120.6(\mathrm{q}, J=275.2 \mathrm{~Hz}), 118.1(\mathrm{~d}, J=93.3 \mathrm{~Hz}), 44.2,24.7 ;{ }^{19} \mathrm{~F}$ NMR $\left(365 \mathrm{MHz}, \mathrm{CDCl}_{3}\right) \delta:-68.54,-78.27 ;{ }^{31} \mathrm{P} \mathrm{NMR}\left(162 \mathrm{MHz}, \mathrm{CDCl}_{3}\right) \delta: 22.98 ; \mathrm{m} / z \mathrm{LRMS}$ $(\mathrm{ESI}+\mathrm{APCI})$ found $[\mathrm{M}-\mathrm{OTf}]^{+} 688.2, \mathrm{C}_{36} \mathrm{H}_{27} \mathrm{ClF}_{3} \mathrm{~N}_{3} \mathrm{O}_{2} \mathrm{PS}^{+}$requires 688.1 .

(8-Chloro-11-(1-(ethoxycarbonyl)piperidin-4-ylidene)-6,11-dihydro-5Hbenzo[5,6]cyclohepta[1,2-b]pyridin-4-yl)diphenyl(6-(trifluoromethyl)pyridin-3yl)phosphonium trifluoromethanesulfonate 


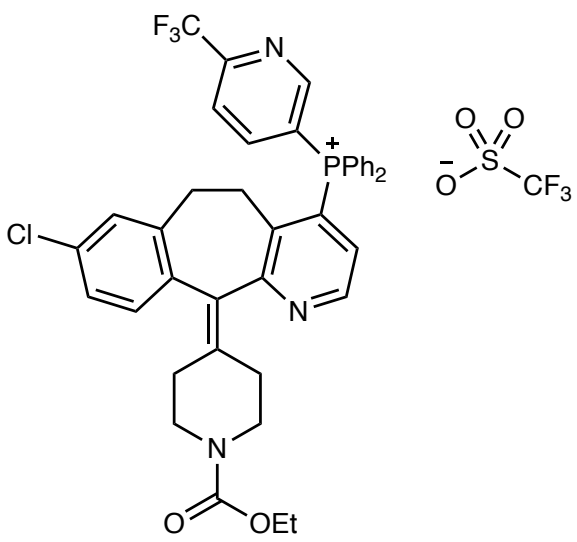

Prepared according to general procedure A using ethyl 4-(8-chloro-5,6-dihydro-11H-benzo[5,6]cyclohepta[1,2-b]pyridin-11-ylidene)piperidine-1-carboxylate (574.5 mg, $1.50 \mathrm{mmol}), \mathrm{Tf}_{2} \mathrm{O}$ (252 $\mu \mathrm{L}, 1.50 \mathrm{mmol}), 5$-(diphenylphosphanyl)-2-(trifluoromethyl)pyridine (546 mg, $1.65 \mathrm{mmol}$ ), DBU $(222 \mu \mathrm{L}, 1.50 \mathrm{mmol})$ and $\mathrm{CH}_{2} \mathrm{Cl}_{2}(15 \mathrm{~mL})$. After purification by the standard procedure, the title compound was isolated as a yellow solid (1.05 g, $1.21 \mathrm{mmol}, 81 \%$ yield). $\mathrm{mp} 150-153$ ${ }^{\circ} \mathrm{C}$; IR $v_{\max } / \mathrm{cm}^{-1}$ (film): 2922, 1688, 1479, 1438, 1336, 1260, 1223, 1143, 1030,724, 636; ${ }^{1} \mathrm{H}$ NMR $\left(400 \mathrm{MHz} \mathrm{CDCl}_{3}\right) \delta: 8.94-8.69(3 \mathrm{H}, \mathrm{m}), 8.24(1 \mathrm{H}, \mathrm{d}, J=7.6 \mathrm{~Hz}), 7.98(2 \mathrm{H}, \mathrm{q}, J=8.0$ $\mathrm{Hz}), 7.92-7.61(8 \mathrm{H}, \mathrm{m}), 7.19-7.07(3 \mathrm{H}, \mathrm{m}), 6.72(1 \mathrm{H}, \mathrm{d}, J=2.0 \mathrm{~Hz}), 4.15(2 \mathrm{H}, \mathrm{q}, J=7.1 \mathrm{~Hz})$, $3.75(2 \mathrm{H}, \mathrm{dd}, J=12.5,6.1 \mathrm{~Hz}), 3.46-3.27(3 \mathrm{H}, \mathrm{m}), 2.81(1 \mathrm{H}, \mathrm{d}, J=17.5 \mathrm{~Hz}), 2.61-2.32(4 \mathrm{H}, \mathrm{m})$, $2.25(1 \mathrm{H}, \mathrm{s}), 1.53(1 \mathrm{H}, \mathrm{ddd}, J=17.0,11.7,4.9 \mathrm{~Hz}), 1.26(3 \mathrm{H}, \mathrm{t}, J=7.1 \mathrm{~Hz}) ;{ }^{13} \mathrm{C} \mathrm{NMR}(100$ $\left.\mathrm{MHz}, \mathrm{CDCl}_{3}\right) \delta: 163.8(\mathrm{~d}, J=8.5 \mathrm{~Hz}), 155.4,153.4(\mathrm{~d}, J=13.2 \mathrm{~Hz}), 153.3(\mathrm{q}, J=36.2 \mathrm{~Hz})$, 149.5 (d, $J=11.7 \mathrm{~Hz}), 146.0(\mathrm{~d}, J=9.5 \mathrm{~Hz}), 139.7,136.9-136.4(\mathrm{~m}), 134.5$ (d, $J=10.9 \mathrm{~Hz})$, $134.3(\mathrm{~d}, J=10.8 \mathrm{~Hz}), 133.9(\mathrm{~d}, J=33.8 \mathrm{~Hz}), 132.2,131.7,131.5$ (dd, $J=13.3,7.4 \mathrm{~Hz}), 129.9$, $127.5(\mathrm{~d}, J=10.4 \mathrm{~Hz}), 126.6,125.3(\mathrm{~d}, J=82.9 \mathrm{~Hz}), 122.8(\mathrm{~d}, J=10.2 \mathrm{~Hz}), 120.6(\mathrm{q}, J=321.0$ $\mathrm{Hz}), 120.5(\mathrm{qd}, J=275.5,2.1 \mathrm{~Hz}), 118.8(\mathrm{~d}, J=86.8 \mathrm{~Hz}), 115.3(\mathrm{~d}, J=55.2 \mathrm{~Hz}), 114.4(\mathrm{~d}, J=$ $55.1 \mathrm{~Hz}), 61.4,44.7,44.6,30.6(\mathrm{dd}, J=15.1,9.6 \mathrm{~Hz}), 29.5,14.6 ;{ }^{19} \mathrm{~F}$ NMR $\left(365 \mathrm{MHz}, \mathrm{CDCl}_{3}\right)$ $\delta:-68.61,-78.38 ;{ }^{31} \mathrm{P}$ NMR $\left(162 \mathrm{MHz}, \mathrm{CDCl}_{3}\right) \delta: 19.14 ; \mathrm{m} / z$ LRMS (ESI + APCI) found [M OTf] $]^{+}$712.3, $\mathrm{C}_{40} \mathrm{H}_{35} \mathrm{ClF}_{3} \mathrm{~N}_{3} \mathrm{O}_{2} \mathrm{P}^{+}$requires 712.2.

(3-((2-Butoxyethoxy)carbonyl)pyridin-4-yl)diphenyl(6-(trifluoromethyl)pyridin-3yl)phosphonium trifluoromethanesulfonate<smiles>CCCCOCCOC(=O)c1cnccc1P(=O)(c1ccccc1)c1ccc(C(F)(F)F)nc1</smiles>

Prepared according to general procedure A using 2-butoxyethyl nicotinate (223 $\mathrm{mg}, 1.00 \mathrm{mmol})$, $\mathrm{Tf}_{2} \mathrm{O}(169 \mu \mathrm{L}, 1.00 \mathrm{mmol})$, 5-(diphenylphosphanyl)-2-(trifluoromethyl)pyridine (382 $\mathrm{mg}, 1.10$ mmol), DBU $(147 \mu \mathrm{L}, 1.00 \mathrm{mmol})$ and $\mathrm{CH}_{2} \mathrm{Cl}_{2}(10 \mathrm{~mL})$. After purification by the standard procedure, the title compound was isolated as a yellow solid (430 mg, $0.62 \mathrm{mmol}, 62 \%$ yield). mp 54-56 ${ }^{\circ} \mathrm{C}$; IR $v_{\max } / \mathrm{cm}^{-1}$ (film): 2960, 2872, 1712, 1440, 1336, 1259, 1142, 1076, 1029, 721, 636; ${ }^{1} \mathrm{H}$ NMR (400 MHz, $\left.\mathrm{CDCl}_{3}\right) \delta: 9.57(1 \mathrm{H}, \mathrm{d}, J=6.4 \mathrm{~Hz}), 9.11(1 \mathrm{H}, \mathrm{s}), 8.77(1 \mathrm{H}, \mathrm{dd}, J=6.1$, $2.1 \mathrm{~Hz}), 8.48(1 \mathrm{H}, \mathrm{ddd}, J=13.2,8.2,2.2 \mathrm{~Hz}), 8.05(1 \mathrm{H}, \mathrm{dd}, J=8.3,2.1 \mathrm{~Hz}), 7.93-7.51(10 \mathrm{H}, \mathrm{m})$, 
$7.41(1 \mathrm{H}, \mathrm{dd}, J=16.2,5.0 \mathrm{~Hz}), 4.15-3.96(2 \mathrm{H}, \mathrm{m}), 3.50-3.39(2 \mathrm{H}, \mathrm{m}), 3.33(2 \mathrm{H}, \mathrm{t}, J=6.6 \mathrm{~Hz})$, $1.46(2 \mathrm{H}, \mathrm{dq}, J=8.4,6.7 \mathrm{~Hz}), 1.37-1.21(2 \mathrm{H}, \mathrm{m}), 0.85(3 \mathrm{H}, \mathrm{t}, J=7.4 \mathrm{~Hz}) ;{ }^{13} \mathrm{C}$ NMR $(100 \mathrm{MHz}$, $\left.\mathrm{CDCl}_{3}\right) \delta: 164.4(\mathrm{~d}, J=1.8 \mathrm{~Hz}), 156.3(\mathrm{~d}, J=10.9 \mathrm{~Hz}), 153.2(\mathrm{~d}, J=5.7 \mathrm{~Hz}), 152.7(\mathrm{~d}, J=12.8$ $\mathrm{Hz}), 152.2(\mathrm{qd}, J=35.9,2.5 \mathrm{~Hz}), 145.1(\mathrm{~d}, J=9.3 \mathrm{~Hz}), 135.6(\mathrm{~d}, J=3.2 \mathrm{~Hz}), 133.8(\mathrm{~d}, J=10.7$ $\mathrm{Hz}), 131.5(\mathrm{~d}, J=9.4 \mathrm{~Hz}), 130.7(\mathrm{~d}, J=13.6 \mathrm{~Hz}), 127.9(\mathrm{~d}, J=84.7 \mathrm{~Hz}), 126.7,122.0(\mathrm{~d}, J=2.3$ $\mathrm{Hz}), 120.7$ (d, $J=92.9 \mathrm{~Hz}), 120.6(\mathrm{qd}, J=283.1,2.3 \mathrm{~Hz}), 120.6(\mathrm{q}, J=321.0 \mathrm{~Hz}), 117.0(\mathrm{~d}, J=$ 93.5 Hz), 71.1, 67.5, 66.5, 31.5, 19.1, 13.8; ${ }^{19} \mathrm{~F} \mathrm{NMR}\left(365 \mathrm{MHz}, \mathrm{CDCl}_{3}\right) \delta:-68.53,-78.36 ;{ }^{31} \mathrm{P}$ NMR (162 MHz, $\left.\mathrm{CDCl}_{3}\right) \delta: 26.70 ; \mathrm{m} / z$ LRMS (ESI + APCI) found [M - OTf] ${ }^{+}$553.3, $\mathrm{C}_{30} \mathrm{H}_{29} \mathrm{~F}_{3} \mathrm{~N}_{2} \mathrm{O}_{3} \mathrm{P}^{+}$requires 553.2.

(3-((3S,8R,9S,10R,13S,14S)-3-acetoxy-10,13-dimethyl-2,3,4,7,8,9,10,11,12,13,14,15dodecahydro-1H-cyclopenta $[a]$ phenanthren-17-yl)pyridin-4-yl)diphenyl(6(trifluoromethyl)pyridin-3-yl)phosphonium trifluoromethanesulfonate

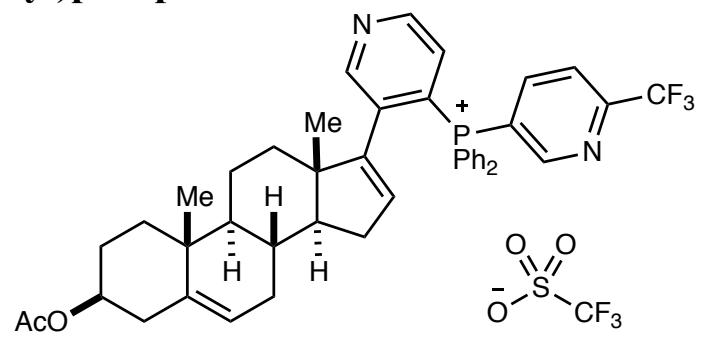

Prepared according to general procedure A, using $(3 S, 9 S, 10 R, 13 S, 14 S)$-10,13-dimethyl-17(pyridin-3-yl)-2,3,4,7,8,9,10,11,12,13,14,15-dodecahydro-1H-cyclopenta[a]phenanthren-3-yl acetate $(587 \mathrm{mg}, 1.50 \mathrm{mmol}), \mathrm{Tf}_{2} \mathrm{O}(252 \mu \mathrm{L}, 1.50 \mathrm{mmol})$, 5-(diphenylphosphaneyl)-2(trifluoromethyl)pyridine (547 mg, $1.65 \mathrm{mmol})$, DBU $(224 \mu \mathrm{L}, 1.50 \mathrm{mmol})$, and $\mathrm{CH}_{2} \mathrm{Cl}_{2}(15$ $\mathrm{mL}$ ). After purification by the standard procedure (except that two crash-outs were required), the title compound was isolated as a white solid (898 mg, $1.03 \mathrm{mmol}, 69 \%$ yield). $\mathrm{mp} 154-158{ }^{\circ} \mathrm{C}$. IR $v_{\max } / \mathrm{cm}^{-1}$ (film): 3503, 2940, 1726, 1258, 1144, 1030, 721, 636; ${ }^{1} \mathrm{H}$ NMR $\left(400 \mathrm{MHz}, \mathrm{CDCl}_{3}\right)$ $\delta$ : $9.04(1 \mathrm{H}, \mathrm{d}, J=7.4 \mathrm{~Hz}), 8.82-8.70(3 \mathrm{H}, \mathrm{m}), 8.18(1 \mathrm{H}, \mathrm{d}, J=6.9 \mathrm{~Hz}), 7.94-7.87$ (2H, m), 7.87$7.77(6 \mathrm{H}, \mathrm{m}), 7.73-7.68(2 \mathrm{H}, \mathrm{m}), 7.32(1 \mathrm{H}, \mathrm{dd}, J=16.3,5.1 \mathrm{~Hz}), 5.54(1 \mathrm{H}, \mathrm{s}), 5.28(1 \mathrm{H}, \mathrm{d}, J=$ $4.6 \mathrm{~Hz}), 4.57(1 \mathrm{H}, \mathrm{m}), 2.33-2.20(2 \mathrm{H}, \mathrm{m}), 2.00(3 \mathrm{H}, \mathrm{s}), 1.87-1.68(5 \mathrm{H}, \mathrm{m}), 1.63-1.32(5 \mathrm{H}, \mathrm{m})$, $1.23-1.01(5 \mathrm{H}, \mathrm{m}), 0.95(3 \mathrm{H}, \mathrm{s}), 0.77(1 \mathrm{H}, \mathrm{td}, J=12.6,4.1 \mathrm{~Hz}), 0.59(1 \mathrm{H}, \mathrm{td}, J=11.3,4.1 \mathrm{~Hz})$, $0.29(1 \mathrm{H}, \mathrm{m}) ;{ }^{13} \mathrm{C} \mathrm{NMR}\left(100 \mathrm{MHz}, \mathrm{CDCl}_{3}\right) \delta: 170.6,152.9(\mathrm{qd}, J=36.2,2.3 \mathrm{~Hz}), 152.8(\mathrm{~d}, J=$ $12.3 \mathrm{~Hz}), 151.0(\mathrm{~d}, J=7.6 \mathrm{~Hz}), 149.7$ (d, $J=4.1 \mathrm{~Hz}), 149.2(\mathrm{~d}, J=11.0 \mathrm{~Hz}), 145.7$ (d, $J=8.8$ Hz), 139.9 (d, $J=20.9 \mathrm{~Hz}), 137.5$ (d, $J=6.2 \mathrm{~Hz}), 136.4(\mathrm{dd}, J=6.9,3.1 \mathrm{~Hz}), 134.7$ (dd, $J=$ 28.9, $10.5 \mathrm{~Hz}), 131.5(\mathrm{dd}, J=13.4,8.8 \mathrm{~Hz}), 130.3(\mathrm{~d}, J=10.7 \mathrm{~Hz}), 123.8(\mathrm{~d}, J=84.9 \mathrm{~Hz})$, $123.0-122.8(\mathrm{~m}), 121.7,120.8(\mathrm{q}, J=321.0 \mathrm{~Hz}), 120.6(\mathrm{~d}, J=86.9 \mathrm{~Hz}), 120.5(\mathrm{qd}, J=273.7,1.8$ Hz), 117.0 (d, $J=89.8 \mathrm{~Hz}), 116.0$ (d, $J=90.0 \mathrm{~Hz}), 73.7,56.1,49.7,49.1,38.1,36.9,36.6,33.6$, 32.6, 31.0, 22.9, 27.7, 21.5, 20.4, 19.1, $18.9 ;{ }^{19} \mathrm{~F} \mathrm{NMR}\left(365 \mathrm{MHz}, \mathrm{CDCl}_{3}\right) \delta$ : $-68.33,-78.29 ;{ }^{31} \mathrm{P}$ NMR (162 MHz, $\left.\mathrm{CDCl}_{3}\right) \delta: 20.59 ; \mathrm{m} / \mathrm{z}$ LRMS (ESI + APCI) found [M - OTf] ${ }^{+}$721.4, $\mathrm{C}_{44} \mathrm{H}_{45} \mathrm{~F}_{3} \mathrm{~N}_{2} \mathrm{O}_{2} \mathrm{P}^{+}$requires 721.3.

(5,7-Dichloro-4-(4-fluorophenoxy)quinolin-2-yl)diphenyl(6-(trifluoromethyl)pyridin-3yl)phosphonium trifluoromethanesulfonate 


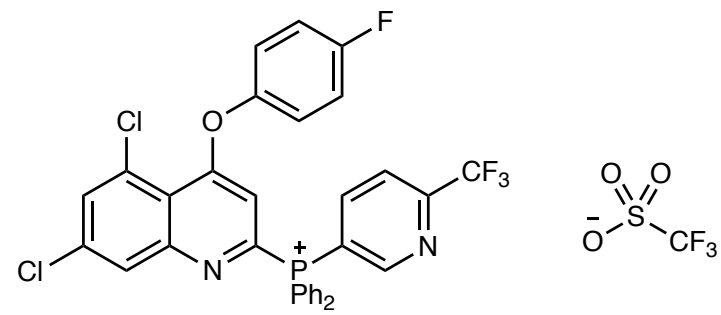

Prepared according to general procedure A (except that EtOAc was used instead of $\mathrm{CH}_{2} \mathrm{Cl}_{2}$ ), using 5,7-dichloro-4-(4-fluorophenoxy)quinoline (308 mg, $1.50 \mathrm{mmol}), \mathrm{Tf}_{2} \mathrm{O}(252 \mu \mathrm{L}, 1.50$ mmol), 5-(diphenylphosphaneyl)-2-(trifluoromethyl)pyridine (547 mg, $1.65 \mathrm{mmol}$ ), DBU (224 $\mu \mathrm{L}, 1.50 \mathrm{mmol})$, and EtOAc $(15 \mathrm{~mL})$. After purification by the standard procedure, the title compound was isolated as a white solid ( $811 \mathrm{mg}, 1.03 \mathrm{mmol}, 69 \%$ yield). mp $143-145^{\circ} \mathrm{C}$. IR $v_{\max } / \mathrm{cm}^{-1}$ (film): $3070,2360,1559,1263,1139,1030,930,725 ;{ }^{1} \mathrm{H}$ NMR $\left(400 \mathrm{MHz}, \mathrm{CDCl}_{3}\right) \delta$ : $8.98(1 \mathrm{H}, \mathrm{d}, J=5.0 \mathrm{~Hz}), 8.64(1 \mathrm{H}, \mathrm{ddd}, J=12.5,8.6,1.8 \mathrm{~Hz}), 8.08-8.02(2 \mathrm{H}, \mathrm{m}), 7.88-7.81(2 \mathrm{H}$, m), $7.75(1 \mathrm{H}, \mathrm{d}, J=1.9 \mathrm{~Hz}), 7.72-7.65(8 \mathrm{H}, \mathrm{m}), 7.17-7.12(2 \mathrm{H}, \mathrm{m}), 7.05(2 \mathrm{H}$, app t, $J=8.1 \mathrm{~Hz})$, $6.56(1 \mathrm{H}, \mathrm{d}, J=6.9 \mathrm{~Hz}) ;{ }^{13} \mathrm{C} \mathrm{NMR}\left(100 \mathrm{MHz}, \mathrm{CDCl}_{3}\right) \delta: 165.2(\mathrm{~d}, J=14.2 \mathrm{~Hz}), 160.7(\mathrm{~d}, J=$ $246.5 \mathrm{~Hz}), 153.8(\mathrm{~d}, J=12.1 \mathrm{~Hz}), 153.2(\mathrm{qd}, J=35.8,2.3 \mathrm{~Hz}), 152.3(\mathrm{~d}, J=25.3 \mathrm{~Hz}), 148.2(\mathrm{~d}$, $J=2.9 \mathrm{~Hz}), 146.8(\mathrm{~d}, J=120.3 \mathrm{~Hz}), 146.0(\mathrm{~d}, J=9.1 \mathrm{~Hz}), 137.9,136.5(\mathrm{~d}, J=8.6 \mathrm{~Hz}), 134.9(\mathrm{~d}$, $J=10.8 \mathrm{~Hz}), 132.9,131.5(\mathrm{~d}, J=1.5 \mathrm{~Hz}), 131.1(\mathrm{~d}, J=13.1 \mathrm{~Hz}), 128.2,123.1(\mathrm{~d}, J=8.6 \mathrm{~Hz})$, 122.2-121.9 (m), $120.7(\mathrm{q}, J=321.1 \mathrm{~Hz}), 120.6(\mathrm{qd}, J=274.4,1.7 \mathrm{~Hz}), 119.0(\mathrm{~d}, J=87.4 \mathrm{~Hz})$, $118.4(\mathrm{~d}, J=2.3 \mathrm{~Hz}), 117.5(\mathrm{~d}, J=23.7 \mathrm{~Hz}), 114.7(\mathrm{~d}, J=88.4 \mathrm{~Hz}), 110.7(\mathrm{~d}, J=30.2 \mathrm{~Hz}) ;{ }^{19} \mathrm{~F}$ NMR (365 MHz, $\left.\mathrm{CDCl}_{3}\right) \delta:-68.69,-78.49,-115.18(\mathrm{~m}) ;{ }^{31} \mathrm{P} \mathrm{NMR}\left(162 \mathrm{MHz}, \mathrm{CDCl}_{3}\right) \delta: 12.91$; $\mathrm{m} / \mathrm{z}$ LRMS (ESI + APCI) found [M - OTf] ${ }^{+} 637.1, \mathrm{C}_{33} \mathrm{H}_{20} \mathrm{Cl}_{2} \mathrm{~F}_{4} \mathrm{~N}_{2} \mathrm{OP}^{+}$requires 637.1.

((6S,10R)-8-(Ethoxycarbonyl)-7,8,9,10-tetrahydro-6H-6,10-methanoazepino[4,5g]quinoxalin-2-yl)diphenyl(6-(trifluoromethyl)pyridin-3-yl)phosphonium trifluoromethanesulfonate

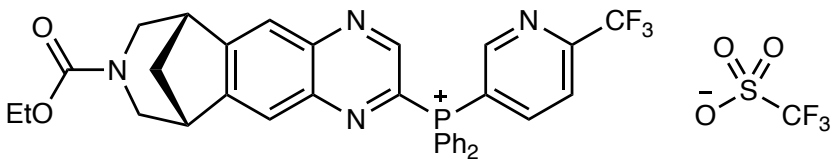

Prepared according to general procedure A, using ethyl (6R,10S)-6,7,9,10-tetrahydro-8H-6,10methanoazepino[4,5-g] quinoxaline-8-carboxylate (185 mg, $0.65 \mathrm{mmol}), \mathrm{Tf}_{2} \mathrm{O}(109 \mu \mathrm{L}, 0.65$ mmol), 5-(diphenylphosphaneyl)-2-(trifluoromethyl)pyridine (237 mg, $0.72 \mathrm{mmol}$ ), DBU (98 $\mu \mathrm{L}, 0.65 \mathrm{mmol})$, and $\mathrm{CH}_{2} \mathrm{Cl}_{2}(7 \mathrm{~mL})$. After purification by the standard procedure (except that two crash-outs were required), the title compound was isolated as a yellow solid (413 $\mathrm{mg}, 0.54$ mmol, $83 \%$ yield). $\mathrm{mp} 133-137^{\circ} \mathrm{C}$. IR $v_{\max } / \mathrm{cm}^{-1}$ (film): 2958, 2870, 1683, 1260, 1142, 1029 , 754, 636; ${ }^{1} \mathrm{H}$ NMR $\left(400 \mathrm{MHz}, \mathrm{CDCl}_{3}\right) \delta: 9.12-8.90(2 \mathrm{H}, \mathrm{m}), 8.86-8.68(1 \mathrm{H}, \mathrm{m}), 8.26-7.90(5 \mathrm{H}$, $\mathrm{m}), 7.88-7.70(8 \mathrm{H}, \mathrm{m}), 4.19-3.74(4 \mathrm{H}, \mathrm{m}), 3.60-3.30(4 \mathrm{H}, \mathrm{m}), 2.52-2.43(1 \mathrm{H}, \mathrm{m}), 2.07(1 \mathrm{H}, \mathrm{d}, J=$ $11.4 \mathrm{~Hz}), 1.06(3 \mathrm{H}, \mathrm{t}, J=7.0 \mathrm{~Hz}) ;{ }^{13} \mathrm{C} \mathrm{NMR}\left(100 \mathrm{MHz}, \mathrm{CDCl}_{3}\right) \delta: 156.4,155.4,153.8(\mathrm{~d}, J=$ $12.1 \mathrm{~Hz}), 152.9(\mathrm{qd}, J=36.1,2.5 \mathrm{~Hz}), 152.8(\mathrm{~d}, J=15.3 \mathrm{~Hz}), 146.0(\mathrm{dd}, J=12.7,9.1 \mathrm{~Hz}), 145.4$ $(\mathrm{dd}, J=27.2,7.6 \mathrm{~Hz}), 144.3(\mathrm{~d}, J=2.7 \mathrm{~Hz}), 143.3(\mathrm{dd}, J=17.4,3.8 \mathrm{~Hz}), 137.9,136.9-136.6$ (m), 135.0-134.7 (m), $131.2(\mathrm{dd}, J=13.3,3.7 \mathrm{~Hz}), 122.5-122.1(2 \mathrm{C}, \mathrm{m}), 120.6(\mathrm{q}, J=321.0 \mathrm{~Hz})$, $120.5(\mathrm{qd}, J=275.2,1.9 \mathrm{~Hz}), 118.8(\mathrm{~d}, J=87.8 \mathrm{~Hz}), 114.6(\mathrm{~d}, J=89.0 \mathrm{~Hz}), 114.3$ (dd, $J=88.9$, 
$7.6 \mathrm{~Hz}), 61.2(\mathrm{~d}, J=4.5 \mathrm{~Hz}), 49.7,49.4(\mathrm{~d}, J=10.6 \mathrm{~Hz}), 40.7-39.8(3 \mathrm{C}, \mathrm{m}), 14.3 ;{ }^{19} \mathrm{~F}$ NMR $(365$ $\left.\mathrm{MHz}, \mathrm{CDCl}_{3}\right) \delta:-68.57,-78.34 ;{ }^{31} \mathrm{P}$ NMR $\left(162 \mathrm{MHz}, \mathrm{CDCl}_{3}\right) \delta: 11.45(\mathrm{~d}, J=7.2 \mathrm{~Hz}) ; \mathrm{m} / \mathrm{z}$ LRMS (ESI + APCI) found [M - OTf] $]^{+} 613.3, \mathrm{C}_{34} \mathrm{H}_{29} \mathrm{~F}_{3} \mathrm{~N}_{4} \mathrm{O}_{2} \mathrm{P}^{+}$requires 613.2.

(5"-chloro-3'-(methoxycarbonyl)-[2,2':5',3"-terpyridin]-4"-yl)triphenylphosphonium trifluoromethanesulfonate<smiles>COC(=O)c1cc(-c2cncc(Cl)c2[PH+](c2ccccc2)c2ccccc2)cnc1-c1ccccn1</smiles>

$>$ 20:1 (Major:Unidentified Phosphonium) Mixture of Isomers

Prepared according to our previous report. ${ }^{2}$ Major isomer: ${ }^{1} \mathrm{H}$ NMR $\left(400 \mathrm{MHz}, \mathrm{CDCl}_{3}\right) \delta: 8.96$ $(1 \mathrm{H}, \mathrm{d}, J=4.5 \mathrm{~Hz}), 8.70(1 \mathrm{H}, \mathrm{d}, J=3.1 \mathrm{~Hz}), 8.61(1 \mathrm{H}, \mathrm{s}), 8.28(1 \mathrm{H}, \mathrm{s}), 8.06-7.46(18 \mathrm{H}, \mathrm{m})$, 7.40-7.29 (1H, m), $3.74(3 \mathrm{H}, \mathrm{s}) ;{ }^{13} \mathrm{C}$ NMR $\left(100 \mathrm{MHz}, \mathrm{CDCl}_{3}\right) \delta: 167.6,155.3(\mathrm{~d}, J=2.2 \mathrm{~Hz})$, 154.7, 152.4 (d, $J=7.2 \mathrm{~Hz}), 151.9(\mathrm{~d}, J=4.8 \mathrm{~Hz}), 149.6,148.6,140.7(\mathrm{~d}, J=5.7 \mathrm{~Hz}), 136.9(\mathrm{~d}$, $J=10.9 \mathrm{~Hz}), 136.8,136.1(\mathrm{~d}, J=2.3 \mathrm{~Hz}), 135.4(\mathrm{~d}, J=2.7 \mathrm{~Hz}), 134.0(\mathrm{~d}, J=10.6 \mathrm{~Hz}), 130.7(\mathrm{~d}$, $J=13.6 \mathrm{~Hz}), 130.0,127.5,125.5(\mathrm{~d}, J=88.0 \mathrm{~Hz}), 124.1,122.6,120.8(\mathrm{q}, J=321.4 \mathrm{~Hz}), 116.9$ $(\mathrm{d}, J=89.1 \mathrm{~Hz}), 52.3 ;{ }^{19} \mathrm{~F} \mathrm{NMR}\left(365 \mathrm{MHz}, \mathrm{CDCl}_{3}\right) \delta:-78.17,{ }^{31} \mathrm{P} \mathrm{NMR}\left(162 \mathrm{MHz}, \mathrm{CDCl}_{3}\right) \delta$ : 20.78 .

(5"-Chloro-3'-(methoxycarbonyl)-[2,2':5',3"'-terpyridin]-4-yl)(phenyl)bis(6(trifluoromethyl)pyridin-3-yl)phosphonium trifluoromethanesulfonate<smiles>COC(=O)c1cc(-c2cncc(Cl)c2)cnc1-c1cc(P(c2ccc(C(F)(F)F)nc2)c2ccc(C(F)(F)F)nc2)ccn1</smiles>

A $25 \mathrm{~mL}$ round bottom flask was charged with methyl 5"-chloro-[2,2':5',3"-terpyridine]-3'carboxylate $(260 \mathrm{mg}, 0.80 \mathrm{mmol}), 5,5^{\prime}$-(phenylphosphanediyl)bis(2-(trifluoromethyl)pyridine (641 mg, $1.60 \mathrm{mmol}, 2.0$ equiv), and $\mathrm{CH}_{2} \mathrm{Cl}_{2}(8 \mathrm{~mL})$. After being cooled to $-50{ }^{\circ} \mathrm{C}, \mathrm{Tf}_{2} \mathrm{O}(269$ $\mu \mathrm{L}, 1.60 \mathrm{mmol}, 2.0$ equiv) was added dropwise. After stirring for 90 minutes, the reaction was cooled to $-78{ }^{\circ} \mathrm{C}$ and N,N-Dimethylcyclohexylamine $(240 \mu \mathrm{L}, 1.60 \mathrm{mmol}, 2$ equiv) was added dropwise. The reaction was warmed to room temperature before being quenched with $\mathrm{H}_{2} \mathrm{O}$. The organic layer was washed with $\mathrm{H}_{2} \mathrm{O}(8 \times 20 \mathrm{~mL})$, dried over $\mathrm{MgSO}_{4}$, concentrated in vacuo, and added dropwise to a $50 / 50$ mixture of $\mathrm{Et}_{2} \mathrm{O} /$ Hexanes before being placed in a $-20{ }^{\circ} \mathrm{C}$ fridge. The mixture was filtered, redisolved in $\mathrm{CH}_{2} \mathrm{Cl}_{2}$, and the crash-out procedure was repeated a second and third time before affording the title compound as a yellow solid (362 $\mathrm{mg}, 0.41 \mathrm{mmol}, 52 \%$ yield). mp $115-119^{\circ} \mathrm{C}$. IR $v_{\max } / \mathrm{cm}^{-1}$ (film): $3061,2955,1726,1440,1334,1258,1029,725 ;{ }^{1} \mathrm{H}$ 
NMR $\left(400 \mathrm{MHz}, \mathrm{CDCl}_{3}\right) \delta: 9.02(1 \mathrm{H}$, app t $J=4.9 \mathrm{~Hz}), 8.96(2 \mathrm{H}, \mathrm{d}, J=5.3 \mathrm{~Hz}), 8.86(1 \mathrm{H}, \mathrm{d}, J$ $=1.5 \mathrm{~Hz}), 8.72(1 \mathrm{H}, \mathrm{s}), 8.67-8.59(3 \mathrm{H}, \mathrm{m}), 8.47(1 \mathrm{H}, \mathrm{d}, J=14.4 \mathrm{~Hz}), 8.15-8.09(3 \mathrm{H}, \mathrm{m}), 7.99$ $(1 \mathrm{H}$, app t, $J=6.5 \mathrm{~Hz}), 7.91(1 \mathrm{H}, \mathrm{s}), 7.87-7.77(5 \mathrm{H}, \mathrm{m}), 3.82(3 \mathrm{H}, \mathrm{s}) ;{ }^{13} \mathrm{C}$ NMR $(100 \mathrm{MHz}$, $\left.\mathrm{CDCl}_{3}\right) \delta: 168.1,157.5(\mathrm{~d}, J=11.0 \mathrm{~Hz}), 153.9(\mathrm{qd}, J=36.0,2.5 \mathrm{~Hz}), 153.8(\mathrm{~d}, J=13.5 \mathrm{~Hz})$, $152.0(\mathrm{~d}, J=2.2 \mathrm{~Hz}), 151.4(\mathrm{~d}, J=11.0 \mathrm{~Hz}), 149.1,148.7,146.2(\mathrm{~d}, J=9.9 \mathrm{~Hz}), 145.8,137.5(\mathrm{~d}$, $J=3.0 \mathrm{~Hz}), 135.8,135.1(\mathrm{~d}, J=11.3 \mathrm{~Hz}), 134.2,132.9,132.8,131.8,131.7,129.3,127.8(\mathrm{~d}, J=$ $9.0 \mathrm{~Hz}), 126.4(\mathrm{~d}, J=86.1 \mathrm{~Hz}), 125.8(\mathrm{~d}, J=9.8 \mathrm{~Hz}), 122.8-122.6(\mathrm{~m}), 120.5(\mathrm{qd}, J=275.5,2.0$ $\mathrm{Hz}), 120.4(\mathrm{q}, J=320.8 \mathrm{~Hz}), 116.5(\mathrm{~d}, J=88.5 \mathrm{~Hz}), 112.5(\mathrm{~d}, J=90.2 \mathrm{~Hz}), 53.1 ;{ }^{19} \mathrm{~F}$ NMR $(365$ $\left.\mathrm{MHz}, \mathrm{CDCl}_{3}\right) \delta:-68.72,-78.70 ;{ }^{31} \mathrm{P}$ NMR $\left(162 \mathrm{MHz}, \mathrm{CDCl}_{3}\right) \delta: 18.51 ; \mathrm{m} / \mathrm{z}$ LRMS (ESI + $\mathrm{APCI}$ ) found $[\mathrm{M}-\mathrm{OTf}]^{+} 724.2, \mathrm{C}_{35} \mathrm{H}_{22} \mathrm{ClF}_{6} \mathrm{~N}_{5} \mathrm{O}_{2} \mathrm{P}^{+}$requires 724.1 . 


\section{Preparation of Chlorinated Heterocycles}

\section{General Procedure B}

An $8 \mathrm{~mL}$ screw-cap vial equipped with a stir bar was charged with the phosphonium salt (1.0 equiv), $\mathrm{LiCl}$ (4.0 equiv), and placed under a nitrogen atmosphere. Dioxane $(0.1 \mathrm{M})$ was added with a syringe. The septa cap was quickly replaced with an unpierced one and the reaction was heated to $80^{\circ} \mathrm{C}$. After the stated time, the reaction was cooled to room temperature, concentrated in vacuo, and purified by column chromatography under the stated conditions to provide the chlorinated heterocycle.

\section{4-Chloro-2-phenylpyridine (3)}<smiles>Clc1ccncc1-c1ccccc1</smiles>

Prepared according to general procedure B, using diphenyl(3-phenylpyridin-4-yl)(6(trifluoromethyl)pyridin-3-yl)phosphonium trifluoromethanesulfonate (32 mg, $0.05 \mathrm{mmol}), \mathrm{LiCl}$ $(9 \mathrm{mg}, 0.20 \mathrm{mmol})$, and Dioxane $(0.5 \mathrm{~mL})$. The reaction was heated to $80{ }^{\circ} \mathrm{C}$ for 24 hours. Flash column chromatography (silica gel: 10\% EtOAc in Hexanes) was used to isolate a pure sample of the product (NMR Yield $=85 \%) .{ }^{1} \mathrm{H}$ NMR $\left(400 \mathrm{MHz}, \mathrm{CDCl}_{3}\right) \delta: 8.56(1 \mathrm{H}, \mathrm{s}), 8.48(1 \mathrm{H}, \mathrm{d}, J=$ $5.3 \mathrm{~Hz}), 7.52-7.41(6 \mathrm{H}, \mathrm{m}) ;{ }^{13} \mathrm{C} \mathrm{NMR}\left(100 \mathrm{MHz}, \mathrm{CDCl}_{3}\right) \delta: 151.6,149.3,142.4,136.6,135.6$, $129.6,128.6,128.6,124.9$. The spectroscopic data is in agreement with the literature. ${ }^{3}$

\section{4-Chloro-2-phenylpyridine (4)}<smiles>Clc1ccnc(-c2ccccc2)c1</smiles>

Prepared according to general procedure B, using phenyl(2-phenylpyridin-4-yl)bis(6(trifluoromethyl)pyridin-3-yl)phosphonium trifluoromethanesulfonate (35 mg, $0.05 \mathrm{mmol}), \mathrm{LiCl}$ $(9 \mathrm{mg}, 0.20 \mathrm{mmol})$, and Dioxane $(0.5 \mathrm{~mL})$. The reaction was heated to $80^{\circ} \mathrm{C}$ for 24 hours. Flash column chromatography (silica gel: $7.5 \%$ EtOAc in Hexanes) was used to isolate a pure sample of the product (GC Yield: $70 \%) .{ }^{1} \mathrm{H}$ NMR $\left(400 \mathrm{MHz}, \mathrm{CDCl}_{3}\right) \delta: 8.59(1 \mathrm{H}, \mathrm{d}, J=5.3 \mathrm{~Hz}), 8.00-$ $7.96(2 \mathrm{H}, \mathrm{m}), 7.74(1 \mathrm{H}, \mathrm{d}, J=1.9 \mathrm{~Hz}), 7.52-7.42(3 \mathrm{H}, \mathrm{m}), 7.25(1 \mathrm{H}, \mathrm{dd}, J=5.4,1.9 \mathrm{~Hz}) ;{ }^{13} \mathrm{C}$ NMR (100 MHz, $\left.\mathrm{CDCl}_{3}\right) \delta: 159.2,150.6,144.9,138.3,129.8,129.0,127.1,122.4,121.0$. The spectroscopic data is in agreement with the literature. ${ }^{4}$

\section{3-Bromo-4-chloro-5-methylpyridine (9)}<smiles>Cc1cncc(Br)c1Cl</smiles> 
Prepared according to general procedure B using (3-bromo-5-methylpyridin-4-yl)

triphenylphosphonium trifluoromethanesulfonate $(175 \mathrm{mg}, 0.30 \mathrm{mmol}), \mathrm{LiCl}(51 \mathrm{mg}, 1.20$ $\mathrm{mmol})$, and Dioxane $(3 \mathrm{~mL})$. The reaction was heated at $80^{\circ} \mathrm{C}$ for 24 hours. Flash column chromatography (silica gel: $33 \%$ EtOAc in Hexanes) afforded the title compound as a colorless oil (42 mg, $0.20 \mathrm{mmol}, 68 \%$ yield). IR $v_{\max } / \mathrm{cm}^{-1}$ (film): 2921, 1584, 1458, 1422, 1146, 876; ${ }^{1} \mathrm{H}$ NMR $\left(400 \mathrm{MHz}, \mathrm{CDCl}_{3}\right) \delta: 8.58(1 \mathrm{H}, \mathrm{s}), 8.33(1 \mathrm{H}, \mathrm{s}), 2.41(3 \mathrm{H}, \mathrm{s}) ;{ }^{13} \mathrm{C} \mathrm{NMR}\left(100 \mathrm{MHz}, \mathrm{CDCl}_{3}\right)$ $\delta: 150.4,149.5,143.9,134.1,121.5,18.0 ; \mathrm{m} / z$ LRMS (ESI + APCI) found $[\mathrm{M}+\mathrm{H}]^{+}$206.0, $\mathrm{C}_{6} \mathrm{H}_{6} \mathrm{BrClN}^{+}$requires 205.9.

\section{Tert-butyl ((4-chloropyridin-3-yl)methyl)(methyl)carbamate (11)}<smiles>CN(Cc1cnccc1Cl)C(=O)O</smiles>

Prepared according to general procedure B, using (3-(((tertbutoxycarbonyl)(methyl)amino)methyl)pyridin-4-yl)diphenyl(6-(trifluoromethyl)pyridin-3yl)phosphonium trifluoromethanesulfonate $(211 \mathrm{mg}, 0.30 \mathrm{mmol}), \mathrm{LiCl}(51 \mathrm{mg}, 1.20 \mathrm{mmol})$, and Dioxane $(3 \mathrm{~mL})$. The reaction was heated to $80{ }^{\circ} \mathrm{C}$ for 24 hours. Flash column chromatography (silica gel: 40\% EtOAc in Hexanes) afforded the title compound as a colorless oil (43 mg, 0.17 mmol, 56\% yield). IR $v_{\max } / \mathrm{cm}^{-1}$ (film): 2976, 2930, 1690, 1390, 1143, 729, 646; ${ }^{1} \mathrm{H}$ NMR (400 $\left.\mathrm{MHz} \mathrm{CDCl}_{3}\right) \delta: 8.41(1 \mathrm{H}, \mathrm{s}), 8.38(1 \mathrm{H}, \mathrm{d}, J=5.0 \mathrm{~Hz}), 7.27(1 \mathrm{H}, \mathrm{d}, J=5.7 \mathrm{~Hz}), 4.53(2 \mathrm{H}, \mathrm{d}, J=$ $10.6 \mathrm{~Hz}) 2.86(3 \mathrm{H}, \mathrm{s}), 1.42(9 \mathrm{H}, \mathrm{d}, J=14.7 \mathrm{~Hz}) ;{ }^{13} \mathrm{C} \mathrm{NMR}\left(100 \mathrm{MHz}, \mathrm{CDCl}_{3}\right) \delta: 155.7(\mathrm{~d}, J=$ $45.7 \mathrm{~Hz}), 150.1,149.4,143.3(\mathrm{~d}, J=12.6 \mathrm{~Hz}) 131.5,124.5,80.3,48.0(\mathrm{~d}, J=71.8 \mathrm{~Hz}), 34.7$, 28.4; m/z LRMS (ESI + APCI) found $[\mathrm{M}+\mathrm{H}]^{+} 257.1, \mathrm{C}_{12} \mathrm{H}_{18} \mathrm{ClN}_{2} \mathrm{O}_{2}{ }^{+}$requires 257.1.

\section{4-Chloro-3-(1-methyl-1H-pyrazol-5-yl)pyridine (12)}<smiles>[X]n1nccc1-c1cnccc1Cl</smiles>

Prepared according to general procedure B, using (3-(1-methyl-1H-pyrazol-5-yl)pyridin-4yl)diphenyl(6-(trifluoromethyl)pyridin-3-yl)phosphonium trifluoromethanesulfonate (192 mg, $0.30 \mathrm{mmol}), \mathrm{LiCl}(51 \mathrm{mg}, 1.20 \mathrm{mmol})$, and Dioxane $(3 \mathrm{~mL})$. The reaction was heated to $80{ }^{\circ} \mathrm{C}$ for 24 hours. Flash column chromatography (silica gel: $80 \% \mathrm{Et}_{2} \mathrm{O}$ in Hexanes) afforded the title compound as a clear oil (42 mg, $0.22 \mathrm{mmol}, 73 \%$ yield). IR $v_{\max } / \mathrm{cm}^{-1}$ (film): 3038, 2920, 2231, 1551, 1391, 1095, 927, 729; ${ }^{1} \mathrm{H}$ NMR (400 MHz, $\left.\mathrm{CDCl}_{3}\right) \delta: 8.58(1 \mathrm{H}, \mathrm{d}, J=4.4 \mathrm{~Hz}), 8.55(1 \mathrm{H}$, s), $7.59(1 \mathrm{H}, \mathrm{s}), 7.48(1 \mathrm{H}, \mathrm{d}, J=5.0 \mathrm{~Hz}), 6.36(1 \mathrm{H}, \mathrm{s}), 3.76(3 \mathrm{H}, \mathrm{s}) ;{ }^{13} \mathrm{C} \mathrm{NMR}\left(100 \mathrm{MHz}, \mathrm{CDCl}_{3}\right)$ $\delta: 151.9,151.0,144.2,138.8,136.7,126.7,124.8,108.1,37.3 ; \mathrm{m} / \mathrm{z}$ LRMS (ESI + APCI) found $[\mathrm{M}+\mathrm{H}]^{+}$194.1, $\mathrm{C}_{9} \mathrm{H}_{9} \mathrm{ClN}_{3}{ }^{+}$requires 194.0. 


\section{4-Chloro-3-(phenylethynyl)pyridine (13)}

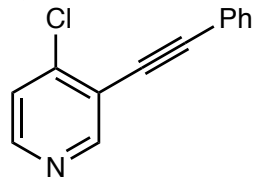

Prepared according to general procedure B, using diphenyl(3-(phenylethynyl)pyridin-4-yl)(6(trifluoromethyl)pyridin-3-yl)phosphonium trifluoromethanesulfonate (198 $\mathrm{mg}, 0.30 \mathrm{mmol}$ ), $\mathrm{LiCl}(51 \mathrm{mg}, 1.20 \mathrm{mmol})$, and Dioxane $(3 \mathrm{~mL})$. The reaction was heated to $80{ }^{\circ} \mathrm{C}$ for 48 hours. Flash column chromatography (silica gel: $2 \% \mathrm{Et}_{2} \mathrm{O}$ in Hexanes) afforded the title compound as a clear oil (41 mg, $0.19 \mathrm{mmol}, 64 \%$ yield). IR $v_{\max } / \mathrm{cm}^{-1}$ (film): 3057, 2922, 2219, 1492, 1261, 851, 688, 564; ${ }^{1} \mathrm{H}$ NMR $\left(400 \mathrm{MHz}, \mathrm{CDCl}_{3}\right) \delta: 8.74(1 \mathrm{H}, \mathrm{s}), 8.42(1 \mathrm{H}, \mathrm{d}, J=5.3 \mathrm{~Hz}), 7.61-7.56(2 \mathrm{H}$, m), 7.43-7.34 (4H, m); ${ }^{13} \mathrm{C}$ NMR (100 MHz, $\left.\mathrm{CDCl}_{3}\right) \delta: 153.5,149.0,145.2,132.0,129.3,128.6$, 124.1, 122.3, 120.9, 97.8, 83.0; m/z LRMS (ESI + APCI) found $[\mathrm{M}+\mathrm{H}]^{+} 214.1, \mathrm{C}_{13} \mathrm{H}_{9} \mathrm{ClN}^{+}$ requires 214.0 .

\section{4-Chloro-3-(pyridin-2-yloxy)pyridine (14)}<smiles>Clc1ccncc1Oc1ccccn1</smiles>

Prepared according to general procedure B, using diphenyl(3-(pyridin-2-yloxy)pyridin-4-yl)(6(trifluoromethyl)pyridin-3-yl)phosphonium trifluoromethanesulfonate (195 $\mathrm{mg}, 0.30 \mathrm{mmol}$ ), $\mathrm{LiCl}(51 \mathrm{mg}, 1.20 \mathrm{mmol})$, and Dioxane $(3 \mathrm{~mL})$. The reaction was heated to $80{ }^{\circ} \mathrm{C}$ for 48 hours. Flash column chromatography (silica gel: 50\% EtOAc in Hexanes) afforded the title compound as a clear oil (24 mg, $0.12 \mathrm{mmol}, 39 \%$ yield). IR $v_{\max } / \mathrm{cm}^{-1}$ (film): 3060, 2924, 2360, 2342, 1478 , 1243, 776, 693; ${ }^{1} \mathrm{H}$ NMR (400 MHz, $\left.\mathrm{CDCl}_{3}\right) \delta: 8.51(1 \mathrm{H}, \mathrm{s}), 8.39(1 \mathrm{H}, \mathrm{d}, J=5.2 \mathrm{~Hz}), 8.11(1 \mathrm{H}$, $\mathrm{dd}, J=4.9,1.8 \mathrm{~Hz}), 7.78-7.72(1 \mathrm{H}, \mathrm{m}), 7.43(1 \mathrm{H}, \mathrm{d}, J=5.2 \mathrm{~Hz}), 7.08-7.02(2 \mathrm{H}, \mathrm{m}) ;{ }^{13} \mathrm{C} \mathrm{NMR}$ $\left(100 \mathrm{MHz}, \mathrm{CDCl}_{3}\right) \delta: 162.5,147.4,147.2,146.8,146.0,139.9,137.1,125.4,119.3,111.3 ; \mathrm{m} / \mathrm{z}$ LRMS (ESI + APCI) found $[\mathrm{M}+\mathrm{H}]^{+} 207.1, \mathrm{C}_{10} \mathrm{H}_{8} \mathrm{ClN}_{2} \mathrm{O}^{+}$requires 207.0.

\section{4'-Chloro-5-(trifluoromethyl)-2,3'-bipyridine (15)}<smiles>FC(F)(F)c1ccc(-c2cnccc2Cl)nc1</smiles>

Prepared according to general procedure B using diphenyl(5-(trifluoromethyl)-[2,3'-bipyridin]4'-yl) (6-(trifluoromethyl)pyridin-3-yl)phosphonium trifluoromethanesulfonate (141 mg, 0.20 $\mathrm{mmol}), \mathrm{LiCl}(34 \mathrm{mg}, 0.80 \mathrm{mmol})$, and Dioxane $(2 \mathrm{~mL})$. The reaction was heated at $80{ }^{\circ} \mathrm{C}$ for 24 hours. Flash column chromatography (silica gel: 33\% EtOAc in Hexanes) afforded the title compound as a white solid (32 mg, $0.13 \mathrm{mmol}, 63 \%$ yield). $\mathrm{mp} 155-157^{\circ} \mathrm{C}$; IR $v_{\text {max }} / \mathrm{cm}^{-1}$ (film): $1625,1604,1575,1484,1323,1126,1083,1015,835 ;{ }^{1} \mathrm{H}$ NMR $\left(400 \mathrm{MHz}, \mathrm{CDCl}_{3}\right) \delta: 9.02(1 \mathrm{H}$, $\mathrm{d}, J=2.2 \mathrm{~Hz}), 8.83(1 \mathrm{H}, \mathrm{s}), 8.73-8.47(1 \mathrm{H}, \mathrm{m}), 8.05(1 \mathrm{H}, \mathrm{dd}, J=8.3,2.4 \mathrm{~Hz}), 7.83(1 \mathrm{H}, \mathrm{d}, J=$ 
$8.3 \mathrm{~Hz}), 7.46(1 \mathrm{H}, \mathrm{d}, J=5.4 \mathrm{~Hz}) ;{ }^{13} \mathrm{C}$ NMR $\left(100 \mathrm{MHz}, \mathrm{CDCl}_{3}\right) \delta: 157.2,152.0,150.9,147.0(\mathrm{q}$, $J=4.0 \mathrm{~Hz}), 142.1,133.9,133.6(\mathrm{q}, J=3.5 \mathrm{~Hz}), 126.1(\mathrm{q}, J=33.2 \mathrm{~Hz}), 125.2,124.8,123.5(\mathrm{q}, J$ $=272.6 \mathrm{~Hz}) ;{ }^{19} \mathrm{~F}$ NMR $\left(365 \mathrm{MHz}, \mathrm{CDCl}_{3}\right) \delta:-62.46 ; \mathrm{m} / z \mathrm{LRMS}(\mathrm{ESI}+\mathrm{APCI})$ found $[\mathrm{M}+\mathrm{H}]^{+}$ 259.1, $\mathrm{C}_{11} \mathrm{H}_{7} \mathrm{ClF}_{3} \mathrm{~N}_{2}{ }^{+}$requires 259.0.

\section{4-Chloro-6,7-dihydro-5H-cyclopenta[b]pyridine (16)}<smiles>Clc1ccnc2c1CCC2</smiles>

Prepared according to general procedure B using (6,7-dihydro-5H-cyclopenta [b]pyridin-4yl)diphenyl(6-(trifluoromethyl)pyridin-3-yl)phosphonium trifluoromethanesulfonate (120 mg, $0.20 \mathrm{mmol}), \mathrm{LiCl}(34 \mathrm{mg}, 0.80 \mathrm{mmol})$, and Dioxane $(2 \mathrm{~mL})$. The reaction was heated at $80{ }^{\circ} \mathrm{C}$ for 40 hours. Flash column chromatography (silica gel: 33\% EtOAc in Hexanes) afforded the title compound as a colorless oil (12 mg, $0.08 \mathrm{mmol}, 41 \%$ yield, NMR yield is $66 \%)$. IR v $v_{\max } / \mathrm{cm}^{-}$ 1 (film): 2961, 2924, 2853, 1559, 1457, 1259, 1088, 798; ${ }^{1} \mathrm{H}$ NMR (400 MHz, CDCl 3 ) $\delta: 8.22$ $(1 \mathrm{H}, \mathrm{d}, J=5.4 \mathrm{~Hz}), 7.04(1 \mathrm{H}, \mathrm{d}, J=5.4 \mathrm{~Hz}), 3.08(2 \mathrm{H}, \mathrm{t}, J=7.8 \mathrm{~Hz}), 2.99(2 \mathrm{H}, \mathrm{t}, J=7.5 \mathrm{~Hz})$, $2.14(2 \mathrm{H}, \mathrm{p}, J=7.7 \mathrm{~Hz}) ;{ }^{13} \mathrm{C}$ NMR $\left(100 \mathrm{MHz}, \mathrm{CDCl}_{3}\right) \delta: 167.4,148.6,140.8,136.0,121.6,35.2$, $30.1,22.1 ; \mathrm{m} / z$ LRMS (ESI $+\mathrm{APCI})$ found $[\mathrm{M}+\mathrm{H}]^{+} 154.1, \mathrm{C}_{8} \mathrm{H}_{9} \mathrm{ClN}^{+}$requires 154.0.

\section{3-Bromo-4-chloro-2-methylpyridine (17)}<smiles>O=[W]c1nccc(Cl)c1Br</smiles>

Prepared according to general procedure B using (3-bromo-2-methylpyridin-4-yl) diphenyl(6(trifluoromethyl)pyridin-3-yl)phosphonium trifluoromethanesulfonate (130 mg, $0.20 \mathrm{mmol}$ ), $\mathrm{LiCl}(34 \mathrm{mg}, 0.80 \mathrm{mmol})$, and Dioxane $(2 \mathrm{~mL})$. The reaction was heated at $80{ }^{\circ} \mathrm{C}$ for 40 hours. Flash column chromatography (silica gel: 33\% EtOAc in Hexanes) afforded the title compound as a colorless oil (26 mg, $0.13 \mathrm{mmol}, 63 \%$ yield). IR $v_{\max } / \mathrm{cm}^{-1}$ (film): $2921,1547,1424,1384$, 1182, 854, 706; ${ }^{1} \mathrm{H}$ NMR (400 MHz, $\left.\mathrm{CDCl}_{3}\right) \delta: 8.28(1 \mathrm{H}, \mathrm{d}, J=5.3 \mathrm{~Hz}), 7.23(1 \mathrm{H}, \mathrm{d}, J=5.2$ $\mathrm{Hz}), 2.73(3 \mathrm{H}, \mathrm{s}) ;{ }^{13} \mathrm{C} \mathrm{NMR}\left(100 \mathrm{MHz}, \mathrm{CDCl}_{3}\right) \delta: 160.1,147.6,144.6,123.1,122.0,26.5 ; \mathrm{m} / z$ LRMS (ESI + APCI) found $[\mathrm{M}+\mathrm{H}]^{+}$206.0, $\mathrm{C}_{6} \mathrm{H}_{6} \mathrm{BrClN}^{+}$requires 205.9.

\section{4-Chloro-2-methyl-3-(thiophen-3-yl)pyridine (18)}<smiles>Cc1nccc(Cl)c1-c1ccsc1</smiles>

Prepared according to general procedure B, using (2-methyl-3-(thiophen-3-yl)pyridin-4yl)diphenyl(6-(trifluoromethyl)pyridin-3-yl)phosphonium trifluoromethanesulfonate (196 mg, $0.30 \mathrm{mmol}), \mathrm{LiCl}(51 \mathrm{mg}, 1.20 \mathrm{mmol})$, and Dioxane $(3 \mathrm{~mL})$. The reaction was heated to $80{ }^{\circ} \mathrm{C}$ for 28 hours. Flash column chromatography (silica gel: 20\% EtOAc in Hexanes) afforded the 
title compound as a yellow oil (54 mg, $0.26 \mathrm{mmol}, 86 \%$ yield). IR $v_{\max } / \mathrm{cm}^{-1}$ (film): 3105,2925 , 2217, 1550, 1418, 907, 726, 656; ${ }^{1} \mathrm{H}$ NMR (400 MHz, $\left.\mathrm{CDCl}_{3}\right) \delta: 8.35(1 \mathrm{H}, \mathrm{d}, J=5.4 \mathrm{~Hz}), 7.44$ $(1 \mathrm{H}, \mathrm{dd}, J=4.9,3.0 \mathrm{~Hz}), 7.25(1 \mathrm{H}, \mathrm{d}, J=5.6 \mathrm{~Hz}), 7.22(1 \mathrm{H}, \mathrm{dd}, J=3.0,1.3 \mathrm{~Hz}), 7.02(1 \mathrm{H}, \mathrm{dd}, J$ $=5.0,1.3 \mathrm{~Hz}), 2.40(3 \mathrm{H}, \mathrm{s}) ;{ }^{13} \mathrm{C} \mathrm{NMR}\left(100 \mathrm{MHz}, \mathrm{CDCl}_{3}\right) \delta: 159.4,148.5,144.1,136.1,131.4$, 128.5, 125.9, 124.6, 122.3, 24.2; m/z LRMS (ESI + APCI) found $[\mathrm{M}+\mathrm{H}]^{+} 210.1, \mathrm{C}_{10} \mathrm{H}_{8} \mathrm{ClNS}^{+}$ requires 210.1 .

\section{2,4-Dichloro-5-phenylpyridine (20)}<smiles>Clc1cc(Cl)c(-c2ccccc2)cn1</smiles>

Prepared according to general procedure B, using (2-chloro-5-phenylpyridin-4-yl)diphenyl(6(trifluoromethyl)pyridin-3-yl)phosphonium trifluoromethanesulfonate (196 mg, $0.30 \mathrm{mmol}$ ), $\mathrm{LiCl}(51 \mathrm{mg}, 1.20 \mathrm{mmol})$, and Dioxane $(3 \mathrm{~mL})$. The reaction was heated to $80{ }^{\circ} \mathrm{C}$ for 72 hours. Flash column chromatography (silica gel gradient elution: $2 \% \mathrm{Et}_{2} \mathrm{O}$ in $\mathrm{Hexanes}$ to $10 \% \mathrm{Et}_{2} \mathrm{O}$ in Hexanes) afforded the title compound as a white solid (32 mg, $0.14 \mathrm{mmol}, 48 \%$ yield). mp 74-78 ${ }^{\circ} \mathrm{C}$. IR $v_{\max } / \mathrm{cm}^{-1}$ (film): 3085, 2923, 1732, 1539, 1441, 1127, 826, 696; ${ }^{1} \mathrm{H}$ NMR (400 MHz, $\left.\mathrm{CDCl}_{3}\right) \delta: 8.34(1 \mathrm{H}, \mathrm{s}), 7.52-7.40(6 \mathrm{H}, \mathrm{m}) ;{ }^{13} \mathrm{C} \mathrm{NMR}\left(100 \mathrm{MHz}, \mathrm{CDCl}_{3}\right) \delta: 150.8,150.7,144.3$, 135.6, 134.4, 129.5, 128.9, 128.7, 124.9; m/z LRMS (ESI + APCI) found $[\mathrm{M}+\mathrm{H}]^{+}$224.0, $\mathrm{C}_{11} \mathrm{H}_{8} \mathrm{Cl}_{2} \mathrm{~N}^{+}$requires 224.0.

\section{4-Chloro-5-(3-chloro-5-fluorophenyl)-2-methylpyridine (21)}<smiles>Cc1cc(Cl)c(-c2cc(F)cc(Cl)c2)cn1</smiles>

Prepared according to general procedure B using (5-(3-chloro-5-fluorophenyl)- 2-methylpyridin4-yl)diphenyl(6-(trifluoromethyl)pyridin-3-yl)phosphonium trifluoromethanesulfonate (140 mg, $0.20 \mathrm{mmol}), \mathrm{LiCl}(34 \mathrm{mg}, 0.80 \mathrm{mmol})$, and Dioxane $(2 \mathrm{~mL})$. The reaction was heated at $80{ }^{\circ} \mathrm{C}$ for 24 hours. Flash column chromatography (silica gel: 15\% EtOAc in Hexanes) afforded the title compound as a white solid (33 mg, $0.13 \mathrm{mmol}, 65 \%$ yield). $\mathrm{mp} 108-110{ }^{\circ} \mathrm{C}$; IR $v_{\max } / \mathrm{cm}^{-1}$ (film): 3027, 1608, 1577, 1449, 1407, 1335, 1220, 873, 794; ${ }^{1} \mathrm{H}$ NMR (400 MHz, $\left.\mathrm{CDCl}_{3}\right) \delta: 8.39$ $(1 \mathrm{H}, \mathrm{s}), 7.29(1 \mathrm{H}, \mathrm{s}), 7.21(1 \mathrm{H}, \mathrm{q}, J=1.2,0.7 \mathrm{~Hz}), 7.15(1 \mathrm{H}, \mathrm{dt}, J=8.4,2.1 \mathrm{~Hz}), 7.06(1 \mathrm{H}, \mathrm{ddd}, J$ $=9.0,2.4,1.5 \mathrm{~Hz}), 2.58(3 \mathrm{H}, \mathrm{s}) ;{ }^{13} \mathrm{C} \mathrm{NMR}\left(100 \mathrm{MHz}, \mathrm{CDCl}_{3}\right) \delta: 162.5(\mathrm{~d}, J=250.2 \mathrm{~Hz}), 159.8$, 150.3, 142.3, $138.7(\mathrm{~d}, J=9.1 \mathrm{~Hz}), 135.2(\mathrm{~d}, J=10.9 \mathrm{~Hz}), 131.4(\mathrm{~d}, J=2.3 \mathrm{~Hz}), 125.8(\mathrm{~d}, J=$ $3.3 \mathrm{~Hz}), 124.3,116.2$ (d, $J=24.6 \mathrm{~Hz}), 115.4(\mathrm{~d}, J=22.3 \mathrm{~Hz}), 24.1 ;{ }^{19} \mathrm{~F}$ NMR $\left(365 \mathrm{MHz}, \mathrm{CDCl}_{3}\right)$ $\delta:-110.53 ; m / z$ LRMS (ESI + APCI) found $[\mathrm{M}+\mathrm{H}]^{+} 256.1, \mathrm{C}_{12} \mathrm{H}_{9} \mathrm{Cl}_{2} \mathrm{FN}^{+}$requires 256.0.

\section{2-Butyl-4-chloro-5-(trifluoromethyl)pyridine (22)}


<smiles>FC(F)(F)c1cnc(Br)cc1Cl</smiles>

Prepared according to general procedure B, using (2-butyl-5-(trifluoromethyl)pyridin-4yl)diphenyl(6-(trifluoromethyl)pyridin-3-yl)phosphonium trifluoromethanesulfonate (205 $\mathrm{mg}$, $0.30 \mathrm{mmol}), \mathrm{LiCl}(51 \mathrm{mg}, 1.20 \mathrm{mmol})$, and Dioxane $(3 \mathrm{~mL})$. The reaction was heated to $80{ }^{\circ} \mathrm{C}$ for 24 hours. The ${ }^{1} \mathrm{H}$ NMR yield was measured using triphenylmethane $(73 \mathrm{mg}, 0.30 \mathrm{mmol}, 1.0$ equiv) as an internal standard (83\% NMR yield). PTLC (2\% $\mathrm{Et}_{2} \mathrm{O}$ in Hexanes) was used to obtain approximately $25 \mathrm{mg}$ of product (with solvent impurities) for characterization. IR $v_{\max } / \mathrm{cm}^{-}$ 1 (film): 2956, 2921, 1724, 1591, 1463, 1148, 1024; ${ }^{1} \mathrm{H} \mathrm{NMR}\left(400 \mathrm{MHz}, \mathrm{CDCl}_{3}\right) \delta: 8.75(1 \mathrm{H}, \mathrm{s})$, $7.31(1 \mathrm{H}, \mathrm{s}), 2.83(2 \mathrm{H}, \mathrm{t}, J=7.7 \mathrm{~Hz}), 1.77-1.67(2 \mathrm{H}, \mathrm{m}), 1.44-1.33(2 \mathrm{H}, \mathrm{m}), 0.95(3 \mathrm{H}, \mathrm{t}, J=7.4$ $\mathrm{Hz}) ;{ }^{13} \mathrm{C} \mathrm{NMR}\left(100 \mathrm{MHz}, \mathrm{CDCl}_{3}\right) \delta: 168.2,147.8$ (q, $\left.J=5.7 \mathrm{~Hz}\right), 142.6(\mathrm{q}, J=1.6 \mathrm{~Hz}), 124.8$, $122.7(\mathrm{~d}, J=273.0 \mathrm{~Hz}), 122.3(\mathrm{q}, J=31.7 \mathrm{~Hz}), 38.0,31.6,22.5,14.0 ;{ }^{19} \mathrm{~F} \mathrm{NMR}(365 \mathrm{MHz}$, $\left.\mathrm{CDCl}_{3}\right) \delta:-62.15 ; \mathrm{m} / \mathrm{z}$ LRMS (ESI $+\mathrm{APCI}$ ) found $[\mathrm{M}+\mathrm{H}]^{+} 238.1, \mathrm{C}_{10} \mathrm{H}_{12} \mathrm{ClF}_{3} \mathrm{~N}^{+}$requires 238.1 .

\section{7-Bromo-4-chloroquinoline (23)}<smiles>Clc1ccnc2cc(Br)ccc12</smiles>

Prepared according to general procedure B using (7-bromoquinolin-4-yl)diphenyl(6(trifluoromethyl)pyridin-3-yl)phosphonium trifluoromethanesulfonate (137.6 mg, $0.20 \mathrm{mmol}$ ), $\mathrm{LiCl}(34 \mathrm{mg}, 0.8 \mathrm{mmol})$, and Dioxane $(2.0 \mathrm{~mL})$. The reaction was heated to $80{ }^{\circ} \mathrm{C}$ for 14 hours. Flash column chromatography (silica gel: 20\% EtOAc in Hexanes) afforded the title compound as a white solid (36 mg, $0.15 \mathrm{mmol}, 75 \%$ yield). $\mathrm{mp} 100-102{ }^{\circ} \mathrm{C}$; IR $v_{\max } / \mathrm{cm}^{-1}$ (film): 3080, 1602, 1551, 1483, 1059, 971, 812; ${ }^{\mathrm{H}} \mathrm{NMR}\left(400 \mathrm{MHz}, \mathrm{CDCl}_{3}\right) \delta: 8.76(1 \mathrm{H}, \mathrm{d}, J=4.7 \mathrm{~Hz}), 8.29(1 \mathrm{H}$, $\mathrm{d}, J=1.9 \mathrm{~Hz}), 8.07(1 \mathrm{H}, \mathrm{d}, J=9.0 \mathrm{~Hz}), 7.70(1 \mathrm{H}, \mathrm{dd}, J=8.9,2.0 \mathrm{~Hz}), 7.48(1 \mathrm{H}, \mathrm{d}, J=4.7 \mathrm{~Hz})$; ${ }^{13} \mathrm{C}$ NMR $\left(100 \mathrm{MHz}, \mathrm{CDCl}_{3}\right) \delta: 151.0,149.8,142.9,132.2,131.3,125.7,125.4,124.9,121.7$; $m / z$ LRMS (ESI + APCI) found $[\mathrm{M}+\mathrm{H}]^{+}$242.0, $\mathrm{C}_{9} \mathrm{H}_{6} \mathrm{BrClN}^{+}$requires 241.9.

\section{4-Chloro-6-(thiophen-3-yl)quinoline (24)}<smiles>Clc1ccnc2ccc(-c3ccsc3)cc12</smiles>

Prepared according to general procedure B using diphenyl(6- (thiophen-3-yl)quinolin-4-yl)(6(trifluoromethyl)pyridin-3-yl)phosphonium trifluoromethanesulfonate (138 $\mathrm{mg}, 0.20 \mathrm{mmol})$, $\mathrm{LiCl}(34 \mathrm{mg}, 0.80 \mathrm{mmol})$, and Dioxane $(2 \mathrm{~mL})$. The reaction was heated at $80{ }^{\circ} \mathrm{C}$ for 17 hours. Flash column chromatography (silica gel: 33\% EtOAc in Hexanes) afforded the title compound as a white solid (35 mg, $0.14 \mathrm{mmol}, 72 \%$ yield). $\mathrm{mp} 114-116^{\circ} \mathrm{C}$; IR $v_{\max } / \mathrm{cm}^{-1}$ (film): 3064,1581 , 1556, 1500, 1432, 1366, 834, 821; ${ }^{1} \mathrm{H}$ NMR (400 MHz, $\left.\mathrm{CDCl}_{3}\right) \delta: 8.73(1 \mathrm{H}, \mathrm{d}, J=4.7 \mathrm{~Hz}), 8.36$ $(1 \mathrm{H}, \mathrm{d}, J=2.0 \mathrm{~Hz}), 8.12(1 \mathrm{H}, \mathrm{d}, J=8.7 \mathrm{~Hz}), 8.01(1 \mathrm{H}, \mathrm{dd}, J=8.8,2.0 \mathrm{~Hz}), 7.64(1 \mathrm{H}, \mathrm{dd}, J=3.0$, 
$1.4 \mathrm{~Hz}), 7.54(1 \mathrm{H}, \mathrm{dd}, J=5.0,1.4 \mathrm{~Hz}), 7.50-7.38(2 \mathrm{H}, \mathrm{m}) ;{ }^{13} \mathrm{C} \mathrm{NMR}\left(100 \mathrm{MHz}, \mathrm{CDCl}_{3}\right) \delta$ : $149.6,148.5,142.6,141.3,135.1,130.5,129.6,127.0,126.9,126.5,122.1,121.8,120.7 ; \mathrm{m} / z$ LRMS (ESI + APCI) found $[\mathrm{M}+\mathrm{H}]^{+} 246.0, \mathrm{C}_{13} \mathrm{H}_{9} \mathrm{ClNS}^{+}$requires 246.0.

2-Chloro-4-methylquinoline (25)<smiles>O=[N+]([O-])c1cc(Cl)nc2ccccc12</smiles>

Prepared according to general procedure B, using (4-methylquinolin-2-yl)diphenyl(6(trifluoromethyl)pyridin-3-yl)phosphonium trifluoromethanesulfonate $(187 \mathrm{mg}, 0.30 \mathrm{mmol})$, $\mathrm{LiCl}(51 \mathrm{mg}, 1.20 \mathrm{mmol})$, and Dioxane $(3 \mathrm{~mL})$. The reaction was heated to $80{ }^{\circ} \mathrm{C}$ for 72 hours. Flash column chromatography (silica gel: $2 \% \mathrm{Et}_{2} \mathrm{O}$ in Hexanes) afforded the title compound as a white solid (17 mg, $0.10 \mathrm{mmol}, 32 \%$ yield). IR $v_{\max } / \mathrm{cm}^{-1}$ (film): 2924, 2853, 1730, 1558, 1291, 1099, 844, 756; ${ }^{1} \mathrm{H}$ NMR $\left(400 \mathrm{MHz}, \mathrm{CDCl}_{3}\right) \delta: 8.01(1 \mathrm{H}, \mathrm{d}, J=8.4 \mathrm{~Hz}), 7.95(1 \mathrm{H}, \mathrm{d}, J=8.3$ $\mathrm{Hz}), 7.75-7.69(1 \mathrm{H}, \mathrm{m}), 7.60-7.54(1 \mathrm{H}, \mathrm{m}), 7.23(1 \mathrm{H}, \mathrm{s}), 2.68(3 \mathrm{H}, \mathrm{s}) ;{ }^{13} \mathrm{C}$ NMR $(100 \mathrm{MHz}$, $\left.\mathrm{CDCl}_{3}\right) \delta: 150.7,147.8,147.8,130.4,129.3,127.1,126.8,123.9,122.6,18.7 ; \mathrm{m} / \mathrm{z}$ LRMS (ESI + $\mathrm{APCI}$ ) found $[\mathrm{M}+\mathrm{H}]^{+} 178.1, \mathrm{C}_{10} \mathrm{H}_{9} \mathrm{ClN}^{+}$requires 178.0 .

\section{1-Chloro-5-nitroisoquinoline (26)}

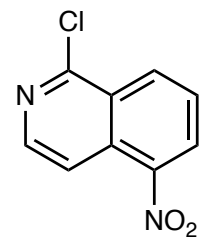

Prepared according to general procedure B, using (5-nitroisoquinolin-1-yl)diphenyl(6(trifluoromethyl)pyridin-3-yl)phosphonium trifluoromethanesulfonate (196 mg, $0.30 \mathrm{mmol}$ ), $\mathrm{LiCl}(51 \mathrm{mg}, 1.20 \mathrm{mmol})$, and Dioxane $(3 \mathrm{~mL})$. The reaction was heated to $80{ }^{\circ} \mathrm{C}$ for 12 hours. Flash column chromatography (silica gel: 10\% EtOAc in Hexanes) afforded the title compound as a yellow solid (48 mg, $0.23 \mathrm{mmol}, 76 \%$ yield). mp $183-185^{\circ} \mathrm{C}$. IR $v_{\max } / \mathrm{cm}^{-1}$ (film): 3055 , 2923, 1622, 1519, 1315, 1048, 813, 725; ${ }^{1} \mathrm{H}$ NMR (400 MHz, $\left.\mathrm{CDCl}_{3}\right) \delta: 8.73(1 \mathrm{H}, \mathrm{d}, J=8.5 \mathrm{~Hz}$ ), $8.55(1 \mathrm{H}, \mathrm{d}, J=7.7 \mathrm{~Hz}), 8.48(1 \mathrm{H}, \mathrm{d}, J=6.1 \mathrm{~Hz}), 8.39(1 \mathrm{H}, \mathrm{d}, J=6.1 \mathrm{~Hz}), 7.81(1 \mathrm{H}$, app t,$J=$ $8.0 \mathrm{~Hz}) ;{ }^{13} \mathrm{C}$ NMR $\left(100 \mathrm{MHz}, \mathrm{CDCl}_{3}\right) \delta: 152.7,145.5,144,9,133.4,130.4,129.0,127.6,127.1$, 115.9; m/z LRMS (ESI + APCI) found $[\mathrm{M}+\mathrm{H}]^{+}$209.0, $\mathrm{C}_{9} \mathrm{H}_{6} \mathrm{ClN}_{2} \mathrm{O}_{2}{ }^{+}$requires 209.0.

\section{4-Chloro-1,5-naphthyridine (27)}<smiles>Clc1ccnc2cccnc12</smiles>

Prepared according to general procedure B using (1,5-naphthyridin-4-yl)diphenyl (6(trifluoromethyl)pyridin-3-yl)phosphonium trifluoromethanesulfonate (122 $\mathrm{mg}, 0.20 \mathrm{mmol}$ ), $\mathrm{LiCl}\left(34 \mathrm{mg}, 0.8 \mathrm{mmol}\right.$ ), and Dioxane (2.0). The reaction was heated at $80{ }^{\circ} \mathrm{C}$ for 34 hours. Flash 
column chromatography (silica gel: $50 \%$ EtOAc in Hexanes) afforded the title compound as a colorless oil (8 mg, $0.05 \mathrm{mmol}, 25 \%$ yield). IR $v_{\max } / \mathrm{cm}^{-1}$ (film): 2961, 2855, 1560, 1457, 1160 , 1088, 942, 796; ${ }^{1} \mathrm{H}$ NMR $\left(400 \mathrm{MHz}, \mathrm{CDCl}_{3}\right) \delta: 9.10(1 \mathrm{H}, \mathrm{dd}, J=4.2,1.6 \mathrm{~Hz}), 8.86(1 \mathrm{H}, \mathrm{d}, J=$ $4.7 \mathrm{~Hz}), 8.45(1 \mathrm{H}, \mathrm{dd}, J=8.5,1.7 \mathrm{~Hz}), 7.82-7.66(\mathrm{~m}, 2 \mathrm{H}) ;{ }^{13} \mathrm{C}$ NMR $\left(100 \mathrm{MHz}, \mathrm{CDCl}_{3}\right) \delta$ : $151.8,150.9,145.1,144.3,141.1,138.2,125.5,124.7 ; \mathrm{m} / z$ LRMS (ESI + APCI) found $[\mathrm{M}+\mathrm{H}]^{+}$ 164.1, $\mathrm{C}_{8} \mathrm{H}_{5} \mathrm{ClN}_{2}^{+}$requires 164.0.

\section{2-Chloroquinoxaline (28)}<smiles>Clc1cnc2ccccc2n1</smiles>

Prepared according to general procedure B, using diphenyl(quinoxalin-2-yl)(6(trifluoromethyl)pyridin-3-yl)phosphonium trifluoromethanesulfonate (244 mg, $0.40 \mathrm{mmol}$ ), $\mathrm{LiCl}(68 \mathrm{mg}, 1.60 \mathrm{mmol})$, and Dioxane $(4 \mathrm{~mL})$. The reaction was heated to $80{ }^{\circ} \mathrm{C}$ for 24 hours. Flash column chromatography (silica gel: $2 \% \mathrm{Et}_{2} \mathrm{O}$ in Hexanes) afforded the title compound as a white solid (36 mg, $0.24 \mathrm{mmol}, 60 \%$ yield). $\mathrm{mp} 43-45^{\circ} \mathrm{C} \mathrm{IR} v_{\max } / \mathrm{cm}^{-1}$ (film): 3048, 2920, 1542, 1153, 1092, 957, 758, 592; ${ }^{1} \mathrm{H}$ NMR (400 MHz, $\left.\mathrm{CDCl}_{3}\right) \delta: 8.77(1 \mathrm{H}, \mathrm{s}), 8.13-8.08(1 \mathrm{H}, \mathrm{m}), 8.04-$ $7.98(1 \mathrm{H}, \mathrm{m})$ 7.83-7.74 $(2 \mathrm{H}, \mathrm{m}) ;{ }^{13} \mathrm{C}$ NMR $\left(100 \mathrm{MHz}, \mathrm{CDCl}_{3}\right) \delta: 147.5,145.1,142.1,141.1$, 131.4, 130.3, 129.4, 128.7; m/z LRMS (ESI + APCI) found $[\mathrm{M}+\mathrm{H}]^{+} 165.1, \mathrm{C}_{8} \mathrm{H}_{6} \mathrm{ClN}_{2}{ }^{+}$requires 165.0 .

\section{4-Chloropyridazine-3-carbonitrile (29)}<smiles>N#Cc1nnccc1Cl</smiles>

A $50 \mathrm{~mL}$ pressure tube was charged with pyridazine-3-carbonitrile $(53 \mathrm{mg}, 0.50 \mathrm{mmol}), 5$ (diphenylphosphaneyl)-2-(trifluoromethyl)pyridine $(182 \mathrm{mg}, 0.55 \mathrm{mmol})$, and EtOAc $(5 \mathrm{~mL})$. The pressure tube was cooled to $-50{ }^{\circ} \mathrm{C}$ and $\mathrm{Tf}_{2} \mathrm{O}(84 \mu \mathrm{L}, 0.50 \mathrm{mmol})$ was added dropwise. After stirring for 1 hour, the flask was cooled to $-78{ }^{\circ} \mathrm{C}$, DBU $(75 \mu \mathrm{L}, 0.50 \mathrm{mmol})$ was added dropwise, and the flask was allowed to warm to room temperature. After 30 minutes, $\mathrm{LiCl}$ (85 $\mathrm{mg}, 2.00 \mathrm{mmol}$ ) was added, and the pressure tube was heated to $80{ }^{\circ} \mathrm{C}$. After 5 hours, the reaction was cooled to room temperature, concentrated in vacuo, and purified by flash column chromatography (silica gel: $60 \% \mathrm{Et}_{2} \mathrm{O}$ in Hexanes) to afford the title compound as a an amorphous solid (32 mg, $0.23 \mathrm{mmol}, 45 \%$ yield). IR $v_{\max } / \mathrm{cm}^{-1}$ (film): 3101, 2921, 1531, 1081, 852, 818, 756, 732; ${ }^{1} \mathrm{H}$ NMR $\left(400 \mathrm{MHz}, \mathrm{CDCl}_{3}\right) \delta: 9.27(1 \mathrm{H}, \mathrm{d}, J=5.6 \mathrm{~Hz}), 7.74(1 \mathrm{H}, \mathrm{d}, J=5.8$ $\mathrm{Hz}) ;{ }^{13} \mathrm{C} \mathrm{NMR}\left(100 \mathrm{MHz}, \mathrm{CDCl}_{3}\right) \delta: 152.0,141.3,140.4,126.9,112.9 ; \mathrm{m} / \mathrm{z}$ LRMS (ESI + APCI) found $[\mathrm{M}+\mathrm{H}]^{+} 140.1, \mathrm{C}_{5} \mathrm{H}_{3} \mathrm{ClN}_{3}{ }^{+}$requires 140.0.

7-Chloropyrazolo[1,5-a]pyrimidine (30)<smiles>Clc1ccnc2ccnn12</smiles> 
Prepared according to general procedure B, using diphenyl(pyrazolo[1,5-a]pyrimidin-7-yl)(6(trifluoromethyl)pyridin-3-yl)phosphonium trifluoromethanesulfonate (180 $\mathrm{mg}, 0.30 \mathrm{mmol})$, $\mathrm{LiCl}(51 \mathrm{mg}, 1.20 \mathrm{mmol})$, and Dioxane $(3 \mathrm{~mL})$. The reaction was heated to $80{ }^{\circ} \mathrm{C}$ for 48 hours. Flash column chromatography (silica gel: 50\% EtOAc in Hexanes) afforded the title compound as a yellow solid (23 mg, $0.06 \mathrm{mmol}, 21 \%$ yield). The NMR spectra obtained match the reported literature values. ${ }^{5}{ }^{1} \mathrm{H}$ NMR $\left(400 \mathrm{MHz}, \mathrm{CDCl}_{3}\right) \delta: 8.40(1 \mathrm{H}, \mathrm{d}, J=4.5 \mathrm{~Hz}), 8.25(1 \mathrm{H}, \mathrm{d}, J=2.3$ $\mathrm{Hz}), 6.99(1 \mathrm{H}, \mathrm{d}, J=4.5 \mathrm{~Hz}), 6.84(\mathrm{~d}, J=2.3 \mathrm{~Hz}) ;{ }^{13} \mathrm{C} \mathrm{NMR}\left(100 \mathrm{MHz}, \mathrm{CDCl}_{3}\right) \delta: 150.2,148.3$, 145.6, 139.1, 108.2, 98.8; m/z LRMS (ESI + APCI) found $[\mathrm{M}+\mathrm{H}]^{+} 154.1, \mathrm{C}_{6} \mathrm{H}_{5} \mathrm{ClN}_{3}{ }^{+}$requires 154.0.

\section{8-Chloroimidazo[1,5-a]pyrazine (31)}<smiles>Clc1nccn2ccnc12</smiles>

Prepared according to general procedure B, using imidazo[1,5-a]pyrazin-8-yldiphenyl(6(trifluoromethyl)pyridin-3-yl)phosphonium trifluoromethanesulfonate (180 mg, $0.30 \mathrm{mmol}$ ), $\mathrm{LiCl}(51 \mathrm{mg}, 1.20 \mathrm{mmol})$, and Dioxane $(3 \mathrm{~mL})$. The reaction was heated to $80{ }^{\circ} \mathrm{C}$ for 48 hours. Flash column chromatography (silica gel: $2 \% \mathrm{CH}_{3} \mathrm{OH}, 49 \%$ EtOAc, 49\% Hexanes) afforded the title compound as a white solid (23 mg, $0.15 \mathrm{mmol}, 50 \%$ yield). $\mathrm{mp} 175-176{ }^{\circ} \mathrm{C}$. IR $v_{\max } / \mathrm{cm}^{-1}$ (film): 3120, 2922, 1613, 1431, 1329, 1197, 905, 738, 592; ${ }^{1} \mathrm{H} \mathrm{NMR}\left(400 \mathrm{MHz}, \mathrm{CDCl}_{3}\right) \delta: 8.06$ $(1 \mathrm{H}, \mathrm{d}, J=4.6 \mathrm{~Hz}), 7.82(1 \mathrm{H}, \mathrm{d}, J=1.0 \mathrm{~Hz}), 7.77(1 \mathrm{H}, \mathrm{d}, J=1.1 \mathrm{~Hz}), 7.67(1 \mathrm{H}, \mathrm{d}, J=4.5 \mathrm{~Hz})$; ${ }^{13} \mathrm{C}$ NMR $\left(100 \mathrm{MHz}, \mathrm{CDCl}_{3}\right) \delta: 144.1,138.2,135.9,128.1,118.9,115.7 ; \mathrm{m} / \mathrm{z}$ LRMS (ESI + APCI) found $[\mathrm{M}+\mathrm{H}]^{+} 154.1, \mathrm{C}_{6} \mathrm{H}_{5} \mathrm{ClN}_{3}{ }^{+}$requires 154.0 .

\section{4-Chloro-2-(3-(pentafluoro- $\lambda^{6}$-sulfaneyl)phenyl)pyridine (32)}<smiles>FS(F)(F)(F)(F)c1cccc(-c2cc(Cl)ccn2)c1</smiles>

Prepared according to general procedure B, using (2-(3-(pentafluoro- $\lambda 6$ sulfaneyl)phenyl)pyridin-4-yl)(phenyl)bis(6-(trifluoromethyl)pyridin-3-yl)phosphonium trifluoromethanesulfonate (249 $\mathrm{mg}, 0.30 \mathrm{mmol}), \mathrm{LiCl}(51 \mathrm{mg}, 1.20 \mathrm{mmol})$, and Dioxane $(3 \mathrm{~mL})$. The reaction was heated to $80{ }^{\circ} \mathrm{C}$ for 48 hours. Flash column chromatography (silica gel: $20 \%$ $\mathrm{CH}_{2} \mathrm{Cl}_{2}$ in Hexanes, ran twice) afforded the title compound as an amorphous solid (76 $\mathrm{mg}, 0.24$ mmol, 80\% yield). IR $v_{\max } / \mathrm{cm}^{-1}$ (film): 3081, 2926, 1572, 1459, 1099, 827, 713, 595; ${ }^{1} \mathrm{H} \mathrm{NMR}$ $\left(400 \mathrm{MHz}, \mathrm{CDCl}_{3}\right) \delta: 8.61(1 \mathrm{H}, \mathrm{d}, J=5.2 \mathrm{~Hz}), 8.43(1 \mathrm{H}, \mathrm{s}), 8.10(1 \mathrm{H}, \mathrm{d}, J=7.8 \mathrm{~Hz}), 7.82(1 \mathrm{H}$, $\mathrm{d}, J=8.2 \mathrm{~Hz}), 7.72(1 \mathrm{H}, \mathrm{s}), 7.58(1 \mathrm{H}$, app t, $J=8.0 \mathrm{~Hz}), 7.33-7.29(1 \mathrm{H}, \mathrm{m}) ;{ }^{13} \mathrm{C}$ NMR $(100$ $\left.\mathrm{MHz}, \mathrm{CDCl}_{3}\right) \delta: 156.9,154.8(\mathrm{~m}), 150.9,145.3,139.3,129.9,129.4,127.0$ (qn, $J=4.7 \mathrm{~Hz}$ ), 124.9 (qn, $J=4.7 \mathrm{~Hz}$ ), 123.4, 121.1; ${ }^{19} \mathrm{~F}$ NMR (365 MHz, $\mathrm{CDCl}_{3}$ ) $\delta: 83.96$ (1F, qn, $J=152.1$ $\mathrm{Hz}), 62.70(4 \mathrm{~F}, \mathrm{~d}, J=150.1 \mathrm{~Hz}) ; \mathrm{m} / \mathrm{z}$ LRMS (ESI + APCI) found $[\mathrm{M}+\mathrm{H}]^{+}$316.0, $\mathrm{C}_{11} \mathrm{H}_{8} \mathrm{ClF}_{5} \mathrm{NS}^{+}$requires 316.0 . 


\section{4-chloro-2-(1,3-dioxolan-2-yl)pyridine (33)}

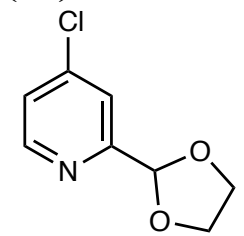

Prepared according to general procedure B, using (2-(1,3-dioxolan-2-yl)pyridin-4yl)(phenyl)bis(6-(trifluoromethyl)pyridin-3-yl)phosphonium trifluoromethanesulfonate (210 $\mathrm{mg}$, $0.30 \mathrm{mmol}), \mathrm{LiCl}(51 \mathrm{mg}, 1.20 \mathrm{mmol})$, and Dioxane $(3 \mathrm{~mL})$. The reaction was heated to $80{ }^{\circ} \mathrm{C}$ for 24 hours. Flash column chromatography (silica gel: 30\% EtOAc in Hexanes) afforded the title compound as a yellow oil (34 $\mathrm{mg}, 0.18 \mathrm{mmol}, 61 \%$ yield). IR $v_{\max } / \mathrm{cm}^{-1}$ (film): 2956, 2889, 1578, 1382, 1227, 1113, 831, 670; ${ }^{1} \mathrm{H} \mathrm{NMR}\left(400 \mathrm{MHz}, \mathrm{CDCl}_{3}\right) \delta: 8.51(1 \mathrm{H}, \mathrm{d}, J=5.3 \mathrm{~Hz}), 7.55$ $(1 \mathrm{H}, \mathrm{d}, J=1.8 \mathrm{~Hz}), 7.29(1 \mathrm{H}, \mathrm{dd}, J=5.3,2.0 \mathrm{~Hz}), 5.84(1 \mathrm{H}, \mathrm{s}), 4.19-4.04(4 \mathrm{H}, \mathrm{m}) ;{ }^{13} \mathrm{C} \mathrm{NMR}$ $\left(100 \mathrm{MHz}, \mathrm{CDCl}_{3}\right) \delta: 158.9,150.4,145.1,124.4,121.4,103.1,65.8 ; \mathrm{m} / \mathrm{z}$ LRMS (ESI + APCI) found $[\mathrm{M}+\mathrm{H}]^{+}$186.1, $\mathrm{C}_{8} \mathrm{H}_{9} \mathrm{ClNO}_{2}{ }^{+}$requires 186.0.

\section{2-(((Tert-butyldimethylsilyl)oxy)(phenyl)methyl)-4-chloropyridine (34)}<smiles>CCCCCOC(c1ccccc1)c1cc(Cl)ccn1</smiles>

Prepared according to general procedure B using (2-(((tertbutyldimethylsilyl)oxy)(phenyl)methyl)pyridin-4-yl)(phenyl)bis(6-(trifluoromethyl)pyridin-3yl)phosphonium trifluoromethanesulfonate ( $85 \mathrm{mg}, 0.10 \mathrm{mmol}), \mathrm{LiCl}(17 \mathrm{mg}, 0.40 \mathrm{mmol})$, and Dioxane $(1 \mathrm{~mL})$. The reaction was heated at $80^{\circ} \mathrm{C}$ for 44 hours. Flash column chromatography (silica gel: 15\% EtOAc in Hexanes) afforded the title compound as a colorless oil (18 $\mathrm{mg}, 0.12$ mmol, 62\% yield). IR $v_{\max } / \mathrm{cm}^{-1}$ (film): 2954, 2928, 2856, 1973, 1556, 1462, 1390, 1252, 1112 , $1068,863,835,702 ;{ }^{1} \mathrm{H}$ NMR $\left(400 \mathrm{MHz}, \mathrm{CDCl}_{3}\right) \delta: 8.34(1 \mathrm{H}, \mathrm{d}, J=5.3 \mathrm{~Hz}), 7.60(1 \mathrm{H}, \mathrm{d}, J=$ $2.1 \mathrm{~Hz}), 7.47-7.37(2 \mathrm{H}, \mathrm{m}), 7.31-7.25(2 \mathrm{H}, \mathrm{m}), 7.21(1 \mathrm{H}, \mathrm{d}, J=7.3 \mathrm{~Hz}), 7.10(1 \mathrm{H}, \mathrm{dd}, J=5.3$, $2.1 \mathrm{~Hz}), 5.83(1 \mathrm{H}, \mathrm{s}), 0.91(10 \mathrm{H}, \mathrm{s}),-0.01(6 \mathrm{H}, \mathrm{d}, J=8.7 \mathrm{~Hz}) ;{ }^{13} \mathrm{C} \mathrm{NMR}\left(100 \mathrm{MHz}, \mathrm{CDCl}_{3}\right) \delta$ : $166.3,149.7,145.0,143.3,128.5,127.6,126.4,122.5,120.6,77.6,26.0,18.4,-4.7,-4.8 ; \mathrm{m} / z$ LRMS (ESI + APCI) found $[\mathrm{M}+\mathrm{H}]^{+} 334.2, \mathrm{C}_{18} \mathrm{H}_{25} \mathrm{ClONSi}^{+}$requires 334.1.

\section{4-Chloro-3,5-dimethylpyridine (35)}<smiles>[N+]c1cncc([N+](=O)[O-])c1Cl</smiles>

A $25 \mathrm{~mL}$ round bottom flask was charged with 3,5-dimethylpyridine (57 $\mu \mathrm{L}, 0.50 \mathrm{mmol})$, triphenylphosphine $(131 \mathrm{mg}, 0.55 \mathrm{mmol})$, and $\mathrm{CH}_{2} \mathrm{Cl}_{2}(5 \mathrm{~mL})$. The flask was cooled to $-50{ }^{\circ} \mathrm{C}$ 
and $\mathrm{Tf}_{2} \mathrm{O}(84 \mu \mathrm{L}, 0.50 \mathrm{mmol})$ was added dropwise. After 1 hour, the flask was cooled to $-78{ }^{\circ} \mathrm{C}$, DBU $(75 \mu \mathrm{L}, 0.50 \mathrm{mmol})$ was added dropwise, and the flask was allowed to warm to room temperature. After 30 minutes, the reaction was quenched with $\mathrm{H}_{2} \mathrm{O}$ and the organic layer was washed $(3 \times 10 \mathrm{~mL})$. The organic layer was dried over $\mathrm{MgSO}_{4}$, concentrated in vacuo, and dried under high-vacuum for 20 minutes. Dioxane $(5 \mathrm{~mL})$ was added, followed by the dropwise addition of $\mathrm{HCl}\left(4 \mathrm{M}\right.$ in Dioxanes, $125 \mu \mathrm{L}, 0.50 \mathrm{mmol}$ ). The reaction was heated at $80{ }^{\circ} \mathrm{C}$ for 24 hours before being cooled to room temperature and quenched with aqueous $\mathrm{NaHCO}_{3}$. The aqueous layer was extracted from with $\mathrm{CH}_{2} \mathrm{Cl}_{2}(3 \times 10 \mathrm{~mL})$, and the organic extracts were dried with $\mathrm{MgSO}_{4}$ and concentrated in vacuo. The ${ }^{1} \mathrm{H}$ NMR yield was measured using triphenylmethane (122 mg, $0.50 \mathrm{mmol}, 1.0$ equiv) as an internal standard (33\% NMR yield). IR $v_{\max } / \mathrm{cm}^{-1}$ (film): 3021, 2954, 1562, 1408, 1078, 880, 762; ${ }^{1} \mathrm{H}$ NMR (400 MHz, CD $\left.{ }_{3} \mathrm{OD}\right) \delta: 8.23$ $(2 \mathrm{H}, \mathrm{s}), 2.38(6 \mathrm{H}, \mathrm{s}) ;{ }^{13} \mathrm{C} \mathrm{NMR}\left(100 \mathrm{MHz}, \mathrm{CDCl}_{3}\right) \delta: 148.9,144.2,131.6,17.1 ; \mathrm{m} / \mathrm{z}$ LRMS (ESI $+\mathrm{APCI})$ found $[\mathrm{M}+\mathrm{H}]^{+} 142.1, \mathrm{C}_{7} \mathrm{H}_{9} \mathrm{ClN}^{+}$requires 142.0 .

\section{4-Chloro-5-phenylnicotinonitrile (36)}<smiles>N#Cc1cncc(-c2ccccc2)c1Cl</smiles>

Prepared according to general procedure B, using (3-cyano-5-phenylpyridin-4yl)triphenylphosphonium trifluoromethanesulfonate $(177 \mathrm{mg}, 0.30 \mathrm{mmol}), \mathrm{LiCl}(51 \mathrm{mg}, 1.20$ $\mathrm{mmol})$, and Dioxane $(3 \mathrm{~mL})$. The reaction was heated to $80{ }^{\circ} \mathrm{C}$ for 40 hours. Flash column chromatography (silica gel: 20\% EtOAc in Hexanes) afforded the title compound as a white solid (57 mg, $0.27 \mathrm{mmol}, 89 \%$ yield). $\mathrm{mp} 64-66^{\circ} \mathrm{C}$; IR $v_{\max } / \mathrm{cm}^{-1}$ (film): $3035,2921,2360,2236,1544$, 1422, 1095, 764, 698; ${ }^{1} \mathrm{H}$ NMR $\left(400 \mathrm{MHz}, \mathrm{CDCl}_{3}\right) \delta: 8.81(1 \mathrm{H}, \mathrm{s}), 8.72(1 \mathrm{H}, \mathrm{s}), 7.55-7.39(5 \mathrm{H}$, $\mathrm{m}) ;{ }^{13} \mathrm{C} \mathrm{NMR}\left(100 \mathrm{MHz}, \mathrm{CDCl}_{3}\right) \delta: 154.2,152.4,144.5,137.2,133.7,129.4,129.4,128.9$, 114.3, 112.0; $\mathrm{m} / \mathrm{z}$ LRMS (ESI + APCI) found $[\mathrm{M}+\mathrm{H}]^{+} 215.0, \mathrm{C}_{12} \mathrm{H}_{8} \mathrm{ClN}_{2}{ }^{+}$requires 215.0.

\section{4-Chloro-5-(4-methoxyphenyl)pyrimidine (37)}<smiles>COc1ccc(-c2cncnc2Cl)cc1</smiles>

Prepared according to general procedure B using (5-(4-methoxyphenyl)pyrimidin-4-yl) triphenylphosphonium trifluoromethanesulfonate $(119 \mathrm{mg}, 0.20 \mathrm{mmol}), \mathrm{LiCl}(34 \mathrm{mg}, 0.80$ $\mathrm{mmol})$, and Dioxane $(2 \mathrm{~mL})$. The reaction was heated at $80{ }^{\circ} \mathrm{C}$ for 40 hours. Flash column chromatography (silica gel: 15\% EtOAc in Hexanes) afforded the title compound as a white solid (17 mg, $0.08 \mathrm{mmol}, 40 \%$ yield). $\mathrm{mp} \mathrm{90-91}{ }^{\circ} \mathrm{C}$; IR $\mathrm{v}_{\max } / \mathrm{cm}^{-1}$ (film): 2934, 1608, 1531, 1514, 1246, 1189, 1111, 1031, 824, 764, 885; ${ }^{1} \mathrm{H}$ NMR (400 MHz, $\left.\mathrm{CDCl}_{3}\right) \delta: 8.94(1 \mathrm{H}, \mathrm{s}), 8.64(1 \mathrm{H}$, s), 7.48-7.35 (2H, m), 7.10-6.93 (2H, m), $\left.3.87(3 \mathrm{H}, \mathrm{s}) ;{ }^{13} \mathrm{C} \mathrm{NMR} \mathrm{(100} \mathrm{MHz,} \mathrm{CDCl}_{3}\right) \delta: 160.5$, $159.4,158.1,157.0,134.7,130.7,125.8,114.4,55.5 ; \mathrm{m} / z$ LRMS (ESI + APCI) found $[\mathrm{M}+\mathrm{H}]^{+}$ 221.1, $\mathrm{C}_{11} \mathrm{H}_{10} \mathrm{ClN}_{2} \mathrm{O}^{+}$requires 221.0.

\section{4-Chloro-5-(3-fluoro-4-(trifluoromethoxy)phenyl)pyrimidine (38)}




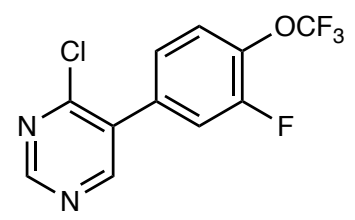

Prepared according to general procedure B using (5-(3-fluoro-4-(trifluoromethoxy) phenyl)pyrimidin-4-yl)triphenylphosphonium (134 mg, $0.20 \mathrm{mmol}$ ), LiCl (34 mg, $0.80 \mathrm{mmol}$ ), and Dioxane $(2 \mathrm{~mL})$. The reaction was heated at $80{ }^{\circ} \mathrm{C}$ for 24 hours. Flash column chromatography (silica gel: 15\% EtOAc in Hexanes) afforded the title compound as a colorless oil (44 mg, $0.15 \mathrm{mmol}, 76 \%$ yield). IR $v_{\max } / \mathrm{cm}^{-1}$ (film): 1625, 1567, 1530, 1506, 1396, 1249, 1210, 1169, 1101, 788, 687; ${ }^{1} \mathrm{H}$ NMR $\left(400 \mathrm{MHz}, \mathrm{CDCl}_{3}\right) \delta: 9.03(1 \mathrm{H}, \mathrm{s}), 8.64(1 \mathrm{H}, \mathrm{s}), 7.41(1 \mathrm{H}$, $\mathrm{t}, J=8.2 \mathrm{~Hz}), 7.28-7.00(2 \mathrm{H}, \mathrm{m}) ;{ }^{13} \mathrm{C}$ NMR $\left(100 \mathrm{MHz}, \mathrm{CDCl}_{3}\right) \delta: 160.8(\mathrm{~d}, J=68.5 \mathrm{~Hz}), 158.7$ $(\mathrm{d}, J=1.7 \mathrm{~Hz}), 158.6,158.5,150.9(\mathrm{dd}, J=11.1,1.7 \mathrm{~Hz}), 132.3(\mathrm{~d}, J=1.7 \mathrm{~Hz}), 128.7,120.4(\mathrm{q}$, $J=259.3 \mathrm{~Hz}), 120.1(\mathrm{~d}, J=15.5 \mathrm{~Hz}), 116.9(\mathrm{~d}, J=3.3 \mathrm{~Hz}), 109.5(\mathrm{~d}, J=25.7 \mathrm{~Hz}) ;{ }^{19} \mathrm{~F}$ NMR $\left(365 \mathrm{MHz}, \mathrm{CDCl}_{3}\right) \delta:-57.99,-109.03 ; \mathrm{m} / z$ LRMS (ESI + APCI) found $[\mathrm{M}+\mathrm{H}]^{+}$293.1, $\mathrm{C}_{11} \mathrm{H}_{6} \mathrm{ClF}_{4} \mathrm{~N}_{2} \mathrm{O}^{+}$requires 293.0.

Ethyl 4-((4-chlorophenyl)(4-chloropyridin-2-yl)methoxy)piperidine-1-carboxylate (49)<smiles>CCN1CCC(OC(c2ccc(Cl)cc2)c2cc(Cl)ccn2)CC1</smiles>

Prepared according to general procedure B using (2-((4-chlorophenyl)((1-(ethoxycarbonyl) piperidin-4-yl)oxy)methyl)pyridin-4-yl)(phenyl)bis(6-(trifluoromethyl)pyridin-3-

yl)phosphonium trifluoromethanesulfonate (185 mg, $0.20 \mathrm{mmol}), \mathrm{LiCl}(34 \mathrm{mg}, 0.80 \mathrm{mmol})$, and Dioxane $(2 \mathrm{~mL})$. The reaction was heated at $80^{\circ} \mathrm{C}$ for 24 hours. Flash column chromatography (silica gel: $33 \%$ EtOAc in Hexanes) afforded the title compound as a colorless oil (54 mg, 0.13 mmol, 66\% yield). IR $v_{\max } / \mathrm{cm}^{-1}$ (film): 2929, 2867, 1692, 1572, 1431, 1383, 1227, 1085, 1029, 823,$756 ;{ }^{1} \mathrm{H}$ NMR $\left(400 \mathrm{MHz}, \mathrm{CDCl}_{3}\right) \delta: 8.37(1 \mathrm{H}, \mathrm{d}, J=5.3 \mathrm{~Hz}), 7.54(1 \mathrm{H}, \mathrm{d}, J=2.0 \mathrm{~Hz}), 7.44-$ $7.23(4 \mathrm{H}, \mathrm{m}), 7.15(1 \mathrm{H}, \mathrm{dd}, J=5.3,2.1 \mathrm{~Hz}), 5.57(1 \mathrm{H}, \mathrm{s}), 4.10(2 \mathrm{H}, \mathrm{q}, J=7.1 \mathrm{~Hz}), 3.75(2 \mathrm{H}, \mathrm{dt}$, $J=11.6,4.6 \mathrm{~Hz}), 3.60(1 \mathrm{H}, \mathrm{tt}, J=7.6,3.6 \mathrm{~Hz}), 3.22-3.10(2 \mathrm{H}, \mathrm{m}), 1.81(2 \mathrm{H}$, ddd, $J=11.6,6.8$, $3.4 \mathrm{~Hz}), 1.63(2 \mathrm{H}$, ddd, $J=13.1,8.5,4.3 \mathrm{~Hz}), 1.23(3 \mathrm{H}, \mathrm{t}, J=7.1 \mathrm{~Hz}) ;{ }^{13} \mathrm{C}$ NMR $(100 \mathrm{MHz}$, $\left.\mathrm{CDCl}_{3}\right) \delta: 163.7,155.6,150.0,145.1,139.5,133.8,128.8,128.3,123.0,120.9,80.5,72.9,61.4$, $41.1,41.1,31.3,31.0,14.8 ; \mathrm{m} / z$ LRMS (ESI + APCI) found $[\mathrm{M}+\mathrm{H}]^{+} 409.2, \mathrm{C}_{20} \mathrm{H}_{23} \mathrm{Cl}_{2} \mathrm{~N}_{2} \mathrm{O}_{3}{ }^{+}$ requires 409.1 .

((3S,4R)-1-(Ethoxycarbonyl)-4-(4-fluorophenyl)piperidin-3-yl)methyl 4-chloro-2methylnicotinate (51) 


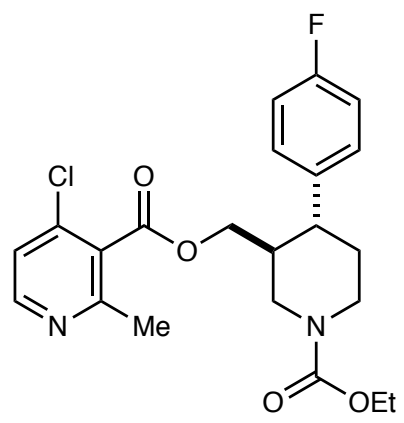

Prepared according to general procedure B, using (3-((( $3 \mathrm{~S}, 4 \mathrm{R})-1$-(ethoxycarbonyl)-4-(4fluorophenyl)piperidin-3-yl)methoxy)carbonyl)-2-methylpyridin-4-yl)diphenyl(6-

(trifluoromethyl)pyridin-3-yl)phosphonium trifluoromethanesulfonate (176 mg, $0.20 \mathrm{mmol}$ ), $\mathrm{LiCl}(34 \mathrm{mg}, 0.80 \mathrm{mmol})$, and Dioxane $(2 \mathrm{~mL})$. The reaction was heated to $80{ }^{\circ} \mathrm{C}$ for 24 hours. Flash column chromatography (silica gel gradient elution: 40\% EtOAc in Hexanes to 50\% EtOAc in Hexanes) afforded the title compound as an amorphous solid (71 $\mathrm{mg}, 0.16 \mathrm{mmol}, 82 \%$ yield). IR $v_{\max } / \mathrm{cm}^{-1}$ (film): 2920, 2864, 1689, 1438, 1273, 1222, 832, 726; ${ }^{1} \mathrm{H}$ NMR (400 MHz, $\left.\mathrm{CDCl}_{3}\right) \delta: 8.42(1 \mathrm{H}, \mathrm{d}, J=5.3 \mathrm{~Hz}), 7.21(1 \mathrm{H}, \mathrm{d}, J=5.4 \mathrm{~Hz}), 7.15(2 \mathrm{H}, \mathrm{dd}, J=13.8 \mathrm{~Hz}, 5.5 \mathrm{~Hz})$, $7.01(2 \mathrm{H}$, app t, $J=8.6 \mathrm{~Hz}), 4.49(1 \mathrm{H}$, br s), $4.27(1 \mathrm{H}, \mathrm{br} \mathrm{s}), 4.19-4.09(3 \mathrm{H}, \mathrm{m}), 3.96(1 \mathrm{H}$, dd, $J=$ 11.4, $7.3 \mathrm{~Hz}), 2.88-2.67(2 \mathrm{H}, \mathrm{m}), 2.60-2.44(4 \mathrm{H}, \mathrm{m}), 2.19-2.07(1 \mathrm{H}, \mathrm{m}), 1.81(1 \mathrm{H}, \mathrm{m}), 1.69(1 \mathrm{H}$, $\mathrm{qd}, J=12.7,4.2 \mathrm{~Hz}), 1.26(3 \mathrm{H}, \mathrm{t}, J=7.1 \mathrm{~Hz}) ;{ }^{13} \mathrm{C} \mathrm{NMR}\left(100 \mathrm{MHz}, \mathrm{CDCl}_{3}\right) \delta: 166.0,161.9(\mathrm{~d}, J$ $=245.7 \mathrm{~Hz}), 156.9,155.5,150.4,141.1,138.4(\mathrm{~d}, J=3.2 \mathrm{~Hz}), 129.1,128.8(\mathrm{~d}, J=7.8 \mathrm{~Hz})$, $122.02,115.9(\mathrm{~d}, J=21.3 \mathrm{~Hz}), 66.3,61.6,47.1,44.5,41.0,41.0,34.2,23.1,14.8 ;{ }^{19} \mathrm{~F}$ NMR $(365$ $\left.\mathrm{MHz}, \mathrm{CDCl}_{3}\right) \delta:-115.60(\mathrm{~m}) ; \mathrm{m} / \mathrm{z}$ LRMS (ESI + APCI) found $[\mathrm{M}+\mathrm{H}]^{+} 435.2, \mathrm{C}_{22} \mathrm{H}_{25} \mathrm{ClFN}_{2} \mathrm{O}_{4}{ }^{+}$ requires 435.1 .

\section{(R)-1-(3,5-Bis(trifluoromethyl)phenyl)ethyl 4-chloro-5-methylpicolinate (52)}<smiles>COc1cnc(C(=O)OC(C)c2cc(C(F)(F)F)cc(C(F)(F)F)c2)cc1Cl</smiles>

Prepared according to general procedure $\mathrm{B}$, using (R)-(2-((1-)(3,5bis(trifluoromethyl)phenyl)ethoxy)carbonyl)-5-methylpyridin-4-yl)diphenyl(6(trifluoromethyl)pyridin-3-yl)phosphonium trifluoromethanesulfonate (257 $\mathrm{mg}, 0.30 \mathrm{mmol})$, $\mathrm{LiCl}(51 \mathrm{mg}, 1.20 \mathrm{mmol})$, and Dioxane $(3 \mathrm{~mL})$. The reaction was heated to $80{ }^{\circ} \mathrm{C}$ for 48 hours. Flash column chromatography (silica gel: 15\% EtOAc in Hexanes) afforded the title compound as a clear oil (71 mg, $0.16 \mathrm{mmol}, 82 \%$ yield). IR $v_{\max } / \mathrm{cm}^{-1}$ (film): 3469, 2976, 1724, 1276, 1130 , 899, 754, 637; ${ }^{1} \mathrm{H}$ NMR $\left(400 \mathrm{MHz}, \mathrm{CDCl}_{3}\right) \delta: 8.58(1 \mathrm{H}, \mathrm{s}), 8.07(1 \mathrm{H}, \mathrm{s}), 7.91(2 \mathrm{H}, \mathrm{s}), 7.81(1 \mathrm{H}$, s), $6.24(1 \mathrm{H}, \mathrm{q}, J=6.7 \mathrm{~Hz}), 2.43(3 \mathrm{H}, \mathrm{s}), 1.77(3 \mathrm{H}, \mathrm{d}, J=6.7 \mathrm{~Hz}) ;{ }^{13} \mathrm{C} \mathrm{NMR}\left(100 \mathrm{MHz}, \mathrm{CDCl}_{3}\right)$ $\delta: 163.6,151.7,146.6,145.2,143.8,136.5,133.2(\mathrm{q}, J=33.6 \mathrm{~Hz}), 126.7(\mathrm{~d}, J=3.7 \mathrm{~Hz}), 125.9$, 123.3 (q, $J=272.7 \mathrm{~Hz}), 122.3(\mathrm{~m}), 72.7,22.1,17.1 ;{ }^{19} \mathrm{~F} \mathrm{NMR}\left(365 \mathrm{MHz}, \mathrm{CDCl}_{3}\right) \delta:-62.93 ; \mathrm{m} / \mathrm{z}$ LRMS (ESI + APCI) found $[\mathrm{M}+\mathrm{H}]^{+} 412.1, \mathrm{C}_{17} \mathrm{H}_{13} \mathrm{ClF}_{6} \mathrm{NO}_{2}{ }^{+}$requires 412.1 .

\section{4-Chloro-3-methyl-5-(((6-methylpyridin-3-yl)oxy)methyl)pyridine (54)}




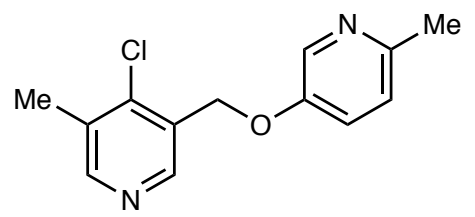

Prepared according to general procedure B using (3-methyl-5-(((6-methylpyridin-3-yl)oxy) methyl)pyridin-4-yl)triphenylphosphonium trifluoromethanesulfonate $(125 \mathrm{mg}, 0.20 \mathrm{mmol})$, $\mathrm{LiCl}(34 \mathrm{mg}, 0.80 \mathrm{mmol})$, and Dioxane $(2 \mathrm{~mL})$. The reaction was heated to $80{ }^{\circ} \mathrm{C}$ for 24 hours. Flash column chromatography (silica gel: $33 \%$ EtOAc in Hexanes) afforded the title compound as a white solid (17 mg, $0.07 \mathrm{mmol}, 34 \%$ yield). $\mathrm{mp} 88-90{ }^{\circ} \mathrm{C}$; IR $v_{\max } / \mathrm{cm}^{-1}$ (film): 2922, 2854, $1742,1697,1574,1484,1455,1368,1271,1243,1065,814,762 ;{ }^{1} \mathrm{H} \mathrm{NMR}\left(400 \mathrm{MHz}, \mathrm{CDCl}_{3}\right) \delta$ : 8.55 (s, 1H), 8.44 (s, 1H), 8.29 (d, $J=3.0 \mathrm{~Hz}, 1 \mathrm{H}), 7.21$ (dd, $J=8.5,3.0 \mathrm{~Hz}, 1 \mathrm{H}), 7.09$ (d, $J=$ $8.4 \mathrm{~Hz}, 1 \mathrm{H}), 5.18(\mathrm{~s}, 2 \mathrm{H}), 2.50(\mathrm{~s}, 3 \mathrm{H}), 2.40(\mathrm{~s}, 3 \mathrm{H}) ;{ }^{13} \mathrm{C} \mathrm{NMR}\left(100 \mathrm{MHz}, \mathrm{CDCl}_{3}\right) \delta: 152.7$, $151.4,151.1,147.8,143.5,136.7,132.3,129.8,123.8,123.0,66.3,23.3,17.1 ; \mathrm{m} / z$ LRMS (ESI + APCI) found $[\mathrm{M}+\mathrm{H}]^{+} 249.1, \mathrm{C}_{13} \mathrm{H}_{14} \mathrm{ClN}_{2} \mathrm{O}^{+}$requires 249.1 .

4-Chloro-3-(3-fluoro-5-(5-(trifluoromethyl)pyridin-2-yl)phenyl)pyridine (55)<smiles>Fc1cc(-c2cc(C(F)(F)F)ccn2)cc(-c2cnccc2Cl)c1</smiles>

Prepared according to general procedure B, using (3-(3-fluoro-5-(5-(trifluoromethyl)pyridin-2yl)phenyl)pyridin-4-yl)diphenyl(6-(trifluoromethyl)pyridin-3-yl)phosphonium trifluoromethanesulfonate (239 $\mathrm{mg}, 0.30 \mathrm{mmol}), \mathrm{LiCl}(51 \mathrm{mg}, 1.20 \mathrm{mmol})$, and Dioxane $(3 \mathrm{~mL})$. The reaction was heated to $80{ }^{\circ} \mathrm{C}$ for 24 hours. Flash column chromatography (silica gel: $15 \%$ EtOAc in Hexanes) afforded the title compound as a white solid ( $82 \mathrm{mg}, 0.23 \mathrm{mmol}, 78 \%$ yield). mp 112-113 ${ }^{\circ} \mathrm{C}$. IR $v_{\max } / \mathrm{cm}^{-1}$ (film): 3044, 2924, 1596, 1328, 1114, 1082, 920, 838; ${ }^{1} \mathrm{H}$ NMR $\left(400 \mathrm{MHz}, \mathrm{CDCl}_{3}\right) \delta: 8.96(1 \mathrm{H}, \mathrm{s}), 8.62(1 \mathrm{H}, \mathrm{s}), 8.53(1 \mathrm{H}, \mathrm{d}, J=5.3 \mathrm{~Hz}), 8.02(1 \mathrm{H}, \mathrm{dd}, J=8.3$, $2.2 \mathrm{~Hz}), 7.92(1 \mathrm{H}$, app t, $J=8.3 \mathrm{~Hz}), 7.89-7.83(2 \mathrm{H}, \mathrm{m}), 7.46(1 \mathrm{H}, \mathrm{d}, J=5.3 \mathrm{~Hz}), 7.29(1 \mathrm{H}, \mathrm{ddd}$, $J=8.8,6.4,1.5 \mathrm{~Hz}) ;{ }^{13} \mathrm{C} \mathrm{NMR}\left(100 \mathrm{MHz}, \mathrm{CDCl}_{3}\right) \delta: 163.1(\mathrm{~d}, J=247.5 \mathrm{~Hz}), 158.7(\mathrm{~m}), 151.3$, $150.1,146.9(\mathrm{q}, J=4.1 \mathrm{~Hz}), 142.4,140.5(\mathrm{~d}, J=8.2 \mathrm{~Hz}), 138.2(\mathrm{~d}, J=8.1 \mathrm{~Hz}), 135.1(\mathrm{~d}, J=2.2$ $\mathrm{Hz}), 134.4(\mathrm{q}, J=3.6 \mathrm{~Hz}), 125.8(\mathrm{q}, J=33.2 \mathrm{~Hz}), 125.0,124.3(\mathrm{~d}, J=2.6 \mathrm{~Hz}), 123.7(\mathrm{q}, J=$ $274.3 \mathrm{~Hz}), 120.2,118.2(\mathrm{~d}, J=22.9 \mathrm{~Hz}), 114.4(\mathrm{~d}, J=23.0 \mathrm{~Hz}) ;{ }^{19} \mathrm{~F} \mathrm{NMR}\left(365 \mathrm{MHz}, \mathrm{CDCl}_{3}\right) \delta$ : $-62.36,-111.67(\mathrm{t}, J=9.5 \mathrm{~Hz}) ; \mathrm{m} / \mathrm{z}$ LRMS (ESI + APCI) found $[\mathrm{M}+\mathrm{H}]^{+} 353.1, \mathrm{C}_{17} \mathrm{H}_{10} \mathrm{ClF}_{4} \mathrm{~N}_{2}{ }^{+}$ requires 353.0.

\section{((4-Chloropyridin-2-yl)methylene)bis(4,1-phenylene) diacetate (57)}


<smiles>CC(=O)Oc1ccc(C(c2ccc(OC(C)=O)cc2)c2cc(Cl)ccn2)cc1</smiles>

Prepared according to general procedure B using (2-(bis(4-acetoxyphenyl)methyl) pyridin-4yl)(phenyl)bis(6-(trifluoromethyl)pyridin-3-yl)phosphonium trifluoromethanesulfonate (190 mg, $0.20 \mathrm{mmol}), \mathrm{LiCl}(34 \mathrm{mg}, 0.80 \mathrm{mmol})$, and Dioxane $(2 \mathrm{~mL})$. The reaction was heated to $80{ }^{\circ} \mathrm{C}$ for 24 hours. Flash column chromatography (silica gel: 33\% EtOAc in Hexanes) afforded the title compound as a colorless oil $\left(51 \mathrm{mg}, 0.13 \mathrm{mmol}, 65 \%\right.$ yield). IR $v_{\max } / \mathrm{cm}^{-1}$ (film): 1754 , $1572,1555,1503,1368,1191,1164,1016,910,733 ;{ }^{1} \mathrm{H}$ NMR $\left(400 \mathrm{MHz}, \mathrm{CDCl}_{3}\right) \delta: 8.48(1 \mathrm{H}, \mathrm{d}$, $J=5.3 \mathrm{~Hz}), 7.23-7.10(6 \mathrm{H}, \mathrm{m}), 7.10-6.96(4 \mathrm{H}, \mathrm{m}), 5.62(1 \mathrm{H}, \mathrm{s}), 2.27(6 \mathrm{H}, \mathrm{s}) ;{ }^{13} \mathrm{C} \mathrm{NMR}(100$ $\left.\mathrm{MHz}, \mathrm{CDCl}_{3}\right) \delta: 169.5,164.4,150.6,149.6,144.8,139.3,130.3,124.1,122.2,121.7,58.0,21.2$; $m / z$ LRMS (ESI + APCI) found $[\mathrm{M}+\mathrm{H}]^{+} 396.2, \mathrm{C}_{22} \mathrm{H}_{19} \mathrm{ClNO}_{4}{ }^{+}$requires 396.1.

Ethyl (4-chloro-3-(4-chloropyridin-2-yl)phenyl)(2-chloro-4(methylsulfonyl)benzoyl)carbamate (58)<smiles>CCOC(=O)N(C(=O)c1ccc(S(C)(=O)=O)cc1Cl)c1ccc(Cl)c(-c2cc(Cl)ccn2)c1</smiles>

Prepared according to general procedure B using (2-(3-(2-chloro-N-(ethoxycarbonyl)-4(methylsulfonyl)benz amido)phenyl)pyridin-4-yl)(phenyl)bis(6-(trifluoromethyl)pyridin-3yl)phosphonium trifluoromethanesulfonate (208 $\mathrm{mg}, 0.20 \mathrm{mmol}), \mathrm{LiCl}(34 \mathrm{mg}, 0.80 \mathrm{mmol})$, and Dioxane $(2 \mathrm{~mL})$. The reaction was heated at $80{ }^{\circ} \mathrm{C}$ for 26 hours. Flash column chromatography (silica gel: $50 \%$ EtOAc in Hexanes) afforded the title compound as a white solid (62 mg, 0.12 mmol, 59\% yield). mp 104-105 ${ }^{\circ} \mathrm{C}$; IR $v_{\max } / \mathrm{cm}^{-1}$ (film): 2980, 2929, 1744, 1697, 1572, 1453, 1312, 1259, 1152, 1074, 919, 726, 667; ${ }^{1} \mathrm{H}$ NMR (400 MHz, $\left.\mathrm{CDCl}_{3}\right) \delta: 8.63(1 \mathrm{H}, \mathrm{d}, J=5.4 \mathrm{~Hz})$, 8.09-7.85 (2H, m), $7.76(1 \mathrm{H}, \mathrm{s}), 7.68-7.51(3 \mathrm{H}, \mathrm{m}), 7.38-7.28(2 \mathrm{H}, \mathrm{m}), 4.10(2 \mathrm{H}, \mathrm{q}, J=7.1 \mathrm{~Hz})$, $3.08(3 \mathrm{H}, \mathrm{s}), 1.07(3 \mathrm{H}, \mathrm{t}, J=7.1 \mathrm{~Hz}) ;{ }^{13} \mathrm{C} \mathrm{NMR}\left(100 \mathrm{MHz}, \mathrm{CDCl}_{3}\right) \delta: 167.9,157.0,152.8,150.6$, 144.2 , 142.6, 142.1, 139.1, 135.8, 132.8, 131.5, 131.4, 131.1, 129.9, 128.9, 128.5, 126.2, 125.4, 123.3, 64.3, 44.6, 13.9; m/z LRMS (ESI + APCI) found $[\mathrm{M}+\mathrm{H}]^{+} 527.1, \mathrm{C}_{22} \mathrm{H}_{18} \mathrm{Cl}_{3} \mathrm{~N}_{2} \mathrm{O}_{5} \mathrm{~S}^{+}$ requires 527.0 .

4',5-Dichloro-6'-methyl-3-(4-(methylsulfonyl)phenyl)-2,3'-bipyridine (59) 
<smiles>Cc1cc(Cl)c(-c2ncc(Cl)cc2-c2ccc(S(C)(=O)=O)cc2)cn1</smiles>

Prepared according to general procedure B using (5-chloro-6'-methyl-3-(4(methylsulfonyl)phenyl)- [2,3'-bipyridin]-4'-yl)diphenyl(6-(trifluoromethyl)pyridin-3yl)phosphonium trifluoromethanesulfonate (168 $\mathrm{mg}, 0.20 \mathrm{mmol}), \mathrm{LiCl}(34 \mathrm{mg}, 0.80 \mathrm{mmol})$, and Dioxane $(2 \mathrm{~mL})$. The reaction was heated to $80{ }^{\circ} \mathrm{C}$ for 24 hours. Flash column chromatography (silica gel: 70\% EtOAc in Hexanes) afforded the title compound as a colorless oil (52 mg, 0.13 mmol, 66\% yield). IR $v_{\max } / \mathrm{cm}^{-1}$ (film): 2923, 2852, 1587, 1538, 1431, 1311, 1149, 1088, 1011 , 836, 785, 727; ${ }^{1} \mathrm{H}$ NMR $\left(400 \mathrm{MHz}, \mathrm{CDCl}_{3}\right) \delta: 8.71(1 \mathrm{H}, \mathrm{d}, J=2.4 \mathrm{~Hz}), 8.34(1 \mathrm{H}, \mathrm{s}), 7.88-7.72$ $(3 \mathrm{H}, \mathrm{m}), 7.38-7.30(2 \mathrm{H}, \mathrm{m}), 7.12(1 \mathrm{H}, \mathrm{s}), 3.03(3 \mathrm{H}, \mathrm{s}), 2.52(3 \mathrm{H}, \mathrm{s}) ;{ }^{13} \mathrm{C}$ NMR $\left(100 \mathrm{MHz}, \mathrm{CDCl}_{3}\right)$ $\delta: 160.2,151.0,150.7,148.3,143.0,142.7,140.3,137.4,137.2,132.2,131.2,130.1,127.7$, 124.0, 44.5, 24.2; $\mathrm{m} / z$ LRMS (ESI + APCI) found $[\mathrm{M}+\mathrm{H}]^{+} 393.1, \mathrm{C}_{18} \mathrm{H}_{15} \mathrm{Cl}_{2} \mathrm{~N}_{2} \mathrm{O}_{2} \mathrm{~S}^{+}$requires 393.0.

\section{Ethyl 4-(4,8-dichloro-5,6-dihydro-11H-benzo[5,6]cyclohepta[1,2-b]pyridin-11-} ylidene)piperidine- 1-carboxylate (61)<smiles>CCOC(=O)N1CCC(=C2c3ccc(Cl)cc3CCc3c(Cl)ccnc32)CC1</smiles>

Prepared according to general procedure B using (8-chloro-11-(1-(ethoxycarbonyl) piperidin-4ylidene)-6,11-dihydro-5H-benzo[5,6]cyclohepta[1,2-b]pyridin-4-yl)diphenyl(6-

(trifluoromethyl)pyridin-3-yl)phosphonium trifluoromethanesulfonate (172 $\mathrm{mg}, 0.20 \mathrm{mmol}$ ), $\mathrm{LiCl}(34 \mathrm{mg}, 0.80 \mathrm{mmol})$, and Dioxane $(2 \mathrm{~mL})$. The reaction was heated to $80{ }^{\circ} \mathrm{C}$ for 24 hours. Flash column chromatography (silica gel: 50\% EtOAc in Hexanes) afforded the title compound as a white solid (59 mg, $0.14 \mathrm{mmol}, 70 \%$ yield). $\mathrm{mp} 140-142{ }^{\circ} \mathrm{C}$; IR $v_{\max } / \mathrm{cm}^{-1}$ (film): 2977,2907 , $1695,1542,1423,1384,1215,1118,1057,836,766 ;{ }^{1} \mathrm{H}$ NMR $\left(400 \mathrm{MHz}, \mathrm{CDCl}_{3}\right) \delta: 8.27(1 \mathrm{H}, \mathrm{d}$, $J=5.3 \mathrm{~Hz}), 7.25-6.93(4 \mathrm{H}, \mathrm{m}), 4.12(2 \mathrm{H}, \mathrm{q}, J=7.1 \mathrm{~Hz}), 3.78(2 \mathrm{H}, \mathrm{t}, J=16.5 \mathrm{~Hz}), 3.38(1 \mathrm{H}$, ddd, $J=14.9,10.3,4.4 \mathrm{~Hz}), 3.20$ (3H, dddt, $J=32.4,13.4,8.7,4.4 \mathrm{~Hz}), 3.03(1 \mathrm{H}$, ddd, $J=16.7,10.3$, $4.5 \mathrm{~Hz}), 2.82(1 \mathrm{H}, \mathrm{ddd}, J=15.0,7.4,4.5 \mathrm{~Hz}), 2.48(1 \mathrm{H}, \mathrm{ddd}, J=14.0,9.3,4.6 \mathrm{~Hz}), 2.31(3 \mathrm{H}, \mathrm{pt}$, $J=5.4,2.9 \mathrm{~Hz}), 1.23(3 \mathrm{H}, \mathrm{t}, J=7.1 \mathrm{~Hz}) ;{ }^{3} \mathrm{C} \mathrm{NMR}\left(100 \mathrm{MHz}, \mathrm{CDCl}_{3}\right) \delta: 158.5,155.5,147.0$, 144.8, 139.5, 138.4, 137.6, 133.7, 133.3, 131.8, 130.4, 128.9, 126.5, 123.4, 61.4, 44.8, 30.8, 30.7, 29.3, 14.8; $m / z$ LRMS (ESI + APCI) found $[\mathrm{M}+\mathrm{H}]^{+} 417.2, \mathrm{C}_{22} \mathrm{H}_{23} \mathrm{Cl}_{2} \mathrm{~N}_{2} \mathrm{O}_{2}{ }^{+}$requires 417.1 .

\section{2-Butoxyethyl 4-chloronicotinate (64)}




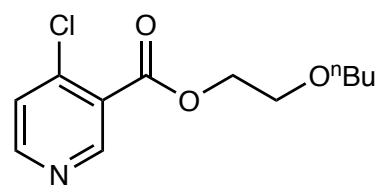

Prepared according to general procedure B using (3-((2-butoxyethoxy)carbonyl)pyridin- 4yl)diphenyl(6-(trifluoromethyl)pyridin-3-yl)phosphonium trifluoromethanesulfonate $(140 \mathrm{mg}$, $0.20 \mathrm{mmol}), \mathrm{LiCl}(34 \mathrm{mg}, 0.80 \mathrm{mmol})$, and Dioxane $(2 \mathrm{~mL})$. The reaction was heated to $80^{\circ} \mathrm{C}$ for 24 hours. Flash column chromatography (silica gel: 33\% EtOAc in Hexanes) afforded the title compound as a colorless oil ( $34 \mathrm{mg}, 0.13 \mathrm{mmol}, 66 \%$ yield). IR $v_{\max } / \mathrm{cm}^{-1}$ (film): 2933 , 2862, 1711, 1640, 1600, 1465, 1292, 1203, 1092, 819; ${ }^{1} \mathrm{H} \mathrm{NMR}\left(400 \mathrm{MHz}, \mathrm{CDCl}_{3}\right) \delta$ : $9.05(1 \mathrm{H}$, s), $8.58(1 \mathrm{H}, \mathrm{s}), 7.40(1 \mathrm{H}, \mathrm{d}, J=5.3 \mathrm{~Hz}), 4.55-4.42(2 \mathrm{H}, \mathrm{m}), 3.83-3.69(2 \mathrm{H}, \mathrm{m}), 3.49(2 \mathrm{H}, \mathrm{t}, J=$ $6.6 \mathrm{~Hz}), 1.56(2 \mathrm{H}, \mathrm{tt}, J=8.5,6.4 \mathrm{~Hz}), 1.43-1.30(2 \mathrm{H}, \mathrm{m}), 0.89(3 \mathrm{H}, \mathrm{t}, J=7.4 \mathrm{~Hz}) ;{ }^{13} \mathrm{C}$ NMR $(100$ $\left.\mathrm{MHz}, \mathrm{CDCl}_{3}\right) \delta: 164.0,152.8,152.5,144.3,126.3,126.0,71.3,68.4,65.1,31.8,19.4,14.0 ; \mathrm{m} / \mathrm{z}$ LRMS (ESI + APCI) found $[\mathrm{M}+\mathrm{H}]^{+} 258.1, \mathrm{C}_{12} \mathrm{H}_{17} \mathrm{ClNO}_{3}{ }^{+}$requires 258.1 .

(3S,8R,9S,10R,13S,14S)-17-(4-Chloropyridin-3-yl)-10,13-dimethyl$2,3,4,7,8,9,10,11,12,13,14,15-d o d e c a h y d r o-1 H$-cyclopenta $[a]$ phenanthren-3-yl acetate (65)

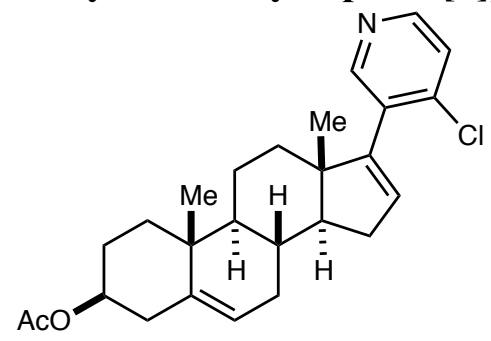

Prepared according to general procedure B, using (3-((3S,8R,9S,10R,13S,14S)-3-acetoxy-10,13dimethyl-2,3,4,7,8,9,10,11,12,13,14,15-dodecahydro-1H-cyclopenta[a]phenanthren-17-

yl)pyridin-4-yl)diphenyl(6-(trifluoromethyl)pyridin-3-yl)phosphonium trifluoromethanesulfonate (218 $\mathrm{mg}, 0.25 \mathrm{mmol}), \mathrm{LiCl}(42 \mathrm{mg}, 1.00 \mathrm{mmol})$, and Dioxane $(2.5 \mathrm{~mL})$. The reaction was heated to $80{ }^{\circ} \mathrm{C}$ for 24 hours. Flash column chromatography (silica gel: $15 \%$ EtOAc in Hexanes) afforded the title compound as a white solid (78 mg, $0.18 \mathrm{mmol}, 73 \%$ yield). $\mathrm{mp} 188-190{ }^{\circ} \mathrm{C}$. IR $v_{\max } / \mathrm{cm}^{-1}$ (film): 2942, 2911, 1730, 1367, 1235, 1036, 816, 729; ${ }^{1} \mathrm{H}$ NMR (400 MHz, $\mathrm{CDCl}_{3}$ ) $\delta: ~ 8.36-8.33(2 \mathrm{H}, \mathrm{m}), 7.32(1 \mathrm{H}, \mathrm{d}, J=5.2 \mathrm{~Hz}), 5.83(1 \mathrm{H}, \mathrm{s}), 5.40(1 \mathrm{H}, \mathrm{d}, J=3.3 \mathrm{~Hz}), 4.65-4.55$ $(1 \mathrm{H}, \mathrm{m}), 2.38-2.26(3 \mathrm{H}, \mathrm{m}), 2.15-1.98(5 \mathrm{H}, \mathrm{m}), 1.89-1.79(2 \mathrm{H}, \mathrm{m}), 1.77-1.43(8 \mathrm{H}, \mathrm{m}), 1.25-0.84$ $(8 \mathrm{H}, \mathrm{m}) ;{ }^{13} \mathrm{C}$ NMR $\left(100 \mathrm{MHz}, \mathrm{CDCl}_{3}\right) \delta: 170.6,150.7,148.7,148.4,143.5,140.1,133.1,132.5$, 124.7, 122.4, 74.0, 56.9, 50.5, 49.8, 38.2, 37.0, 36.9, 34.8, 32.4, 31.7, 30.8, 27.8, 21.5, 20.8, 19.3, 16.4; $\mathrm{m} / \mathrm{z}$ LRMS (ESI + APCI) found $[\mathrm{M}+\mathrm{H}]^{+} 426.3, \mathrm{C}_{26} \mathrm{H}_{32} \mathrm{ClNO}_{2}{ }^{+}$requires 426.2.

\section{2,5,7-Trichloro-4-(4-fluorophenoxy)quinolone (67)}<smiles>Fc1ccc(Oc2cc(Cl)nc3cc(Cl)cc(Cl)c23)cc1</smiles> 
Prepared according to general procedure B, using (5,7-dichloro-4-(4-fluorophenoxy)quinolin-2yl)diphenyl(6-(trifluoromethyl)pyridin-3-yl)phosphonium trifluoromethanesulfonate (236 $\mathrm{mg}$, $0.3 \mathrm{mmol}), \mathrm{LiCl}(51 \mathrm{mg}, 1.20 \mathrm{mmol})$, and Dioxane $(3 \mathrm{~mL})$. The reaction was heated to $80{ }^{\circ} \mathrm{C}$ for 48 hours. Flash column chromatography (silica gel: 2\% EtOAc in Hexanes) afforded the title compound as a white solid (42 mg, $0.12 \mathrm{mmol}, 41 \%$ yield). Mp $143-145^{\circ} \mathrm{C}$. IR $v_{\mathrm{max}} / \mathrm{cm}^{-1}$ (film): 3095, 2923, 1560, 1365, 1189, 917, 767, 620; ${ }^{1} \mathrm{H}$ NMR $\left(400 \mathrm{MHz}, \mathrm{CDCl}_{3}\right) \delta: 7.88(1 \mathrm{H}, \mathrm{d}, J=2.0$ $\mathrm{Hz}), 7.58(1 \mathrm{H}, \mathrm{d}, J=2.0 \mathrm{~Hz}), 7.23-7.10(4 \mathrm{H}, \mathrm{m}), 6.52(1 \mathrm{H}, \mathrm{s}) ;{ }^{13} \mathrm{C} \mathrm{NMR}\left(100 \mathrm{MHz}, \mathrm{CDCl}_{3}\right) \delta$ : $164.2,166.6(\mathrm{~d}, J=248.0 \mathrm{~Hz}), 153.0,150.1,149.2(\mathrm{~d}, J=2.9 \mathrm{~Hz}), 136.4,130.8,130.1,127.2$, $122.7(\mathrm{~d}, J=8.7 \mathrm{~Hz}), 117.6(\mathrm{~d}, J=23.7 \mathrm{~Hz}), 117.0,107.0 ;{ }^{19} \mathrm{~F}$ NMR $\left(365 \mathrm{MHz}, \mathrm{CDCl}_{3}\right) \delta$ : 155.57-(-155.65); m/z LRMS (ESI + APCI) found $[\mathrm{M}+\mathrm{H}]^{+} 342.0, \mathrm{C}_{15} \mathrm{H}_{8} \mathrm{Cl}_{3} \mathrm{FNO}^{+}$requires 342.0 .

Ethyl (6R,10S)-2-chloro-6,7,9,10-tetrahydro-8H-6,10-methanoazepino[4,5-g]quinoxaline-8carboxylate (68)

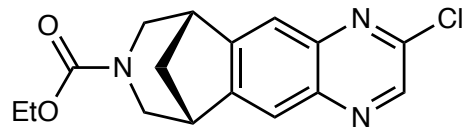

Prepared according to general procedure B, using ((6S,10R)-8-(ethoxycarbonyl)-7,8,9,10tetrahydro-6H-6,10-methanoazepino[4,5-g]quinoxalin-2-yl)diphenyl(6-(trifluoromethyl)-114pyridin-3-yl)phosphonium trifluoromethanesulfonate (191 mg, $0.25 \mathrm{mmol}), \mathrm{LiCl}$ (42 $\mathrm{mg}, 1.00$ $\mathrm{mmol})$, and Dioxane $(2.5 \mathrm{~mL})$. The reaction was heated to $80{ }^{\circ} \mathrm{C}$ for 72 hours. Flash column chromatography (silica gel: 50\% EtOAc in Hexanes) afforded the title compound as a yellow oil (36 mg, $0.11 \mathrm{mmol}, 46 \%$ yield). IR $v_{\max } / \mathrm{cm}^{-1}$ (film): 2955, 2867, 2245, 1686, 1213, 1094, 910, 727; ${ }^{1} \mathrm{H}$ NMR $\left(400 \mathrm{MHz}, \mathrm{CDCl}_{3}\right) \delta: 8.69(1 \mathrm{H}, \mathrm{d}, J=3.8 \mathrm{~Hz}), 7.87(1 \mathrm{H}, \mathrm{d}, J=6.3 \mathrm{~Hz}), 7.77(1 \mathrm{H}$, $\mathrm{d}, J=6.5 \mathrm{~Hz}), 4.15-3.76(4 \mathrm{H}, \mathrm{m}), 3.48-3.25(4 \mathrm{H}, \mathrm{m}), 2.45-2.38(1 \mathrm{H}, \mathrm{m}), 2.00(1 \mathrm{H}, \mathrm{d}, J=11.1$ $\mathrm{Hz}), 1.04(3 \mathrm{H}, \mathrm{t}, J=7.1 \mathrm{~Hz}) ;{ }^{13} \mathrm{C}$ NMR $\left(100 \mathrm{MHz}, \mathrm{CDCl}_{3}\right) \delta: 156.6,150.0,148.9,146.5(\mathrm{~d}, J=$ $1.9 \mathrm{~Hz}), 143.7,142.4(\mathrm{~d}, J=7.2 \mathrm{~Hz}), 141.4(\mathrm{~d}, J=5.3 \mathrm{~Hz}), 122.2(\mathrm{~d}, J=43.9 \mathrm{~Hz}), 141.4(\mathrm{~d}, J=$ $42.6 \mathrm{~Hz}), 61.3,49.7,49.4,41.2,40.1,39.4,14.5 ; \mathrm{m} / \mathrm{z}$ LRMS (ESI + APCI) found $[\mathrm{M}+\mathrm{H}]^{+}$ 318.2, $\mathrm{C}_{16} \mathrm{H}_{17} \mathrm{ClN}_{3} \mathrm{O}_{2}{ }^{+}$requires 318.1 .

Methyl 4",5"'-dichloro-[2,2':5',3"'-terpyridine]-3'-carboxylate (69)<smiles>COC(=O)c1cc(-c2cncc(Cl)c2Cl)cnc1-c1ccccn1</smiles>

Prepared according to general procedure B, using (5"-chloro-3'-(methoxycarbonyl)-[2,2':5',3"terpyridin]-4"-yl)triphenylphosphonium trifluoromethanesulfonate (147 mg, $0.20 \mathrm{mmol}), \mathrm{LiCl}$ (34 mg, $0.80 \mathrm{mmol}$ ), and Dioxane $(2 \mathrm{~mL})$. The reaction was heated to $80{ }^{\circ} \mathrm{C}$ for 72 hours. Flash column chromatography (silica gel: 70\% EtOAc in Hexanes, run twice) afforded the title compound as an amorphous solid (24 mg, $0.07 \mathrm{mmol}, 33 \%$ yield). IR $v_{\max } / \mathrm{cm}^{-1}$ (film): 2948 , 2853, 1728, 1421, 1269, 1118, 908, 726; ${ }^{1} \mathrm{H}$ NMR (400 MHz, $\left.\mathrm{CDCl}_{3}\right) \delta: 8.83(1 \mathrm{H}, \mathrm{d}, J=2.2$ $\mathrm{Hz}), 8.71(1 \mathrm{H}, \mathrm{s}), 8.65(1 \mathrm{H}, \mathrm{ddd}, J=4.8,3.1,0.9 \mathrm{~Hz}), 8.48(1 \mathrm{H}, \mathrm{s}), 8.23(1 \mathrm{H}$ app dt, $J=7.9,1.0$ $\mathrm{Hz}), 8.06(1 \mathrm{H}, \mathrm{d}, J=2.2 \mathrm{~Hz}), 7.88(1 \mathrm{H}, \mathrm{app} \mathrm{td}, J=7.8,1.8 \mathrm{~Hz}), 7.36(1 \mathrm{H}, \mathrm{ddd}, J=7.6,2.8,1.1$ 
$\mathrm{Hz}), 3.84(3 \mathrm{H}, \mathrm{s}) ;{ }^{13} \mathrm{C} \mathrm{NMR}\left(100 \mathrm{MHz}, \mathrm{CDCl}_{3}\right) \delta: 169.0,155.6,155.5,150.3,150.2,148.8$, 148.7, 141.0, 137.8, 137.1, 133.6, 132.0, 130.3, 128.4, 124.2, 123.0, 52.8; m/z LRMS (ESI + APCI) found $[\mathrm{M}+\mathrm{H}]^{+} 360.1, \mathrm{C}_{17} \mathrm{H}_{12} \mathrm{Cl}_{2} \mathrm{~N}_{3} \mathrm{O}_{2}{ }^{+}$requires 360.0 .

Methyl 4,5''-dichloro-[2,2':5',3''-terpyridine]-3'-carboxylate (70)<smiles>COC(=O)c1cc(-c2cncc(Cl)c2)cnc1-c1cc(Cl)ccn1</smiles>

Prepared according to general procedure B, using (5"'-chloro-3'-(methoxycarbonyl)-[2,2':5',3"terpyridin]-4-yl)(phenyl)bis(6-(trifluoromethyl)pyridin-3-yl)phosphonium trifluoromethanesulfonate (131 mg, $0.15 \mathrm{mmol}), \mathrm{LiCl}(25 \mathrm{mg}, 0.60 \mathrm{mmol})$, and Dioxane (1.5 $\mathrm{mL}$ ). The reaction was heated to $80{ }^{\circ} \mathrm{C}$ for 72 hours. Flash column chromatography (silica gel: $30 \%$ EtOAc in Hexanes) afforded the title compound as a white solid (28 $\mathrm{mg}, 0.08 \mathrm{mmol}, 51 \%$ yield). mp $140-144{ }^{\circ} \mathrm{C}$. IR $v_{\max } / \mathrm{cm}^{-1}$ (film): 2919, 2850, 1732, 1376, 1265, 1129, 891, 632; ${ }^{1} \mathrm{H}$ NMR $\left(400 \mathrm{MHz}, \mathrm{CDCl}_{3}\right) \delta: 8.95(1 \mathrm{H}, \mathrm{d}, J=2.3 \mathrm{~Hz}), 8.80(1 \mathrm{H}, \mathrm{d}, J=2.0 \mathrm{~Hz}), 8.67(1 \mathrm{H}, \mathrm{d}, J=$ $2.3 \mathrm{~Hz}), 8.52(1 \mathrm{H}, \mathrm{d}, J=5.3 \mathrm{~Hz}), 8.28(1 \mathrm{H}, \mathrm{d}, J=1.9 \mathrm{~Hz}), 8.13(1 \mathrm{H}, \mathrm{d}, J=2.3 \mathrm{~Hz}), 7.95(1 \mathrm{H}$, app t, $J=2.1 \mathrm{~Hz}), 7.36(1 \mathrm{H}, \mathrm{dd}, J=5.3,2.0 \mathrm{~Hz}), 3.85(3 \mathrm{H}, \mathrm{s}) ;{ }^{13} \mathrm{C}$ NMR $\left(100 \mathrm{MHz}, \mathrm{CDCl}_{3}\right) \delta$ : $168.9,156.8,153.9,149.5,148.9,148.4,145.9,145.2,135.5,134.2,133.4,132.8,131.9,129.2$, 124.4, 123.4, 52.8; m/z LRMS (ESI + APCI) found $[\mathrm{M}+\mathrm{H}]^{+} 360.1, \mathrm{C}_{17} \mathrm{H}_{12} \mathrm{Cl}_{2} \mathrm{~N}_{3} \mathrm{O}_{2}{ }^{+}$requires 360.0 .

\section{Preparation of Brominated Heterocycles}

\section{General Procedure C}

An $8 \mathrm{~mL}$ screw-cap vial equipped with a stir bar was charged with the phosphonium salt (1.0 equiv), $\mathrm{LiBr}$ (4.0 equiv), and placed under a nitrogen atmosphere. Dioxane (0.1M) was added, followed by a dropwise addition of $\mathrm{TfOH}$ (1.0 equiv). The septa cap was quickly replaced with an unpierced one and the reaction was heated to $80^{\circ} \mathrm{C}$. After the stated time, the reaction was quenched with a saturated aqueous solution of $\mathrm{Na}_{2} \mathrm{CO}_{3}$ and the aqueous layer was extracted with $\mathrm{CH}_{2} \mathrm{Cl}_{2}(3 \mathrm{x})$. The combined organic extracts were dried $\left(\mathrm{MgSO}_{4}\right)$, filtered, and concentrated in vасио. The residue was purified by flash column chromatography under the stated conditions to provide the brominated heterocycle.

\section{4-Bromo-2-phenylpyridine (39)}<smiles>Brc1ccnc(-c2ccccc2)c1</smiles> 
Prepared according to general procedure C, using phenyl(2-phenylpyridin-4-yl)bis(6(trifluoromethyl)pyridin-3-yl)phosphonium trifluoromethanesulfonate (35 mg, $0.05 \mathrm{mmol}$ ), $\mathrm{LiBr}$ (17 mg, $0.20 \mathrm{mmol})$, TfOH $(4 \mu \mathrm{L}, 0.05 \mathrm{mmol})$ and Dioxane $(0.5 \mathrm{~mL})$. The reaction was heated to $80{ }^{\circ} \mathrm{C}$ for 24 hours. Flash column chromatography (silica gel: 3\% EtOAc in Hexanes) was used to isolate a pure sample of the product (NMR Yield: 79\%). ${ }^{1} \mathrm{H}$ NMR $\left(400 \mathrm{MHz}, \mathrm{CDCl}_{3}\right) \delta: 8.50$ $(1 \mathrm{H}, \mathrm{dd}, J=5.3,0.4 \mathrm{~Hz}), 7.99-7.95(2 \mathrm{H}, \mathrm{m}), 7.90(2 \mathrm{H}, \mathrm{dd}, J=1.8,0.5 \mathrm{~Hz}), 7.51-7.38(4 \mathrm{H}, \mathrm{m})$; ${ }^{13} \mathrm{C}$ NMR $\left(100 \mathrm{MHz}, \mathrm{CDCl}_{3}\right) \delta: 159.1,150.5,138.2,133.6,129.7,129.0,127.1,125.4,124.0$. The spectroscopic data is in agreement with the literature. ${ }^{4}$

\section{4-Bromo-2-(3-(pentafluoro- $\lambda^{6}$-sulfaneyl)phenyl)pyridine (42)}<smiles>FC(F)(F)c1cccc(-c2cc(Br)ccn2)c1</smiles>

Prepared according to general procedure C, using (2-(3-(pentafluoro- $\lambda 6$ sulfaneyl)phenyl)pyridin-4-yl)(phenyl)bis(6-(trifluoromethyl)pyridin-3-yl)phosphonium trifluoromethanesulfonate (166 mg, $0.20 \mathrm{mmol}), \mathrm{LiBr}(69 \mathrm{mg}, 0.80 \mathrm{mmol})$, TfOH $(18 \mu \mathrm{L}, 0.20$ $\mathrm{mmol})$, and Dioxane $(2 \mathrm{~mL})$. The reaction was heated to $80{ }^{\circ} \mathrm{C}$ for 48 hours. Flash column chromatography (silica gel: $20 \% \mathrm{CH}_{2} \mathrm{Cl}_{2}$ in Hexanes, ran twice) afforded the title compound as an amorphous solid (62 mg, $0.17 \mathrm{mmol}, 86 \%$ yield). IR $v_{\max } / \mathrm{cm}^{-1}$ (film): 3084, 2927, 1566, 1369, 1100, 834, 694, 596; ${ }^{1} \mathrm{H}$ NMR $\left(400 \mathrm{MHz}, \mathrm{CDCl}_{3}\right) \delta: 8.53(1 \mathrm{H}, \mathrm{d}, J=5.2 \mathrm{~Hz}), 8.42(1 \mathrm{H}$, app t, $J$ $=1.9 \mathrm{~Hz}), 8.09(1 \mathrm{H}, \mathrm{d}, J=7.9 \mathrm{~Hz}), 7.91(1 \mathrm{H}, \mathrm{d}, J=1.4 \mathrm{~Hz}), 7.82(1 \mathrm{H}, \mathrm{ddd}, J=8.3,2.2,0.8 \mathrm{~Hz})$, $7.85(1 \mathrm{H}$, app t, $J=8.0 \mathrm{~Hz}), 7.47(1 \mathrm{H}, \mathrm{dd}, J=7.0,1.7 \mathrm{~Hz}) ;{ }^{13} \mathrm{C} \mathrm{NMR}\left(100 \mathrm{MHz}, \mathrm{CDCl}_{3}\right) \delta$ : $156.8,154.8$ (m), 150.8, 139.1, 134.0, 129.9, 129.4, 127.0 (qn, $J=4.6 \mathrm{~Hz}), 126.4,124.9$ (qn, $J=$ $4.7 \mathrm{~Hz}), 124.1 ;{ }^{19} \mathrm{~F}$ NMR $\left(365 \mathrm{MHz}, \mathrm{CDCl}_{3}\right) \delta: 83.99(1 \mathrm{~F}, \mathrm{qn}, J=151.7 \mathrm{~Hz}), 62.73(4 \mathrm{~F}, \mathrm{~d}, J=$ $150.0 \mathrm{~Hz}) ; \mathrm{m} / \mathrm{z} \mathrm{LRMS}(\mathrm{ESI}+\mathrm{APCI})$ found $[\mathrm{M}+\mathrm{H}]^{+} 360.0, \mathrm{C}_{11} \mathrm{H}_{8} \mathrm{BrF}_{5} \mathrm{NS}^{+}$requires 359.9 .

\section{4-Bromo-2-butyl-5-(trifluoromethyl)pyridine (45)}<smiles>FC(F)(F)c1cnc(Br)cc1Br</smiles>

Prepared according to general procedure C, using (2-butyl-5-(trifluoromethyl)pyridin-4yl)diphenyl(6-(trifluoromethyl)pyridin-3-yl)phosphonium trifluoromethanesulfonate (137 mg, $0.20 \mathrm{mmol}), \mathrm{LiBr}(69 \mathrm{mg}, 0.80 \mathrm{mmol})$, TfOH $(18 \mu \mathrm{L}, 0.20 \mathrm{mmol})$, and Dioxane $(2 \mathrm{~mL})$. The reaction was heated to $80{ }^{\circ} \mathrm{C}$ for 24 hours. The ${ }^{1} \mathrm{H}$ NMR yield was measured using triphenylmethane (49 $\mathrm{mg}, 0.20 \mathrm{mmol}, 1.0$ equiv) as an internal standard (90\% NMR yield). PTLC (4\% $\mathrm{Et}_{2} \mathrm{O}$ in Hexanes) was used to obtain approximately $10 \mathrm{mg}$ of product for characterization. IR $v_{\max } / \mathrm{cm}^{-1}$ (film): 2959, 2861, 1583, 1321, 1137, 1019, 870, 690; ${ }^{1} \mathrm{H}$ NMR $\left(400 \mathrm{MHz}, \mathrm{CDCl}_{3}\right) \delta: 8.72(1 \mathrm{H}, \mathrm{s}), 7.51(1 \mathrm{H}, \mathrm{s}), 2.82(2 \mathrm{H}, \mathrm{t}, J=7.7 \mathrm{~Hz}), 1.76-1.67(2 \mathrm{H}, \mathrm{m}), 1.39$ $(2 \mathrm{H}, \mathrm{m}, J=7.5 \mathrm{~Hz}), 0.95(3 \mathrm{H}, \mathrm{t}, J=7.3 \mathrm{~Hz}) ;{ }^{13} \mathrm{C} \mathrm{NMR}\left(100 \mathrm{MHz}, \mathrm{CDCl}_{3}\right) \delta: 167.8,147.7$ (q, $J$ $=5.9 \mathrm{~Hz}), 131.1(\mathrm{q}, J=2.0 \mathrm{~Hz}), 128.4,124.1(\mathrm{q}, J=31.5 \mathrm{~Hz}), 122.9(\mathrm{q}, J=273.1 \mathrm{~Hz}), 37.8$, 
31.6, 22.5, 14.0; ${ }^{19} \mathrm{~F}$ NMR $\left(365 \mathrm{MHz}, \mathrm{CDCl}_{3}\right) \delta:-62.29 ; \mathrm{m} / \mathrm{z}$ LRMS (ESI + APCI) found [M + $\mathrm{H}]^{+} 282.0, \mathrm{C}_{10} \mathrm{H}_{12} \mathrm{BrF}_{3} \mathrm{~N}^{+}$requires 282.0.

\section{3,4-Dibromo-5-methylpyridine (47)}<smiles>Cc1cncc(Br)c1Br</smiles>

Prepared according to general procedure $\mathrm{C}$ using (3-bromo-5-methylpyridin-4-yl) triphenylphosphonium trifluoromethanesulfonate $(175 \mathrm{mg}, 0.30 \mathrm{mmol}), \mathrm{LiBr}(104 \mathrm{mg}, 1.20$ $\mathrm{mmol})$, TfOH $(26 \mu \mathrm{L}, 0.3 \mathrm{mmol})$, and Dioxane $(3 \mathrm{~mL})$. The reaction was heated to $80{ }^{\circ} \mathrm{C}$ for 40 hours. Flash column chromatography (silica gel: $10 \%$ EtOAc in Hexanes) afforded the title compound as a colorless oil (60 mg, $0.24 \mathrm{mmol}, 80 \%$ yield). IR $v_{\max } / \mathrm{cm}^{-1}$ (film): 2924, 1461, 1268, 1120, 1071, 1029; ${ }^{1} \mathrm{H}$ NMR (400 MHz, $\left.\mathrm{CDCl}_{3}\right) \delta: 8.52(1 \mathrm{H}, \mathrm{s}), 8.28(1 \mathrm{H}, \mathrm{s}), 2.43(3 \mathrm{H}, \mathrm{s})$; ${ }^{13} \mathrm{C}$ NMR $\left(100 \mathrm{MHz}, \mathrm{CDCl}_{3}\right) \delta: 149.9,148.7,137.1,136.4,124.2,21.2 ; \mathrm{m} / \mathrm{z}$ LRMS (ESI + APCI) found $[\mathrm{M}+\mathrm{H}]^{+}$249.9, $\mathrm{C}_{6} \mathrm{H}_{6} \mathrm{Br}_{2} \mathrm{~N}^{+}$requires 249.9 .

\section{1-Bromo-5-nitroisoquinoline}<smiles>O=[N+]([O-])c1cccc2c(Br)nccc12</smiles>

Prepared according to general procedure $\mathrm{C}$, using (5-nitroisoquinolin-1-yl)diphenyl(6(trifluoromethyl)pyridin-3-yl)phosphonium trifluoromethanesulfonate $(131 \mathrm{mg}, 0.20 \mathrm{mmol}$ ), $\mathrm{LiBr}(69 \mathrm{mg}, 0.80 \mathrm{mmol})$, TfOH $(18 \mu \mathrm{L}, 0.20 \mathrm{mmol})$, and Dioxane $(2 \mathrm{~mL})$. The reaction was heated to $80{ }^{\circ} \mathrm{C}$ for 24 hours. Flash column chromatography (silica gel: $20 \% \mathrm{CH}_{2} \mathrm{Cl}_{2}$ in Hexanes, ran twice) afforded the title compound as a white solid (37 $\mathrm{mg}, 0.15 \mathrm{mmol}, 70 \%$ yield). IR $v_{\max } / \mathrm{cm}^{-1}$ (film): $3050,2923,1622,1522,1311,1037,812,717 ;{ }^{1} \mathrm{H} \mathrm{NMR}\left(400 \mathrm{MHz}, \mathrm{CDCl}_{3}\right) \delta$ : $8.71(1 \mathrm{H}$, app dt, $J=8.5,0.9 \mathrm{~Hz}), 8.55(1 \mathrm{H}, \mathrm{dd}, J=7.7,1.1 \mathrm{~Hz}), 8.46(1 \mathrm{H}, \mathrm{d}, J=6.1 \mathrm{~Hz}), 8.40$ $(1 \mathrm{H}, \mathrm{dd}, J=6.1,0.8 \mathrm{~Hz}), 7.81(1 \mathrm{H}$, app t, $J=8.0 \mathrm{~Hz}) ;{ }^{13} \mathrm{C} \mathrm{NMR}\left(100 \mathrm{MHz}, \mathrm{CDCl}_{3}\right) \delta: 146.3$, $145.5,145.4,135.8,130.1,129.4,129.0,127.3,116.2 ; \mathrm{m} / \mathrm{z}$ LRMS (ESI + APCI) found $[\mathrm{M}+\mathrm{H}]^{+}$ 253.0, $\mathrm{C}_{9} \mathrm{H}_{6} \mathrm{Br}_{2} \mathrm{~N}_{2} \mathrm{O}_{2}{ }^{+}$requires 253.0.

Ethyl 4-((4-bromopyridin-2-yl)(4-chlorophenyl)methoxy)piperidine-1-carboxylate (50)<smiles>CCON1CCC(OC(c2ccc(Cl)cc2)c2cc(Br)ccn2)CC1</smiles> 
Prepared according to general procedure C using (2-((4-chlorophenyl) ((1(ethoxycarbonyl)piperidin-4-yl)oxy)methyl)pyridin-4-yl)(phenyl)bis(6-(trifluoromethyl)pyridin3-yl)phosphonium trifluoromethanesulfonate $(185 \mathrm{mg}, 0.20 \mathrm{mmol})$, LiBr $(70 \mathrm{mg}, 0.80 \mathrm{mmol})$, TfOH $(18 \mu \mathrm{L}, 0.20 \mathrm{mmol})$, and Dioxane $(2 \mathrm{~mL})$. The reaction was heated at $80{ }^{\circ} \mathrm{C}$ for 40 hours. Flash column chromatography (silica gel: 50\% EtOAc in Hexanes) afforded the title compound as a colorless oil (52 mg, $0.12 \mathrm{mmol}, 58 \%$ yield). IR $v_{\max } / \mathrm{cm}^{-1}$ (film): 2929, 2866, 1691, 1566, 1431, 1381, 1226, 1085, 810; ${ }^{1} \mathrm{H}$ NMR $\left(400 \mathrm{MHz}, \mathrm{CDCl}_{3}\right) \delta: 8.28(1 \mathrm{H}, \mathrm{d}, J=5.3 \mathrm{~Hz}), 7.69(1 \mathrm{H}$, $\mathrm{d}, J=1.9 \mathrm{~Hz}), 7.41-7.18(5 \mathrm{H}, \mathrm{m}), 5.56(1 \mathrm{H}, \mathrm{s}), 4.10(2 \mathrm{H}, \mathrm{q}, J=7.1 \mathrm{~Hz}), 3.75(2 \mathrm{H}, \mathrm{t}, J=9.1 \mathrm{~Hz})$, $3.60(1 \mathrm{H}, \mathrm{dq}, J=7.9,3.8 \mathrm{~Hz}), 3.17(2 \mathrm{H}, \mathrm{ddd}, J=12.9,8.5,3.7 \mathrm{~Hz}), 1.81(2 \mathrm{H}, \mathrm{ddd}, J=14.4,7.1$, $3.5 \mathrm{~Hz}), 1.63(2 \mathrm{H}$, ddt, $J=17.0,12.9,6.1 \mathrm{~Hz}), 1.23(3 \mathrm{H}, \mathrm{t}, J=7.1 \mathrm{~Hz}) ;{ }^{13} \mathrm{C} \mathrm{NMR}(100 \mathrm{MHz}$, $\left.\mathrm{CDCl}_{3}\right) \delta: 163.5,155.6,149.9,139.5,133.9,133.8,128.8,128.3,126.0,123.9,80.5,72.9,61.4$, 41.2, 41.1, 31.3, 31.0, 14.8; $\mathrm{m} / z$ LRMS (ESI + APCI) found $[\mathrm{M}+\mathrm{H}]^{+} 453.1, \mathrm{C}_{20} \mathrm{H}_{23} \mathrm{BrClN}_{2} \mathrm{O}_{3}{ }^{+}$ requires 453.1 .

\section{(R)-1-(3,5-Bis(trifluoromethyl)phenyl)ethyl 4-bromo-5-methylpicolinate (53)}<smiles>Cc1cnc(C(=O)OC(C)c2cc(C(F)(F)F)cc(C(F)(F)F)c2)cc1Br</smiles>

Prepared according to general procedure C, using (R)-(2-((1-(3,5bis(trifluoromethyl)phenyl)ethoxy)carbonyl)-5-methylpyridin-4-yl)diphenyl(6-(trifluoromethyl) pyridin-3-yl)phosphonium trifluoromethanesulfonate $(171 \mathrm{mg}, 0.20 \mathrm{mmol}), \mathrm{LiBr}(69 \mathrm{mg}, 0.80$ $\mathrm{mmol})$, TfOH $(18 \mu \mathrm{L}, 0.20 \mathrm{mmol})$, and Dioxane $(2 \mathrm{~mL})$. The reaction was heated to $80{ }^{\circ} \mathrm{C}$ for 24 hours. Flash column chromatography (silica gel: 15\% EtOAc in Hexanes) afforded the title compound as a clear oil (72 mg, $0.16 \mathrm{mmol}, 79 \%$ yield). IR $v_{\max } / \mathrm{cm}^{-1}$ (film): 2999, 2926, 1717 , 1280, 1196, 1112, 900, 682; ${ }^{1} \mathrm{H} \mathrm{NMR}\left(400 \mathrm{MHz}, \mathrm{CDCl}_{3}\right) \delta: 8.55(1 \mathrm{H}, \mathrm{s}), 8.26(1 \mathrm{H}, \mathrm{s}), 7.91(2 \mathrm{H}$, s), $7.82(1 \mathrm{H}, \mathrm{s}), 6.25(1 \mathrm{H}, \mathrm{q}, J=6.7 \mathrm{~Hz}), 2.45(3 \mathrm{H}, \mathrm{s}), 1.77(3 \mathrm{H}, \mathrm{d}, J=6.7 \mathrm{~Hz}) ;{ }^{13} \mathrm{C}$ NMR $(100$ $\left.\mathrm{MHz} \mathrm{CDCl}_{3}\right) \delta: 163.5,151.1,146.3,143.8,138.6,135.9,132.2(\mathrm{q}, J=33.3 \mathrm{~Hz}), 129.2,126.7$ (d, $J=2.6 \mathrm{~Hz}$ ), 123.3 (q, $J=272.9 \mathrm{~Hz}), 122.3$ (qn, $J=3.6 \mathrm{~Hz}), 72.8,22.11,20.0 ;{ }^{19} \mathrm{~F}$ NMR $(365$ $\mathrm{MHz}, \mathrm{CDCl}_{3}$ ) $\delta$ : $-62.88 ; \mathrm{m} / \mathrm{z}$ LRMS (ESI + APCI) found $[\mathrm{M}+\mathrm{H}]^{+} 456.1, \mathrm{C}_{17} \mathrm{H}_{13} \mathrm{BrF}_{6} \mathrm{NO}_{2}{ }^{+}$ requires 456.0 .

\section{4-Bromo-3-(3-fluoro-5-(5-(trifluoromethyl)pyridin-2-yl)phenyl)pyridine (56)}

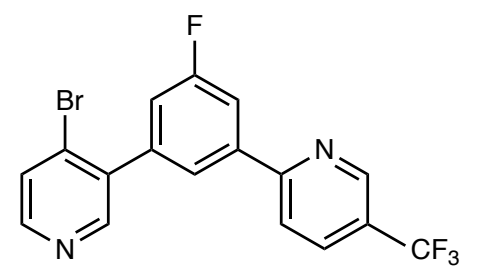

Prepared according to general procedure C, using (3-(3-fluoro-5-(5-(trifluoromethyl)pyridin-2yl)phenyl)pyridin-4-yl)diphenyl(6-(trifluoromethyl)pyridin-3-yl)phosphonium

trifluoromethanesulfonate $(239 \mathrm{mg}, 0.30 \mathrm{mmol}), \mathrm{LiBr}(104 \mathrm{mg}, 1.20 \mathrm{mmol})$, TfOH $(27 \mu \mathrm{L}, 0.30$ 
mmol), and Dioxane ( $3 \mathrm{~mL})$. The reaction was heated to $80^{\circ} \mathrm{C}$ for 48 hours. Flash column chromatography (silica gel: 20\% EtOAc in Hexanes) afforded the title compound as a yellow solid (37 mg, $0.15 \mathrm{mmol}, 70 \%$ yield). $\mathrm{mp} 115-118{ }^{\circ} \mathrm{C}$. IR $v_{\max } / \mathrm{cm}^{-1}$ (film): $3038,2924,1603$, 1329, 1109, 922, 840, 691; ${ }^{\mathrm{H}} \mathrm{NMR}\left(400 \mathrm{MHz}, \mathrm{CDCl}_{3}\right) \delta: 8.96(1 \mathrm{H}, \mathrm{s}), 8.58(1 \mathrm{H}, \mathrm{s}), 8.43(1 \mathrm{H}$, $\mathrm{d}, J=4.9 \mathrm{~Hz}), 8.03(1 \mathrm{H}, \mathrm{d}, J=8.3 \mathrm{~Hz}), 7.92-7.84(3 \mathrm{H}, \mathrm{m}), 7.66(1 \mathrm{H}, \mathrm{d}, J=4.9 \mathrm{~Hz}), 7.27(1 \mathrm{H}, \mathrm{d}$, $J=7.6 \mathrm{~Hz}) ;{ }^{13} \mathrm{C} \mathrm{NMR}\left(100 \mathrm{MHz}, \mathrm{CDCl}_{3}\right) \delta: 163.1(\mathrm{~d}, J=247.7 \mathrm{~Hz}), 158.7(\mathrm{~m}), 150.8,149.8$, $146.9(\mathrm{~d}, J=4.0 \mathrm{~Hz}), 140.4(\mathrm{~d}, J=8.1 \mathrm{~Hz}), 139.8(\mathrm{~d}, J=8.4 \mathrm{~Hz}), 137.2(\mathrm{~d}, J=1.4 \mathrm{~Hz}), 134.4(\mathrm{q}$, $J=3.4 \mathrm{~Hz}), 132.9,128.3,125.8(\mathrm{q}, J=33.2 \mathrm{~Hz}), 124.2(\mathrm{~d}, J=2.6 \mathrm{~Hz}), 123.7(\mathrm{q}, J=272.1 \mathrm{~Hz})$, 120.2, $118.2(\mathrm{~d}, J=22.7 \mathrm{~Hz}), 114.4(\mathrm{~d}, J=23.0 \mathrm{~Hz}) ;{ }^{19} \mathrm{~F} \mathrm{NMR}\left(365 \mathrm{MHz}, \mathrm{CDCl}_{3}\right) \delta:-62.36(3 \mathrm{~F}$, s), $-111.63(1 \mathrm{~F}, \mathrm{t}, J=9.3 \mathrm{~Hz}) ; \mathrm{m} / \mathrm{z}$ LRMS (ESI + APCI) found $[\mathrm{M}+\mathrm{H}]^{+} 397.1, \mathrm{C}_{17} \mathrm{H}_{10} \mathrm{BrF}_{4} \mathrm{~N}_{2}{ }^{+}$ requires 397.0 .

\section{4'-Bromo-5-chloro-6'-methyl-3-(4-(methylsulfonyl)phenyl)-2,3'-bipyridine (60)}<smiles>Cc1cc(Br)c(-c2ncc(Cl)cc2-c2ccc(S(C)(=O)=O)cc2)cn1</smiles>

Prepared according to general procedure C using (5-chloro-6'-methyl-3-(4(methylsulfonyl)phenyl)-[2,3'-bipyridin]-4'-yl)diphenyl(6-(trifluoromethyl)pyridin-3-

yl)phosphonium trifluoromethanesulfonate (168 $\mathrm{mg}, 0.20 \mathrm{mmol}), \mathrm{LiBr}(70 \mathrm{mg}, 0.80 \mathrm{mmol})$, and Dioxane $(2.0 \mathrm{~mL})$. The reaction was heated at $80^{\circ} \mathrm{C}$ for 36 hours. Flash column chromatography (silica gel: 50\% EtOAc in Hexanes) afforded the title compound as a colorless oil (51 mg, 0.12 mmol, 58\% yield). IR $v_{\max } / \mathrm{cm}^{-1}$ (film): 2924, 1581, 1431, 1399, 1311, 1284, 1149, 1090, 789, 727; ${ }^{1} \mathrm{H}$ NMR $\left(400 \mathrm{MHz}, \mathrm{CDCl}_{3}\right) \delta: 8.73(1 \mathrm{H}, \mathrm{d}, J=2.3 \mathrm{~Hz}), 8.27(1 \mathrm{H}, \mathrm{s}), 7.89-7.75(3 \mathrm{H}, \mathrm{m})$, 7.40-7.32 (3H, m), $3.04(3 \mathrm{H}, \mathrm{s}), 2.53(3 \mathrm{H}, \mathrm{s}) ;{ }^{13} \mathrm{C}$ NMR $\left(100 \mathrm{MHz}, \mathrm{CDCl}_{3}\right) \delta: 159.9,152.1$, $150.6,148.3,142.9,140.3,137.5,137.0,133.3,133.2,132.2,130.3,127.8,127.3,44.6,24.1 ; \mathrm{m} / z$ LRMS (ESI + APCI) found $[\mathrm{M}+\mathrm{H}]^{+} 437.1, \mathrm{C}_{18} \mathrm{H}_{15} \mathrm{BrClO}_{2} \mathrm{~N}_{2} \mathrm{~S}^{+}$requires 437.0.

Ethyl 4-(4-bromo-8-chloro-5,6-dihydro-11H-benzo[5,6]cyclohepta[1,2-b]pyridin-11ylidene) piperidine-1-carboxylate (62)<smiles>CCOC(=O)N1CCC(=C2c3ccc(Cl)cc3CCc3c(Br)ccnc32)CC1</smiles>

Prepared according to general procedure $\mathrm{C}$ using 8-chloro-11-(1-(ethoxycarbonyl) piperidin-4ylidene)-6,11-dihydro-5H-benzo[5,6]cyclohepta[1,2-b]pyridin-4-yl)diphenyl(6-

(trifluoromethyl)pyridin-3-yl)phosphonium trifluoromethanesulfonate (172 $\mathrm{mg}, 0.20 \mathrm{mmol}$ ), 
$\mathrm{LiBr}(69 \mathrm{mg}, 0.80 \mathrm{mmol})$, TfOH $(18 \mu \mathrm{L}, 0.20 \mathrm{mmol})$, and Dioxane $(2 \mathrm{~mL})$. The reaction was heated to $80{ }^{\circ} \mathrm{C}$ for 36 hours. Flash column chromatography (silica gel: 50\% EtOAc in Hexanes) afforded the title compound as a white solid (76 mg, $0.17 \mathrm{mmol}, 83 \%$ yield). $\mathrm{mp} 143-144{ }^{\circ} \mathrm{C}$; IR $v_{\max } / \mathrm{cm}^{-1}$ (film): 2976, 2907, 1695, 1541, 1422, 1383, 1215, 1120, 995, 835, 767; ${ }^{1} \mathrm{H}$ NMR (400 $\left.\mathrm{MHz} \mathrm{CDCl}_{3}\right) \delta: 8.15(1 \mathrm{H}, \mathrm{d}, J=5.3 \mathrm{~Hz}), 7.35(1 \mathrm{H}, \mathrm{d}, J=5.2 \mathrm{~Hz}), 7.20-7.02(3 \mathrm{H}, \mathrm{m}), 4.11(2 \mathrm{H}$, q, $J=7.1 \mathrm{~Hz}), 3.86-3.70(2 \mathrm{H}, \mathrm{m}), 3.44-3.10(4 \mathrm{H}, \mathrm{m}), 3.02(1 \mathrm{H}, \mathrm{ddd}, J=16.3,9.6,4.5 \mathrm{~Hz}), 2.82$ (1H, ddd, $J=15.3,8.0,4.5 \mathrm{~Hz}), 2.46(1 \mathrm{H}, \mathrm{ddd}, J=14.0,9.0,4.4 \mathrm{~Hz}), 2.39-2.22(3 \mathrm{H}, \mathrm{m}), 1.22$ $(3 \mathrm{H}, \mathrm{t}, J=7.1 \mathrm{~Hz}) ;{ }^{13} \mathrm{C} \mathrm{NMR}\left(100 \mathrm{MHz}, \mathrm{CDCl}_{3}\right) \delta: 158.6,155.5,147.0,139.2,138.2,137.2$, $136.0,133.7,133.4,133.3,130.5,129.0,126.8,126.4,61.4,44.8,44.7,32.1,31.0,30.8,30.7$, 14.7; $m / z$ LRMS (ESI + APCI) found $[\mathrm{M}+\mathrm{H}]^{+} 461.2, \mathrm{C}_{22} \mathrm{H}_{23} \mathrm{ClBrN}_{2} \mathrm{O}_{2}{ }^{+}$requires 461.1.

\section{(3S,8R,9S,10R,13S,14S)-17-(4-Bromopyridin-3-yl)-10,13-dimethyl-}

$2,3,4,7,8,9,10,11,12,13,14,15-d o d e c a h y d r o-1 H$-cyclopenta $[a]$ phenanthren-3-yl acetate (66)

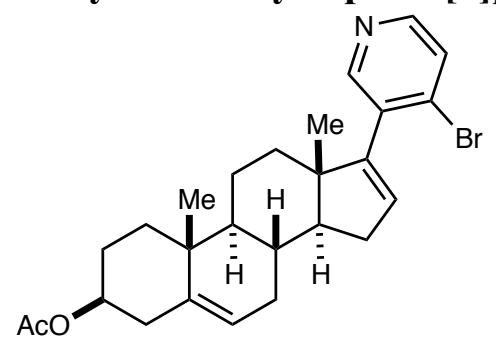

Prepared according to general procedure C, using using (3-((3S,8R,9S,10R,13S,14S)-3-acetoxy10,13-dimethyl-2,3,4,7,8,9,10,11,12,13,14,15-dodecahydro-1H-cyclopenta[a]phenanthren-17yl)pyridin-4-yl)diphenyl(6-(trifluoromethyl)pyridin-3-yl)phosphonium trifluoromethanesulfonate (174 mg, $0.20 \mathrm{mmol}), \mathrm{LiBr}(69 \mathrm{mg}, 0.80 \mathrm{mmol})$, TfOH $(18 \mu \mathrm{L}, 0.20 \mathrm{mmol})$, and Dioxane $(2$ $\mathrm{mL}$ ). The reaction was heated to $80{ }^{\circ} \mathrm{C}$ for 24 hours. Flash column chromatography (silica gel: $15 \%$ EtOAc in Hexanes) afforded the title compound as a white solid (78 mg, $0.17 \mathrm{mmol}, 83 \%$ yield). IR $v_{\max } / \mathrm{cm}^{-1}$ (film): 2951, 2854, 1729, 1366, 1234, 1036, 815, 740; ${ }^{1} \mathrm{H}$ NMR (400 MHz, $\left.\mathrm{CDCl}_{3}\right) \delta: 8.32(1 \mathrm{H}, \mathrm{s}), 8.25(1 \mathrm{H}, \mathrm{d}, J=5.0 \mathrm{~Hz}), 7.52(1 \mathrm{H}, \mathrm{d}, J=5.2 \mathrm{~Hz}), 5.82(1 \mathrm{H}, \mathrm{s}), 5.41(1 \mathrm{H}$, $\mathrm{d}, J=4.8 \mathrm{~Hz}), 4.66-4.56(1 \mathrm{H}, \mathrm{m}), 2.40-2.29(3 \mathrm{H}, \mathrm{m}), 2.15-2.00(5 \mathrm{H}, \mathrm{m}), 1.90-1.80(2 \mathrm{H}, \mathrm{m})$, 1.77-1.45 (8H, m), 1.28-0.90 (8H, m); $\left.{ }^{13} \mathrm{C} \mathrm{NMR} \mathrm{(100} \mathrm{MHz,} \mathrm{CDCl}_{3}\right) \delta: 170.6,150.1,150.0$, $148.5,140.1,135.3,134.5,132.4,128.1,122.4,74.0,57.0,50.5,49.9,38.2,37.0,36.9,34.8$, $32.4,31.7,30.8,27.8,21.5,20.8,19.4,16.4 ; \mathrm{m} / \mathrm{z}$ LRMS (ESI + APCI) found $[\mathrm{M}+\mathrm{H}]^{+} 470.3$, $\mathrm{C}_{26} \mathrm{H}_{32} \mathrm{BrNO}_{2}{ }^{+}$requires 470.2 .

\section{Preparation of Iodinated Heterocycles}

\section{General Procedure D}

An $8 \mathrm{~mL}$ screw-cap vial equipped with a stir bar was charged with the phosphonium salt (1.0 equiv), LiI (4.0 equiv), and placed under a nitrogen atmosphere. Dioxane (0.1M) was added, followed by a dropwise addition of $\mathrm{TfOH}$ (1.0 equiv). The septa cap was quickly replaced with an unpierced one and the reaction was heated to $120^{\circ} \mathrm{C}$. After the stated time, the reaction was quenched with a saturated aqueous solution of $\mathrm{Na}_{2} \mathrm{CO}_{3}$ and the aqueous layer was extracted with $\mathrm{CH}_{2} \mathrm{Cl}_{2}(3 \mathrm{x})$. The combined organic extracts were dried $\left(\mathrm{MgSO}_{4}\right)$, filtered, and concentrated in vасио. The residue was purified by flash column chromatography under the stated conditions to provide the iodinated heterocycle. 


\section{4-Iodo-2-phenylpyridine (40)}

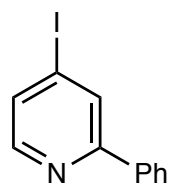

Prepared according to general procedure B, using phenyl(2-phenylpyridin-4-yl)bis(6(trifluoromethyl)pyridin-3-yl)phosphonium trifluoromethanesulfonate (1.06 g, $1.50 \mathrm{mmol})$, LiI $(803 \mathrm{mg}, 6.00 \mathrm{mmol})$, and Dioxane $(15 \mathrm{~mL})$. The reaction was heated to $120{ }^{\circ} \mathrm{C}$ for 63 hours Flash column chromatography (silica gel: 5\% EtOAc in Hexanes, then 3\% EtOAc in Hexanes) afforded the title compound as a clear oil $\left(320 \mathrm{mg}, 1.14 \mathrm{mmol}, 76 \%\right.$ yield). IR $v_{\max } / \mathrm{cm}^{-1}$ (film): 3036, 2921, 1558, 1442, 1374, 1049, 771, 691, 632; ${ }^{1} \mathrm{H}$ NMR (400 MHz, $\left.\mathrm{CDCl}_{3}\right) \delta: 8.34(1 \mathrm{H}, \mathrm{d}$, $J=5.1 \mathrm{~Hz}), 8.11(1 \mathrm{H}, \mathrm{s}), 7.59(2 \mathrm{H}, \mathrm{d}, J=8.0 \mathrm{~Hz}), 7.60(1 \mathrm{H}, \mathrm{d}, J=5.2 \mathrm{~Hz}), 7.50-7.41(3 \mathrm{H}, \mathrm{m})$; ${ }^{13} \mathrm{C}$ NMR $\left(100 \mathrm{MHz}, \mathrm{CDCl}_{3}\right) \delta: 158.5,150.1,138.1,131.3,130.1,129.7,129.0,127.1,106.3$; $\mathrm{m} / \mathrm{z}$ LRMS (ESI + APCI) found $[\mathrm{M}+\mathrm{H}]^{+}$282.0, $\mathrm{C}_{11} \mathrm{H}_{9} \mathrm{IN}^{+}$requires 282.1 .

\section{4-Iodo-2-(3-(pentafluoro-l6-sulfaneyl)phenyl)pyridine (43)}<smiles>FS(F)(F)(F)(F)c1cccc(-c2cc(I)ccn2)c1</smiles>

Prepared according to general procedure D, using (2-(3-(pentafluoro- $\lambda 6$ sulfaneyl)phenyl)pyridin-4-yl)(phenyl)bis(6-(trifluoromethyl)pyridin-3-yl)phosphonium trifluoromethanesulfonate (166 mg, $0.20 \mathrm{mmol})$, LiI (107 mg, $0.80 \mathrm{mmol})$, TfOH (18 $\mu \mathrm{L}, 0.20$ $\mathrm{mmol})$, and Dioxane $(2 \mathrm{~mL})$. The reaction was heated to $120{ }^{\circ} \mathrm{C}$ for 72 hours. Flash column chromatography (silica gel: $20 \% \mathrm{CH}_{2} \mathrm{Cl}_{2}$ in Hexanes, ran twice) afforded the title compound as a white solid (50 mg, $0.12 \mathrm{mmol}, 61 \%$ yield). $\mathrm{mp} \mathrm{33-34}{ }^{\circ} \mathrm{C}$. IR $v_{\max } / \mathrm{cm}^{-1}$ (film): $3086,2923,1560$, 1364, 1100, 823, 781, 593; ${ }^{1} \mathrm{H}$ NMR (400 MHz, $\left.\mathrm{CDCl}_{3}\right) \delta: 8.41-8.36(2 \mathrm{H}, \mathrm{m}), 8.13-8.07$ (2H, m), $7.82(1 \mathrm{H}, \mathrm{ddd}, J=8.3,6.0,0.8 \mathrm{~Hz}), 7.68(1 \mathrm{H}, \mathrm{dd}, J=5.1,1.5 \mathrm{~Hz}), 7.58(1 \mathrm{H}$, app t, $J=8.1 \mathrm{~Hz})$; ${ }^{13} \mathrm{C}$ NMR $\left(100 \mathrm{MHz}, \mathrm{CDCl}_{3}\right) \delta: 156.3,154.9-154.5$ (m), 150.3, 139.0, 132.3, 130.1, 129.9, 129.3, 126.9 (qn, $J=4.6 \mathrm{~Hz}$ ), 124.9 (qn, $J=4.7 \mathrm{~Hz}$ ), $106.5 ;{ }^{19} \mathrm{~F}$ NMR $\left(365 \mathrm{MHz}, \mathrm{CDCl}_{3}\right) \delta$ : $84.00(1 \mathrm{~F}, \mathrm{qn}, J=150.8 \mathrm{~Hz}), 62.67(4 \mathrm{~F}, \mathrm{~d}, J=150.0 \mathrm{~Hz}) ; \mathrm{m} / \mathrm{z}$ LRMS (ESI + APCI) found [M + $\mathrm{H}]^{+}$408.0, $\mathrm{C}_{11} \mathrm{H}_{8} \mathrm{~F}_{5} \mathrm{INS}^{+}$requires 407.9.

\section{2-Butyl-4-iodo-5-(trifluoromethyl)pyridine (46)}<smiles>CCCCc1cc(I)c(C(F)(F)F)cn1</smiles> 
Prepared according to general procedure D, using 2-butyl-5-(trifluoromethyl)pyridine (136 mg, $0.20 \mathrm{mmol})$, LiI $(107 \mathrm{mg}, 0.80 \mathrm{mmol})$, TfOH $(18 \mu \mathrm{L}, 0.20 \mathrm{mmol})$, and Dioxane $(2 \mathrm{~mL})$. The reaction was heated to $120{ }^{\circ} \mathrm{C}$ for 24 hours. Flash column chromatography (silica gel: 5\% $\mathrm{CH}_{2} \mathrm{Cl}_{2}$ in Toluene) afforded the title compound as a white solid (47 mg, $0.14 \mathrm{mmol}, 71 \%$ yield). $\mathrm{mp} 44-46{ }^{\circ} \mathrm{C}$. IR $v_{\max } / \mathrm{cm}^{-1}$ (film): 2939, 2866, 1577, 1324, 1105, 1024, 937, 744; ${ }^{1} \mathrm{H}$ NMR (400 $\left.\mathrm{MHz} \mathrm{CDCl}_{3}\right) \delta: 8.65(1 \mathrm{H}, \mathrm{s}), 7.83(1 \mathrm{H}, \mathrm{s}), 2.78(2 \mathrm{H}, \mathrm{t}, J=7.7 \mathrm{~Hz}), 1.75-1.66(2 \mathrm{H}, \mathrm{m}), 1.44-1.33$ $(2 \mathrm{H}, \mathrm{m}), 0.95(3 \mathrm{H}, \mathrm{t}, J=7.3 \mathrm{~Hz}) ;{ }^{13} \mathrm{C} \mathrm{NMR}\left(100 \mathrm{MHz}, \mathrm{CDCl}_{3}\right) \delta: 166.9,146.9(\mathrm{q}, J=6.2 \mathrm{~Hz})$, 135.5, 127.6 (q, $J=31.0 \mathrm{~Hz}), 122.8(\mathrm{q}, J=276.0 \mathrm{~Hz}), 103.4,37.5,31.6,22.5,14.0 ;{ }^{19} \mathrm{~F}$ NMR $\left(365 \mathrm{MHz}, \mathrm{CDCl}_{3}\right) \delta:-62.61 ; \mathrm{m} / \mathrm{z}$ LRMS (ESI $\left.+\mathrm{APCI}\right)$ found $[\mathrm{M}+\mathrm{H}]^{+} 330.1, \mathrm{C}_{11} \mathrm{H}_{8} \mathrm{~F}_{5} \mathrm{INS}^{+}$ requires 330.0 .

\section{3-Bromo-4-iodo-5-methylpyridine (48)}<smiles>Cc1cncc(Br)c1I</smiles>

Prepared according to general procedure D using (3-bromo-5-methylpyridin-4-yl) triphenylphosphonium trifluoromethanesulfonate $(175 \mathrm{mg}, 0.30 \mathrm{mmol})$, LiI (160 mg, 1.20 $\mathrm{mmol})$, TfOH $(26 \mu \mathrm{L}, 0.30 \mathrm{mmol})$, and Dioxane $(3 \mathrm{~mL})$. The reaction was heated at $120^{\circ} \mathrm{C}$ for 3 hours. Flash column chromatography (silica gel: 10\% EtOAc in Hexanes) afforded the title compound as a white solid (60 mg, $0.13 \mathrm{mmol}, 67 \%$ yield). $\mathrm{mp} 110-112{ }^{\circ} \mathrm{C}$; IR $v_{\max } / \mathrm{cm}^{-1}$ (film): 2920, 1545, 1414, 1374, 1182, 1134, 854, 704; ${ }^{1} \mathrm{H}$ NMR (400 MHz, $\left.\mathrm{CDCl}_{3}\right) \delta: 8.44(1 \mathrm{H}, \mathrm{s}), 8.19$ $(1 \mathrm{H}, \mathrm{s}), 2.49(3 \mathrm{H}, \mathrm{s}) ;{ }^{13} \mathrm{C} \mathrm{NMR}\left(100 \mathrm{MHz}, \mathrm{CDCl}_{3}\right) \delta: 148.3,146.6,140.7,129.5,119.2,27.1 ; \mathrm{m} / z$ LRMS (ESI + APCI) found $[\mathrm{M}+\mathrm{H}]^{+} 297.9, \mathrm{C}_{6} \mathrm{H}_{6} \mathrm{BrIN}^{+}$requires 297.9.

\section{Ethyl 4-(8-chloro-4-iodo-5,6-dihydro-11H-benzo[5,6]cyclohepta[1,2-b]pyridin-11-ylidene) piperidine-1-carboxylate (63)}<smiles>CCOC(=O)N1CCC(=C2c3ccc(Cl)cc3CCc3c(I)ccnc32)CC1</smiles>

Prepared according to general procedure D using 8-chloro-11-(1-(ethoxycarbonyl) piperidin-4ylidene)-6,11-dihydro-5H-benzo[5,6]cyclohepta[1,2-b]pyridin-4-yl)diphenyl(6-

(trifluoromethyl)pyridin-3-yl)phosphonium trifluoromethanesulfonate (172 $\mathrm{mg}, 0.20 \mathrm{mmol}$ ), LiI (107 mg, $0.80 \mathrm{mmol})$, TfOH $(18 \mu \mathrm{L}, 0.2 \mathrm{mmol})$, and Dioxane $(2 \mathrm{~mL})$. The reaction was heated at $120{ }^{\circ} \mathrm{C}$ for 16 hours. Flash column chromatography (silica gel: 50\% EtOAc in Hexanes) afforded the title compound as a white solid (64 mg, $0.13 \mathrm{mmol}, 63 \%$ yield). $\mathrm{mp} 149-150{ }^{\circ} \mathrm{C}$; IR $v_{\max } / \mathrm{cm}^{-1}$ (film): 2920, 2855, 1688, 1534, 1471, 1431, 1227, 1114, 997, 832; ${ }^{1} \mathrm{H}$ NMR $\left(400 \mathrm{MHz}, \mathrm{CDCl}_{3}\right)$ $\delta: 7.93(1 \mathrm{H}, \mathrm{d}, J=5.2 \mathrm{~Hz}), 7.64(1 \mathrm{H}, \mathrm{d}, J=5.2 \mathrm{~Hz}), 7.20-7.03(3 \mathrm{H}, \mathrm{m}), 4.13(2 \mathrm{H}, \mathrm{q}, J=7.1 \mathrm{~Hz})$, $3.77(2 \mathrm{H}, \mathrm{dd}, J=19.7,9.9 \mathrm{~Hz}), 3.32(2 \mathrm{H}, \mathrm{tdd}, J=18.9,8.6,5.3 \mathrm{~Hz}), 3.17(\mathrm{tt}, J=2 \mathrm{H}, 9.1,4.6$ 
$\mathrm{Hz}), 3.01-2.76(2 \mathrm{H}, \mathrm{m}), 2.52-2.21(4 \mathrm{H}, \mathrm{m}), 1.24(3 \mathrm{H}, \mathrm{t}, J=7.1 \mathrm{~Hz}) ;{ }^{13} \mathrm{C} \mathrm{NMR}(100 \mathrm{MHz}$, $\left.\mathrm{CDCl}_{3}\right) \delta: 157.7,155.6,146.9,139.0,137.8,136.8,136.6,133.9,133.7,133.3,130.8,129.3$, 126.4, 113.6, 61.5, 44.8, 44.8, 37.4, 31.3, 30.7, 14.8; $\mathrm{m} / z$ LRMS (ESI + APCI) found [M+H] ${ }^{+}$ 509.1, $\mathrm{C}_{22} \mathrm{H}_{23} \mathrm{ClIN}_{2} \mathrm{O}_{2}{ }^{+}$requires 509.0. 


\section{Computational study}

\section{Technical details}

The dispersion-corrected density functional theory (DFT) functional $\omega$ B97X-D was used with the Def2-SVP basis set in the optimization of geometries of all ground and transition structures. We carried out vibrational frequency calculations to obtain thermal corrections to Gibbs free energies $(\mathrm{G})$. Additionally, these frequency calculations were used to confirm that stationary points were either minima or first-order saddle points on the potential energy surface. The connection between intermediates (Int) and their corresponding transition structures (TS) was studied using intrinsic reaction coordinate (IRC) calculations. ${ }^{6}$

$G$ values included a correction for the change in standard state from gas phase at 1 atm to a 1 $\mathrm{M}$ solution. In all the individual calculations, quasi-harmonic (QHA) vibrational corrections were applied to entropies using a frequency cut-off value of $100.0 \mathrm{~cm}^{-1}$, as proposed by Grimme. ${ }^{7}$ In a few cases, systems showed one persistent small imaginary frequency with $v i<50$ $\mathrm{cm}^{-1}$. These imaginary frequencies were inverted to their respective positive values before the QHA entropic corrections were computed as seen in previous examples. ${ }^{8}$ All of these thermodynamic corrections were calculated and automatically applied to the computed results by the GoodVibes program using $\mathrm{T}=353.15 \mathrm{~K}\left(80^{\circ} \mathrm{C}\right)$.

Single-point energy calculations were used to refine electronic energies of optimized geometries. For this, DFT ( $\omega$ B97X-D/Def2-QZVPP) was used. QHA G corrections calculated at the $\omega \mathrm{B} 97 \mathrm{X}-\mathrm{D} / \mathrm{Def} 2-\mathrm{SVP}$ level of theory were applied to these single point energies to obtain the final $\mathrm{G}$ values used to create energy profiles. This process was automated using the GoodVibes program.

All the optimizations, frequency and single-point energy calculations included solvent effects obtained with the integral equation formalism variant of the polarizable continuum model (IEFPCM) with the SMD solvation model (solvent=1,4-dioxane).

A manual conformational search was carried out in all the steps, positioning the different units in multiple interaction sites. Additionally, the different functional groups were rotated in each of the different interaction sites (see section Thermochemical data and absolute energy values for more information about the number of conformers used).

Gaussian $16^{9}$ was used to perform all the DFT calculations with an "ultrafine" pruned $(99,590)$ grid for numerical integration. Atomic charges and Wiberg bond orders (WBO) were computed using natural population analysis (NPA) with NBO 6.0 ${ }^{10}$ interfaced to Gaussian 16. Molecular graphics were generated using $P y M o l ;{ }^{11}$ Our display settings have been made openly accessible. ${ }^{12}$ NCIPlot was used to generate the surfaces of noncovalent interactions using previously DFT-generated WFN files (at the optimization level of theory) and these surfaces were represented using $P y M O L$.

Structures of different initial reagents in step Int-II with halogen atoms 
A
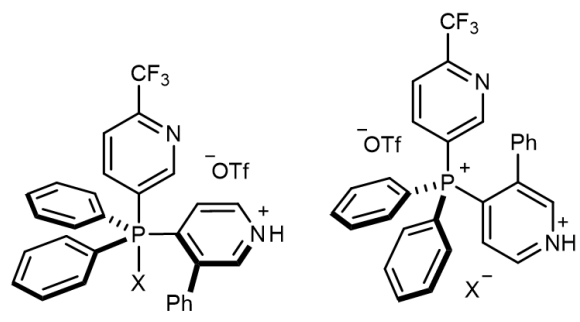

$\mathrm{X}=\mathrm{F}$

\begin{tabular}{|cccc|}
\hline & $\mathrm{F}$ & $\mathrm{Cl}$ & $\mathrm{Br}$ \\
$\mathrm{WBO}_{\mathrm{P}-\mathrm{X}}$ & 0.39 & 0.04 & $<0.01$ \\
$\mathrm{~d}_{\mathrm{P}-\mathrm{X}}(\AA)$ & 1.77 & 3.33 & 5.12 \\
\hline
\end{tabular}

B

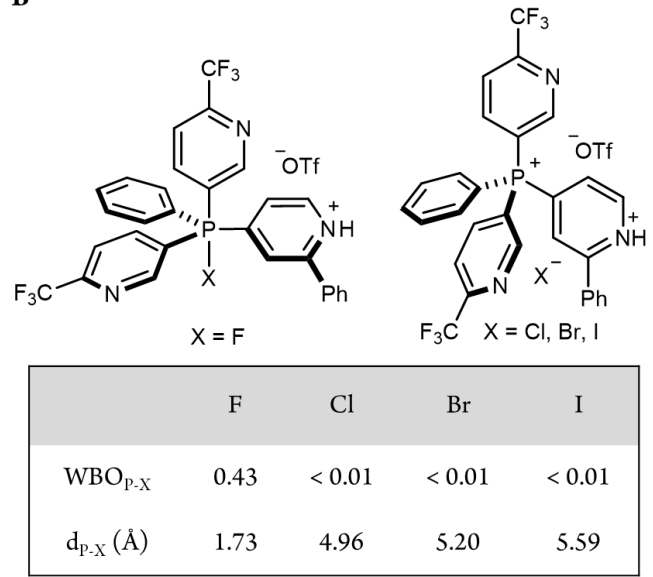

Figure S1. (A) Int-II step when using a phosphonium salt with 3-Ph-Py and one 3-(6-CF $-\mathrm{Py})$ ring. (B) Int-II step when using a phosphonium salt with 2-Ph-Py and two 3-(6-CF $-\mathrm{Py})$ rings.

Electrostatic potential at nucleus (EPN) studies to determine relative electrophilicity

EPN values have previously shown a correlation to the electrophilicity of acceptor atoms in different nucleophilic substitution reactions. ${ }^{13}$ As the EPN value of the acceptor atom becomes higher, the electrophilicity and, therefore, reactivity of that atom increases. As seen in Figure S2, including electron-withdrawing aromatic substituents in the phosphine and using acids that interact with the acceptor Py increase the relative electrophilicity of the acceptor Py.

EPN (kcal/mol): As EPN values becomes higher, electrophilicity increases

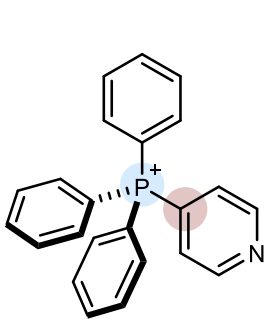

P $\quad 0.0$

C

0.0

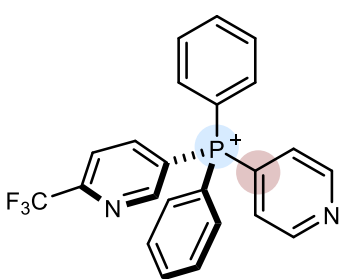

11.6

8.8

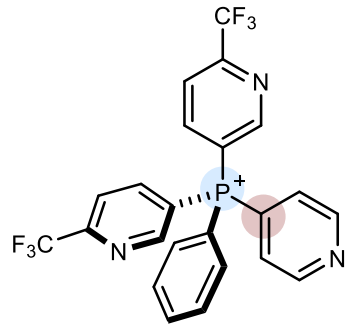

23.3

17.2

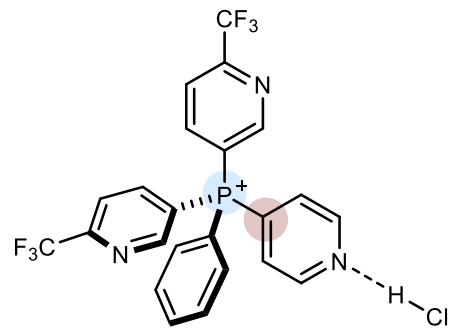

29.5

27.4

Figure S2. Relative EPN values (in $\mathrm{kcal} / \mathrm{mol}$ ) at the $\mathrm{P}$ central atom and the para $\mathrm{C}$ atom of the accepting pyridine group for different phosphines.

\section{Computed reaction pathways}

The names of the calculations included in the xyz and thermochemistry files (provided along with this ESI) contain suffixes that help understanding the type of system studied in each calculation. The suffixes used to separate the different pathways studied are represented in 
reaction schemes below the energy profiles of this section. Also, for each reaction step, the different conformers were differentiated by using letters at the end of their names (i.e. TS-I-Cl2Ph_a, TS-I-Cl-2Ph_b, and so on).

Computed reaction pathways for the chlorination of 3-Ph-Py (5) and 2-Ph-Py (6)

Charge: $0 \quad$ Multiplicity: 1
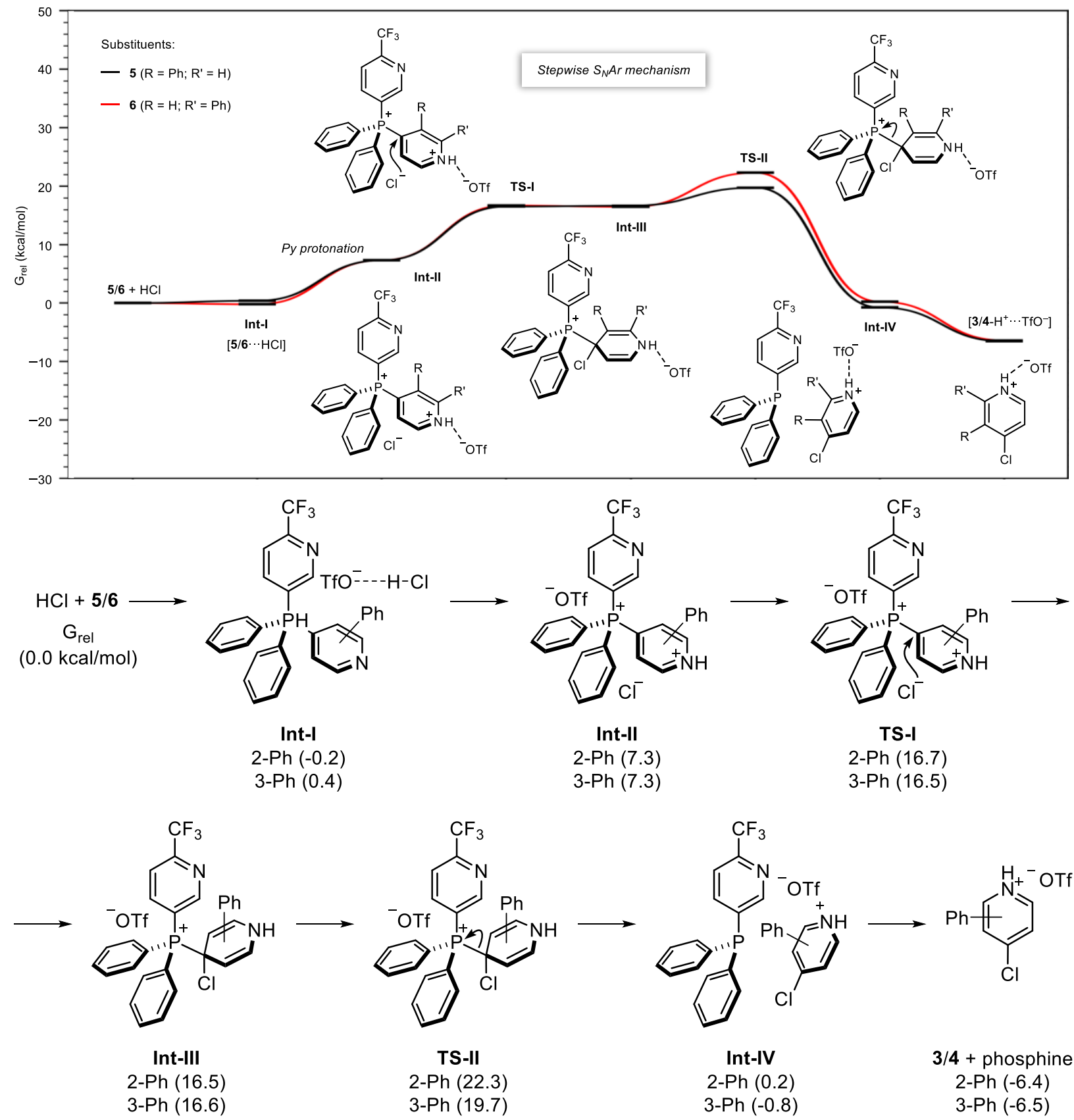

Other names of the structures in the xyz file and thermochemistry data: 
Figure S3. Top: G profiles of pathways with different selectivity in the chlorination of 5 and $\mathbf{6}$ to form products 3 and 4 . G values are Boltzmann weighted $\mathrm{G}$ obtained using all the conformers found in each reaction step (see section Thermochemical data and absolute energy values). Bottom: Schematic representation of the reaction pathways studied.
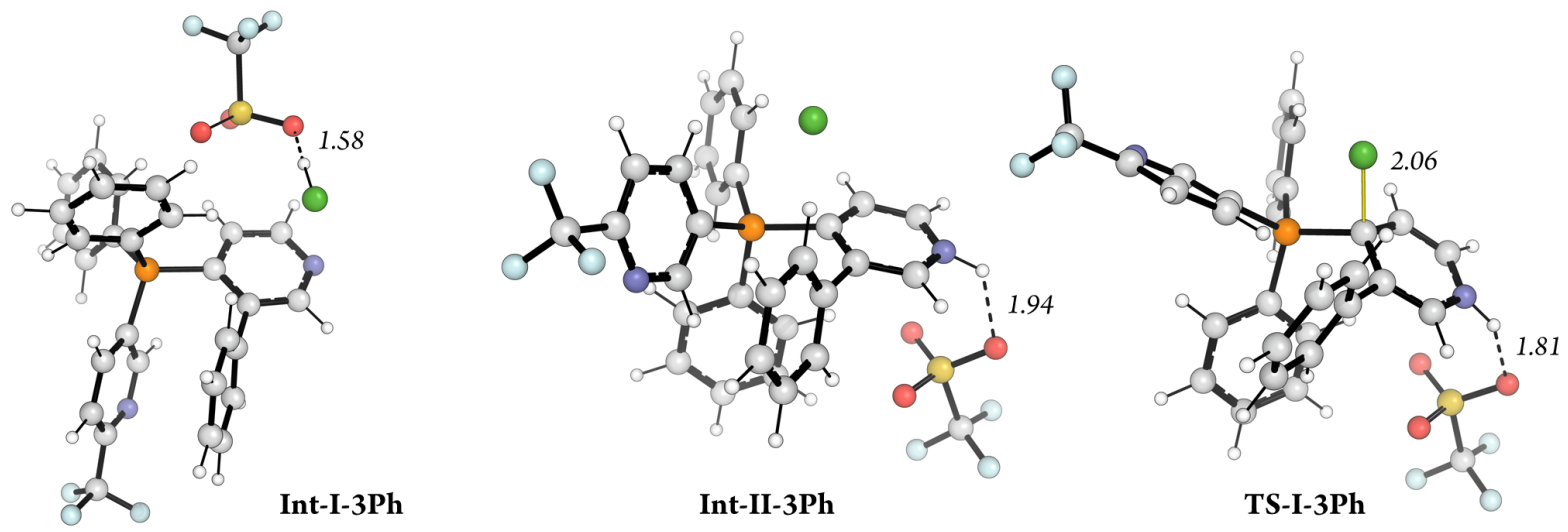

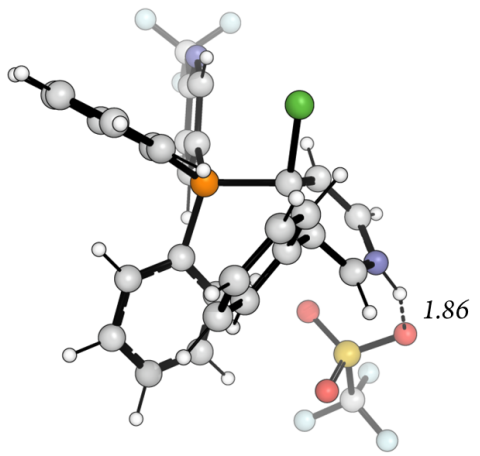

Int-III-3Ph

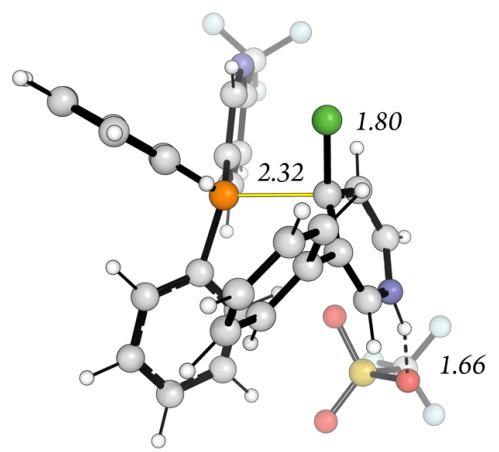

TS-II-3Ph

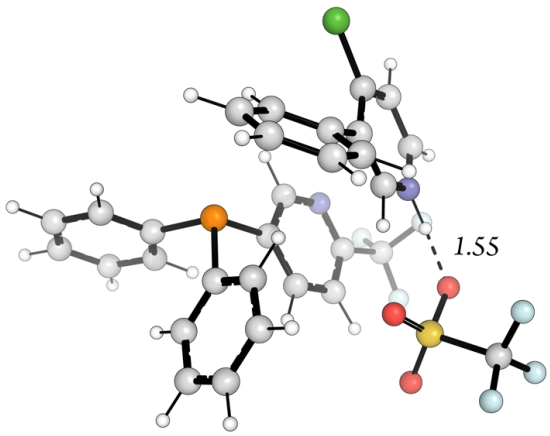

Int-IV-3Ph

Figure S4. Representation of the most stable conformers of each reaction step when using $\mathbf{5}$ as the initial reagent $(3-\mathrm{Ph})$. Bonds involved in the TS and hydrogen bonds are represented as yellow and dotted lines, respectively. Distances are shown in Å. 

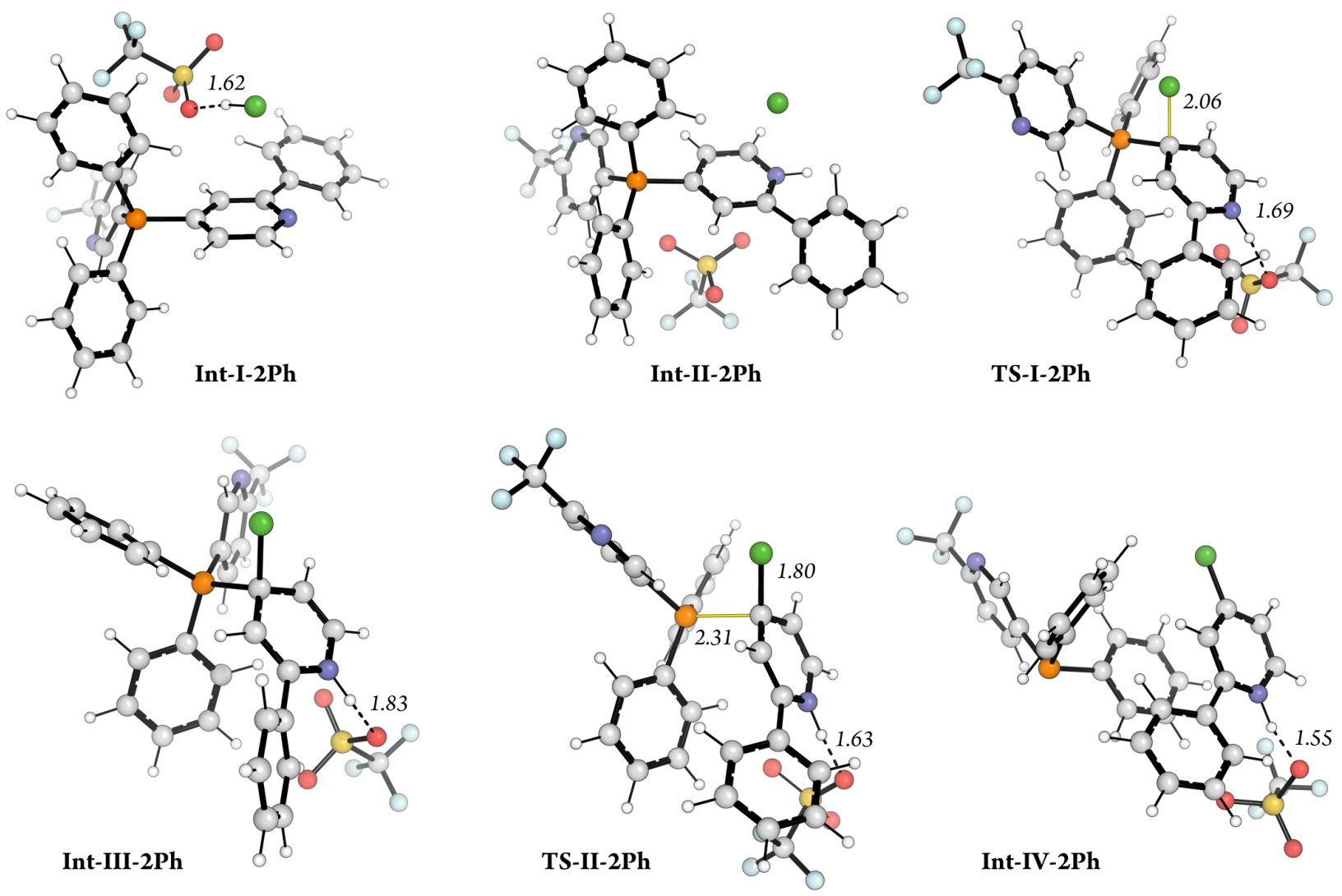

Figure S5. Representation of the most stable conformers of each reaction step when using 6 as the initial reagent (2-Ph). Bonds involved in the TS and hydrogen bonds are represented as yellow and dotted lines, respectively. Distances are shown in $\AA$.

Computed reaction pathways for the chlorination of other substituted pyridines

Charge: $0 \quad$ Multiplicity: 1 

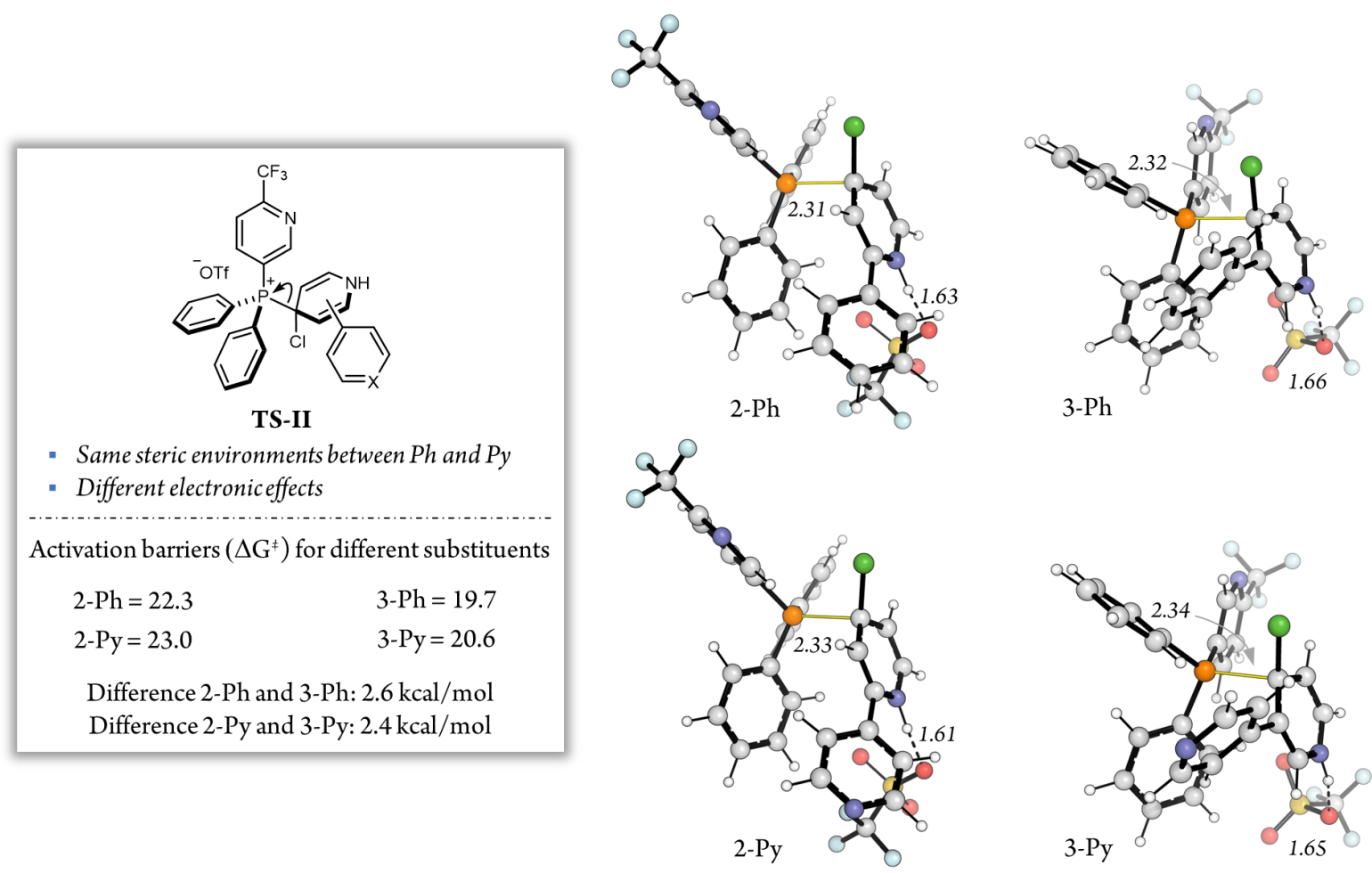

Figure S6. Boltzmann weighted $\mathrm{G}$ barriers from $\mathrm{HCl}+\mathbf{5} / \mathbf{6}$ to TS-II computed when using $\mathrm{Ph}$ and Py groups as substituents in the pyridine acceptors (for the $G$ values of each conformer, see section Thermochemical data and absolute energy values). The geometries of the most stable conformers of TS-II are represented for each substituent. Bonds involved in the TS and hydrogen bonds are represented as yellow and dotted lines, respectively. Distances are shown in $\AA$.

\section{Computed reaction pathway without $\mathrm{TfO}^{-}$}

\section{Charge: 1 Multiplicity: 1}

A model system that did not include the $\mathrm{TfO}^{-}$counteranion was also modeled (Figure $\mathrm{S} 7$ ). In this model, the overall mechanism is similar to the mechanism of the complete system including the counteranion but there are reaction steps that are very difficult to model such as TS-I. One reason why TS-I is challenging to model might be the small energy difference between this step and steps Int-II and Int-III. 


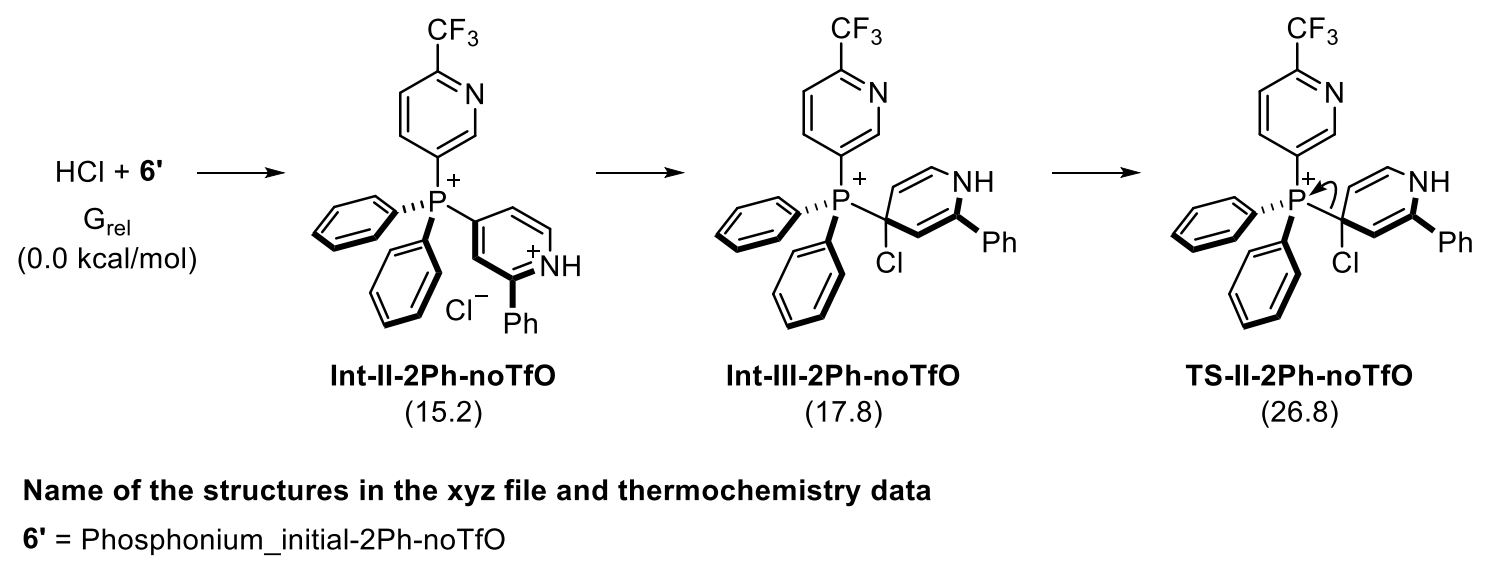

Figure S7. Boltzmann weighted $\mathrm{G}$ values obtained for the model reaction in which the $\mathrm{TfO}^{-}$ counteranion is not included.

Computed reaction pathway without $\mathrm{TfOH}$ and $\mathrm{H}^{+}$

Charge: 0 Multiplicity: 1 (with no TfOH)

Charge: $-1 \quad$ Multiplicity: 1 (with no $\mathrm{H}^{+}$)

Furthermore, model systems that did not include the $\mathrm{H}^{+}$source (excluding either $\mathrm{TfOH}$ or only $\mathrm{H}^{+}$) were also employed to simulate the experimental reactions in which $\mathrm{Cl}$ anions are used without strong Lewis or Brønsted acids (i.e. $\mathrm{NMe}_{4} \mathrm{Cl}$ ). As seen in Figure S8, these reactions show high energy activation barriers that should not be possible to cross under the reaction temperature $\left(80^{\circ} \mathrm{C}\right)$. 


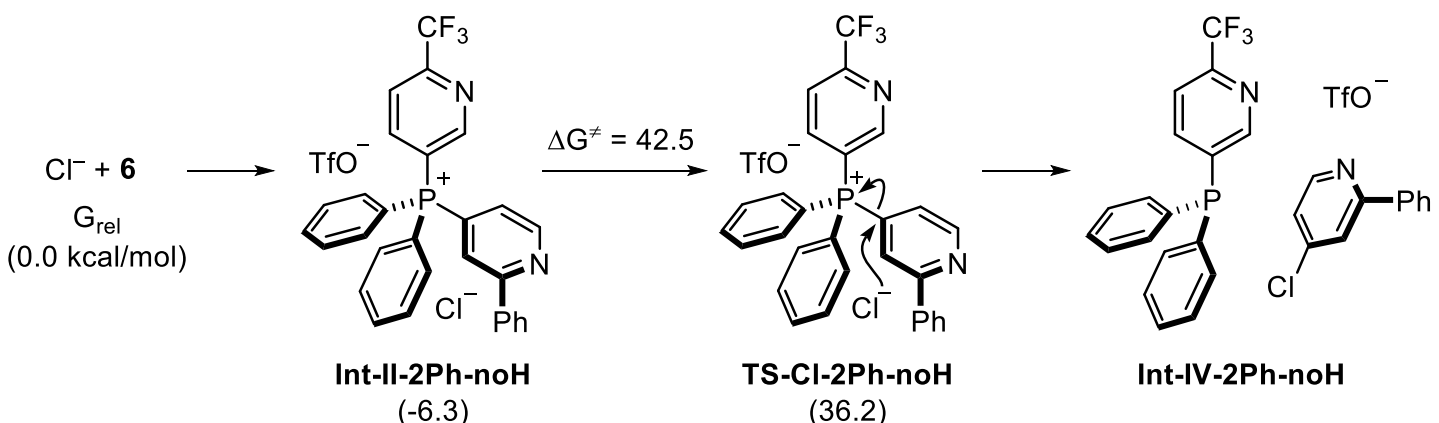

Name of the structures in the xyz file and thermochemistry data $6=$ Phosphonium_initial-2Ph

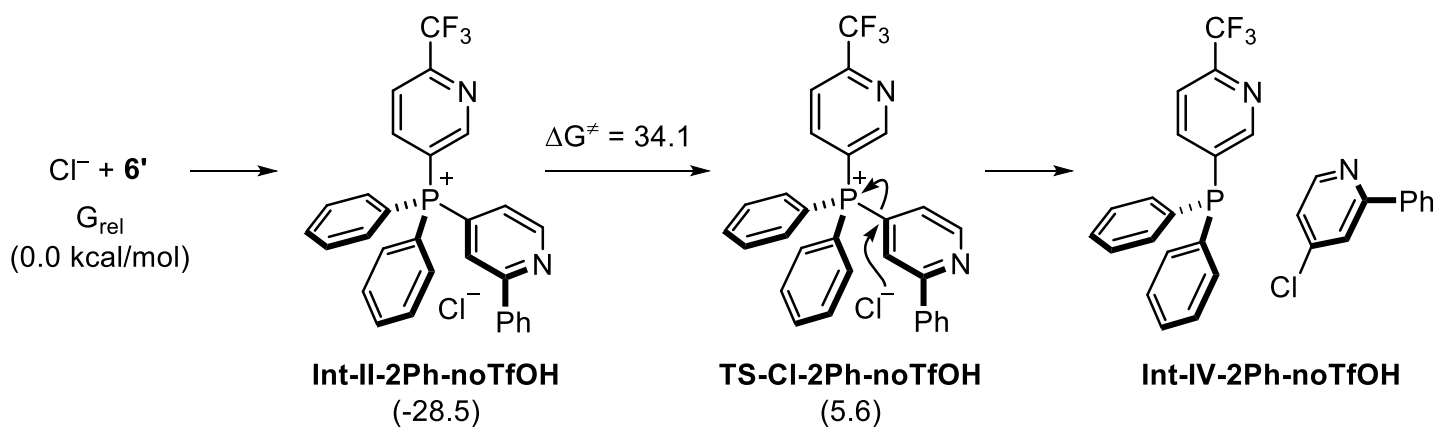

Name of the structures in the xyz file and thermochemistry data 6' = Phosphonium_initial-2Ph-noTfO

Figure S8. Boltzmann weighted $\mathrm{G}$ values obtained for reactions in which either $\mathrm{TfOH}$ or $\mathrm{H}^{+}$ were excluded.

One notorious difference to the mechanism involving acid activation is the concerted nature of these $\mathrm{S}_{N} \mathrm{Ar}$ to nonactivated pyridine acceptors. Figure S9 shows that Int-III is not stable when there are not any $\mathrm{H}^{+}$sources, since this reaction goes directly from Int-II to Int-IV in a single transition step (compared to TS-I, Int-III and TS-II when a $\mathrm{H}^{+}$source is used). 


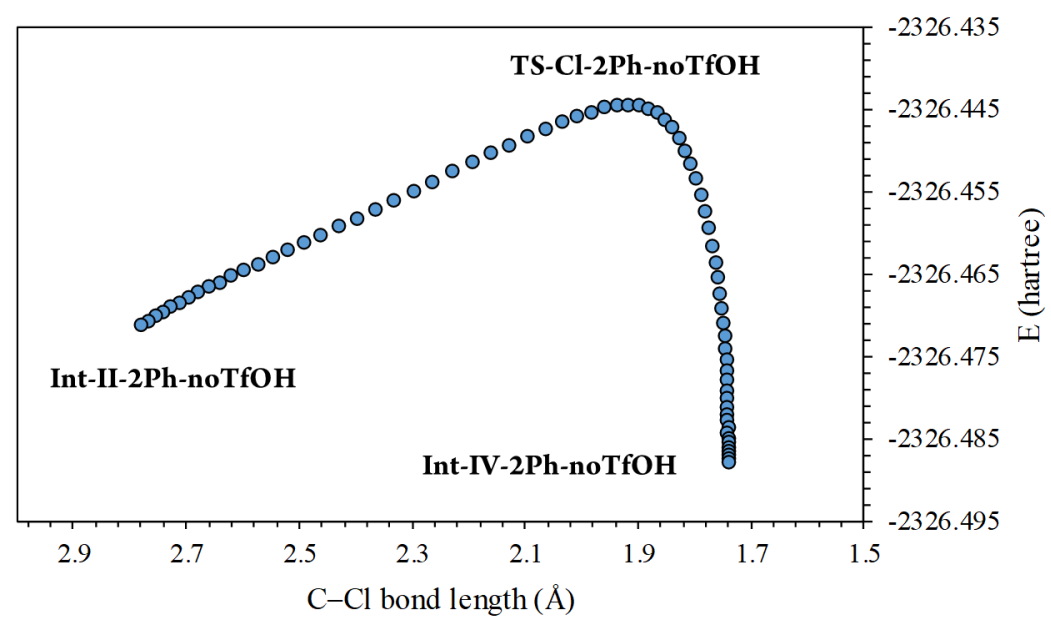

Figure S9. IRC of the TS step of the Cl-Py coupling to nonactivated pyridines.

\section{Studies using larger basis sets with diffuse functions}

For the DFT benchmarking study, larger basis sets were employed for halide anions and $\mathrm{P}$ atoms (Def2-TZVPPD for F, Cl, Br and I atoms and Def2-TZVPP for P atoms) during the optimization process, which was followed by single-point energy corrections at the $\omega$ B97X-D/Def2-QZVPP level. Using a total of 24 calculations including the most stable conformers of the different reaction steps, the geometries and $\mathrm{G}$ values obtained were very similar compared to the initial methodology ( $\mathrm{G}$ mean absolute error (MAE) for both approaches was $0.4 \pm 0.4 \mathrm{kcal} / \mathrm{mol}$, Table $\mathrm{S} 7$ and Figure S10). Thus, it is preferred to use the initial approach since it requires less computation time, the input files are easier to make, and it avoids some SCF convergence problems encountered when using I atoms with Def2-TZVPPD. Additionally, we further tested the robustness of the initial approach to model this reaction by comparing the energy values obtained initially with the values obtained using single-point calculations with diffuse functions $(\omega B 97 X-D / D e f 2-Q Z V P P D)$. For this test, 20 structures chosen from different reaction steps were employed. In all the cases, the two approaches led to nearly identical results (MAE of calculated $\mathrm{G}$ with both methods was $0.04 \pm 0.04 \mathrm{kcal} / \mathrm{mol}$, Table S8) and, therefore, it is preferred to employ Def2-QZVPP over Def2-QZVPPD for single-point energy corrections since the calculation times required for both approaches differ considerably (average job CPU times were $176 \pm 13$ and 578 \pm 107 hours for Def2-QZVPP and Def2-QZVPPD, respectively, Table S9).

Geometry optimization including diffuse functions $^{14}$

In order to use the gen (or genecp when I atoms were included) option in Gaussian for the level $\omega B$ 97X-D/Def2-SVP for C, H, N, O, F (selected atoms for $\mathrm{CF}_{3}$ ) and S; Def2-TZVPPD for F, Cl, $\mathrm{Br}$ and I anions; and Def2-TZVPP for P, we used the following input to specify the basis sets:

Def2-SVP for C, O, S, F, N and $\mathrm{H}$ atoms:

COS F N H 0

$* * * *$

def2SVP 
Def2-TZVPP for $\mathrm{P}$ atoms:

$\mathrm{P} 0$

def2TZVPP

$* * * *$

Def2-TZVPPD for $\mathrm{F}$ atoms:

F 0

S $6 \quad 1.00$

35479.1004410 $\quad 0.21545014888 \mathrm{D}-03$

$5318.4728983 \quad 0.16700686527 \mathrm{D}-02$

$1210.4810975 \quad 0.86733211476 \mathrm{D}-02$

$342.85518140 \quad 0.35049933175 \mathrm{D}-01$

$112.01943181 \quad 0.11165320133$

$40.714740248 \quad 0.25988506647$

S 21.00

$16.039678111 \quad 0.39422966880$

$6.5038186740 \quad 0.24998238551$

S 11.00 $1.5440477509 \quad 1.0000000$

S 11.00 $0.61223452862 \quad 1.0000000$

S 11.00 $0.24027979698 \quad 1.0000000$

S 11.00 $0.90918446478 \mathrm{D}-01 \quad 1.0000000$

P 41.00 $80.233900483 \quad 0.63685999134 \mathrm{D}-02$ $18.594010743 \quad 0.44303143530 \mathrm{D}-01$ $5.6867902653 \quad 0.16867248708$ $1.9511006294 \quad 0.36166346255$

P 111.00 $0.66970211298 \quad .44202901491$

P 11.00 $0.21651300410 \quad .24319875730$

P 111.00 $0.59613282472 \mathrm{D}-01 \quad 1.0000000$

D 11.00 $3.10700000 \quad 1.0000000$

D 11.00 $0.85500000 \quad 1.0000000$

D 11.00 $0.18608388111 \quad 1.0000000$

F 11.00 $\begin{array}{ll}1.91700000 & 1.0000000 \\ * * * * & \end{array}$

Def2-TZVPPD for $\mathrm{Cl}$ atoms:

$\begin{array}{ll}\mathrm{Cl} \quad 0 & \\ \mathrm{~S} 7 \quad 1.00 & \\ 69507.9909450 & 0.54314897497 \mathrm{D}-03 \\ 10426.1568800 & 0.41990463961 \mathrm{D}-02 \\ 2373.2334061 & 0.21592141679 \mathrm{D}-01 \\ 671.56420071 & 0.84598850094 \mathrm{D}-01\end{array}$

\begin{tabular}{|c|c|c|}
\hline & 218.41999790 & 0.24757249724 \\
\hline & 77.572249714 & 0.47016930228 \\
\hline & 28.888815277 & 0.37436370716 \\
\hline \multicolumn{3}{|c|}{$\begin{array}{lll}S & 3 & 1.00\end{array}$} \\
\hline & 127.10527185 & $0.25182166603 \mathrm{D}-01$ \\
\hline & 39.339582961 & 0.10786112456 \\
\hline & 7.6740679989 & -0.27408821574 \\
\hline \multicolumn{3}{|c|}{ S 21.00} \\
\hline & 3.8745627630 & 1.3213875014 \\
\hline & 1.8385832573 & 0.68636955368 \\
\hline \multicolumn{3}{|c|}{$\begin{array}{lll}S & 1 & 1.00\end{array}$} \\
\hline & 0.50229057542 & 1.0000000 \\
\hline \multicolumn{3}{|c|}{$\begin{array}{lll}S & 1 & 1.00\end{array}$} \\
\hline & 0.17962723420 & 1.0000000 \\
\hline \multicolumn{3}{|c|}{$\begin{array}{lll}S & 1 & 1.00\end{array}$} \\
\hline & $0.61133095854 \mathrm{D}-01$ & 1.0000000 \\
\hline \multicolumn{3}{|c|}{$\begin{array}{lll}P & 5 & 1.00\end{array}$} \\
\hline & 666.50423284 & $0.23632663836 \mathrm{D}-02$ \\
\hline & 157.64241690 & $0.18879300374 \mathrm{D}-01$ \\
\hline & 50.262520978 & $0.87206341273 \mathrm{D}-01$ \\
\hline & 18.536078105 & 0.25285612970 \\
\hline & 7.2940532777 & 0.43507154820 \\
\hline \multicolumn{3}{|c|}{$\begin{array}{lll}\mathrm{P} & 1 & 1.00\end{array}$} \\
\hline & 2.9433248995 & .35026513165 \\
\hline \multicolumn{3}{|c|}{$\begin{array}{lll}P & 1 & 1.00\end{array}$} \\
\hline & 1.0404970818 & 1.0000000 \\
\hline \multicolumn{3}{|c|}{$\begin{array}{lll}P & 1 & 1.00\end{array}$} \\
\hline & 0.38456415080 & 1.0000000 \\
\hline \multicolumn{3}{|c|}{$\begin{array}{lll}\mathrm{P} & 1 & 1.00\end{array}$} \\
\hline & 0.13069642732 & 1.0000000 \\
\hline \multicolumn{3}{|c|}{$\begin{array}{lll}P & 1 & 1.00\end{array}$} \\
\hline & $0.35381580722 \mathrm{D}-01$ & 1.0000000 \\
\hline \multicolumn{3}{|c|}{11.00} \\
\hline & 4.61000000 & 1.0000000 \\
\hline \multirow[t]{2}{*}{$\mathrm{D}$} & 11.00 & \\
\hline & 1.01100000 & 1.0000000 \\
\hline \multirow[t]{2}{*}{ D } & 11.00 & \\
\hline & 0.339000000 & 1.0000000 \\
\hline \multicolumn{3}{|c|}{11.00} \\
\hline & 0.99601358790D-01 & 1.0000000 \\
\hline \multicolumn{3}{|c|}{11.00} \\
\hline & $\begin{array}{l}0.706000000 \\
* *\end{array}$ & 1.0000000 \\
\hline
\end{tabular}

Def2-TZVPPD for $\mathrm{Br}$ atoms:

$\begin{array}{ll}\mathrm{Br} \quad 0 & \\ \mathrm{~S} \quad 8 \quad 1.00 & \\ 565073.2525600 & 0.23660314690 \mathrm{D}-03 \\ 84701.7231790 & 0.18348332508 \mathrm{D}-02 \\ 19276.2719000 & 0.95465849860 \mathrm{D}-02 \\ 5456.4284576 & 0.38877142153 \mathrm{D}-01 \\ 1776.9503500 & 0.12718314231 \\ 639.19398276 & 0.30437662191 \\ 248.78823961 & 0.44490940497 \\ 98.678305494 & 0.24381643058\end{array}$




\begin{tabular}{|c|c|c|}
\hline \multicolumn{3}{|c|}{$\begin{array}{lll}S & 4 & 1.00\end{array}$} \\
\hline & 606.07824568 & $-0.26527158709 \mathrm{D}-01$ \\
\hline & 188.45598484 & -0.12484584809 \\
\hline & 31.497144506 & 0.56468683559 \\
\hline & 13.736008320 & 0.55555268564 \\
\hline \multicolumn{3}{|c|}{21.00} \\
\hline & 21.203212766 & -0.24940920493 \\
\hline & 3.7616420178 & 0.71213119743 \\
\hline \multicolumn{3}{|c|}{11.00} \\
\hline & 1.7735933962 & 1.0000000 \\
\hline \multicolumn{3}{|c|}{$\begin{array}{lll}S & 1 & 1.00\end{array}$} \\
\hline & 0.45197413664 & 1.0000000 \\
\hline \multicolumn{3}{|c|}{ S $\quad 1 \quad 1.00$} \\
\hline & 0.16613377099 & 1.0000000 \\
\hline \multicolumn{3}{|c|}{$\begin{array}{lll}\text { S } & 1 & 1.00\end{array}$} \\
\hline & $0.52769579657 \mathrm{D}-0$ & 1.0000000 \\
\hline \multicolumn{3}{|c|}{$\begin{array}{lll}\mathrm{P} & 6 & 1.00\end{array}$} \\
\hline & 3019.6955723 & $0.24971049798 \mathrm{D}-02$ \\
\hline & 715.35481126 & $0.20419267596 \mathrm{D}-01$ \\
\hline & 229.98328751 & 0.96897148309D-01 \\
\hline & 86.167844615 & 0.28053901252 \\
\hline & 34.667870802 & 0.44606390473 \\
\hline & 14.113870307 & 0.24410073923 \\
\hline \multicolumn{3}{|c|}{$4 \quad 1.00$} \\
\hline & 57.085653082 & $-0.21855950710 \mathrm{D}-01$ \\
\hline & 8.8193845840 & 0.32707075320 \\
\hline & 3.9340302872 & 0.57855229520 \\
\hline & 1.7998830384 & 0.33570987698 \\
\hline \multicolumn{3}{|c|}{$\begin{array}{lll}\mathrm{P} & 1 & 1.00\end{array}$} \\
\hline & 0.66899410512 & 1.0000000 \\
\hline \multicolumn{3}{|c|}{11.00} \\
\hline & 0.27136238231 & 1.0000000 \\
\hline \multicolumn{3}{|c|}{$\mathrm{P} \quad 1 \quad 1.00$} \\
\hline & 0.10083790243 & 1.0000000 \\
\hline \multicolumn{3}{|c|}{$\begin{array}{lll}\mathrm{P} & 1 & 1.00\end{array}$} \\
\hline & $0.29005224647 \mathrm{D}-0$ & 1.0000000 \\
\hline \multicolumn{3}{|c|}{ D $5 \quad 1.00$} \\
\hline & 168.85370257 & 0.89663981988D-02 \\
\hline & 49.977949919 & $0.62062059316 \mathrm{D}-01$ \\
\hline & 18.274913338 & 0.21474732384 \\
\hline & 7.2455694631 & 0.40335336746 \\
\hline & 2.8562315025 & 0.42208813080 \\
\hline \multirow[t]{2}{*}{$\mathrm{D}$} & 11.00 & \\
\hline & 1.0459621144 & .17874813267 \\
\hline \multirow[t]{2}{*}{$\mathrm{D}$} & 11.00 & \\
\hline & 0.56865655 & 1.0000000 \\
\hline & 11.00 & \\
\hline & 0.22031490 & 1.0000000 \\
\hline \multicolumn{3}{|c|}{$1 \quad 1.00$} \\
\hline & $0.75865567476 \mathrm{D}-0$ & 1.0000000 \\
\hline $\mathrm{F}$ & 11.00 & \\
\hline & 0.57083312 & 1.0000000 \\
\hline
\end{tabular}

Def2-TZVPPD for I atoms:

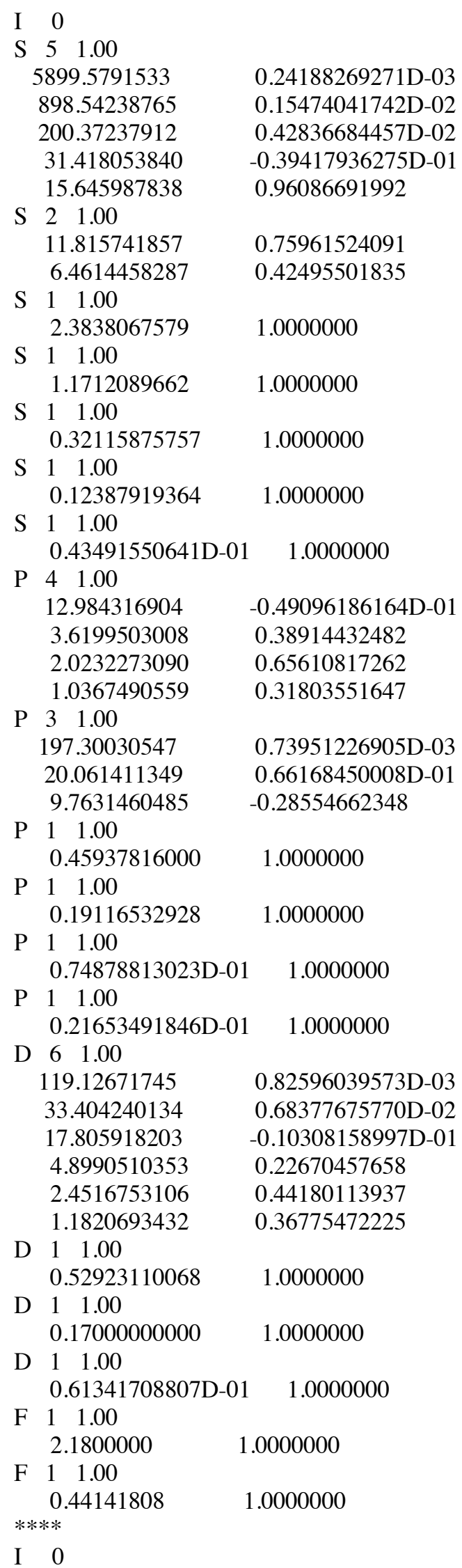




$\begin{array}{lcl}\text { I-ECP } 3 \quad 28 & \\ \text { f potential } & \\ 4 & & \\ 2 & 19.45860900 & -21.84204000 \\ 2 & 19.34926000 & -28.46819100 \\ 2 & 4.82376700 & -0.24371300 \\ 2 & 4.88431500 & -0.32080400 \\ \text { s-f potential } & \\ 7 & & \\ 2 & 40.01583500 & 49.99429300 \\ 2 & 17.42974700 & 281.02531700 \\ 2 & 9.00548400 & 61.57332600 \\ 2 & 19.45860900 & 21.84204000 \\ 2 & 19.34926000 & 28.46819100 \\ 2 & 4.82376700 & 0.24371300 \\ 2 & 4.88431500 & 0.32080400 \\ \text { p-f potential } & \\ 8 & & \\ 2 & 15.35546600 & 67.44284100\end{array}$

\begin{tabular}{ccc}
2 & 14.97183300 & 134.88113700 \\
2 & 8.96016400 & 14.67505100 \\
2 & 8.25909600 & 29.37566600 \\
2 & 19.45860900 & 21.84204000 \\
2 & 19.34926000 & 28.46819100 \\
2 & 4.82376700 & 0.24371300 \\
2 & 4.88431500 & 0.32080400 \\
d-f potential & \\
\multicolumn{1}{l}{10} \\
2 & 15.06890800 & 35.43952900 \\
2 & 14.55532200 & 53.17605700 \\
2 & 6.71864700 & 9.06719500 \\
2 & 6.45639300 & 13.20693700 \\
2 & 1.19177900 & 0.08933500 \\
2 & 1.29115700 & 0.05238000 \\
2 & 19.45860900 & 21.84204000 \\
2 & 19.34926000 & 28.46819100 \\
2 & 4.82376700 & 0.24371300 \\
2 & 4.88431500 & 0.32080400
\end{tabular}

Even in the most difficult cases (i.e. modelling $\mathrm{F}$ anions), ${ }^{15}$ the geometries optimized with diffuse functions did not present substantial differences compared to the geometries obtained with the initial approach ( $\omega$ B97X-D/Def2-SVP for all the atoms) (Figure S10). It is worth to mention that in all cases the geometries and $\mathrm{P}-$ halogen, $\mathrm{P}-\mathrm{C}$ and other bond distances obtained when using diffuse functions do not show significant changes that would affect any of the results obtained when optimizing with $\omega B$ 97X-D/Def2-SVP. 


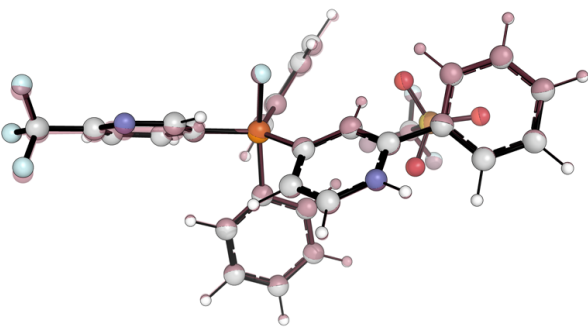

Int-II-F_a

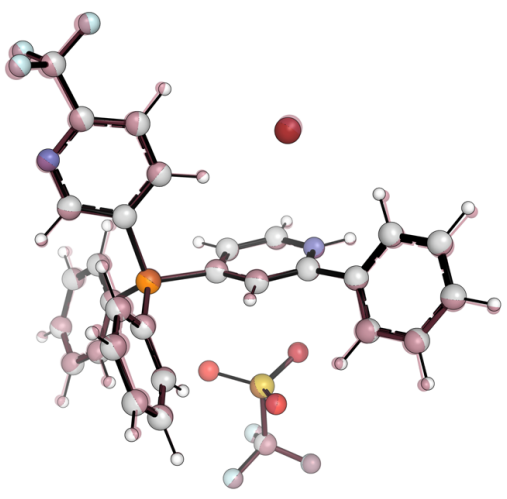

Int-II-Br_h

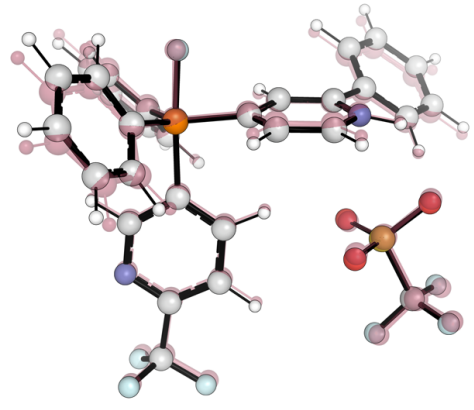

Int-II-F_b

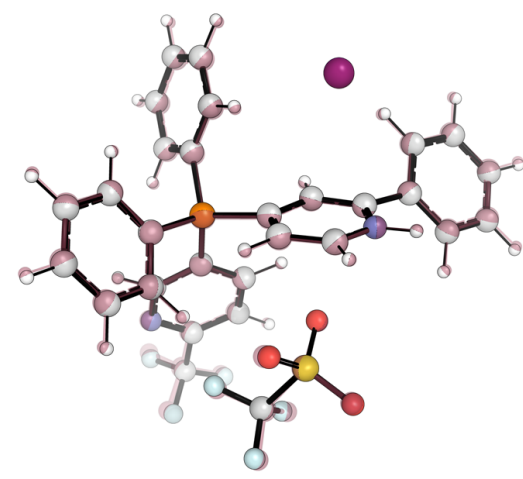

Int-II-I_d

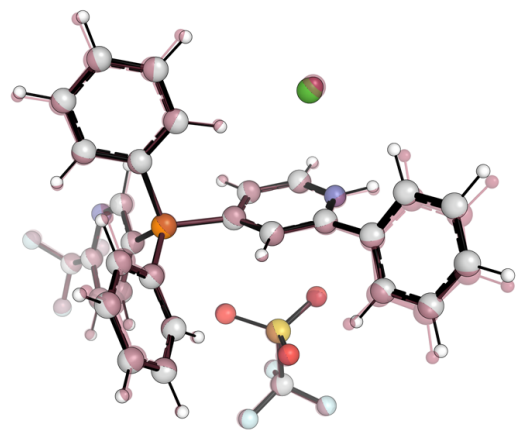

Int-II-Cl-2Ph_a

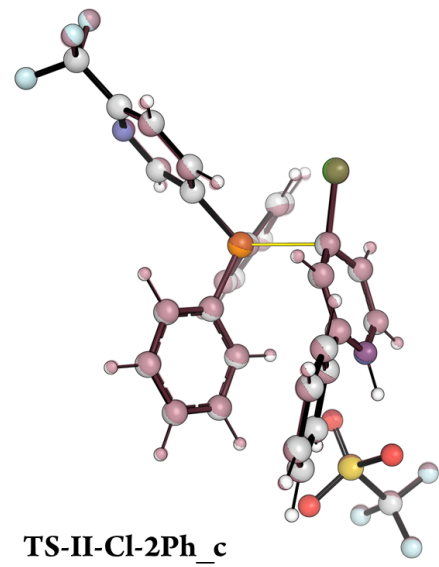

Figure S10. Overlay of geometries optimized with $\omega \mathrm{B} 97 \mathrm{X}-\mathrm{D} / \mathrm{Def2}-\mathrm{SVP}$ for $\mathrm{C}, \mathrm{H}, \mathrm{N}, \mathrm{O}, \mathrm{F}$ (in $\mathrm{CF}_{3}$ ) and S; Def2-TZVPPD for $\mathrm{F}, \mathrm{Cl}, \mathrm{Br}$ and I anions; and Def2-TZVPP for P (red color) and $\omega \mathrm{B} 97 \mathrm{X}-\mathrm{D} / \mathrm{Def} 2-\mathrm{SVP}$ (normal colors).

Table S7. Comparison of relative $\mathrm{G}$ obtained using (i) $\omega \mathrm{B} 97 \mathrm{X}-\mathrm{D} / \mathrm{Def} 2-\mathrm{QZVPP} / / \omega \mathrm{B} 97 \mathrm{X}-$ D/Def2-SVP for all atoms and (ii) $\omega$ B97X-D/Def2-QZVPP// $\omega$ B97X-D/Def2-SVP for C, H, N, $\mathrm{O}, \mathrm{F}$ (in $\mathrm{CF}_{3}$ ) and $\mathrm{S}$; Def2-TZVPPD for $\mathrm{F}, \mathrm{Cl}, \mathrm{Br}$ and I anions; and Def2-TZVPP for P. The difference in the two relative $G$ is also shown. It is worth to mention that the $G$ values represented are the energy of individual conformers (not Boltzmann averages). MAE = mean absolute error. $\mathrm{SD}=$ standard deviation. Product- $3 \mathrm{Ph}=3$. Product- $2 \mathrm{Ph}=4$. Phosphonium_initial-3Ph $=5$. Phosphonium_initial-2Ph $=6$.

\begin{tabular}{|c|c|c|c|}
\hline System & $\begin{array}{c}\mathrm{G}_{\mathrm{rel}}(\mathrm{kcal} / \mathrm{mol}) \text { with } \omega \mathrm{B} 97 \mathrm{X}- \\
\mathrm{D} / \text { Def2-QZVPP// } / \mathrm{B} 97 \mathrm{X}-\mathrm{D} / \text { Def2- } \\
\text { SVP for all atoms }\end{array}$ & 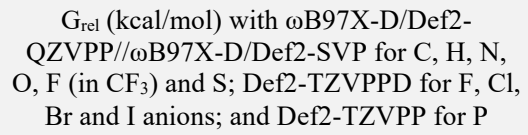 & $\begin{array}{l}\text { Absolute } \\
\text { G diff. } \\
(\mathrm{kcal} / \mathrm{mol})\end{array}$ \\
\hline 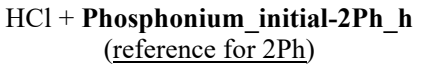 & 0.0 & 0.0 & - \\
\hline $\begin{array}{c}\mathrm{HCl}+ \\
\begin{array}{c}\text { Phosphonium_initial-3Ph_f } \\
\text { (reference for } 3 \mathrm{Ph})\end{array}\end{array}$ & 0.0 & 0.0 & - \\
\hline Int-I-Cl-2Ph_g & 1.7 & 1.7 & 0.0 \\
\hline Int-I-Cl-3Ph_f & 1.4 & 1.5 & 0.1 \\
\hline Int-II-Cl-2Ph_a & 7.4 & 6.9 & 0.5 \\
\hline
\end{tabular}




\begin{tabular}{|c|c|c|c|}
\hline Int-II-Cl-2Ph_f & 8.2 & 7.2 & 1.0 \\
\hline Int-II-Cl-3Ph_c & 8.3 & 6.8 & 1.5 \\
\hline Int-III-Cl-2Ph_b & 21.9 & 22.3 & 0.4 \\
\hline Int-III-Cl-2Ph_e & 16.9 & 17.4 & 0.5 \\
\hline Int-III-Cl-3Ph_a & 20.4 & 20.2 & 0.2 \\
\hline Int-III-Cl-3Ph_b & 17.1 & 17.7 & 0.6 \\
\hline Int-IV-Cl-2Ph_e & 0.2 & 0.9 & 0.7 \\
\hline Int-IV-Cl-3Ph_b & 1.7 & 2.1 & 0.4 \\
\hline Int-IV-Cl-3Ph_c & -0.4 & -0.3 & 0.1 \\
\hline $\mathrm{HCl}+$ Phosphonium_initial-2Ph_f & 0.2 & 0.6 & 0.4 \\
\hline $\mathrm{HCl}+$ Phosphonium_initial-3Ph_k & 4.5 & 3.9 & 0.6 \\
\hline $\begin{array}{c}\text { Product-2Ph_a }+ \\
\text { Phosphine-product_b }\end{array}$ & -5.9 & -5.8 & 0.1 \\
\hline $\begin{array}{c}\text { Product-2Ph_b + } \\
\text { Phosphine-product_b }\end{array}$ & -6.1 & -6.1 & 0.0 \\
\hline $\begin{array}{c}\text { Product-3Ph_a }+ \\
\text { Phosphine-product_b }\end{array}$ & -6.0 & -6.0 & 0.0 \\
\hline $\begin{array}{c}\text { Product-3Ph_c }+ \\
\text { Phosphine-product_b }\end{array}$ & -6.2 & -6.0 & 0.2 \\
\hline TS-II-Cl-2Ph_c & 23.5 & 23.5 & 0.0 \\
\hline TS-II-Cl-2Ph_g & 22.4 & 22.0 & 0.4 \\
\hline TS-II-Cl-3Ph_c & 20.1 & 21.6 & 1.5 \\
\hline TS-II-Cl-3Ph_h & 22.0 & 22.2 & 0.2 \\
\hline \multicolumn{4}{|c|}{$\mathrm{MAE}=0.4 \mathrm{kcal} / \mathrm{mol} ; \mathrm{SD}=0.4 \mathrm{kcal} / \mathrm{mol}$} \\
\hline
\end{tabular}

$\underline{\text { Single-point energy calculations including diffuse functions }}$

In order to use the gen option in Gaussian for the level $\omega$ B97X-D/Def2-QZVPPD for all the atoms, we used the following input to specify the basis sets:

Def2-QZVPPD for H atoms:

$\mathrm{H} \quad 0$

S $\quad 4 \quad 1.00$

$190.6916900 \quad 0.70815167 \mathrm{D}-03$

$28.6055320 \quad 0.54678827 \mathrm{D}-02$

$6.5095943 \quad 0.27966605 \mathrm{D}-01$

$1.8412455 \quad 0.10764538$

S 11.00

$0.59853725 \quad 1.0000000$

S $\quad 1 \quad 1.00$

$0.21397624 \quad 1.0000000$

S $11 \quad 1.00$

$0.80316286 \mathrm{D}-01 \quad 1.0000000$

P $11 \quad 1.00$

2.29200000
$\begin{array}{lll}\mathrm{P} & 1 & 1.00\end{array}$

$0.83800000 \quad 1.0000000$

P $11 \quad 1.00$ $0.29200000 \quad 1.0000000$

P 11.00 0.84063199228D-01 1.0000000

D $\quad 1 \quad 1.00$ $2.06200000 \quad 1.0000000$

D $1 \quad 1.00$ $0.66200000 \quad 1.0000000$

F 11.00 $1.39700000 \quad 1.0000000$

$* * * *$

Def2-QZVPPD for $\mathrm{C}$ atoms: 


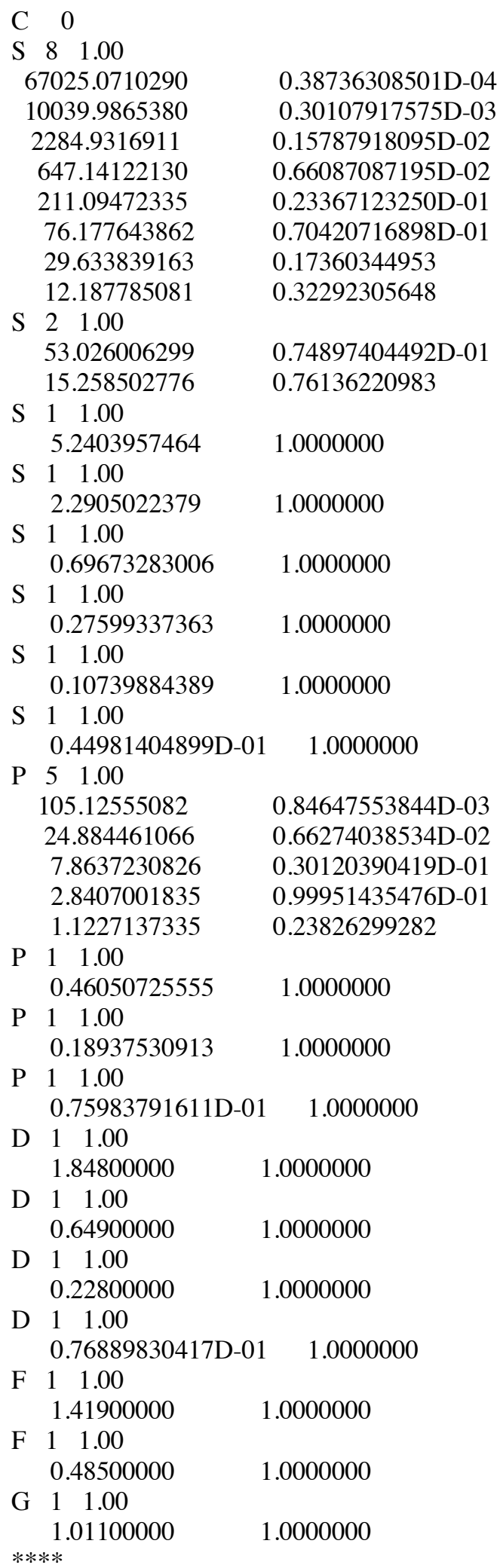

Def2-QZVPPD for $\mathrm{N}$ atoms:

N 0

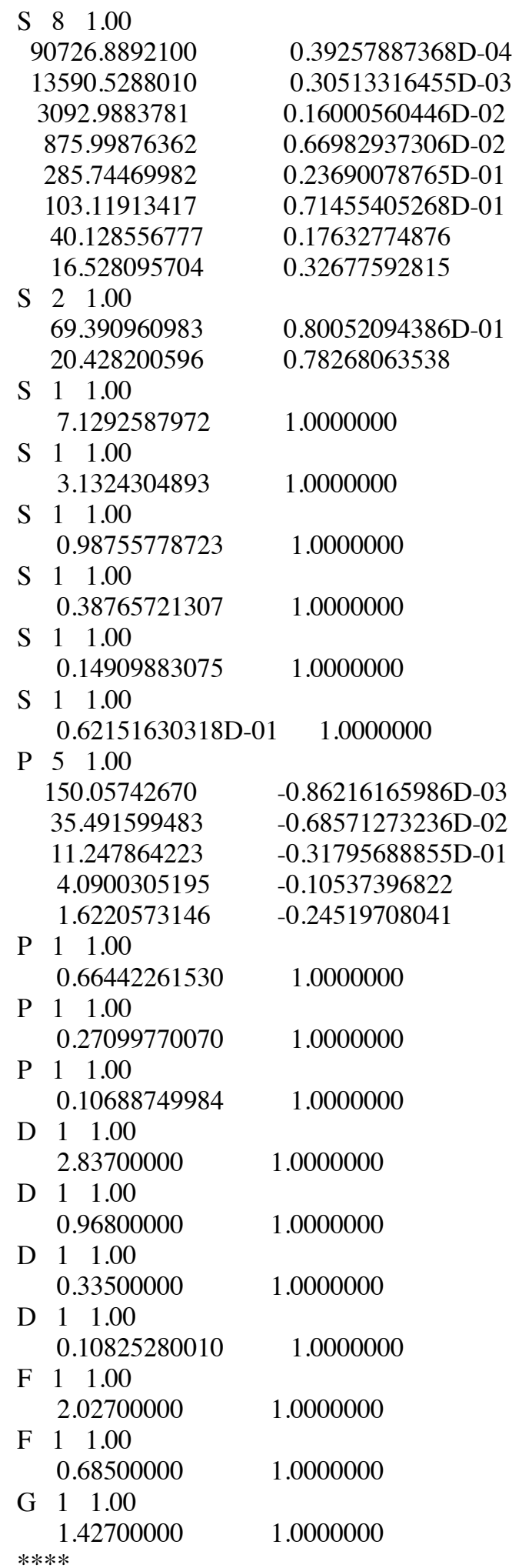

Def2-QZVPPD for O atoms:

O 0

$\begin{array}{lll}\mathrm{S} & 8 & 1.00\end{array}$ 


\begin{tabular}{|c|c|}
\hline 116506.4690800 & 0.40383857939D-04 \\
\hline 17504.3497240 & $0.31255139004 \mathrm{D}-03$ \\
\hline 3993.4513230 & $0.16341473495 \mathrm{D}-02$ \\
\hline 1133.0063186 & $0.68283224757 \mathrm{D}-02$ \\
\hline 369.99569594 & $0.24124410221 \mathrm{D}-01$ \\
\hline 133.62074349 & $0.72730206154 \mathrm{D}-01$ \\
\hline 52.035643649 & 0.17934429892 \\
\hline 21.461939313 & 0.33059588895 \\
\hline $\begin{array}{lll}\text { S } & 2 & 1.00\end{array}$ & \\
\hline 89.835051252 & $0.96468652996 \mathrm{D}-01$ \\
\hline 26.428010844 & 0.94117481120 \\
\hline $\begin{array}{lll}S & 1 & 1.00\end{array}$ & \\
\hline 9.2822824649 & 1.0000000 \\
\hline $\begin{array}{lll}S & 1 & 1.00\end{array}$ & \\
\hline 4.0947728533 & 1.0000000 \\
\hline $\begin{array}{lll}S & 1 & 1.00\end{array}$ & \\
\hline 1.3255349078 & 1.0000000 \\
\hline $\begin{array}{lll}S & 1 & 1.00\end{array}$ & \\
\hline 0.51877230787 & 1.0000000 \\
\hline $\begin{array}{lll}S & 1 & 1.00\end{array}$ & \\
\hline 0.19772676454 & 1.0000000 \\
\hline $\begin{array}{llll}S & 1 & 1.00\end{array}$ & \\
\hline $0.69638535104 \mathrm{D}-01$ & 1.0000000 \\
\hline $\begin{array}{lll}P & 5 & 1.00\end{array}$ & \\
\hline 191.15255810 & $0.25115697705 \mathrm{D}-02$ \\
\hline 45.233356739 & $0.20039240864 \mathrm{D}-01$ \\
\hline 14.353465922 & $0.93609064762 \mathrm{D}-01$ \\
\hline 5.2422371832 & 0.30618127124 \\
\hline 2.0792418599 & 0.67810501439 \\
\hline $\begin{array}{lll}\mathrm{P} & 1 & 1.00\end{array}$ & \\
\hline 0.84282371424 & 1.0000000 \\
\hline $\mathrm{P} \quad 1 \quad 1.00$ & \\
\hline 0.33617694891 & 1.0000000 \\
\hline $\begin{array}{lll}\mathrm{P} & 1 & 1.00\end{array}$ & \\
\hline 0.12863997974 & 1.0000000 \\
\hline $\begin{array}{lll}P & 1 & 1.00\end{array}$ & \\
\hline $0.43598162776 \mathrm{D}-01$ & 1.0000000 \\
\hline D $\quad 111.00$ & \\
\hline 3.77500000 & 1.0000000 \\
\hline D $\quad 1 \quad 1.00$ & \\
\hline 1.30000000 & 1.0000000 \\
\hline D 11.00 & \\
\hline 0.44400000 & 1.0000000 \\
\hline D $\quad 1 \quad 1.00$ & \\
\hline 0.12546378695 & 1.0000000 \\
\hline $\mathrm{F} \quad 1 \quad 1.00$ & \\
\hline 2.66600000 & 1.0000000 \\
\hline $\begin{array}{lll}\mathrm{F} & 1 & 1.00\end{array}$ & \\
\hline 0.85900000 & 1.0000000 \\
\hline G $\quad 1 \quad 1.00$ & \\
\hline 1.84600000 & 1.0000000 \\
\hline
\end{tabular}

Def2-QZVPPD for F atoms:

F $\quad 0$

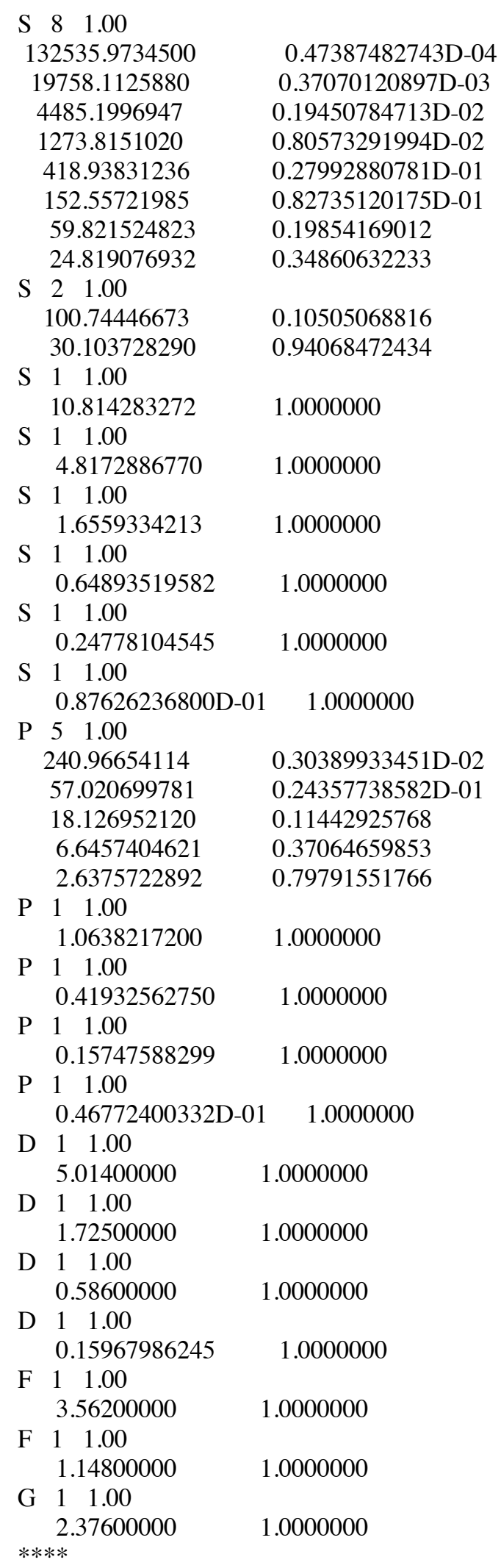

Def2-QZVPPD for P atoms: 


\begin{tabular}{|c|c|}
\hline 0 & \\
\hline S $\quad 10 \quad 1.00$ & \\
\hline 1090561.7138000 & $0.12142449664 \mathrm{D}-04$ \\
\hline 163316.3946100 & $0.94395292614 \mathrm{D}-04$ \\
\hline 37166.6074510 & $0.49622262177 \mathrm{D}-03$ \\
\hline 10526.8809450 & $0.20900040747 \mathrm{D}-02$ \\
\hline 3433.9976028 & $0.75489230025 \mathrm{D}-02$ \\
\hline 1239.5360480 & $0.24010423937 \mathrm{D}-01$ \\
\hline 483.27456199 & $0.67231473697 \mathrm{D}-01$ \\
\hline 200.16911586 & 0.15978669881 \\
\hline 86.960394829 & 0.29735906782 \\
\hline 39.211283369 & 0.36187171850 \\
\hline S $3 \quad 1.00$ & \\
\hline 336.75883662 & $0.19154721050 \mathrm{D}-01$ \\
\hline 103.72179793 & 0.17134079100 \\
\hline 39.771861240 & 0.63689655985 \\
\hline $\begin{array}{lll}S & 1 & 1.00\end{array}$ & \\
\hline 17.888612952 & 1.0000000 \\
\hline S 111.00 & \\
\hline 6.9644556879 & 1.0000000 \\
\hline S 111.00 & \\
\hline 3.2198092087 & 1.0000000 \\
\hline S 111.00 & \\
\hline 1.4669943979 & 1.0000000 \\
\hline S $\quad 1 \quad 1.00$ & \\
\hline 0.47765437532 & 1.0000000 \\
\hline S $\quad 1 \quad 1.00$ & \\
\hline 0.21637789241 & 1.0000000 \\
\hline $\begin{array}{lll}S & 1 & 1.00\end{array}$ & \\
\hline $0.90235894336 \mathrm{D}-01$ & 1.0000000 \\
\hline $\begin{array}{lll}S & 1 & 1.00\end{array}$ & \\
\hline $0.40836372978 \mathrm{D}-01$ & 1.0000000 \\
\hline $\mathrm{P} \quad 8 \quad 1.00$ & \\
\hline 2019.6711374 & $0.21359172406 \mathrm{D}-03$ \\
\hline 478.60125090 & $0.18568771399 \mathrm{D}-02$ \\
\hline 155.14942504 & $0.10070690115 \mathrm{D}-01$ \\
\hline 58.816356575 & $0.39605153679 \mathrm{D}-01$ \\
\hline 24.544512785 & 0.11736067844 \\
\hline 10.883571061 & 0.24950540642 \\
\hline 4.9624791285 & 0.36421287984 \\
\hline 2.3002912343 & 0.31764127123 \\
\hline $\mathrm{P} \quad 2 \quad 1.00$ & \\
\hline 59.371345016 & 0.39432918004 \\
\hline 3.0694590986 & -6.3522960431 \\
\hline $\mathrm{P} \quad 1 \quad 1.00$ & \\
\hline 1.0634401739 & 1.0000000 \\
\hline $\mathrm{P} \quad 1 \quad 1.00$ & \\
\hline 0.45022152161 & 1.0000000 \\
\hline $\mathrm{P} \quad 1 \quad 1.00$ & \\
\hline 0.18267271344 & 1.0000000 \\
\hline $\mathrm{P} \quad 1 \quad 1.00$ & \\
\hline $0.71610333771 \mathrm{D}-01$ & 1.0000000 \\
\hline D $\quad 1 \quad 1.00$ & \\
\hline 3.34300000 & 1.0000000 \\
\hline D $\quad 1 \quad 1.00$ & \\
\hline 0.807000000 & 1.0000000 \\
\hline
\end{tabular}

\begin{tabular}{|c|c|c|}
\hline D & 11.00 & \\
\hline & 0.365000000 & 1.0000000 \\
\hline & 11.00 & \\
\hline & 0.154000000 & 1.0000000 \\
\hline & 11.00 & \\
\hline & $0.59687731846 \mathrm{D}-0$ & 1.0000000 \\
\hline & 11.00 & \\
\hline & 0.703000000 & 1.0000000 \\
\hline & $1 \quad 1.00$ & \\
\hline & 0.280000000 & 1.0000000 \\
\hline & 11.00 & \\
\hline & 0.597000000 & 1.0000000 \\
\hline
\end{tabular}

Def2-QZVPPD for $\mathrm{S}$ atoms:

\begin{tabular}{|c|c|}
\hline \multicolumn{2}{|l|}{$\mathrm{S} \quad 0$} \\
\hline $\begin{array}{lll}S & 10 & 1.00\end{array}$ & \\
\hline 1273410.9023000 & $0.11767088246 \mathrm{D}-04$ \\
\hline 190697.8300700 & $0.91478610166 \mathrm{D}-04$ \\
\hline 43397.8853300 & $0.48090078640 \mathrm{D}-03$ \\
\hline 12291.8096770 & $0.20257193592 \mathrm{D}-02$ \\
\hline 4009.7420824 & $0.73190096406 \mathrm{D}-02$ \\
\hline 1447.3531030 & $0.23300499900 \mathrm{D}-01$ \\
\hline 564.30102913 & $0.65386213610 \mathrm{D}-01$ \\
\hline 233.74506243 & 0.15614449910 \\
\hline 101.56402814 & 0.29318563787 \\
\hline 45.805907187 & 0.36287914289 \\
\hline S $3 \quad 1.00$ & \\
\hline 394.27281503 & $0.18753305081 \mathrm{D}-01$ \\
\hline 121.72249591 & 0.16870726663 \\
\hline 46.754125963 & 0.63806830653 \\
\hline S 111.00 & \\
\hline 20.923008254 & 1.0000000 \\
\hline S 111.00 & \\
\hline 8.2685567800 & 1.0000000 \\
\hline S 111.00 & \\
\hline 3.8629345671 & 1.0000000 \\
\hline $\begin{array}{lll}S & 1 & 1.00\end{array}$ & \\
\hline 1.7794684781 & 1.0000000 \\
\hline $\begin{array}{lll}S & 1 & 1.00\end{array}$ & \\
\hline 0.61064260103 & 1.0000000 \\
\hline S $\quad 1 \quad 1.00$ & \\
\hline 0.27412269445 & 1.0000000 \\
\hline S $\quad 1 \quad 1.00$ & \\
\hline 0.11325939107 & 1.0000000 \\
\hline $\begin{array}{lll}S & 1 & 1.00\end{array}$ & \\
\hline 0.44392624881D-01 & 1.0000000 \\
\hline $\begin{array}{lll}\mathrm{P} & 8 & 1.00\end{array}$ & \\
\hline 2189.8930459 & $0.23912552864 \mathrm{D}-03$ \\
\hline 518.94596592 & $0.20772032158 \mathrm{D}-02$ \\
\hline 168.19560151 & $0.11242420571 \mathrm{D}-01$ \\
\hline 63.745282788 & 0.44069933941D-01 \\
\hline 26.597033077 & 0.12918778608 \\
\hline 11.774251449 & 0.26910820167 \\
\hline 5.3534379024 & 0.37855928620 \\
\hline
\end{tabular}




\begin{tabular}{|c|c|c|}
\hline & 2.4701911802 & 0.29692134655 \\
\hline$P$ & 21.00 & \\
\hline & 82.120288349 & $-0.39420318847 \mathrm{D}-01$ \\
\hline & 4.9523532869 & 0.64048403090 \\
\hline $\mathrm{P}$ & 11.00 & \\
\hline & 1.0828262029 & 1.0000000 \\
\hline $\mathrm{P}$ & 11.00 & \\
\hline & 0.49271277356 & 1.0000000 \\
\hline$P$ & 11.00 & \\
\hline & 0.20483450942 & 1.0000000 \\
\hline $\mathrm{P}$ & 11.00 & \\
\hline & $0.80743615716 \mathrm{D}-0$ & 1.0000000 \\
\hline $\mathrm{P}$ & 11.00 & \\
\hline & $0.25661157833 \mathrm{D}-0$ & 1.0000000 \\
\hline D & 11.00 & \\
\hline & 4.15900000 & 1.0000000 \\
\hline $\mathrm{D}$ & 11.00 & \\
\hline & 1.01900000 & 1.0000000 \\
\hline D & 11.00 & \\
\hline & 0.464000000 & 1.0000000 \\
\hline $\mathrm{D}$ & 11.00 & \\
\hline & 0.194000000 & 1.0000000 \\
\hline $\mathrm{D}$ & 11.00 & \\
\hline & $0.67889829366 \mathrm{D}-0$ & 1.0000000 \\
\hline $\mathrm{F}$ & 11.00 & \\
\hline & 0.869000000 & 1.0000000 \\
\hline $\mathrm{F}$ & 11.00 & \\
\hline & 0.335000000 & 1.0000000 \\
\hline G & 11.00 & \\
\hline & $\underset{* *}{0.683000000}$ & 1.0000000 \\
\hline
\end{tabular}

Def2-QZVPPD for $\mathrm{Cl}$ atoms:

\begin{tabular}{|c|c|}
\hline $\mathrm{Cl}$ & \\
\hline $\begin{array}{lll}S & 10 & 1.00\end{array}$ & \\
\hline 1467459.0095000 & 0.11478257194D-04 \\
\hline 219756.1643300 & 0.89234299775D-04 \\
\hline 50010.7703010 & $0.46911086186 \mathrm{D}-03$ \\
\hline 14164.8239180 & $0.19762446133 \mathrm{D}-02$ \\
\hline 4620.7465525 & 0.71419937783D-02 \\
\hline 1667.8991635 & $0.22753219445 \mathrm{D}-01$ \\
\hline 650.29199265 & $0.63959782953 \mathrm{D}-01$ \\
\hline 269.38037376 & 0.15331059238 \\
\hline 117.06752106 & 0.28986952417 \\
\hline 52.811766843 & 0.36348071452 \\
\hline S $3 \quad 1.00$ & \\
\hline 461.42769988 & $0.18019457578 \mathrm{D}-01$ \\
\hline 142.12665355 & 0.16489442314 \\
\hline 54.437838768 & 0.63891587584 \\
\hline $\begin{array}{lll}S & 1 & 1.00\end{array}$ & \\
\hline 24.160770219 & 1.0000000 \\
\hline $\begin{array}{lll}S & 1 & 1.00\end{array}$ & \\
\hline 9.7083540306 & 1.0000000 \\
\hline $\begin{array}{lll}S & 1 & 1.00\end{array}$ & \\
\hline 4.5640696733 & 1.0000000 \\
\hline
\end{tabular}

S 11.00

$2.1194744832 \quad 1.0000000$

S 11.00

$0.75722365394 \quad 1.0000000$

S 11.00

$0.33747224597 \quad 1.0000000$

$\begin{array}{lll}\text { S } & 1 & 1.00\end{array}$

$0.13860775149 \quad 1.0000000$

S 11.00

$0.54234565513 \mathrm{D}-01 \quad 1.0000000$

P $8 \quad 1.00$

$2501.9457890 \quad 0.24242618410 \mathrm{D}-03$

$592.88059285 \quad 0.21079961749 \mathrm{D}-02$

$192.18089186 \quad 0.11432693869 \mathrm{D}-01$

$72.875710488 \quad 0.44956698060 \mathrm{D}-01$

$30.436358370 \quad 0.13197476145$

$13.490178902 \quad 0.27493639225$

$6.1478071413 \quad 0.38347236372$

$2.8450944820 \quad 0.28871943885$

P 21.00

$105.39397936 \quad-0.34311760144 \mathrm{D}-01$

$6.7369738513 \quad 0.64060818902$

P 11.00

$1.2421095772 \quad 1.0000000$

P 11.00

$0.55669714254 \quad 1.0000000$

$\begin{array}{lll}\mathrm{P} & 1 & 1.00\end{array}$

$0.23387801464 \quad 1.0000000$

P 11.00

$0.93164490890 \mathrm{D}-01 \quad 1.0000000$

P 11.00 $0.27256146018 \mathrm{D}-01 \quad 1.0000000$

D 11.00 $5.19100000 \quad 1.0000000$

D 11.00 $1.27600000 \quad 1.0000000$

D 11.00 $0.583000000 \quad 1.0000000$

D 11.00 $0.243000000 \quad 1.0000000$

D 11.00 $0.83456538402 \mathrm{D}-01 \quad 1.0000000$

F $1 \quad 1.00$ $1.08900000 \quad 1.0000000$

F 11.00 $0.423000000 \quad 1.0000000$

G 11.00 $0.827000000 \quad 1.0000000$ $* * *$ 
We calculated the relative $G$ energies obtained with and without diffuse functions in the singlepoint energy corrections using a variety of structures from different reaction steps (Table S8). The $G$ values obtained with both basis sets are nearly equal (MAE $=0.04 \pm 0.04 \mathrm{kcal} / \mathrm{mol}$ over 20 structures) and the use of diffuse functions do not change any of the conclusions discussed in this study.

Table S8. Comparison of relative $G$ obtained using (i) $\omega B 97 X-D / D e f 2-Q Z V P P / / \omega B 97 X-$

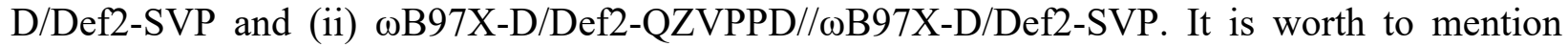
that the $G$ values represented are the energy of individual conformers (not Boltzmann averages). $\mathrm{MAE}=$ mean absolute error. $\mathrm{SD}=$ standard deviation. Product-3Ph $=\mathbf{3}$. Product $-2 \mathrm{Ph}=4$. Phosphonium_initial-2Ph $=\mathbf{6}$.

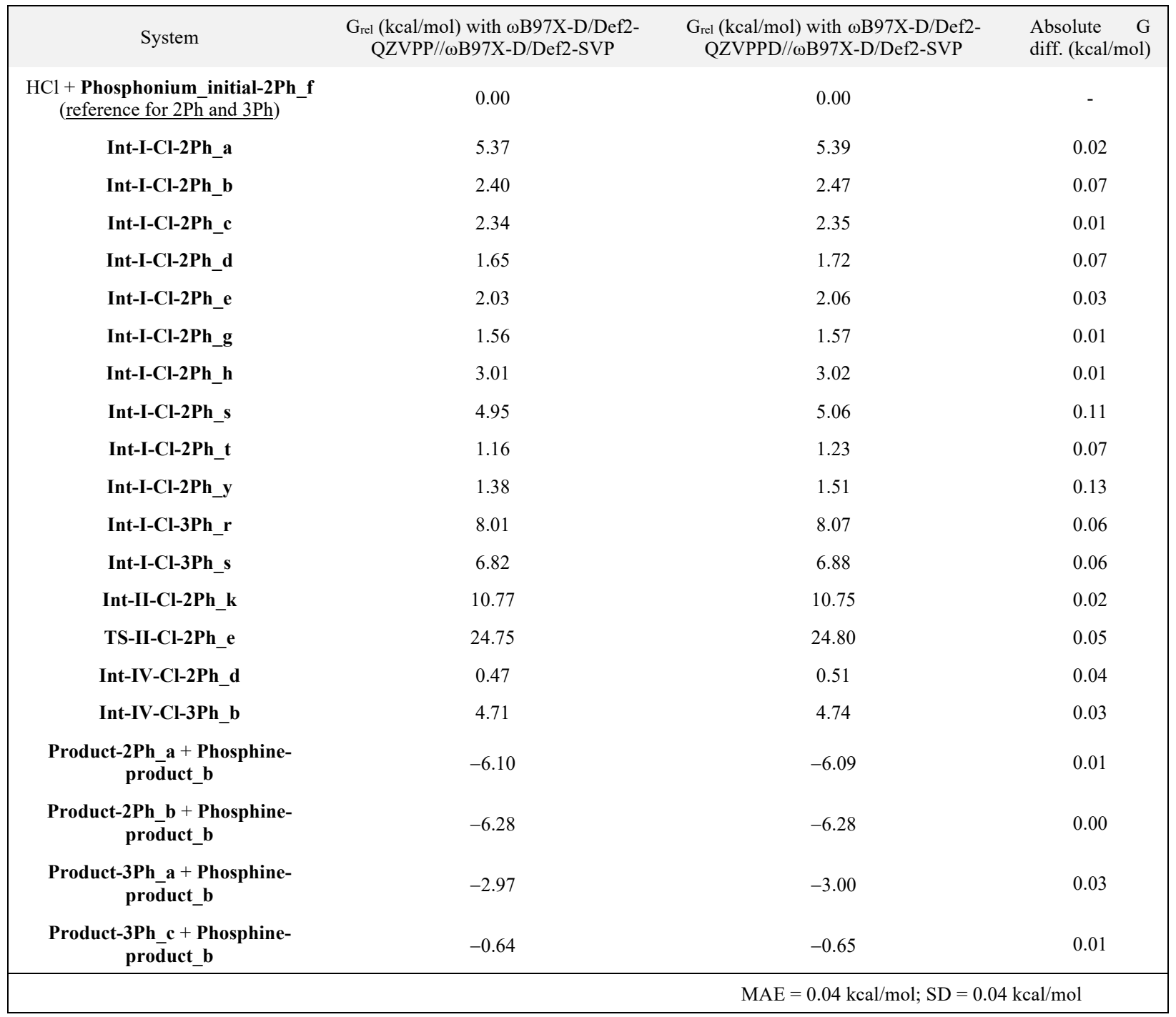


The computation times of the two types of single-point corrections were measured. The results strongly suggested that using Def2-QZVPPD requires considerably longer times than Def2QZVPP while leading to very similar results (Table S9).

Table S9. Comparison of job CPU times required to calculate reaction intermediates and transition states for (i) $\omega$ B97X-D/Def2-QZVPP// $\omega$ B97X-D/Def2-SVP and (ii) $\omega$ B97X-D/Def2QZVPPD// $\omega$ B97X-D/Def2-SVP. MAE = mean absolute error. SD = standard deviation.

\begin{tabular}{|c|c|c|}
\hline System & 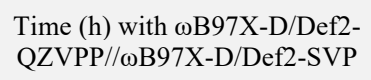 & $\begin{array}{l}\text { Time (h) with } \omega \text { B97X-D/Def2- } \\
\text { QZVPPD// } \omega \text { B } 97 X-D / \text { Def2-SVP }\end{array}$ \\
\hline Int-I-Cl-2Ph_a & 141 & 609 \\
\hline Int-I-Cl-2Ph_b & 175 & 696 \\
\hline Int-I-Cl-2Ph_c & 172 & 697 \\
\hline Int-I-Cl-2Ph_d & 178 & 656 \\
\hline Int-I-Cl-2Ph_e & 173 & 613 \\
\hline Int-I-Cl-2Ph_g & 173 & 594 \\
\hline Int-I-Cl-2Ph_h & 175 & 659 \\
\hline Int-I-Cl-2Ph_s & 176 & 500 \\
\hline Int-I-Cl-2Ph_t & 177 & 500 \\
\hline Int-I-Cl-2Ph_y & 176 & 769 \\
\hline Int-I-Cl-3Ph_r & 198 & 650 \\
\hline Int-I-Cl-3Ph_s & 198 & 429 \\
\hline Int-II-Cl-2Ph_k & 168 & 433 \\
\hline TS-II-Cl-2Ph_e & 185 & 524 \\
\hline Int-IV-Cl-2Ph_d & 174 & 445 \\
\hline Int-IV-Cl-3Ph_b & 181 & 473 \\
\hline & $\mathrm{MAE}=176 \mathrm{~h} ; \mathrm{SD}=13 \mathrm{~h}$ & $\mathrm{MAE}=578 \mathrm{~h} ; \mathrm{SD}=107 \mathrm{~h}$ \\
\hline
\end{tabular}

Calculation of the overall reaction activation energy with different DFT methods

Table S10. Calculation of the overall reaction activation energy ( $\Delta \mathrm{G}^{\ddagger}$, from most to least favorable energies) using different levels of theory and solvents. For this study, the two most stable conformers found at the $\omega$ B97X-D/Def2-QZVPP// $\omega$ B97X-D/Def2-SVP were used.

\begin{tabular}{|c|c|c|c|}
\hline Reaction step & $\begin{array}{c}\mathrm{G}_{\mathrm{rel}}(\mathrm{kcal} / \mathrm{mol}) \omega \mathrm{B} 97 \mathrm{X}-\mathrm{D} / \text { Def2- } \\
\mathrm{QZVPP} / / \omega \mathrm{B} 97 \mathrm{X}-\mathrm{D} / \text { Def2-SVP } \\
\text { (dioxane) }\end{array}$ & $\begin{array}{c}\mathrm{G}_{\text {rel }}(\mathrm{kcal} / \mathrm{mol}) \omega \mathrm{B} 97 \mathrm{X}-\mathrm{D} / \mathrm{Def} 2- \\
\mathrm{QZVPP} / / \omega \mathrm{B} 97 \mathrm{X}-\mathrm{D} / \mathrm{Def} 2-\mathrm{SVP} \\
(\mathrm{AcOEt})\end{array}$ & $\begin{array}{c}\mathrm{G}_{\mathrm{rel}}(\mathrm{kcal} / \mathrm{mol}) \mathrm{M} 06-2 \mathrm{X}-\mathrm{D} 3 / \text { Def2- } \\
\mathrm{QZVPP} / / \omega \mathrm{\omega B} 97 \mathrm{X}-\mathrm{D} / \text { Def2-SVP } \\
\text { (dioxane) }\end{array}$ \\
\hline \multicolumn{4}{|l|}{ For 5} \\
\hline $\mathbf{5}+\mathrm{HCl}$ & 0.0 & 0.0 & 0.0 \\
\hline Int-I-Cl-3Ph & 0.4 & 1.0 & 2.3 \\
\hline Int-II-Cl-3Ph & 7.3 & 6.2 & 5.8 \\
\hline TS-I-Cl-3Ph & 16.5 & 19.3 & 14.7 \\
\hline TS-II-Cl-3Ph & 19.7 & 23.4 & 16.4 \\
\hline$\Delta \mathrm{G}^{\ddagger}(\mathrm{kcal} / \mathrm{mol})$ & 19.7 & 23.4 & 16.4 \\
\hline
\end{tabular}




$\begin{array}{|cccc|}\text { For } \mathbf{6} & 0.0 & 0.0 & 0.0 \\ \mathbf{6}+\mathrm{HCl} & -0.2 & 1.8 & 2.4 \\ \text { Int-I-Cl-2Ph } & 7.3 & 4.8 & 7.4 \\ \text { Int-II-Cl-2Ph } & 16.7 & 18.4 & 16.8 \\ \text { TS-I-Cl-2Ph } & 22.3 & 25.2 & 20.4 \\ \text { TS-II-Cl-2Ph } & 22.5 & 25.2 & 20.4 \\ \Delta \mathrm{G}^{\ddagger}(\mathrm{kcal} / \mathrm{mol}) & & \end{array}$

Thermochemical data and absolute energy values

Boltzmann weighted $\mathrm{G}\left(\mathrm{G}_{\mathrm{av}}\right)$ were calculated with the GoodVibes program (option --pes) as:

$$
G_{a v}=\sum_{i} G_{i} \times p_{i}
$$

where $G_{i}$ is the relative Gibbs free energy of the corresponding conformers of a certain reaction step and $\mathrm{p}_{\mathrm{i}}$ is the probability of each conformer calculated as:

$$
p_{i}=\frac{e^{\frac{-G_{i}}{R T}}}{\sum_{i}\left(e^{\frac{-G_{i}}{R T}}\right)}
$$

$G$ values, including the energies of all the conformers of each reaction step, are detailed in a separate file included in the ESI. Also, the commands and options used by GoodVibes are represented in the same file.

\section{Molecular coordinates}

A separated xyz file containing all the geometries considered in this study is provided along with this ESI. 


\section{References}

(1) Hilton, M. C.; Dolewski, R. D.; McNally, A. Selective Functionalization of Pyridines via Heterocyclic Phosphonium Salts. J. Am. Chem. Soc. 2016, 138 (42), 13806-13809.

(2) Dolewski, R. D.; Fricke, P. J.; McNally, A. Site-Selective Switching Strategies to Functionalize Polyazines. J. Am. Chem. Soc. 2018, 140 (25), 8020-8026.

(3) Yamanaka, H.; Araki, T.; Sakamoto, T. Site-Selectivity in the Reaction of 3-Substituted Pyridine 1-Oxides with Phosphoryl Chloride. Chem. Pharm. Bull. 1988, 36 (6), 2244-2247.

(4) Zhang, X.; Feng, X.; Zhou, C.; Yu, X.; Yamamoto, Y.; Bao, M. Transition-Metal-Free Decarboxylative Arylation of 2-Picolinic Acids with Arenes under Air Conditions. Org. Lett. 2018, 20 (22), 7095-7099.

(5) Gehringer, M.; Forster, M.; Pfaffenrot, E.; Bauer, S. M.; Laufer, S. A. Novel Hinge-Binding Motifs for Janus Kinase 3 Inhibitors: A Comprehensive Structure-Activity Relationship Study on Tofacitinib Bioisosteres. ChemMedChem 2014, 9 (11), 2516-2527.

(6) Fukui, K. The Path of Chemical Reactions - the IRC Approach. Acc. Chem. Res. 1981, 14, 363-368.

(7) Grimme, S. Supramolecular Binding Thermodynamics by Dispersion-corrected Density Functional Theory. Chem. Eur. J. 2012, 18, 9955-9964.

(8) Sure, R.; Grimme, S. Comprehensive Benchmark of Association (Free) Energies of Realistic Host-Guest Complexes. J. Chem. Theory Comput. 2015, 11, 3785-3801.

(9) Gaussian 16, Revision B.01, Frisch, M. J.; Trucks, G. W.; Schlegel, H. B.; Scuseria, G. E.; Robb, M. A.; Cheeseman, J. R.; Scalmani, G.; Barone, V.; Petersson, G. A.; Nakatsuji, H.; Li, X.; Caricato, M.; Marenich, A. V.; Bloino, J.; Janesko, B. G.; Gomperts, R.; Mennucci, B.; Hratchian, H. P.; Ortiz, J. V.; Izmaylov, A. F.; Sonnenberg, J. L.; Williams-Young, D.; Ding, F.; Lipparini, F.; Egidi, F.; Goings, J.; Peng, B.; Petrone, A.; Henderson, T.; Ranasinghe, D.; Zakrzewski, V. G.; Gao, J.; Rega, N.; Zheng, G.; Liang, W.; Hada, M.; Ehara, M.; Toyota, K.; Fukuda, R.; Hasegawa, J.; Ishida, M.; Nakajima, T.; Honda, Y.; Kitao, O.; Nakai, H.; Vreven, T.; Throssell, K.; Montgomery, Jr., J. A.; Peralta, J. E.; Ogliaro, F.; Bearpark, M. J.; Heyd, J. J.; Brothers, E. N.; Kudin, K. N.; Staroverov, V. N.; Keith, T. A.; Kobayashi, R.; Normand, J.; Raghavachari, K.; Rendell, A. P.; Burant, J. C.; Iyengar, S. S.; Tomasi, J.; Cossi, M.; Millam, J. M.; Klene, M.; Adamo, C.; Cammi, R.; Ochterski, J. W.; Martin, R. L.; Morokuma, K.; Farkas, O.; Foresman, J. B.; Fox, D. J. Gaussian, Inc., Wallingford CT, 2016.

(10) NBO, version 6.0, Glendening, E. D.; Badenhoop, J. K.; Reed, A. E.; Carpenter, J. E.; Bohmann, J. A.; Morales, C. M.; Landis, C. R.; Weinhold, F. Theoretical Chemistry Institute, University of Wisconsin, Madison WI, 2013.

(11) The PyMOL Molecular Graphics System, version 2.0.7, Schrödinger, LLC.

(12) https://gist.github.com/bobbypaton (accessed 13 April 2018) 
(13) (a) Galabov, B.; Ilieva, S.; Hadjieva, B.; Atanasov, Y.; Schaefer III, H. F. Predicting Reactivities of Organic Molecules. Theoretical and Experimental Studies on the Aminolysis of Phenyl Acetates. J. Phys. Chem. A 2008, 112, 6700-6707; (b) Galabov, B.; Ilieva, S.; Koleva, G.; Allen, W. D.; Schaefer III, H. F.; Schleyer, P. von R. Structure-Reactivity Relationships for Aromatic Molecules: Electrostatic Potentials at Nuclei and Electrophile Affinity Indices. Wiley Interdiscip. Rev. Comput. Mol. Sci. 2013, 3, 37-55; (c) Alegre-Requena, J. V.; Valero-Tena, A.; Sonsona, I. G.; Uriel, S.; Herrera, R. P. Simple Iodoalkyne-Based Organocatalysts for the Activation of Carbonyl Compounds. Org. Biomol. Chem. 2020, 18, 1594-1601.

(14) Pritchard, B. P.; Altarawy, D.; Didier, B.; Gibson, T. D.; Windus, T. L. A New Basis Set Exchange: An Open, Up-to-date Resource for the Molecular Sciences Community. J. Chem. Inf. Model. 2019, 59, 4814-4820.

(15) Pupo, G.; Ibba, F.; Ascough, D. M. H.; Vicini, A. C.; Ricci, P.; Christensen, K. E.; Pfeifer, L.; Morphy, J. R.; Brown, J. M.; Paton, R. S.; Gouverneur, V. Asymmetric Nucleophilic Fluorination under Hydrogen Bonding Phase-Transfer Catalysis. Science 2018, 360, 638-642. 
${ }^{1} \mathrm{H} \mathrm{NMR}\left(\mathrm{CDCl}_{3}, 400 \mathrm{MHz}\right)$<smiles>FC(F)(F)c1ccc(-c2ccccc2)cn1</smiles>

1.13 눈. 10.7 1.18 
${ }^{13} \mathrm{C} \mathrm{NMR}\left(\mathrm{CDCl}_{3}, 100 \mathrm{MHz}\right)$<smiles>FC(F)(F)c1ccc(P)cn1</smiles> 
${ }^{19} \mathrm{~F} \mathrm{NMR}\left(\mathrm{CDCl}_{3}, 365 \mathrm{MHz}\right)$<smiles>FC(F)(F)c1ccc(-c2ccccc2)cn1</smiles>

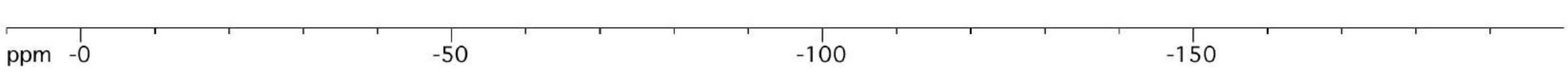


${ }^{31} \mathrm{P}$ NMR $\left(\mathrm{CDCl}_{3}, 162 \mathrm{MHz}\right)$<smiles>FC(F)(F)c1ccc(-c2ccccc2)cn1</smiles> 
${ }^{1} \mathrm{H} \mathrm{NMR}\left(\mathrm{CDCl}_{3}, 400 \mathrm{MHz}\right)$<smiles>FC(F)(F)c1ccc(P(c2ccccc2)c2ccc(C(F)(F)F)nc2)cn1</smiles>

$\mathrm{CDCl}_{3}$

$\begin{aligned} & 2.19 \text { 누 } \\ & 2.27\end{aligned}$

$\begin{array}{ll}4 & 4 \\ 2.27 & 2.17\end{array}$

\section{$\frac{1}{2}$}

9

$8 \quad 7$

1 6 
${ }^{13} \mathrm{C} \mathrm{NMR}\left(\mathrm{CDCl}_{3}, 100 \mathrm{MHz}\right)$<smiles>FC(F)(F)c1ccc(P(c2ccccc2)c2ccc(C(F)(F)F)nc2)cn1</smiles>

II 
${ }^{19} \mathrm{~F} \mathrm{NMR}\left(\mathrm{CDCl}_{3}, 365 \mathrm{MHz}\right)$

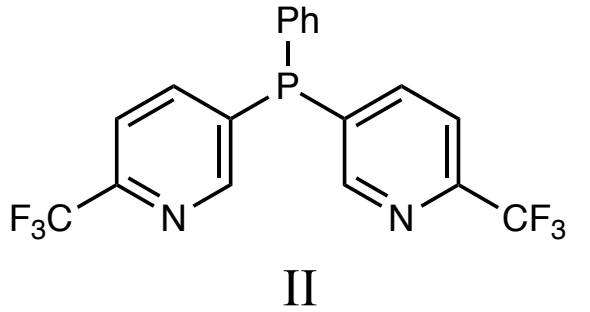

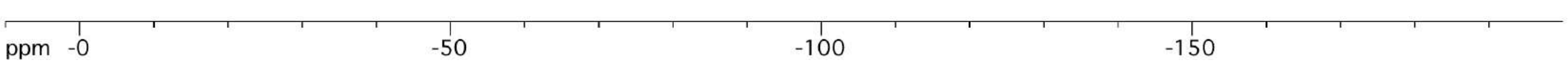


${ }^{31} \mathrm{P}$ NMR $\left(\mathrm{CDCl}_{3}, 162 \mathrm{MHz}\right)$

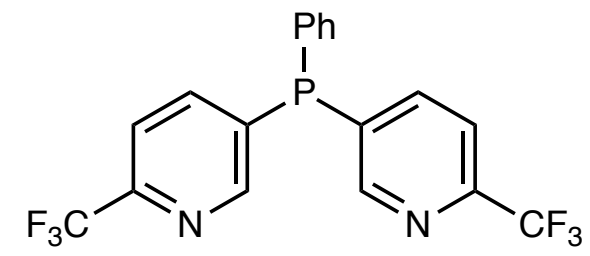

II 
${ }^{1} \mathrm{H} \mathrm{NMR}\left(\mathrm{CDCl}_{3}, 400 \mathrm{MHz}\right)$<smiles>Cn1nccc1-c1cccnc1</smiles>

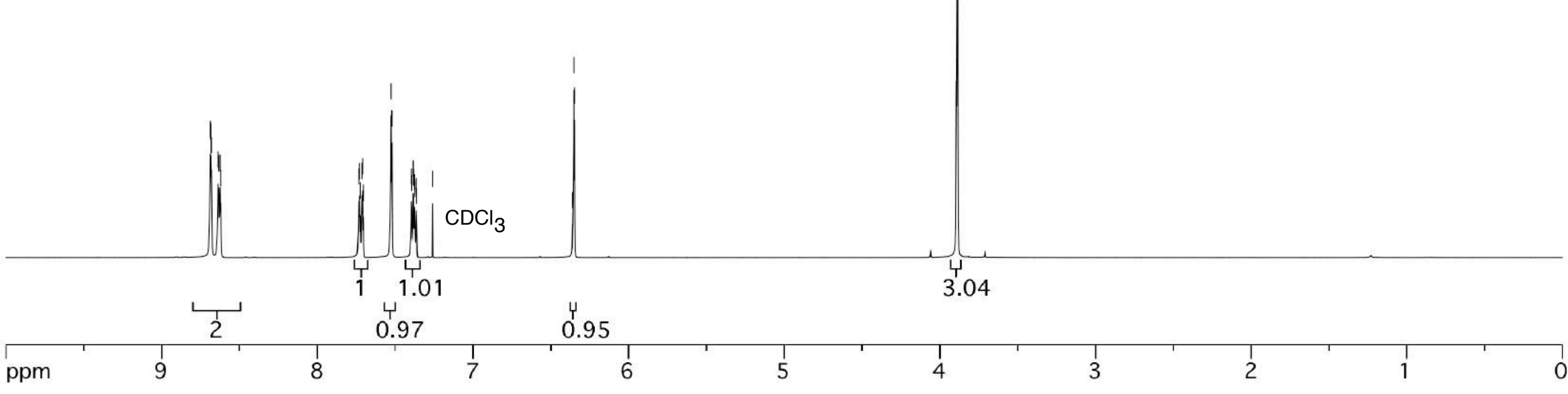




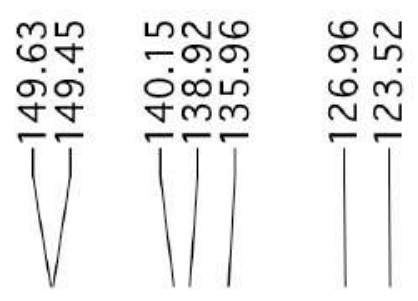

${ }^{13} \mathrm{C} \mathrm{NMR}\left(\mathrm{CDCl}_{3}, 100 \mathrm{MHz}\right)$<smiles>Cn1nccc1-c1cccnc1</smiles> 


\section{${ }^{1} \mathrm{H}$ NMR, $\left(\mathrm{CDCl}_{3}, 400 \mathrm{MHz}\right)$}

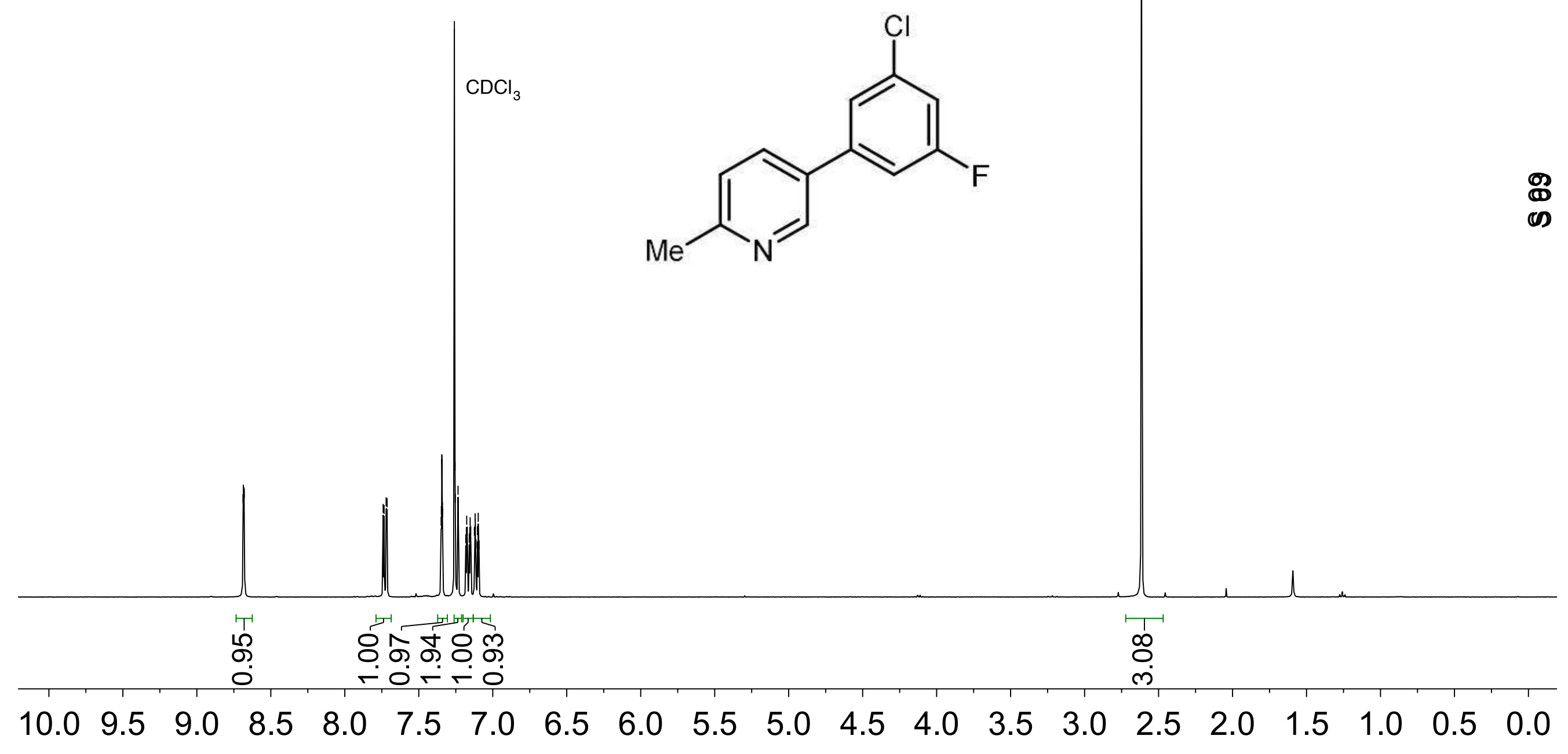


๙ิต

它它吕守守守守

${ }^{13} \mathrm{C} \mathrm{NMR},\left(\mathrm{CDCl}_{3}, 100 \mathrm{MHz}\right)$<smiles>Cc1ccc(-c2cc(F)cc(Cl)c2)cn1</smiles> 
${ }^{19} \mathrm{~F} \mathrm{NMR}\left(\mathrm{CDCl}_{3}, 365 \mathrm{MHz}\right)$<smiles>Cc1ccc(-c2cc(F)cc(Cl)c2)cn1</smiles>

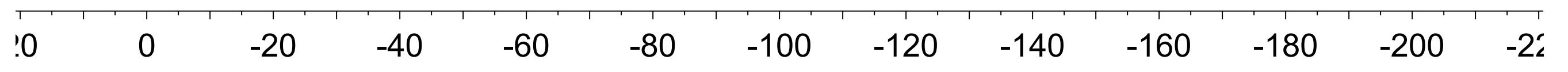


mN-OONO- -

$\infty_{\infty}^{\infty} \infty^{\circ} \infty \infty^{\circ} N$ NNNNNNNNNNNN

${ }^{1} \mathrm{H} \mathrm{NMR}\left(\mathrm{CDCl}_{3}, 400 \mathrm{MHz}\right)$<smiles>FS(F)(F)c1cccc(-c2ccccn2)c1</smiles>

7


${ }^{13} \mathrm{C} \mathrm{NMR}\left(\mathrm{CDCl}_{3}, 100 \mathrm{MHz}\right)$<smiles>FC(F)(F)c1cccc(-c2ccccn2)c1</smiles> 


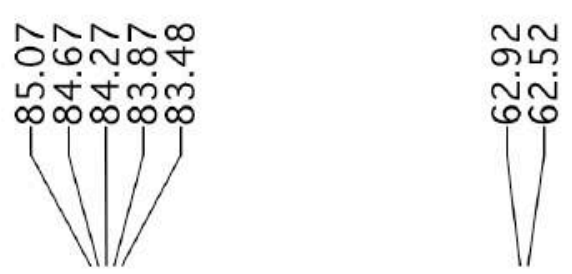

${ }^{19} \mathrm{~F} \mathrm{NMR}\left(\mathrm{CDCl}_{3}, 365 \mathrm{MHz}\right)$

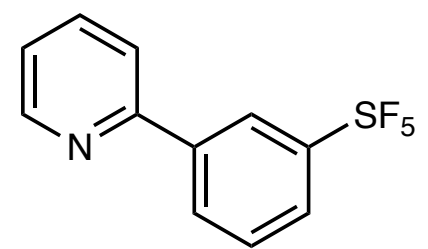

$\frac{8}{\text { is }}$ 


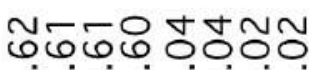

$\infty \infty^{\circ} \infty \infty^{\circ} \infty \infty^{\circ} \infty$

(1) (U)

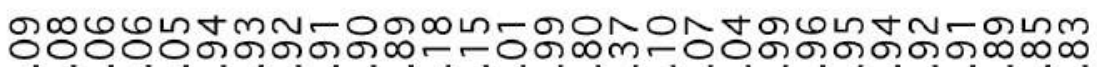

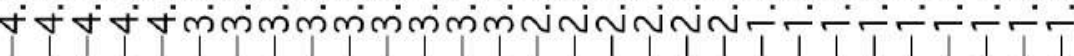

${ }^{1} \mathrm{H} \mathrm{NMR}\left(\mathrm{CDCl}_{3}, 400 \mathrm{MHz}\right)$<smiles>Cc1ncccc1C(=O)OCC1CN(C)CC[C@H]1c1ccc(F)cc1</smiles>

$\mathrm{CDCl}_{3}$
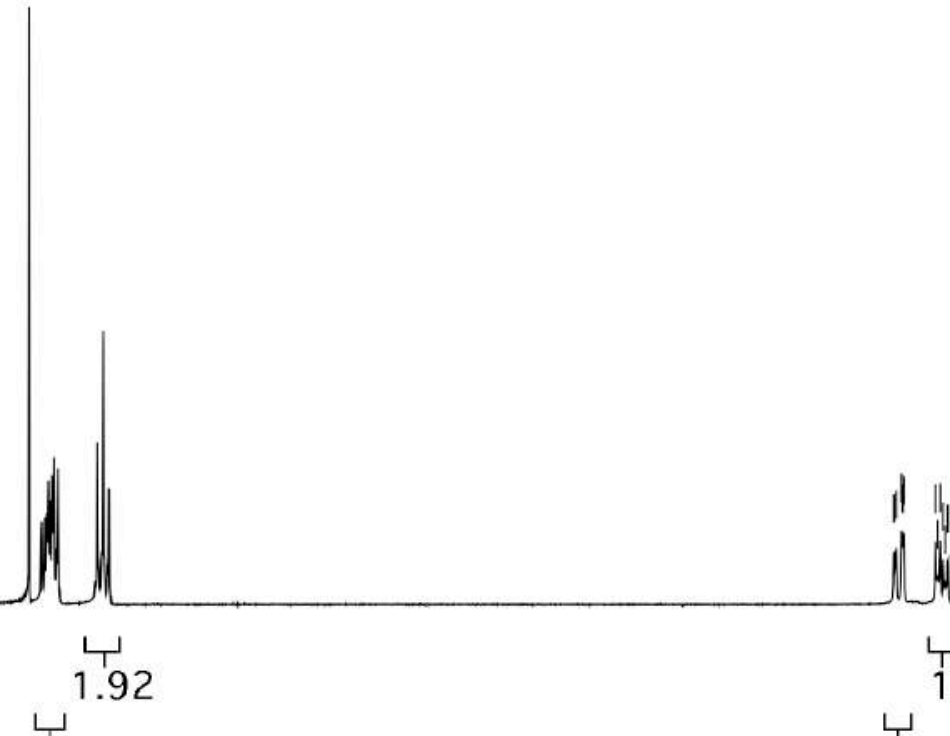

$\|$

|ll|l||l|

0.9525

$\lcm{0.95}$

2.98

누

14

4.58

8

6

5

4

3

2




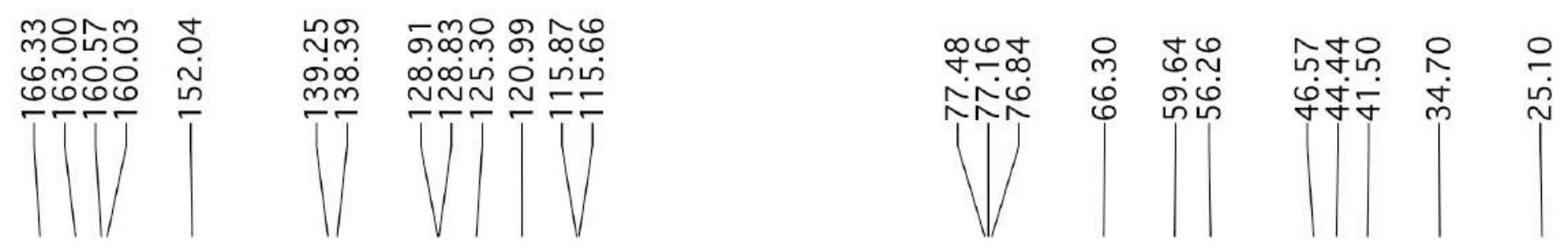

${ }^{13} \mathrm{C} \mathrm{NMR}\left(\mathrm{CDCl}_{3}, 100 \mathrm{MHz}\right)$<smiles>CN1CC[C@H](c2ccc(F)cc2)[C@H](COC(=O)c2cccnc2[N+](=O)[O-])C1</smiles> 
${ }^{19} \mathrm{~F}$ NMR $\left(\mathrm{CDCl}_{3}, 365 \mathrm{MHz}\right)$

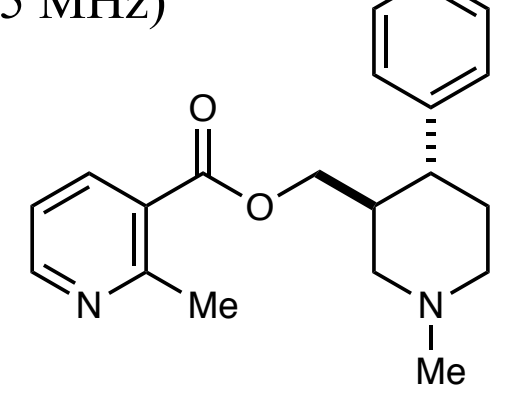




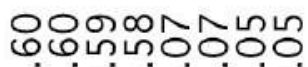
$\infty \infty^{\circ} \infty \infty^{\infty} \infty \infty$

$6000 \wedge \infty$ NNNG

$(1)$

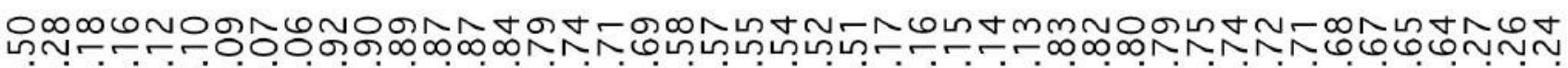

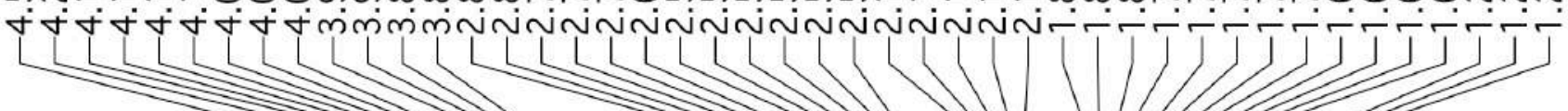

\section{${ }^{1} \mathrm{H} \mathrm{NMR}\left(\mathrm{CDCl}_{3}, 400 \mathrm{MHz}\right)$}<smiles>CCOC(=O)N1CC[C@@H](c2ccc(F)cc2)C(COC(=O)c2cccnc2C)C1</smiles>

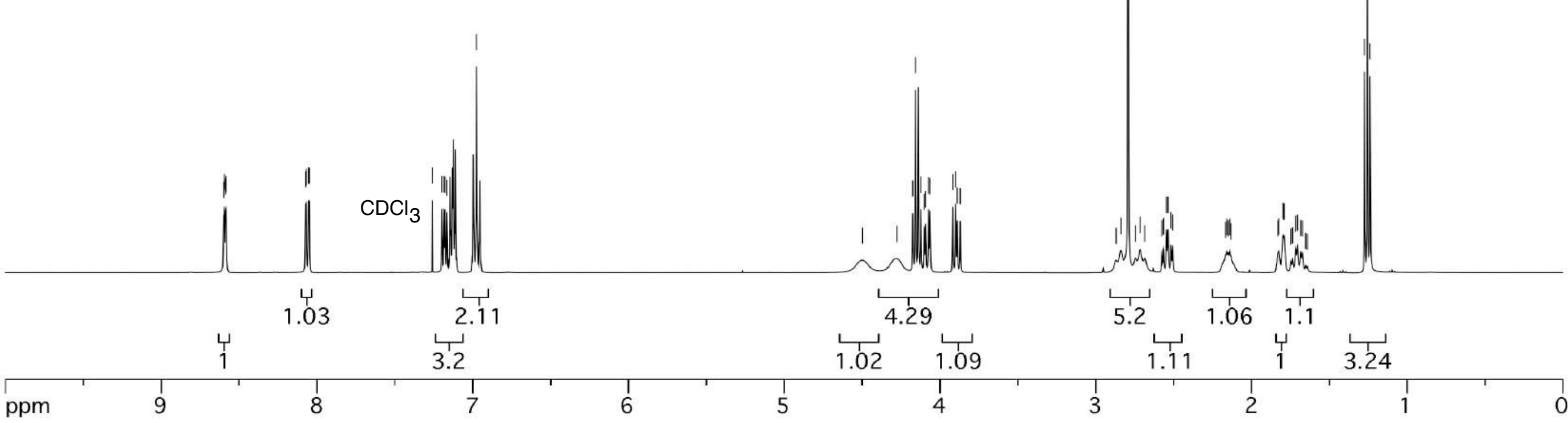




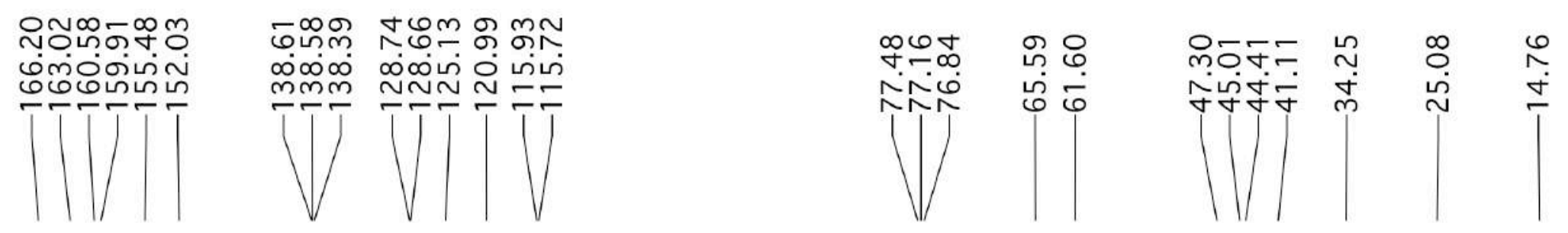

${ }^{13} \mathrm{C} \mathrm{NMR}\left(\mathrm{CDCl}_{3}, 100 \mathrm{MHz}\right)$<smiles>CCOC(=O)N1CC[C@@H](c2ccc(F)cc2)[C@H](COC(=O)c2cccnc2[N+](=O)[O-])C1</smiles>

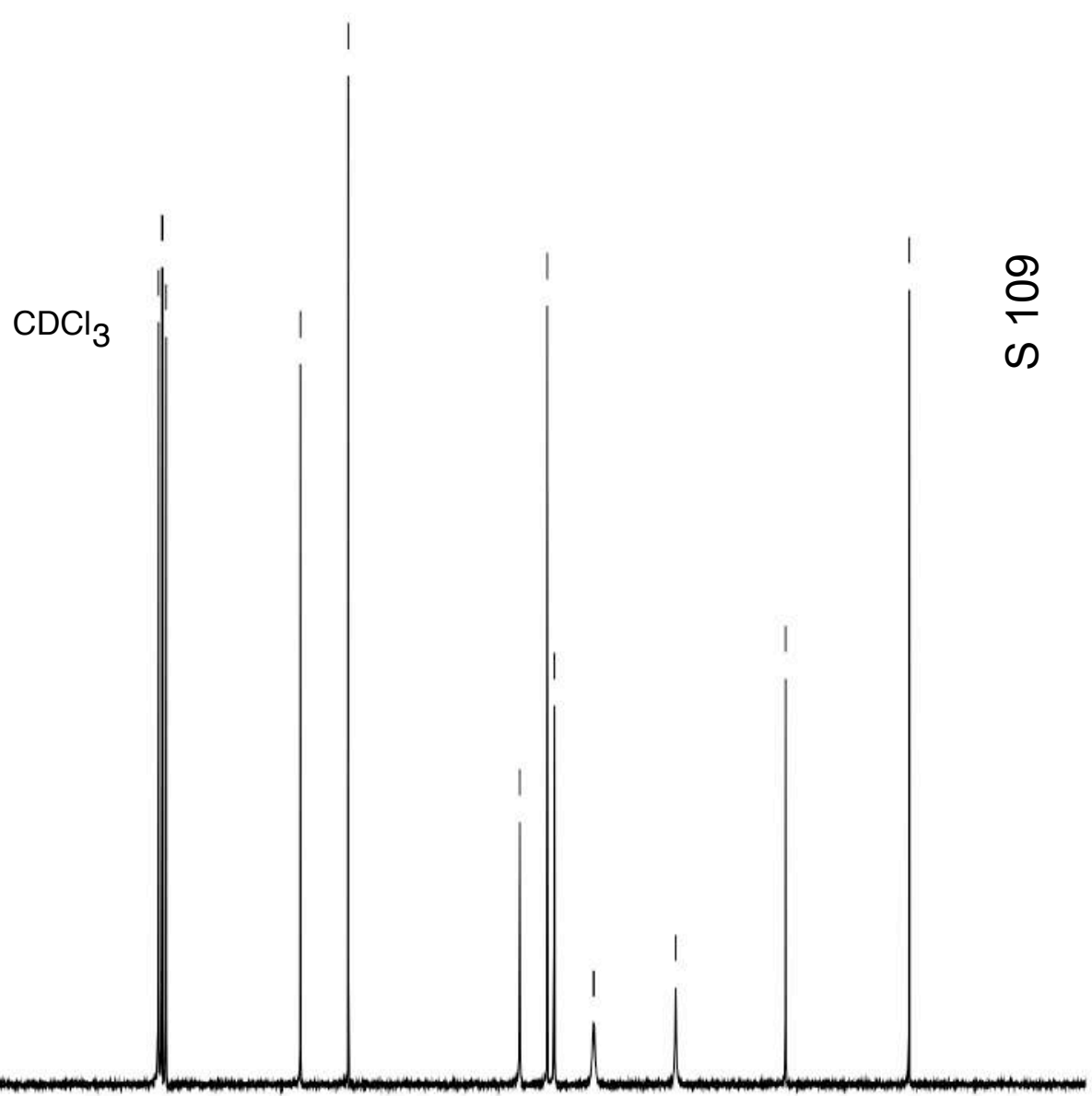


${ }^{13} \mathrm{C} \mathrm{NMR}\left(\mathrm{CDCl}_{3}, 100 \mathrm{MHz}\right)$<smiles>Cc1ccc(C(=O)OC([Al])c2cc(C(F)(F)F)cc(C(F)(F)F)c2)nc1</smiles> 
${ }^{1} \mathrm{H} \mathrm{NMR}\left(\mathrm{CDCl}_{3}, 400 \mathrm{MHz}\right)$<smiles>CCOC(=O)N1CC2CC(C1)c1cc3nccnc3cc12</smiles>

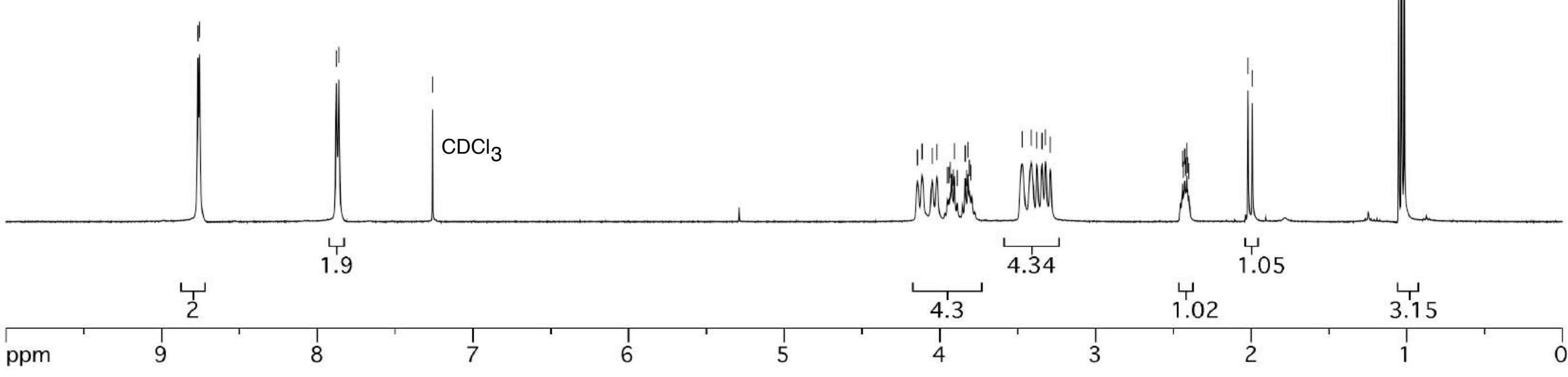




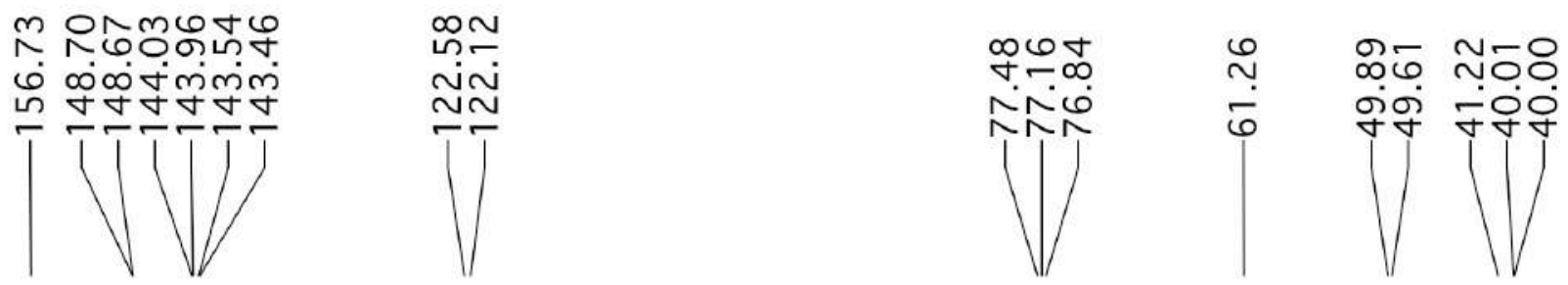

${ }^{13} \mathrm{C} \mathrm{NMR}\left(\mathrm{CDCl}_{3}, 100 \mathrm{MHz}\right)$<smiles>CCOC(=O)N1CC2C[C@@H](C1)c1cc3nccnc3cc12</smiles> 


\section{${ }^{1} \mathrm{H} \mathrm{NMR}\left(\mathrm{CDCl}_{3}, 400 \mathrm{MHz}\right)$}

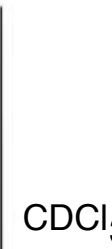

$\mathrm{F}_{3} \mathrm{C}$<smiles>Cc1ccc([Pb]c2ccccc2)cn1</smiles><smiles>O=S(=O)([O-])C(F)(F)F</smiles>

5 
${ }^{13} \mathrm{C} \mathrm{NMR}\left(\mathrm{CDCl}_{3}, 100 \mathrm{MHz}\right)$

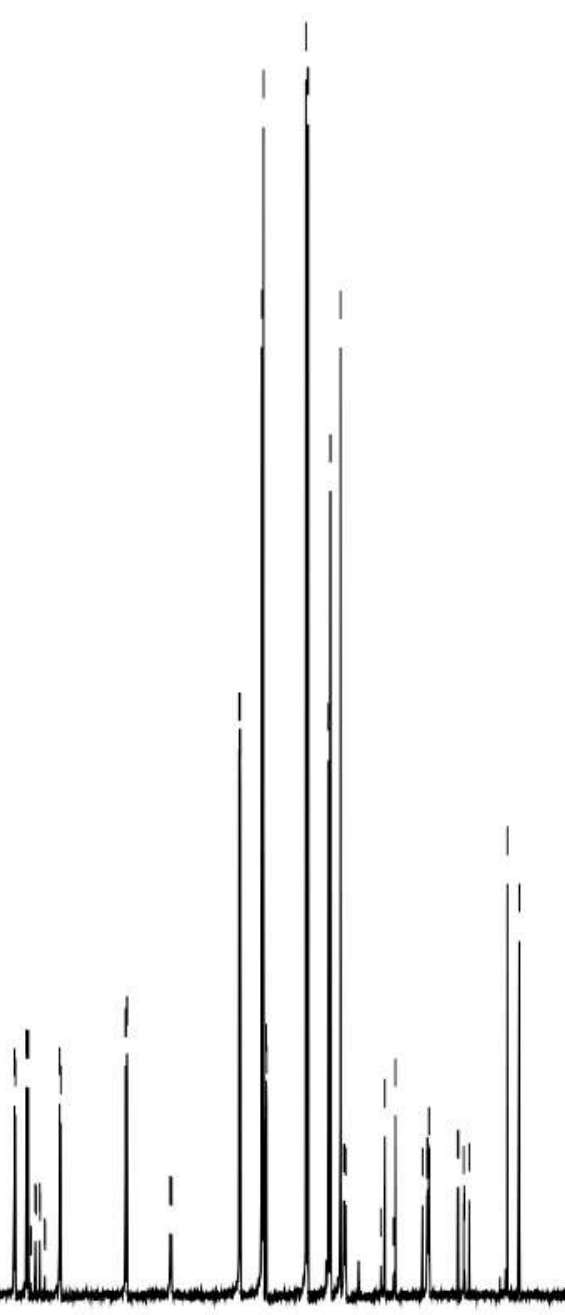


${ }^{19} \mathrm{~F} \mathrm{NMR}\left(\mathrm{CDCl}_{3}, 365 \mathrm{MHz}\right)$

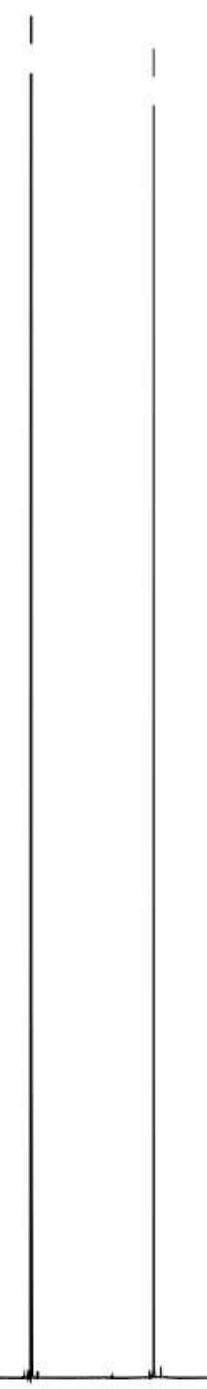

\begin{tabular}{|c|c|c|c|}
\hline \begin{tabular}{l|l} 
& 1 \\
$\mathrm{ppm}$ & -0
\end{tabular} & $\begin{array}{l}1 \\
-50\end{array}$ & $\begin{array}{c}1 \\
-100\end{array}$ & $\begin{array}{r}1 \\
-150\end{array}$ \\
\hline
\end{tabular}


${ }^{31} \mathrm{P} \mathrm{NMR}\left(\mathrm{CDCl}_{3}, 162 \mathrm{MHz}\right)$

5<smiles>FC(F)(F)c1ccc([PH2+]c2ccncc2-c2ccccc2)cn1</smiles>

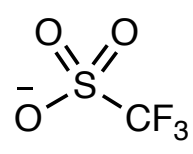


${ }^{1} \mathrm{H} \mathrm{NMR}\left(\mathrm{CDCl}_{3}, 400 \mathrm{MHz}\right)$

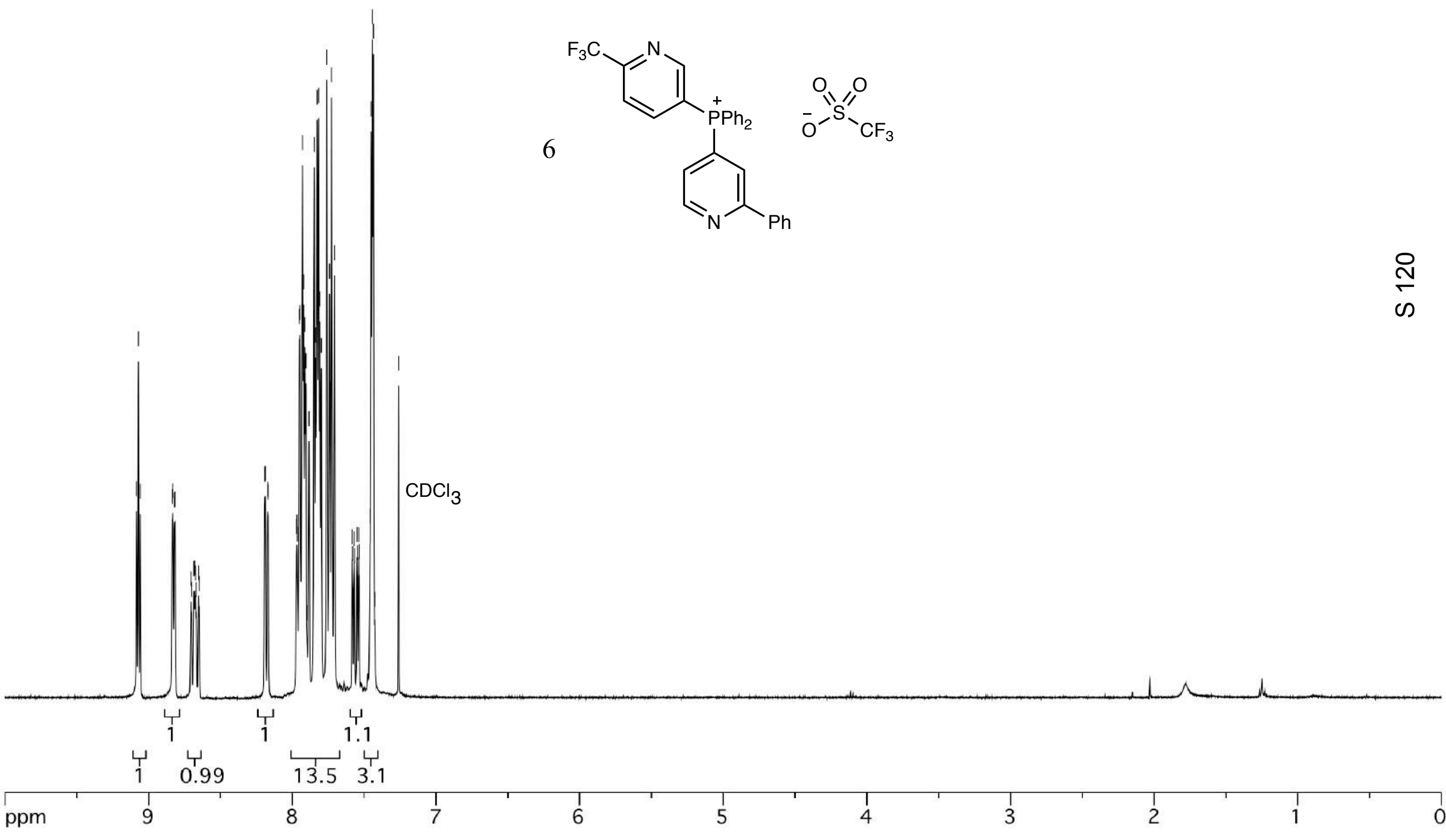


${ }^{13} \mathrm{C} \mathrm{NMR}\left(\mathrm{CDCl}_{3}, 100 \mathrm{MHz}\right)$

6<smiles>O=S(=O)([O-])C(F)(F)F</smiles>

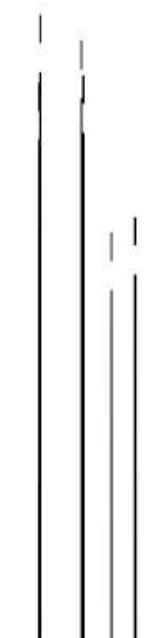


${ }^{19} \mathrm{~F}$ NMR $\left(\mathrm{CDCl}_{3}, 365 \mathrm{MHz}\right)$

$\frac{N}{\sim}$

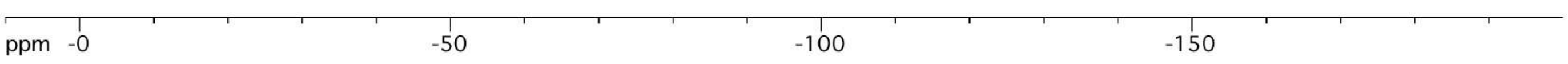


${ }^{31} \mathrm{P}$ NMR $\left(\mathrm{CDCl}_{3}, 162 \mathrm{MHz}\right)$

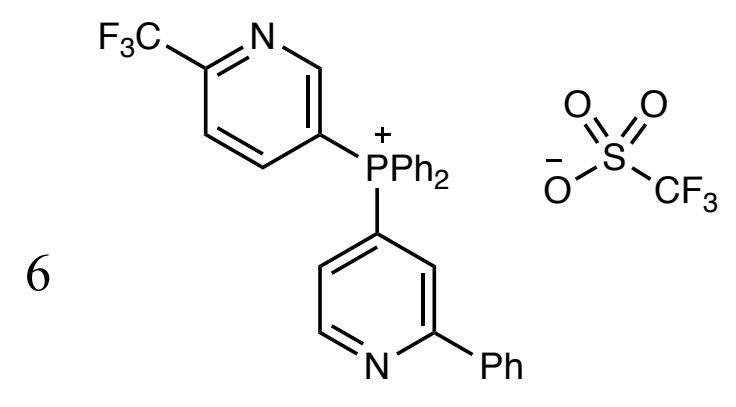


${ }^{13} \mathrm{C} \mathrm{NMR}\left(\mathrm{CDCl}_{3}, 100 \mathrm{MHz}\right)$

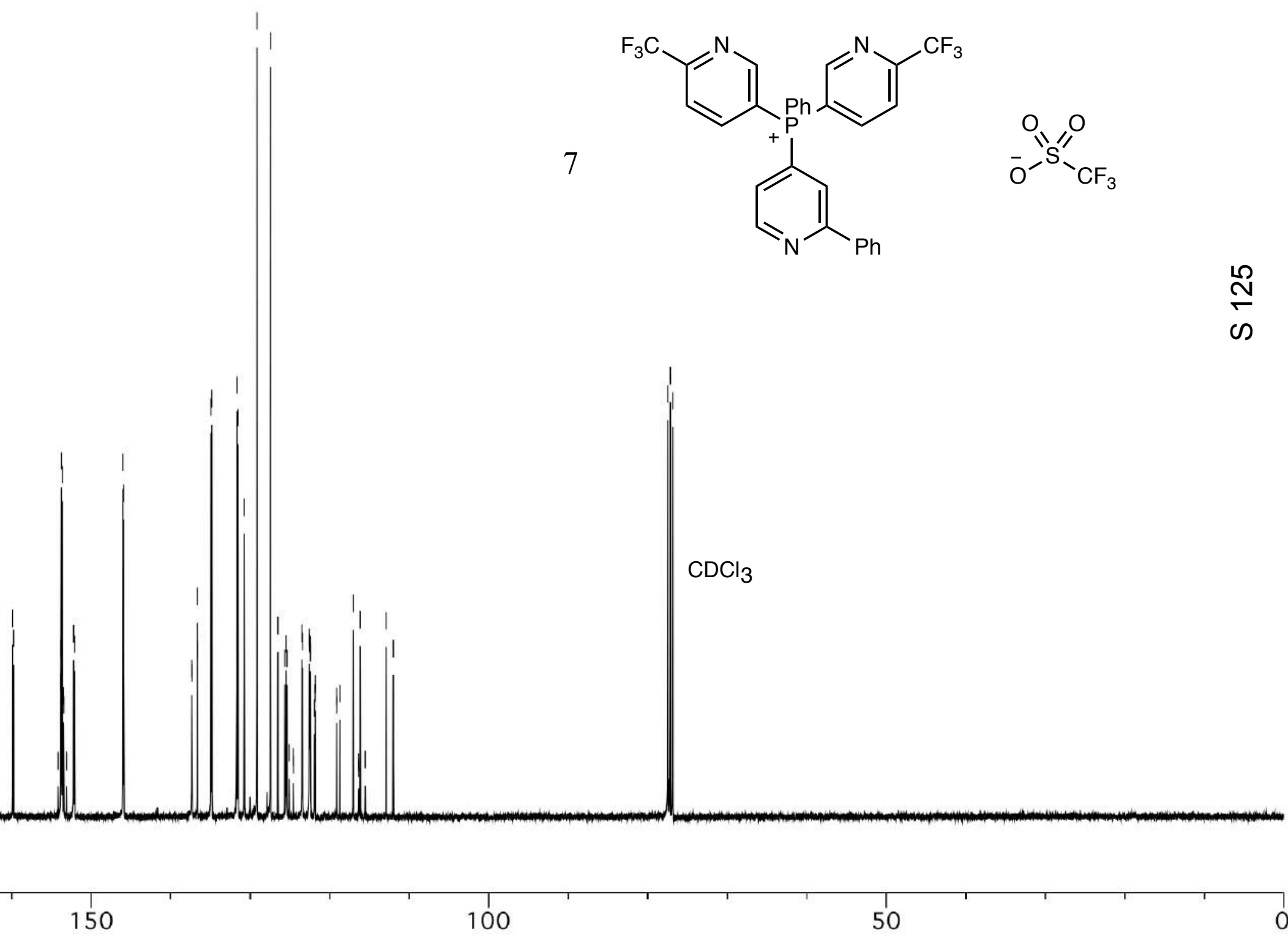


${ }^{19} \mathrm{~F} \mathrm{NMR}\left(\mathrm{CDCl}_{3}, 365 \mathrm{MHz}\right)$

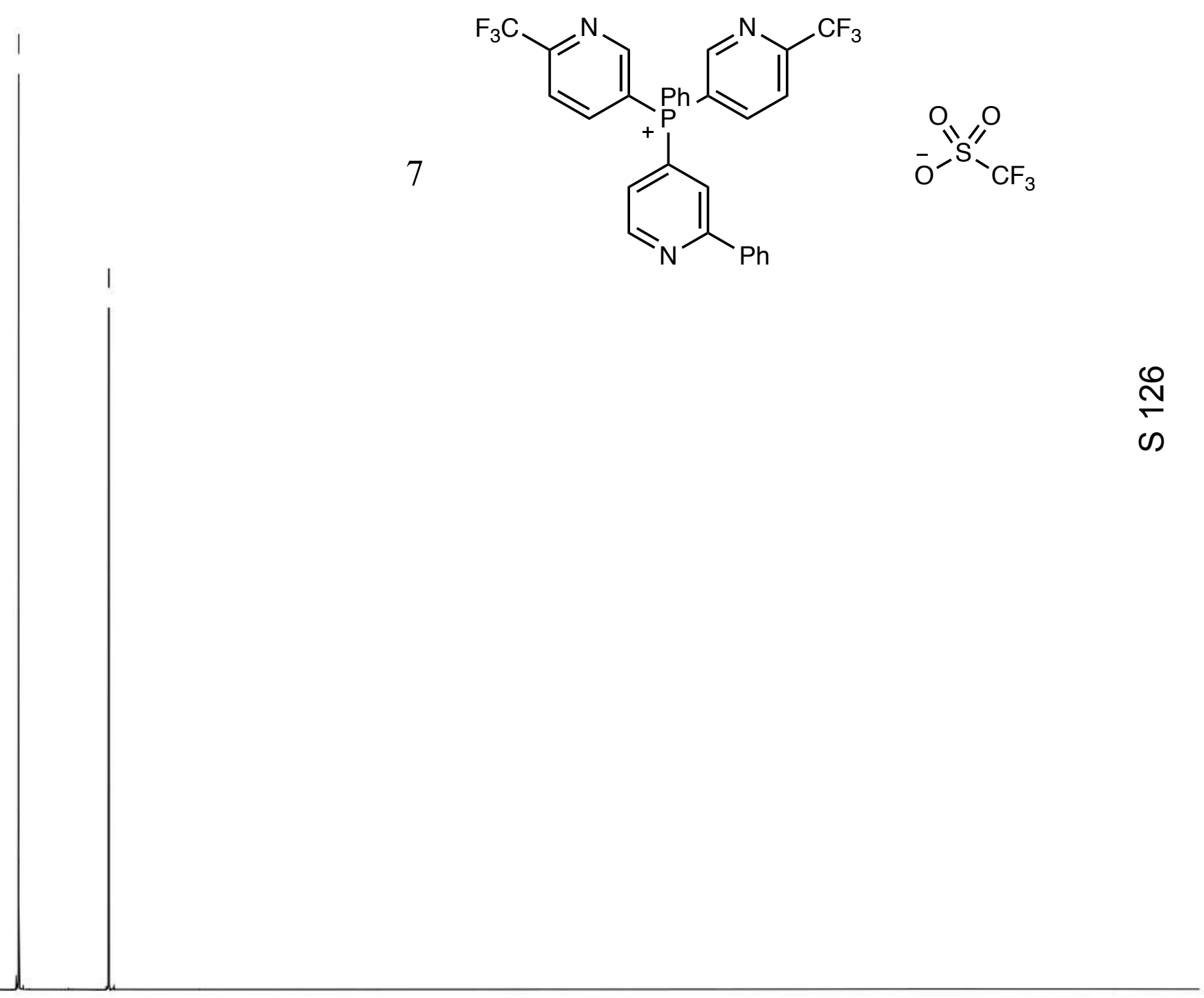

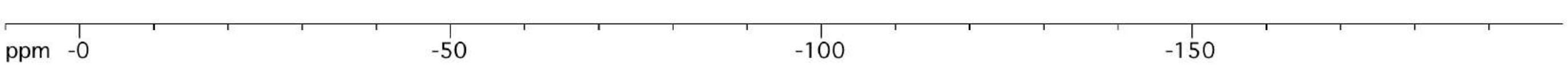


${ }^{31} \mathrm{P}$ NMR $\left(\mathrm{CDCl}_{3}, 162 \mathrm{MHz}\right)$

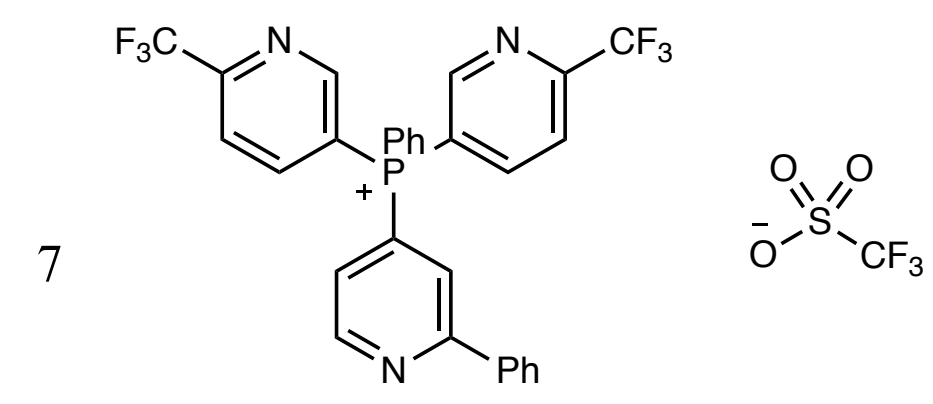


${ }^{1} \mathrm{H} \mathrm{NMR}\left(\mathrm{CDCl}_{3}, 400 \mathrm{MHz}\right)$

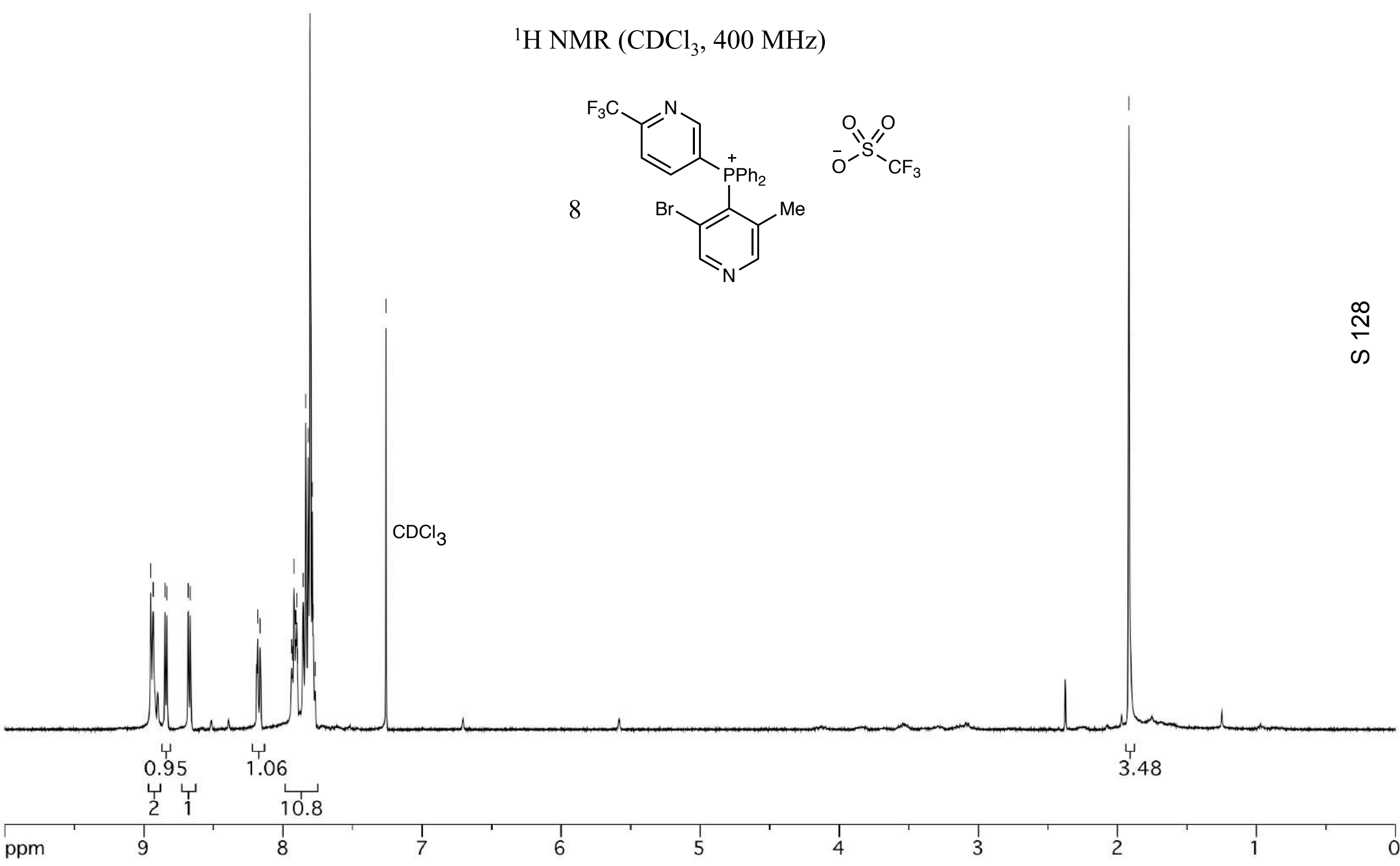


${ }^{13} \mathrm{C} \mathrm{NMR}\left(\mathrm{CDCl}_{3}, 100 \mathrm{MHz}\right)$<smiles>FC(F)(F)c1ccc([Pb+]c2ccccc2)cn1</smiles><smiles>[13CH3]S(=O)(=O)O</smiles>

8<smiles>Cc1cncc(Br)c1C</smiles> 
${ }^{19} \mathrm{~F} \mathrm{NMR}\left(\mathrm{CDCl}_{3}, 365 \mathrm{MHz}\right)$

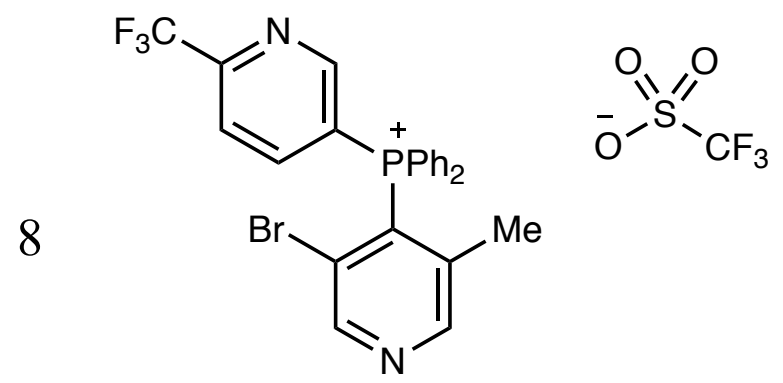


${ }^{31} \mathrm{P} \mathrm{NMR}\left(\mathrm{CDCl}_{3}, 162 \mathrm{MHz}\right)$

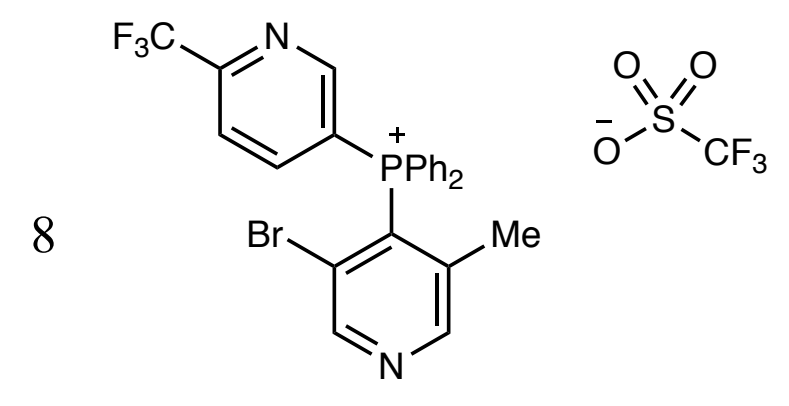


${ }^{1} \mathrm{H} \mathrm{NMR}\left(\mathrm{CDCl}_{3}, 400 \mathrm{MHz}\right)$

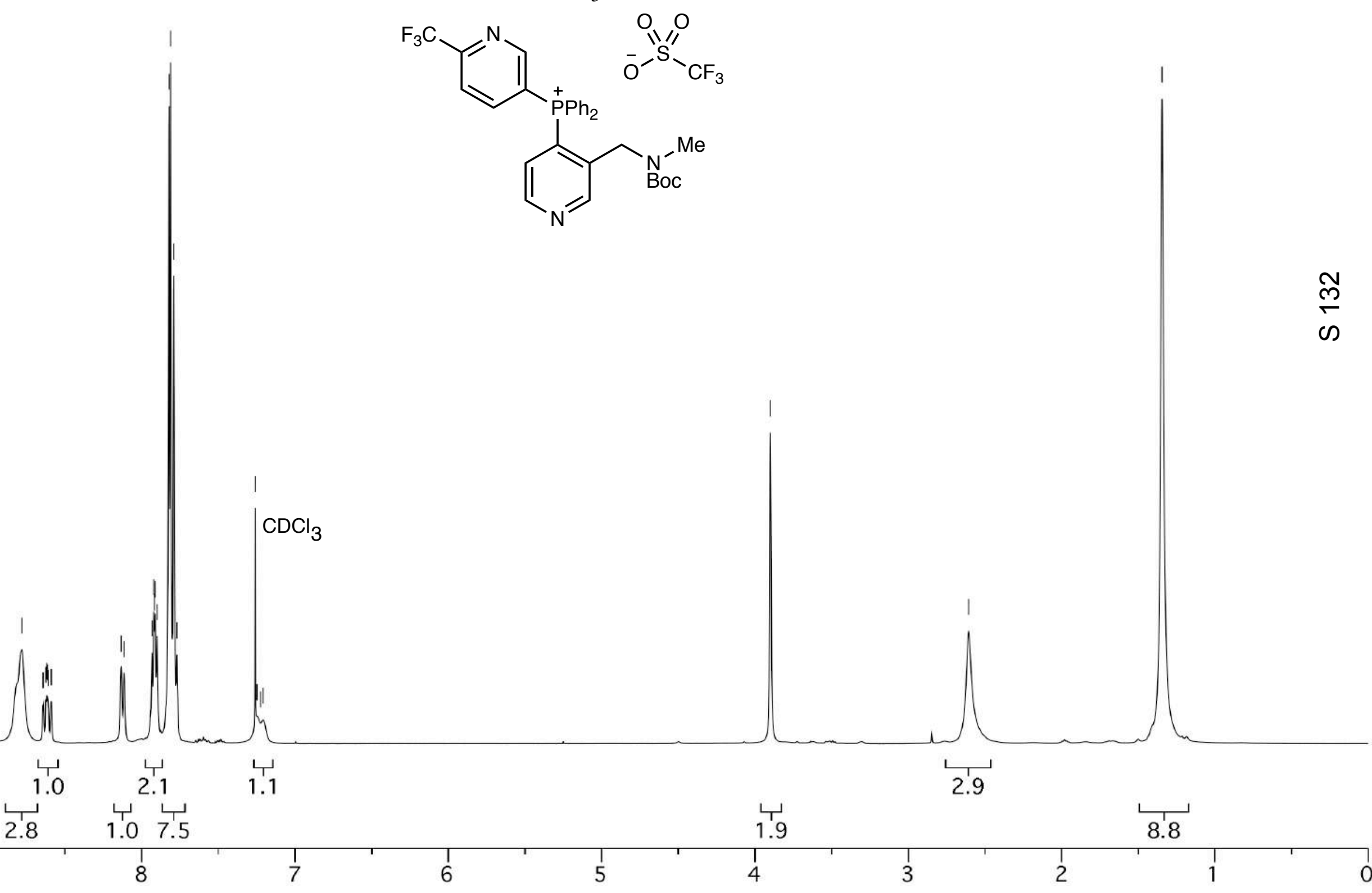

9

.


${ }^{13} \mathrm{C} \mathrm{NMR}\left(\mathrm{CDCl}_{3}, 100 \mathrm{MHz}\right)$<smiles>O=[W]OC(c1ccccc1)c1ccncc1[P+](=O)c1ccc(C(F)(F)F)nc1</smiles> 
${ }^{31} \mathrm{P}$ NMR $\left(\mathrm{CDCl}_{3}, 162 \mathrm{MHz}\right)$

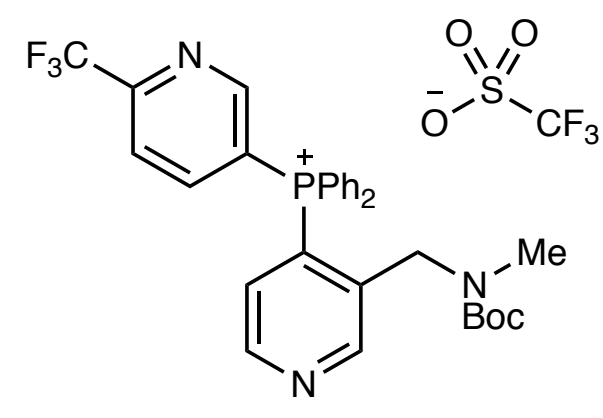



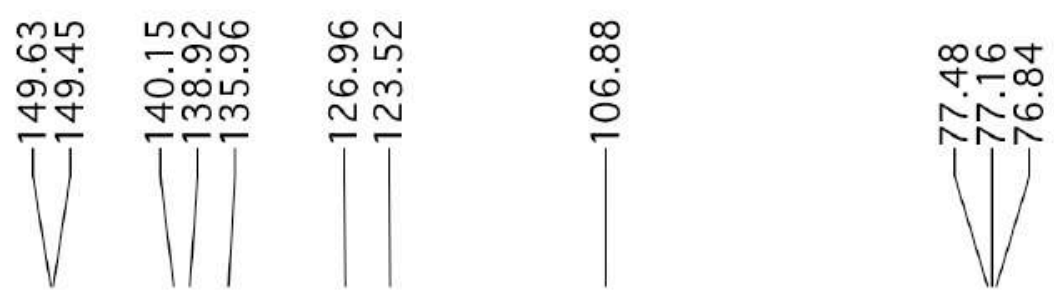

${ }^{13} \mathrm{C}$ NMR $\left(\mathrm{CDCl}_{3}, 100 \mathrm{MHz}\right)$<smiles>Cn1nccc1-c1cnccc1[PH2+](=O)c1ccc(C(F)(F)F)nc1</smiles> 
${ }^{19} \mathrm{~F} \mathrm{NMR}\left(\mathrm{CDCl}_{3}, 365 \mathrm{MHz}\right)$

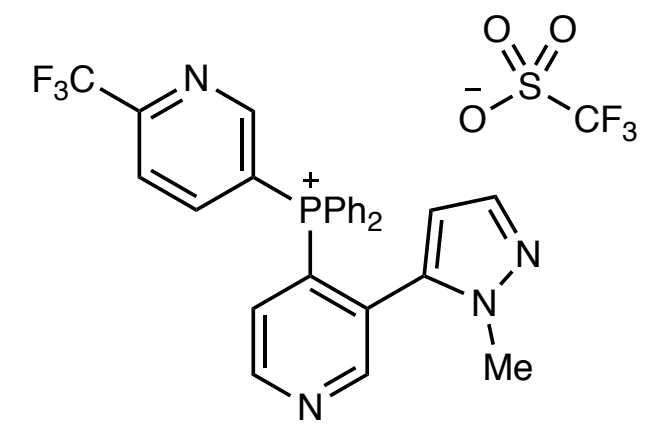

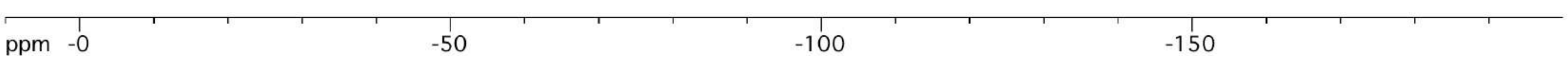


${ }^{31} \mathrm{P}$ NMR $\left(\mathrm{CDCl}_{3}, 162 \mathrm{MHz}\right)$

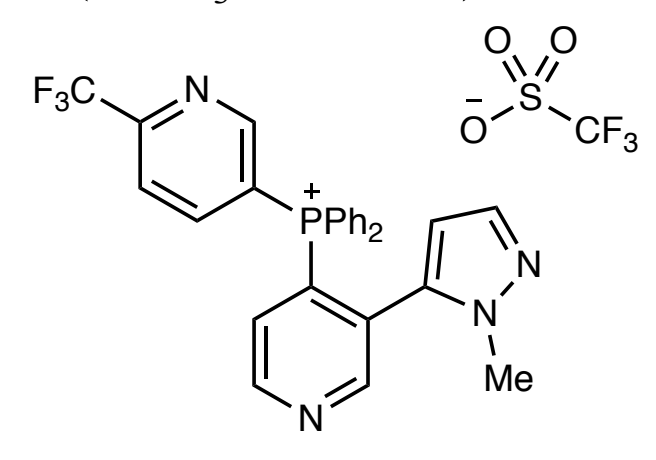


${ }^{1} \mathrm{H} \mathrm{NMR}\left(\mathrm{CDCl}_{3}, 400 \mathrm{MHz}\right)$

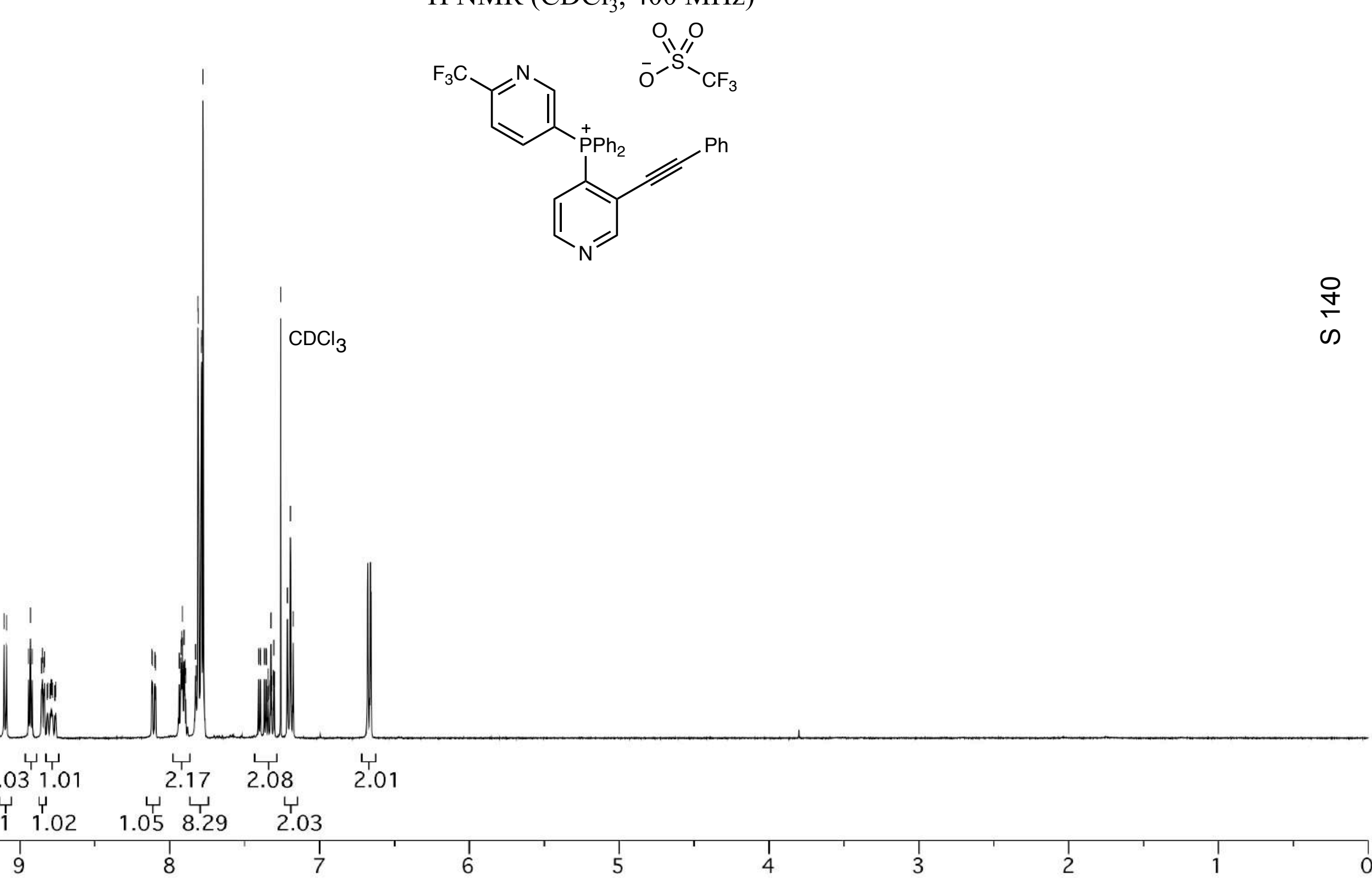


${ }^{13} \mathrm{C} \mathrm{NMR}\left(\mathrm{CDCl}_{3}, 100 \mathrm{MHz}\right)$<smiles>O=[P+](c1ccc(S(=O)(=O)[O-])nc1)c1ccncc1C#Cc1ccccc1</smiles>

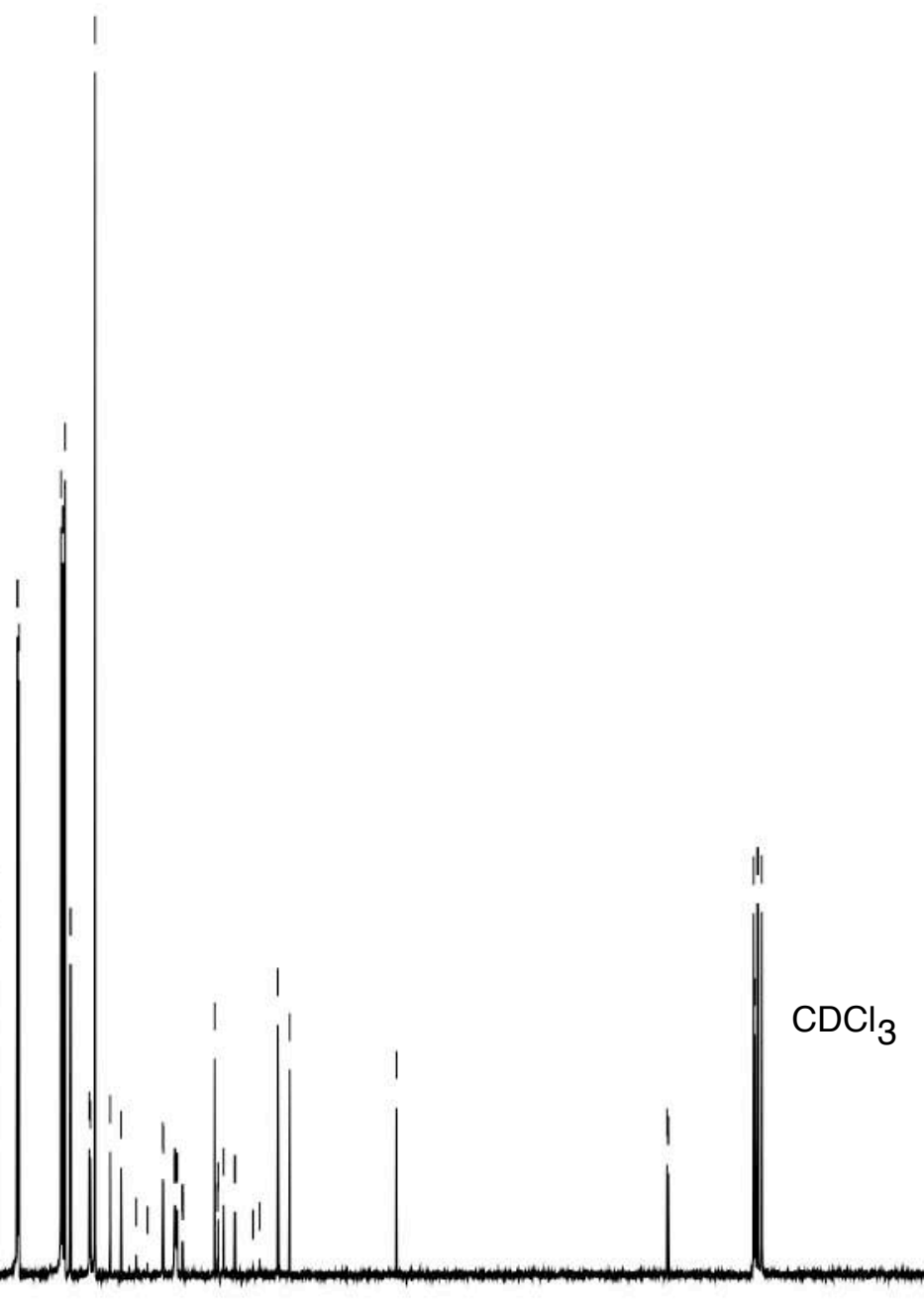


${ }^{19} \mathrm{~F} \mathrm{NMR}\left(\mathrm{CDCl}_{3}, 365 \mathrm{MHz}\right)$

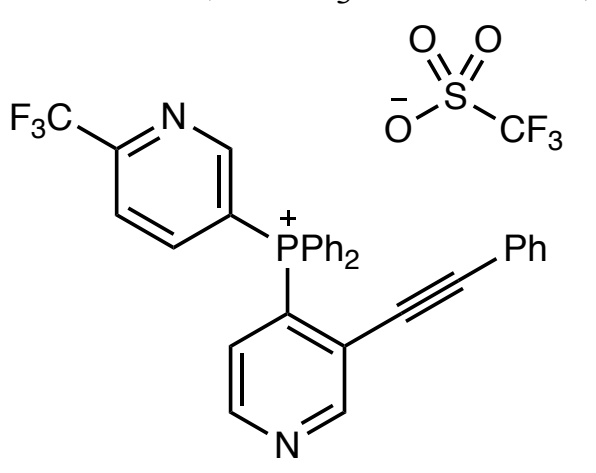

$\frac{\mathfrak{Y}}{\mathrm{I}}$

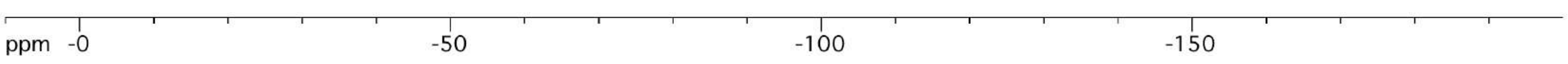


${ }^{31} \mathrm{P}$ NMR $\left(\mathrm{CDCl}_{3}, 162 \mathrm{MHz}\right)$

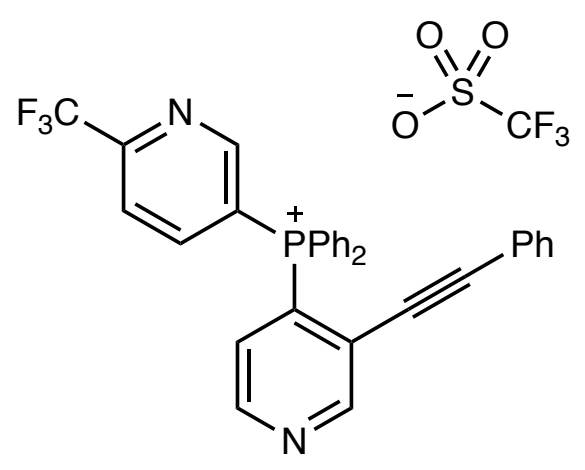


${ }^{1} \mathrm{H} \mathrm{NMR}\left(\mathrm{CDCl}_{3}, 400 \mathrm{MHz}\right)$

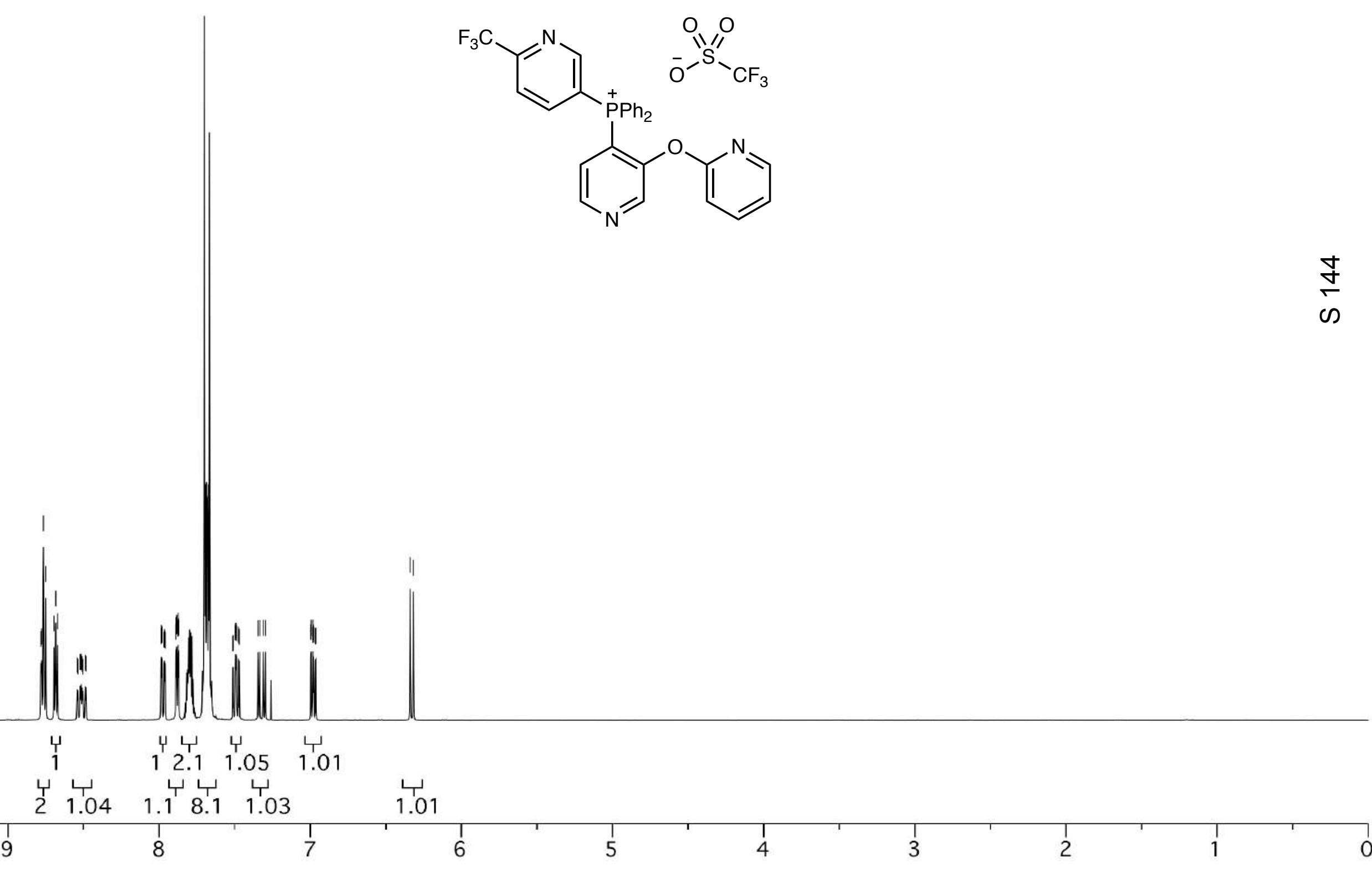




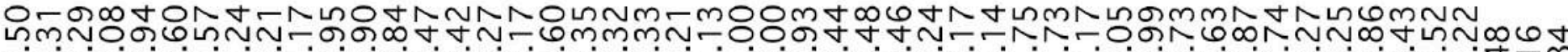

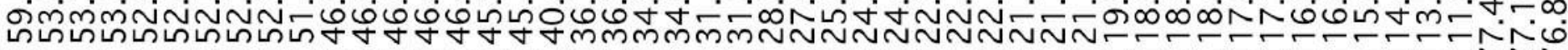
(

${ }^{13} \mathrm{C} \mathrm{NMR}\left(\mathrm{CDCl}_{3}, 100 \mathrm{MHz}\right)$

$\mathrm{F}_{3} \mathrm{C}$<smiles>Cc1ccc([PH+](c2ccccc2)c2ccncc2Oc2ccccn2)cn1</smiles> 
${ }^{19} \mathrm{~F} \mathrm{NMR}\left(\mathrm{CDCl}_{3}, 365 \mathrm{MHz}\right)$

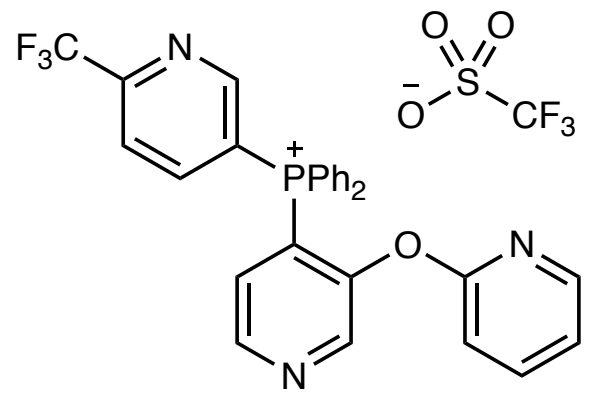

\begin{tabular}{|c|c|c|}
\hline $\begin{array}{ll} & 1 \\
\text { ppm } & -0\end{array}$ & -50 & -100 \\
\hline
\end{tabular}


${ }^{31} \mathrm{P} \mathrm{NMR}\left(\mathrm{CDCl}_{3}, 162 \mathrm{MHz}\right)$

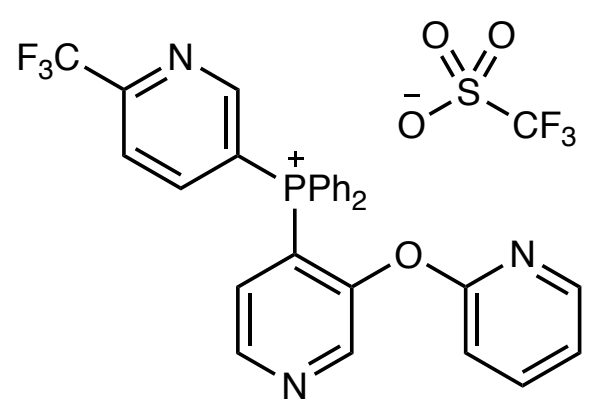




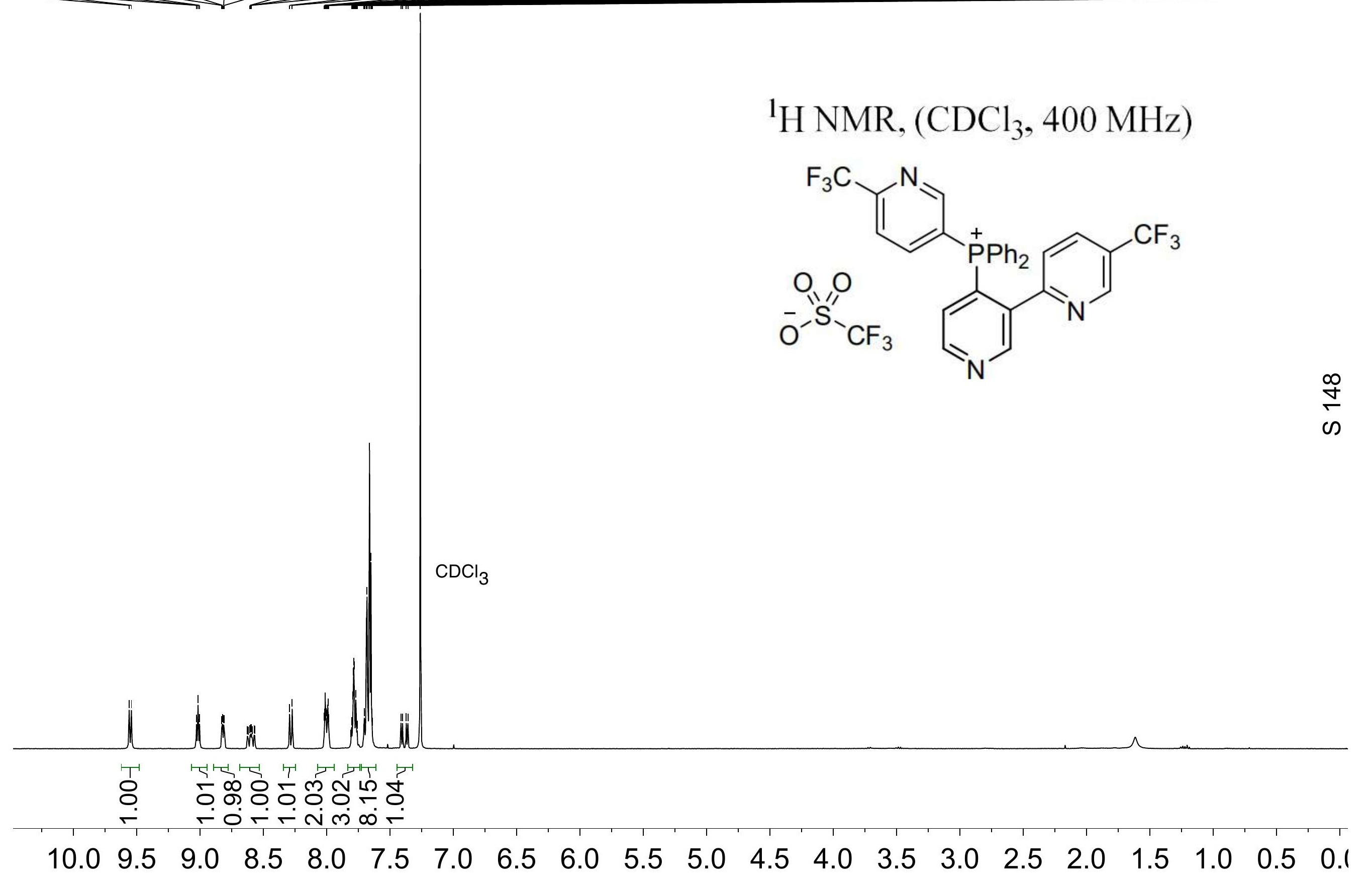




\section{${ }^{13} \mathrm{C} \mathrm{NMR},\left(\mathrm{CDCl}_{3}, 100 \mathrm{MHz}\right)$}<smiles>O=S(=O)([O-])C(F)(F)F</smiles> 


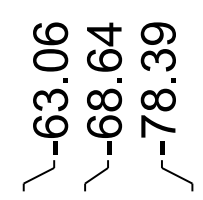

${ }^{19} \mathrm{~F} \mathrm{NMR}\left(\mathrm{CDCl}_{3}, 365 \mathrm{MHz}\right)$

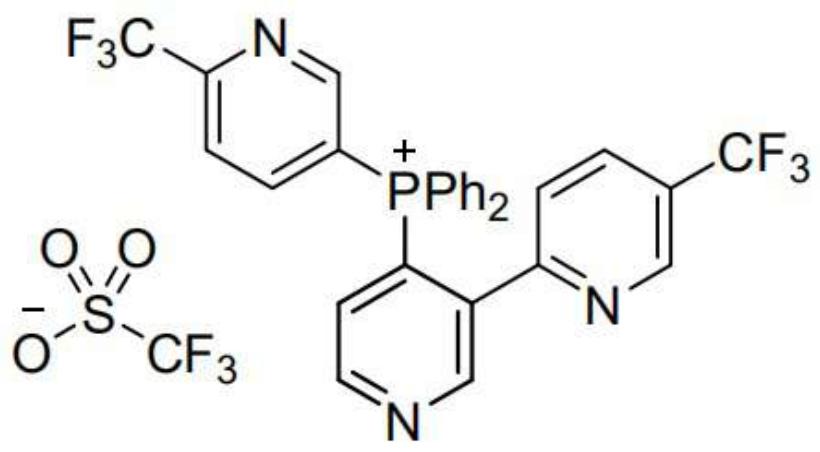

$\begin{array}{lll}0 & -20 & -40\end{array}$

$-40$

$-60$




$$
\text { ষ্ঠ }
$$

${ }^{31} \mathrm{P} \mathrm{NMR}\left(\mathrm{CDCl}_{3}, 162 \mathrm{MHz}\right)$<smiles>O=S(=O)(O)C(F)(F)C(F)(F)c1ccncc1-c1ccncc1-c1ccc(C(F)(F)F)cn1</smiles>

$\begin{array}{llllllllllllllll}190 & 170 & 150 & 130 & 110 & 90 & 70 & 50 & 30 & 10 & -10 & -30 & -50 & -70 & -90\end{array}$




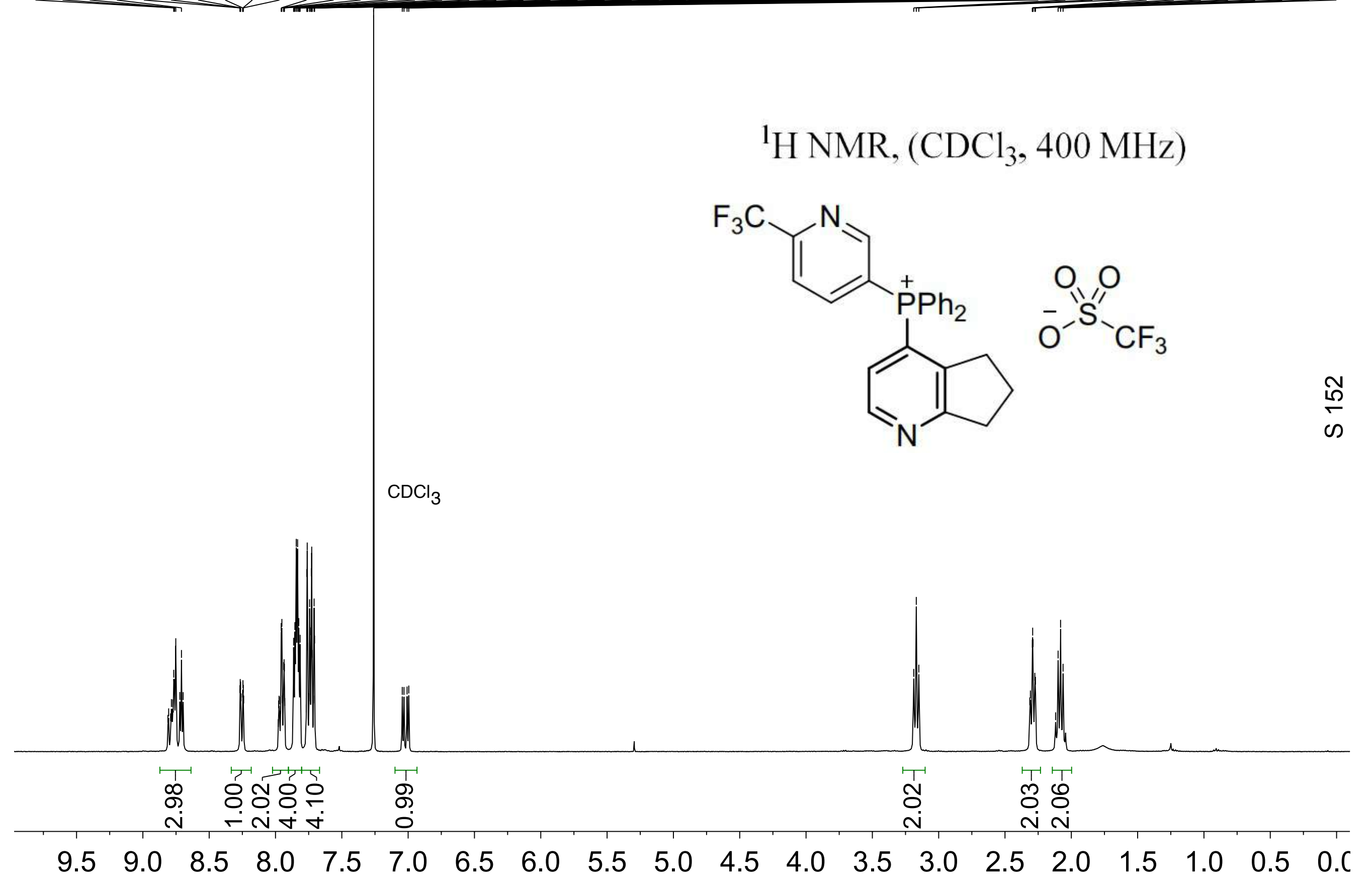




\section{${ }^{13} \mathrm{C} \mathrm{NMR},\left(\mathrm{CDCl}_{3}, 100 \mathrm{MHz}\right)$}

$\mathrm{F}_{3} \mathrm{C}$

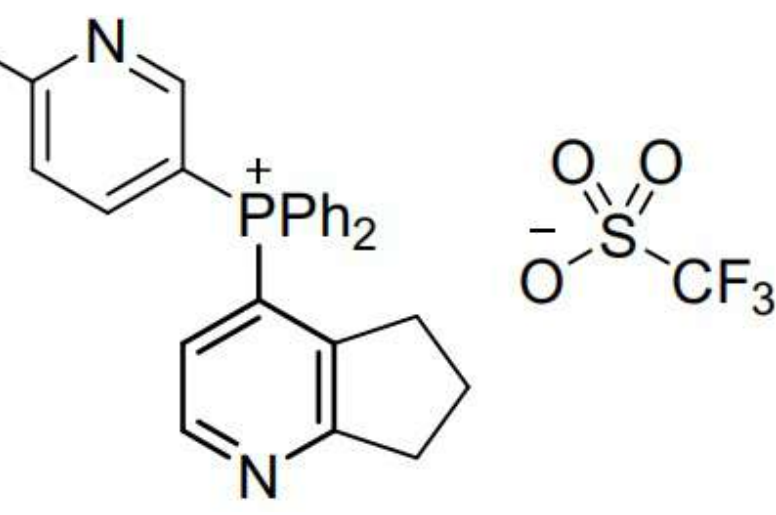




\section{${ }^{19} \mathrm{~F} \mathrm{NMR}\left(\mathrm{CDCl}_{3}, 365 \mathrm{MHz}\right)$}

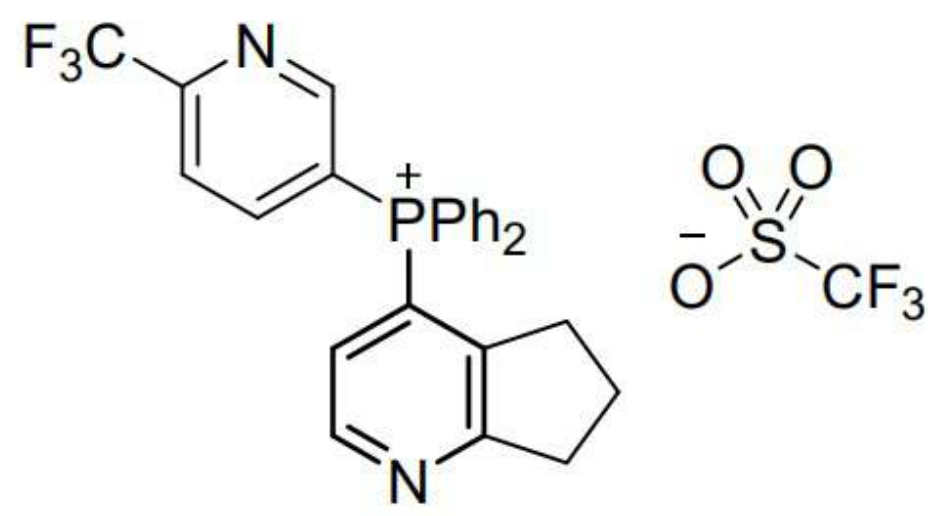

$\frac{10}{\infty}$

$0 \quad-2$

$\begin{array}{lll}-20 & -40 \quad-60\end{array}$

$-80$

$-100$

$-120$

$-140$

$-160$

$-180$

$-200-2 i$ 
${ }^{19} \mathrm{~F} \mathrm{NMR}\left(\mathrm{CDCl}_{3}, 365 \mathrm{MHz}\right)$<smiles>O=S(=O)(O)C(F)(F)F</smiles> 


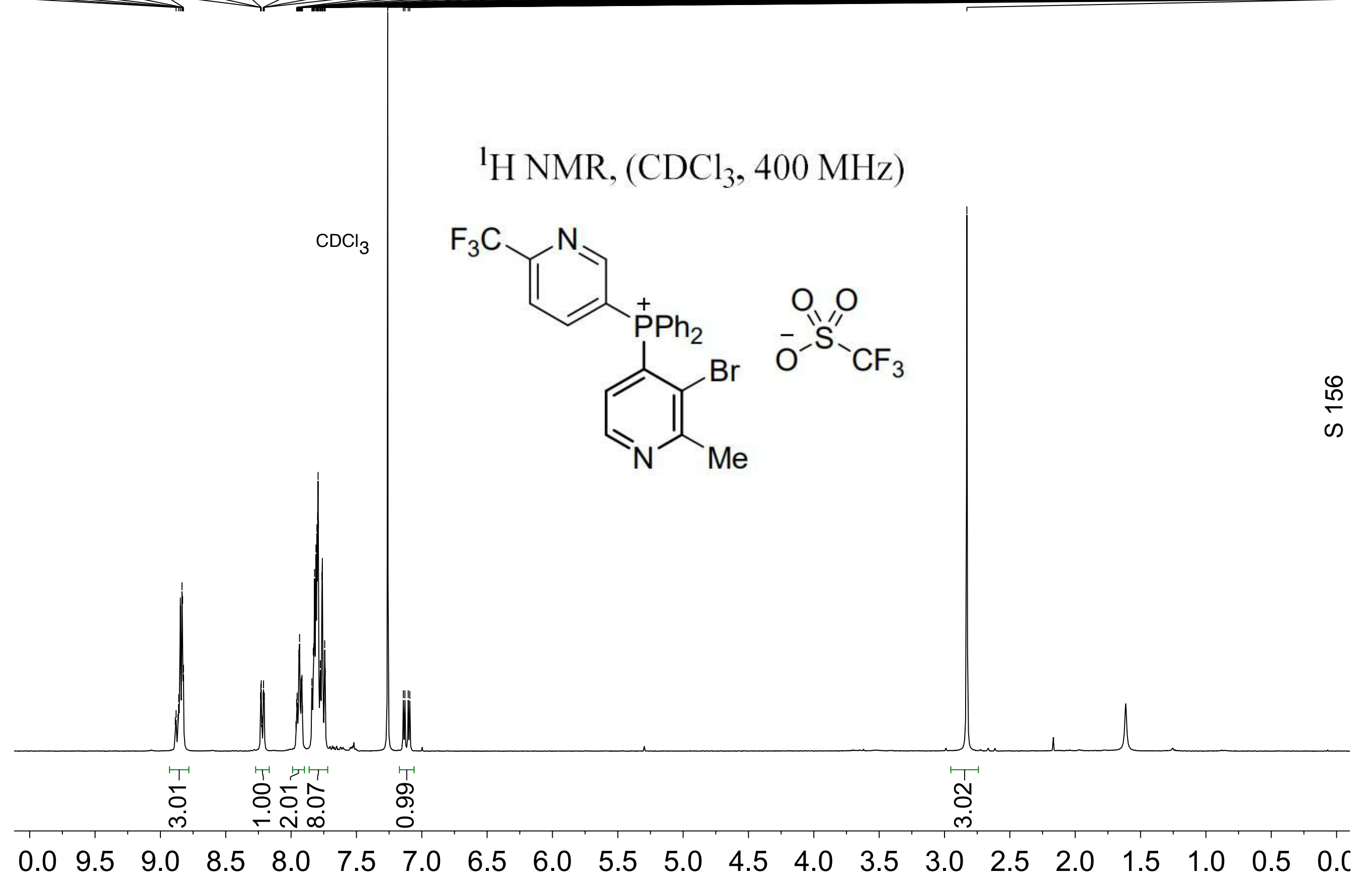




\section{${ }^{13} \mathrm{C} \mathrm{NMR},\left(\mathrm{CDCl}_{3}, 100 \mathrm{MHz}\right)$}<smiles></smiles> 


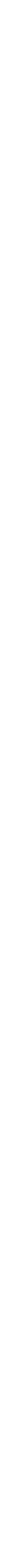

\begin{tabular}{|c|c|c|c|c|c|c|c|c|c|c|c|}
\hline .0 & 0 & -20 & -40 & -60 & -80 & -100 & -120 & -140 & -160 & -180 & -200 \\
\hline
\end{tabular}




\section{${ }^{31} \mathrm{P} \mathrm{NMR}\left(\mathrm{CDCl}_{3}, 162 \mathrm{MHz}\right)$}<smiles>Cc1nccc([P+](=O)c2ccc(C(F)(F)F)nc2)c1Br</smiles>

$\begin{array}{llllllllllllllll} & 170 & 150 & 130 & 110 & 90 & 70 & 50 & 30 & 10 & -10 & -30 & -50 & -70 & -9\end{array}$


${ }^{1} \mathrm{H} \mathrm{NMR}\left(\mathrm{CDCl}_{3}, 400 \mathrm{MHz}\right)$<smiles>Cc1nccc(-c2ccc(C(F)(F)F)nc2)c1-c1ccsc1</smiles>

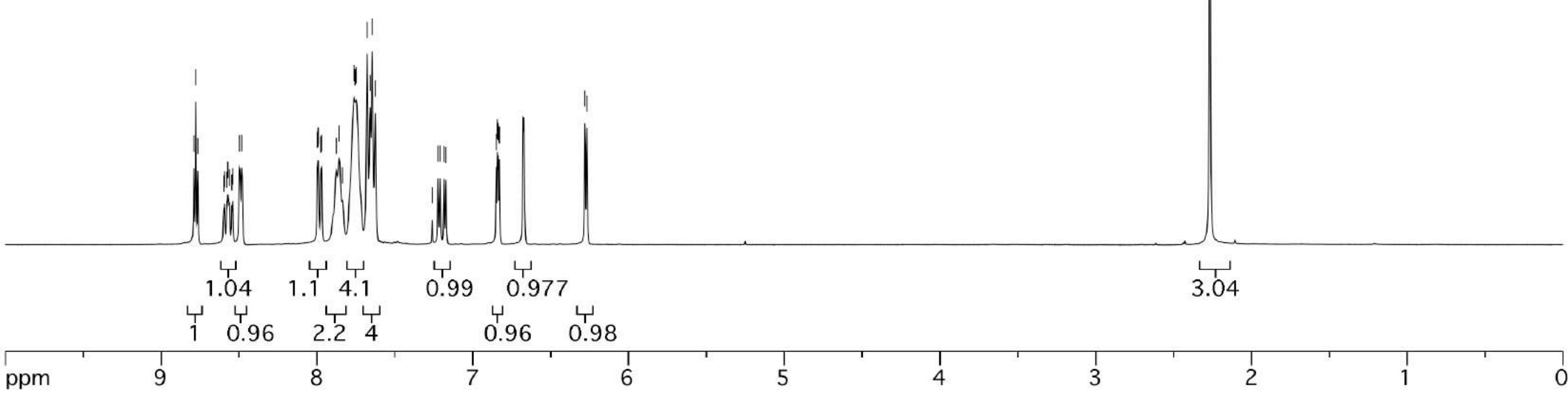


${ }^{13} \mathrm{C} \mathrm{NMR}\left(\mathrm{CDCl}_{3}, 100 \mathrm{MHz}\right)$<smiles>Cc1nccc([PH+]([O-])c2ccc(C(F)(F)F)nc2)c1-c1ccsc1</smiles> 
${ }^{19} \mathrm{~F} \mathrm{NMR}\left(\mathrm{CDCl}_{3}, 365 \mathrm{MHz}\right)$

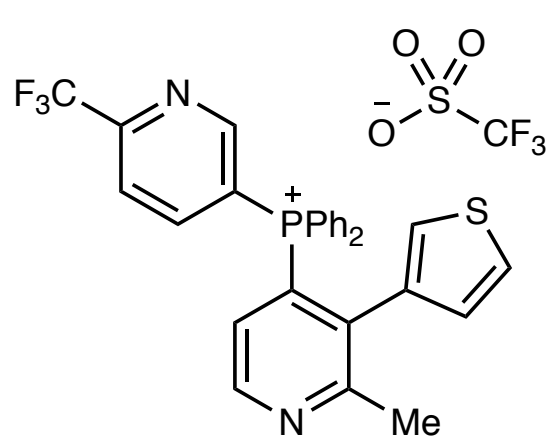

\begin{tabular}{|c|c|c|}
\hline $\begin{array}{ll} & 1 \\
\text { ppm }-0\end{array}$ & $\begin{array}{l}1 \\
-50\end{array}$ & $\begin{array}{l}1 \\
-100\end{array}$ \\
\hline
\end{tabular}


${ }^{31} \mathrm{P} \mathrm{NMR}\left(\mathrm{CDCl}_{3}, 162 \mathrm{MHz}\right)$

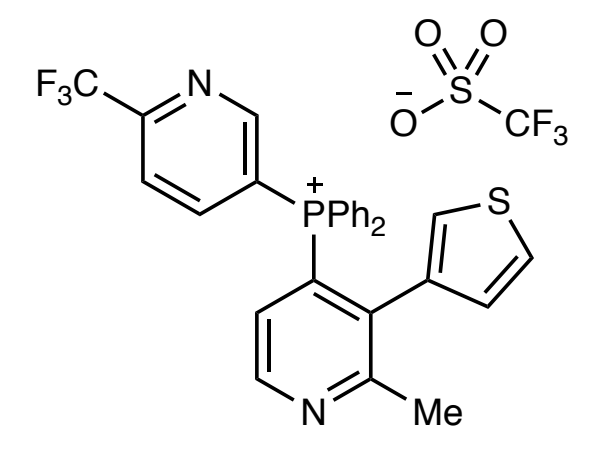


${ }^{1} \mathrm{H} \mathrm{NMR}\left(\mathrm{CDCl}_{3}, 400 \mathrm{MHz}\right)$<smiles>Cc1c(-c2ccc(C(F)(F)F)nc2)ccnc1C#N</smiles><smiles>[O]S(=O)(=O)C(F)(F)F</smiles>

17:1 mixture of product:impurity

$\mathrm{CDCl}_{3}$

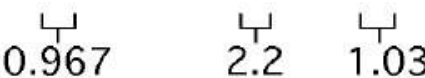

1.94 난

누요 
${ }^{13} \mathrm{C}$ NMR $\left(\mathrm{CD}_{3} \mathrm{CN}, 100 \mathrm{MHz}\right)$<smiles>N#Cc1nccc([P+]([O-])([O-])c2ccc(C(F)(F)F)nc2)c1[N+](=O)[O-]</smiles>

17:1 mixture of product:impurity

\section{$6=$}

- Rogiñ NN-

1

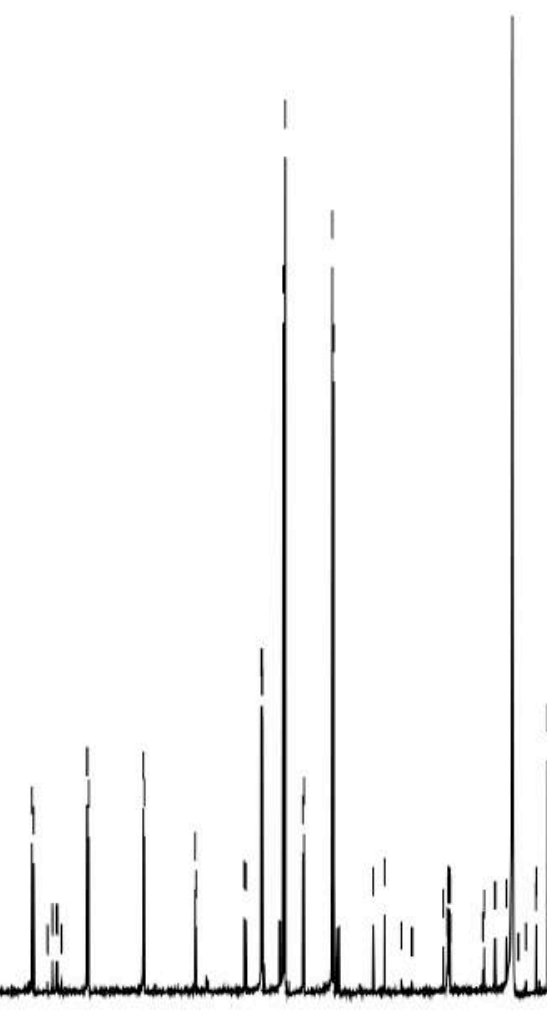


밍

${ }^{19} \mathrm{~F}$ NMR $\left(\mathrm{CD}_{3} \mathrm{CN}, 365 \mathrm{MHz}\right)$<smiles>FC(F)(F)c1ccc(P)cn1</smiles><smiles>[13CH3]S(=O)(=O)[O-]</smiles><smiles>N#Cc1nccc(I)c1N</smiles>

17:1 mixture of product:impurity

\begin{tabular}{|c|c|c|}
\hline $\begin{array}{ll} & 1 \\
\text { ppm }-0\end{array}$ & $\begin{array}{l}1 \\
-50\end{array}$ & $\begin{array}{l}1 \\
-100\end{array}$ \\
\hline
\end{tabular}


${ }^{31} \mathrm{P}$ NMR $\left(\mathrm{CD}_{3} \mathrm{CN}, 162 \mathrm{MHz}\right)$

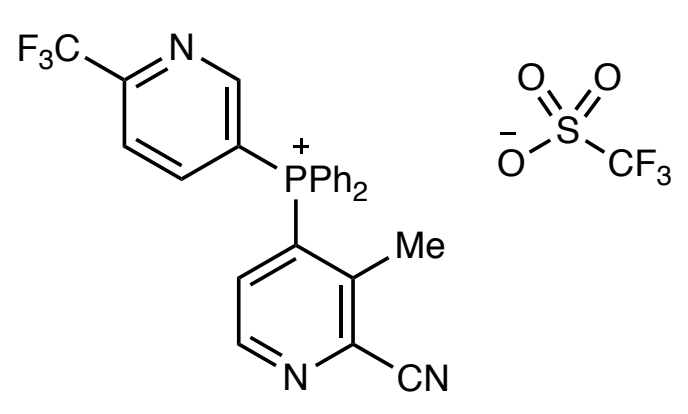

17:1 mixture of product:impurity 
${ }^{1} \mathrm{H} \mathrm{NMR}\left(\mathrm{CDCl}_{3}, 400 \mathrm{MHz}\right)$

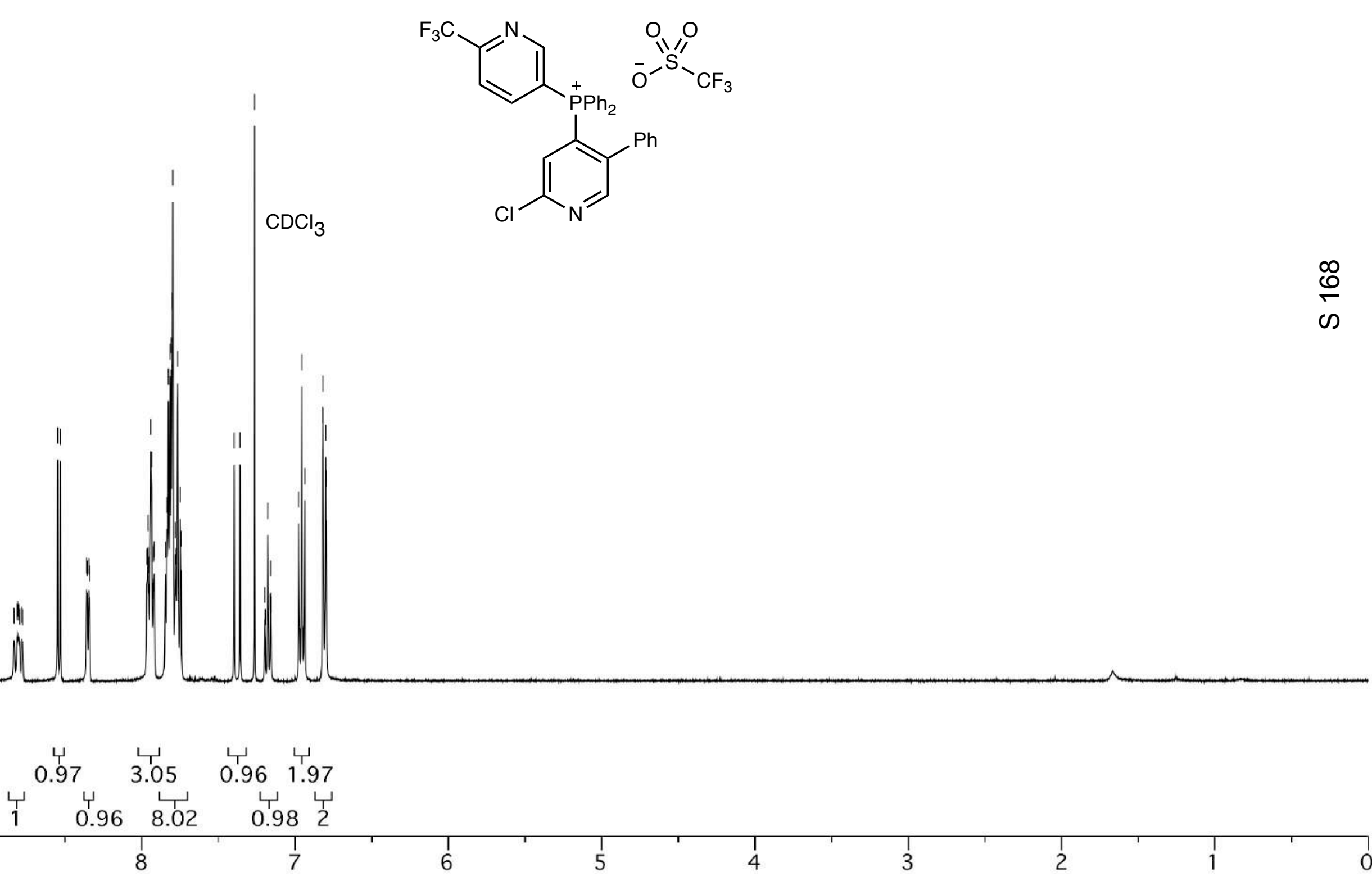


NmG

の

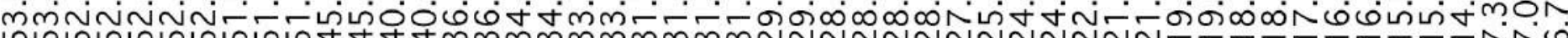

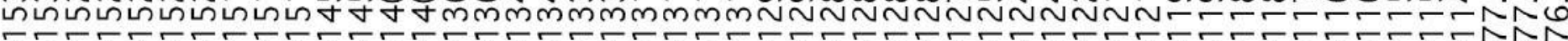

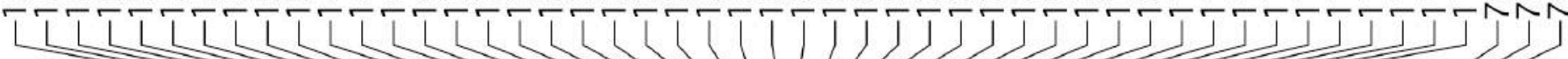

${ }^{13} \mathrm{C} \mathrm{NMR}\left(\mathrm{CDCl}_{3}, 100 \mathrm{MHz}\right)$<smiles>O=S(=O)([O-])c1ccccc1-c1cc(Cl)ncc1[PH2+]c1ccc(C(F)(F)F)nc1</smiles> 
${ }^{31} \mathrm{P}$ NMR $\left(\mathrm{CDCl}_{3}, 162 \mathrm{MHz}\right)$

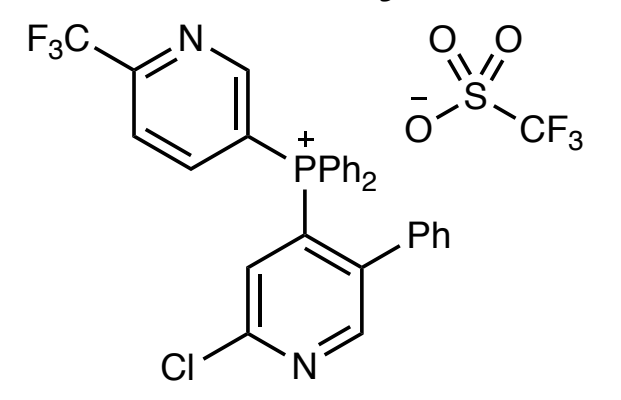




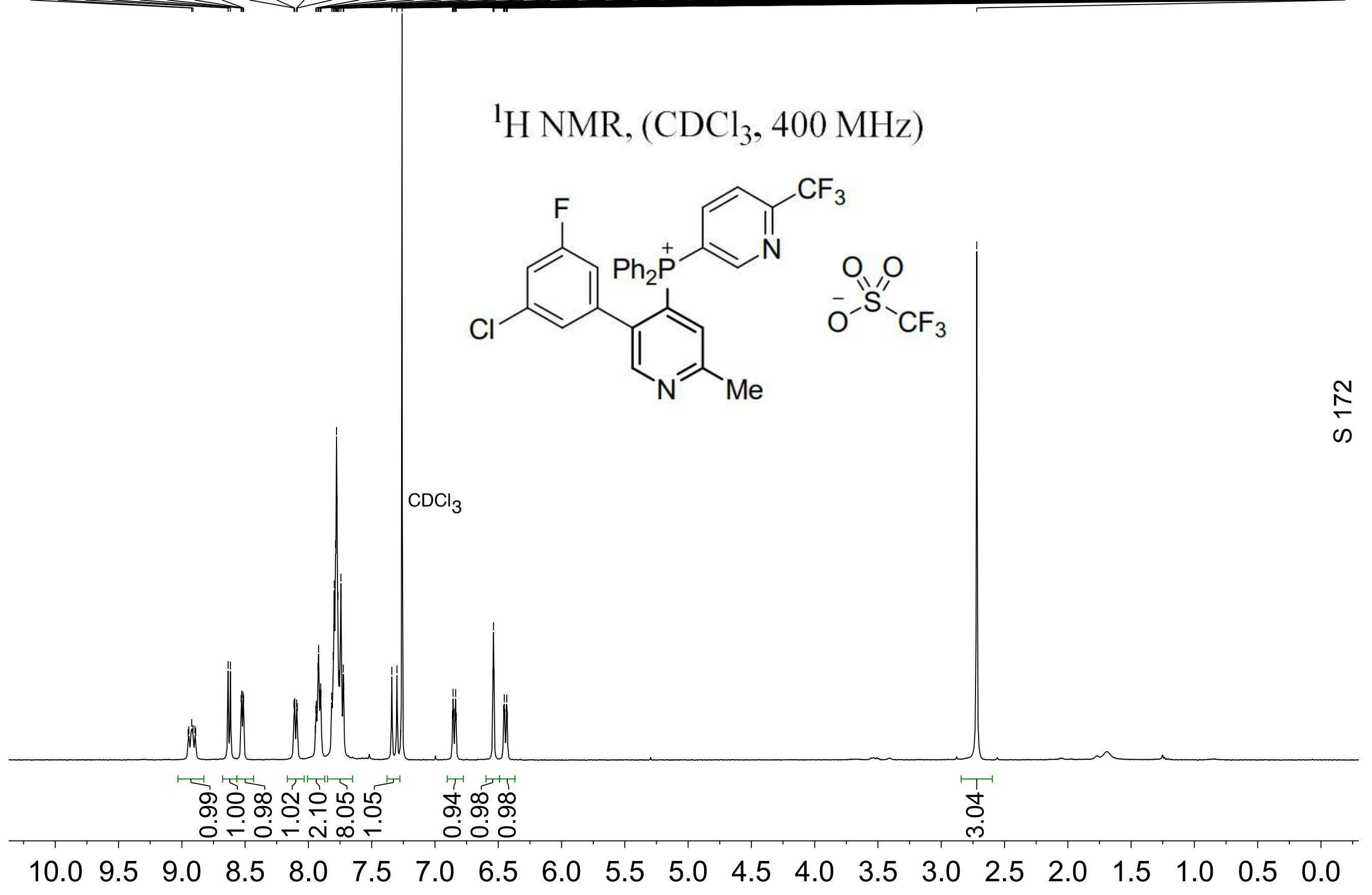




\section{${ }^{13} \mathrm{C} \mathrm{NMR},\left(\mathrm{CDCl}_{3}, 100 \mathrm{MHz}\right)$}<smiles>Cc1cc(P(c2ccccc2)c2ccc(C(F)(F)F)nc2)c(-c2cc(F)cc(Cl)c2)cn1</smiles> 


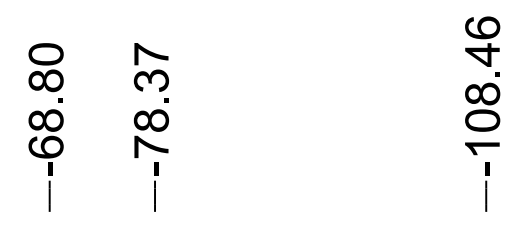

${ }^{19} \mathrm{~F} \mathrm{NMR}\left(\mathrm{CDCl}_{3}, 365 \mathrm{MHz}\right)$

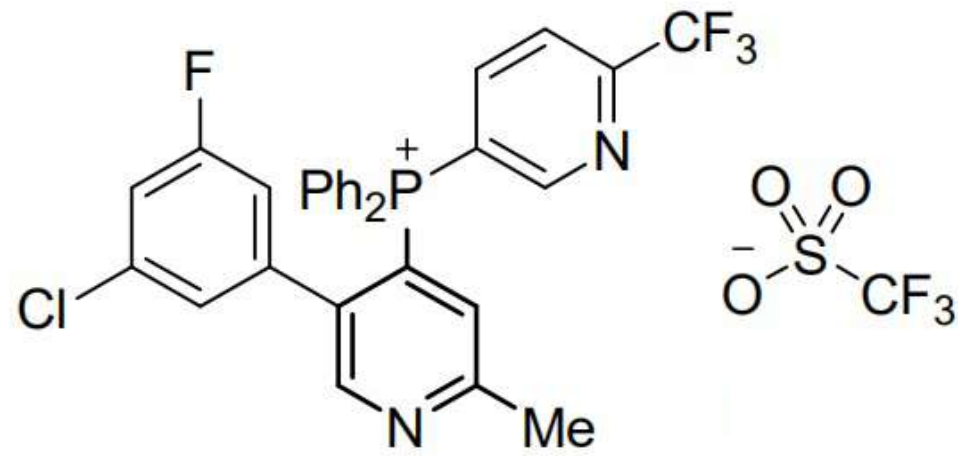

$\frac{\pi}{\infty}$

$0 \quad-20$

$-20 \quad-40 \quad-60$

$-80 \quad-100$

$-120$

$-140$

$-160$

$-180$

$-200-2 i$ 
${ }^{31} \mathrm{P} \mathrm{NMR}\left(\mathrm{CDCl}_{3}, 162 \mathrm{MHz}\right)$<smiles>Cc1cc(P(c2ccccc2)c2ccc(C(F)(F)F)cn2)c(-c2cc(F)cc(Cl)c2)cn1</smiles>

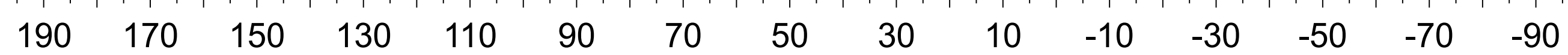


กัฒळ

กำ

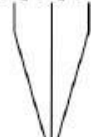

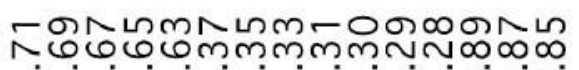

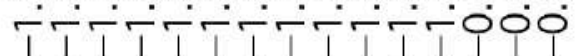

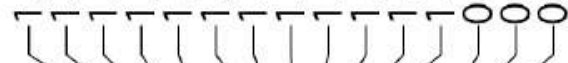

${ }^{1} \mathrm{H} \mathrm{NMR}\left(\mathrm{CDCl}_{3}, 400 \mathrm{MHz}\right)$

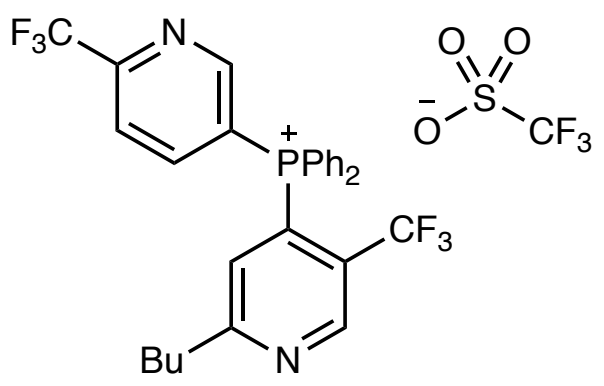

44

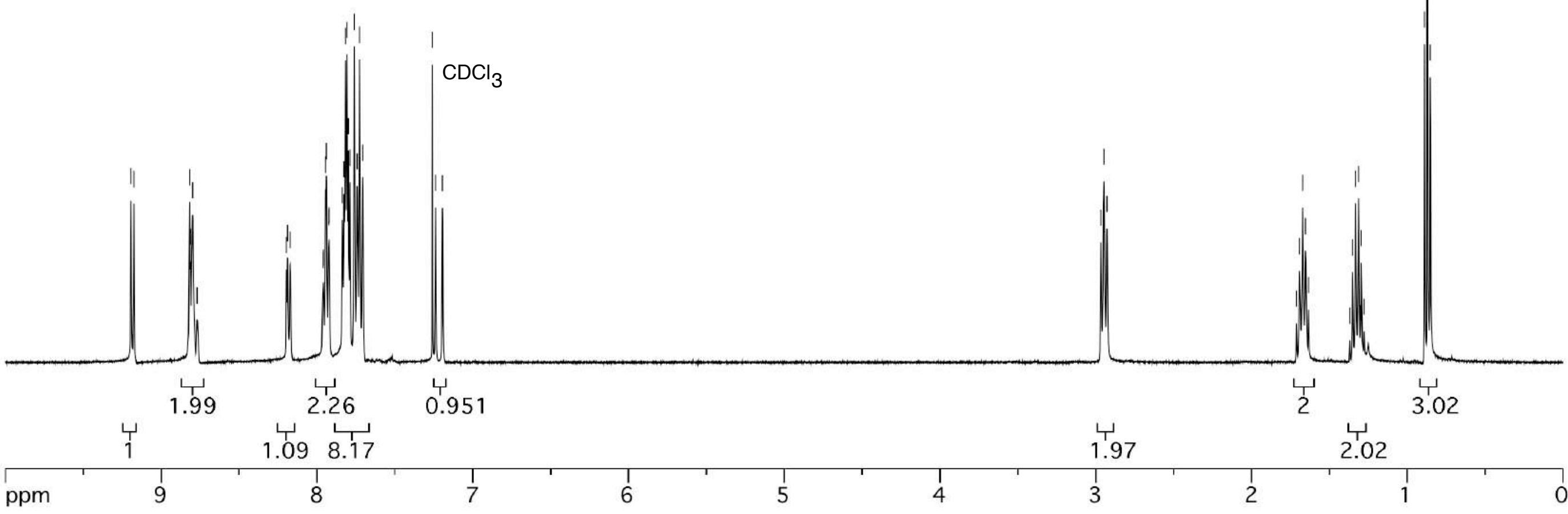


${ }^{13} \mathrm{C} \mathrm{NMR}\left(\mathrm{CDCl}_{3}, 100 \mathrm{MHz}\right)$<smiles>O=S(=O)([O-])C(F)(F)C(F)(F)c1cnc(Br)cc1[P+](c1ccc(Br)cc1)c1ccc(C(F)(F)F)nc1</smiles>

44 
${ }^{31} \mathrm{P}$ NMR $\left(\mathrm{CDCl}_{3}, 162 \mathrm{MHz}\right)$

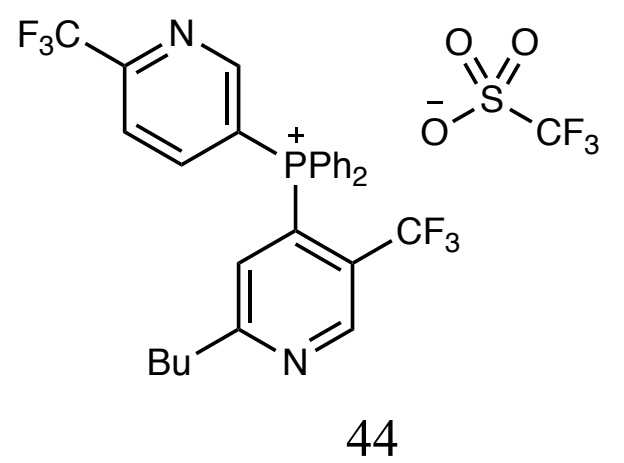

$\frac{\infty}{2}$ 


\section{${ }^{1} \mathrm{H} \mathrm{NMR},\left(\mathrm{CDCl}_{3}, 400 \mathrm{MHz}\right)$}

$\mathrm{CDCl}_{3}$

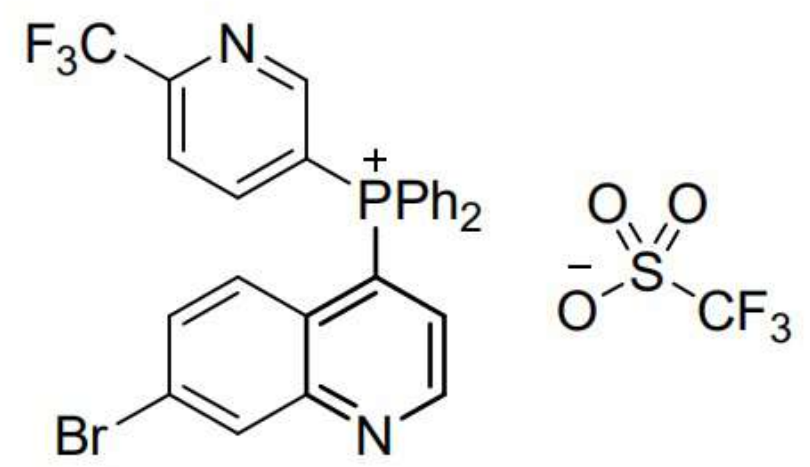

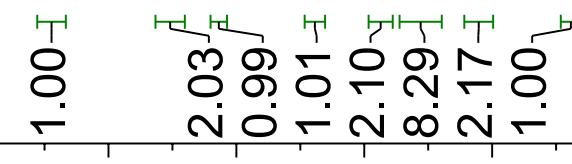

$\begin{array}{lllllllllllllllllllll}10.0 & 9.5 & 9.0 & 8.5 & 8.0 & 7.5 & 7.0 & 6.5 & 6.0 & 5.5 & 5.0 & 4.5 & 4.0 & 3.5 & 3.0 & 2.5 & 2.0 & 1.5 & 1.0 & 0.5 & 0.0\end{array}$ 
${ }^{13} \mathrm{C} \mathrm{NMR},\left(\mathrm{CDCl}_{3}, 100 \mathrm{MHz}\right)$<smiles>FC(F)(F)c1ccc([PH2+]c2ccnc3cc(Br)ccc23)cn1</smiles><smiles>O=S(=O)([O-])C(F)(F)F</smiles> 


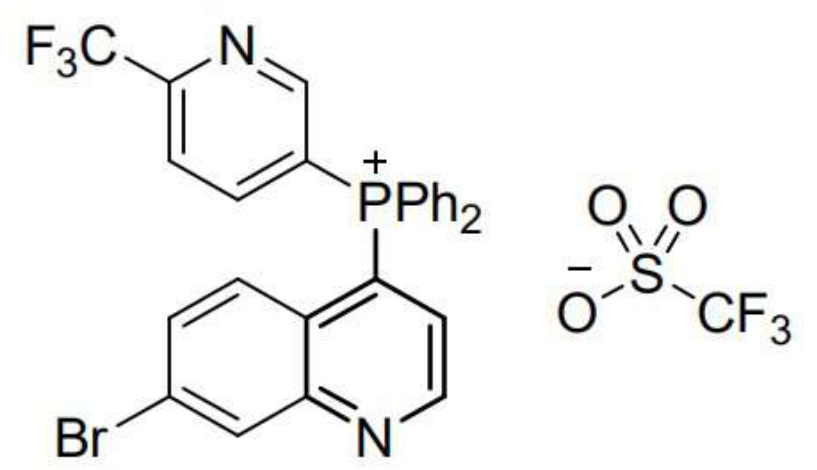

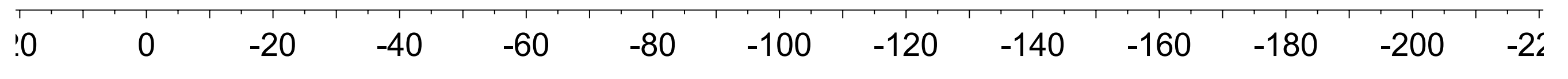




\section{${ }^{31} \mathrm{P} \mathrm{NMR}\left(\mathrm{CDCl}_{3}, 162 \mathrm{MHz}\right)$}<smiles>O=S(=O)(O)C(F)(F)F</smiles> 


\section{${ }^{1} \mathrm{H} \mathrm{NMR},\left(\mathrm{CDCl}_{3}, 400 \mathrm{MHz}\right)$}

$\mathrm{CDCl}_{3}$<smiles>O=S(=O)([O-])C(F)(F)F</smiles>

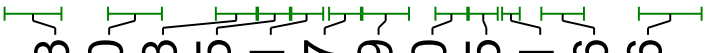

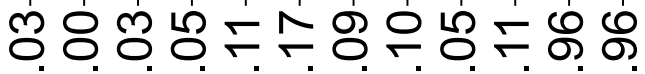

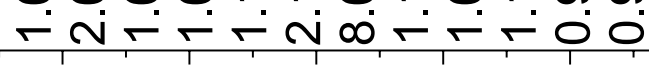


ᄋ 官

\section{${ }^{13} \mathrm{C} \mathrm{NMR},\left(\mathrm{CDCl}_{3}, 100 \mathrm{MHz}\right)$}

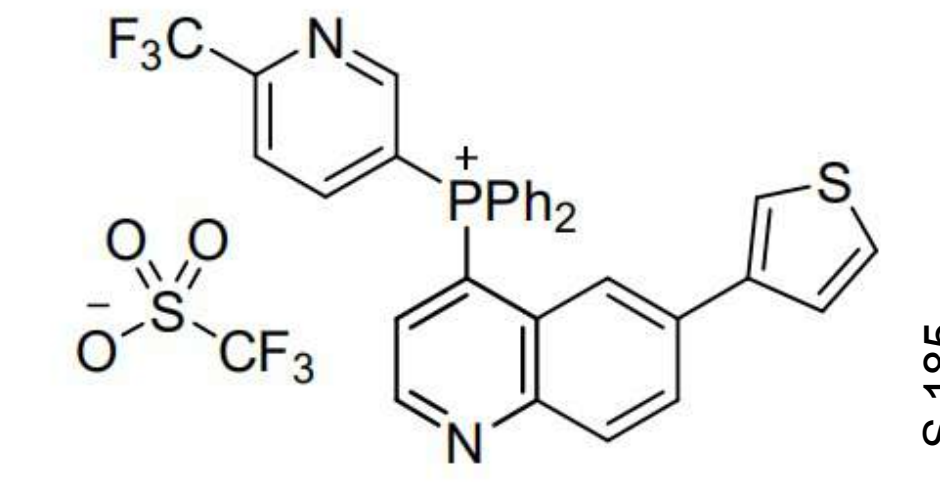


${ }^{19} \mathrm{~F}$ NMR $\left(\mathrm{CDCl}_{3}, 365 \mathrm{MHz}\right)$

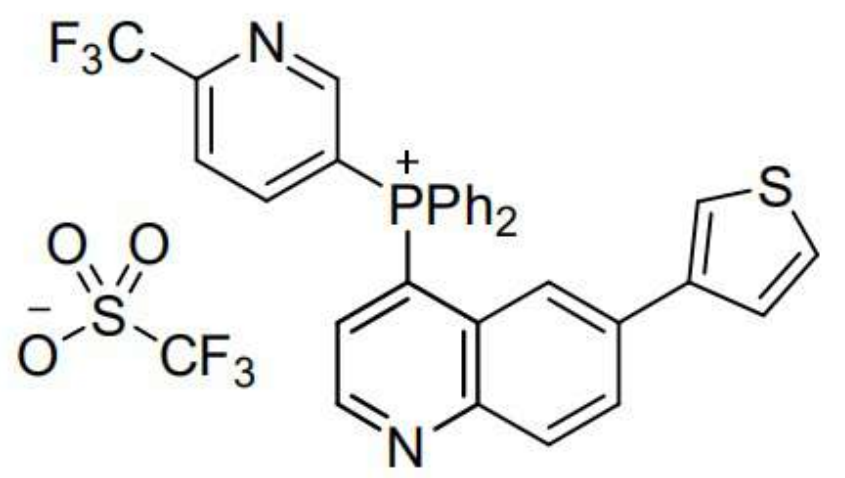

$0 \quad-20$

$-20 \quad-40 \quad-60$

$-80 \quad-100$

$-120$

$-140$

$-160$

$-180$

$-200-2 i$ 


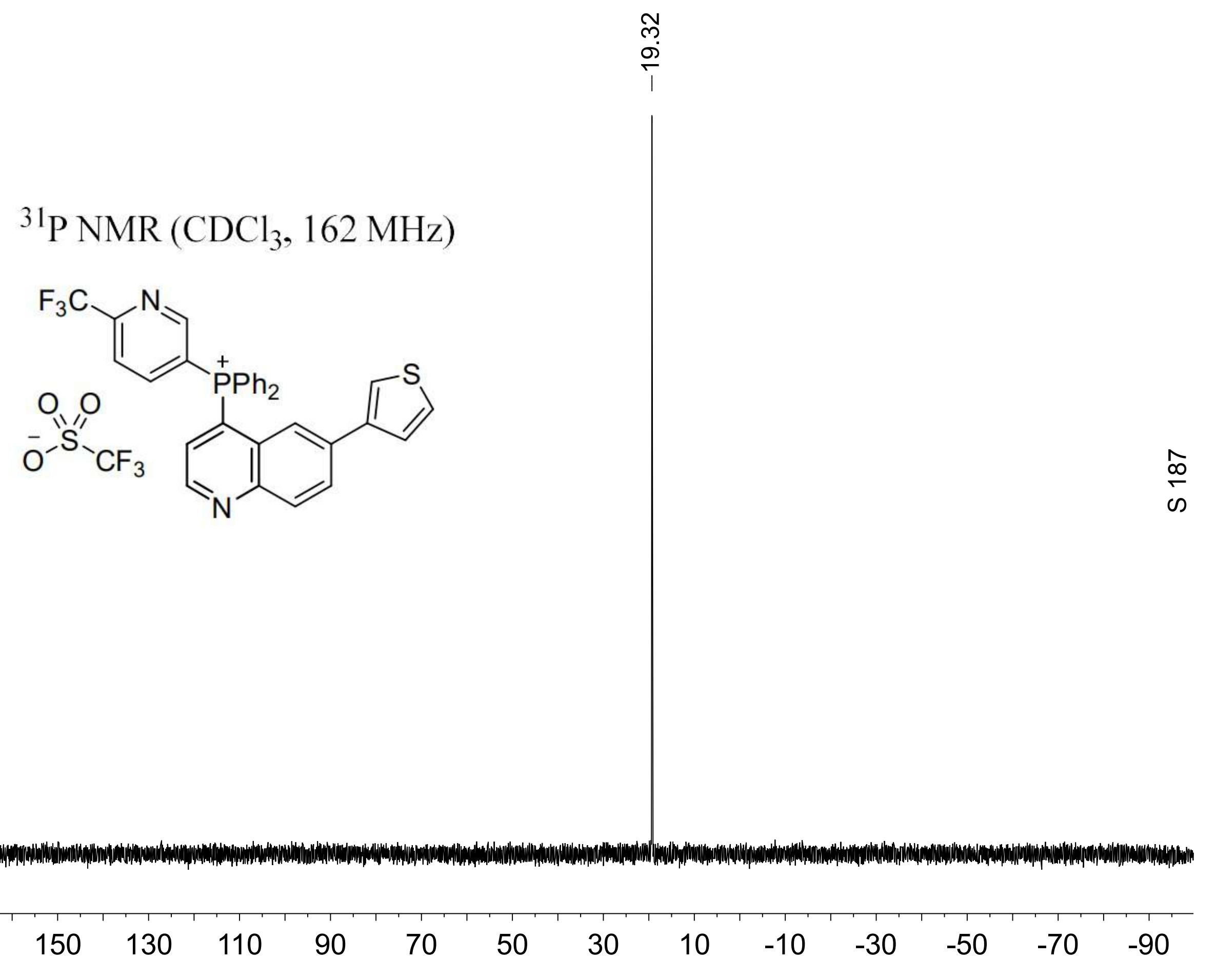




\section{${ }^{1} \mathrm{H} \mathrm{NMR}\left(\mathrm{CDCl}_{3}, 400 \mathrm{MHz}\right)$}<smiles>Cc1cc([PH+](c2ccccc2)c2ccc(C(F)(F)F)nc2)nc2ccccc12</smiles>

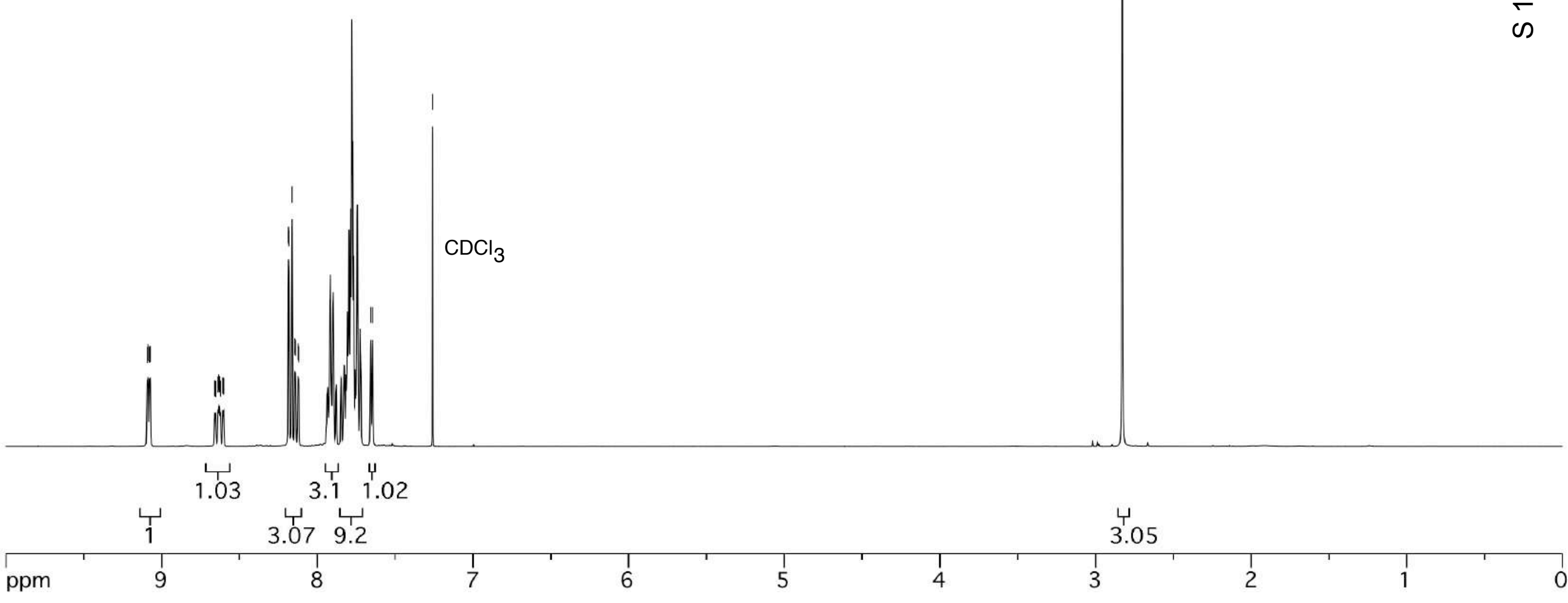


№-

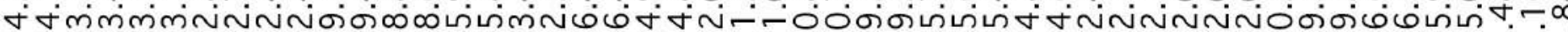

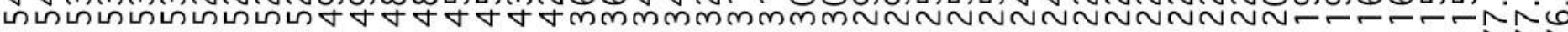

${ }^{13} \mathrm{C} \mathrm{NMR}\left(\mathrm{CDCl}_{3}, 100 \mathrm{MHz}\right)$<smiles>O=[N+]([O-])c1cc(P(c2ccccc2)c2ccc(C(F)(F)F)nc2)nc2ccccc12</smiles> 
${ }^{19} \mathrm{~F} \mathrm{NMR}\left(\mathrm{CDCl}_{3}, 365 \mathrm{MHz}\right)$

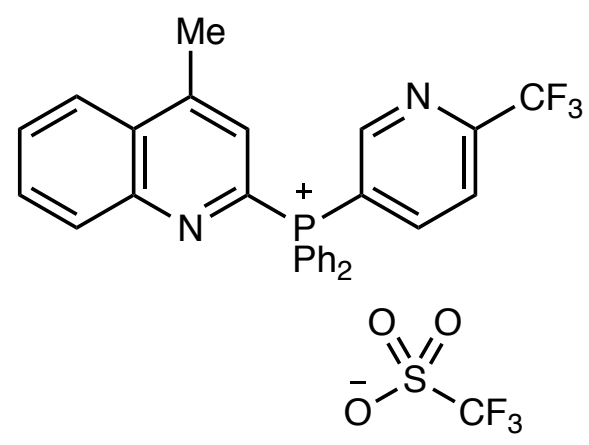

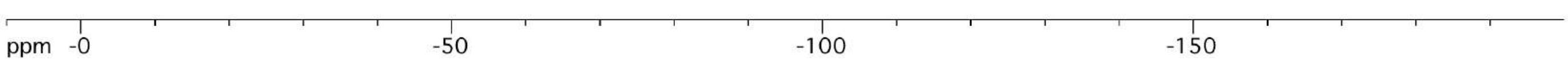


${ }^{31} \mathrm{P} \mathrm{NMR}\left(\mathrm{CDCl}_{3}, 162 \mathrm{MHz}\right)$

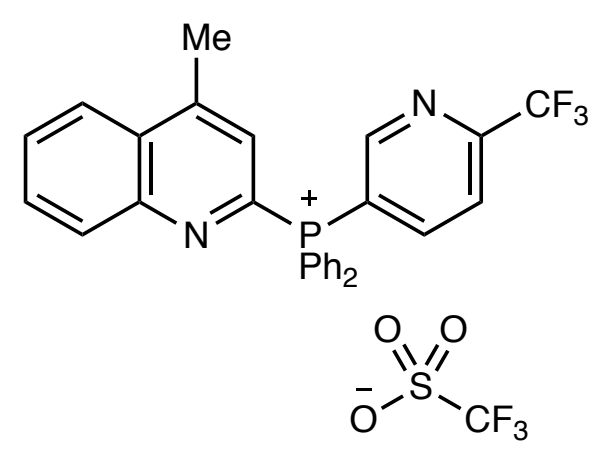


${ }^{1} \mathrm{H} \mathrm{NMR}\left(\mathrm{CDCl}_{3}, 400 \mathrm{MHz}\right)$

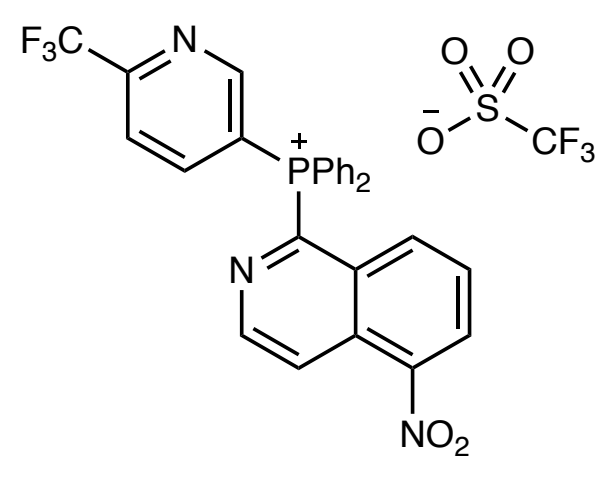


${ }^{13} \mathrm{C} \mathrm{NMR}\left(\mathrm{CDCl}_{3}, 100 \mathrm{MHz}\right)$<smiles>O=[N+]([O-])c1cccc2c([PH+]([O-])c3ccc(C(F)(F)F)nc3)nccc12</smiles> 
${ }^{19} \mathrm{~F} \mathrm{NMR}\left(\mathrm{CDCl}_{3}, 365 \mathrm{MHz}\right)$

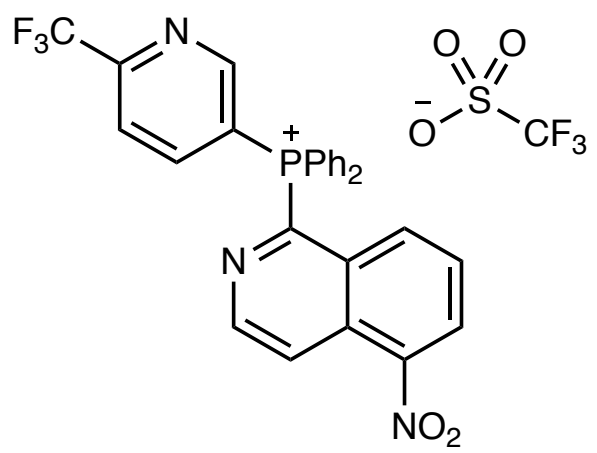

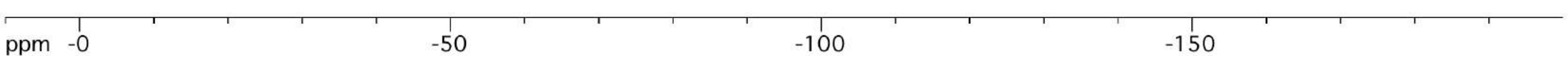


${ }^{31} \mathrm{P}$ NMR $\left(\mathrm{CDCl}_{3}, 162 \mathrm{MHz}\right)$

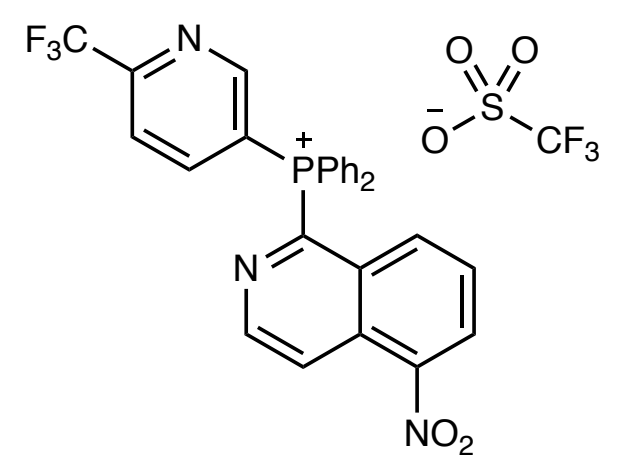




\section{${ }^{1} \mathrm{H} \mathrm{NMR},\left(\mathrm{CDCl}_{3}, 400 \mathrm{MHz}\right)$}<smiles>O=S(=O)([O-])C(F)(F)F</smiles>

$7: 1$ mixture of product:impurity

O

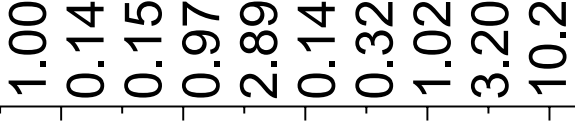




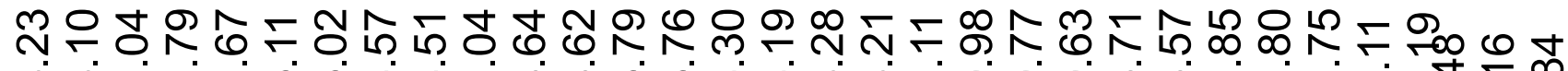

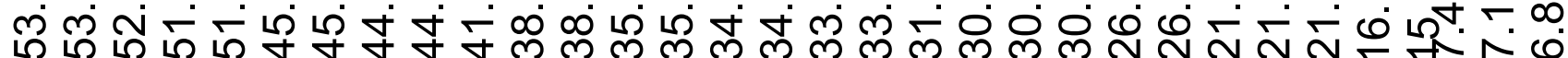
员

\section{${ }^{13} \mathrm{C} \mathrm{NMR},\left(\mathrm{CDCl}_{3}, 100 \mathrm{MHz}\right)$}

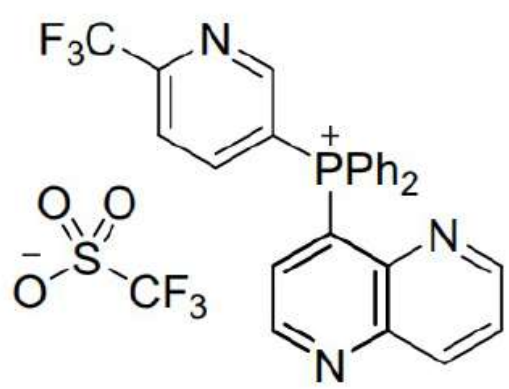

7:1 mixture of product:impurity 


\section{${ }^{19} \mathrm{~F} \mathrm{NMR}\left(\mathrm{CDCl}_{3}, 365 \mathrm{MHz}\right)$}

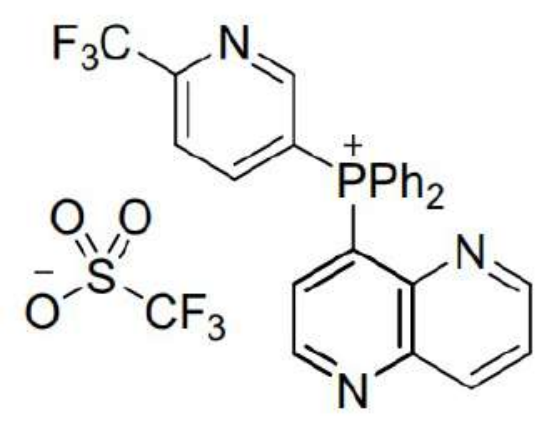

$7: 1$ mixture of product:impurity

$0 \quad-20$

$\begin{array}{lll}-20 & -40 \quad-60\end{array}$

$-80 \quad-100$

$-120$

$-140$

$-160$

$-180$

$-200$

$-2$ 
${ }^{31} \mathrm{P} \mathrm{NMR}\left(\mathrm{CDCl}_{3}, 162 \mathrm{MHz}\right)$<smiles>O=S(=O)([O-])C(F)(F)F</smiles>

7:1 mixture of product:impurity 

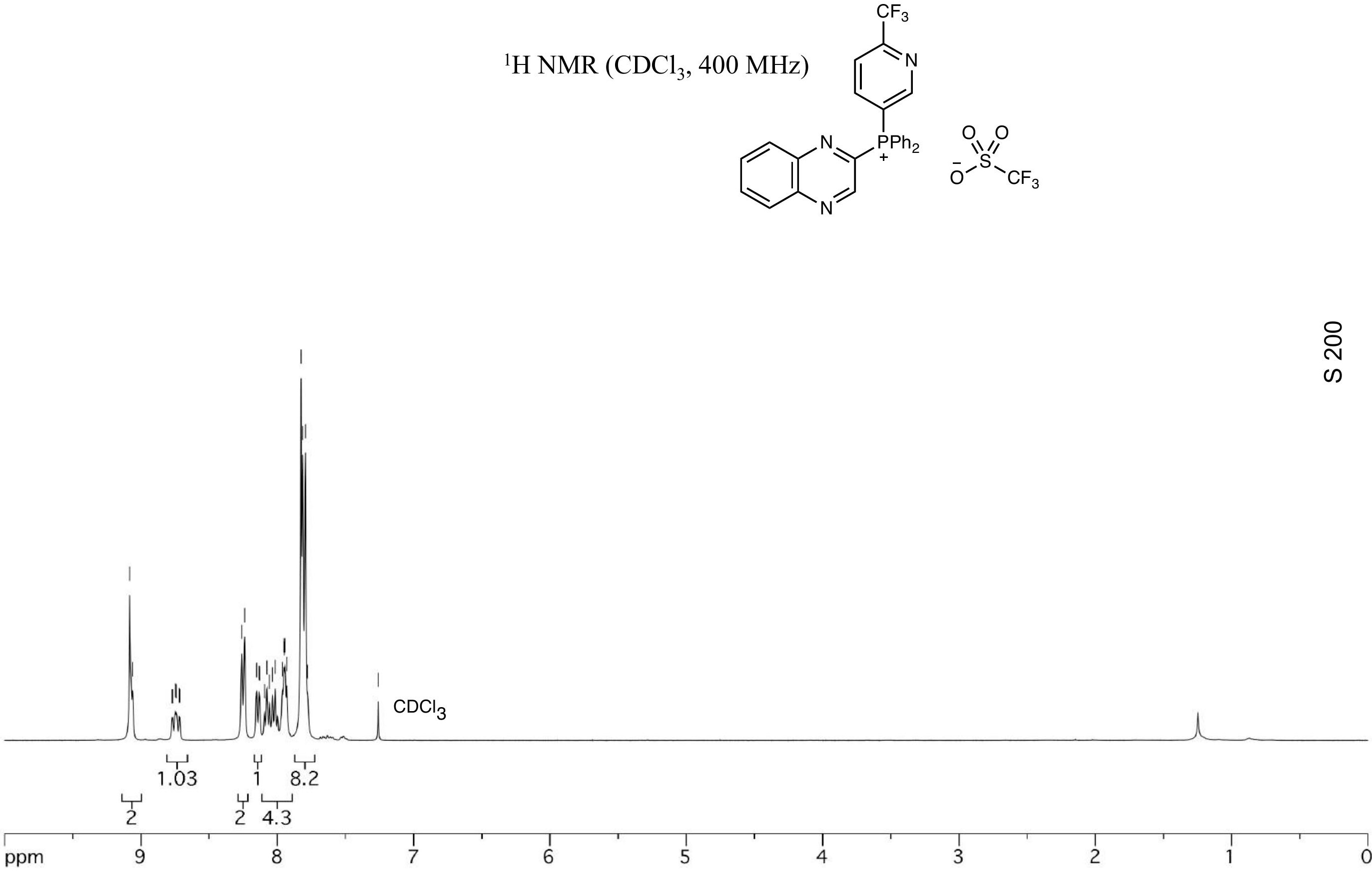
M-N

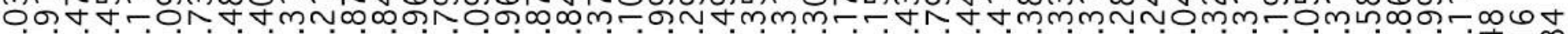

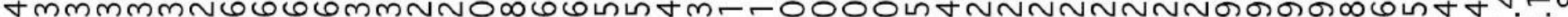

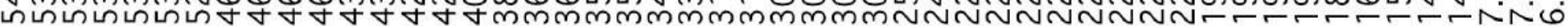
$-r-N \wedge N$

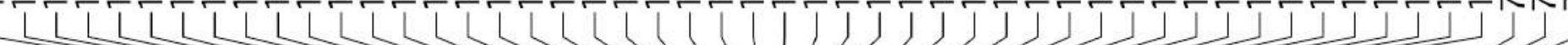

${ }^{13} \mathrm{C} \mathrm{NMR}\left(\mathrm{CDCl}_{3}, 100 \mathrm{MHz}\right)$<smiles>O=S(=O)([O-])C(F)(F)F</smiles>

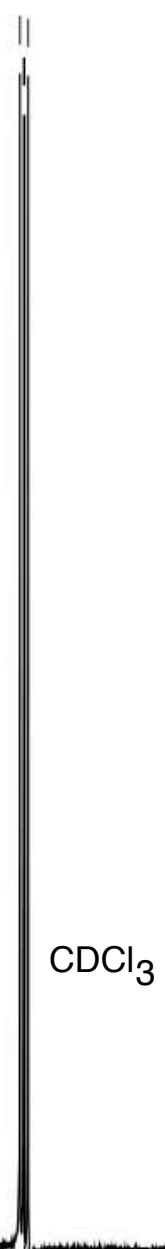


${ }^{19} \mathrm{~F} \mathrm{NMR}\left(\mathrm{CDCl}_{3}, 365 \mathrm{MHz}\right)$

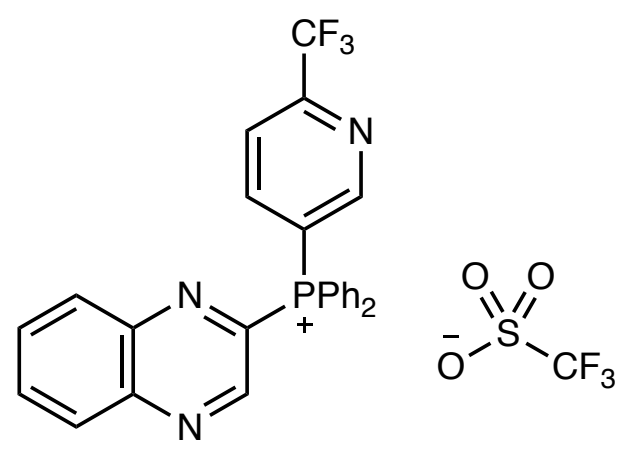

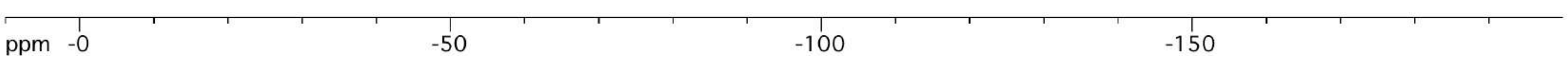


${ }^{31} \mathrm{P} \mathrm{NMR}\left(\mathrm{CDCl}_{3}, 162 \mathrm{MHz}\right)$

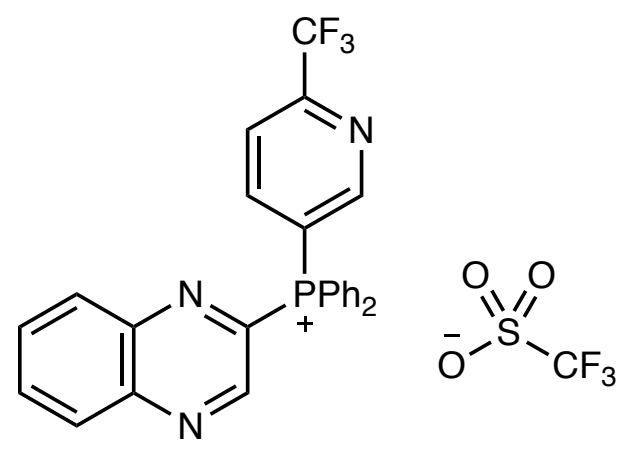


${ }^{13} \mathrm{C} \mathrm{NMR}\left(\mathrm{CDCl}_{3}, 100 \mathrm{MHz}\right)$<smiles>C[Pb](c1ccc(C(F)(F)F)nc1)c1ccnc2ccnn12</smiles> 
${ }^{31} \mathrm{P} \mathrm{NMR}\left(\mathrm{CDCl}_{3}, 162 \mathrm{MHz}\right)$<smiles>O=S(=O)(O)C(F)(F)F</smiles> 
${ }^{1} \mathrm{H} \mathrm{NMR}\left(\mathrm{CDCl}_{3}, 400 \mathrm{MHz}\right)$

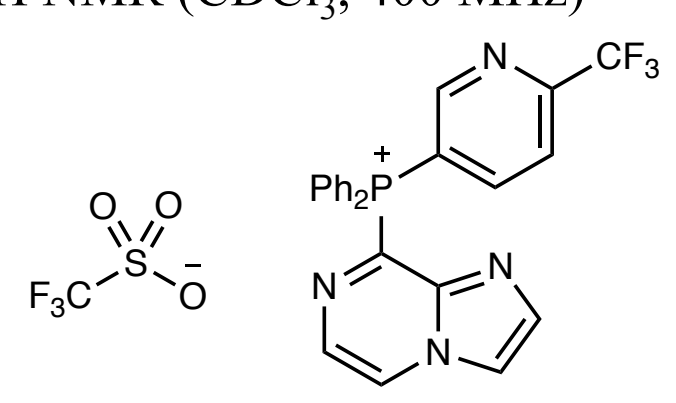

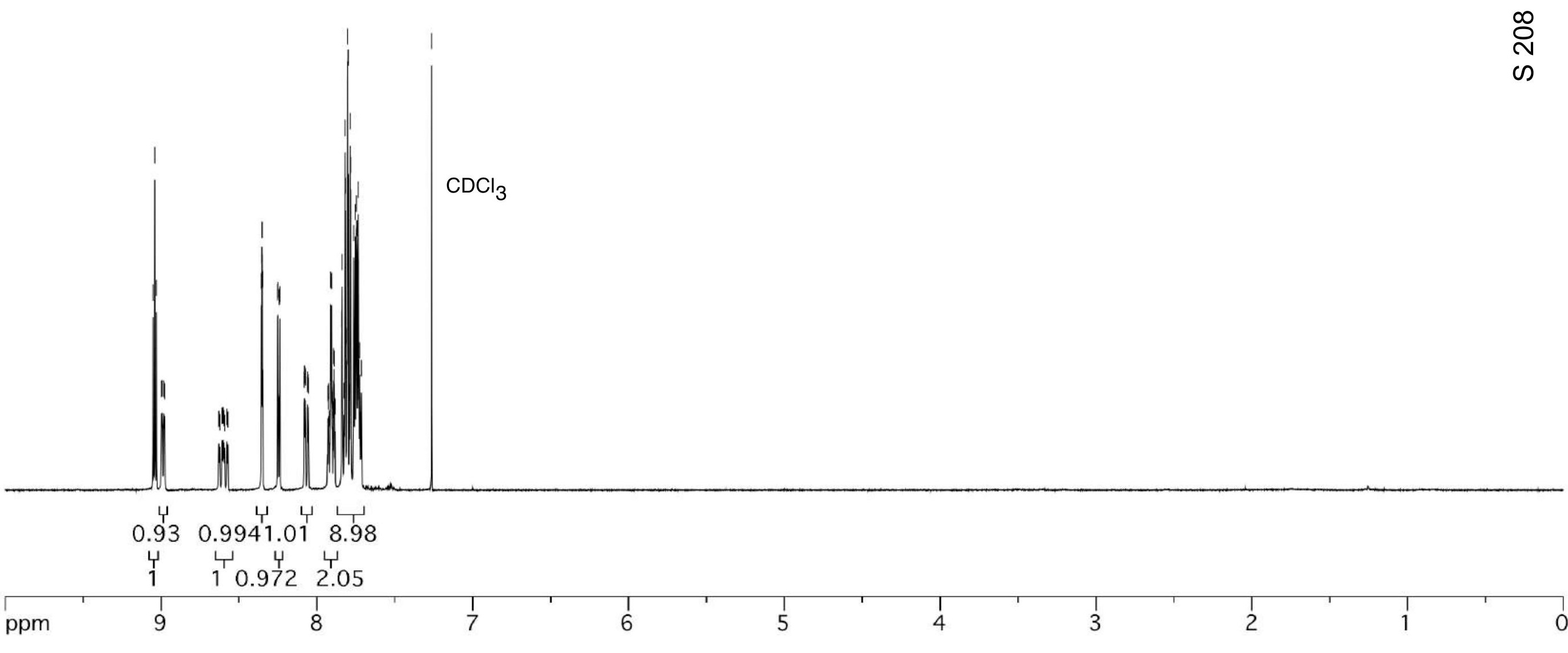


ํำ

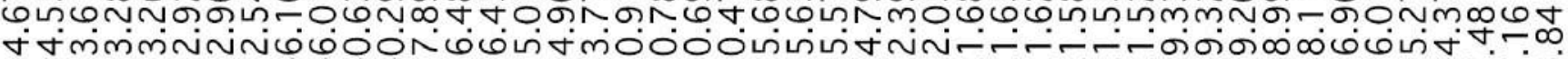

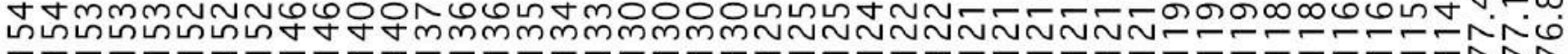

${ }^{13} \mathrm{C} \mathrm{NMR}\left(\mathrm{CDCl}_{3}, 100 \mathrm{MHz}\right)$

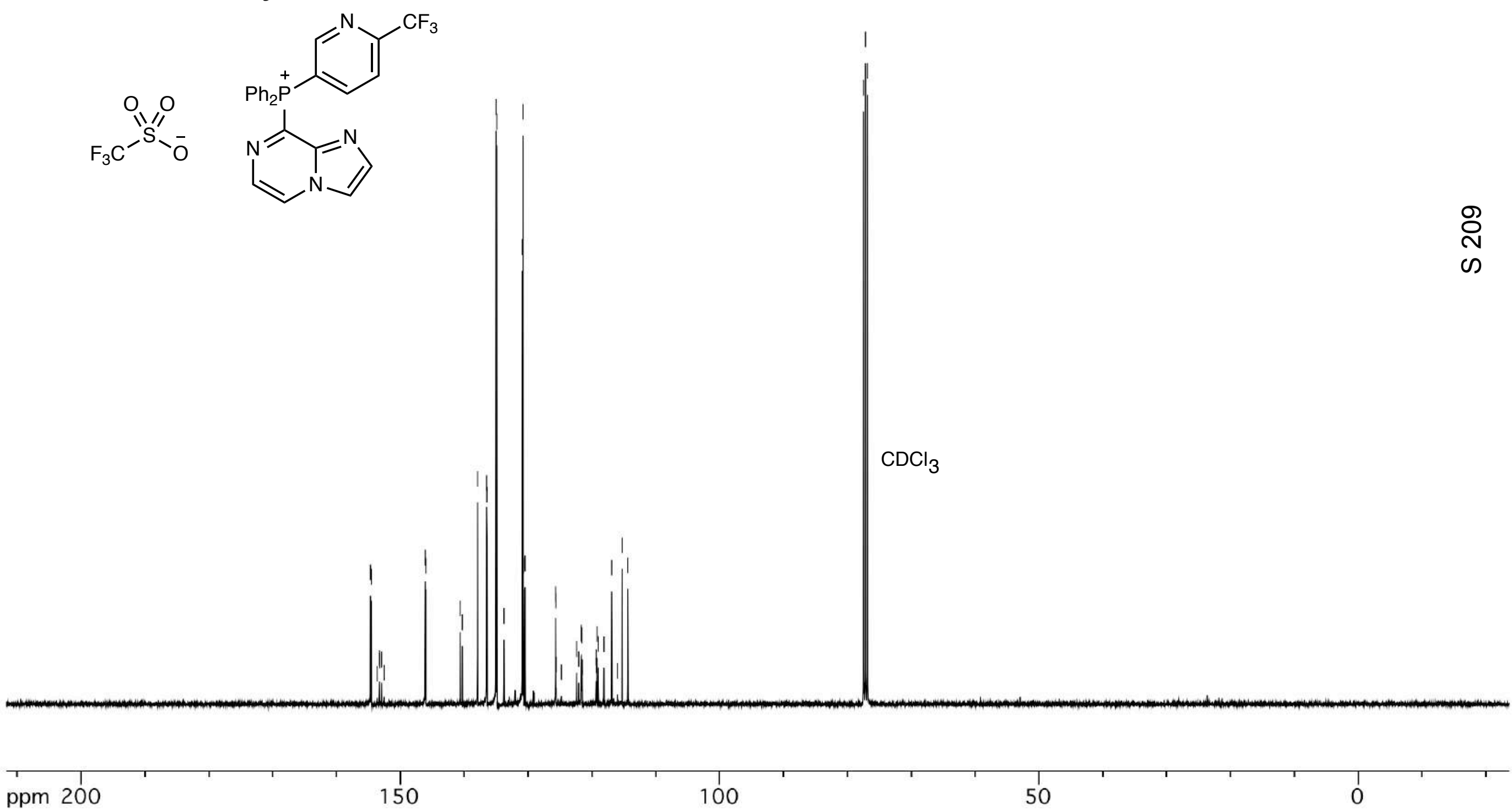


${ }^{19} \mathrm{~F} \mathrm{NMR}\left(\mathrm{CDCl}_{3}, 365 \mathrm{MHz}\right)$

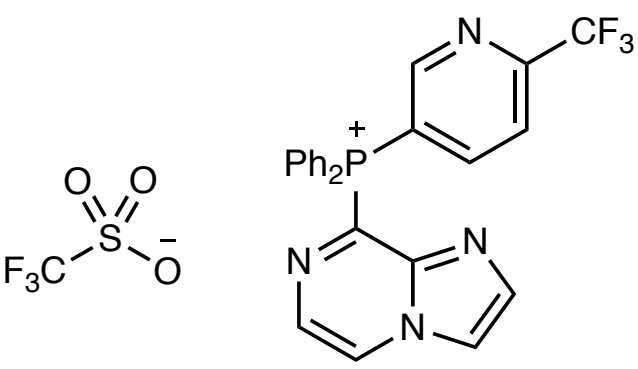

\begin{tabular}{|c|c|c|}
\hline $\begin{array}{ll} & 1 \\
\mathrm{ppm} & -0\end{array}$ & -50 & -100 \\
\hline
\end{tabular}


${ }^{31} \mathrm{P} \mathrm{NMR}\left(\mathrm{CDCl}_{3}, 162 \mathrm{MHz}\right)$

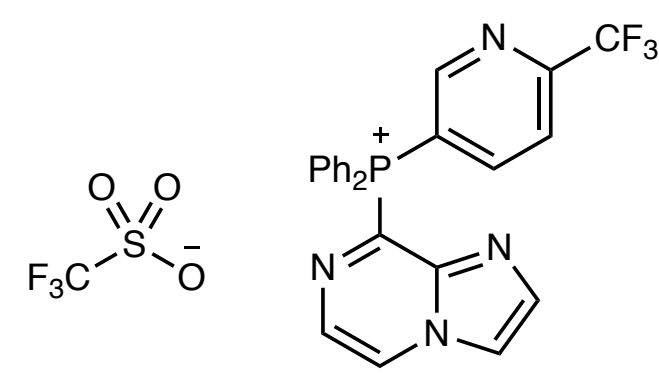


${ }^{1} \mathrm{H} \mathrm{NMR}\left(\mathrm{CDCl}_{3}, 400 \mathrm{MHz}\right)$<smiles>O=S(=O)([O-])C(F)(F)F</smiles>

41

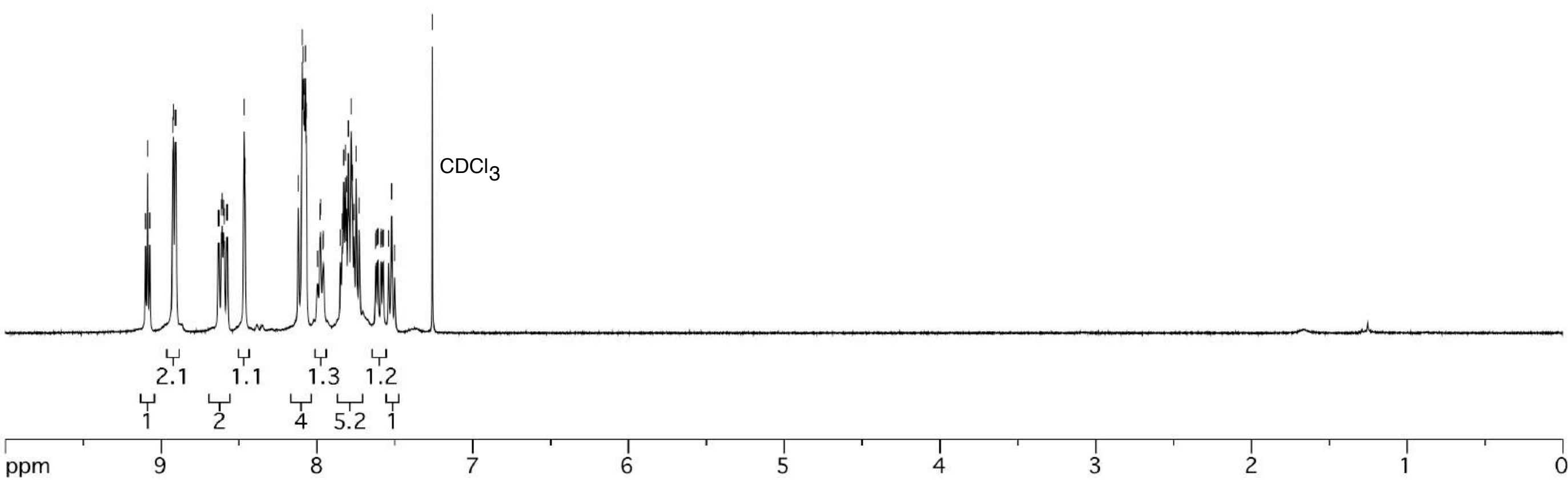


${ }^{13} \mathrm{C} \mathrm{NMR}\left(\mathrm{CDCl}_{3}, 100 \mathrm{MHz}\right)$<smiles>O=S(=O)(O)C(F)(F)F</smiles> 
${ }^{19} \mathrm{~F}$ NMR $\left(\mathrm{CDCl}_{3}, 365 \mathrm{MHz}\right)$

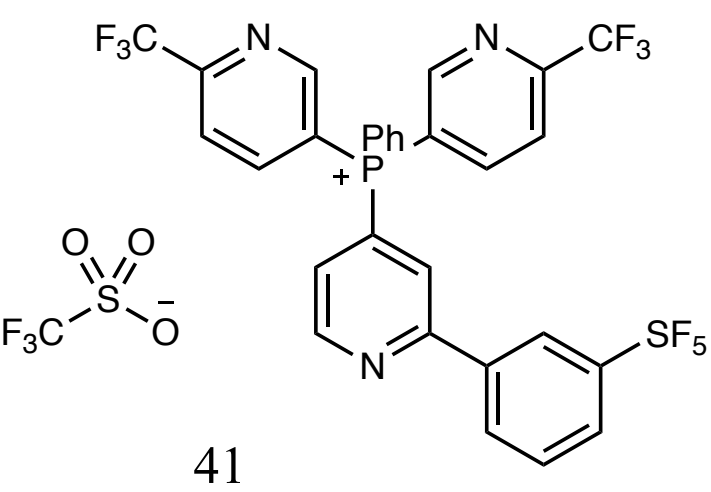


${ }^{31} \mathrm{P} \mathrm{NMR}\left(\mathrm{CDCl}_{3}, 162 \mathrm{MHz}\right)$

41

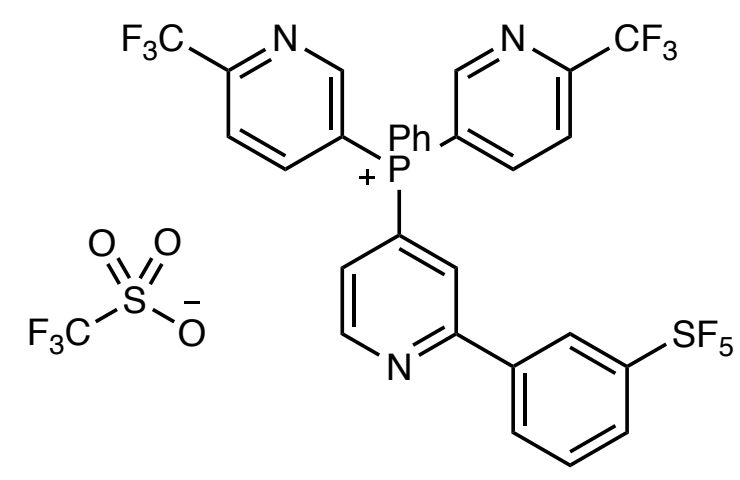




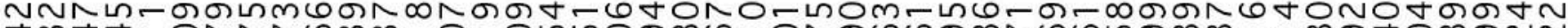

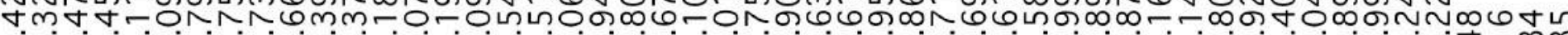

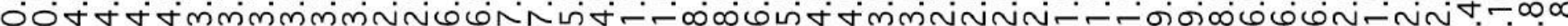
6ढn -NN

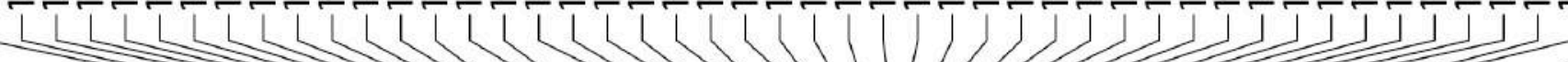

${ }^{13} \mathrm{C} \mathrm{NMR}\left(\mathrm{CDCl}_{3}, 100 \mathrm{MHz}\right)$<smiles>O=S(=O)(O)C(F)(F)C(F)(F)F</smiles> 
${ }^{19} \mathrm{~F} \mathrm{NMR}\left(\mathrm{CDCl}_{3}, 365 \mathrm{MHz}\right)$

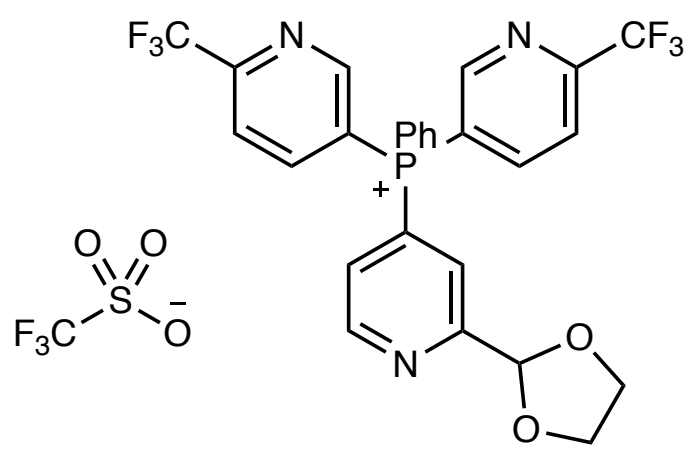

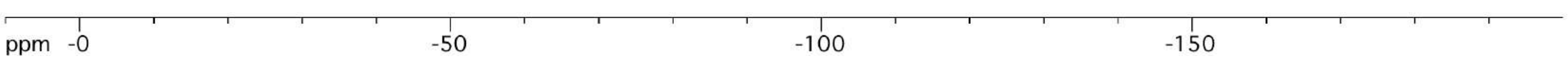


${ }^{31} \mathrm{P} \mathrm{NMR}\left(\mathrm{CDCl}_{3}, 162 \mathrm{MHz}\right)$

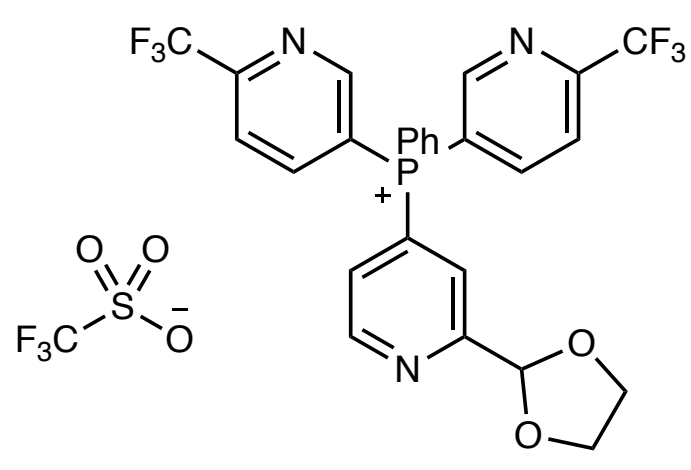


${ }^{1} \mathrm{H} \mathrm{NMR},\left(\mathrm{CDCl}_{3}, 400 \mathrm{MHz}\right)$<smiles>O=S(=O)([O-])C(c1ccccc1)c1cc([P+](c2ccc(C(F)(F)F)nc2)c2ccc(C(F)(F)F)nc2)ccn1</smiles>

$\mathrm{CDCl}_{3}$

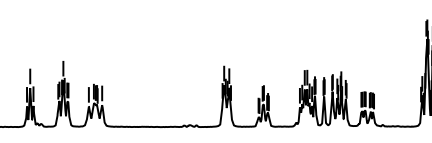

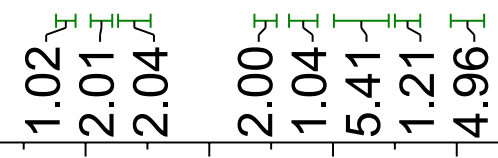




\section{${ }^{13} \mathrm{C} \mathrm{NMR},\left(\mathrm{CDCl}_{3}, 100 \mathrm{MHz}\right)$}<smiles>O=S(=O)(O)C(F)(F)F</smiles> 


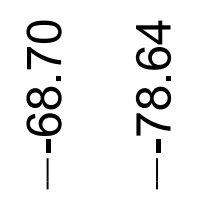

\section{${ }^{19} \mathrm{~F} \mathrm{NMR}\left(\mathrm{CDCl}_{3}, 365 \mathrm{MHz}\right)$}

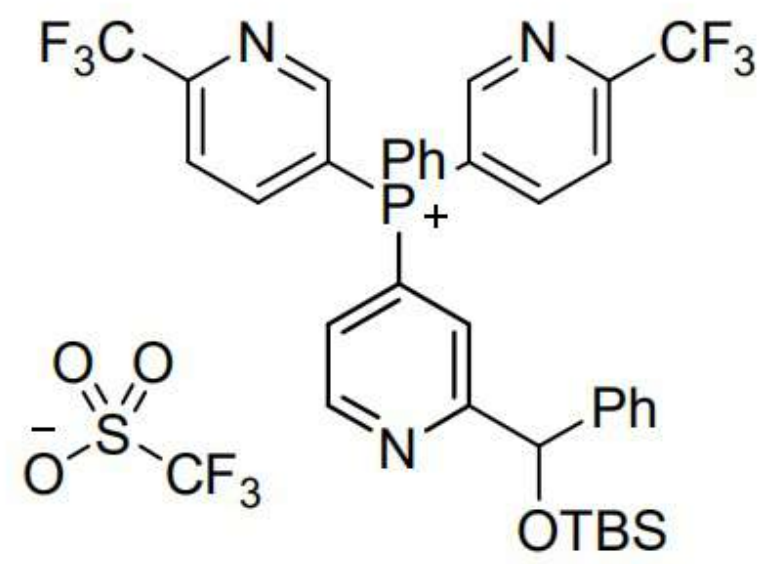

$0-20-40$

$-40-60$

$\begin{array}{ll}-80 & -100\end{array}$

$-120$

$-140$

$-160$

$-180$

$-200-2 i$ 
${ }^{31} \mathrm{P} \mathrm{NMR}\left(\mathrm{CDCl}_{3}, 162 \mathrm{MHz}\right)$<smiles>O=S(=O)([O-])C(F)(F)F</smiles> 


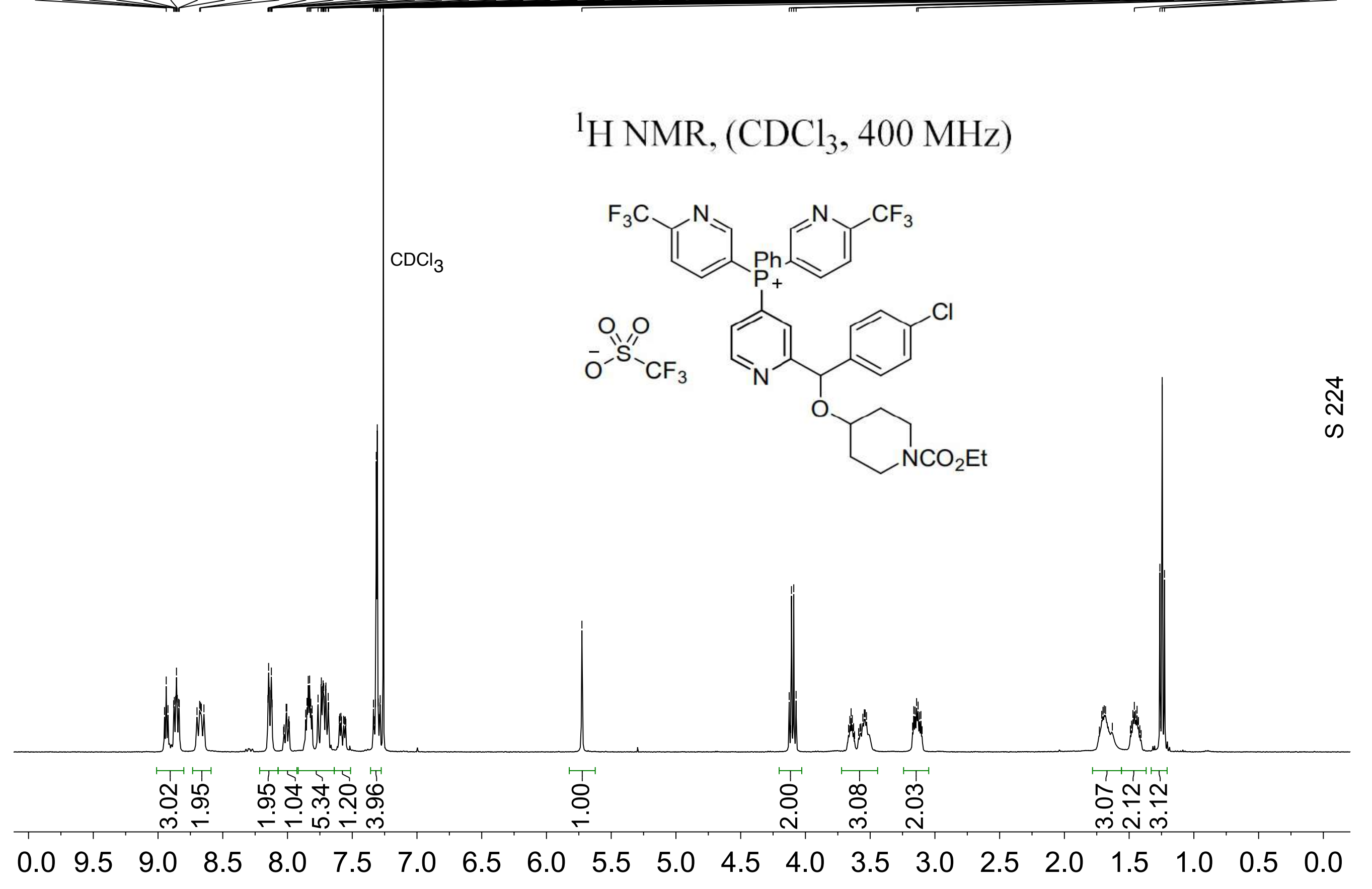




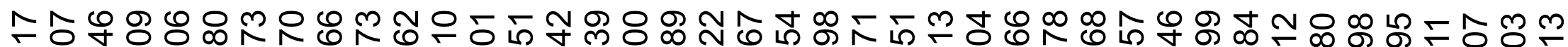

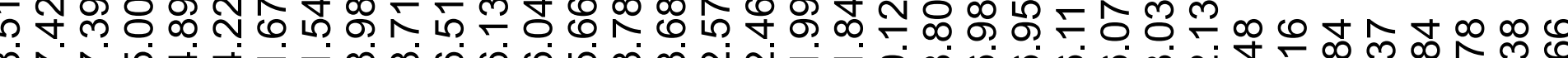
น

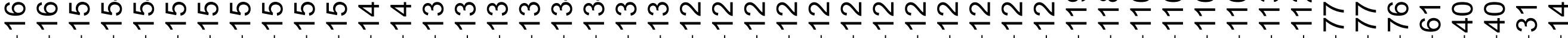

${ }^{13} \mathrm{C} \mathrm{NMR},\left(\mathrm{CDCl}_{3}, 100 \mathrm{MHz}\right)$<smiles>CCON1CCC(OC(c2ccc(Cl)cc2)c2cc([P+](c3ccc(C(F)(F)F)nc3)c3ccc(C(F)(F)F)cn3)ccn2)CC1</smiles> 


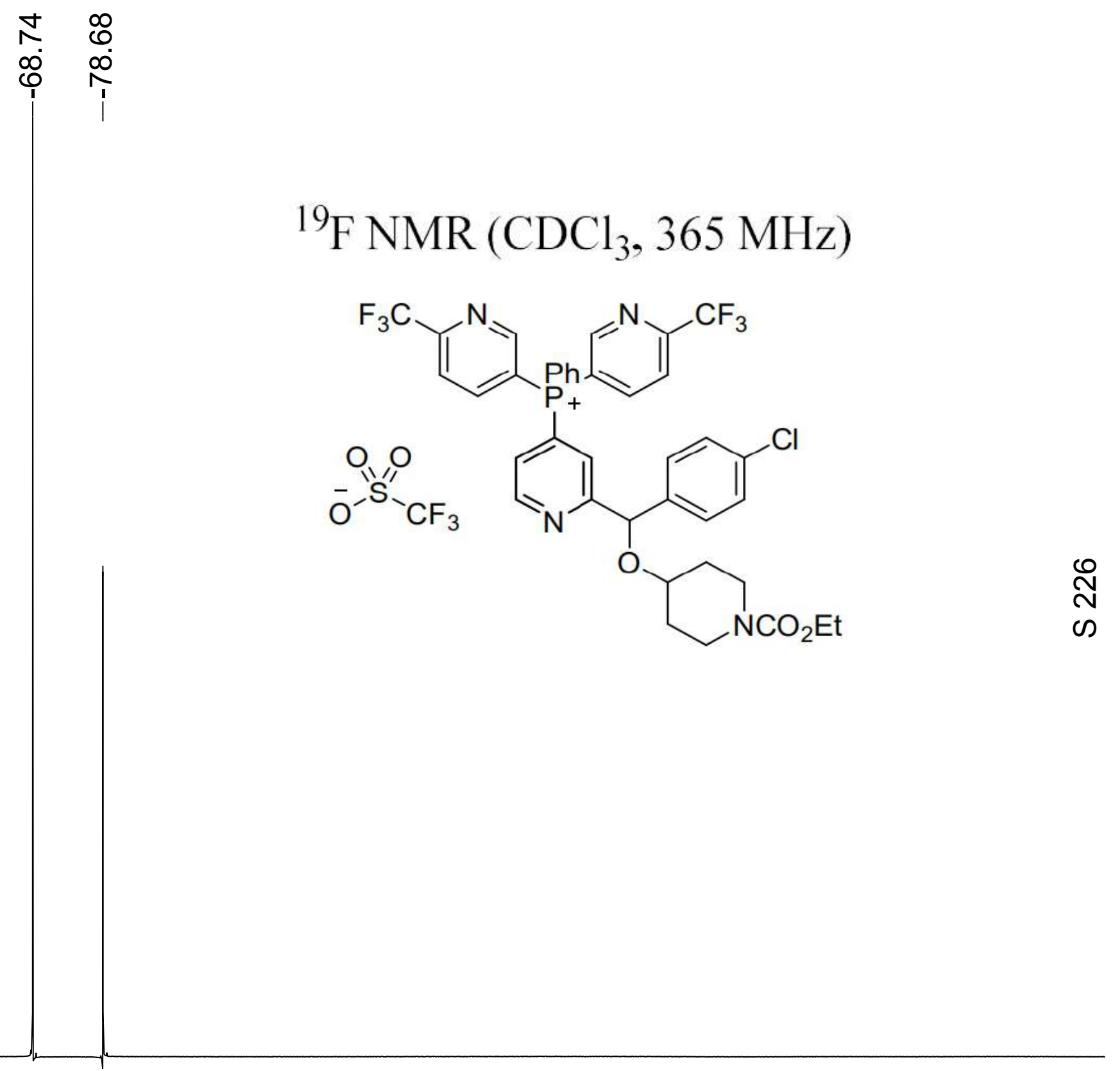

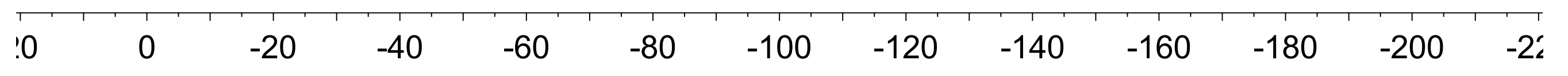




\section{${ }^{31} \mathrm{P} \mathrm{NMR}\left(\mathrm{CDCl}_{3}, 162 \mathrm{MHz}\right)$}

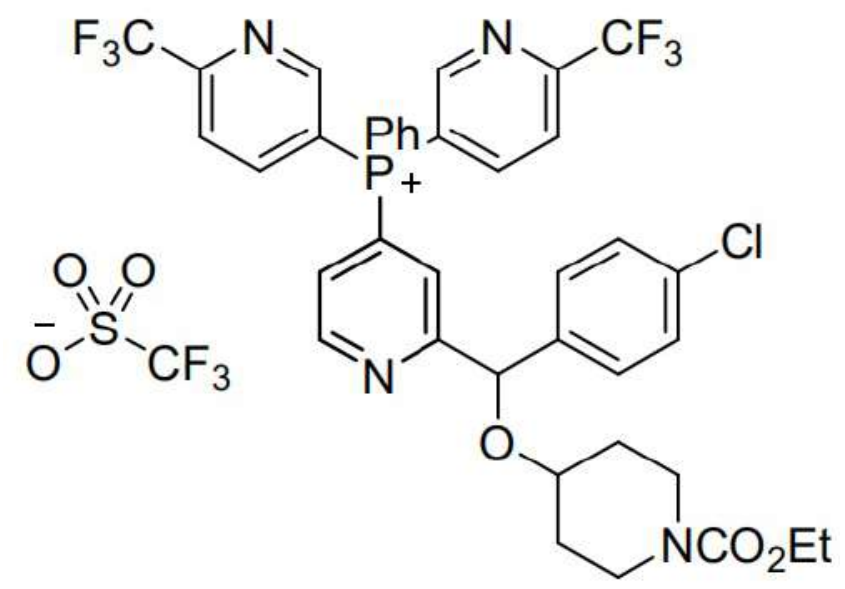

$\begin{array}{llllllllllllllll}190 & 170 & 150 & 130 & 110 & 90 & 70 & 50 & 30 & 10 & -10 & -30 & -50 & -70 & -90\end{array}$




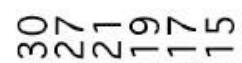
ச்சுச்ச்ச் mmก̃̄̃ minimin

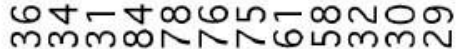

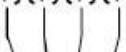

\section{${ }^{1} \mathrm{H} \mathrm{NMR}\left(\mathrm{CDCl}_{3}, 400 \mathrm{MHz}\right)$}<smiles>CCOC(=O)N1CC[C@@H](c2ccc(F)cc2)[C@H](COC(=O)c2c([PH+](c3ccc(F)cc3)c3ccc(C(F)(F)F)nc3)ccnc2C)C1</smiles>
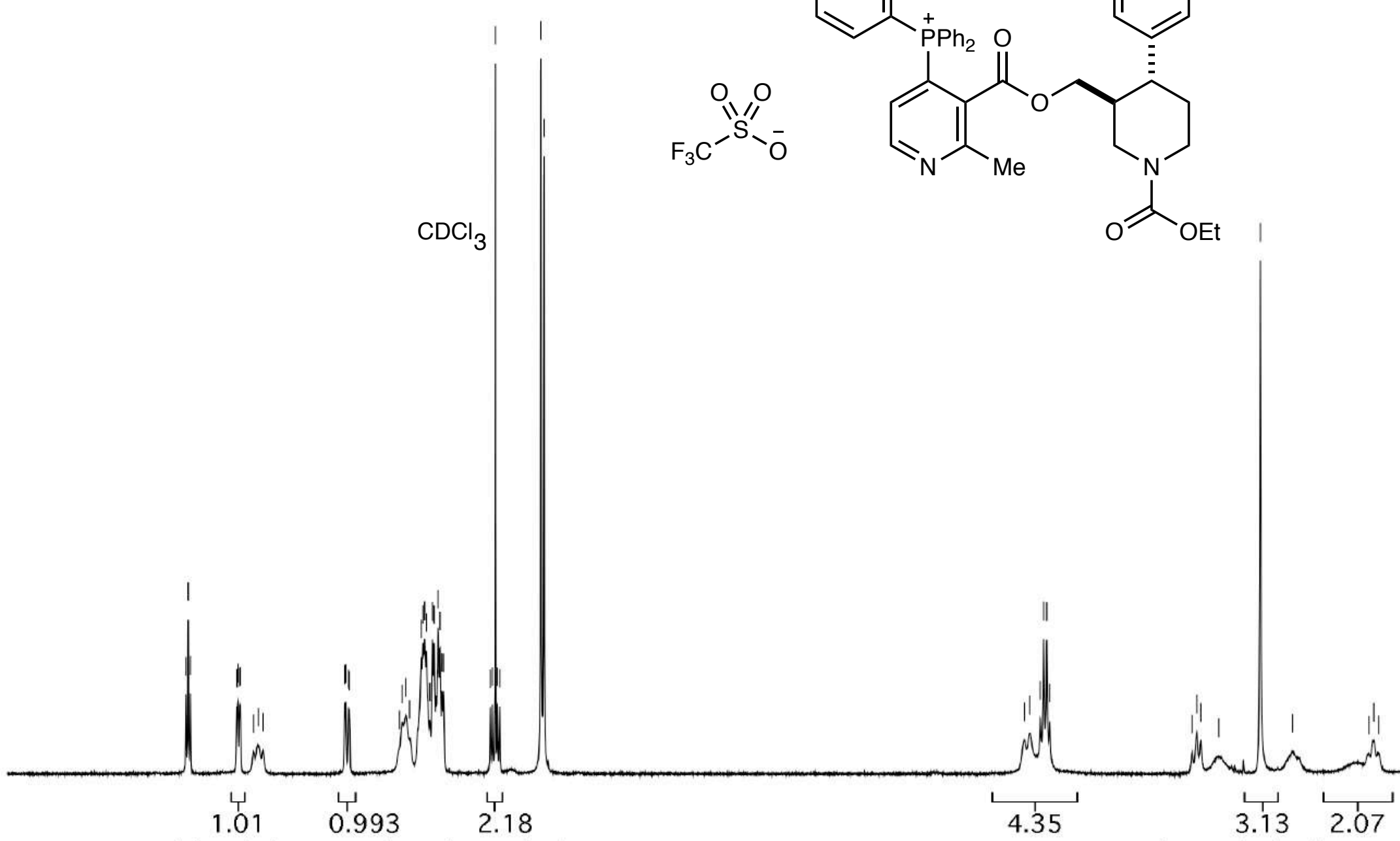
${ }^{13} \mathrm{C} \mathrm{NMR}\left(\mathrm{CDCl}_{3}, 100 \mathrm{MHz}\right)$<smiles>CCOC(=O)N1CC[C@@H](c2ccc(F)cc2)[C@H](COC(=O)c2c([PH+](c3ccc(F)cc3)c3ccc(C(F)(F)F)cn3)ccnc2C)C1</smiles> 

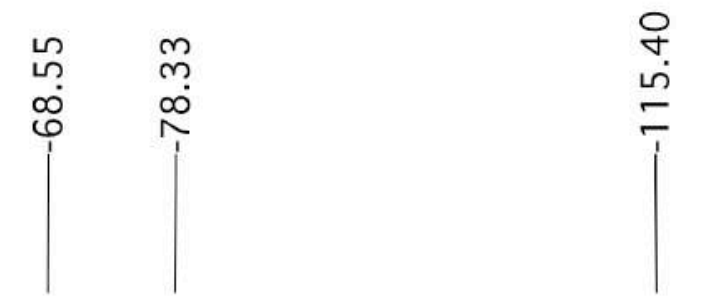

${ }^{19} \mathrm{~F}$ NMR $\left(\mathrm{CDCl}_{3}, 365 \mathrm{MHz}\right)$

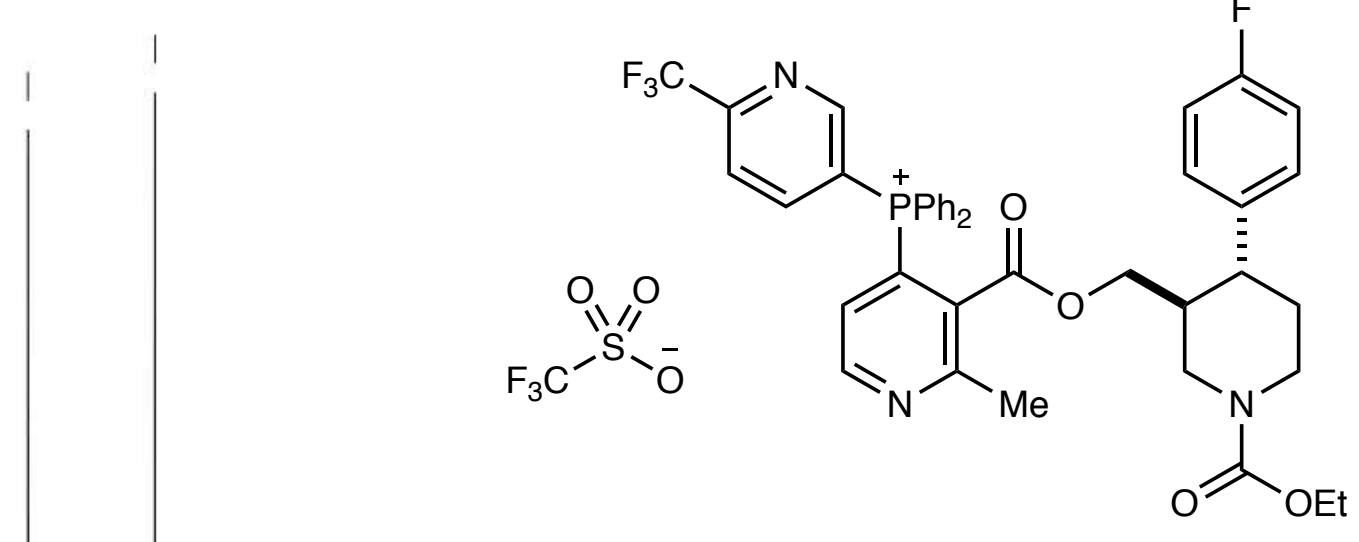

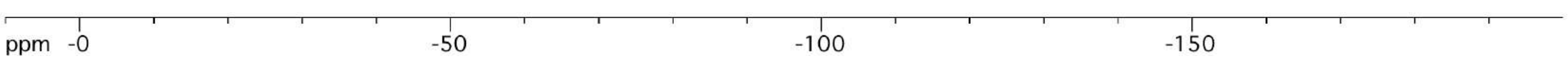


${ }^{31} \mathrm{P} \mathrm{NMR}\left(\mathrm{CDCl}_{3}, 162 \mathrm{MHz}\right)$

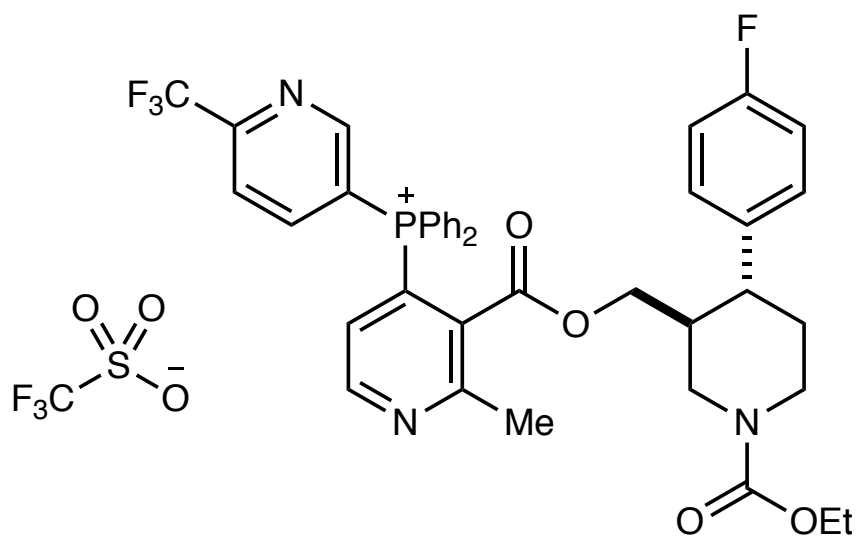




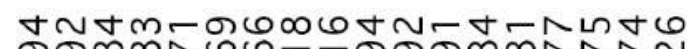

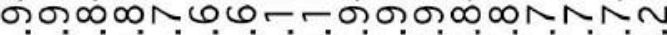
$\infty \infty^{\circ} \infty \infty^{\circ} \infty \infty^{\circ} N N N N N$

${ }^{1} \mathrm{H} \mathrm{NMR}\left(\mathrm{CDCl}_{3}, 400 \mathrm{MHz}\right)$

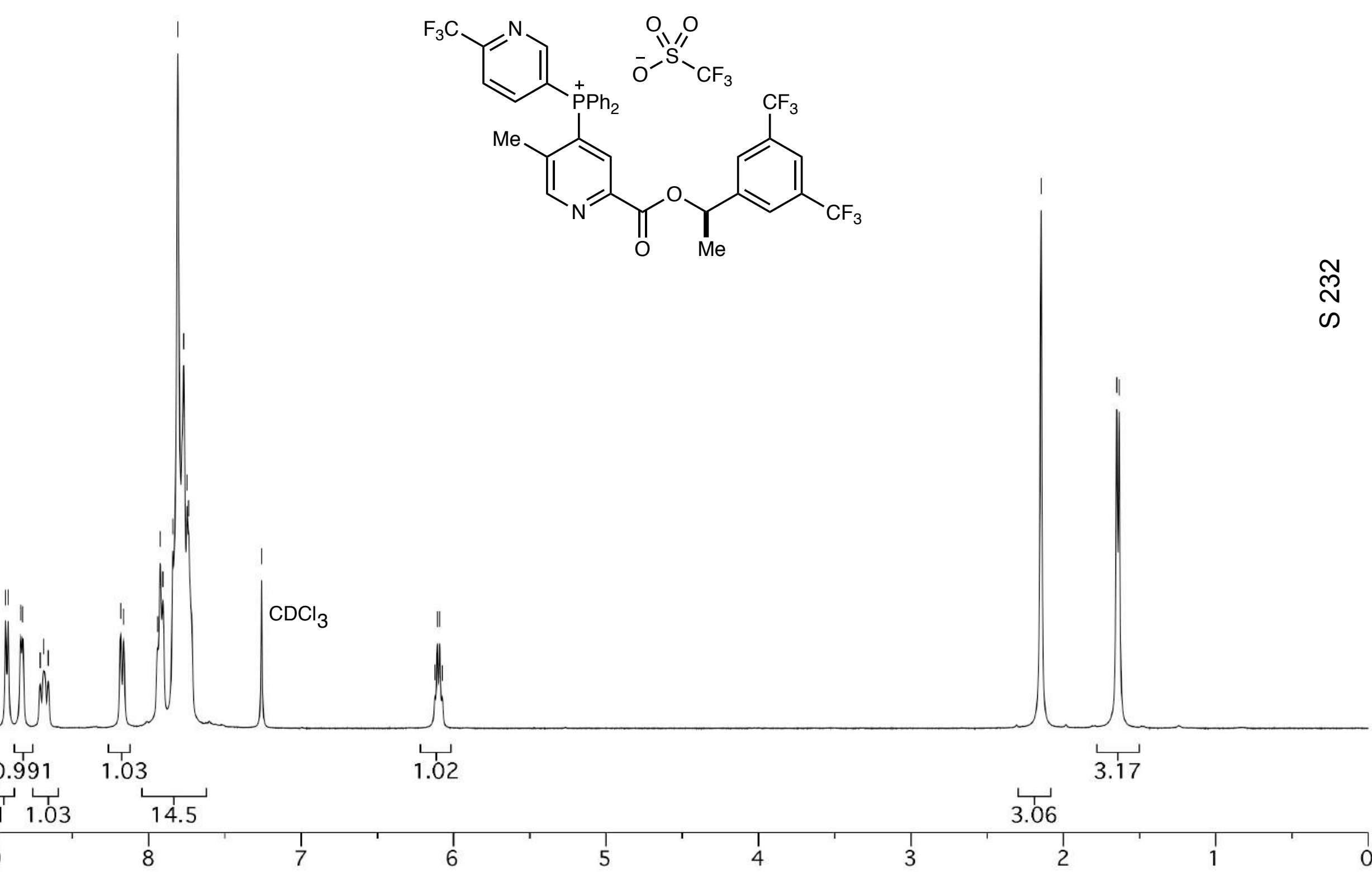


${ }^{13} \mathrm{C} \mathrm{NMR}\left(\mathrm{CDCl}_{3}, 100 \mathrm{MHz}\right)$<smiles>[Y4]C(OC(=O)c1cc([PH+]([O-])c2ccc(C(F)(F)F)nc2)c(C)cn1)c1cc(C(F)(F)F)cc(C(F)(F)F)c1</smiles> 


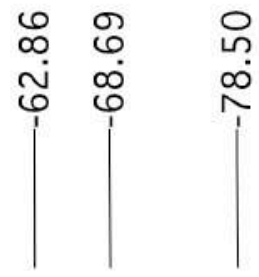

${ }^{19} \mathrm{~F} \mathrm{NMR}\left(\mathrm{CDCl}_{3}, 365 \mathrm{MHz}\right)$

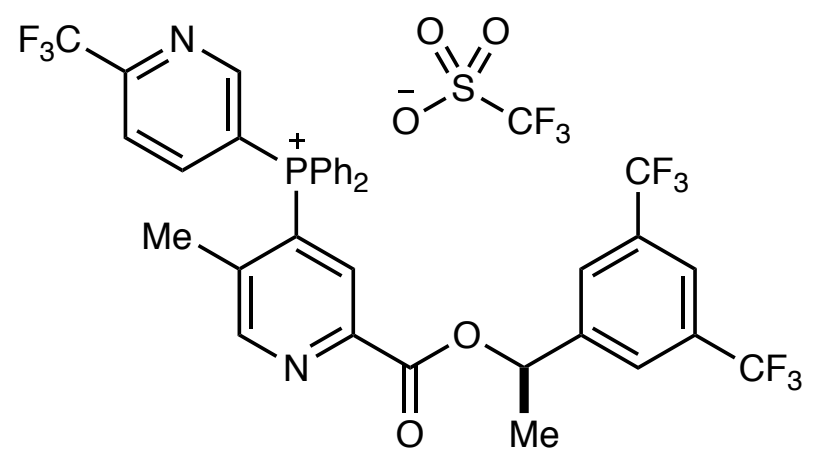

ָั

\begin{tabular}{|c|c|c|}
\hline $\begin{array}{ll} & 1 \\
\text { ppm }-0\end{array}$ & $\begin{array}{l}1 \\
-50\end{array}$ & $\begin{array}{l}1 \\
-100\end{array}$ \\
\hline
\end{tabular}


${ }^{31} \mathrm{P} \mathrm{NMR}\left(\mathrm{CDCl}_{3}, 162 \mathrm{MHz}\right)$

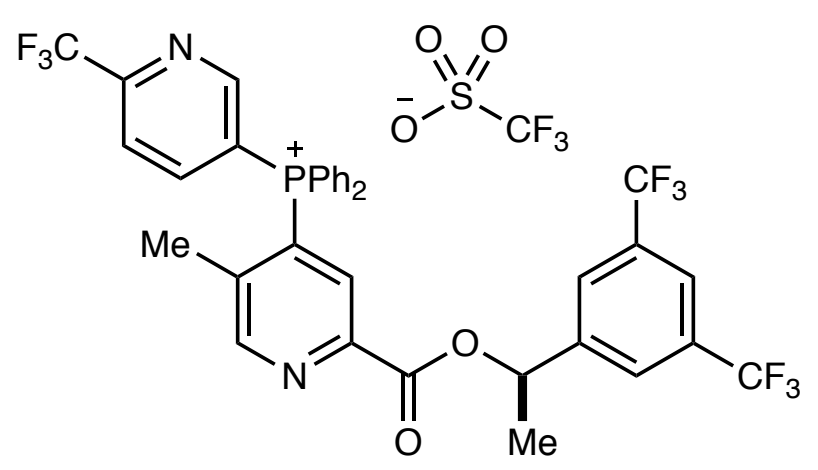


${ }^{13} \mathrm{C} \mathrm{NMR}\left(\mathrm{CDCl}_{3}, 100 \mathrm{MHz}\right)$

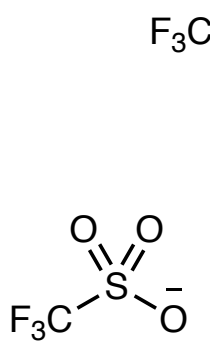

$\mathrm{F}_{3} \mathrm{C}$
$\mathrm{O}$<smiles>[BH3-]c1ccc(C)nc1</smiles><smiles>Cc1cc(F)cc(-c2ccc(C(F)(F)F)cn2)c1</smiles> 


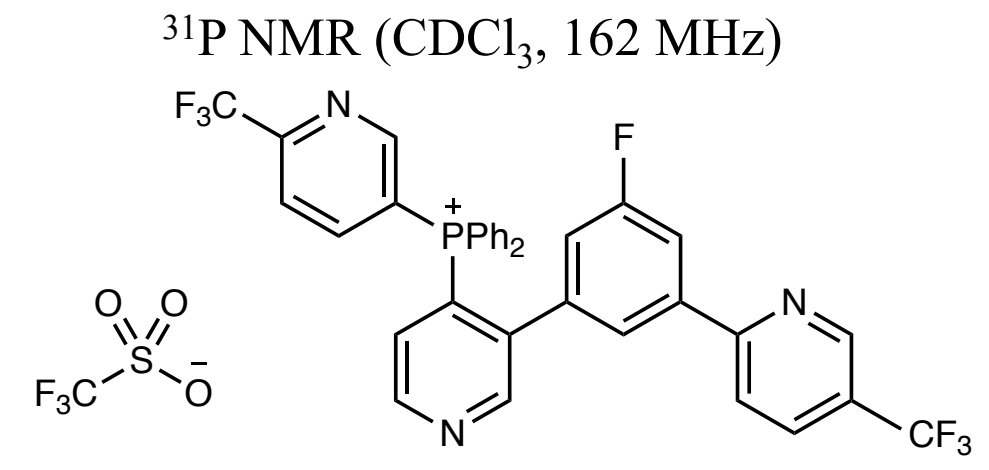




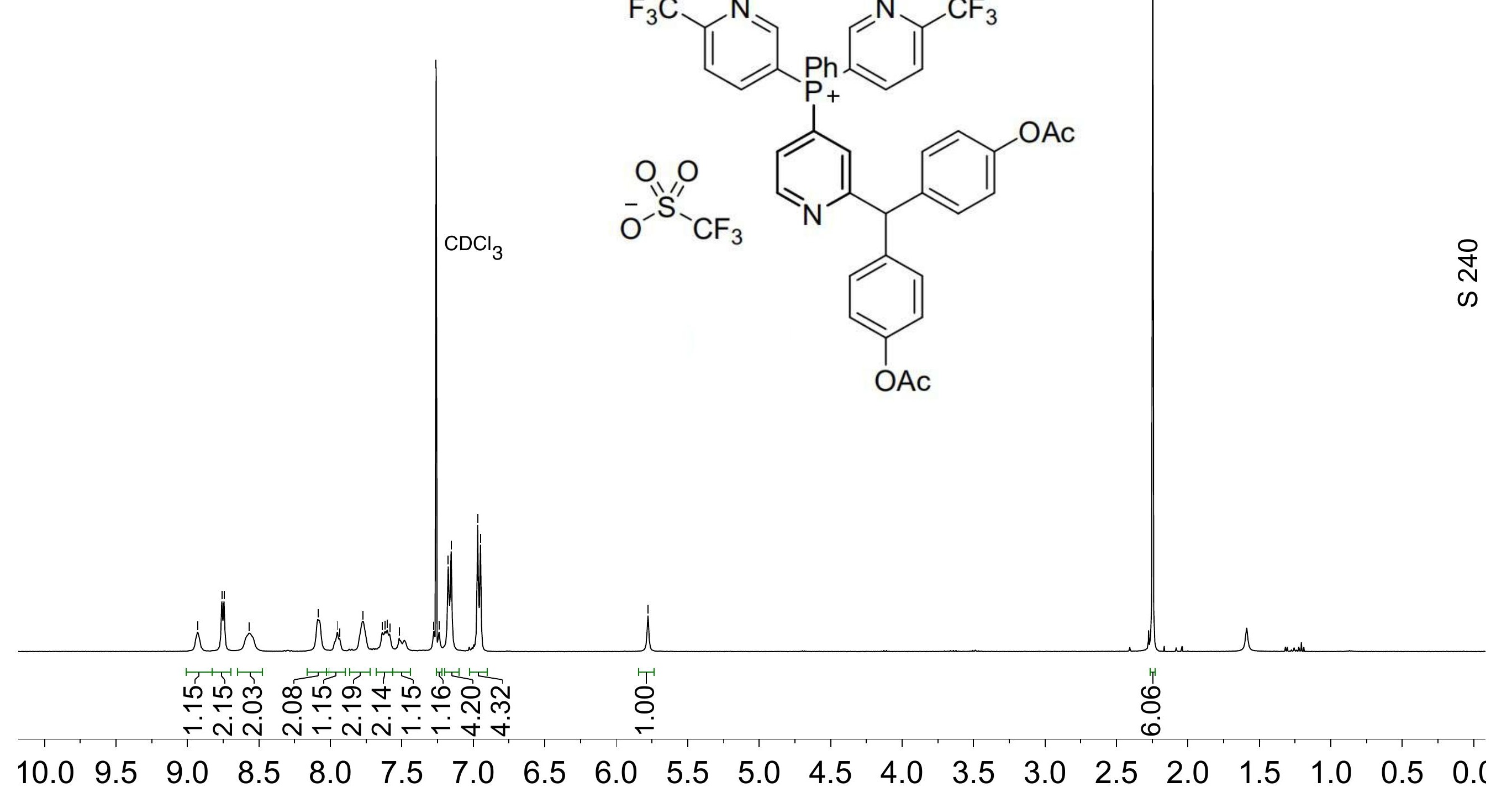


苟

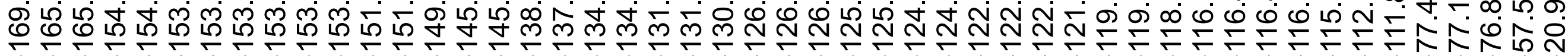

\section{${ }^{13} \mathrm{C} \mathrm{NMR},\left(\mathrm{CDCl}_{3}, 100 \mathrm{MHz}\right)$}
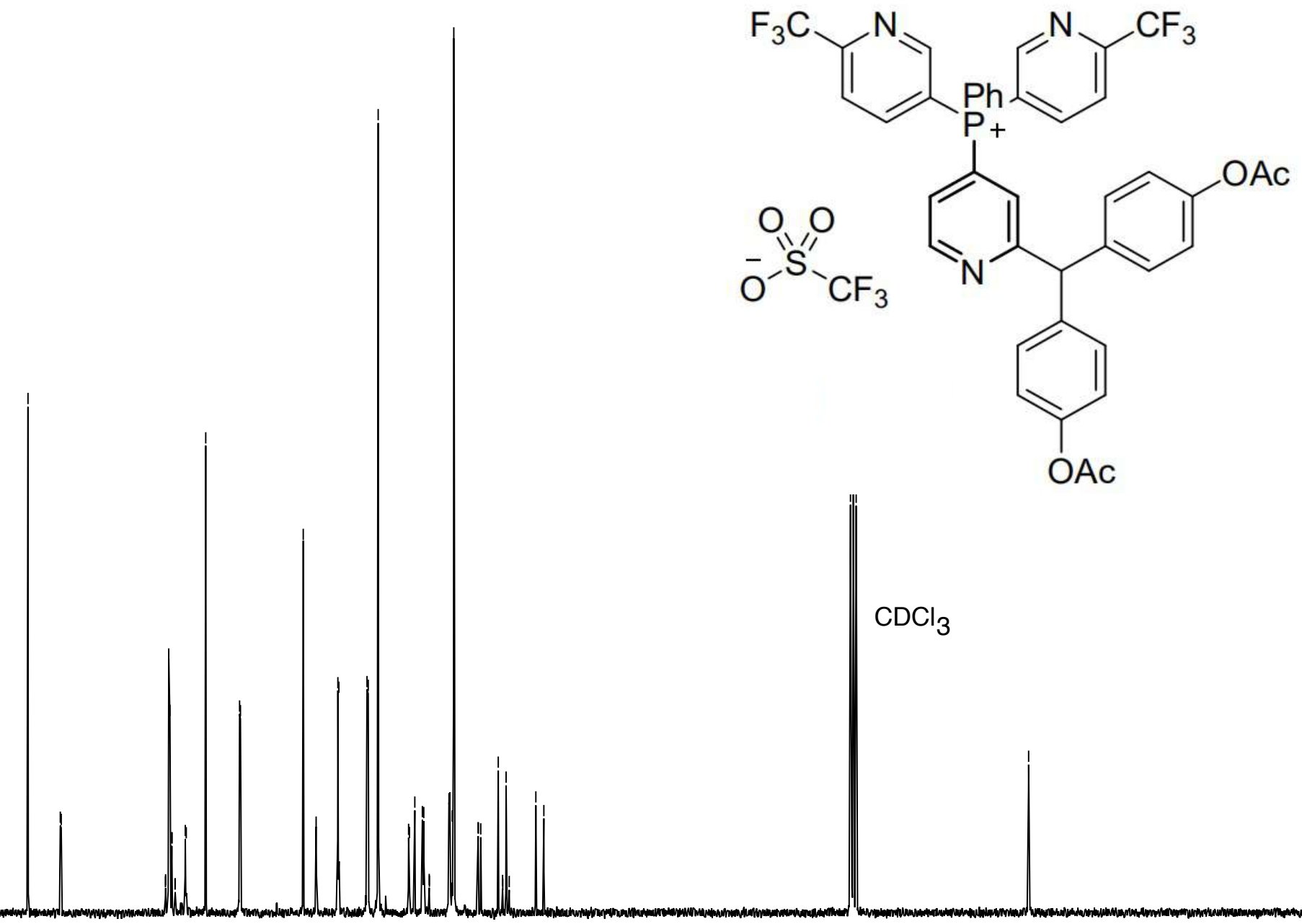
$\begin{array}{ll}0 & \infty \\ 0 & 0 \\ 0 & \infty \\ 0 & 1 \\ 1 & 1\end{array}$

\section{${ }^{19} \mathrm{~F} \mathrm{NMR}\left(\mathrm{CDCl}_{3}, 365 \mathrm{MHz}\right)$}

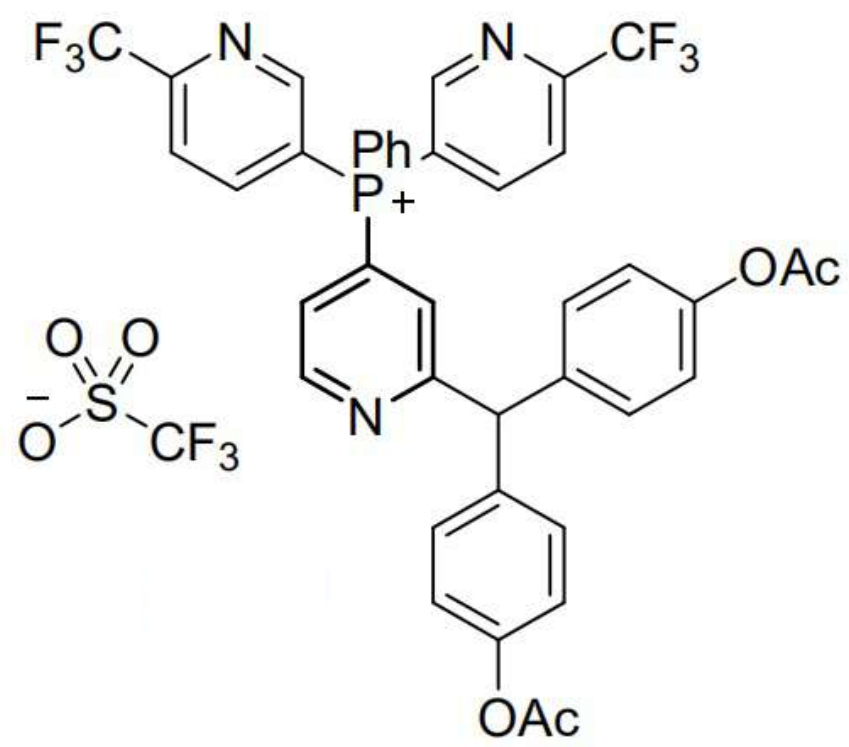

$0 \quad-20 \quad-40$

$-40 \quad-60$

$-80$

$-100$

$-120$

$-140$

$-160$

$-180$

$-200$

$-2$ 


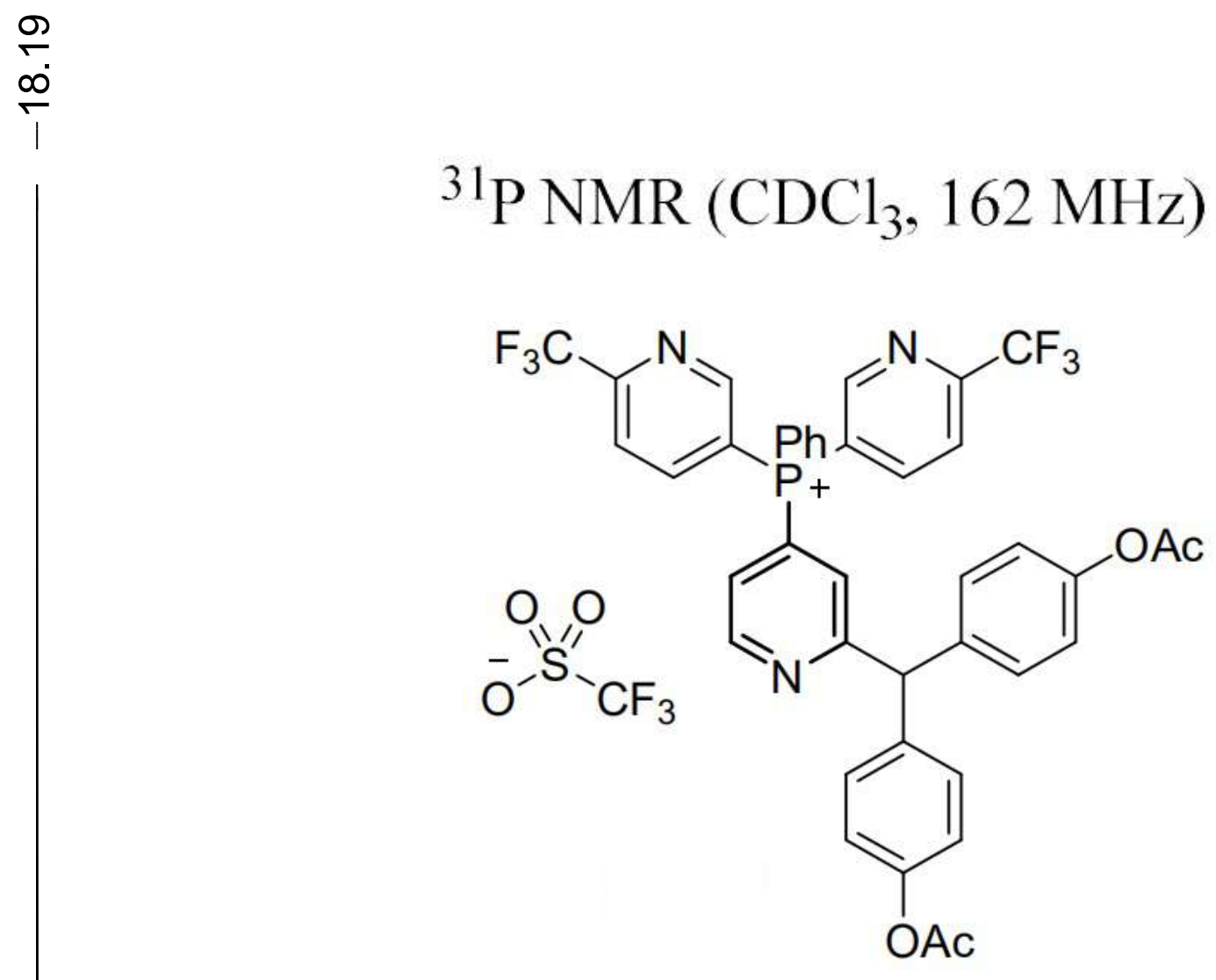

$\begin{array}{llllllllllllll}140 & 110 & 80 & 50 & 20 & -10 & -40 & -70 & -100 & -130 & -160 & -190 & -220 & -25\end{array}$




\section{${ }^{1} \mathrm{H} \mathrm{NMR},\left(\mathrm{CDCl}_{3}, 400 \mathrm{MHz}\right)$}<smiles>O=S(=O)([O-])C(F)(F)F</smiles>

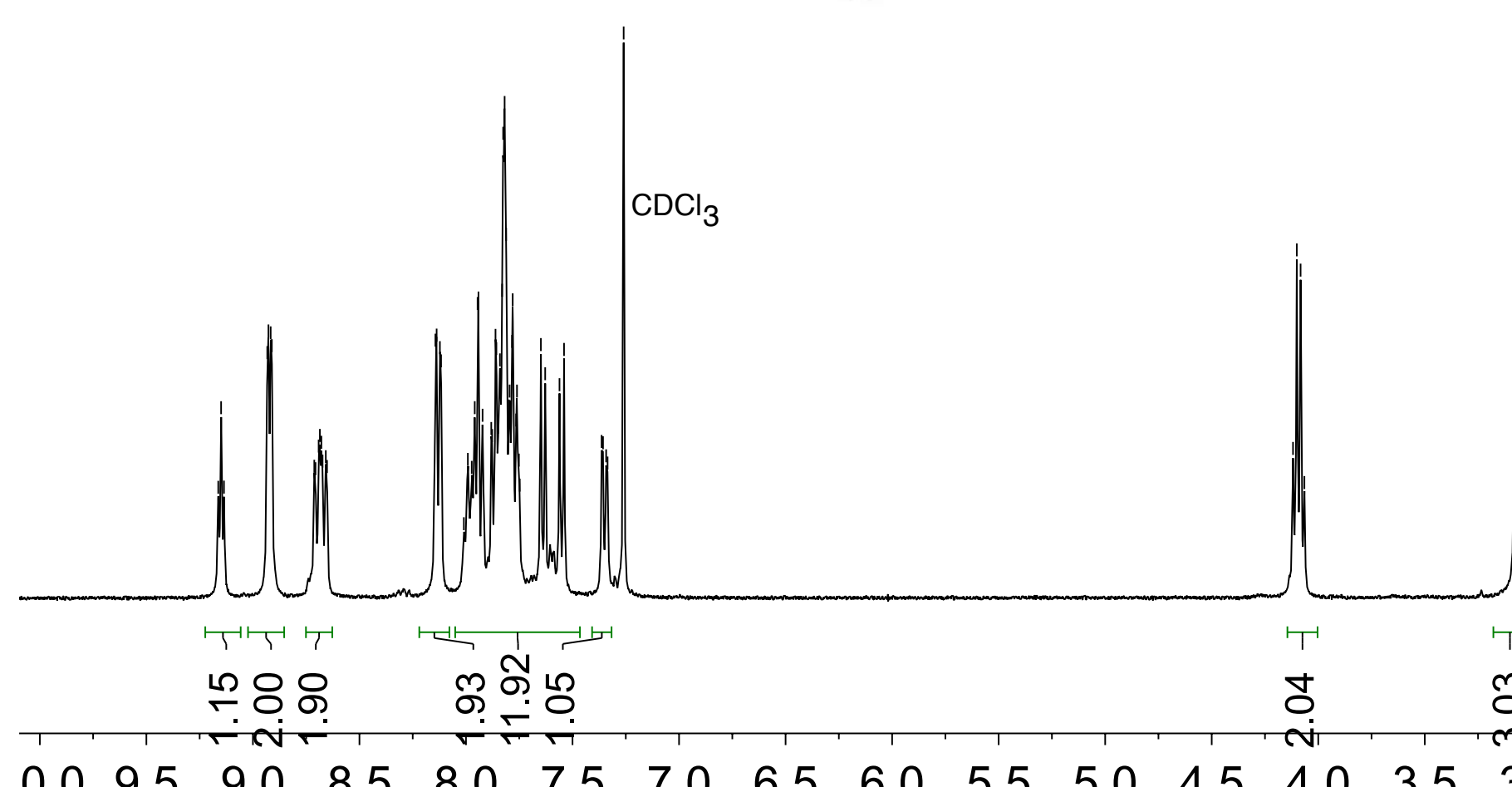

$\begin{array}{llllllllllllllllllllll}0.0 & 9.5 & 9.0 & 8.5 & 8.0 & 7.5 & 7.0 & 6.5 & 6.0 & 5.5 & 5.0 & 4.5 & 4.0 & 3.5 & 3.0 & 2.5 & 2.0 & 1.5 & 1.0 & 0.5 & 0.0\end{array}$




\section{${ }^{13} \mathrm{C} \mathrm{NMR},\left(\mathrm{CDCl}_{3}, 100 \mathrm{MHz}\right)$}<smiles>CCOC(=O)N(C(=O)c1ccc(S(=O)(=O)O)cc1Cl)c1ccc(Cl)c(-c2cc([PH+](c3ccc(C(F)(F)F)nc3)c3ccc(F)cn3)ccn2)c1</smiles> 


\section{${ }^{19} \mathrm{~F} \mathrm{NMR}\left(\mathrm{CDCl}_{3}, 365 \mathrm{MHz}\right)$}

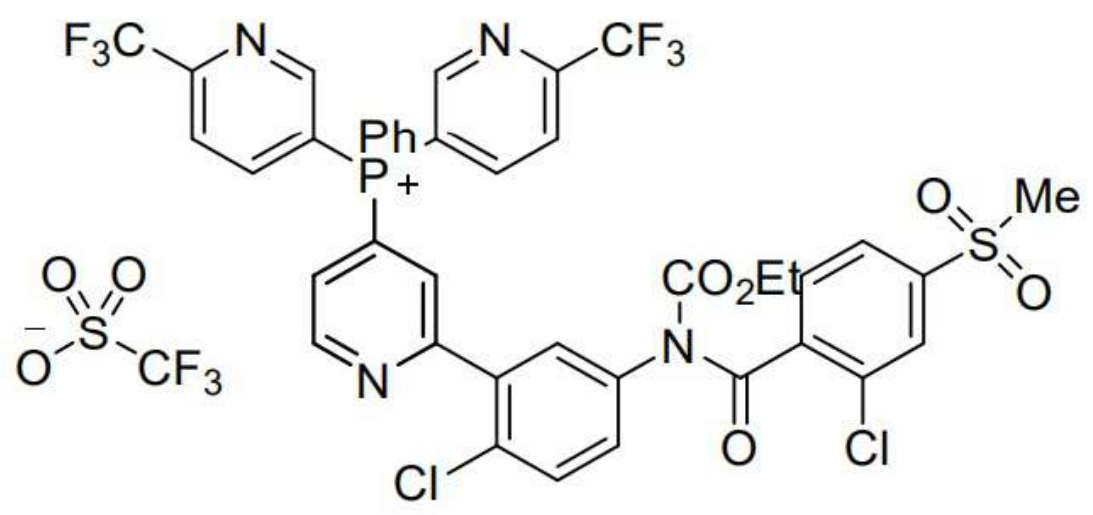

$0 \quad-20$

$-20 \quad-40 \quad-60$

$\begin{array}{ll}-80 & -100\end{array}$

$-120$

$-140$

$-160$

$-180$

$-200$

$-2 i$ 
${ }^{31} \mathrm{P} \mathrm{NMR}\left(\mathrm{CDCl}_{3}, 162 \mathrm{MHz}\right)$<smiles>CCc1ccc(S(=O)(=O)O)cc1Cl</smiles>

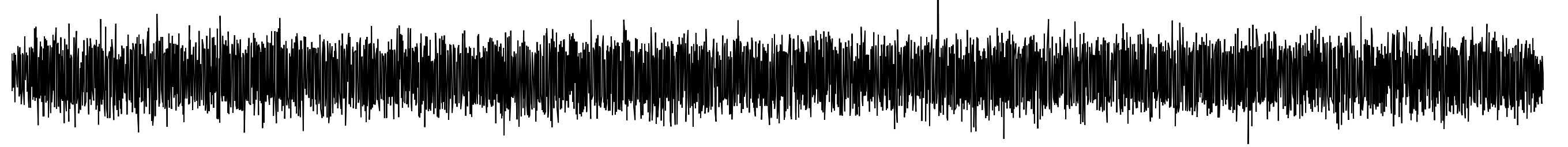

$\begin{array}{lllllllllllllll}190 & 170 & 150 & 130 & 110 & 90 & 70 & 50 & 30 & 10 & -10 & -30 & -50 & -70 & -90\end{array}$


ஊ ஊ

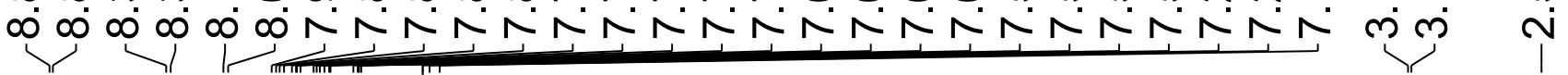

\section{${ }^{1} \mathrm{H} \mathrm{NMR},\left(\mathrm{CDCl}_{3}, 400 \mathrm{MHz}\right)$}

\section{क्ष}

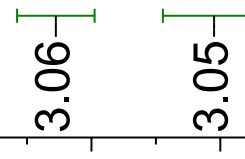


${ }^{13} \mathrm{C} \mathrm{NMR},\left(\mathrm{CDCl}_{3}, 100 \mathrm{MHz}\right)$<smiles>Cc1cc([PH+](c2ccc(S(C)(=O)=O)cc2)c2ccc(C(F)(F)F)cn2)c(-c2cnc(Cl)cc2-c2ccc(S(=O)(=O)C(F)(F)F)cc2)cn1</smiles> 


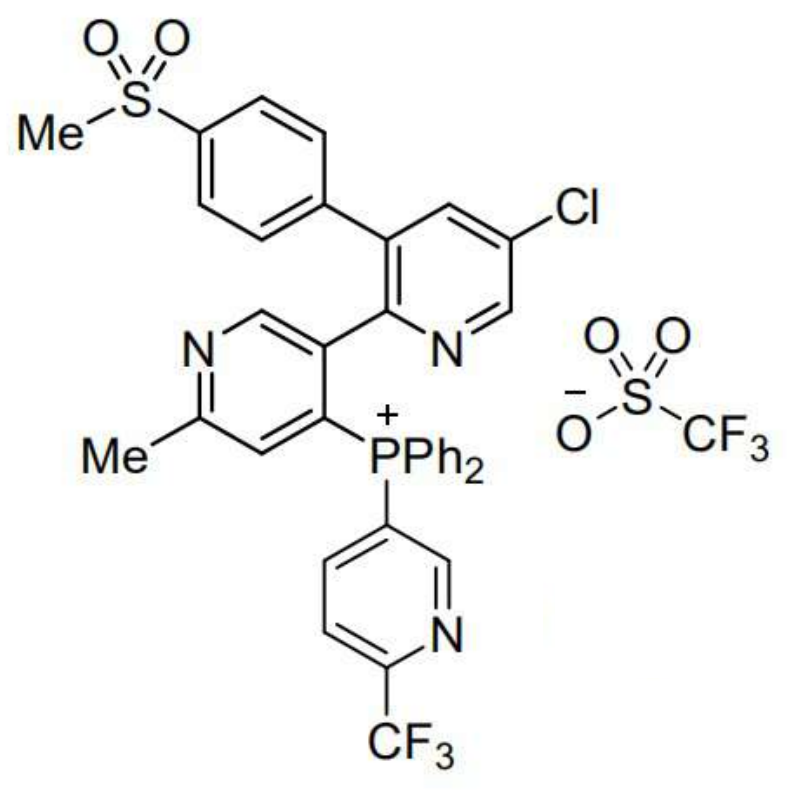

$\begin{array}{lllllllllllll}0 & -20 & -40 & -60 & -80 & -100 & -120 & -140 & -160 & -180 & -200 & -2\end{array}$


${ }^{31} \mathrm{P} \mathrm{NMR}\left(\mathrm{CDCl}_{3}, 162 \mathrm{MHz}\right)$<smiles>Cc1ccc(-c2cncc(Cl)c2)c([PH+](c2ccc(S(C)(=O)=O)cc2)c2ccc(C(F)(F)F)cn2)c1</smiles>

$\begin{array}{lllllllllllllll}190 & 170 & 150 & 130 & 110 & 90 & 70 & 50 & 30 & 10 & -10 & -30 & -50 & -70 & -90\end{array}$




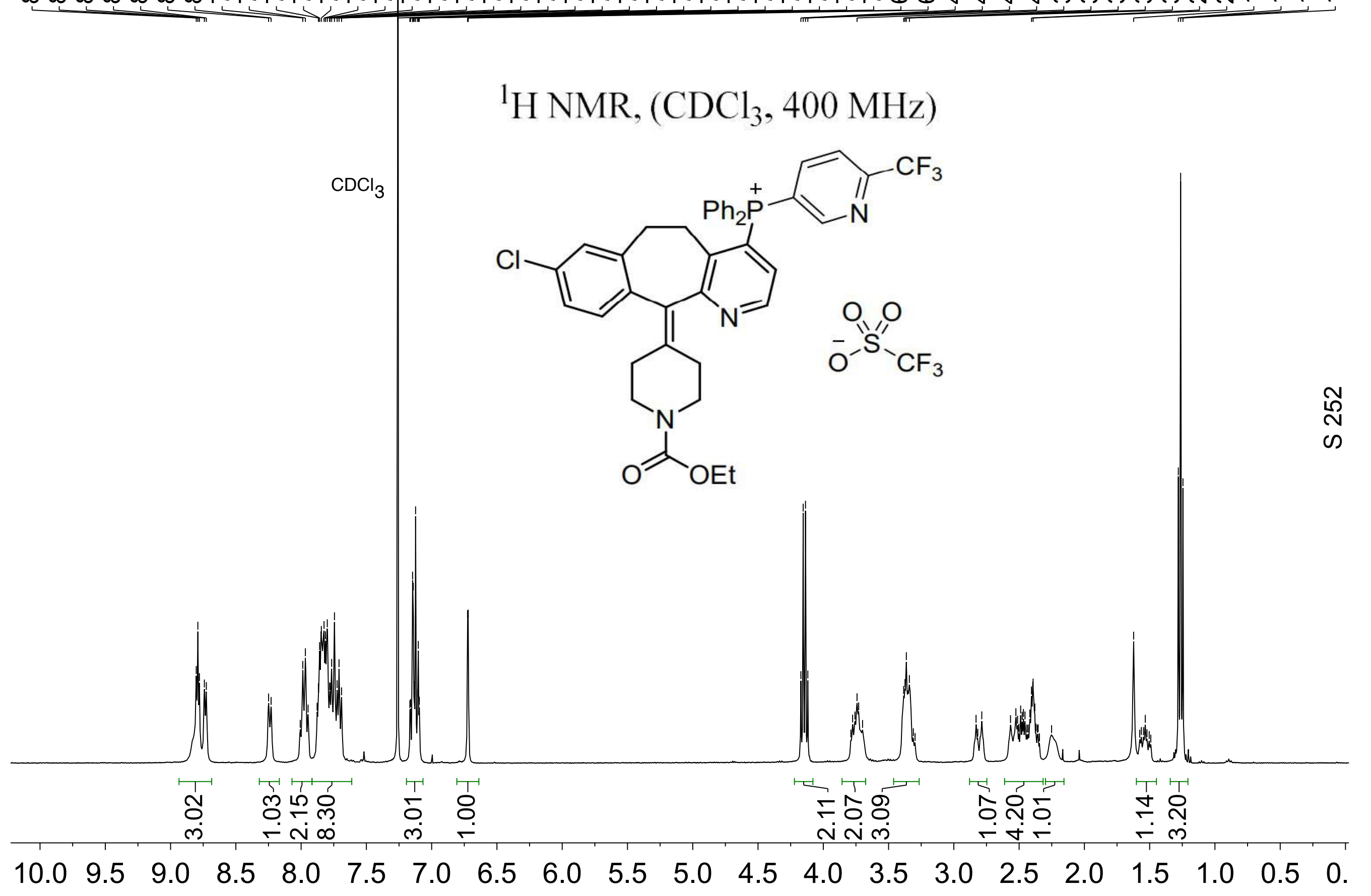


๓

ம்

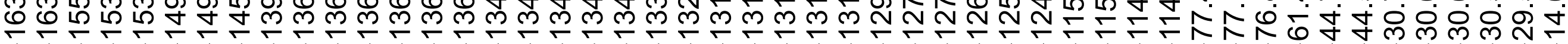

${ }^{13} \mathrm{C} \mathrm{NMR},\left(\mathrm{CDCl}_{3}, 100 \mathrm{MHz}\right)$<smiles>CCOC(=O)N1CCC(=C2c3ccc(Cl)cc3CCc3c([P@](C)(=O)c4ccc(C(F)(F)F)nc4)ccnc32)CC1</smiles> 
${ }^{19} \mathrm{~F} \mathrm{NMR}\left(\mathrm{CDCl}_{3}, 365 \mathrm{MHz}\right)$

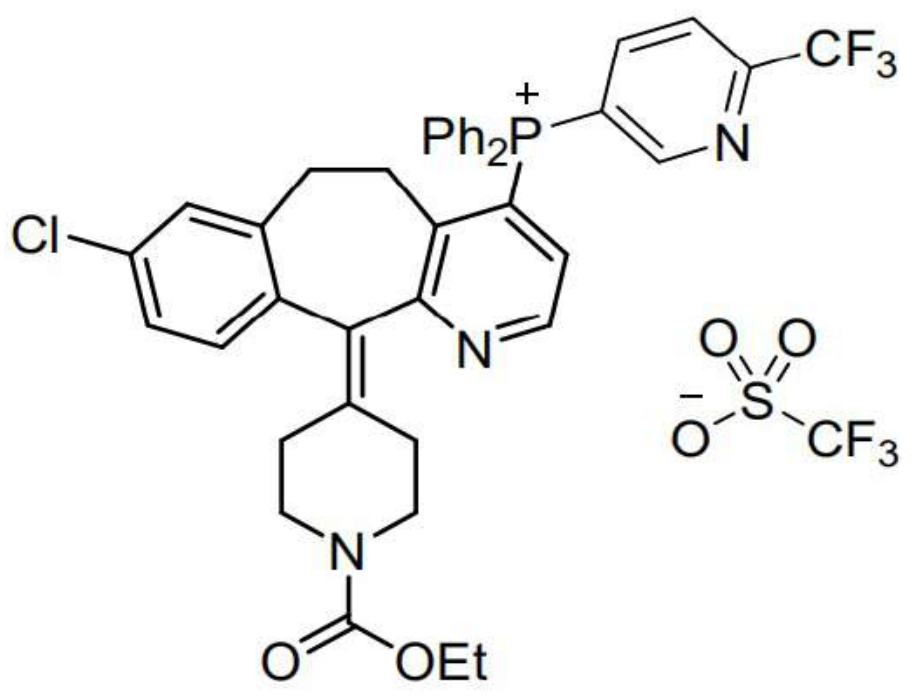

$0 \quad-20 \quad-40$

$-40-60$

$\begin{array}{ll}-80 & -100\end{array}$

$-120$

$-140$

$-160$

$-180$

$-200-2 i$ 
${ }^{31} \mathrm{P} \mathrm{NMR}\left(\mathrm{CDCl}_{3}, 162 \mathrm{MHz}\right)$

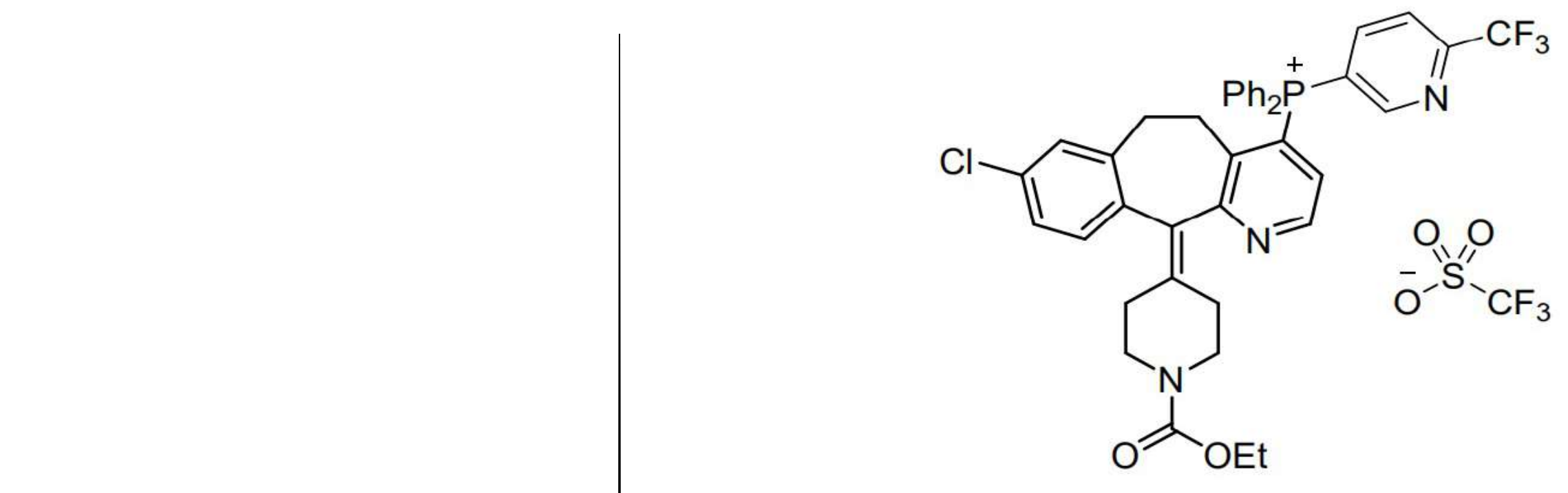

$\begin{array}{llllllllllllll}140 & 110 & 80 & 50 & 20 & -10 & -40 & -70 & -100 & -130 & -160 & -190 & -220 & -25\end{array}$




\section{${ }^{1} \mathrm{H} \mathrm{NMR},\left(\mathrm{CDCl}_{3}, 400 \mathrm{MHz}\right)$}

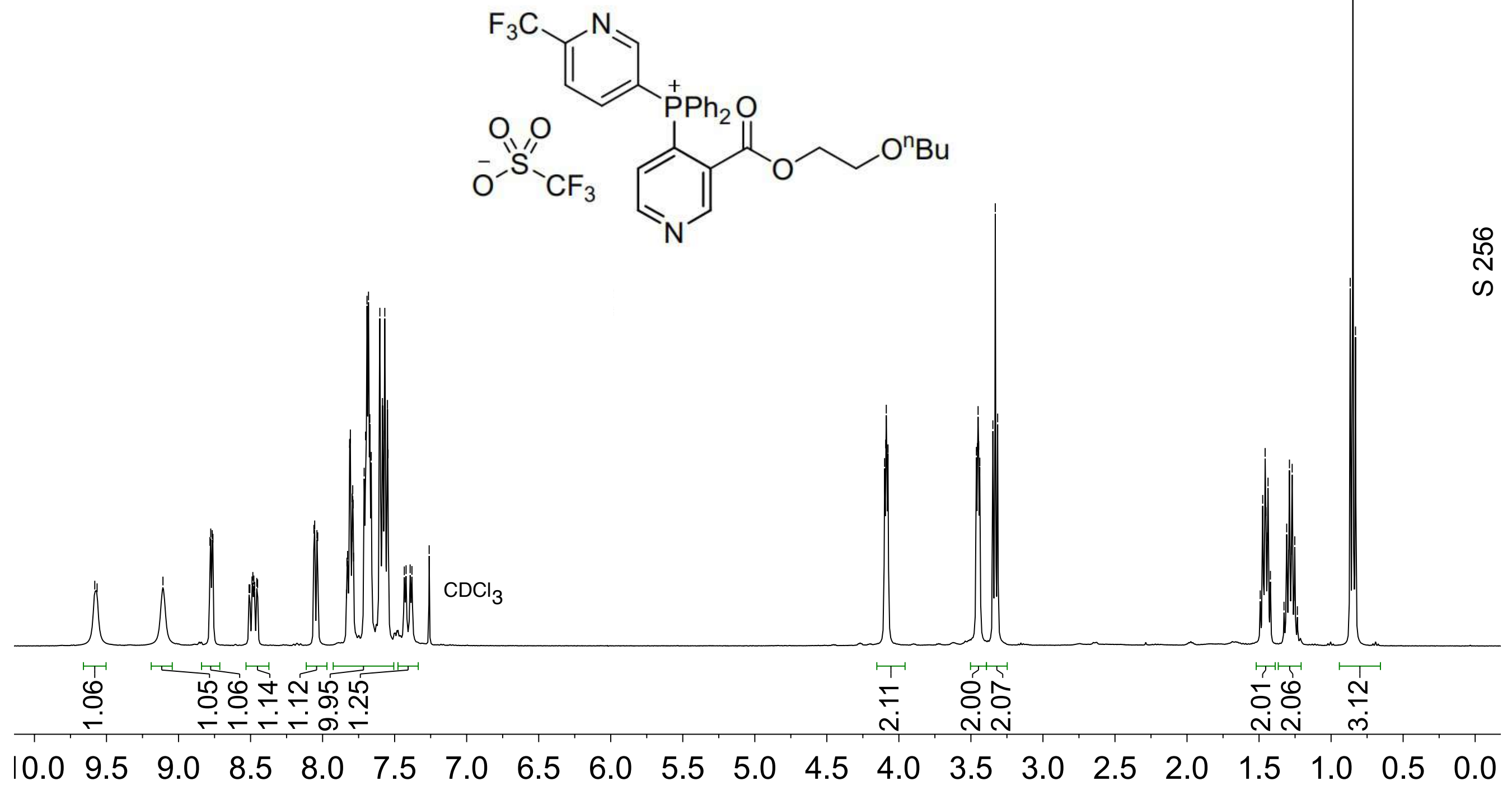


${ }^{13} \mathrm{C} \mathrm{NMR},\left(\mathrm{CDCl}_{3}, 100 \mathrm{MHz}\right)$

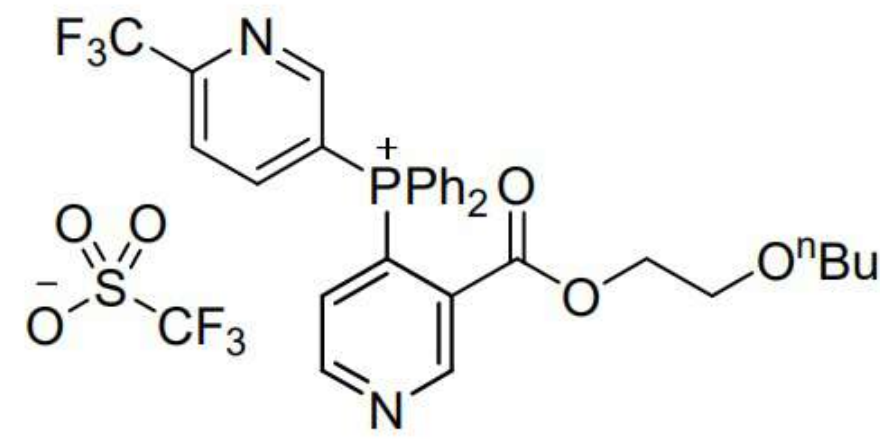




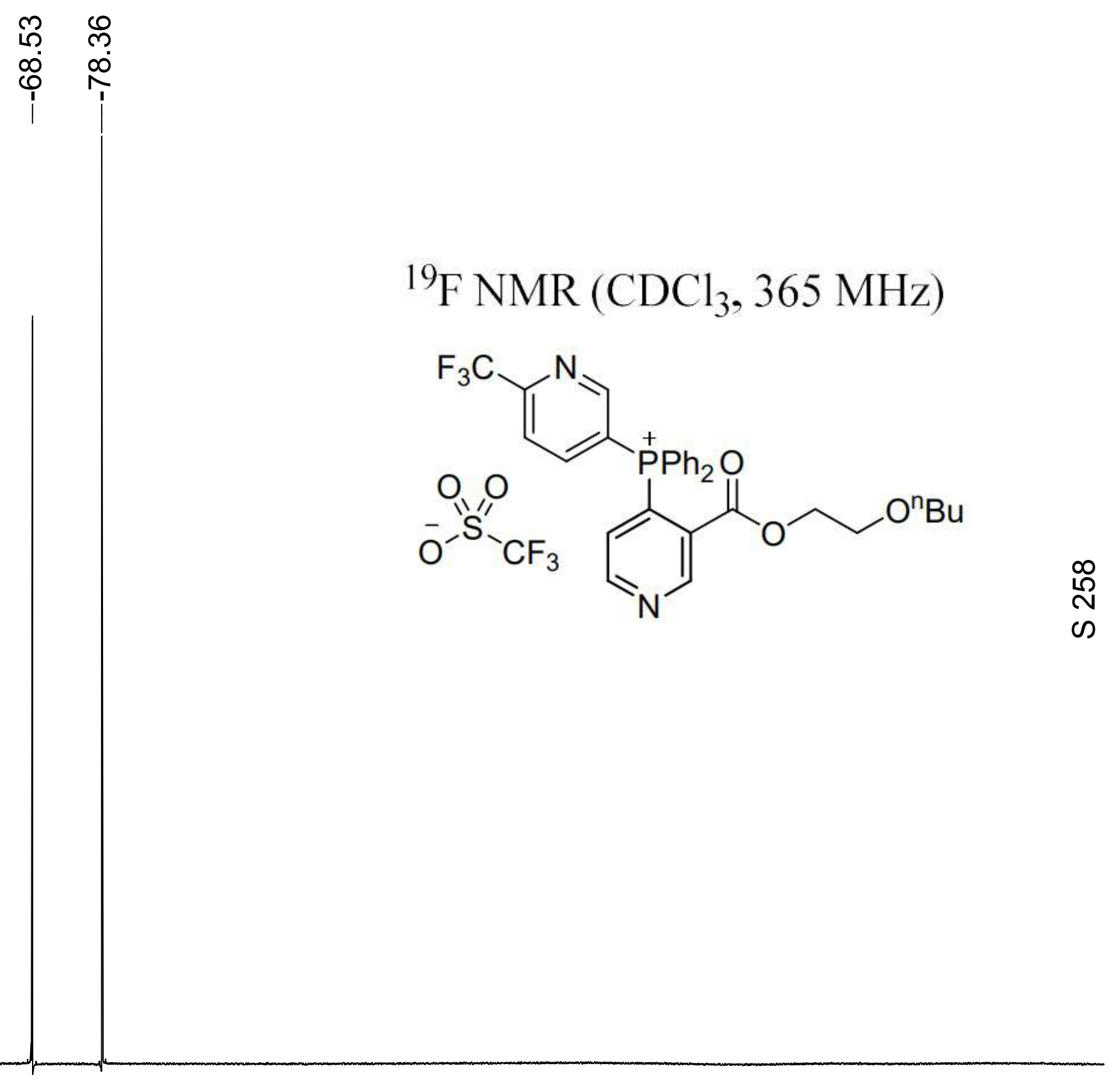

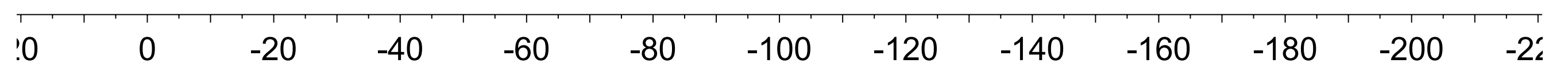




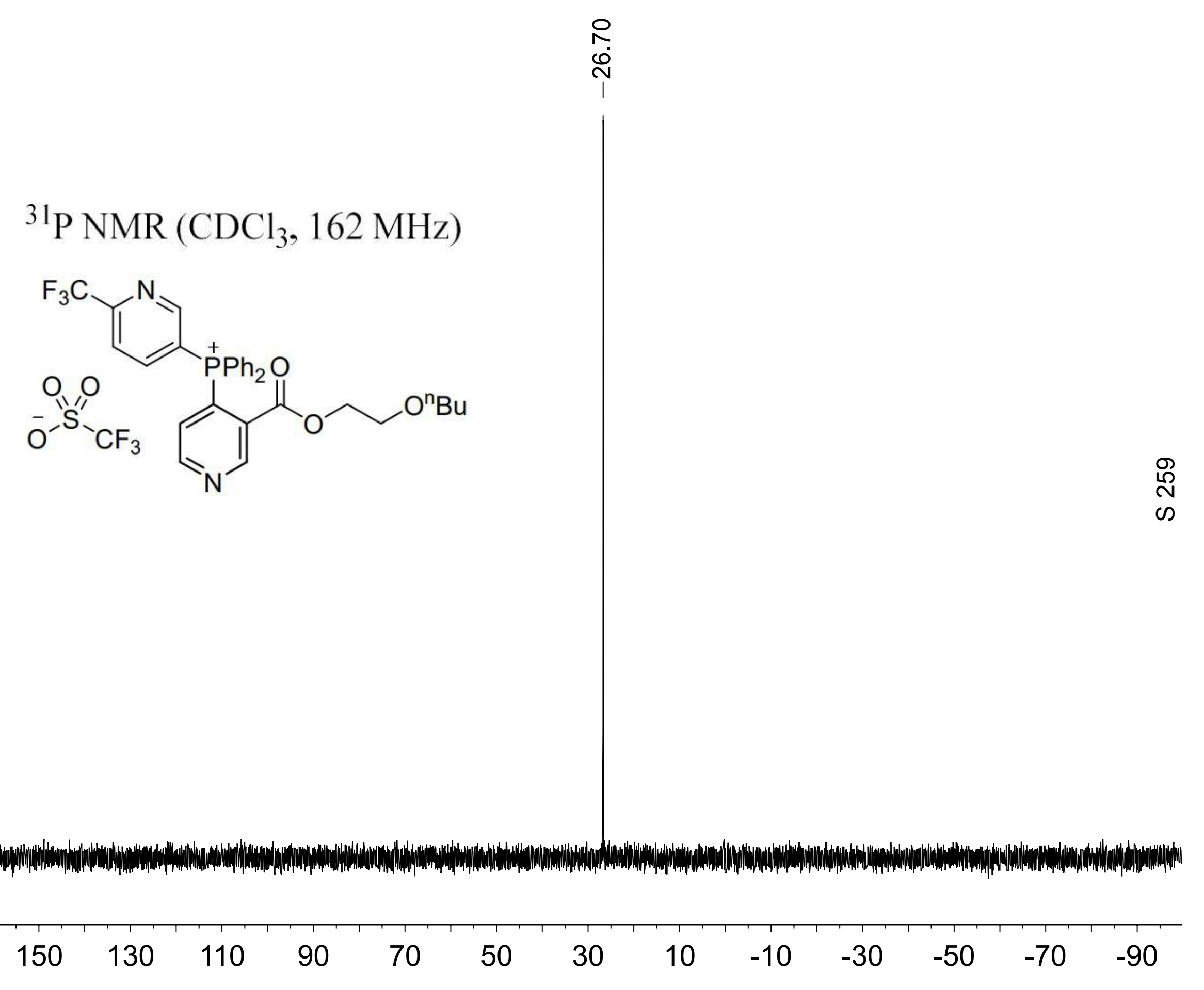


${ }^{1} \mathrm{H} \mathrm{NMR}\left(\mathrm{CDCl}_{3}, 400 \mathrm{MHz}\right)$

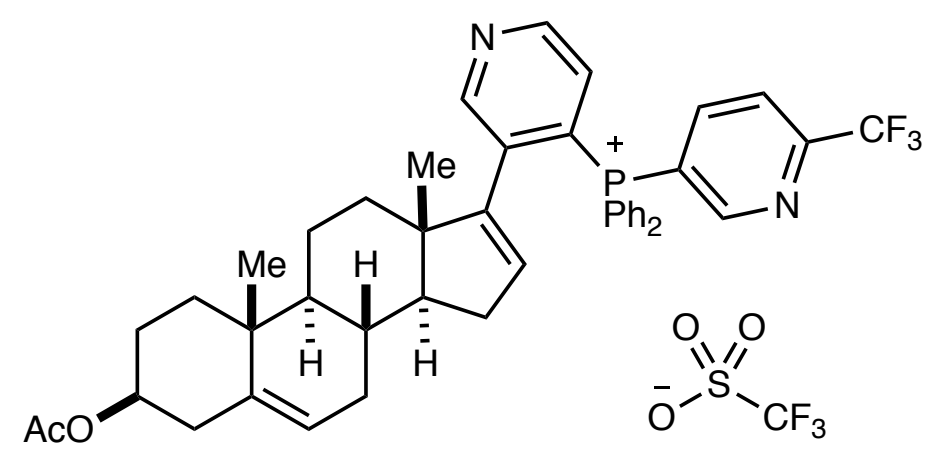
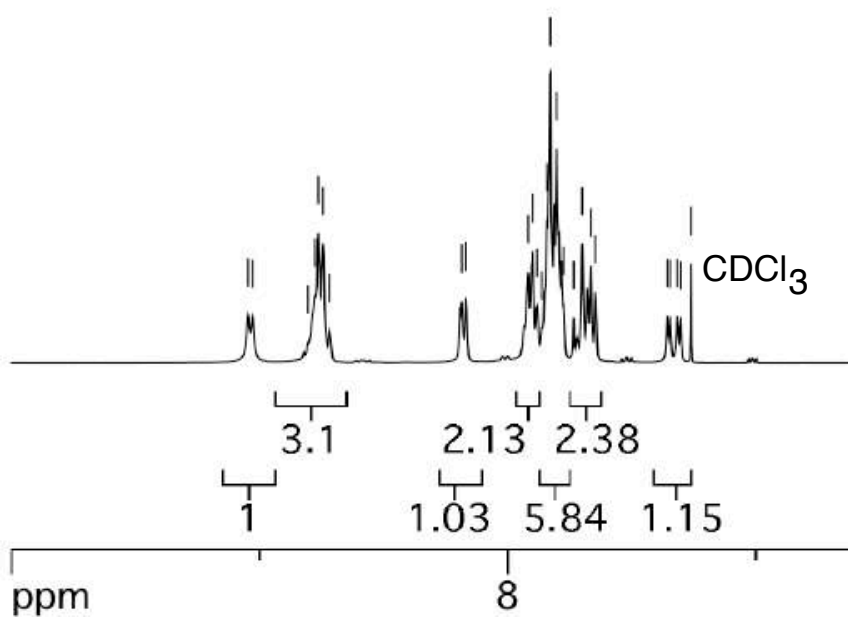


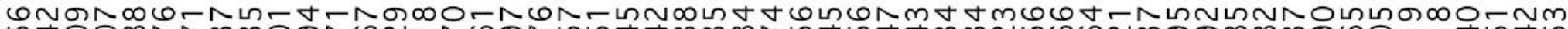

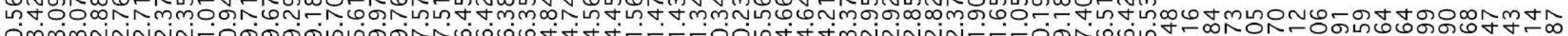
○mmก N N N 든ㄷㄴ

${ }^{13} \mathrm{C} \mathrm{NMR}\left(\mathrm{CDCl}_{3}, 100 \mathrm{MHz}\right)$
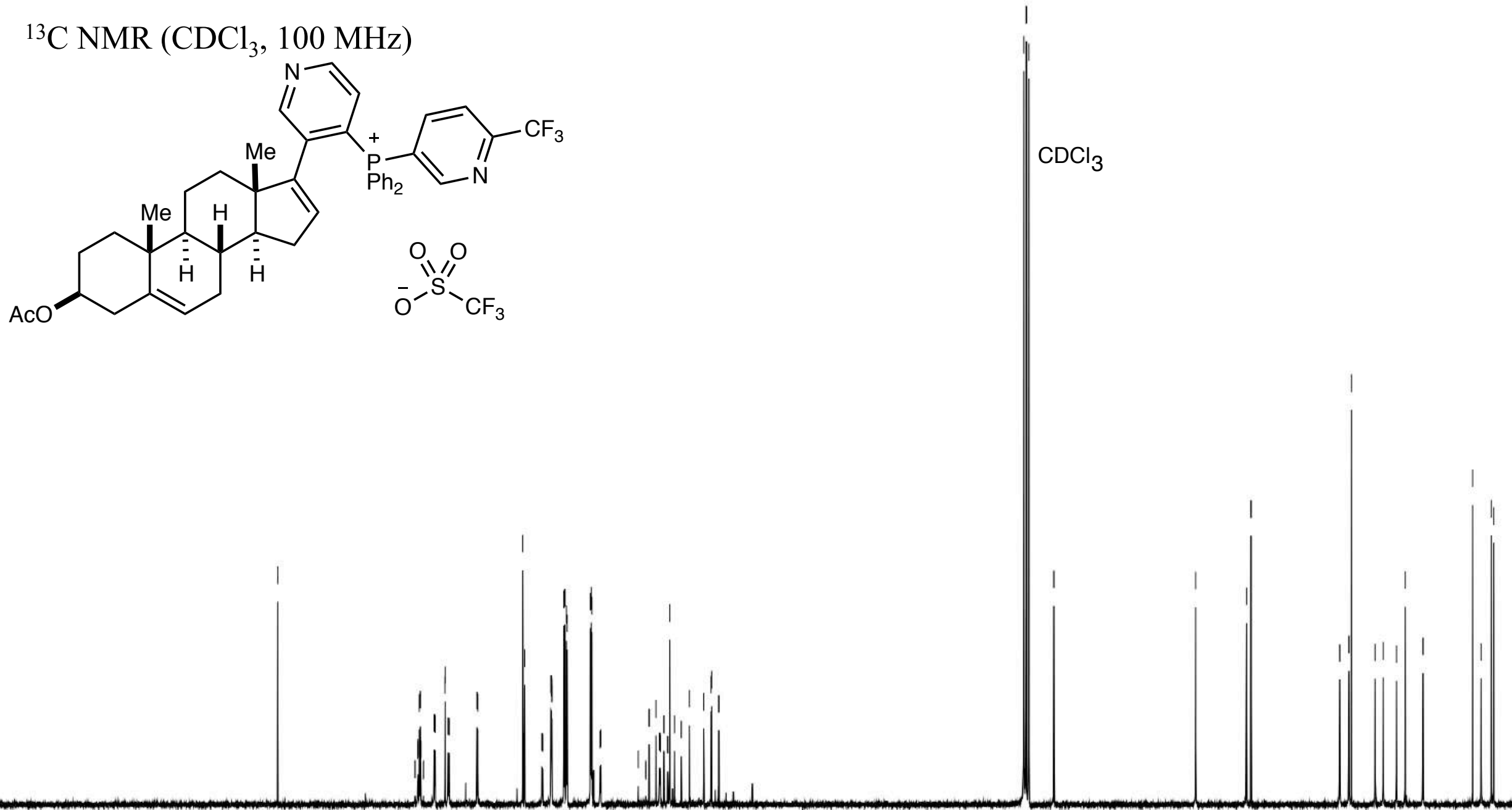
${ }^{19} \mathrm{~F} \mathrm{NMR}\left(\mathrm{CDCl}_{3}, 365 \mathrm{MHz}\right)$

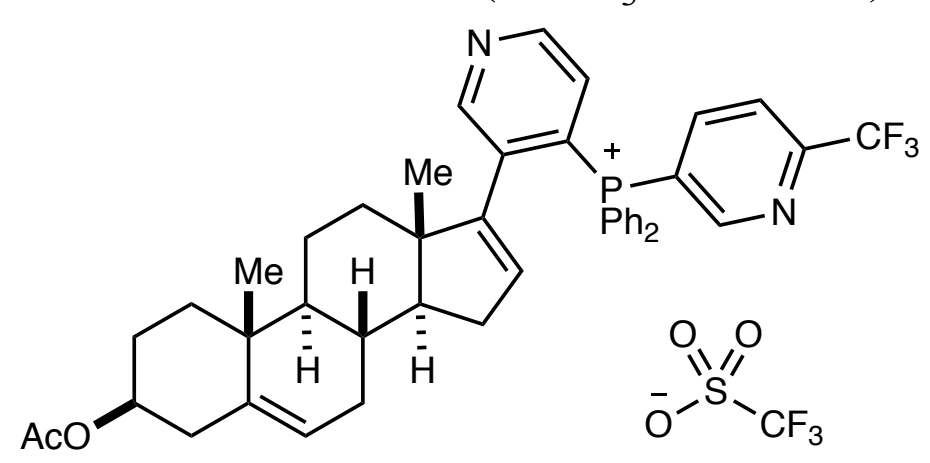


${ }^{31} \mathrm{P} \mathrm{NMR}\left(\mathrm{CDCl}_{3}, 162 \mathrm{MHz}\right)$

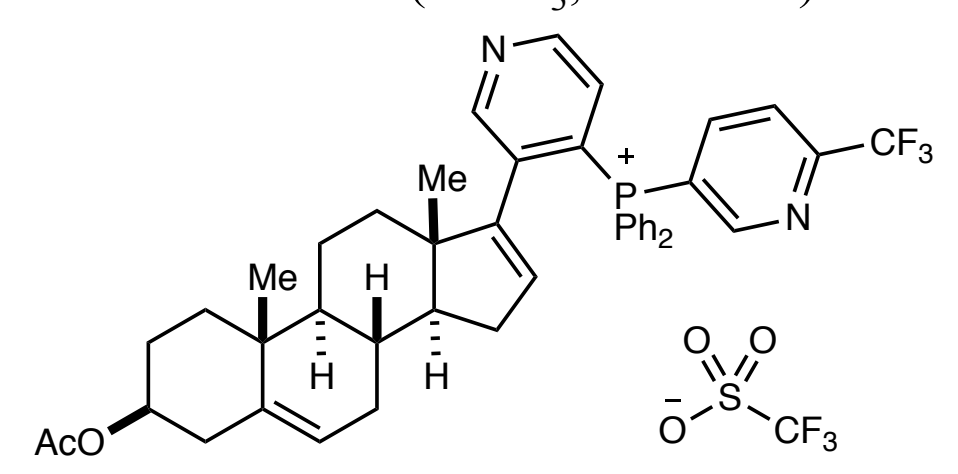


${ }^{1} \mathrm{H} \mathrm{NMR}\left(\mathrm{CDCl}_{3}, 400 \mathrm{MHz}\right)$

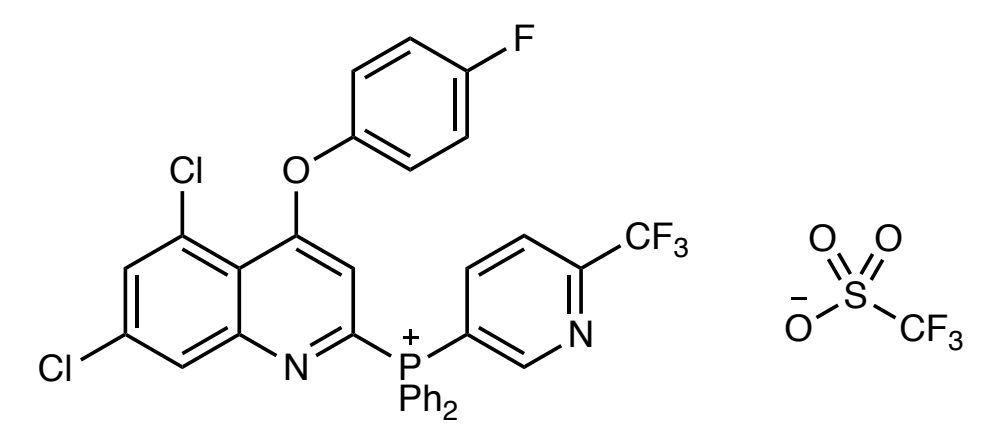

$\begin{array}{rrrr}4.03 & 1.06 & 2.08\end{array}$


${ }^{13} \mathrm{C} \mathrm{NMR}\left(\mathrm{CDCl}_{3}, 100 \mathrm{MHz}\right)$<smiles>Fc1ccc(Oc2cc(P(c3ccc(F)cc3)c3ccc(C(F)(F)F)nc3)nc3cc(Cl)cc(Cl)c23)cc1</smiles><smiles>O=S(=O)([O-])C(F)(F)F</smiles> 
${ }^{31} \mathrm{P} \mathrm{NMR}\left(\mathrm{CDCl}_{3}, 162 \mathrm{MHz}\right)$

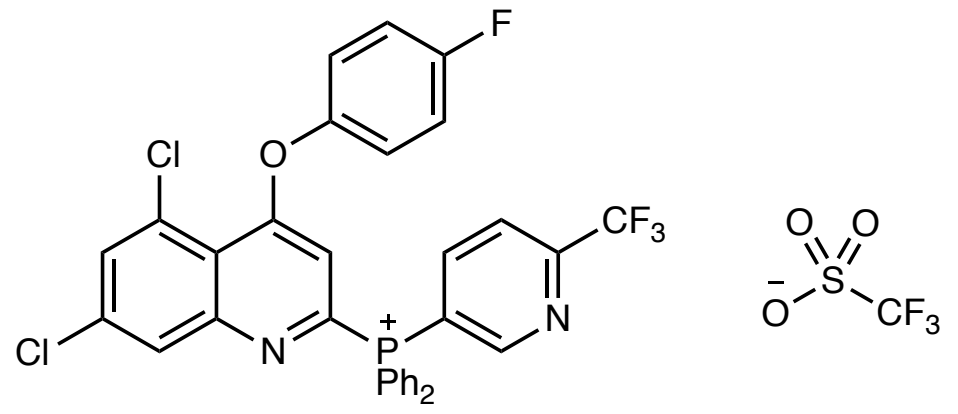


${ }^{13} \mathrm{C} \mathrm{NMR}\left(\mathrm{CDCl}_{3}, 100 \mathrm{MHz}\right)$
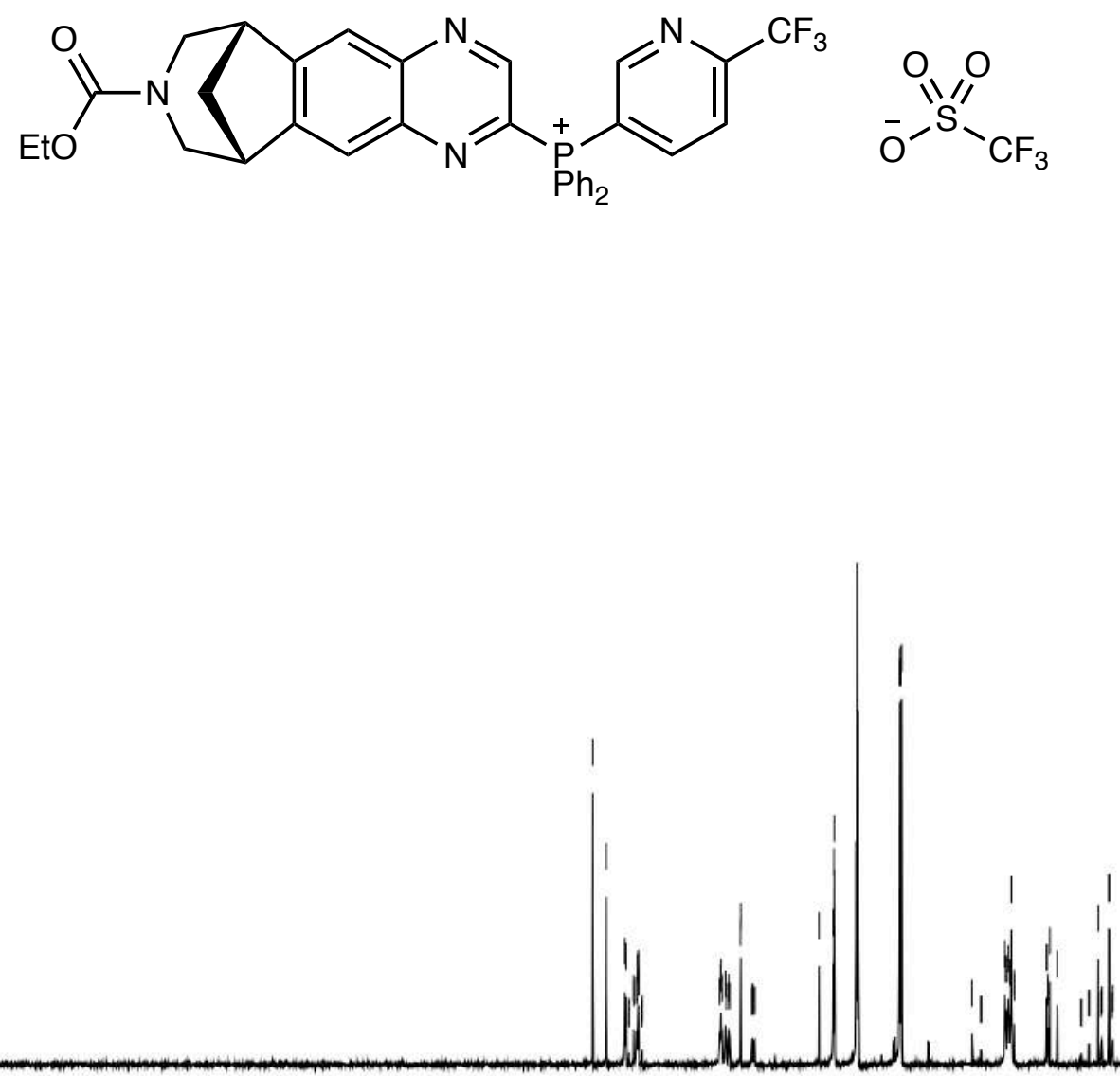


$$
\text { V) }
$$

${ }^{31} \mathrm{P} \mathrm{NMR}\left(\mathrm{CDCl}_{3}, 162 \mathrm{MHz}\right)$<smiles>CCOC(=O)N1CC2C[C@@H](C1)c1cc3ncc([PH+](c4ccc(C(F)(F)F)cn4)c4ccc(C(F)(F)F)cn4)nc3cc12</smiles> 
${ }^{1} \mathrm{H} \mathrm{NMR}\left(\mathrm{CDCl}_{3}, 400 \mathrm{MHz}\right)$
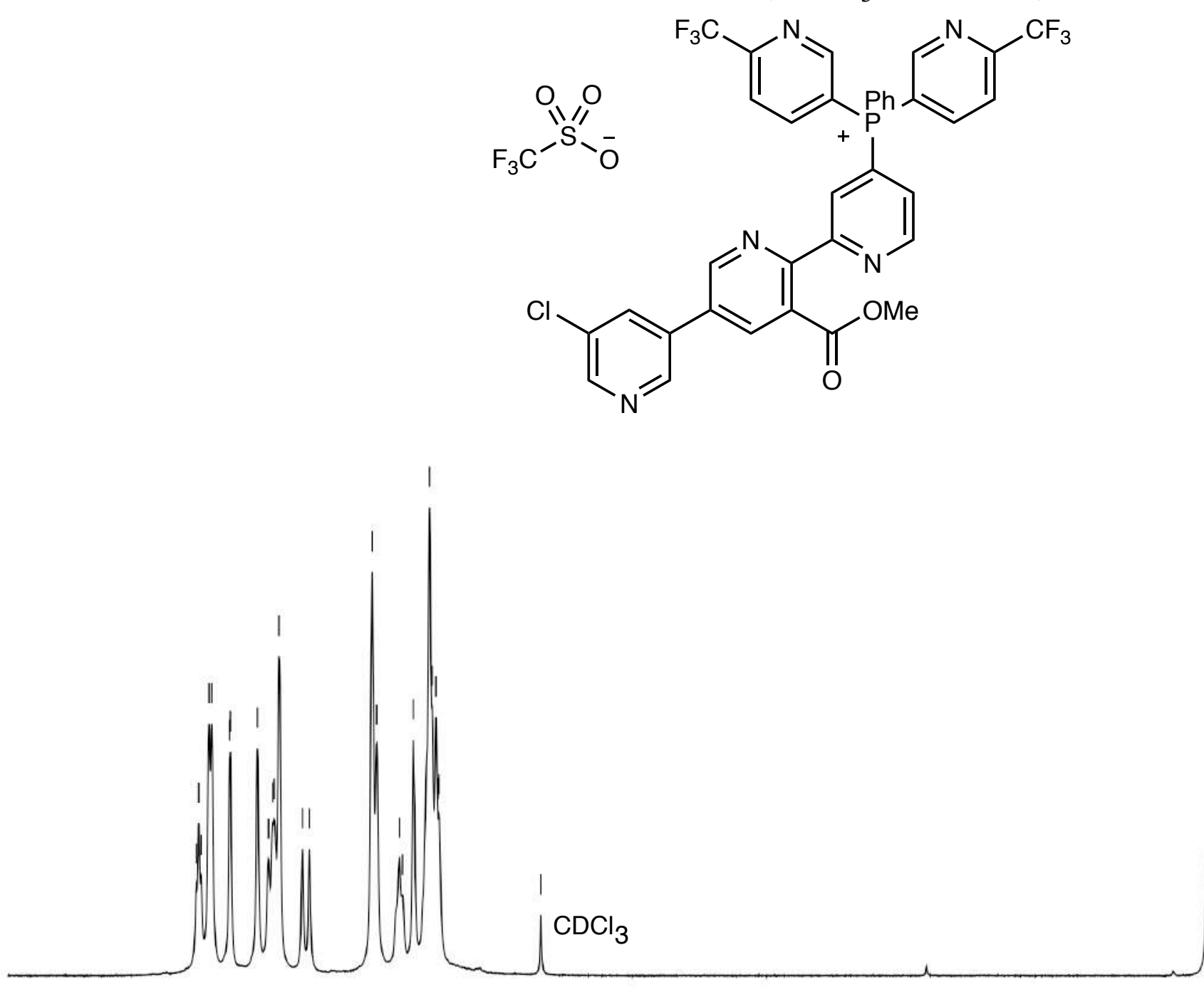

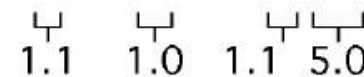

$$
\begin{aligned}
& \text { 난 눈 }
\end{aligned}
$$$$
3.0
$$

8

$\frac{1}{7}, \quad \frac{1}{6}, \frac{1}{3.0}$

$+1$


${ }^{13} \mathrm{C} \mathrm{NMR}\left(\mathrm{CDCl}_{3}, 100 \mathrm{MHz}\right)$

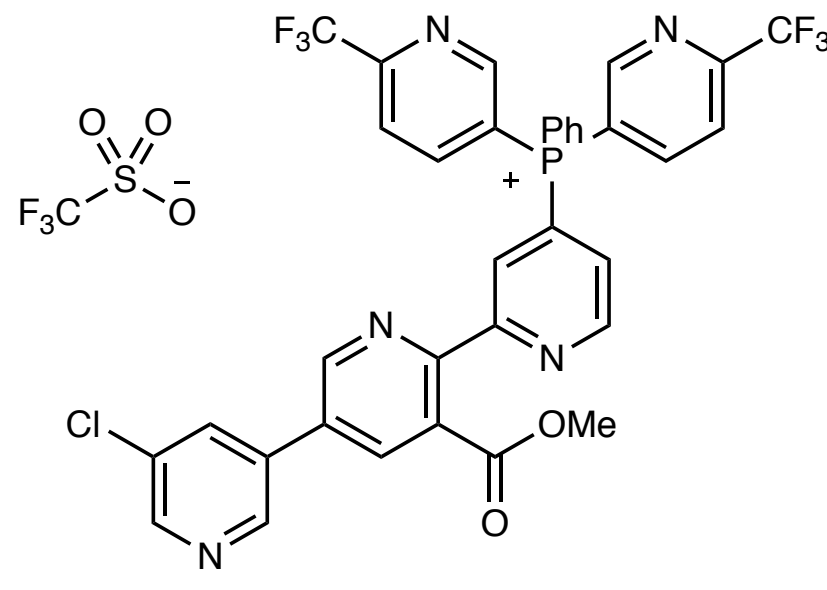


${ }^{19} \mathrm{~F} \mathrm{NMR}\left(\mathrm{CDCl}_{3}, 365 \mathrm{MHz}\right)$

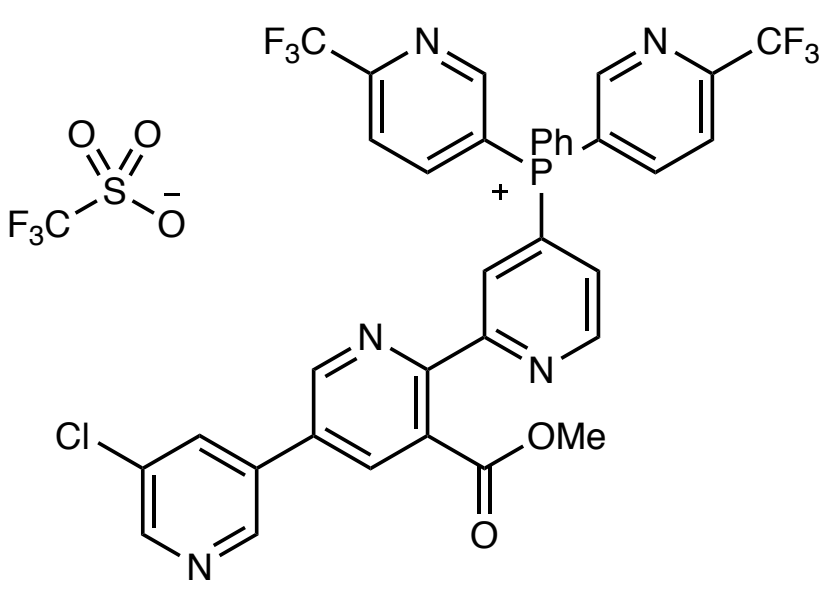

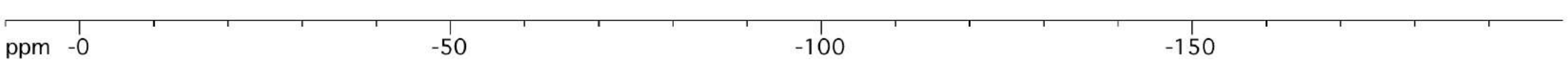


${ }^{31} \mathrm{P}$ NMR $\left(\mathrm{CDCl}_{3}, 162 \mathrm{MHz}\right)$

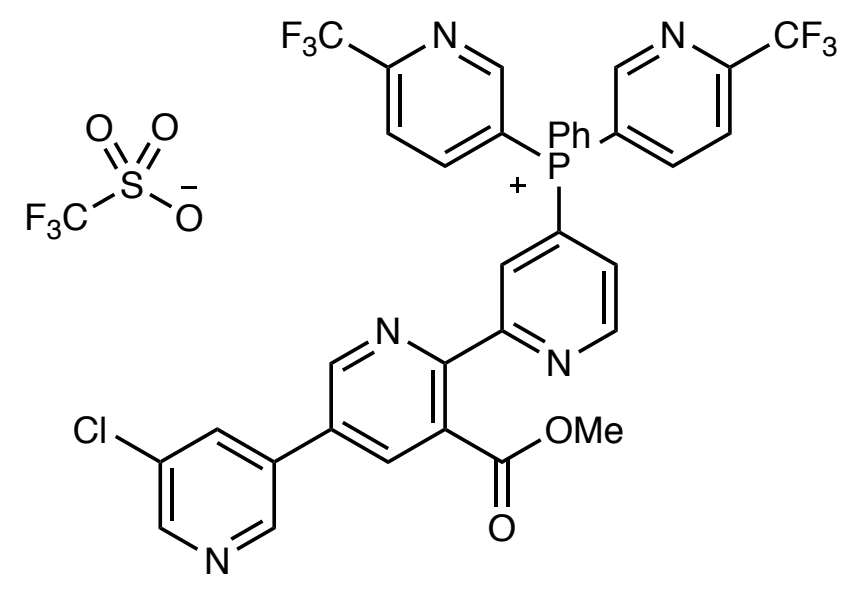




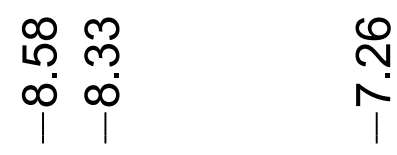

\section{${ }^{1} \mathrm{H} \mathrm{NMR},\left(\mathrm{CDCl}_{3}, 400 \mathrm{MHz}\right)$}<smiles>Cc1cncc(Br)c1Cl</smiles>

9

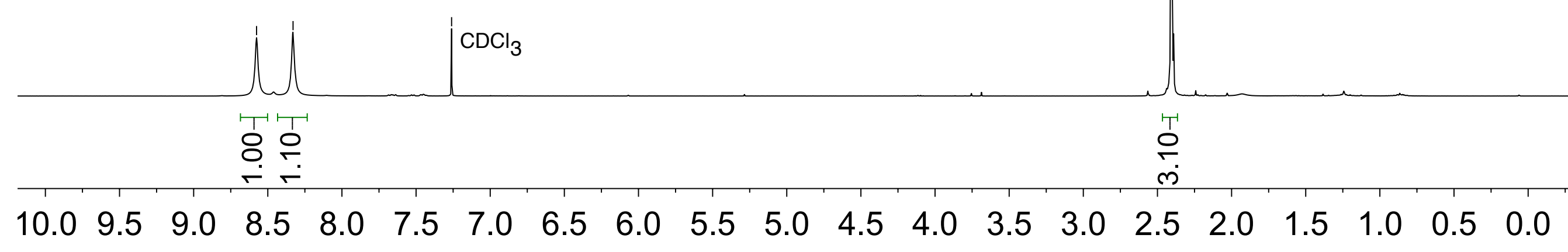




\begin{tabular}{|c|c|c|c|}
\hline 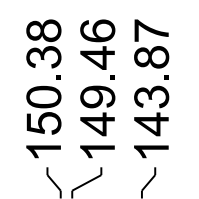 & $\begin{array}{l}n \\
0 \\
\frac{1}{1}\end{array}$ & $\frac{m}{i}$ & 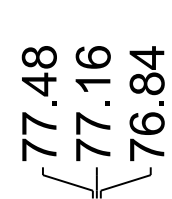 \\
\hline
\end{tabular}

${ }^{13} \mathrm{C} \mathrm{NMR},\left(\mathrm{CDCl}_{3}, 100 \mathrm{MHz}\right)$<smiles>Cc1cncc(Br)c1Cl</smiles> 
${ }^{1} \mathrm{H} \mathrm{NMR}\left(\mathrm{CDCl}_{3}, 400 \mathrm{MHz}\right)$<smiles>C[14C](=O)NCc1cnccc1Cl</smiles>

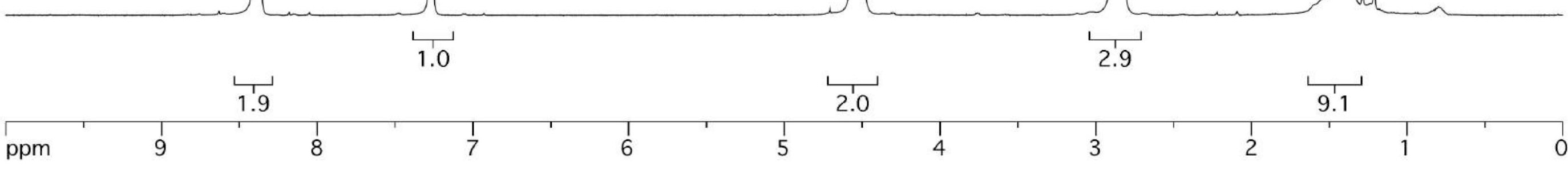



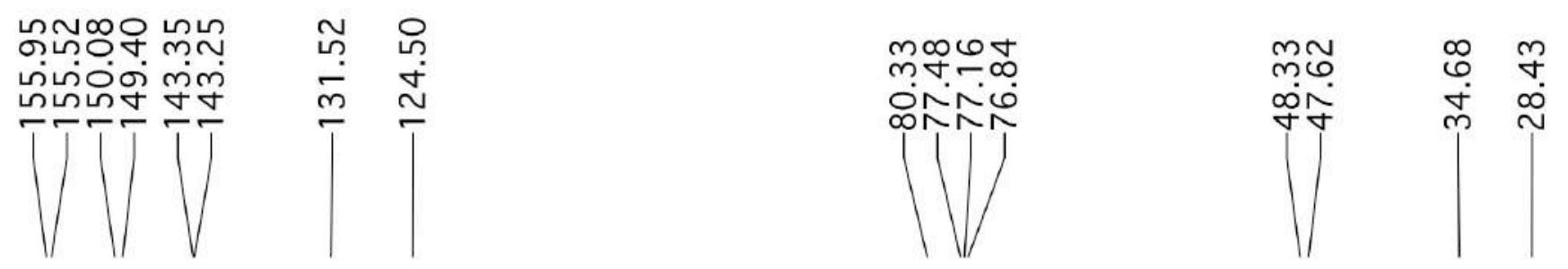

${ }^{13} \mathrm{C} \mathrm{NMR}\left(\mathrm{CDCl}_{3}, 100 \mathrm{MHz}\right)$<smiles>Clc1ccncc1[14CH2][Nb]</smiles> 


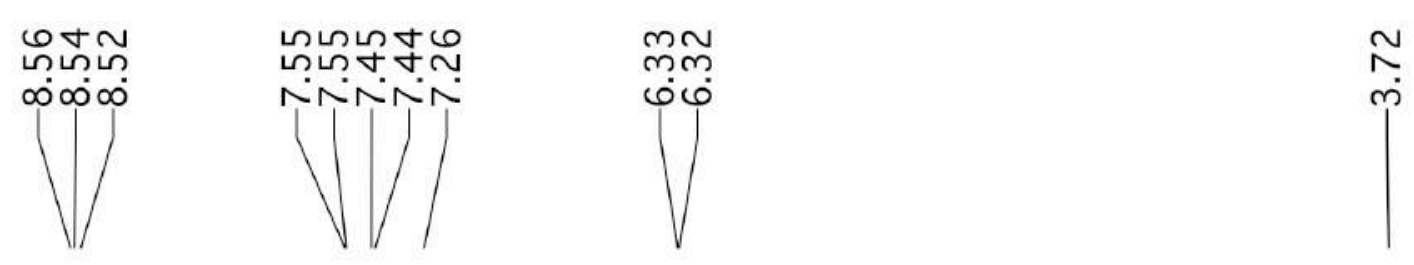

${ }^{1} \mathrm{H} \mathrm{NMR}\left(\mathrm{CDCl}_{3}, 400 \mathrm{MHz}\right)$<smiles>Cn1nccc1-c1cnccc1Cl</smiles>

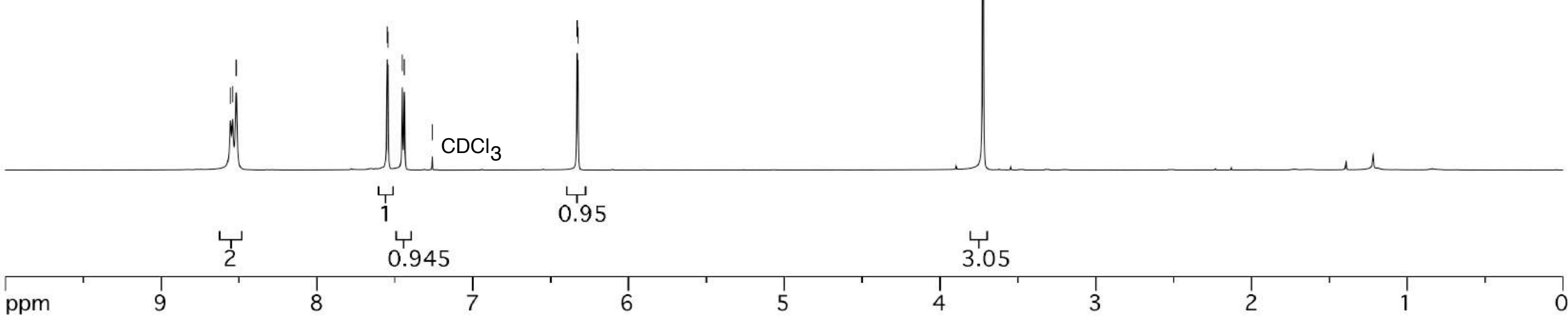



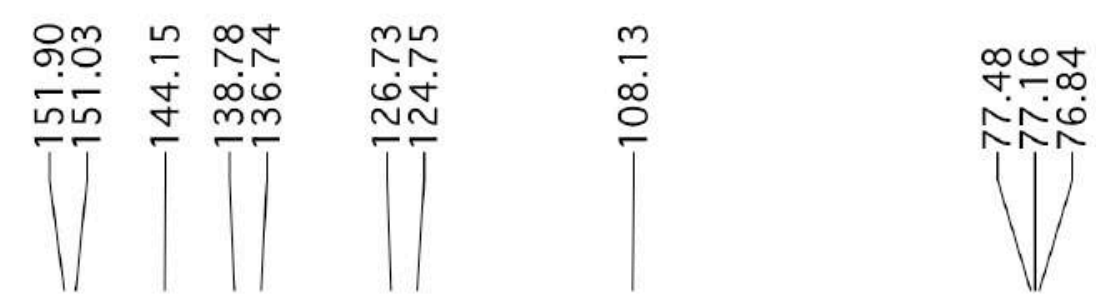

${ }^{13} \mathrm{C} \mathrm{NMR}\left(\mathrm{CDCl}_{3}, 100 \mathrm{MHz}\right)$

12<smiles>Cn1nccc1-c1cnccc1Cl</smiles>

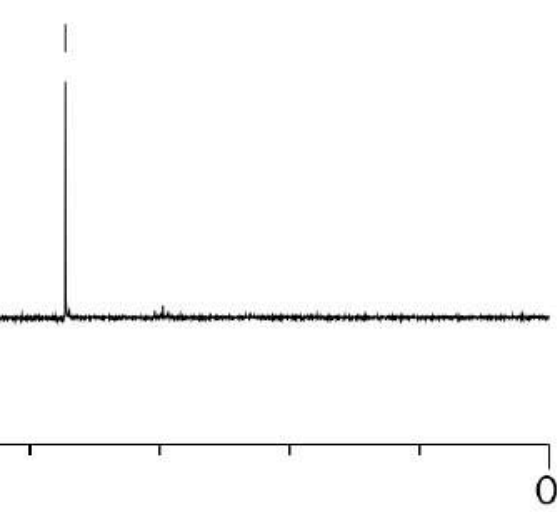


广

$\infty \infty^{\circ} \times N N$

${ }^{1} \mathrm{H} \mathrm{NMR}\left(\mathrm{CDCl}_{3}, 400 \mathrm{MHz}\right)$

13<smiles>Clc1ccncc1C#Cc1ccccc1</smiles>

$\stackrel{\text { N }}{\mathcal{N}}$

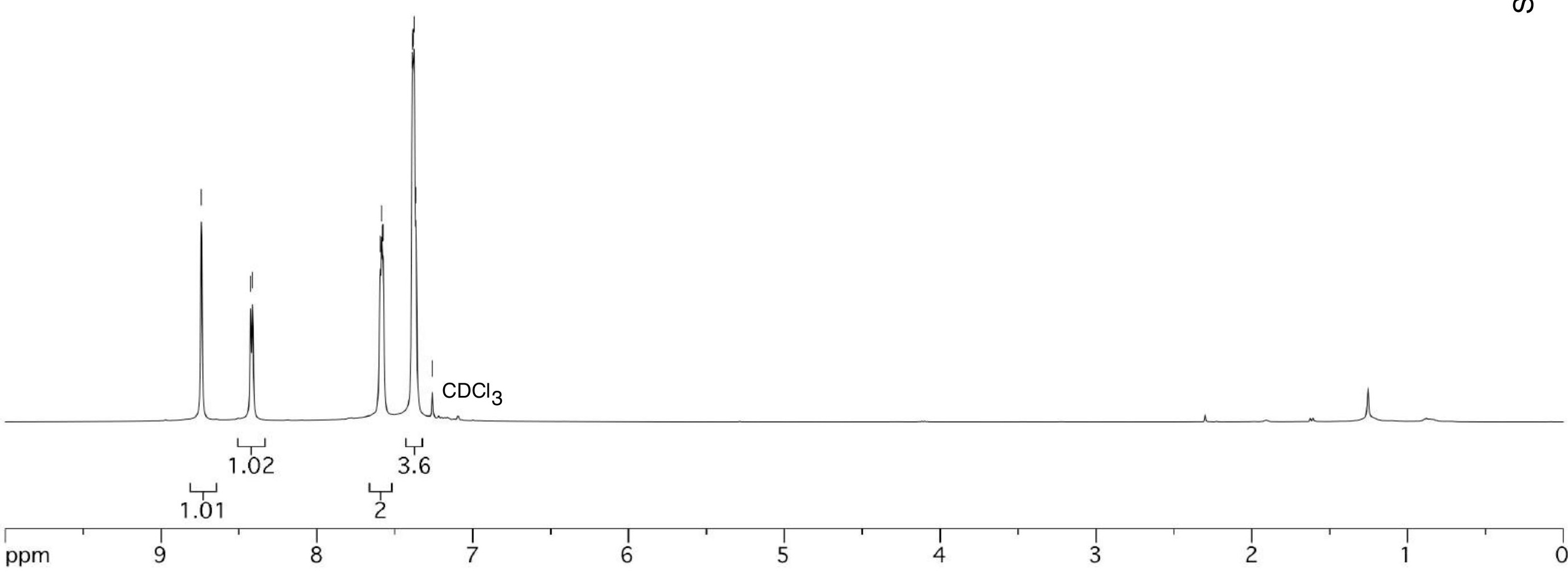




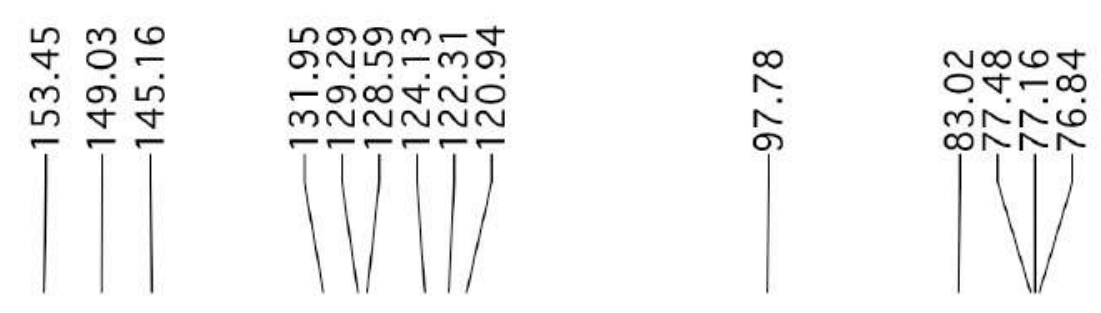

${ }^{13} \mathrm{C} \mathrm{NMR}\left(\mathrm{CDCl}_{3}, 100 \mathrm{MHz}\right)$

13<smiles>Clc1ccncc1C#Cc1ccccc1</smiles> 
${ }^{1} \mathrm{H} \mathrm{NMR}\left(\mathrm{CDCl}_{3}, 400 \mathrm{MHz}\right)$

14<smiles>Clc1ccncc1Oc1ccccn1</smiles>

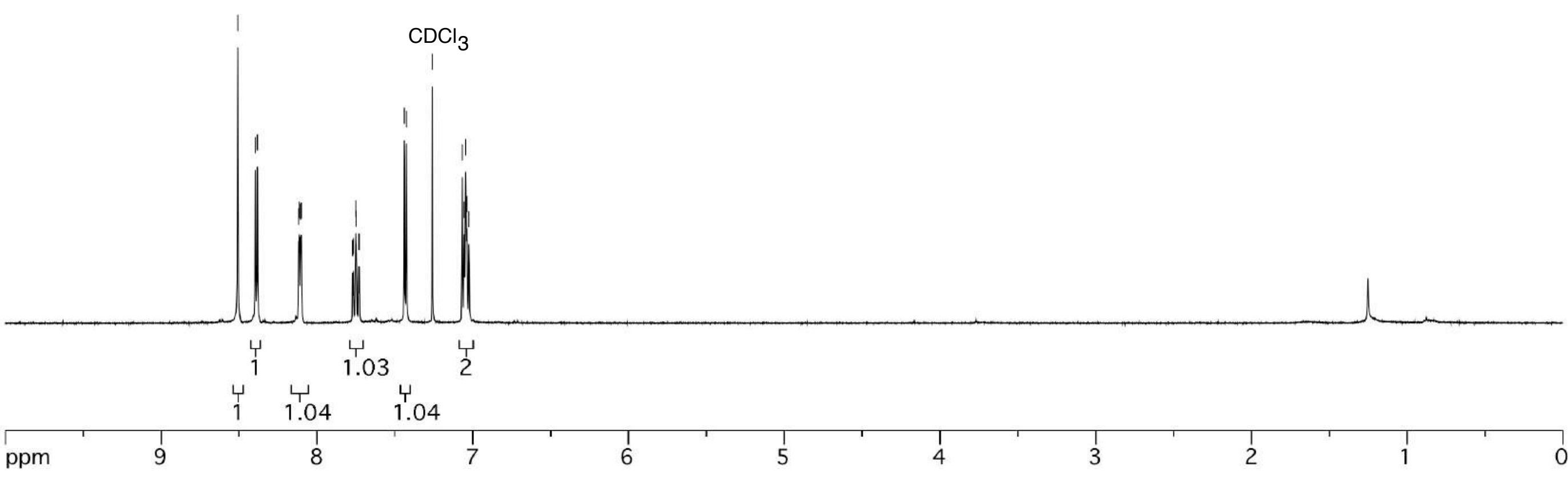



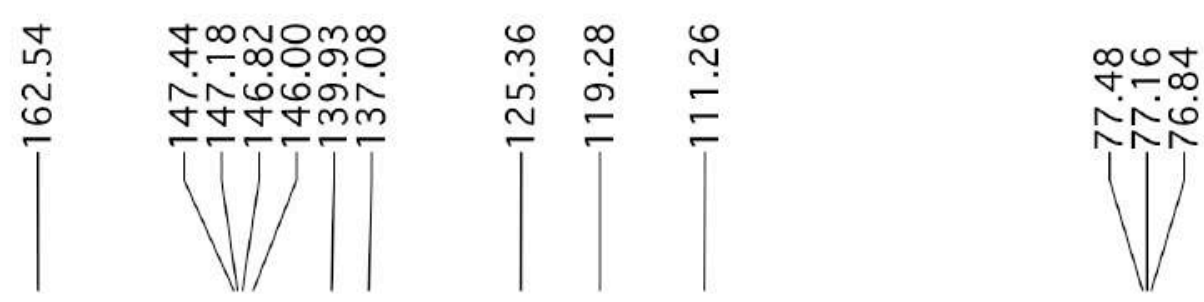

${ }^{13} \mathrm{C} \mathrm{NMR}\left(\mathrm{CDCl}_{3}, 100 \mathrm{MHz}\right)$<smiles>Clc1ccncc1Oc1ccccn1</smiles> 
ํํำ ఝ

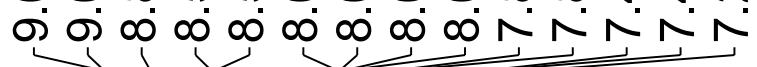

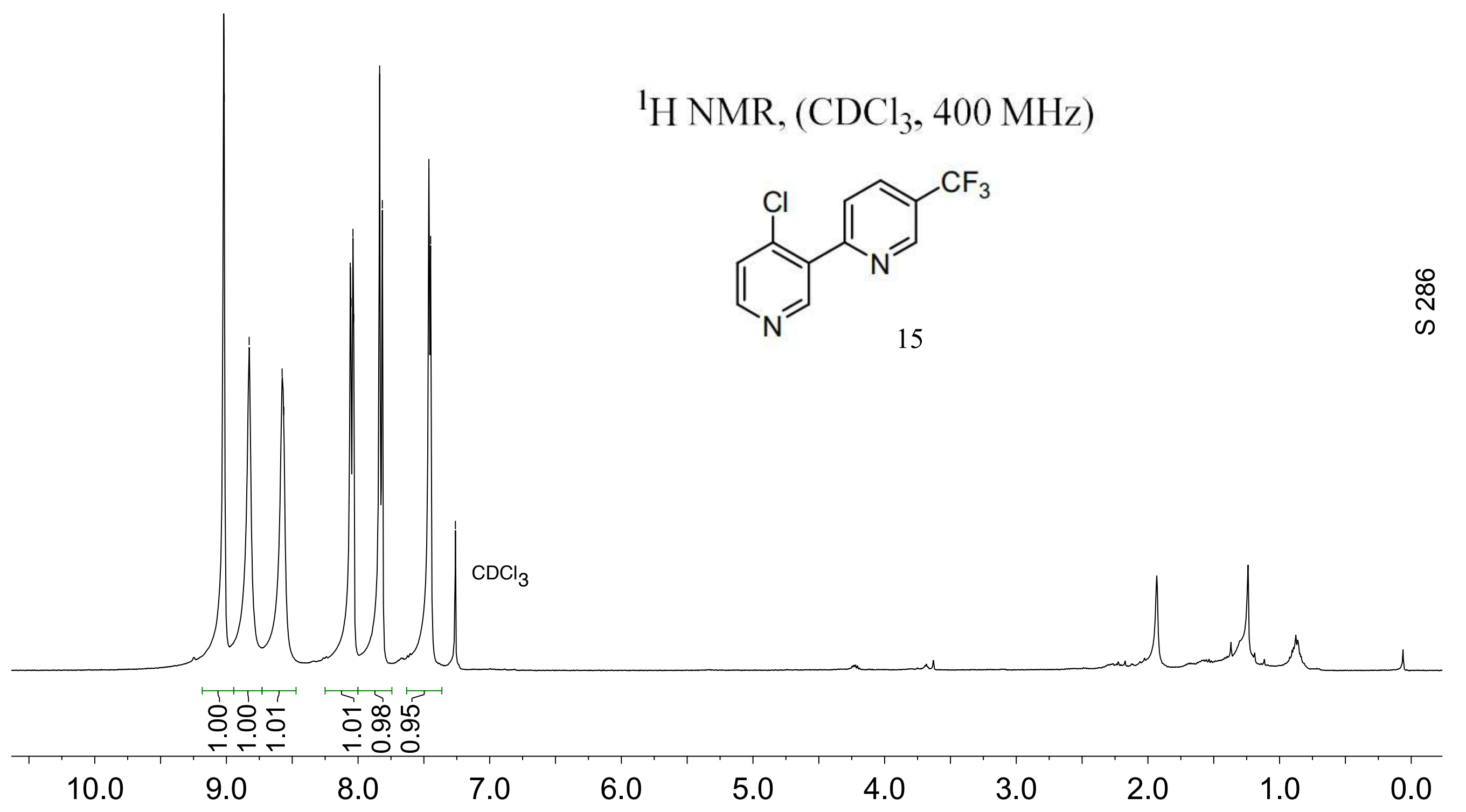


<smiles>FC(F)(F)c1ccc(-c2cnccc2Cl)nc1</smiles>

15 


\section{${ }^{19} \mathrm{~F} \mathrm{NMR}\left(\mathrm{CDCl}_{3}, 365 \mathrm{MHz}\right)$}

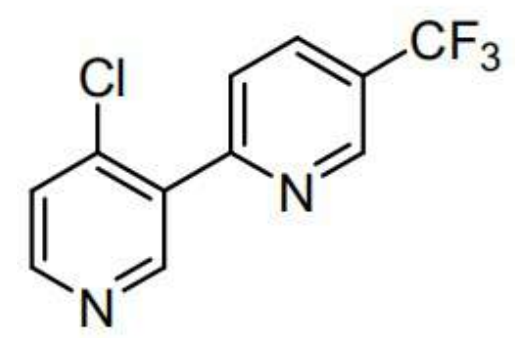

15

$0 \quad-2$

$-20 \quad-40 \quad-60$

$-80$

$-100$

$-120$

$-140$

$-160$

$-180$

$-200-2 i$ 


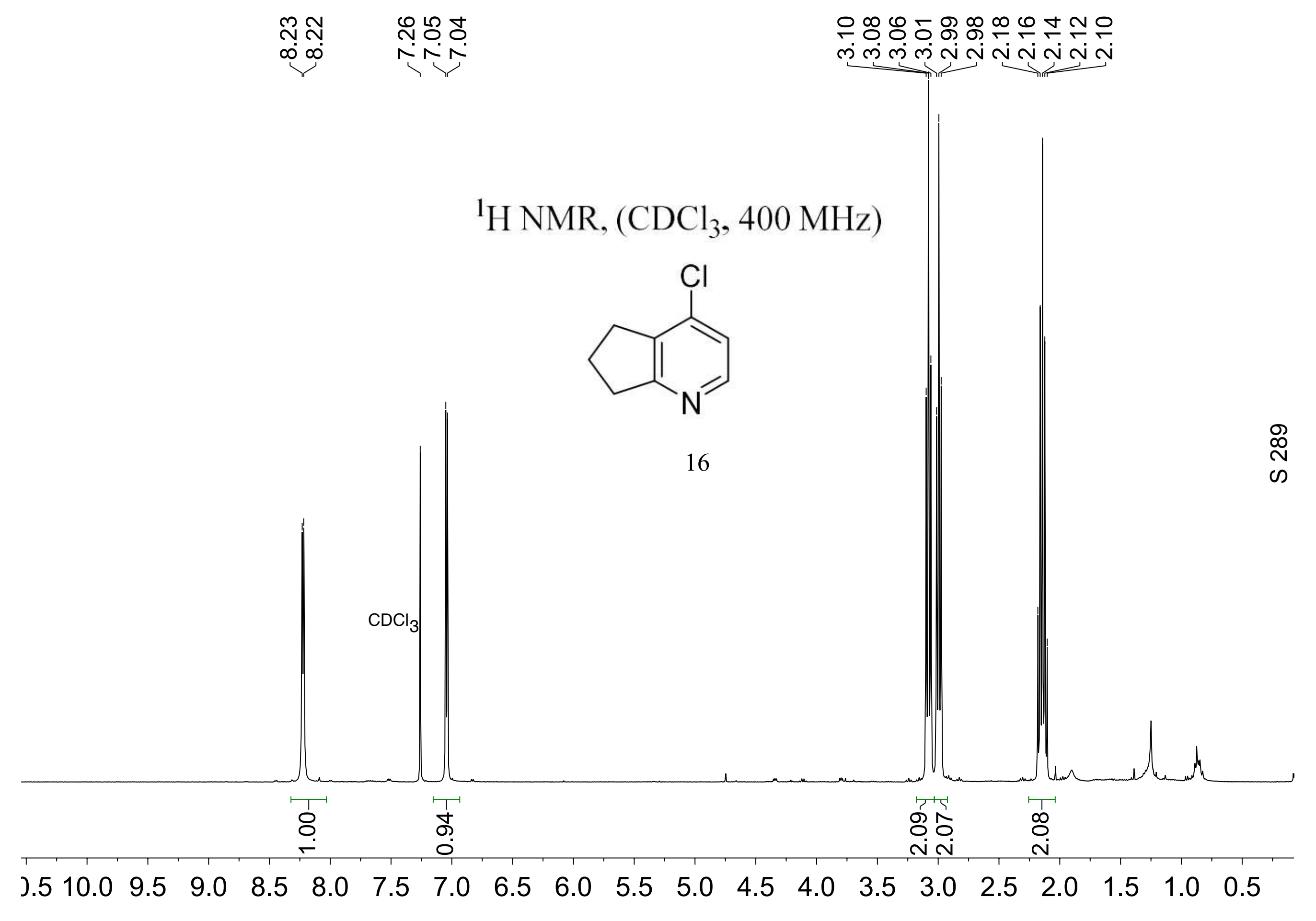




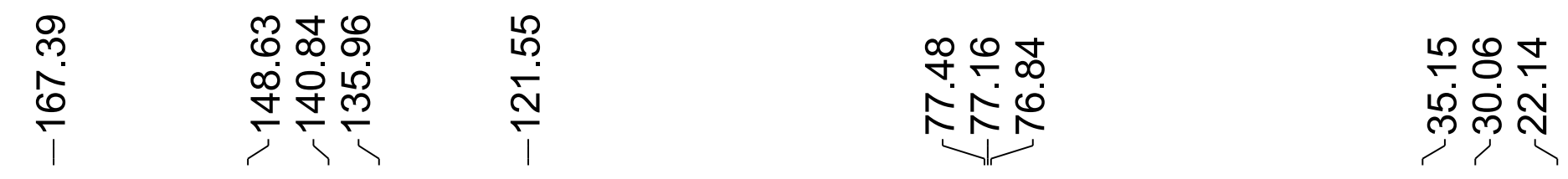

${ }^{13} \mathrm{C} \mathrm{NMR},\left(\mathrm{CDCl}_{3}, 100 \mathrm{MHz}\right)$<smiles>Clc1ccnc2c1CCC2</smiles>

16

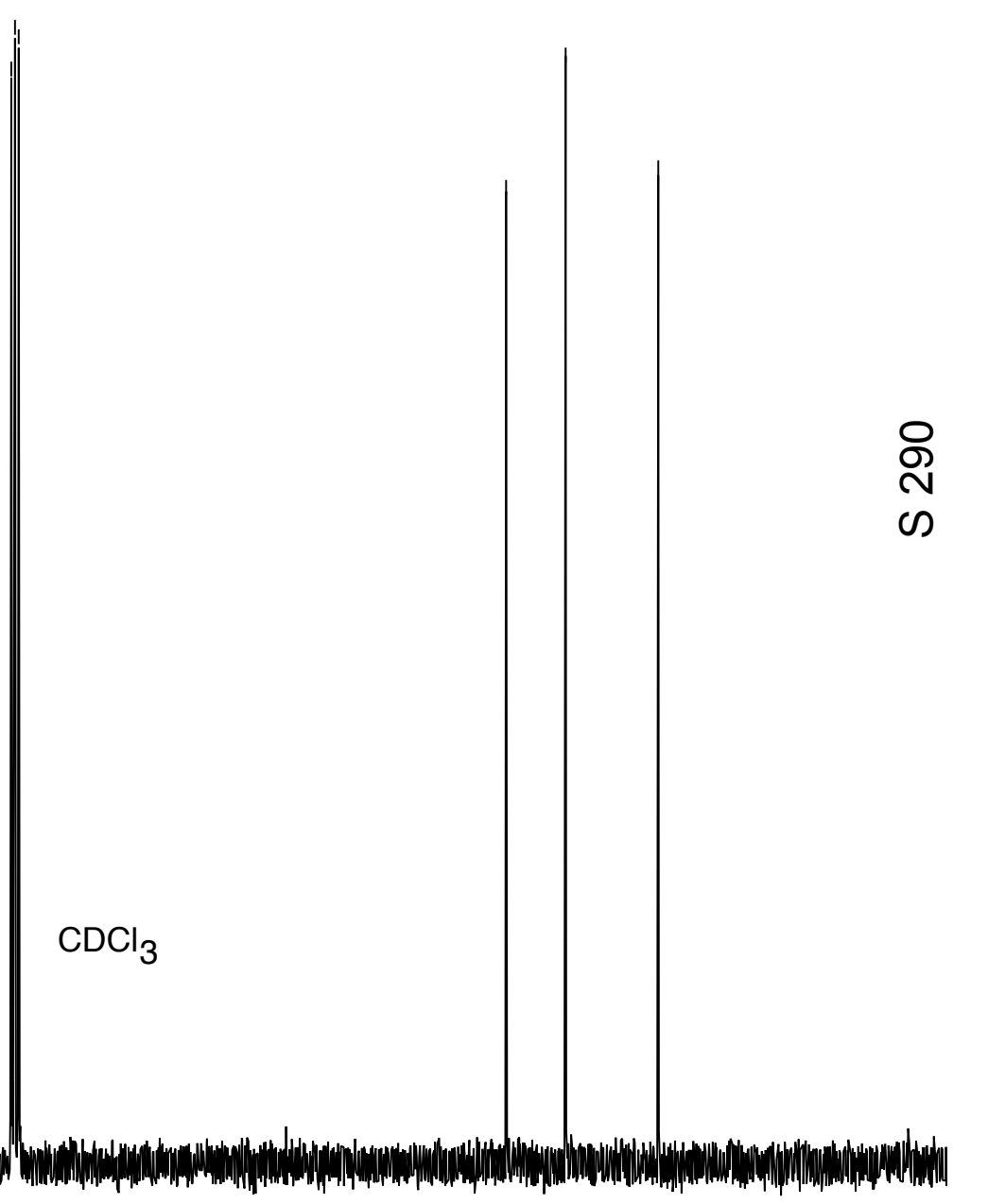

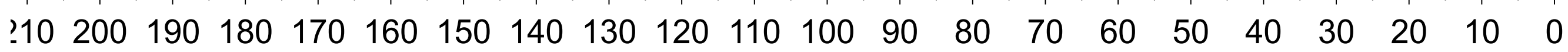




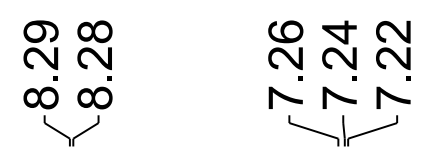

\section{${ }^{1} \mathrm{H} \mathrm{NMR},\left(\mathrm{CDCl}_{3}, 400 \mathrm{MHz}\right)$}<smiles>Cc1nccc(Cl)c1Br</smiles>

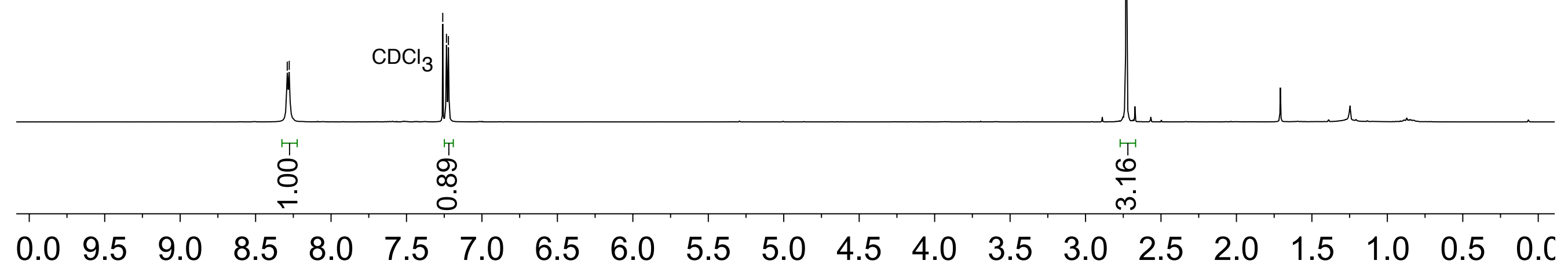




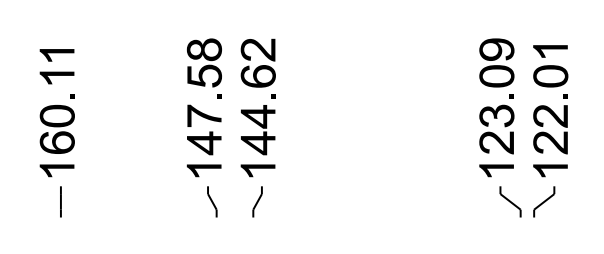

+

$\sim N$

NN

${ }^{13} \mathrm{C} \mathrm{NMR},\left(\mathrm{CDCl}_{3}, 100 \mathrm{MHz}\right)$<smiles>Cc1nccc(Cl)c1Br</smiles> 


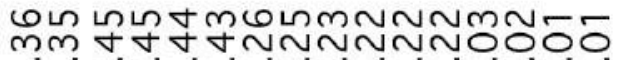

$\infty \infty N-N N N N N$ NNNN

^ิ

${ }^{1} \mathrm{H} \mathrm{NMR}\left(\mathrm{CDCl}_{3}, 400 \mathrm{MHz}\right)$

18<smiles>Cc1nccc(Cl)c1-c1ccsc1</smiles>

$\mathrm{CDCl}_{3}$

I

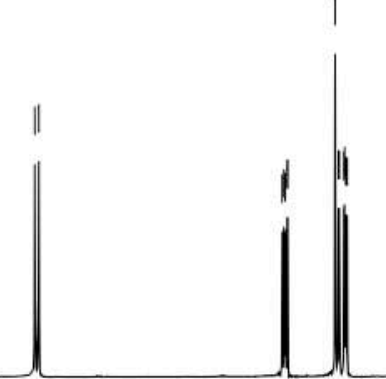

$\begin{array}{lll}4 & 4 \\ 1 & 0.946\end{array}$

3.3

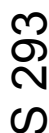

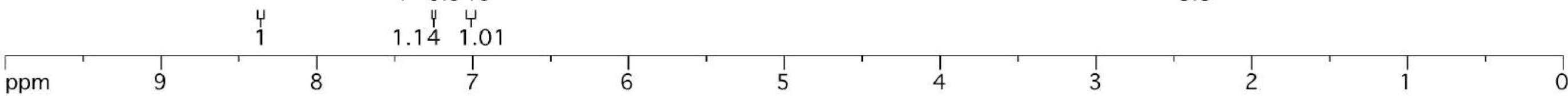



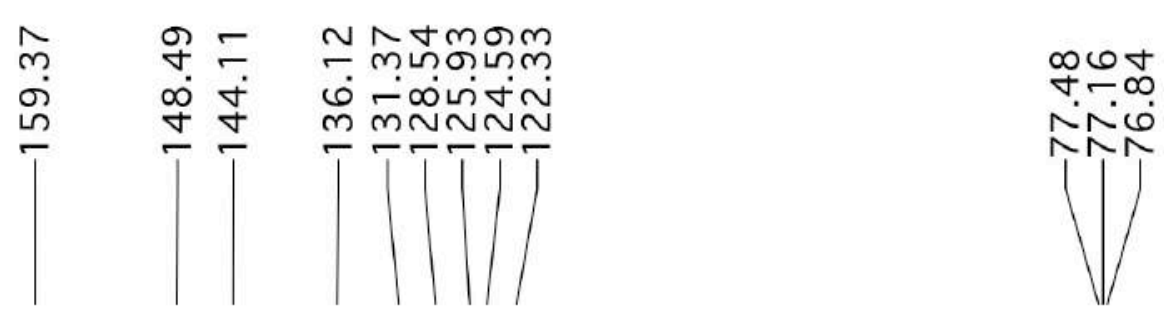

${ }^{13} \mathrm{C} \mathrm{NMR}\left(\mathrm{CDCl}_{3}, 100 \mathrm{MHz}\right)$<smiles>[N-]=[N+]=[N+]=[N+]=[N+]=[N+]=O</smiles>

$\mathrm{CDCl}_{3}$ 


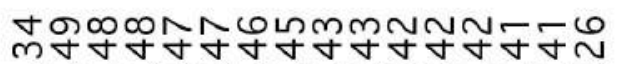

कíñ

${ }^{1} \mathrm{H} \mathrm{NMR}\left(\mathrm{CDCl}_{3}, 400 \mathrm{MHz}\right)$

20<smiles>Clc1cc(Cl)c(-c2ccccc2)cn1</smiles> 


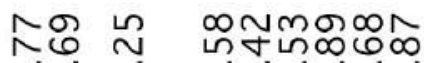

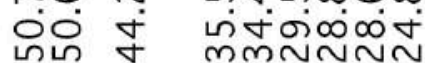

丁T T

$\stackrel{\substack{+\infty \\ \infty}}{\infty}$

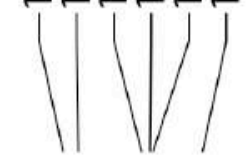

${ }^{13} \mathrm{C} \mathrm{NMR}\left(\mathrm{CDCl}_{3}, 100 \mathrm{MHz}\right)$<smiles>Clc1cc(Cl)c(-c2ccccc2)cn1</smiles> 


\section{${ }^{1} \mathrm{H} \mathrm{NMR},\left(\mathrm{CDCl}_{3}, 400 \mathrm{MHz}\right)$}<smiles>Cc1cc(Cl)c(-c2cc(F)cc(Cl)c2)cn1</smiles>

21

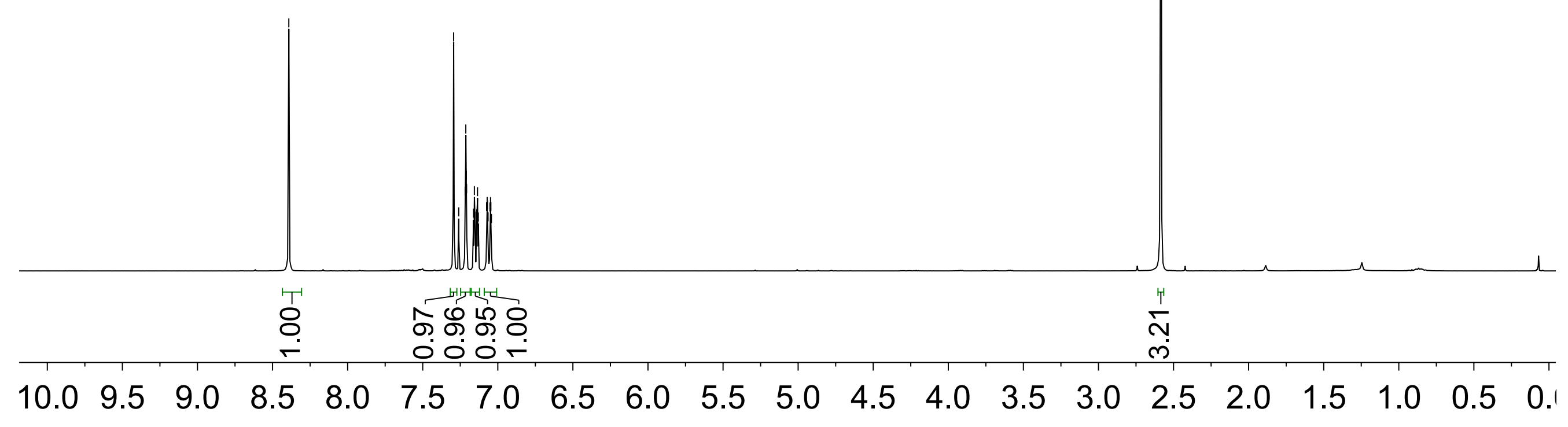




\section{${ }^{13} \mathrm{C} \mathrm{NMR,}\left(\mathrm{CDCl}_{3}, 100 \mathrm{MHz}\right)$}

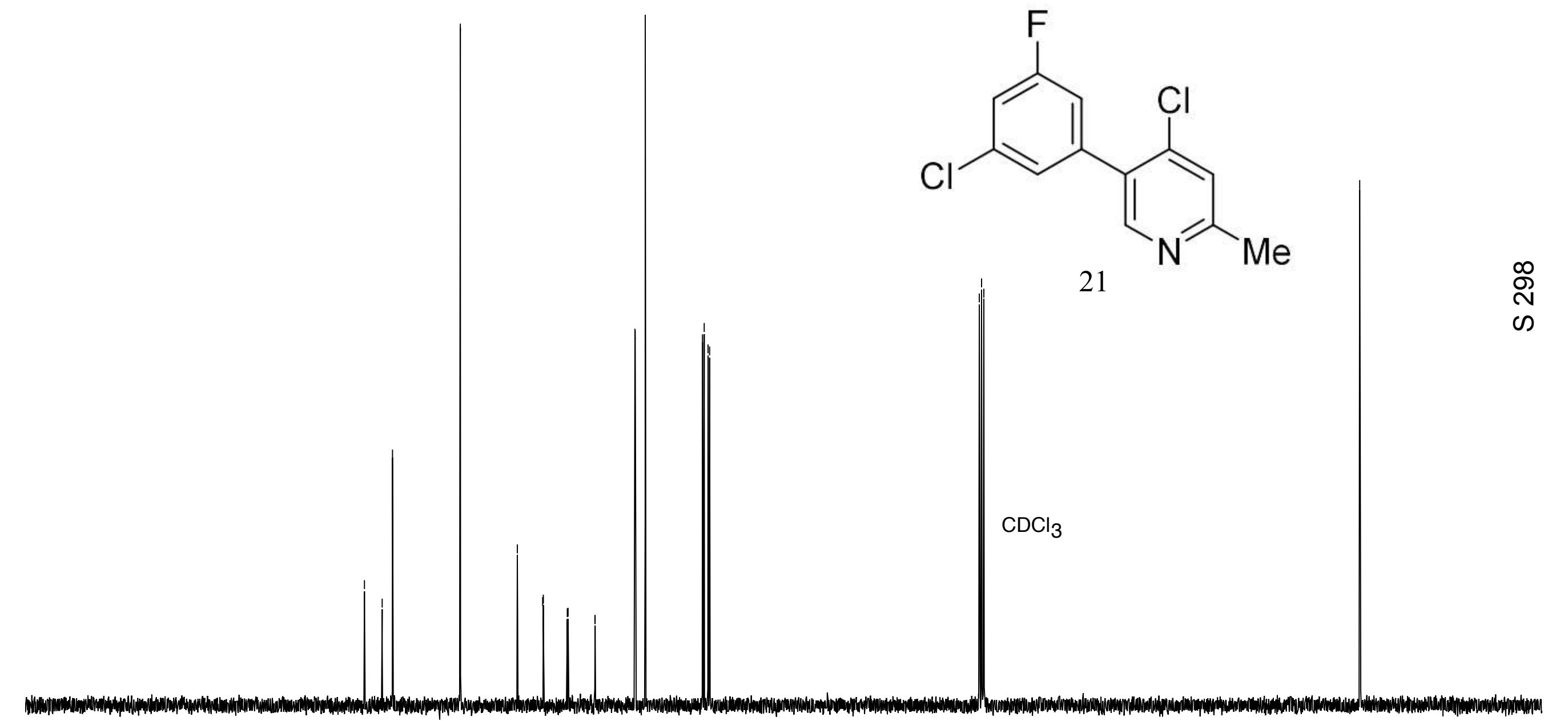

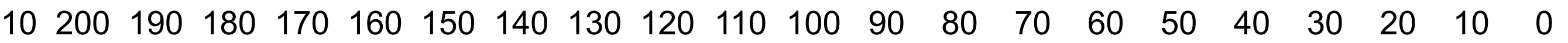




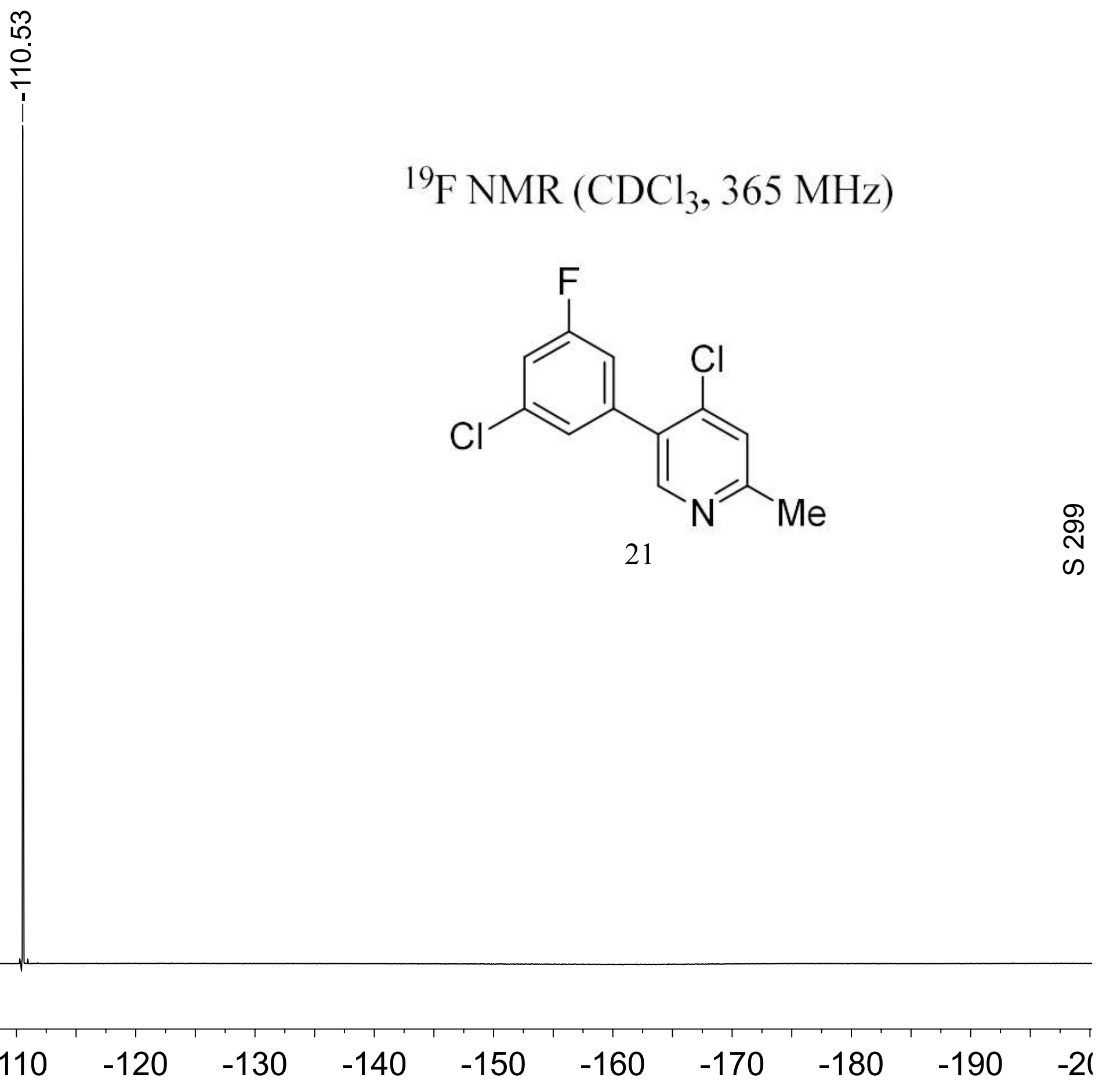



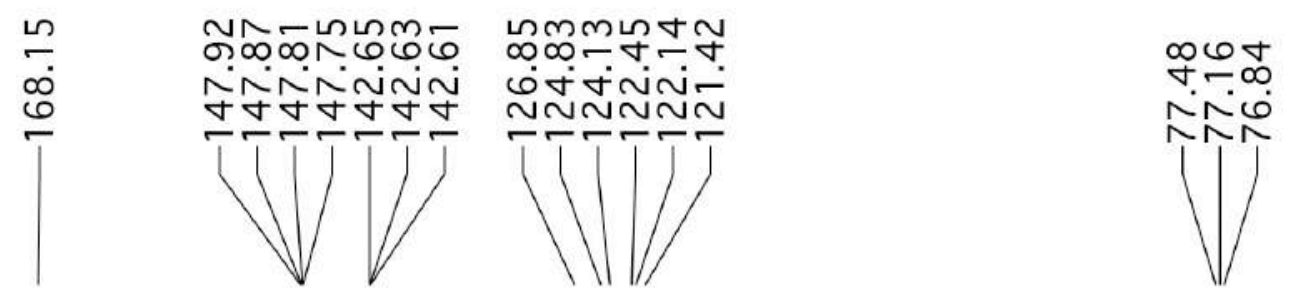

ஸे ம

${ }^{13} \mathrm{C}$ NMR $\left(\mathrm{CDCl}_{3}, 100 \mathrm{MHz}\right)$<smiles>FC(F)(F)c1cnc(Br)cc1Cl</smiles> 
${ }^{19} \mathrm{~F} \mathrm{NMR}\left(\mathrm{CDCl}_{3}, 365 \mathrm{MHz}\right)$<smiles>O=C(c1ccccc1)c1cc(Cl)c(C(F)(F)F)cn1</smiles>

\begin{tabular}{|c|c|c|}
\hline $\begin{array}{ll}\text { ppm } & -0\end{array}$ & $\begin{array}{c}1 \\
-50\end{array}$ & $\begin{array}{r}1 \\
-100\end{array}$ \\
\hline
\end{tabular}




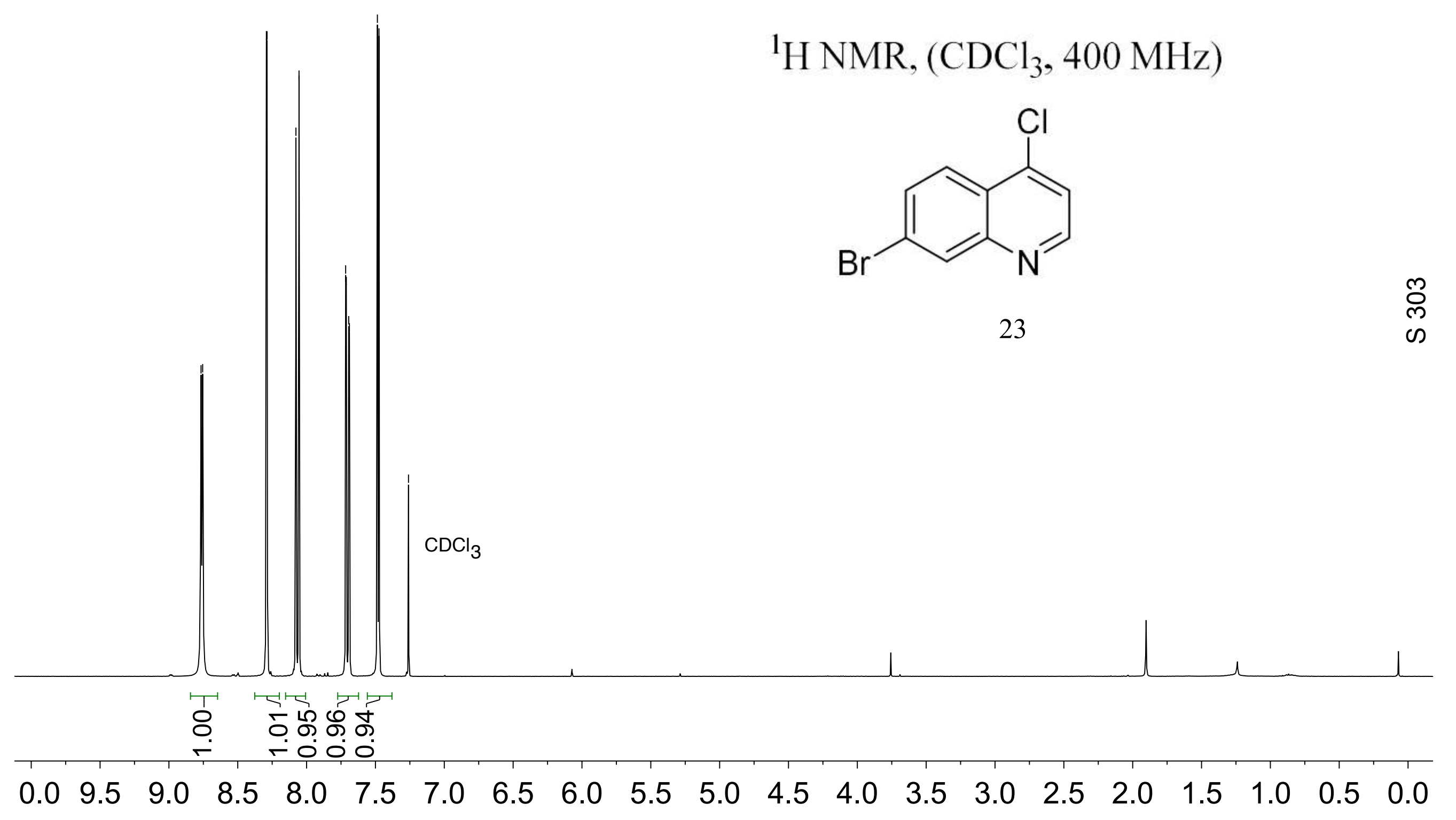


소유ำ 웅워

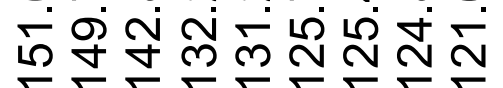

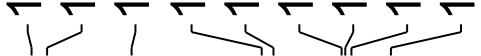

$\stackrel{\infty}{+} \stackrel{+}{\sim}$

Nं

솟

\section{${ }^{13} \mathrm{C}$ NMR, $\left(\mathrm{CDCl}_{3}, 100 \mathrm{MHz}\right)$}<smiles>Clc1ccnc2cc(Br)ccc12</smiles> 


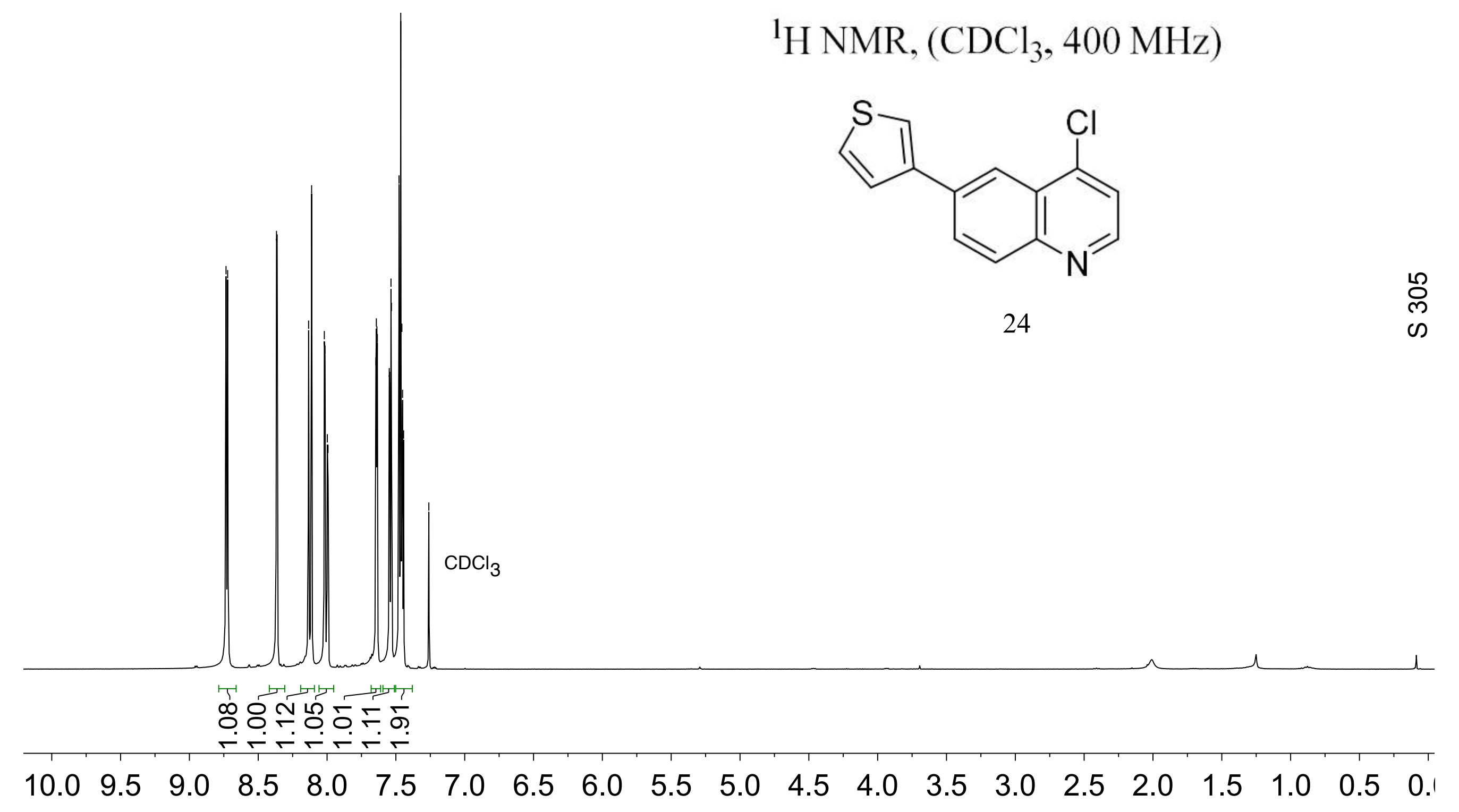




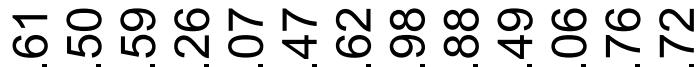

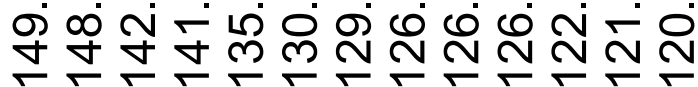

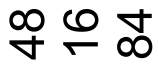 \\ Nंกั \\ 西年}

\section{${ }^{13} \mathrm{C} \mathrm{NMR},\left(\mathrm{CDCl}_{3}, 100 \mathrm{MHz}\right)$}<smiles>Clc1ccnc2ccc(-c3ccsc3)cc12</smiles>

24

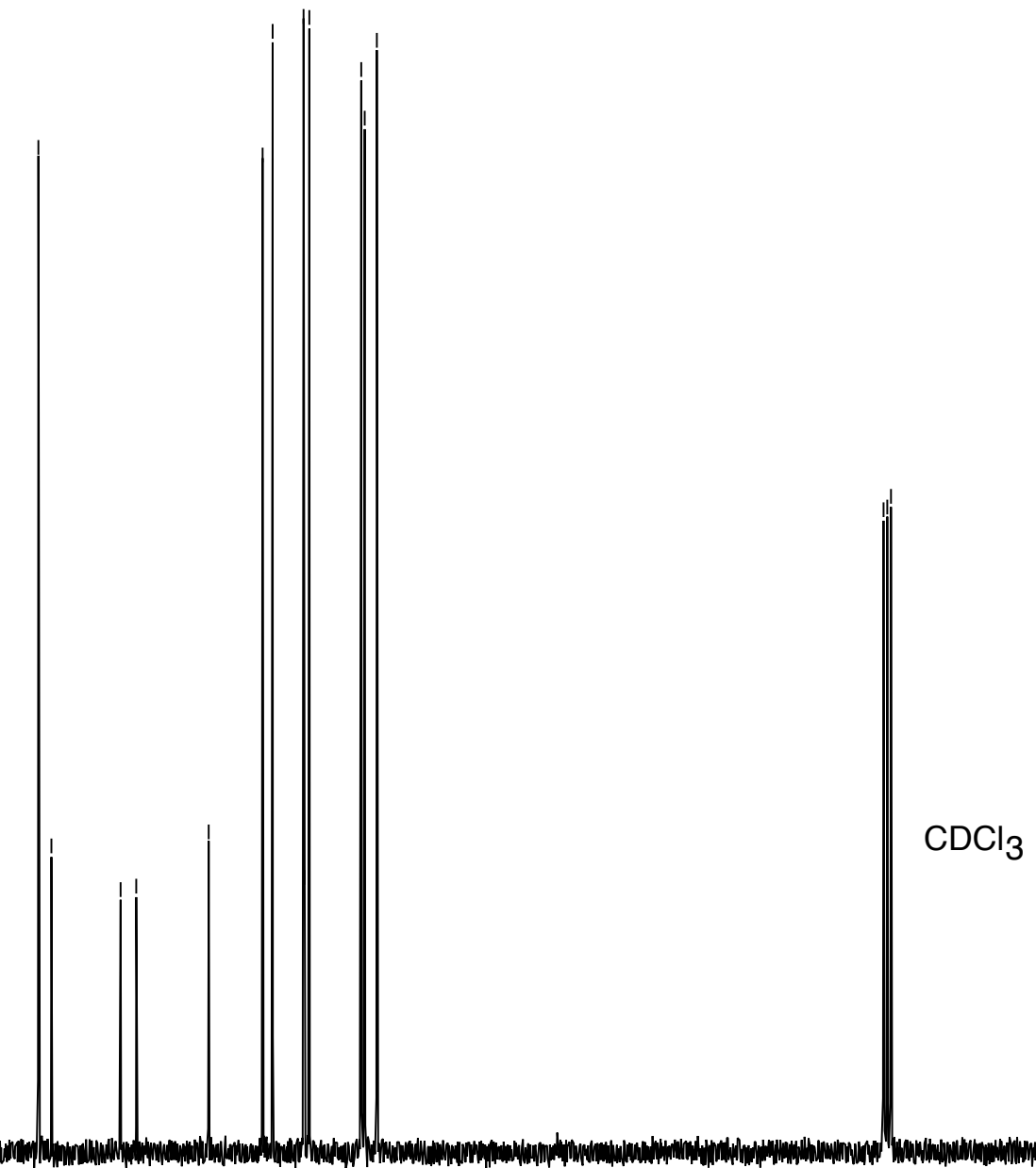



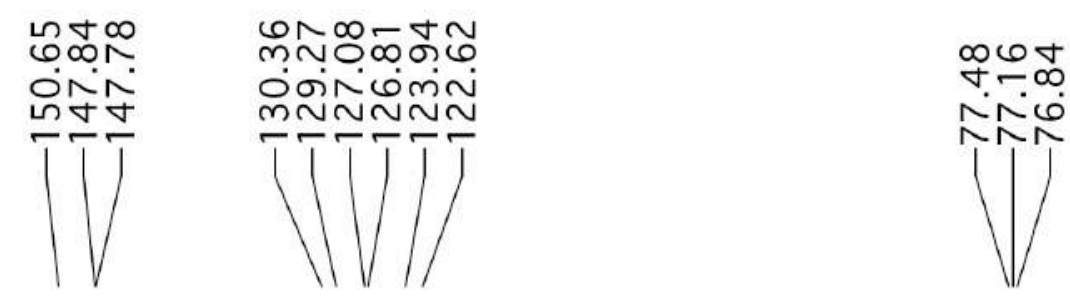

${ }^{13} \mathrm{C} \mathrm{NMR}\left(\mathrm{CDCl}_{3}, 100 \mathrm{MHz}\right)$<smiles>Cc1cc(Cl)nc2ccccc12</smiles> 

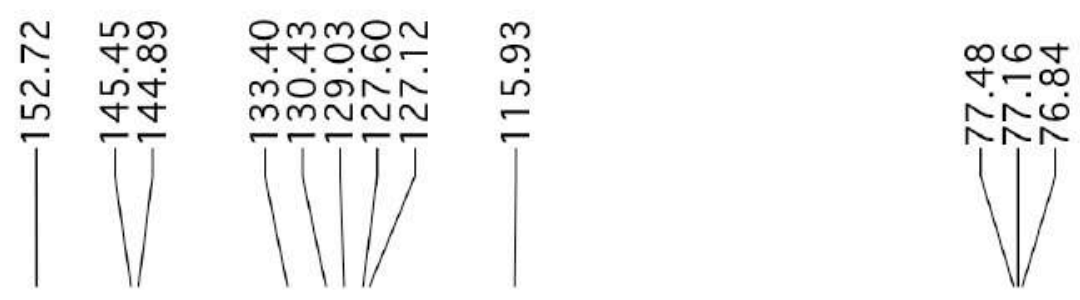

${ }^{13} \mathrm{C}$ NMR $\left(\mathrm{CDCl}_{3}, 100 \mathrm{MHz}\right)$

26<smiles>O=[N+]([O-])c1cccc2c(Cl)nccc12</smiles> 


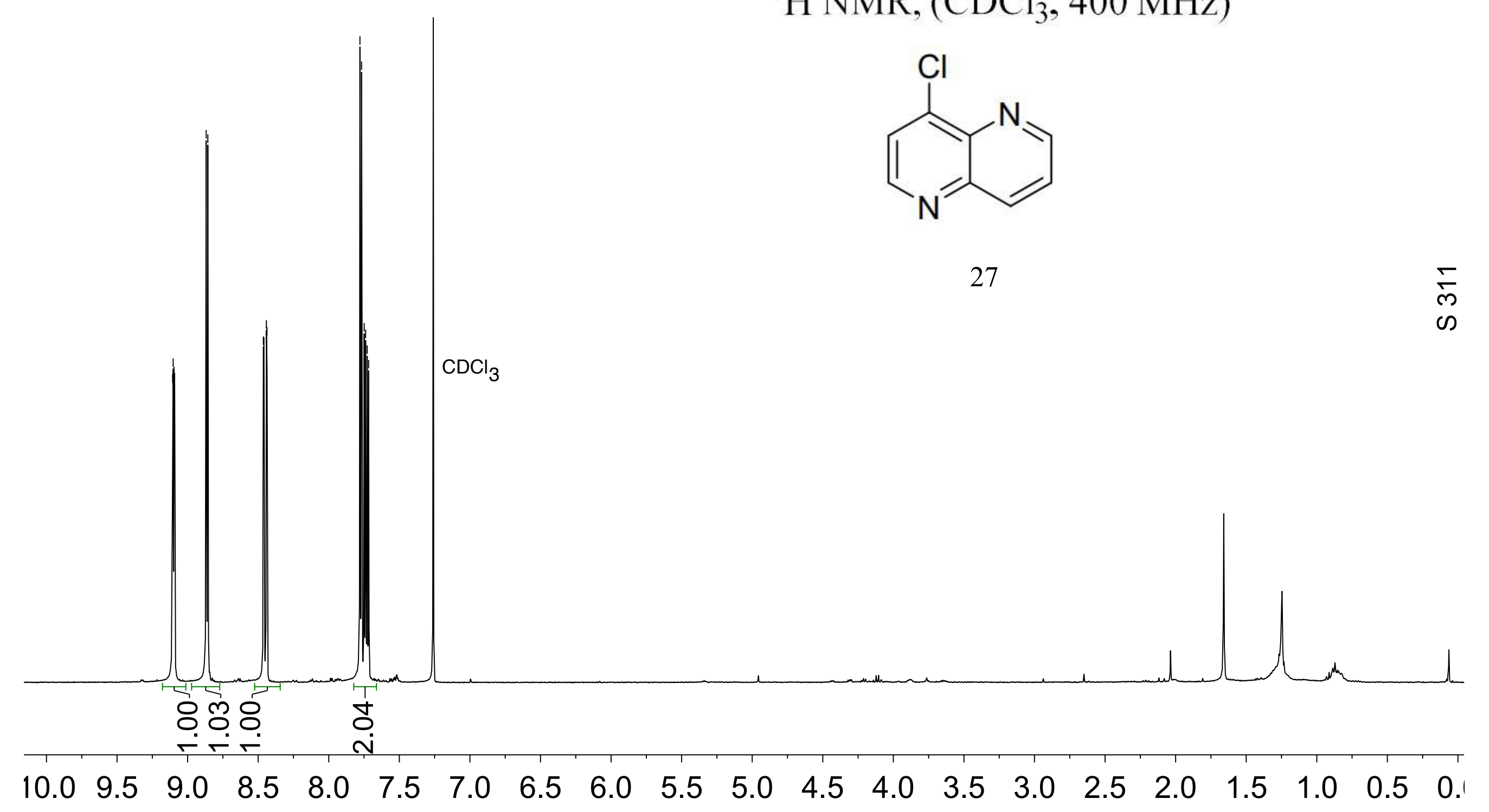




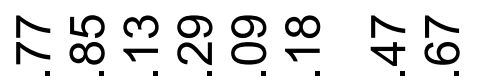

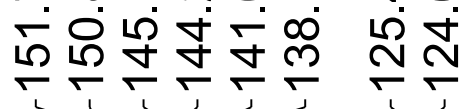

䎹 $N N$

\section{${ }^{13} \mathrm{C} \mathrm{NMR},\left(\mathrm{CDCl}_{3}, 100 \mathrm{MHz}\right)$}<smiles>Clc1ccnc2cccnc12</smiles>

27 
${ }^{1} \mathrm{H} \mathrm{NMR}\left(\mathrm{CDCl}_{3}, 400 \mathrm{MHz}\right)$

28<smiles>Clc1cnc2ccccc2n1</smiles>

$\stackrel{m}{m}$

$\mathrm{CDCl}_{3}$

눈다.1

$1.0 \quad 1.1$ 

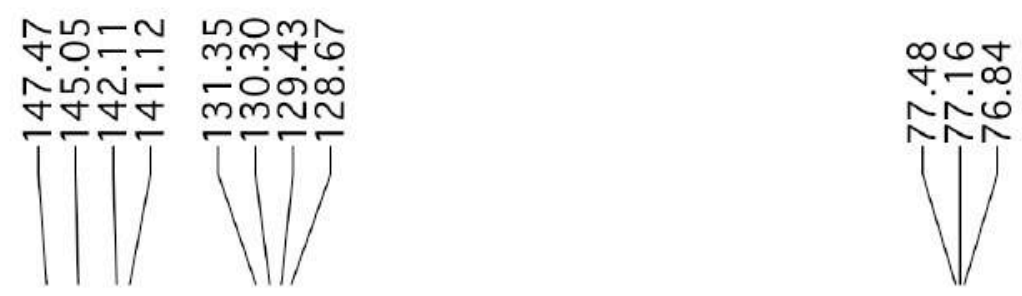

${ }^{13} \mathrm{C}$ NMR $\left(\mathrm{CDCl}_{3}, 100 \mathrm{MHz}\right)$

28<smiles>Clc1cnc2ccccc2n1</smiles> 


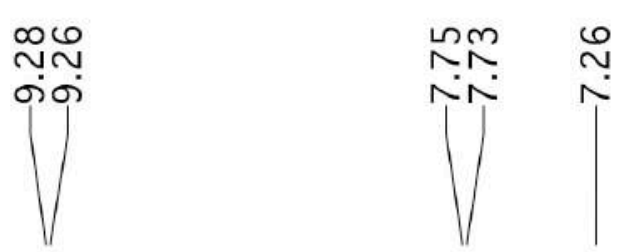

${ }^{1} \mathrm{H} \mathrm{NMR}\left(\mathrm{CDCl}_{3}, 400 \mathrm{MHz}\right)$

29<smiles>N#Cc1nnccc1Cl</smiles>

$\stackrel{\infty}{\infty}$

$\mathrm{CDCl}_{3}$

0.96

1

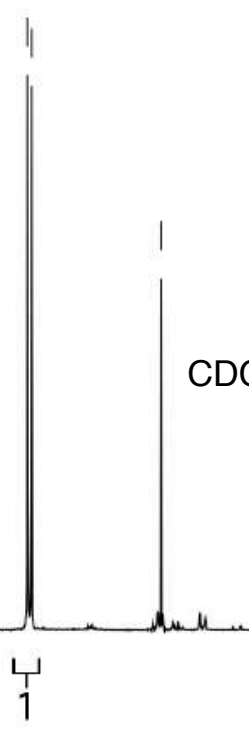

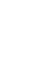




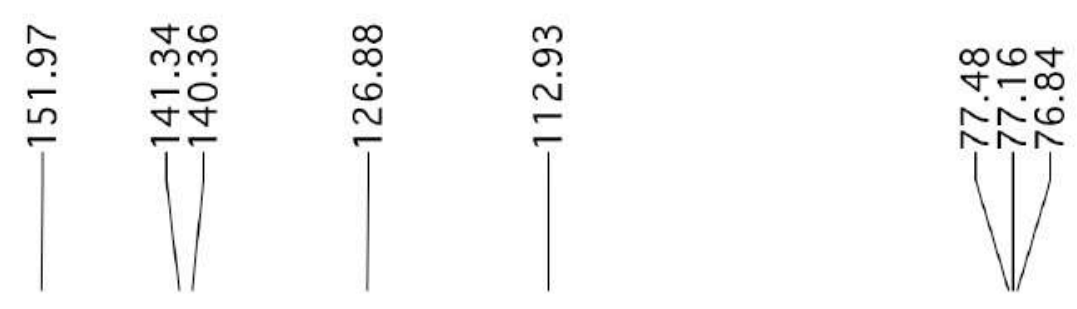

${ }^{13} \mathrm{C} \mathrm{NMR}\left(\mathrm{CDCl}_{3}, 100 \mathrm{MHz}\right)$

29<smiles>N#Cc1nnccc1Cl</smiles> 
${ }^{1} \mathrm{H} \mathrm{NMR}\left(\mathrm{CDCl}_{3}, 400 \mathrm{MHz}\right)$<smiles>Clc1nccn2ccnc12</smiles>

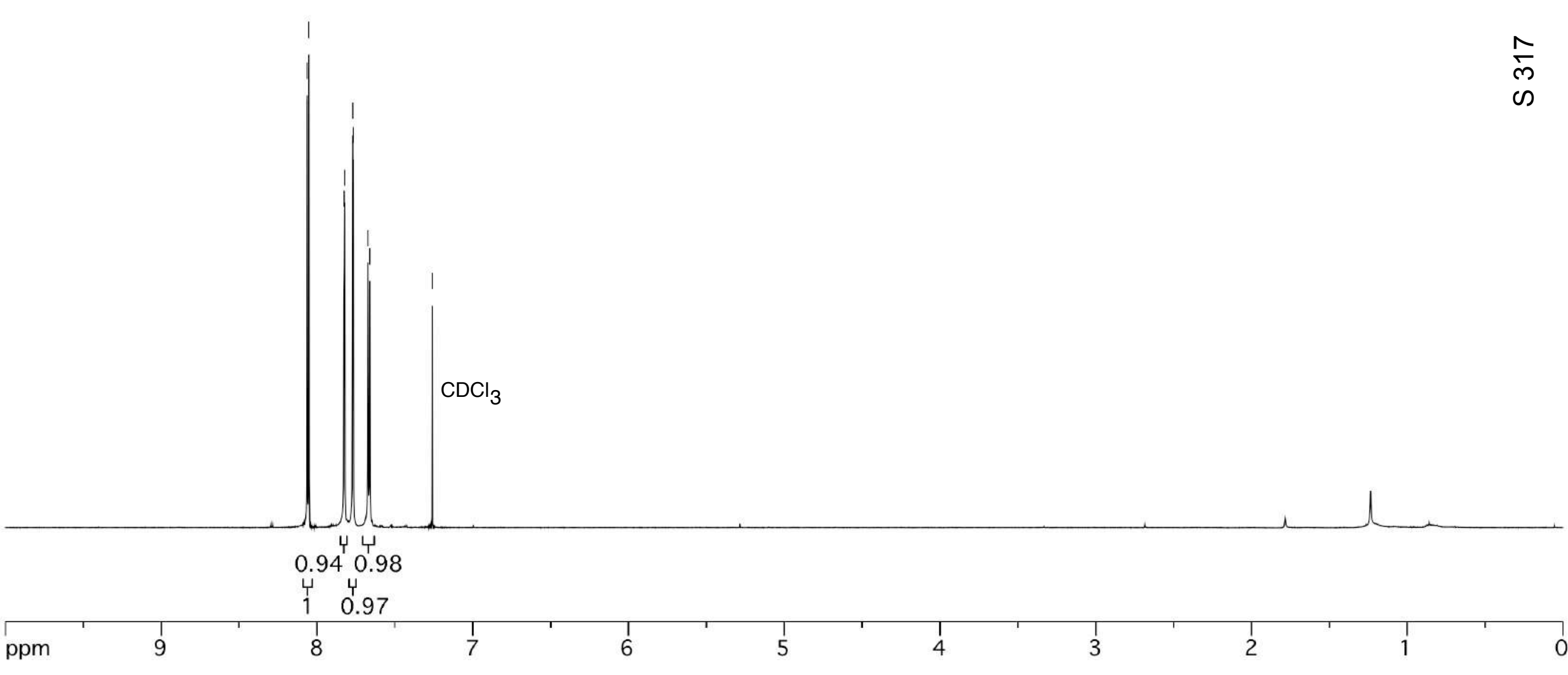



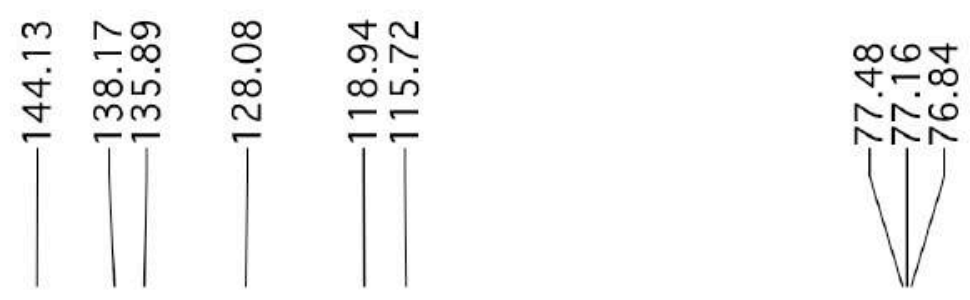

${ }^{13} \mathrm{C} \mathrm{NMR}\left(\mathrm{CDCl}_{3}, 100 \mathrm{MHz}\right)$<smiles>Clc1nccn2ccnc12</smiles>

31 


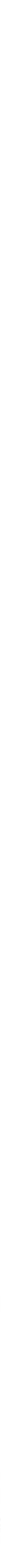




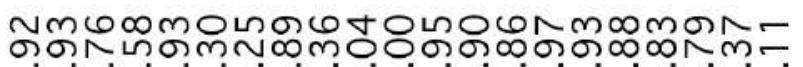

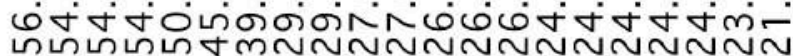
促

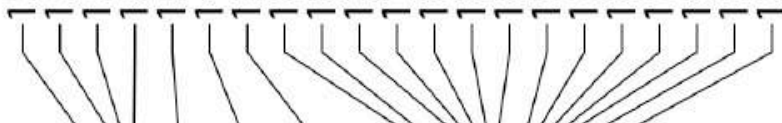

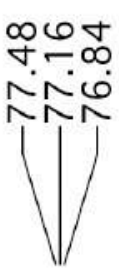

${ }^{13} \mathrm{C} \mathrm{NMR}\left(\mathrm{CDCl}_{3}, 100 \mathrm{MHz}\right)$

32<smiles>FS(F)(F)c1cccc(-c2cc(Cl)ccn2)c1</smiles> 
${ }^{19} \mathrm{~F}$ NMR $\left(\mathrm{CDCl}_{3}, 365 \mathrm{MHz}\right)$

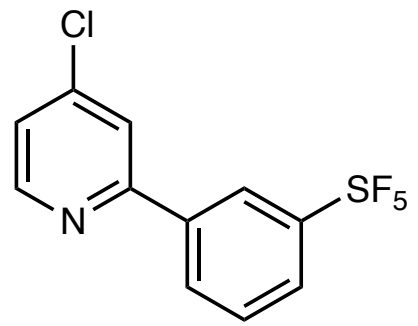


${ }^{1} \mathrm{H} \mathrm{NMR}\left(\mathrm{CDCl}_{3}, 400 \mathrm{MHz}\right)$

33<smiles>Clc1ccnc(C2OCCO2)c1</smiles>

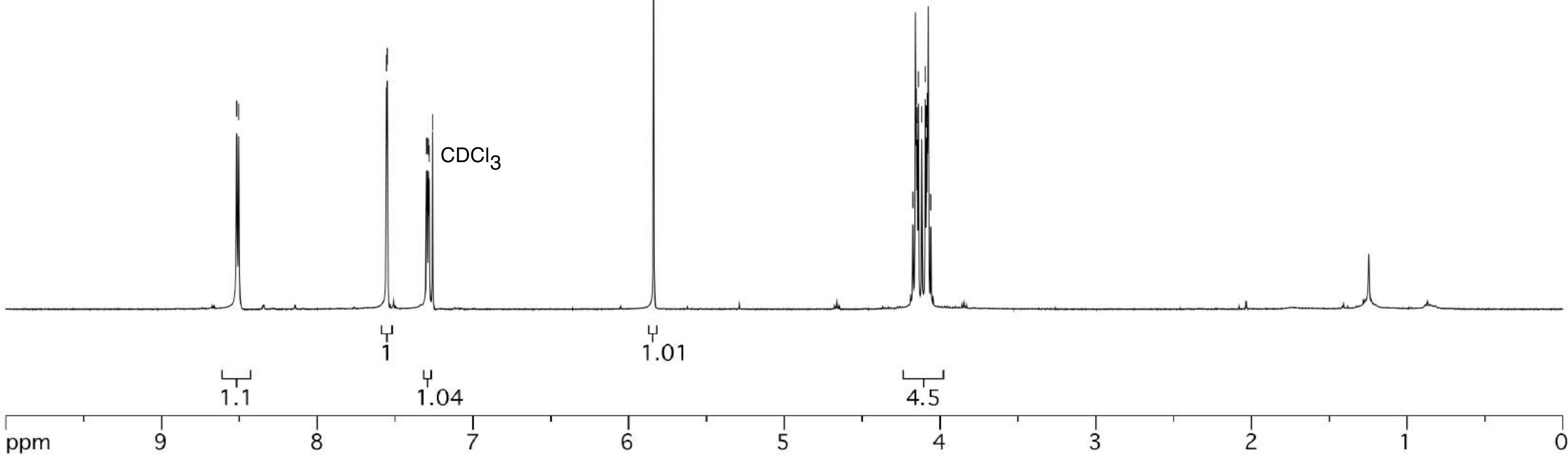




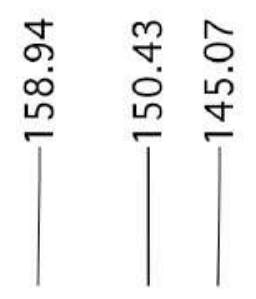

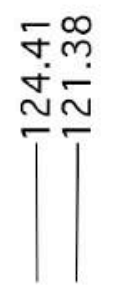

${ }^{13} \mathrm{C} \mathrm{NMR}\left(\mathrm{CDCl}_{3}, 100 \mathrm{MHz}\right)$

33

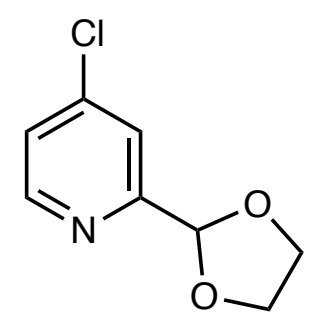

$\infty$
0
0
1

$\infty 6 \downarrow$ ن.

NNE

1)

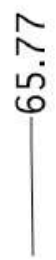

10

N
$\omega$

$\mathrm{CDCl}_{3}$ 


\section{${ }^{1} \mathrm{H} \mathrm{NMR},\left(\mathrm{CDCl}_{3}, 400 \mathrm{MHz}\right)$}<smiles>[O+]C(c1ccccc1)c1cc(Cl)ccn1</smiles>

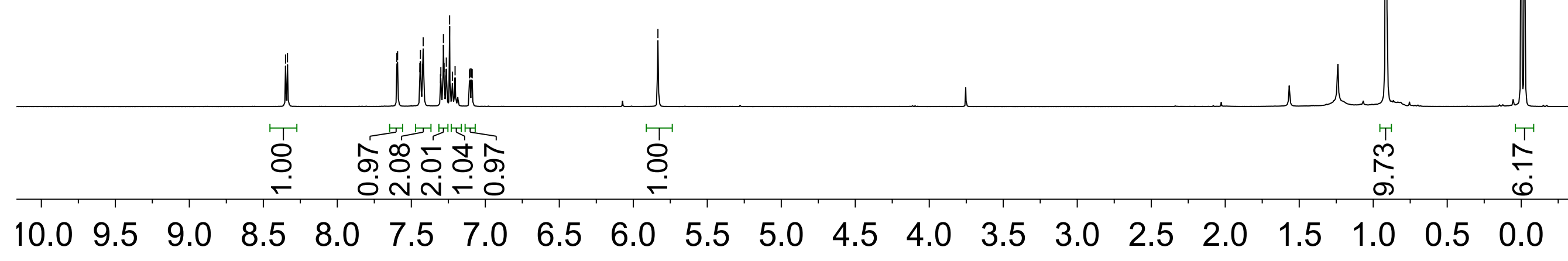




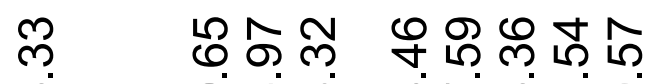

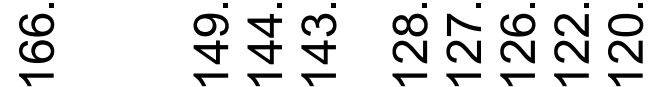 \\ F एक}

${ }^{13} \mathrm{C} \mathrm{NMR},\left(\mathrm{CDCl}_{3}, 100 \mathrm{MHz}\right)$

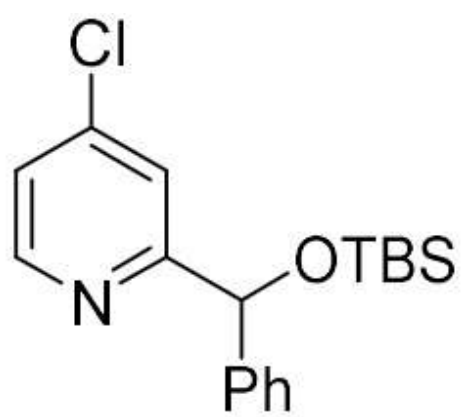

34

\section{ষ্ণে \\ ÑN่ \\ 손}

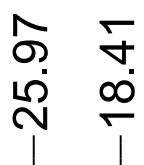

זঙ
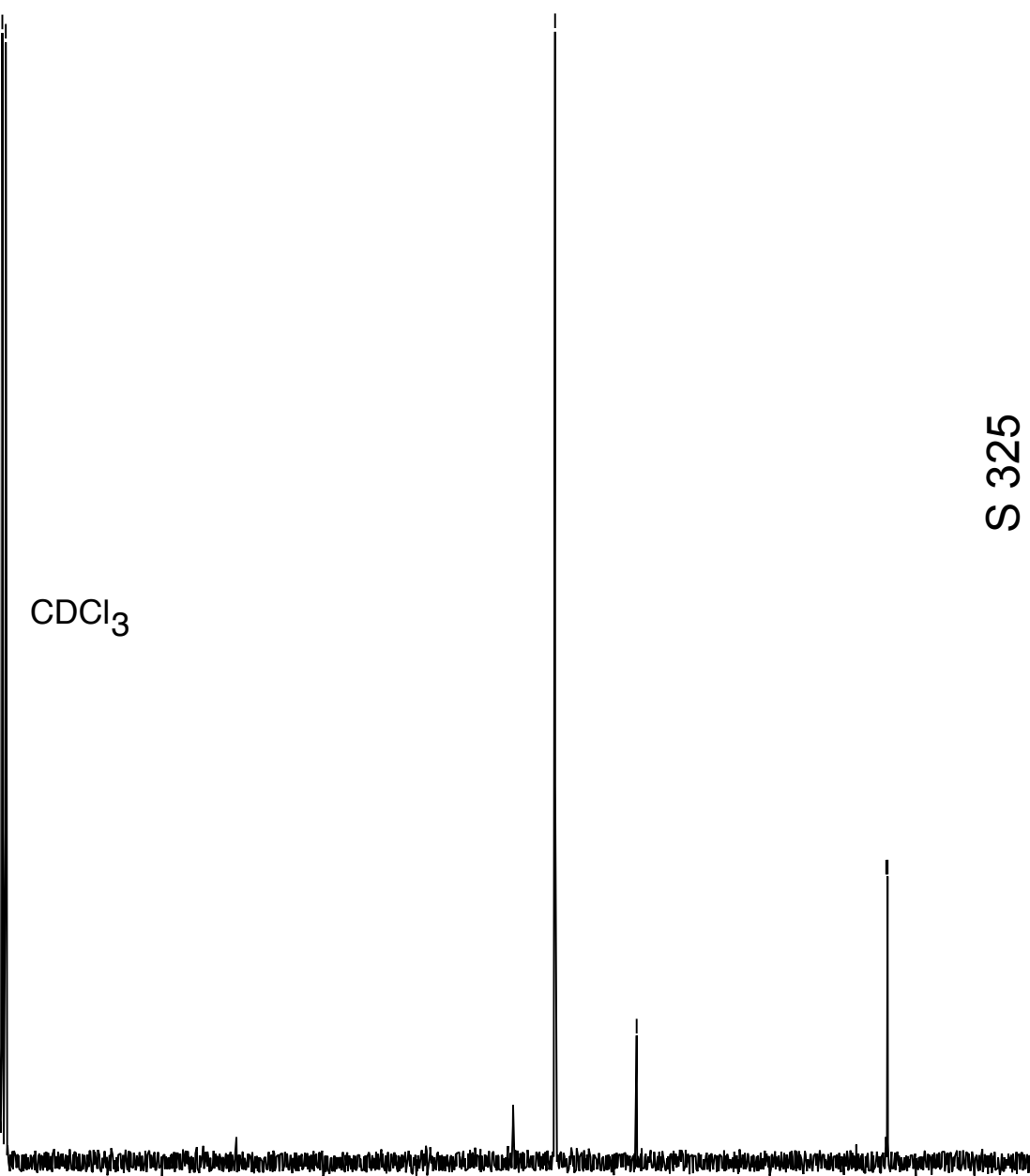

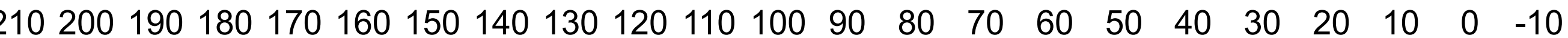


${ }^{1} \mathrm{H} \mathrm{NMR}\left(\mathrm{CD}_{3} \mathrm{OD}, 400 \mathrm{MHz}\right)$
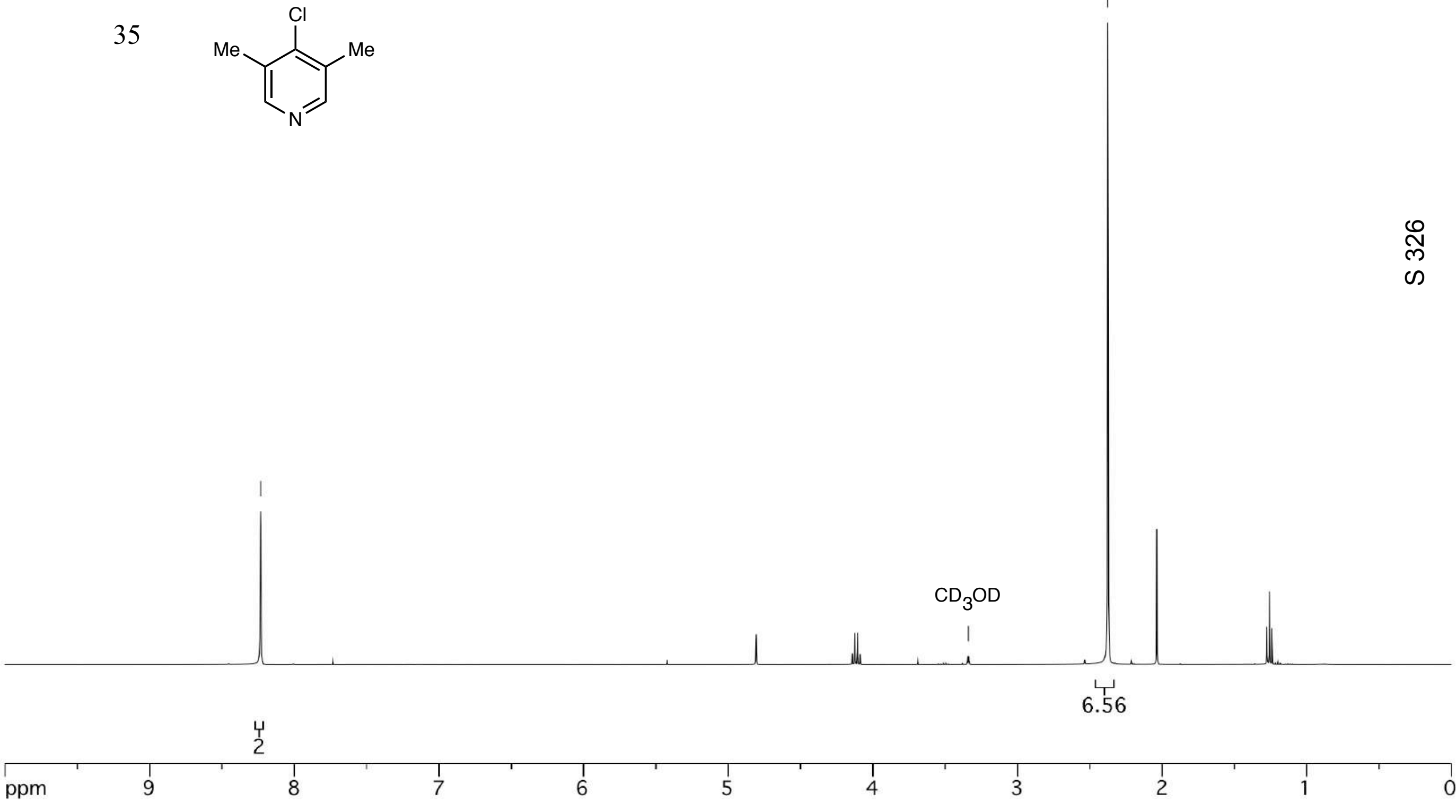

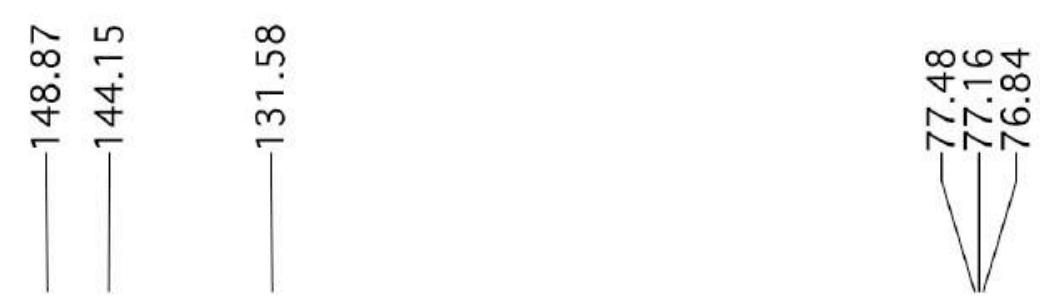

${ }^{13} \mathrm{C}$ NMR $\left(\mathrm{CDCl}_{3}, 100 \mathrm{MHz}\right)$

35<smiles>Cc1cncc([N+](=O)[O-])c1Cl</smiles> 


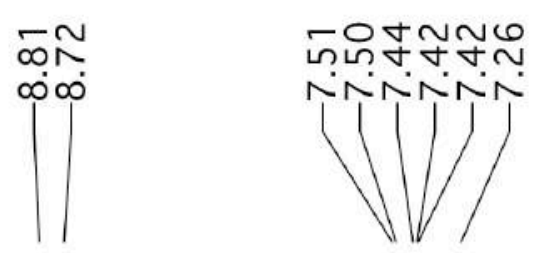

${ }^{1} \mathrm{H} \mathrm{NMR}\left(\mathrm{CDCl}_{3}, 400 \mathrm{MHz}\right)$

$\stackrel{\infty}{\sim}$

$\mathrm{CDCl}_{3}$

36<smiles>N#Cc1cncc(-c2ccccc2)c1Cl</smiles>

5.3

\begin{tabular}{l|l|l}
1 & 1 & 1 \\
8 & 7 & 6
\end{tabular}



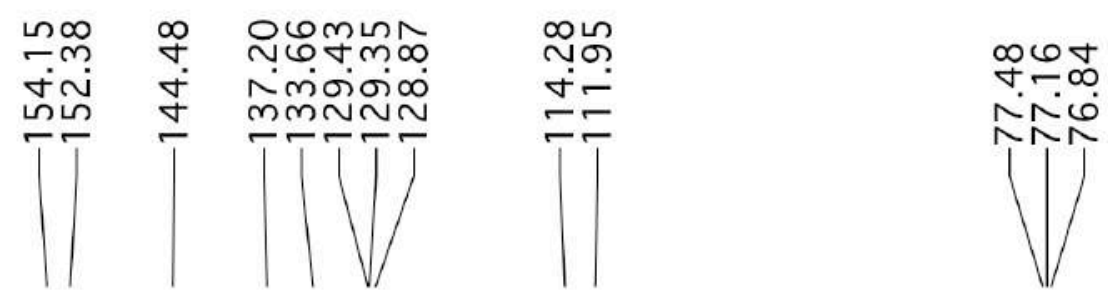

${ }^{13} \mathrm{C} \mathrm{NMR}\left(\mathrm{CDCl}_{3}, 100 \mathrm{MHz}\right)$

36<smiles>N#Cc1cncc(-c2ccccc2)c1Cl</smiles> 


\section{${ }^{1} \mathrm{H}$ NMR, $\left(\mathrm{CDCl}_{3}, 400 \mathrm{MHz}\right)$}
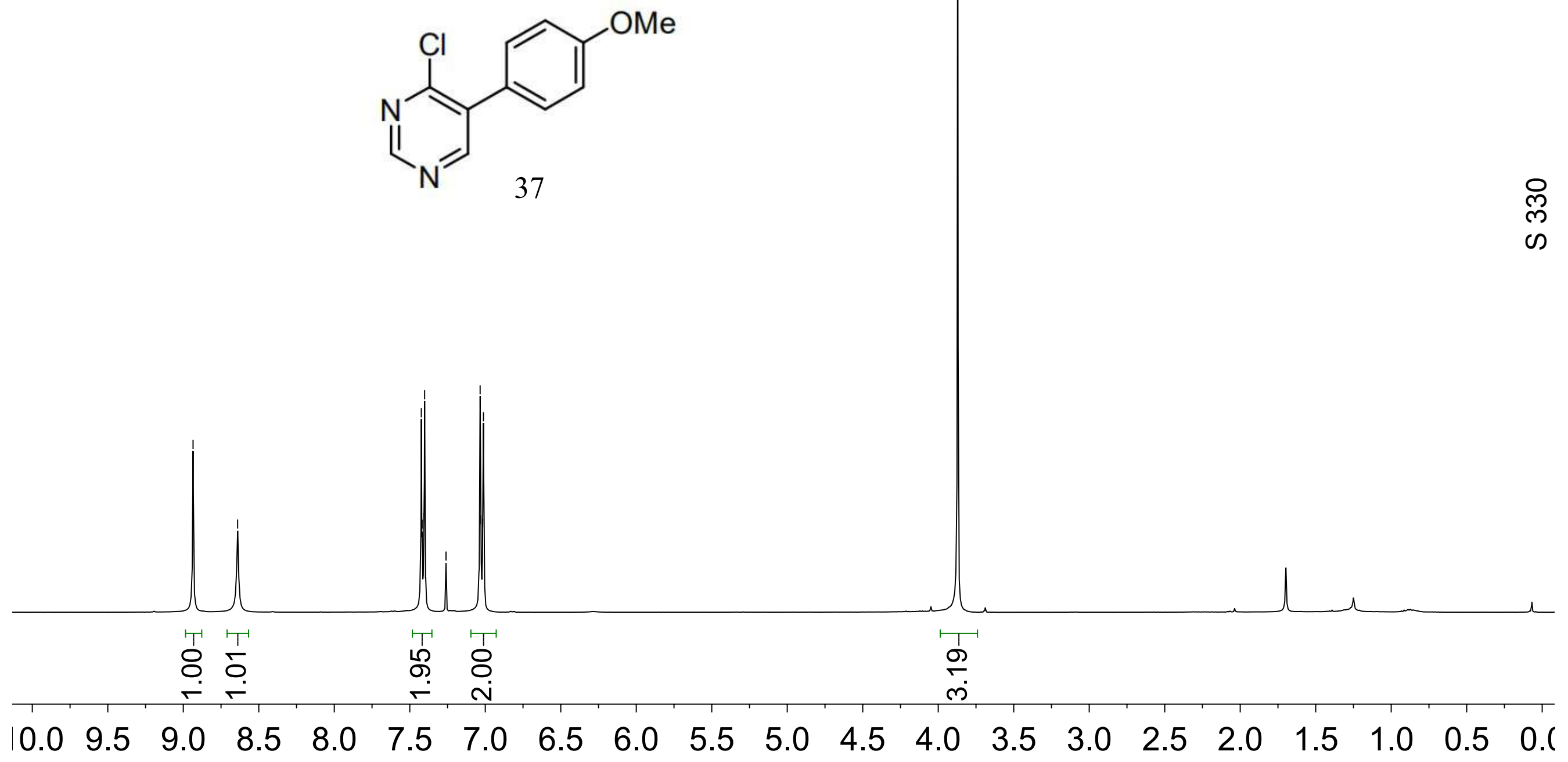


\section{年 \\ ㅇํㄴ \\ 和

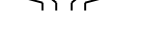

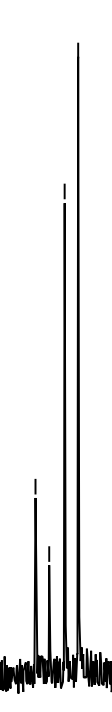

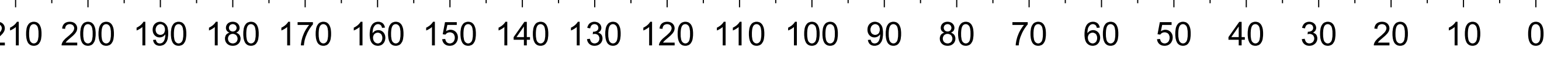




\section{${ }^{1} \mathrm{H} \mathrm{NMR},\left(\mathrm{CDCl}_{3}, 400 \mathrm{MHz}\right)$}

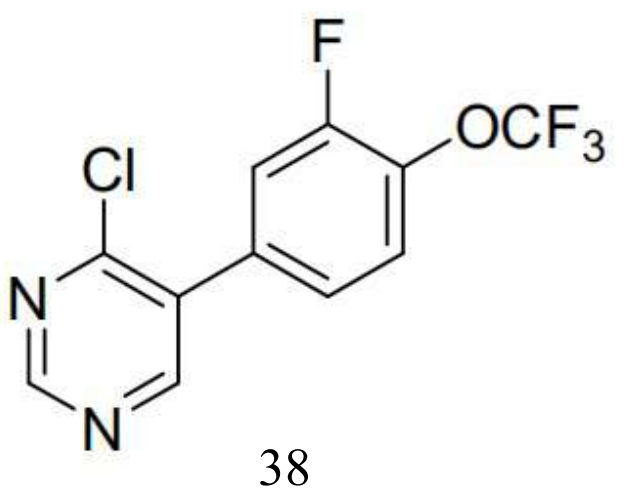


<smiles>Fc1cc(-c2cncnc2Cl)ccc1OC(F)(F)F</smiles> 


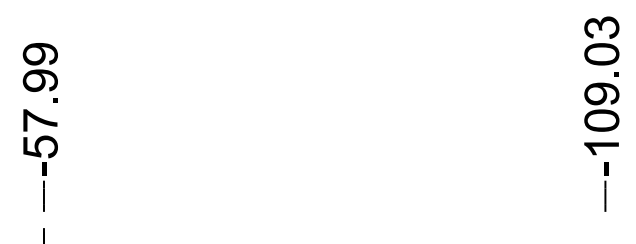

${ }^{19} \mathrm{~F} \mathrm{NMR}\left(\mathrm{CDCl}_{3}, 365 \mathrm{MHz}\right)$

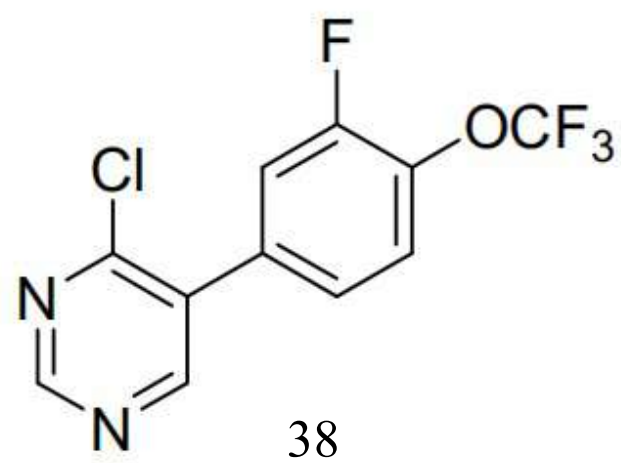

$\begin{array}{lllllllllllll}0 & 0 & -20 & -40 & -60 & -80 & -100 & -120 & -140 & -160 & -180 & -200 & -2 \%\end{array}$




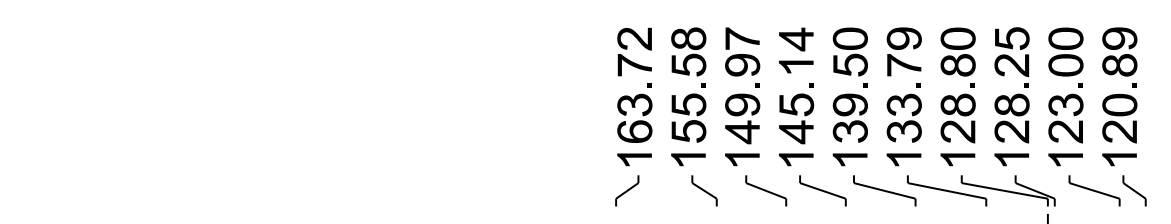

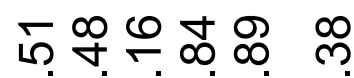 余走昌}

${ }^{13} \mathrm{C} \mathrm{NMR},\left(\mathrm{CDCl}_{3}, 100 \mathrm{MHz}\right)$<smiles>CCON1CCC(OC(c2ccc(Cl)cc2)c2cc(Cl)ccn2)CC1</smiles>

49 
${ }^{1} \mathrm{H} \mathrm{NMR}\left(\mathrm{CDCl}_{3}, 400 \mathrm{MHz}\right)$

51<smiles>CCOC(=O)N1CC[C@@H](c2ccc(F)cc2)[C@H](COC(=O)c2c(Cl)ccnc2C)C1</smiles>

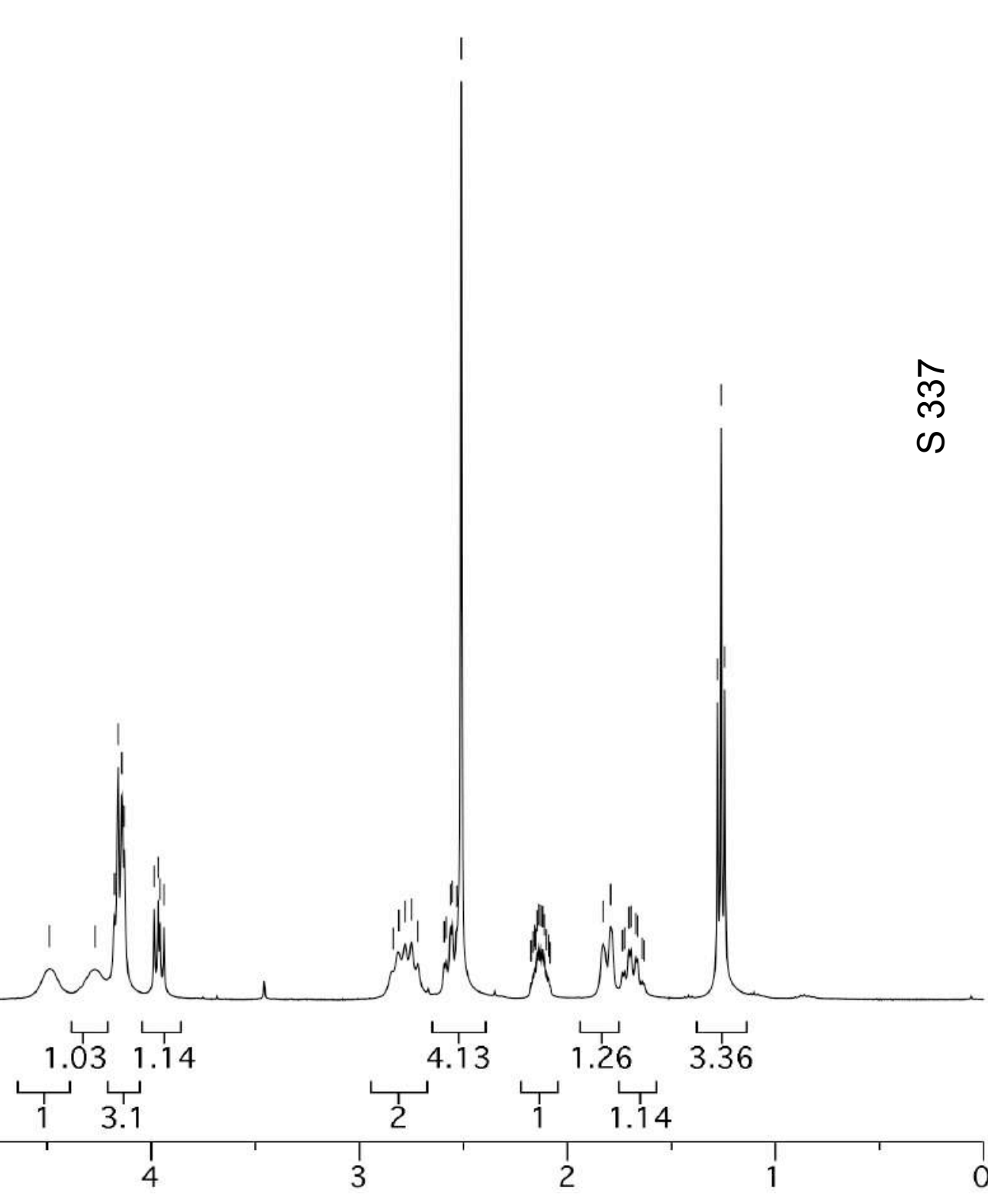



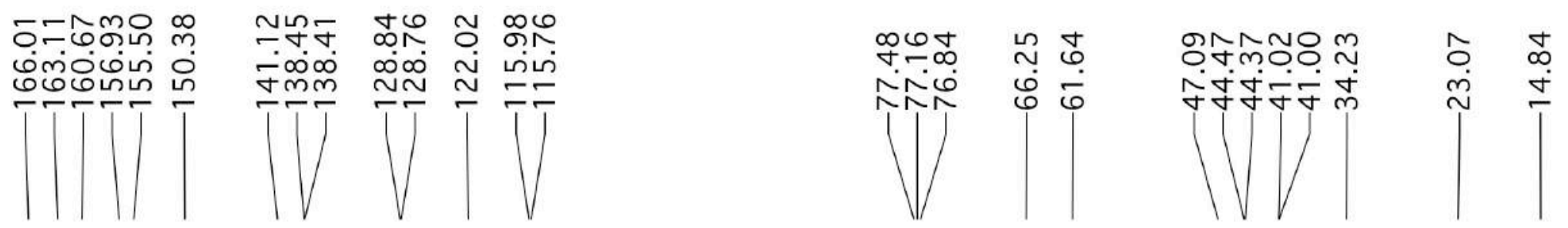

${ }^{13} \mathrm{C} \mathrm{NMR}\left(\mathrm{CDCl}_{3}, 100 \mathrm{MHz}\right)$

51<smiles>[C]c1nccc(Cl)c1C(=O)OC[C@@H]1CN(C(=O)OCC)CC[C@H]1c1ccc(F)cc1</smiles>

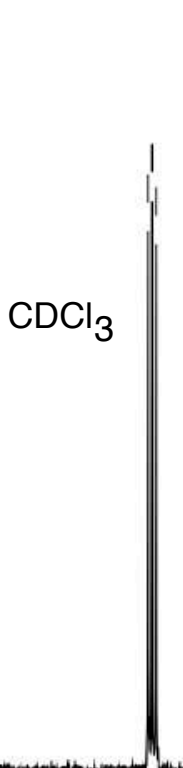

$\stackrel{\infty}{m}$

$\circlearrowleft$ 


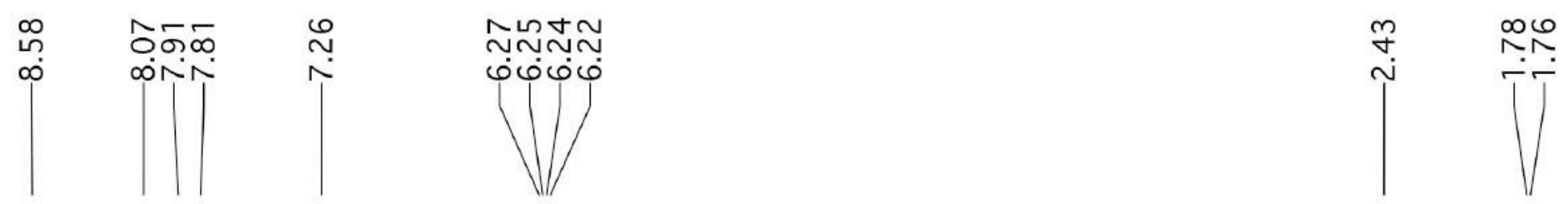

${ }^{1} \mathrm{H} \mathrm{NMR}\left(\mathrm{CDCl}_{3}, 400 \mathrm{MHz}\right)$

52<smiles>[M]C(OC(=O)c1cc(Cl)c(OC)cn1)c1cc(C(F)(F)F)cc(C(F)(F)F)c1</smiles>

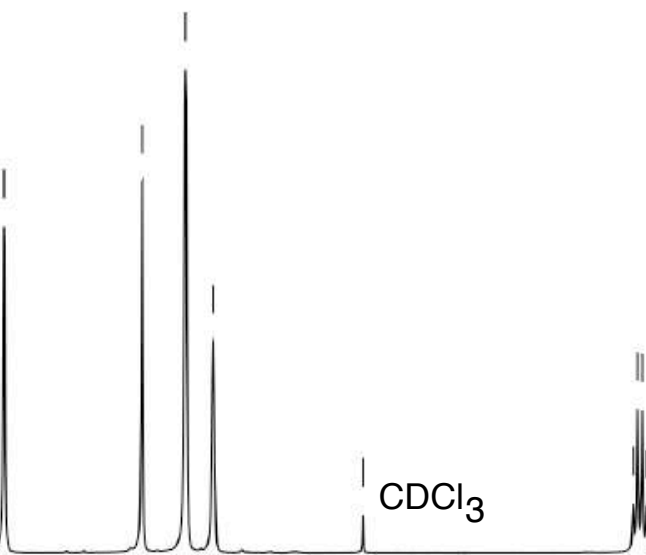

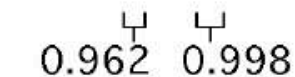

눈.16

$\begin{array}{ll}4 & \text { 누 } \\ 1 & 2.07\end{array}$

1.04

3.24 


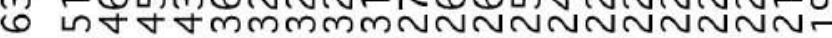

${ }^{13} \mathrm{C} \mathrm{NMR}\left(\mathrm{CDCl}_{3}, 100 \mathrm{MHz}\right)$<smiles>Cc1cnc(C(=O)OC([14CH3])c2cc(C(F)(F)F)cc(C(F)(F)F)c2)cc1Cl</smiles>

52

$$
\text { (n) }
$$


${ }^{1} \mathrm{H} \mathrm{NMR},\left(\mathrm{CDCl}_{3}, 400 \mathrm{MHz}\right)$<smiles>Cc1ccc(OCc2cncc(C)c2Cl)cn1</smiles>

54

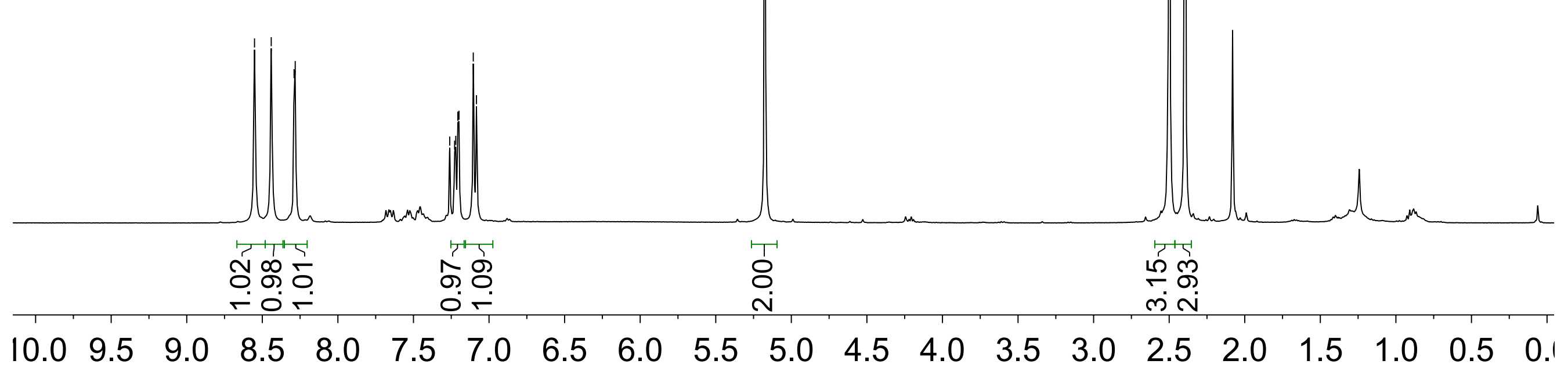




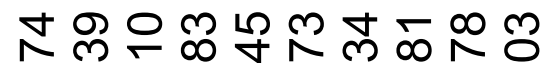

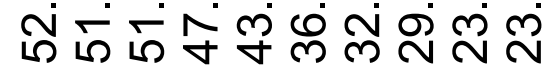

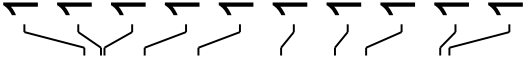

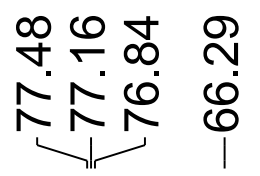

$\begin{array}{ll}\bar{m} & 8 \\ \stackrel{\sim}{\infty} & \stackrel{\Gamma}{F} \\ 1 & 1\end{array}$

\section{${ }^{13} \mathrm{C} \mathrm{NMR}\left(\mathrm{CDCl}_{3}, 100 \mathrm{MHz}\right)$}<smiles>Cc1ccc(OCc2cncc(C)c2Cl)cn1</smiles>

54

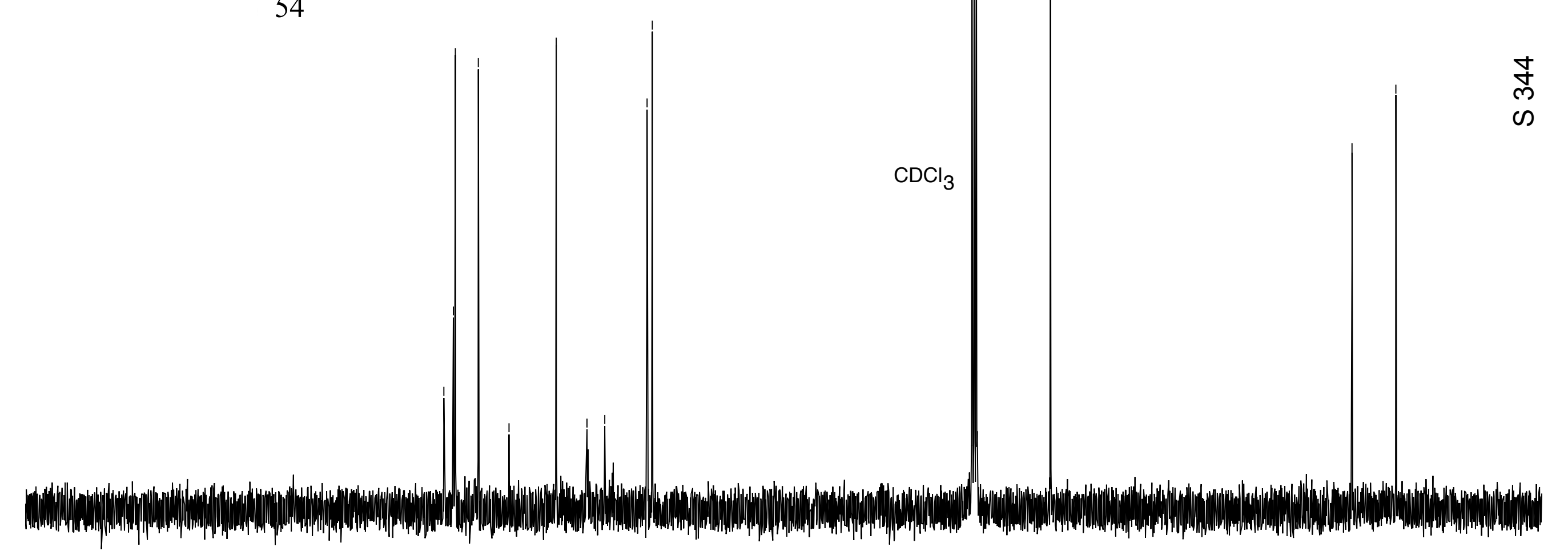

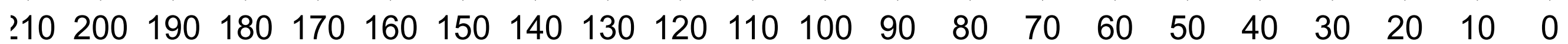


ตื๋น

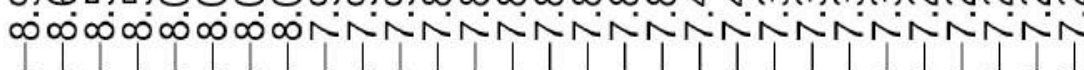
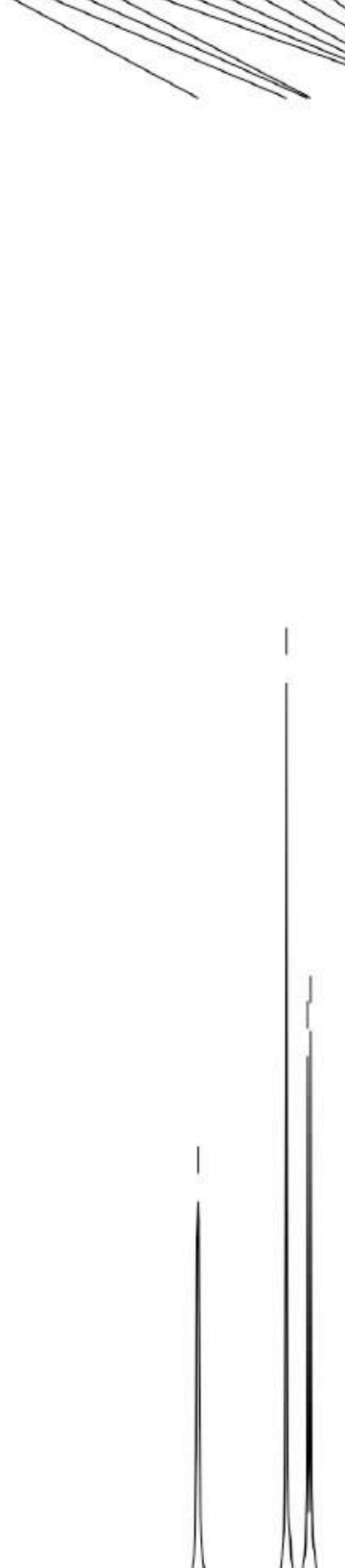

55

${ }^{1} \mathrm{H} \mathrm{NMR}\left(\mathrm{CDCl}_{3}, 400 \mathrm{MHz}\right)$

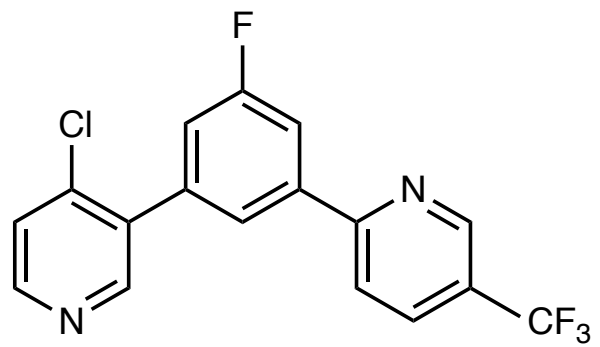

ก)

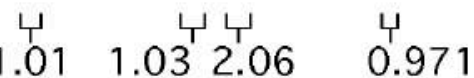

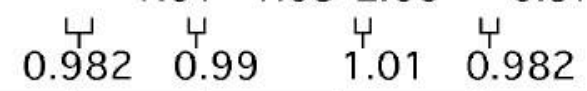

9

8

7

5

3


${ }^{13} \mathrm{C} \mathrm{NMR}\left(\mathrm{CDCl}_{3}, 100 \mathrm{MHz}\right)$
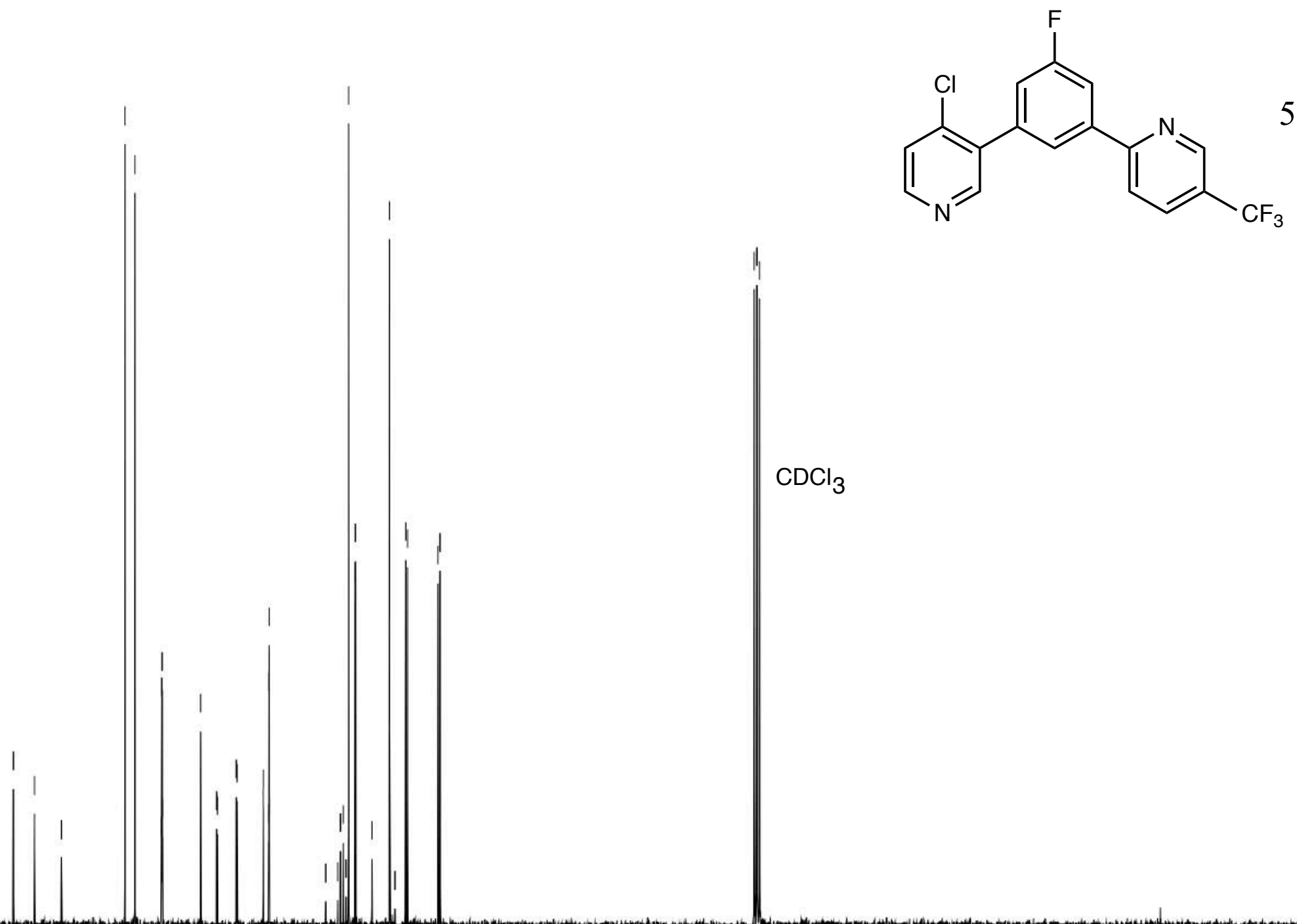
${ }^{19} \mathrm{~F}$ NMR $\left(\mathrm{CDCl}_{3}, 365 \mathrm{MHz}\right)$<smiles>Fc1cc(-c2cncc(C(F)(F)F)c2)cc(-c2cnccc2Cl)c1</smiles>

55

\begin{tabular}{|c|c|c|}
\hline $\begin{array}{ll}\text { ppm }-0 & \end{array}$ & $\begin{array}{l}1 \\
-50\end{array}$ & $\begin{array}{r}1 \\
-100\end{array}$ \\
\hline
\end{tabular}




\section{${ }^{1} \mathrm{H} \mathrm{NMR},\left(\mathrm{CDCl}_{3}, 400 \mathrm{MHz}\right)$}<smiles>CC(=O)Oc1ccc(C(c2ccc(OC(C)=O)cc2)c2cc(Cl)ccn2)cc1</smiles>

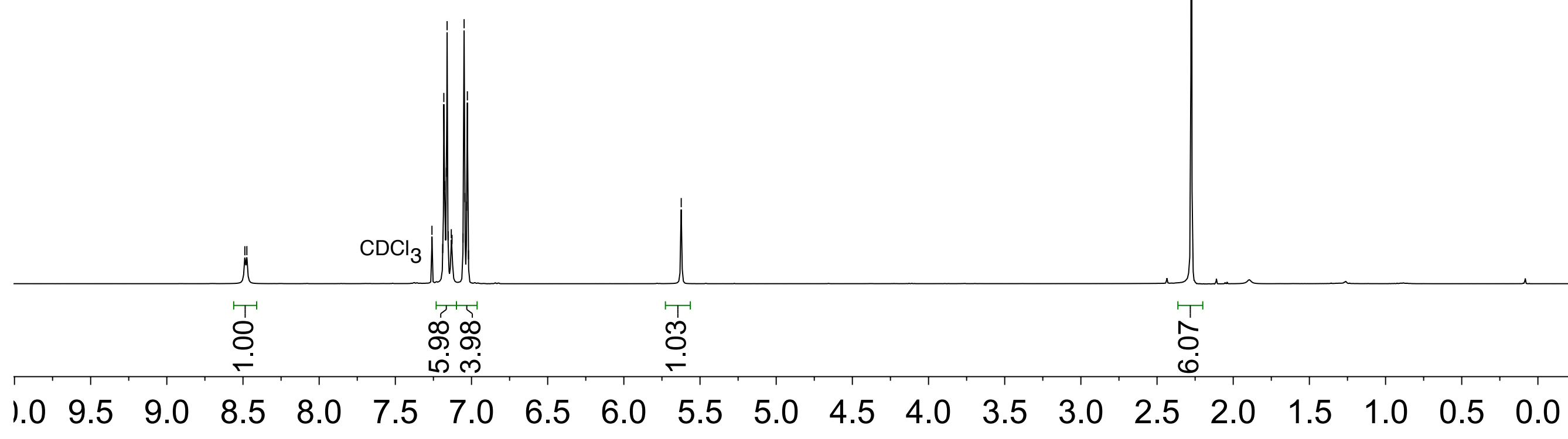




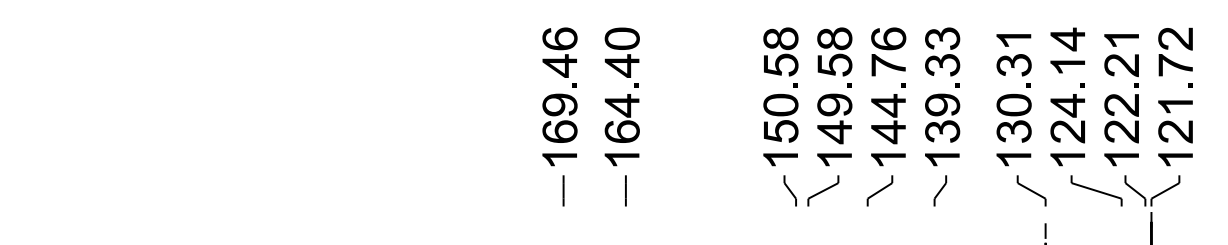

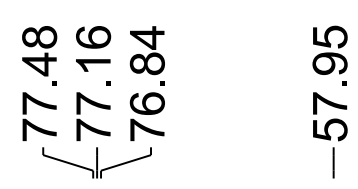

$\underset{\text { N }}{\stackrel{N}{1}}$

${ }^{13} \mathrm{C} \mathrm{NMR},\left(\mathrm{CDCl}_{3}, 100 \mathrm{MHz}\right)$

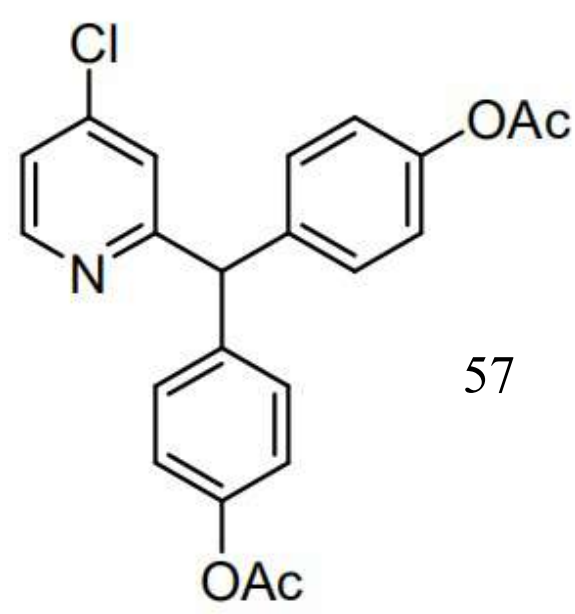


${ }^{1} \mathrm{H} \mathrm{NMR},\left(\mathrm{CDCl}_{3}, 400 \mathrm{MHz}\right)$<smiles>CCOc1cc(S(C)(=O)=O)cc(Cl)c1C(=O)N(C)c1ccc(Cl)c(-c2cc(Cl)ccn2)c1</smiles>

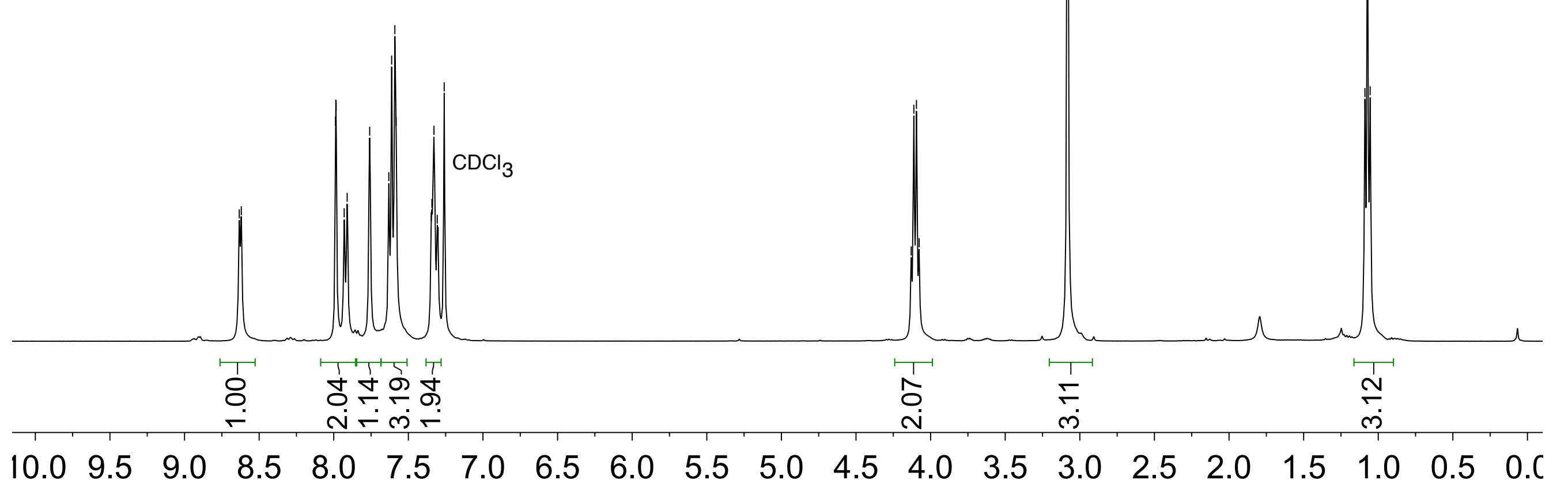


${ }^{13} \mathrm{C} \mathrm{NMR},\left(\mathrm{CDCl}_{3}, 100 \mathrm{MHz}\right)$<smiles>CCOC(=O)N(C(=O)c1cc(S(C)(=O)=O)ccc1Cl)c1ccc(Cl)c(-c2cc(Cl)ccn2)c1</smiles>

58

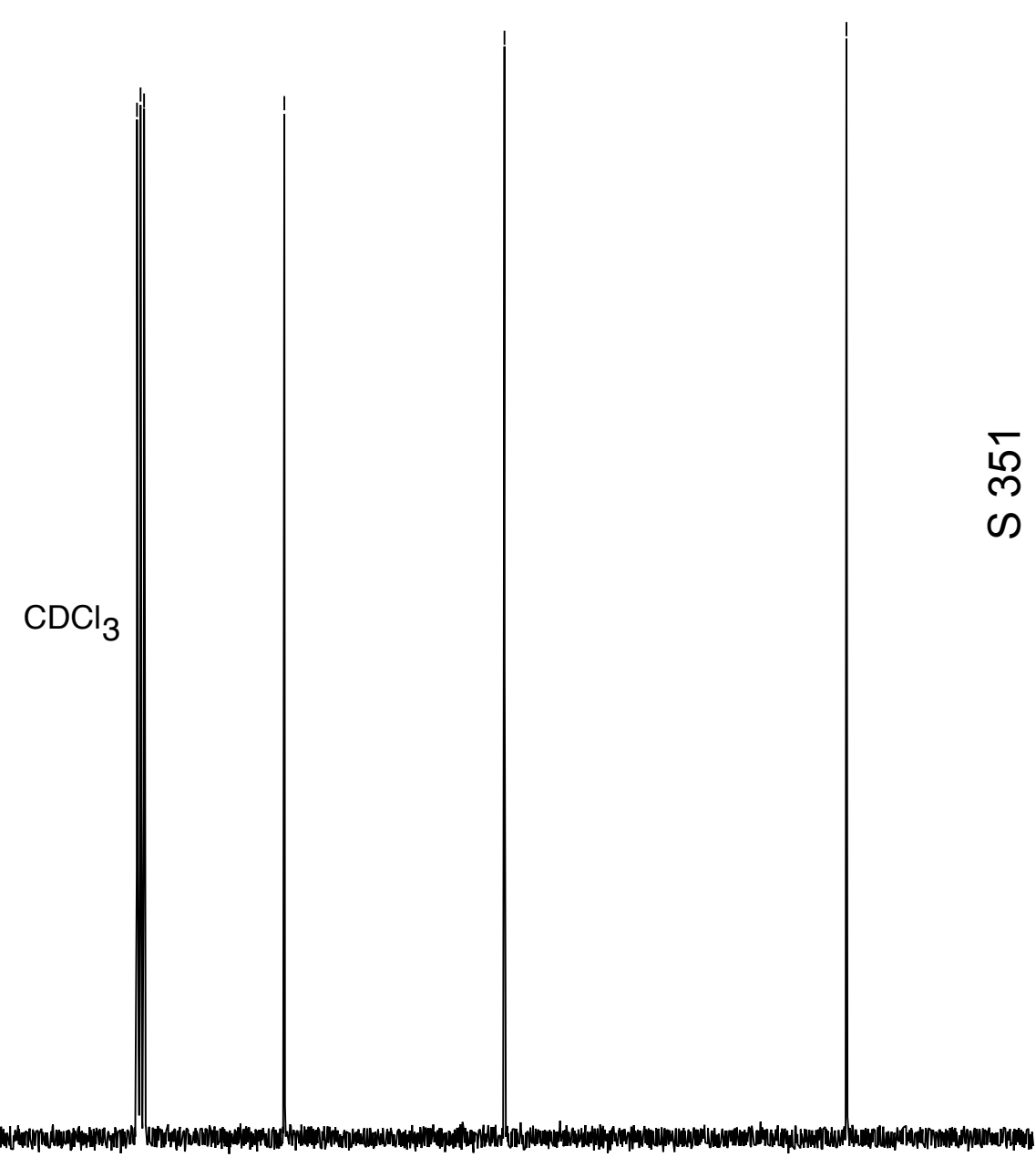

$\begin{array}{llllllllllllllllllllll}210 & 200 & 190 & 180 & 170 & 160 & 150 & 140 & 130 & 120 & 110 & 100 & 90 & 80 & 70 & 60 & 50 & 40 & 30 & 20 & 10 & 0\end{array}$ 


\section{${ }^{1} \mathrm{H} \mathrm{NMR},\left(\mathrm{CDCl}_{3}, 400 \mathrm{MHz}\right)$}<smiles>Cc1cc(Cl)c(-c2ncc(Cl)cc2-c2ccc(S(C)(=O)=O)cc2)cn1</smiles>

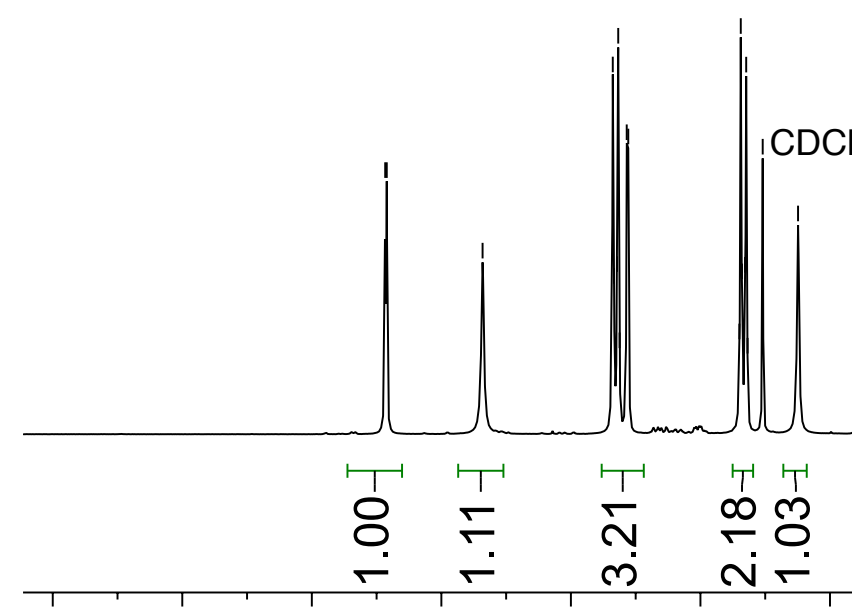


${ }^{13} \mathrm{C} \mathrm{NMR},\left(\mathrm{CDCl}_{3}, 100 \mathrm{MHz}\right)$<smiles>Cc1cc(Cl)c(-c2ncc(Cl)cc2-c2ccc(S(C)(=O)=O)cc2)cn1</smiles> 


\section{${ }^{1} \mathrm{H} \mathrm{NMR},\left(\mathrm{CDCl}_{3}, 400 \mathrm{MHz}\right)$}

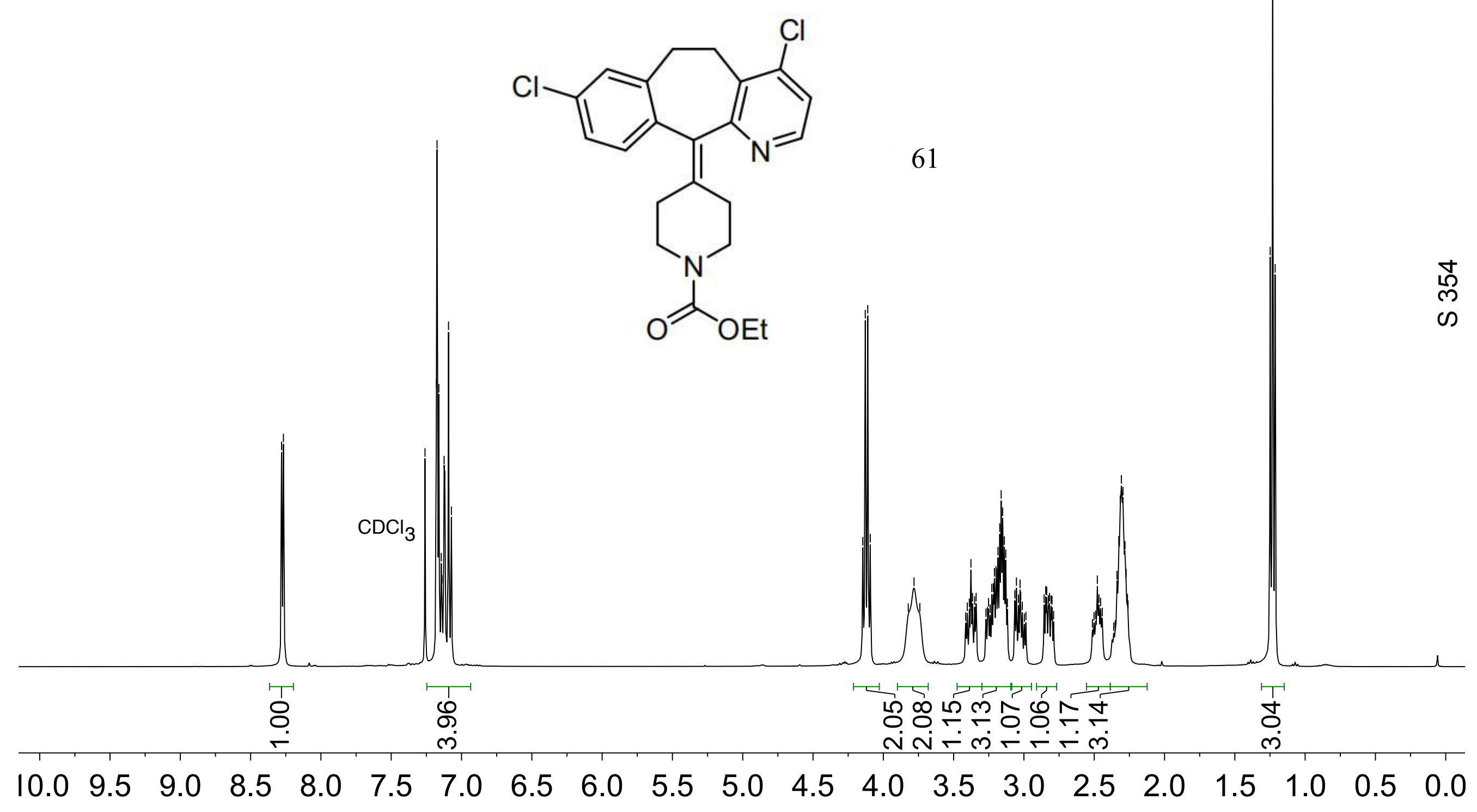




\section{${ }^{13} \mathrm{C} \mathrm{NMR},\left(\mathrm{CDCl}_{3}, 100 \mathrm{MHz}\right)$}<smiles>CCOC(=O)N1CCC(=C2c3ccc(Cl)cc3CCc3c(Cl)ccnc32)CC1</smiles>
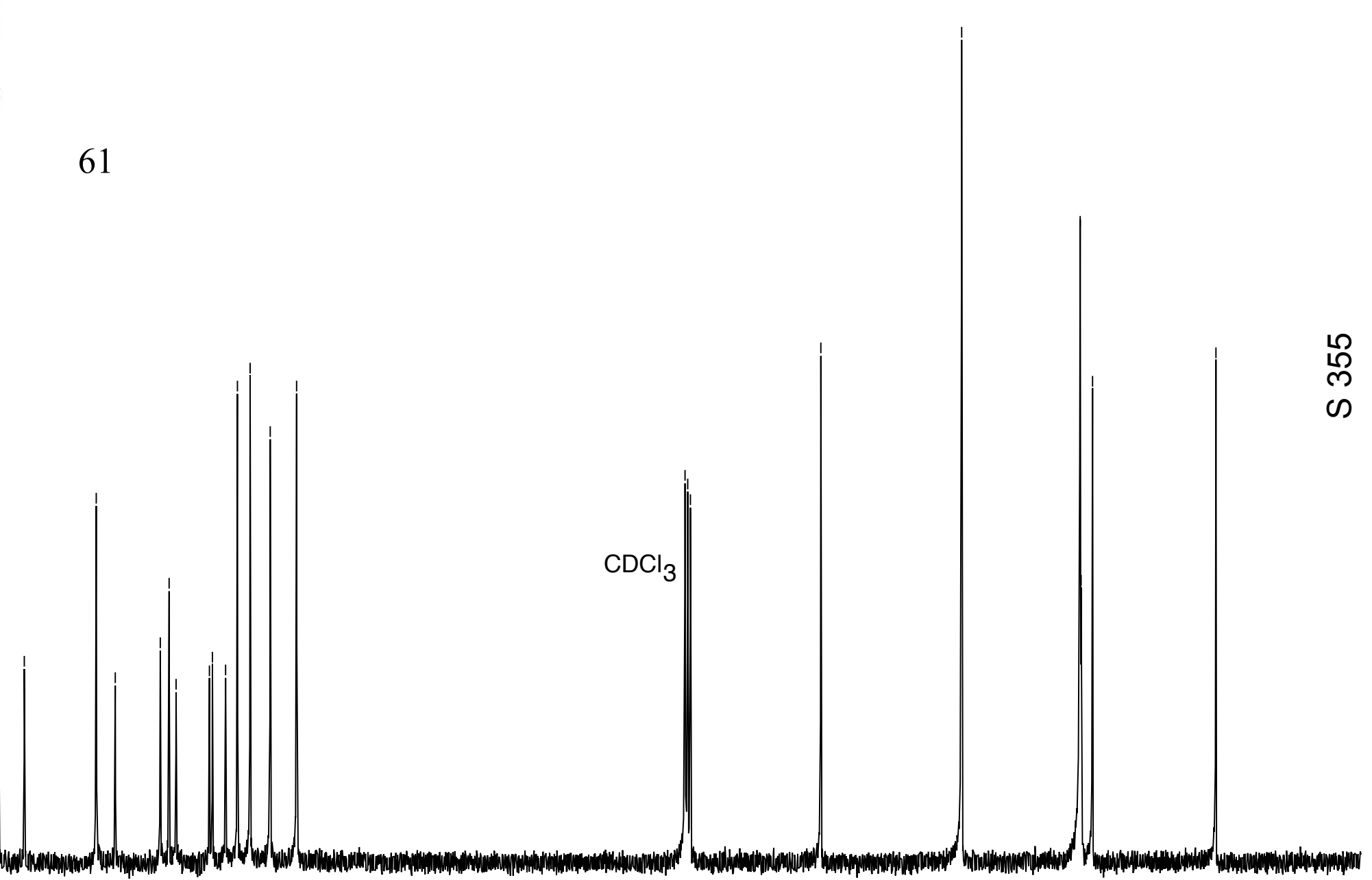

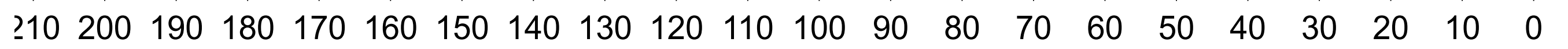




\section{${ }^{1} \mathrm{H} \mathrm{NMR},\left(\mathrm{CDCl}_{3}, 400 \mathrm{MHz}\right)$}<smiles>O=C(OCCOPCBr)c1cnccc1Cl</smiles>

64

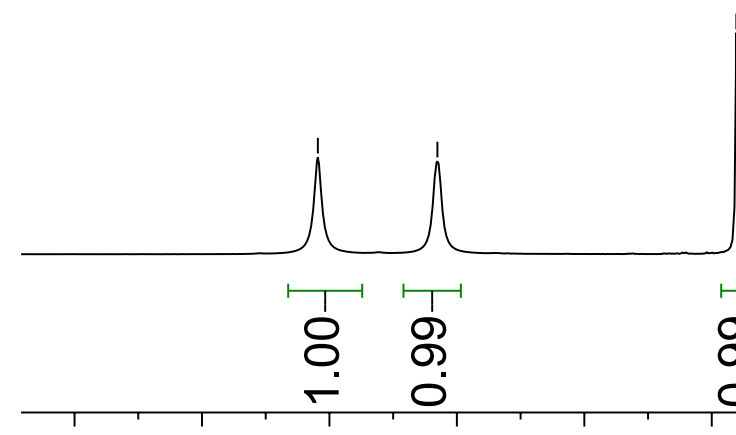

$\mathrm{CDCl}_{3}$

$\begin{array}{lllllllllllllllllllll}10.0 & 9.5 & 9.0 & 8.5 & 8.0 & 7.5 & 7.0 & 6.5 & 6.0 & 5.5 & 5.0 & 4.5 & 4.0 & 3.5 & 3.0 & 2.5 & 2.0 & 1.5 & 1.0 & 0.5 & 0.0\end{array}$ 


\section{员

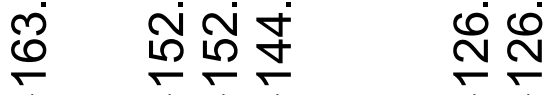 \\ Г}

${ }^{13} \mathrm{C} \mathrm{NMR},\left(\mathrm{CDCl}_{3}, 100 \mathrm{MHz}\right)$<smiles>O=C(OCCOPBr)c1cnccc1Cl</smiles>

64

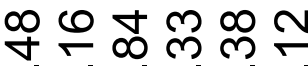

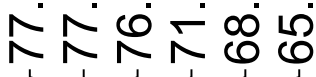

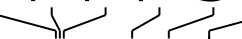

ก)

$\begin{array}{llllllllllllllllllllll}! 10 & 200 & 190 & 180 & 170 & 160 & 150 & 140 & 130 & 120 & 110 & 100 & 90 & 80 & 70 & 60 & 50 & 40 & 30 & 20 & 10 & 0\end{array}$ 
${ }^{1} \mathrm{H} \mathrm{NMR}\left(\mathrm{CDCl}_{3}, 400 \mathrm{MHz}\right)$

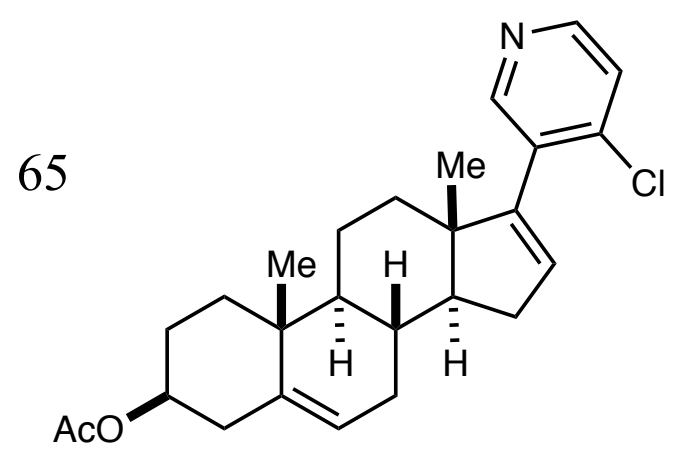



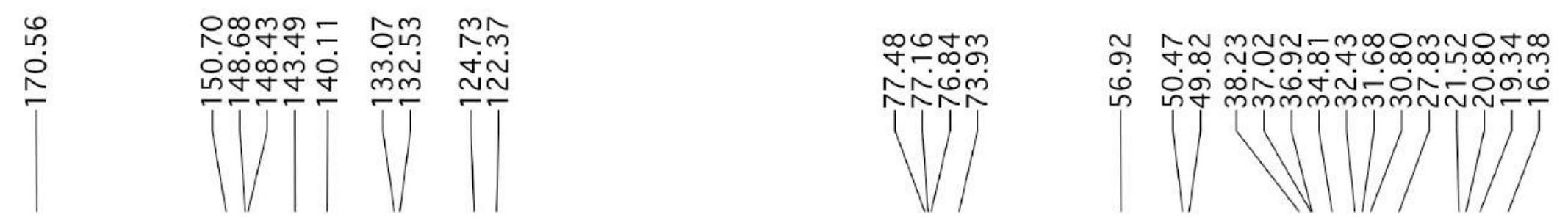

${ }^{13} \mathrm{C} \mathrm{NMR}\left(\mathrm{CDCl}_{3}, 100 \mathrm{MHz}\right)$

65

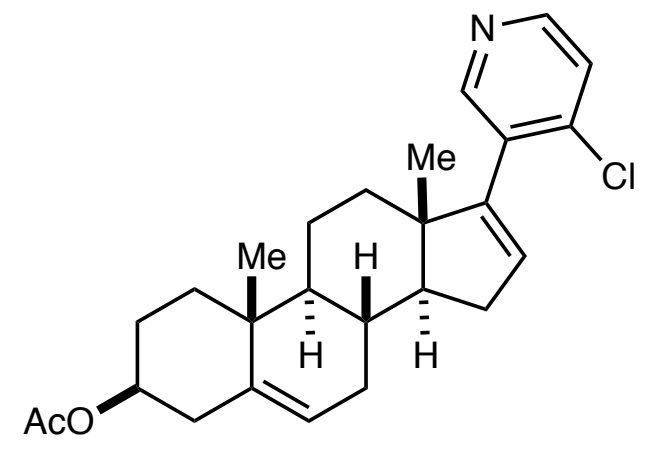




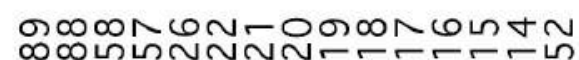

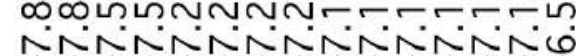

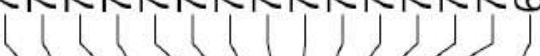

$\mathrm{H}$ NMR $\left(\mathrm{CDCl}_{3}, 400 \mathrm{MHz}\right)$
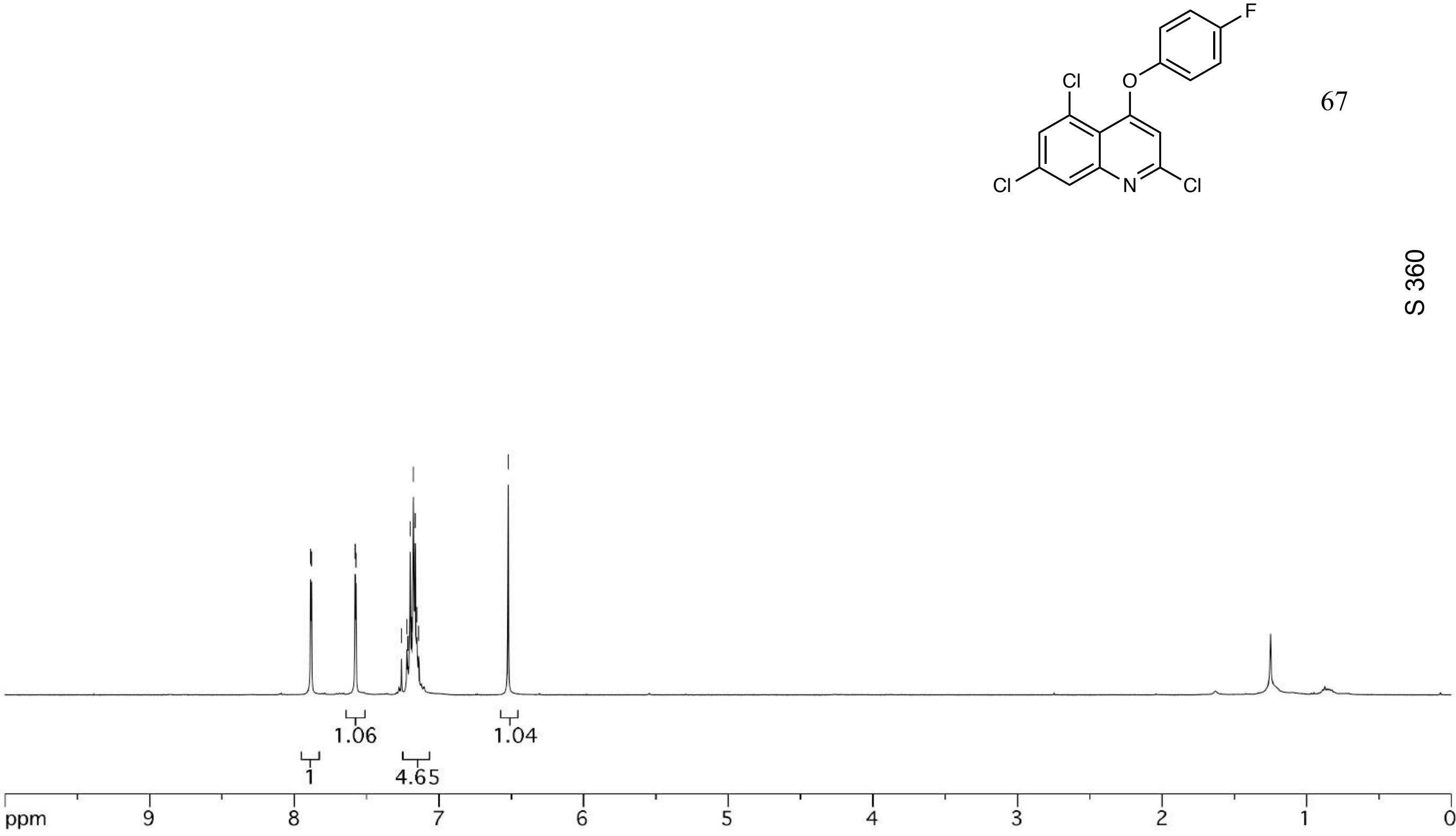

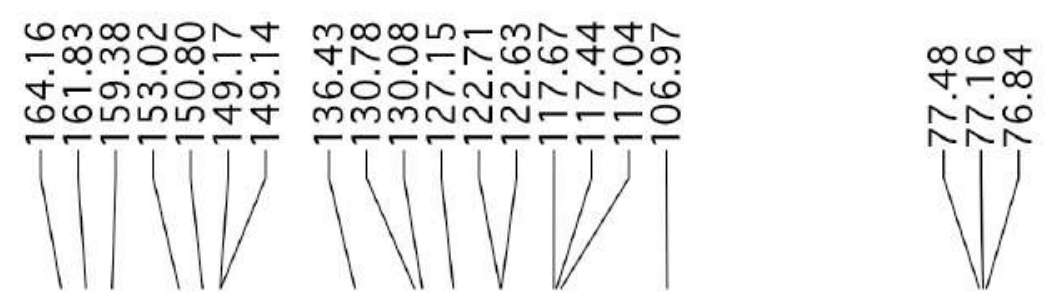

${ }^{13} \mathrm{C} \mathrm{NMR}\left(\mathrm{CDCl}_{3}, 100 \mathrm{MHz}\right)$

67<smiles>Fc1ccc(Oc2cc(Cl)nc3cc(Cl)cc(Cl)c23)cc1</smiles> 


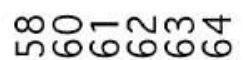

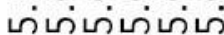

モニニニニニ

(i)

${ }^{19} \mathrm{~F} \mathrm{NMR}\left(\mathrm{CDCl}_{3}, 365 \mathrm{MHz}\right)$

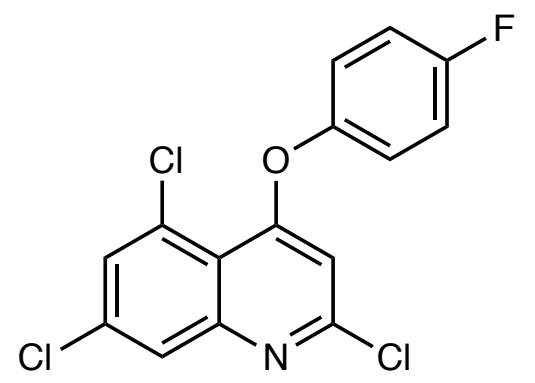


${ }^{1} \mathrm{H} \mathrm{NMR}\left(\mathrm{CDCl}_{3}, 400 \mathrm{MHz}\right)$

68<smiles>CCOC(=O)N1CC2CC(C1)c1cc3nc(Cl)cnc3cc12</smiles>

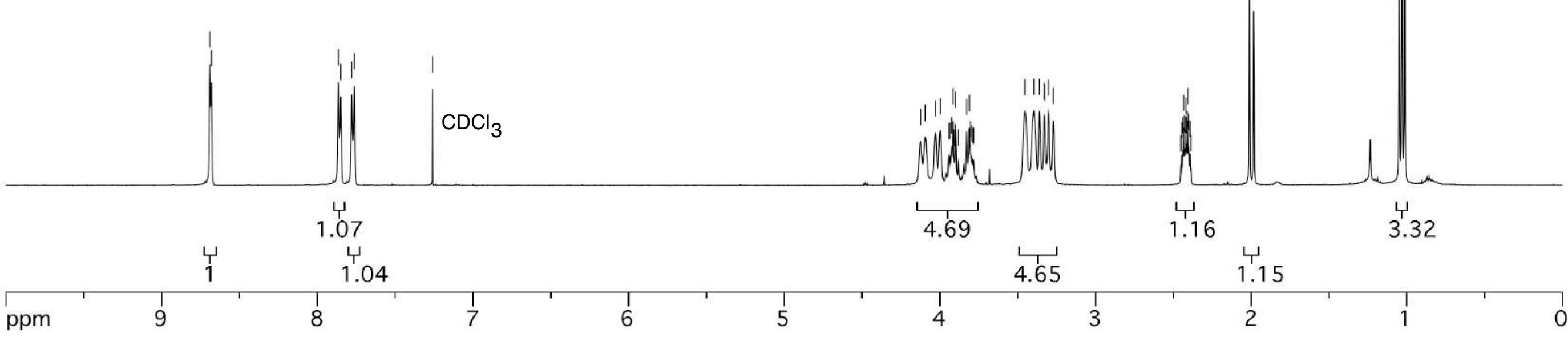


68

${ }^{13} \mathrm{C} \mathrm{NMR}\left(\mathrm{CDCl}_{3}, 100 \mathrm{MHz}\right)$

68 
${ }^{1} \mathrm{H} \mathrm{NMR}\left(\mathrm{CDCl}_{3}, 400 \mathrm{MHz}\right)$

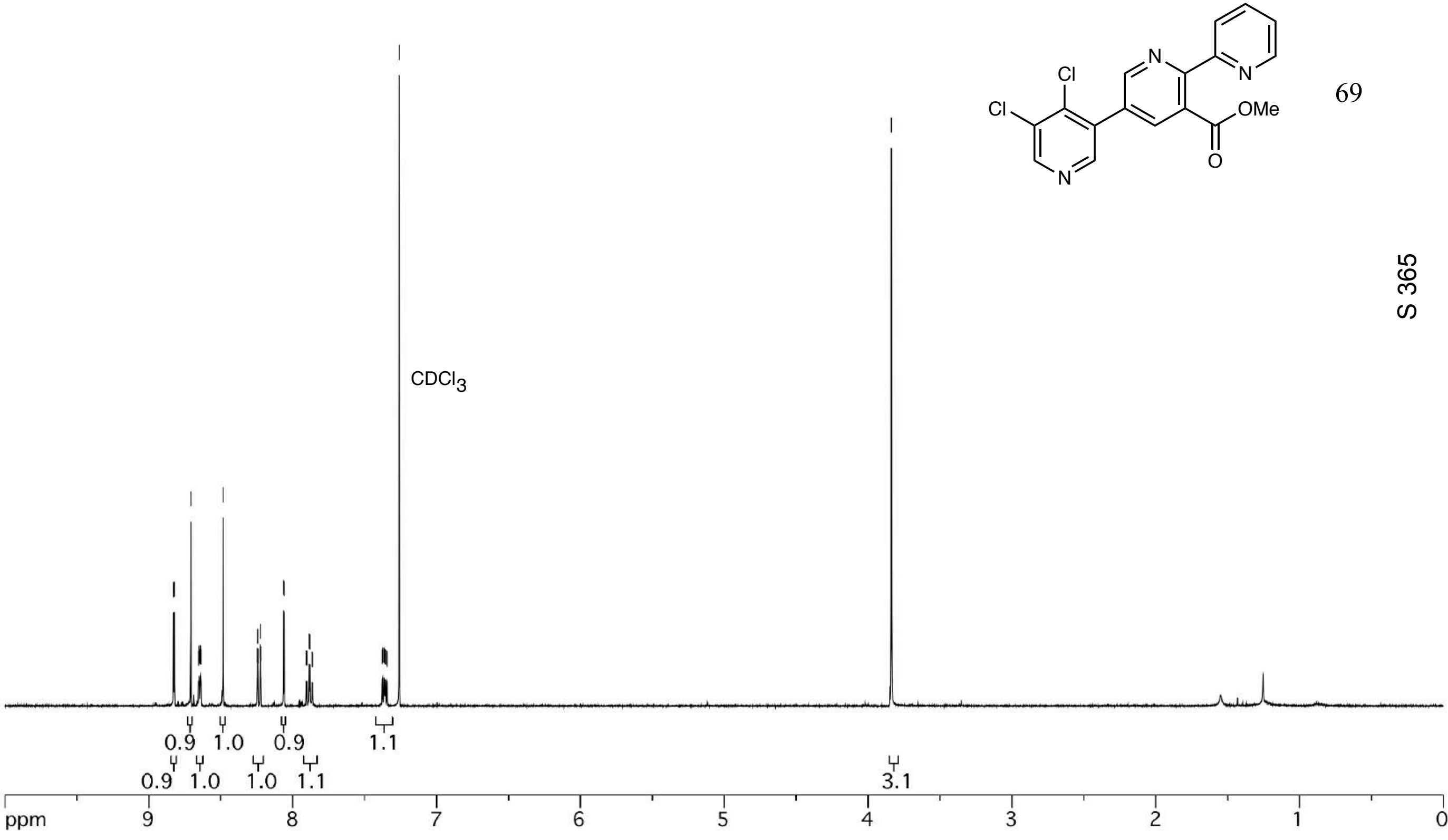



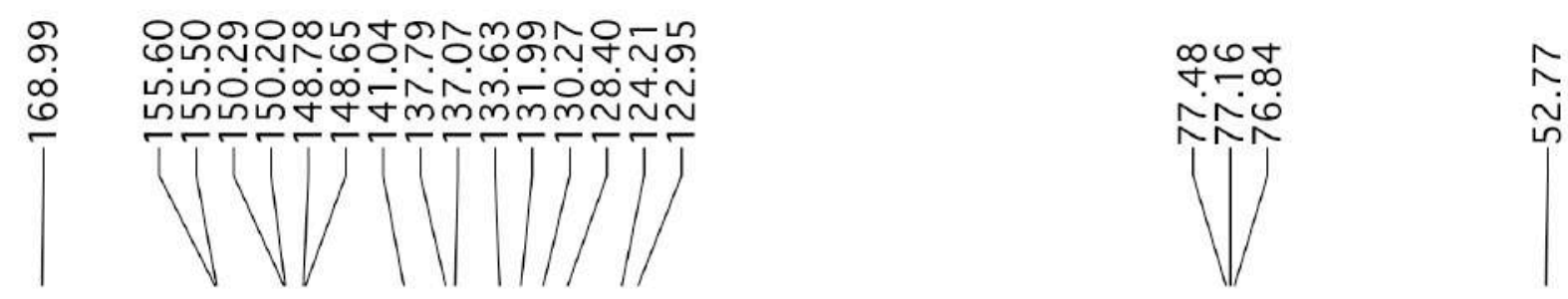

69

${ }^{13} \mathrm{C} \mathrm{NMR}\left(\mathrm{CDCl}_{3}, 100 \mathrm{MHz}\right)$<smiles>COC(=O)c1cc(-c2cncc(Cl)c2Cl)cnc1-c1ccccn1</smiles> 
${ }^{1} \mathrm{H} \mathrm{NMR}\left(\mathrm{CDCl}_{3}, 400 \mathrm{MHz}\right)$

70<smiles>COC(=O)c1cc(-c2cncc(Cl)c2)cnc1-c1cc(Cl)ccn1</smiles>

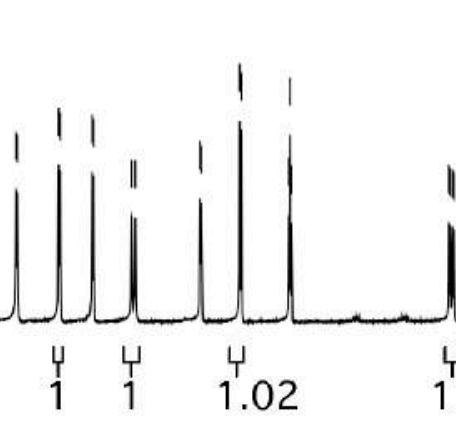

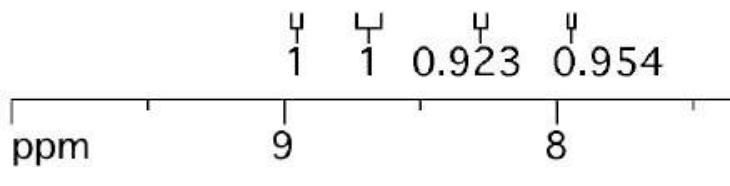

3.1 

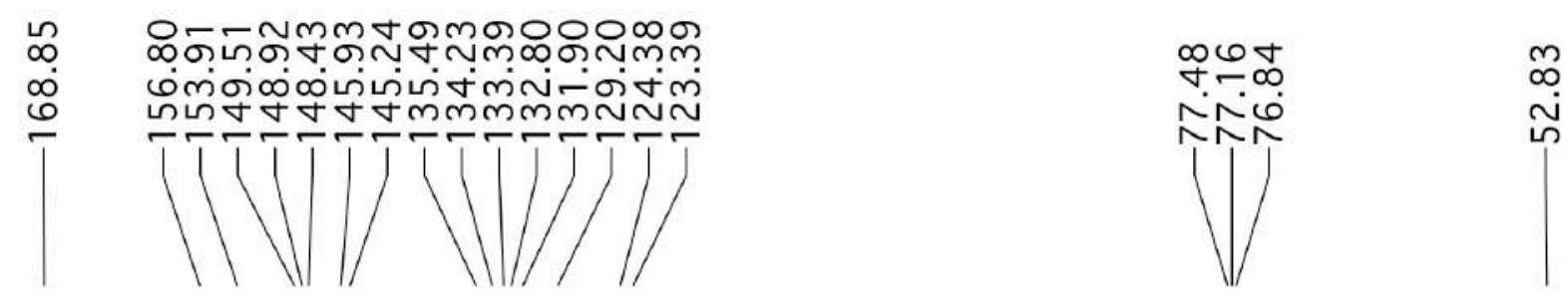

${ }^{13} \mathrm{C} \mathrm{NMR}\left(\mathrm{CDCl}_{3}, 100 \mathrm{MHz}\right)$<smiles>COC(=O)c1cc(-c2cncc(Cl)c2)cnc1-c1cc(Cl)ccn1</smiles>

70 
${ }^{1} \mathrm{H} \mathrm{NMR}\left(\mathrm{CDCl}_{3}, 400 \mathrm{MHz}\right)$

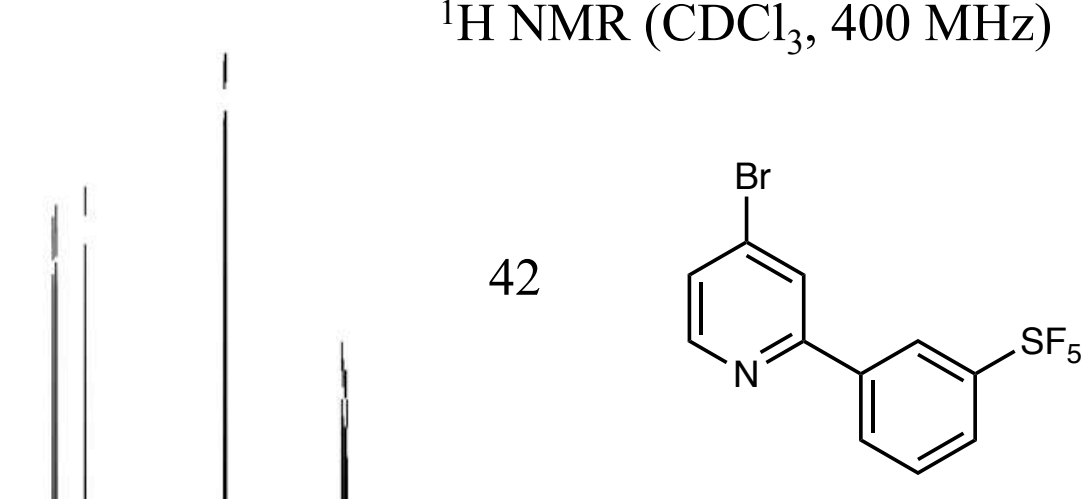




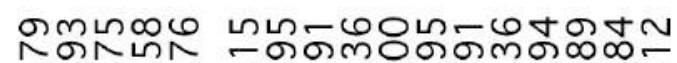

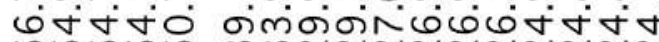

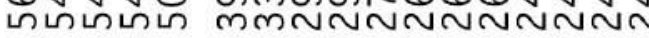

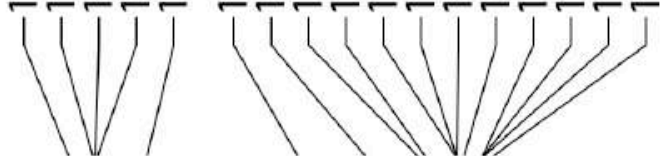

ㄱ/

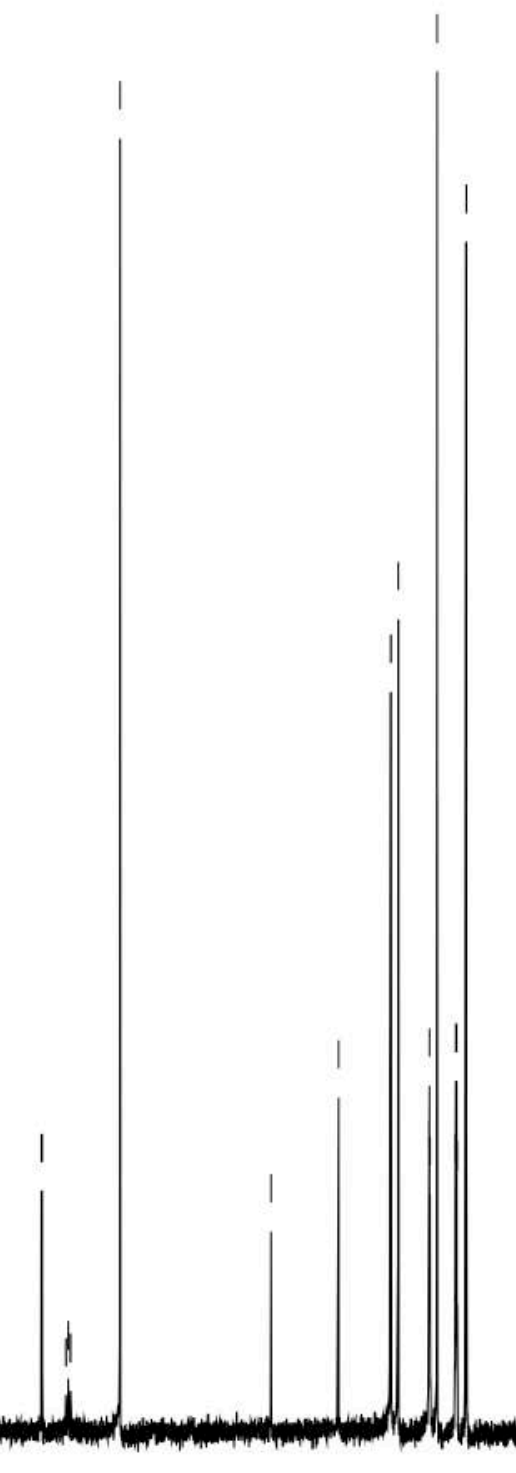

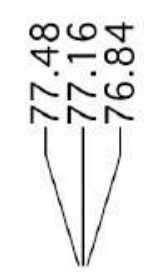

${ }^{13} \mathrm{C} \mathrm{NMR}\left(\mathrm{CDCl}_{3}, 100 \mathrm{MHz}\right)$<smiles>FS(F)(F)(F)(F)c1cccc(-c2cc(Br)ccn2)c1</smiles> 
${ }^{19} \mathrm{~F}$ NMR $\left(\mathrm{CDCl}_{3}, 365 \mathrm{MHz}\right)$

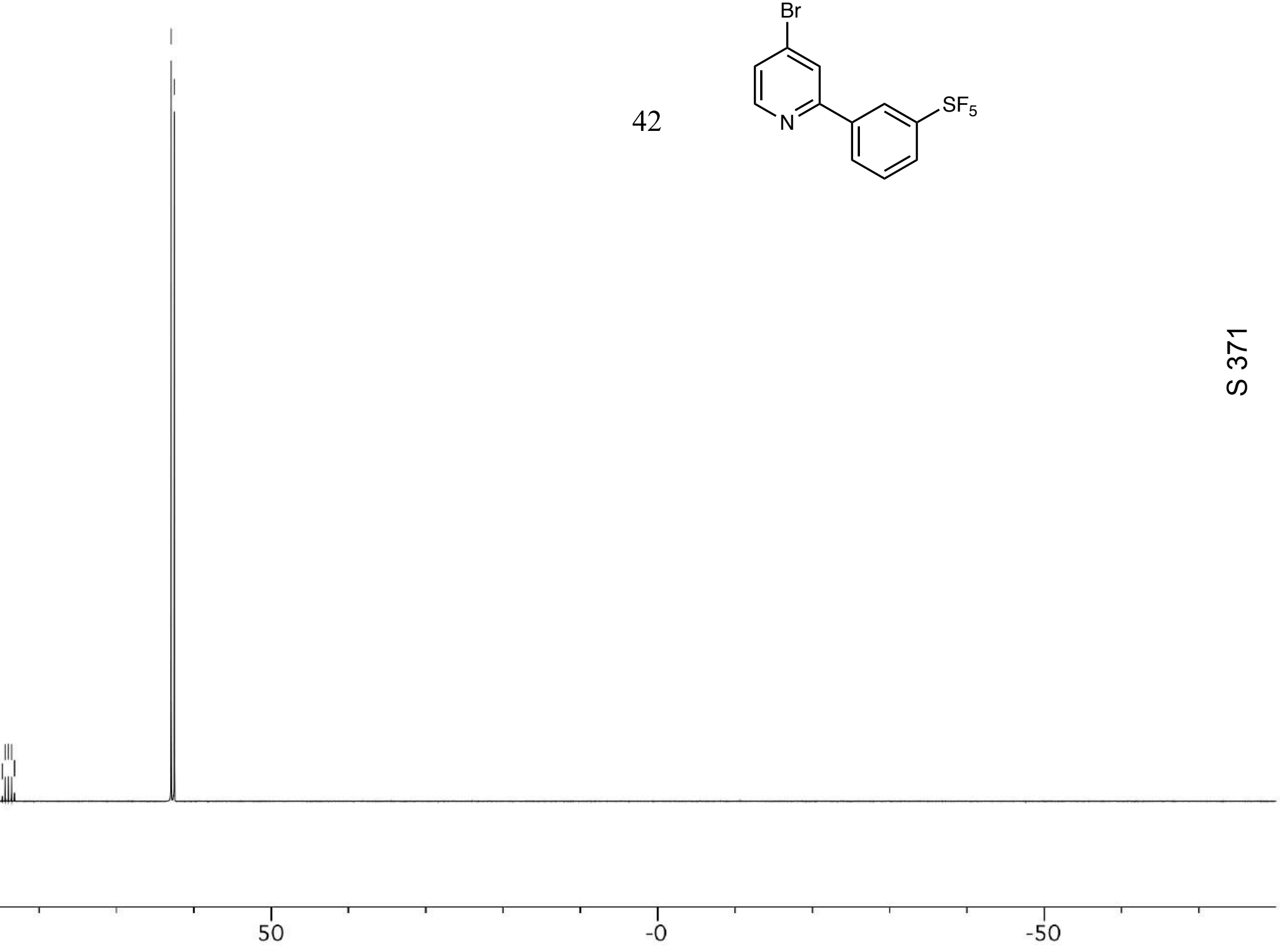

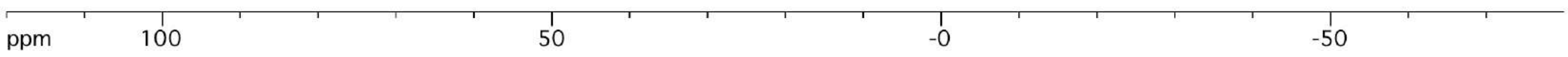



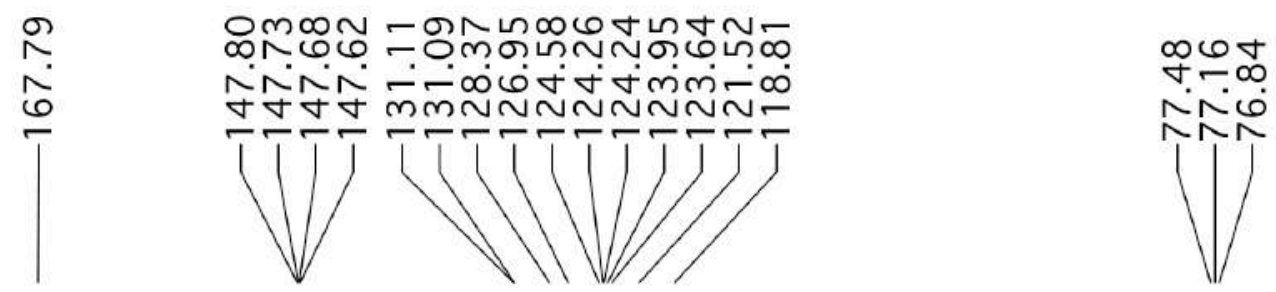

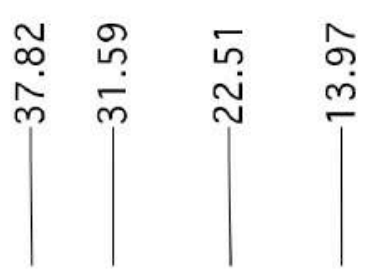

${ }^{13} \mathrm{C} \mathrm{NMR}\left(\mathrm{CDCl}_{3}, 100 \mathrm{MHz}\right)$

45<smiles>FC(F)(F)c1cnc(Br)cc1Br</smiles>

$\mathrm{CDCl}_{3}$ 
${ }^{19} \mathrm{~F} \mathrm{NMR}\left(\mathrm{CDCl}_{3}, 365 \mathrm{MHz}\right)$

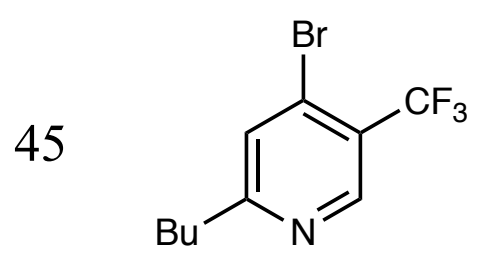

$\underset{N}{N}$

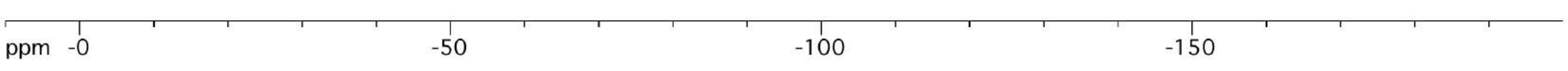




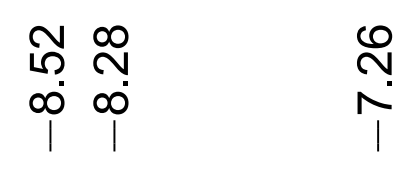

\section{${ }^{1} \mathrm{H}$ NMR, $\left(\mathrm{CDCl}_{3}, 400 \mathrm{MHz}\right)$}

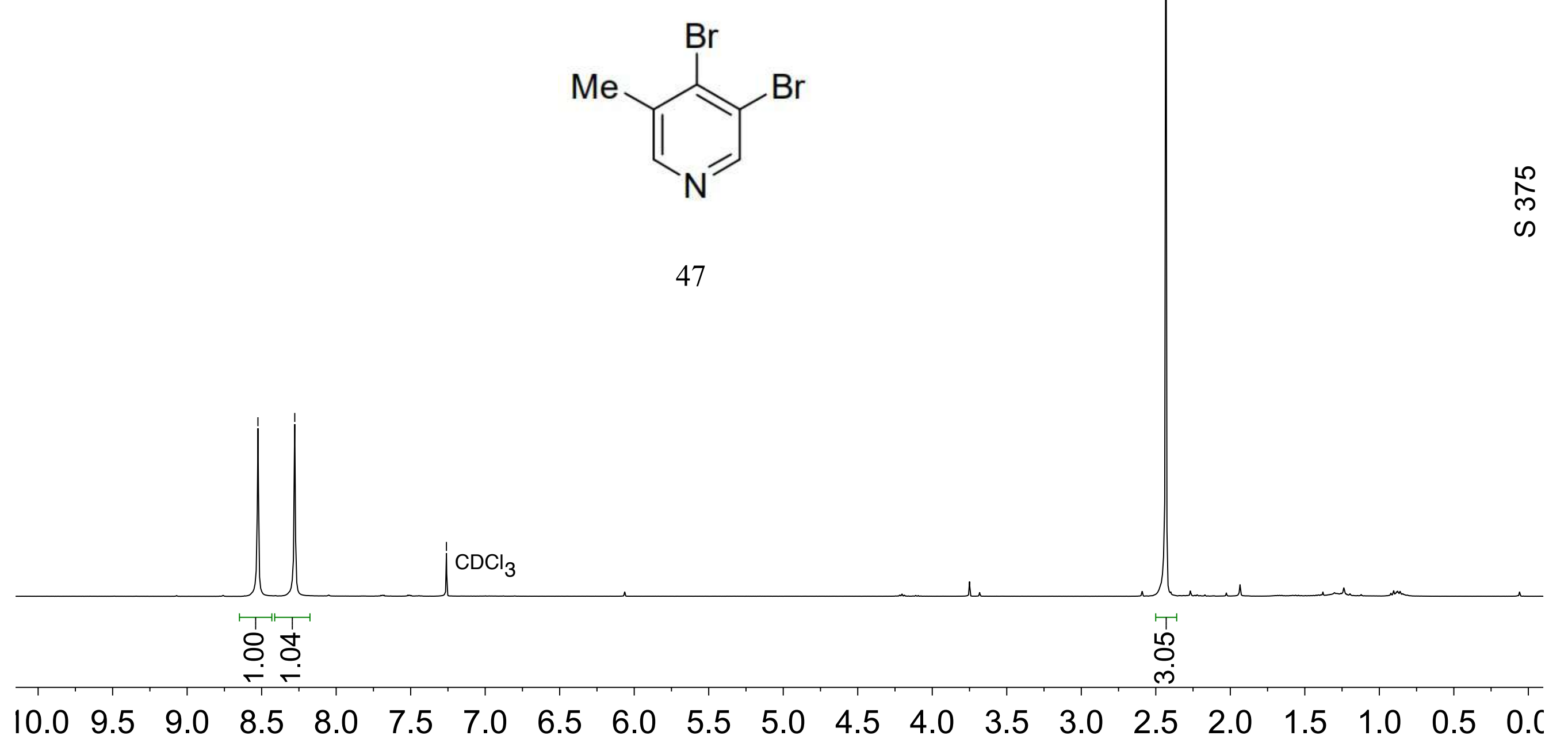




\section{${ }^{13} \mathrm{C} \mathrm{NMR},\left(\mathrm{CDCl}_{3}, 100 \mathrm{MHz}\right)$}<smiles>Cc1cncc(Br)c1Br</smiles> 


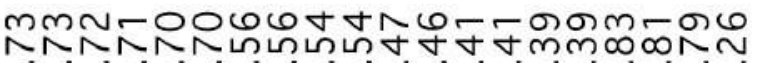

$\infty \infty^{\circ} \infty \infty^{\circ} \infty \infty^{\circ} \infty \infty^{\circ} \infty N^{\circ}$

${ }^{1} \mathrm{H} \mathrm{NMR}\left(\mathrm{CDCl}_{3}, 400 \mathrm{MHz}\right)$<smiles>O=[N+]([O-])c1cccc2c(Br)nccc12</smiles>

\section{1 눈. 0.984}

1.090 .96

1.12

\begin{tabular}{l|l}
1 & 1 \\
7 & 6
\end{tabular}




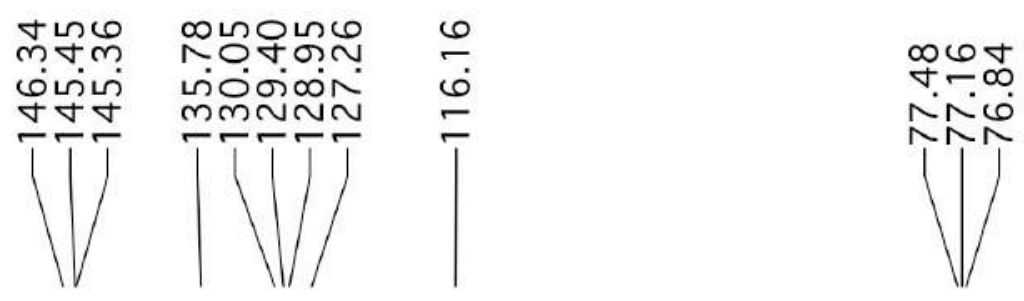

${ }^{13} \mathrm{C} \mathrm{NMR}\left(\mathrm{CDCl}_{3}, 100 \mathrm{MHz}\right)$<smiles>O=[N+]([O-])c1cccc2c(Br)nccc12</smiles> 


\section{${ }^{1} \mathrm{H} \mathrm{NMR},\left(\mathrm{CDCl}_{3}, 400 \mathrm{MHz}\right)$}

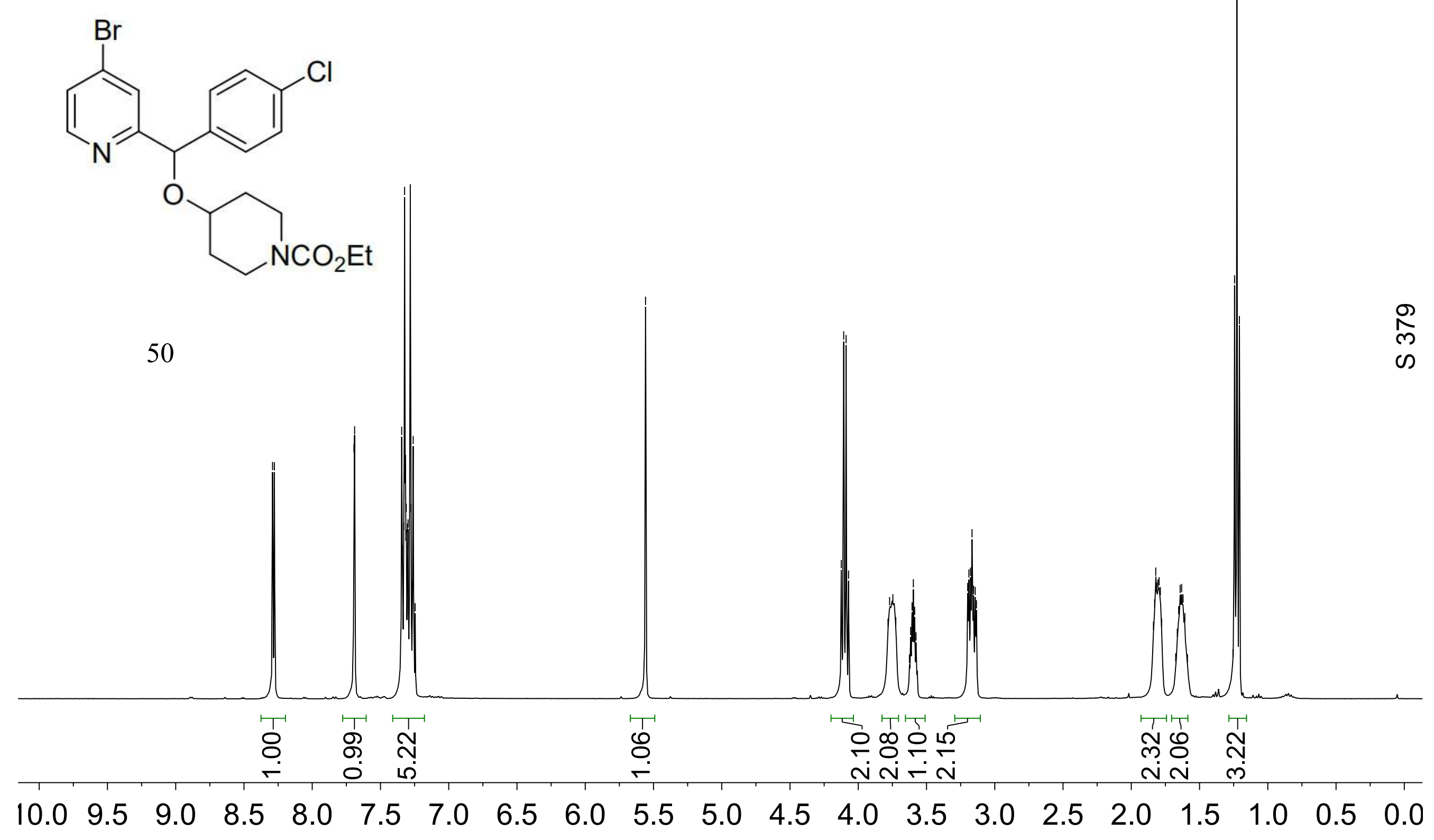




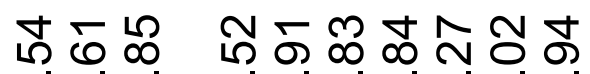

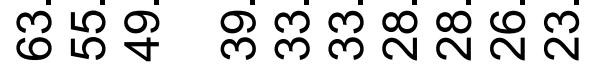

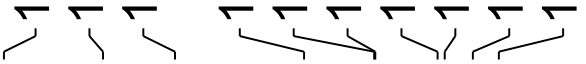

${ }^{13} \mathrm{C} \mathrm{NMR},\left(\mathrm{CDCl}_{3}, 100 \mathrm{MHz}\right)$<smiles>CCON1CCC(OC(c2ccc(Cl)cc2)c2cc(Br)ccn2)CC1</smiles> 
-

6 in

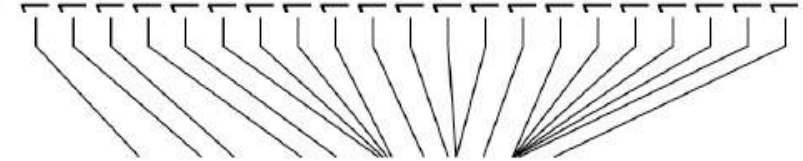

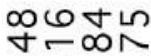

inion

โே

NN

No

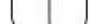

${ }^{13} \mathrm{C} \mathrm{NMR}\left(\mathrm{CDCl}_{3}, 100 \mathrm{MHz}\right)$<smiles>Cc1cnc(C(=O)OC(c2cc(C(F)(F)F)cc(C(F)(F)F)c2)[N+](=O)[O-])cc1Br</smiles>

53 
${ }^{19} \mathrm{~F} \mathrm{NMR}\left(\mathrm{CDCl}_{3}, 365 \mathrm{MHz}\right)$

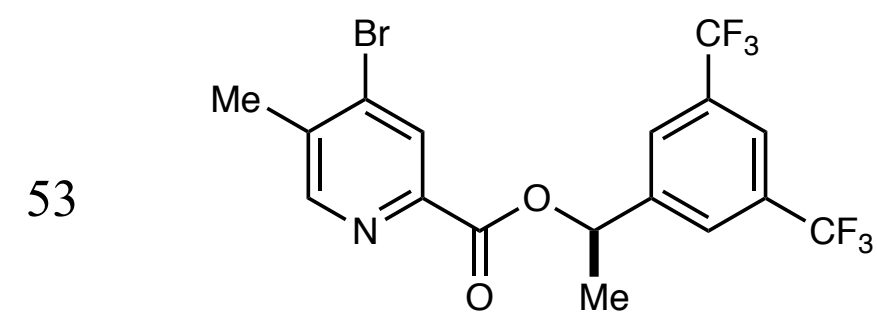

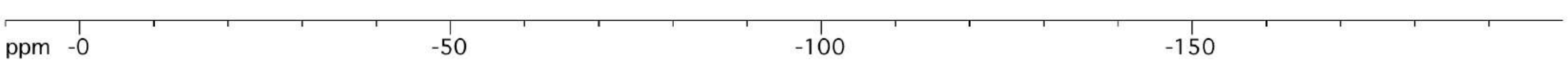


${ }^{1} \mathrm{H} \mathrm{NMR}\left(\mathrm{CDCl}_{3}, 400 \mathrm{MHz}\right)$<smiles>Fc1cc(-c2ccc(C(F)(F)F)cn2)cc(-c2cnccc2Br)c1</smiles>

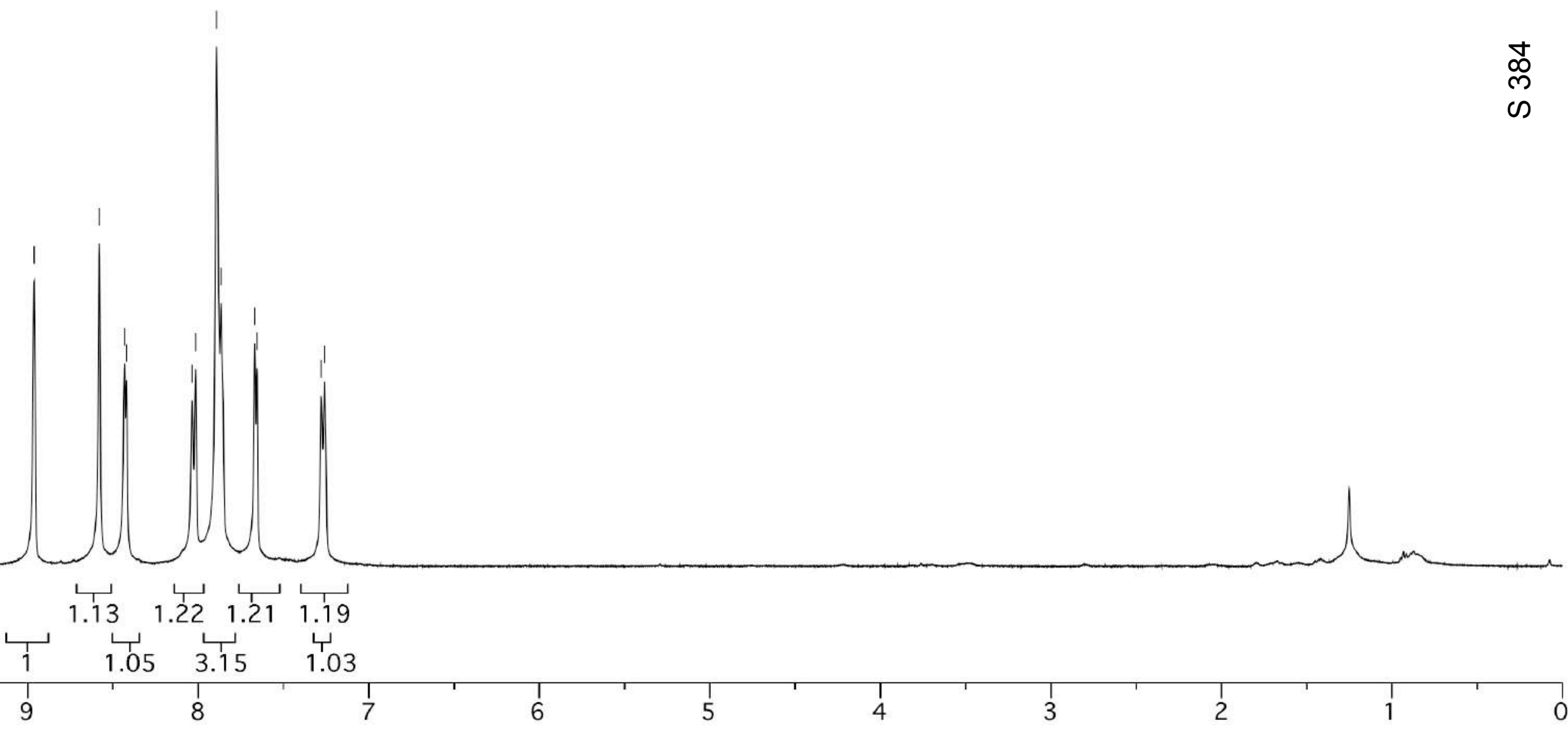




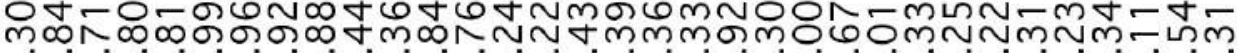
オ-

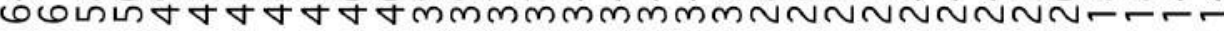

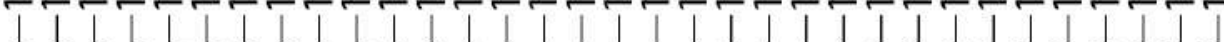

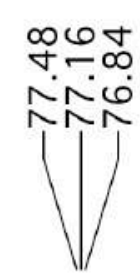

${ }^{13} \mathrm{C} \mathrm{NMR}\left(\mathrm{CDCl}_{3}, 100 \mathrm{MHz}\right)$<smiles>Fc1cc(-c2ccc(C(F)(F)F)cn2)ccc1-c1cnccc1Br</smiles> 
56

${ }^{19} \mathrm{~F} \mathrm{NMR}\left(\mathrm{CDCl}_{3}, 365 \mathrm{MHz}\right)$

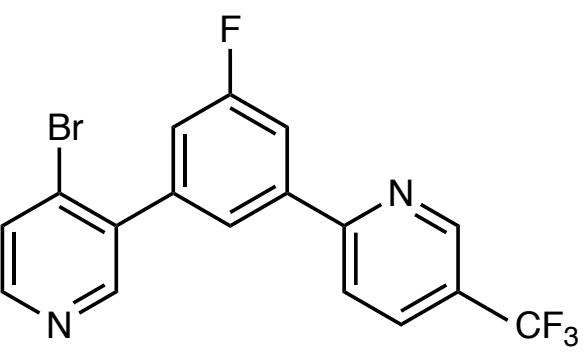

\begin{tabular}{|c|c|c|}
\hline $\begin{array}{ll} & 1 \\
\mathrm{ppm} & -0\end{array}$ & -50 & -100 \\
\hline
\end{tabular}




\section{${ }^{1} \mathrm{H} \mathrm{NMR},\left(\mathrm{CDCl}_{3}, 400 \mathrm{MHz}\right)$}<smiles>Cc1cc(Br)c(-c2ncc(Cl)cc2-c2ccc(S(C)(=O)=O)cc2)cn1</smiles>

60

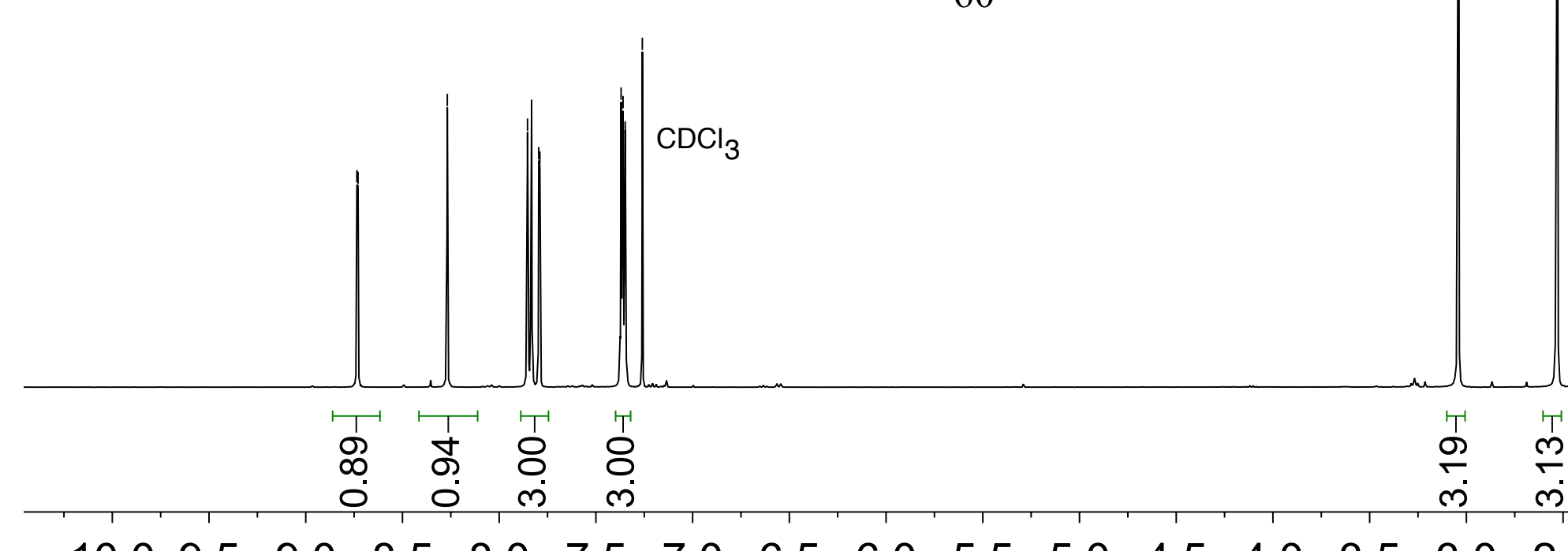


${ }^{13} \mathrm{C} \mathrm{NMR},\left(\mathrm{CDCl}_{3}, 100 \mathrm{MHz}\right)$<smiles>Cc1cc(Br)c(-c2ncc(Cl)cc2-c2ccc(S(C)(=O)=O)cc2)cn1</smiles>

60 


\section{${ }^{1} \mathrm{H} \mathrm{NMR},\left(\mathrm{CDCl}_{3}, 400 \mathrm{MHz}\right)$}<smiles></smiles>

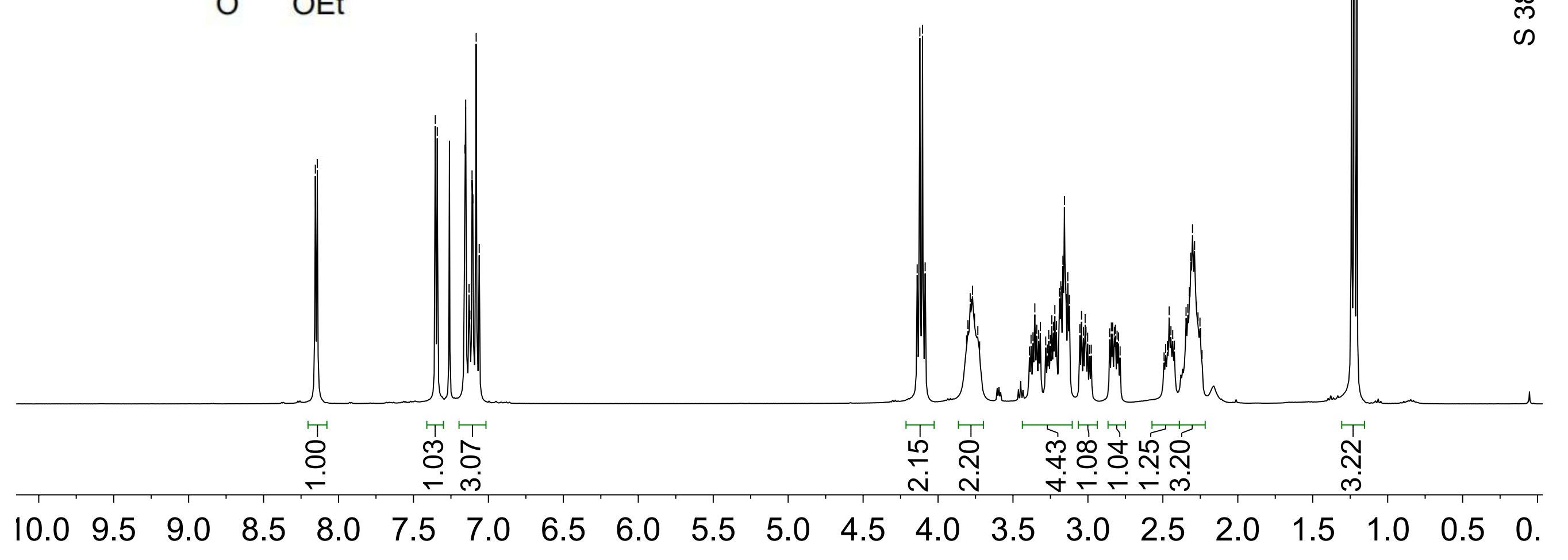




\section{${ }^{13} \mathrm{C} \mathrm{NMR,}\left(\mathrm{CDCl}_{3}, 100 \mathrm{MHz}\right)$}<smiles>CCOC(=O)N1CCC(=C2c3ccc(Cl)cc3CCc3c(Br)ccnc32)CC1</smiles>

$\begin{array}{llllllllllllllllllllll}.10 & 200 & 190 & 180 & 170 & 160 & 150 & 140 & 130 & 120 & 110 & 100 & 90 & 80 & 70 & 60 & 50 & 40 & 30 & 20 & 10 & 0\end{array}$ 


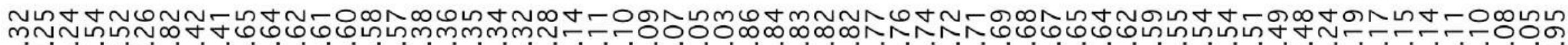

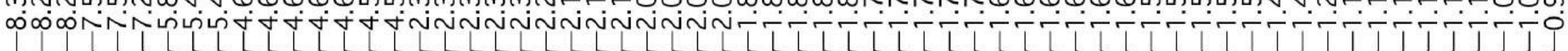

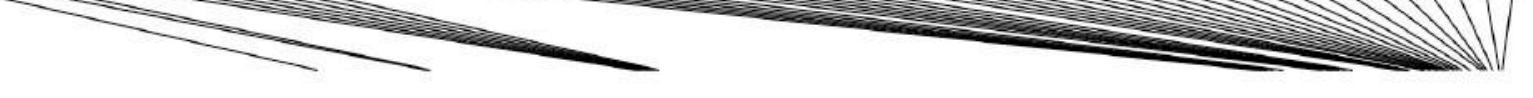

${ }^{1} \mathrm{H} \mathrm{NMR}\left(\mathrm{CDCl}_{3}, 400 \mathrm{MHz}\right)$
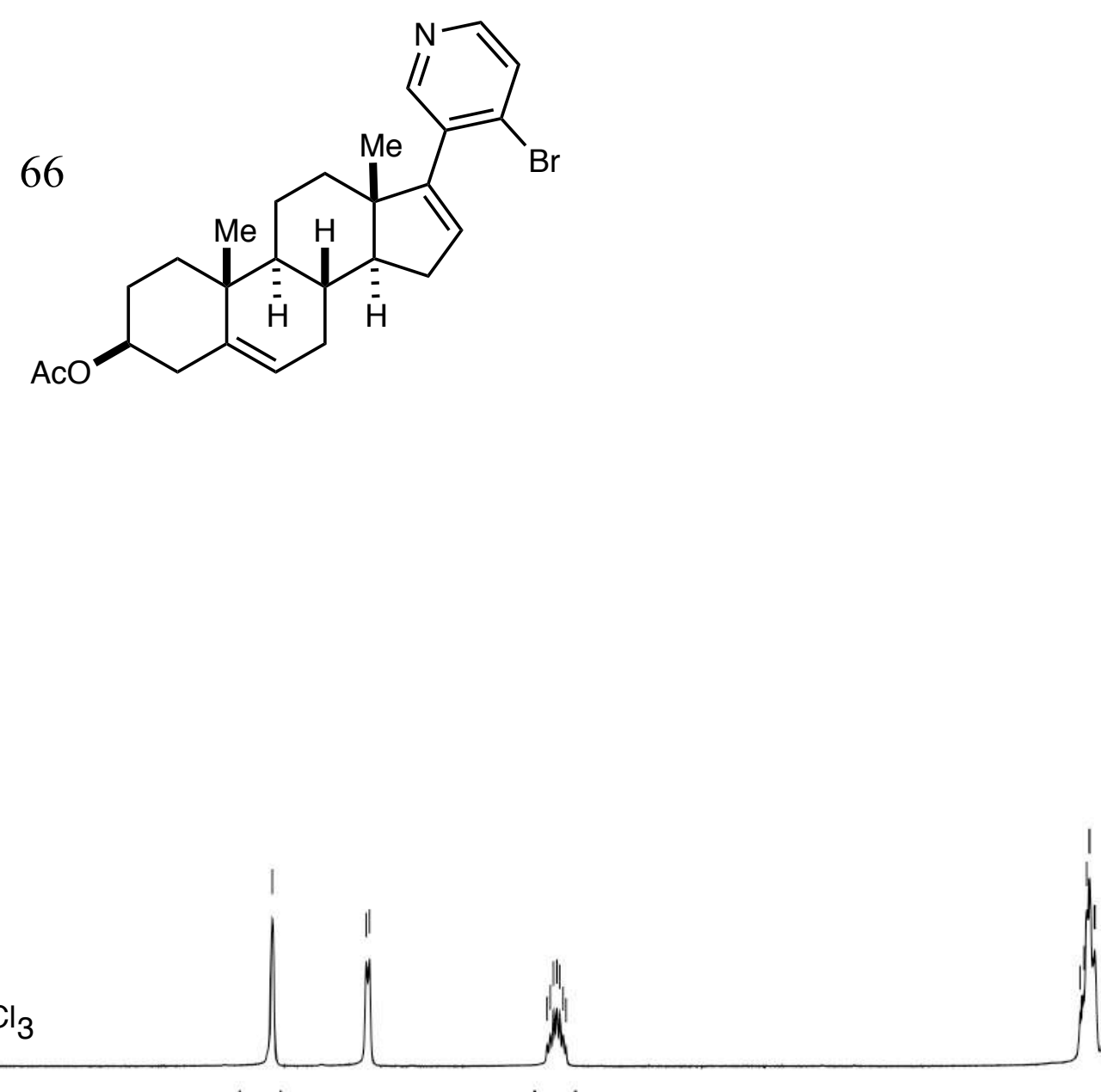

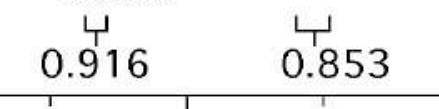

0.929

$$
0.913
$$

2.91

$2.15 \quad 8.66$



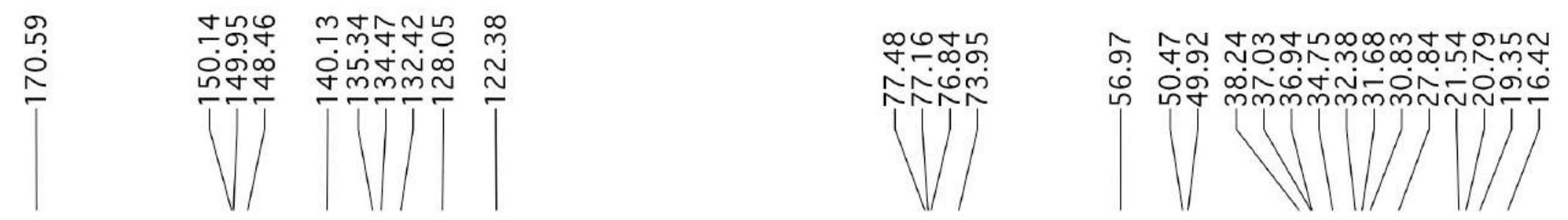

${ }^{13} \mathrm{C} \mathrm{NMR}\left(\mathrm{CDCl}_{3}, 100 \mathrm{MHz}\right)$

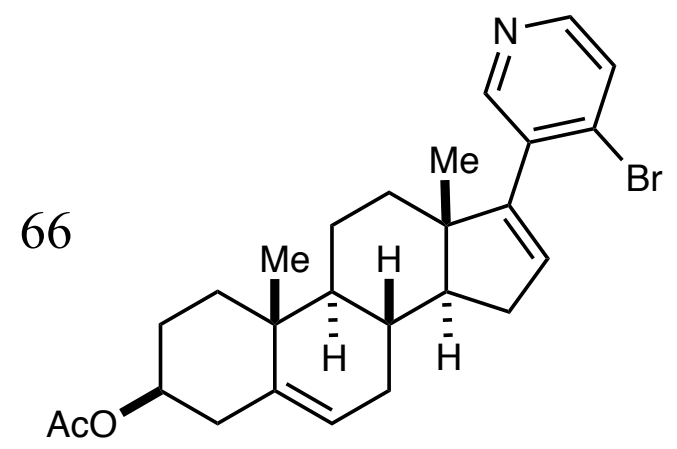




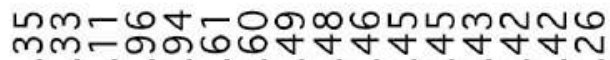
$\infty \infty \infty N-N$

${ }^{1} \mathrm{H}$ NMR $\left(\mathrm{CDCl}_{3}, 400 \mathrm{MHz}\right)$

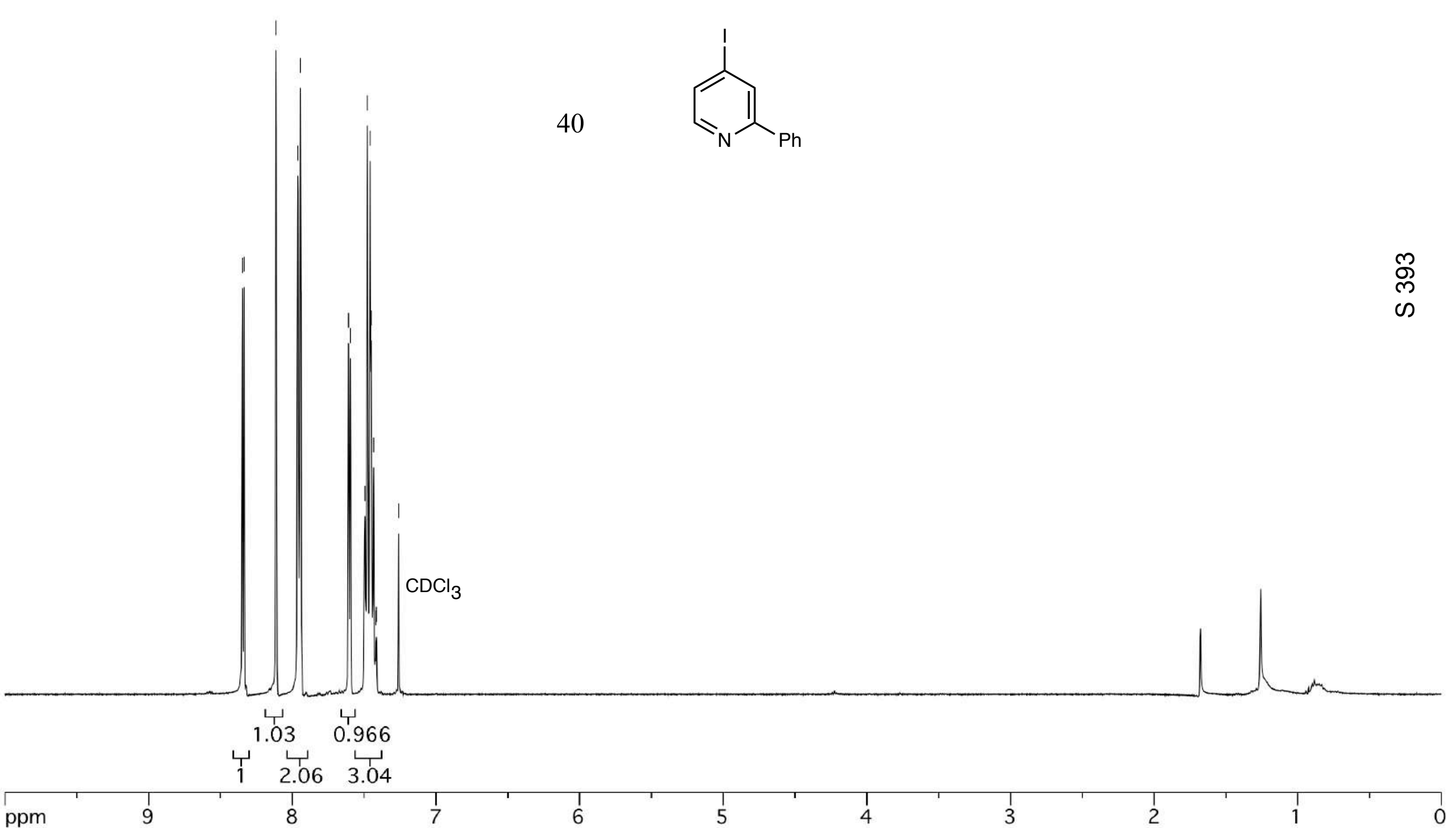




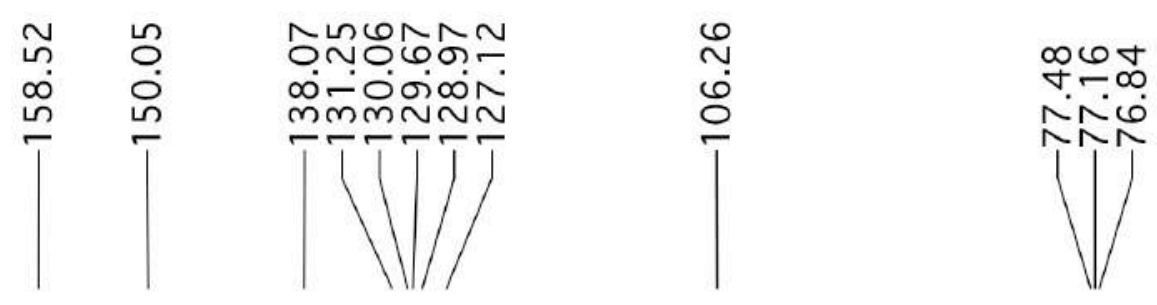

${ }^{13} \mathrm{C} \mathrm{NMR}\left(\mathrm{CDCl}_{3}, 100 \mathrm{MHz}\right)$

40<smiles>Ic1ccnc(-c2ccccc2)c1</smiles> 
ব아에

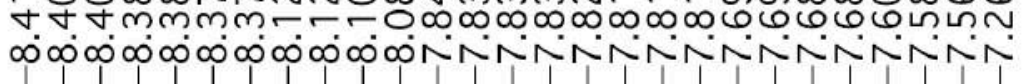

${ }^{1} \mathrm{H} \mathrm{NMR}\left(\mathrm{CDCl}_{3}, 400 \mathrm{MHz}\right)$

43

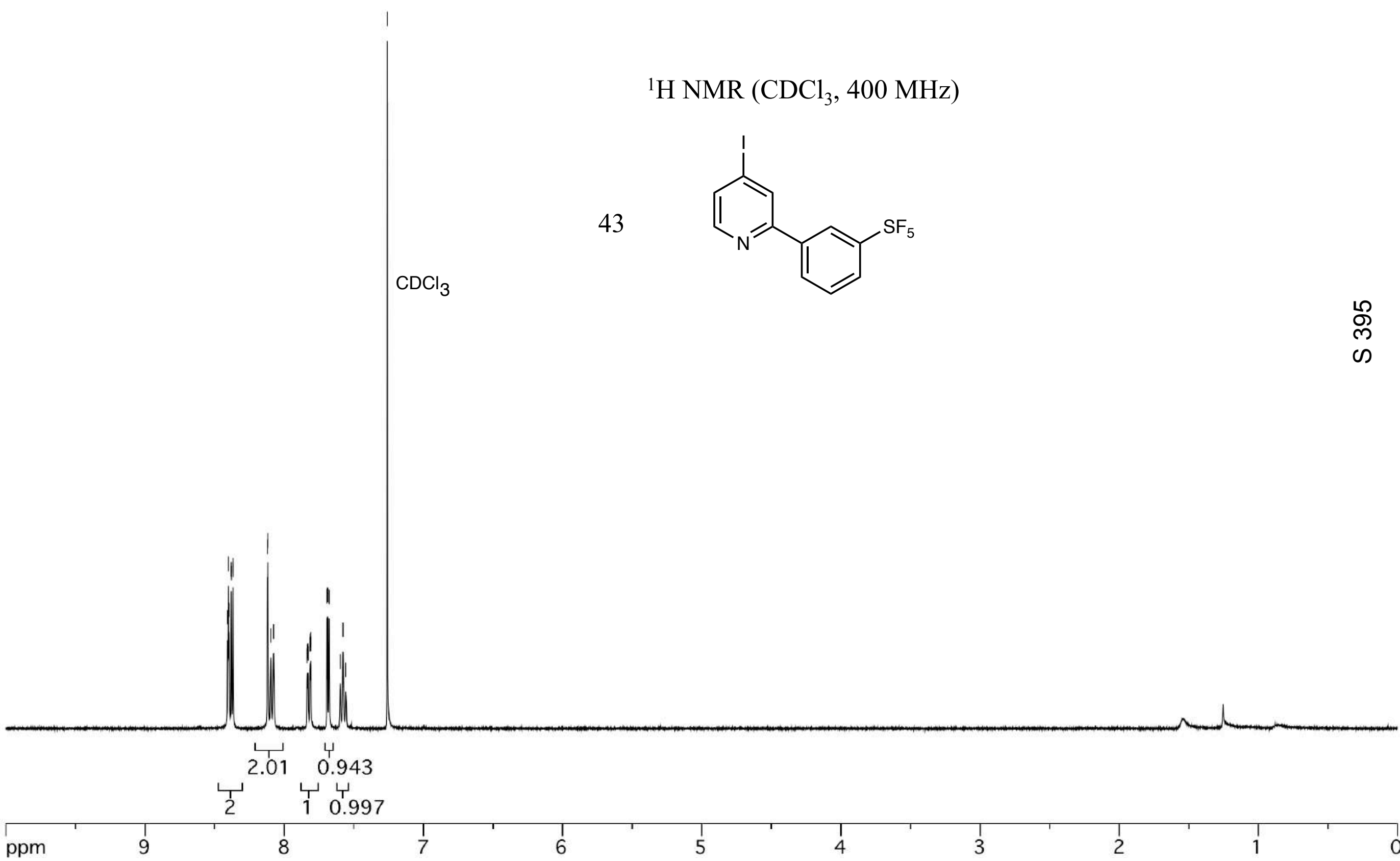

คి 


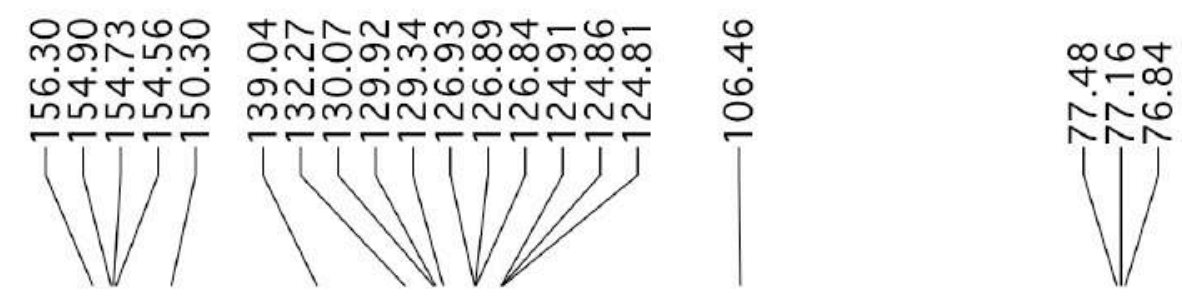

${ }^{13} \mathrm{C} \mathrm{NMR}\left(\mathrm{CDCl}_{3}, 100 \mathrm{MHz}\right)$

43<smiles>FC(F)(S)c1cccc(-c2cc(I)ccn2)c1</smiles> 
${ }^{19} \mathrm{~F} \mathrm{NMR}\left(\mathrm{CDCl}_{3}, 365 \mathrm{MHz}\right)$<smiles>FC(F)(F)c1cccc(-c2cc(I)ccn2)c1</smiles>

43 
${ }^{1} \mathrm{H} \mathrm{NMR}\left(\mathrm{CDCl}_{3}, 400 \mathrm{MHz}\right)$<smiles>CCCCc1cc(I)c(C(F)(F)F)cn1</smiles>

46

$\mathrm{CDCl}_{3}$

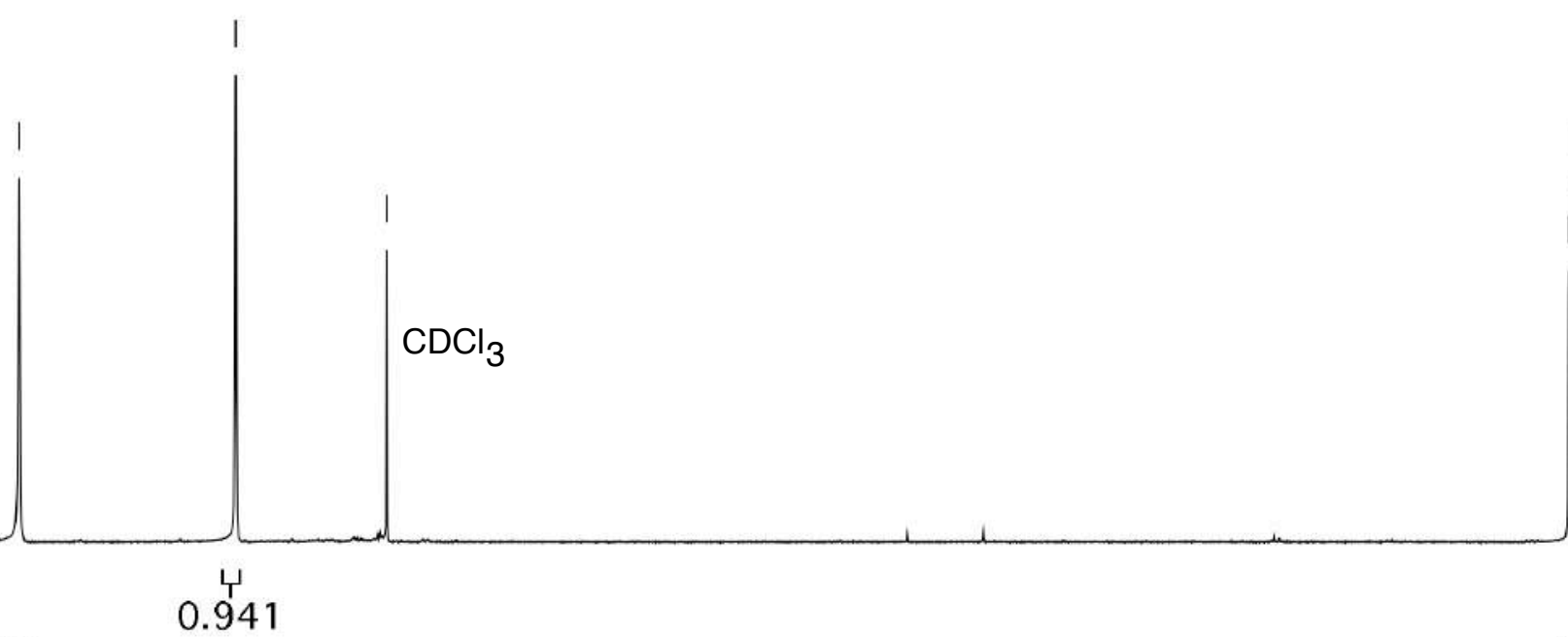

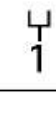

2.09 2.14 3.08

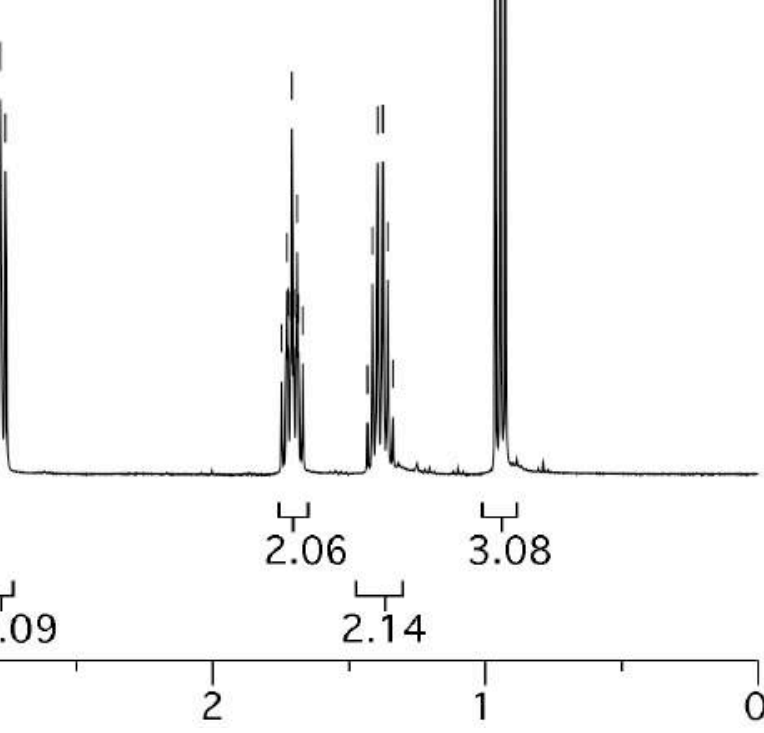




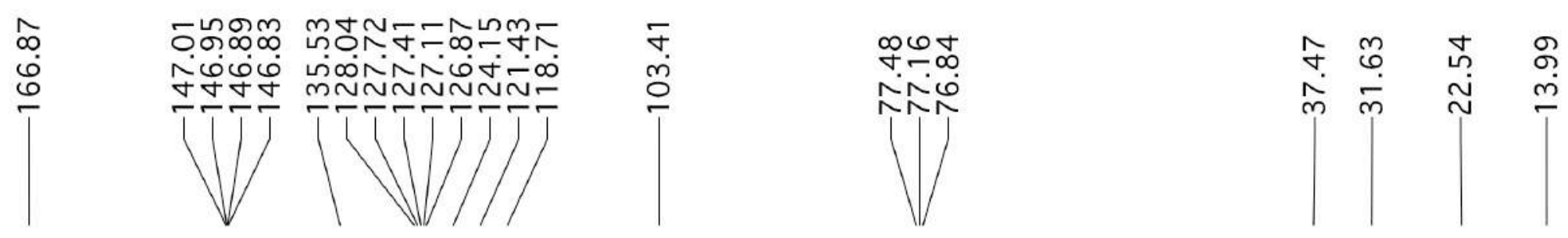

${ }^{13} \mathrm{C} \mathrm{NMR}\left(\mathrm{CDCl}_{3}, 100 \mathrm{MHz}\right)$<smiles>CCCCc1cc(I)c(C(F)(F)F)cn1</smiles>

46

$\mathrm{CDCl}_{3}$ 


$\begin{array}{lll}0 & 0 \\ 0 & 0 & 0 \\ 0 & 1 & 0\end{array}$

\section{${ }^{1} \mathrm{H} \mathrm{NMR},\left(\mathrm{CDCl}_{3}, 400 \mathrm{MHz}\right)$}

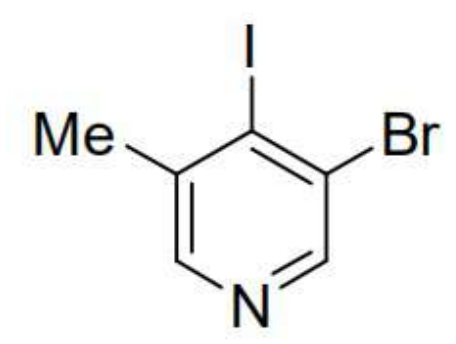

48

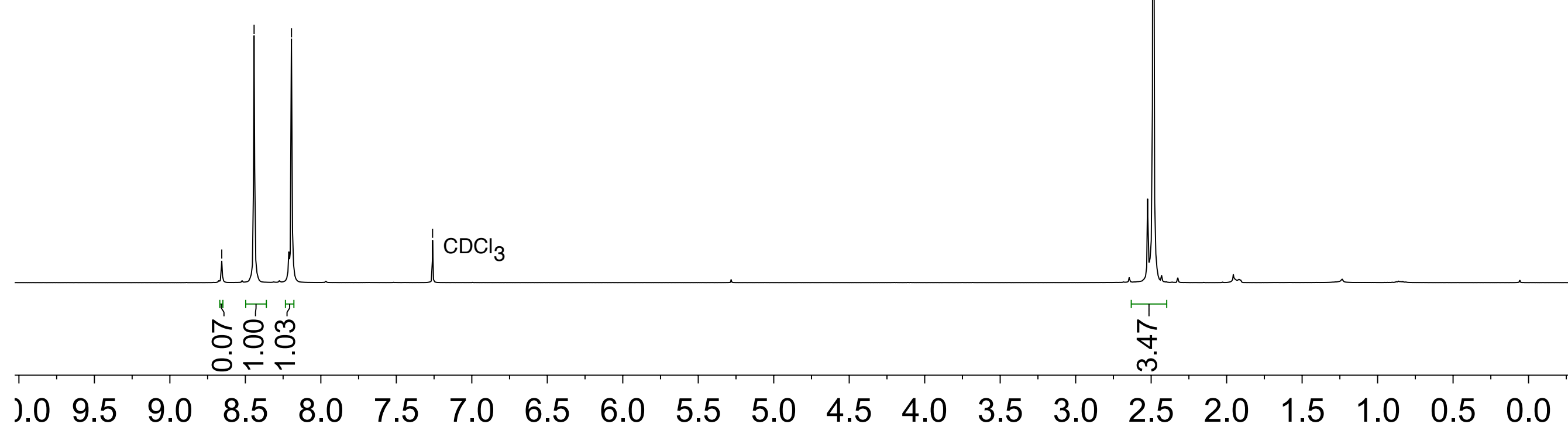




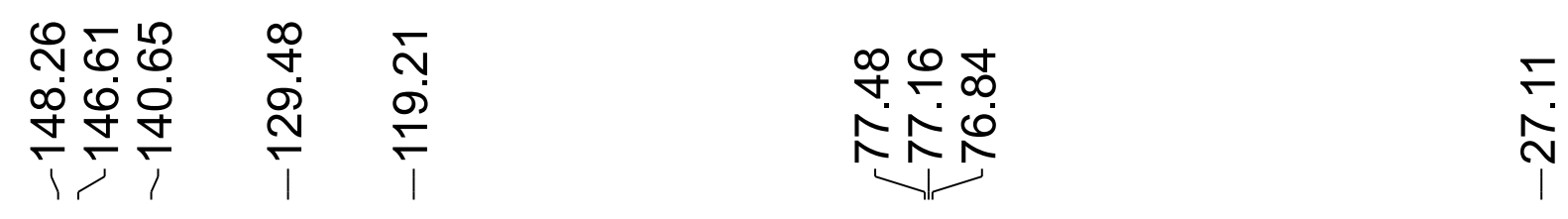

${ }^{13} \mathrm{C} \mathrm{NMR},\left(\mathrm{CDCl}_{3}, 100 \mathrm{MHz}\right)$<smiles>Cc1cncc(Br)c1I</smiles>

48 


\section{${ }^{1} \mathrm{H} \mathrm{NMR},\left(\mathrm{CDCl}_{3}, 400 \mathrm{MHz}\right)$}<smiles></smiles>

63

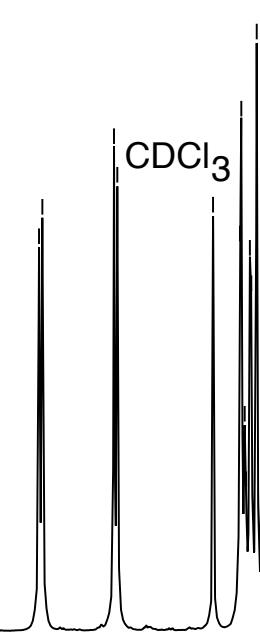

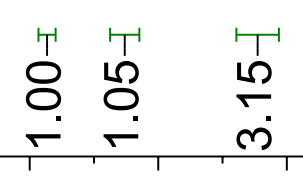

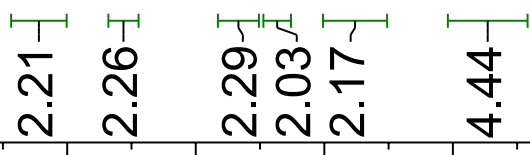




\section{${ }^{13} \mathrm{C} \mathrm{NMR,}\left(\mathrm{CDCl}_{3}, 100 \mathrm{MHz}\right)$}

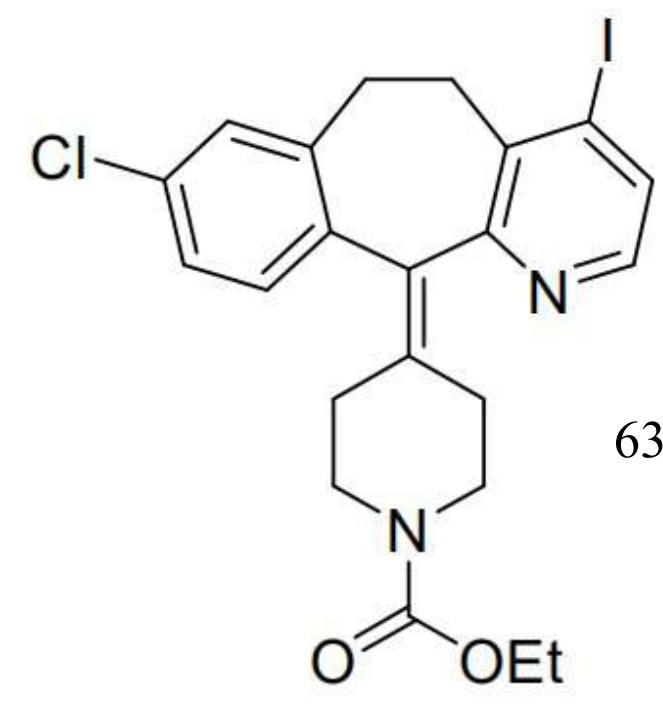

63
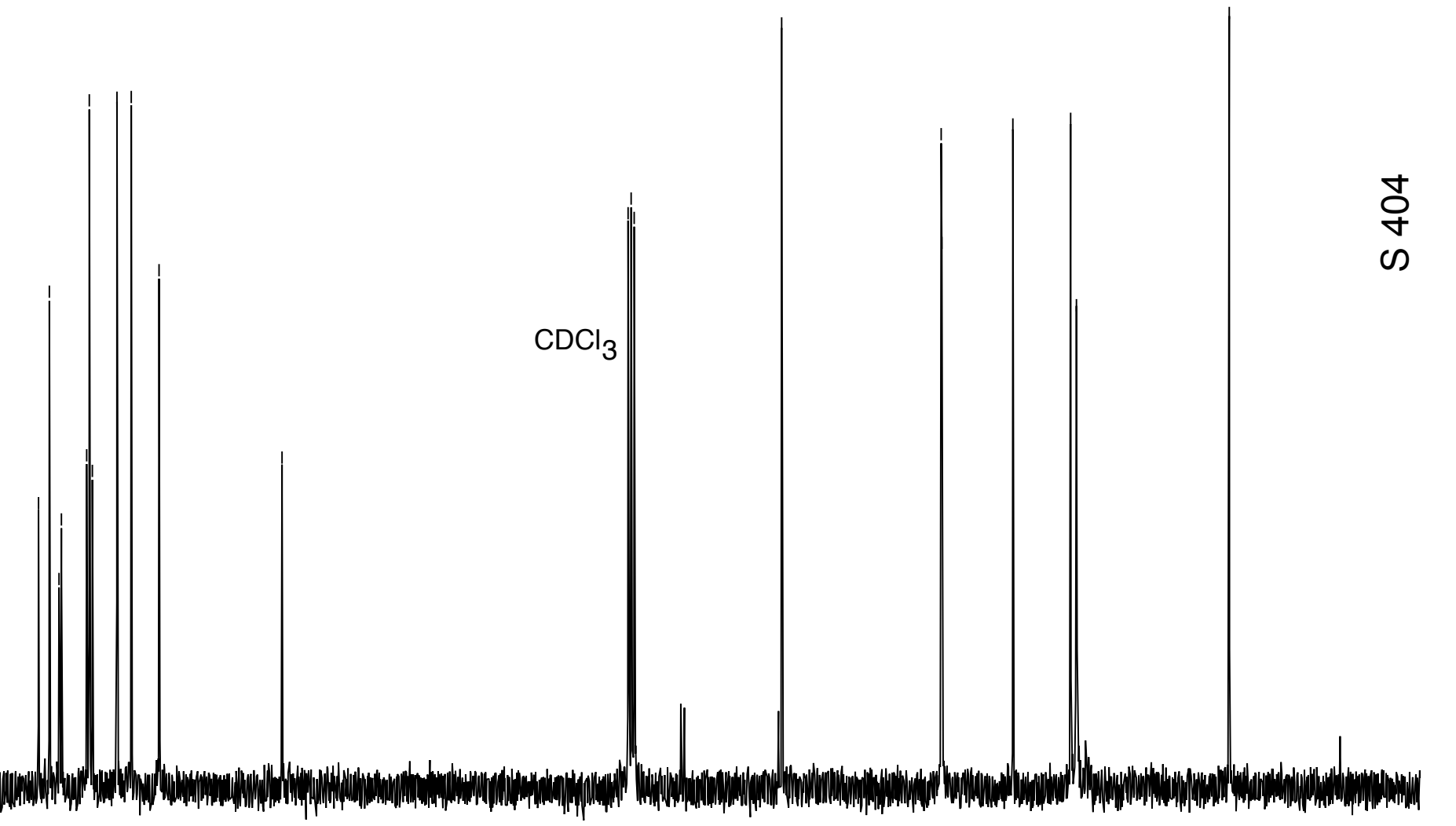

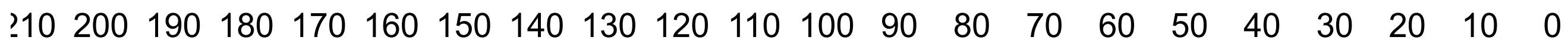

